\title{
Catalytic Asymmetric Carbon-Carbon Bond Formation Using Alkenes as Alkylmetal Equivalents
}

Rebecca M. Maksymowicz

A dissertation presented in partial fulfilment of the requirements for the award of the degree of

Doctor of Philosophy

of the

University of Oxford

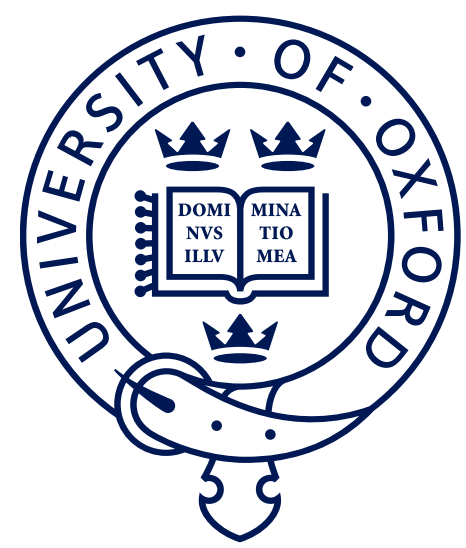

Lincoln College

February 2014 


\section{Declaration}

This thesis describes work carried out in the Chemistry Research Laboratory, Oxford, between January 2010 and January 2014 under the supervision of Dr Stephen Fletcher. The thesis is a result of my own work, except where otherwise stated, and has not been submitted for any other degree at this or any other university.

\section{Rebecca M. Maksymowicz}




\section{Abstract: Catalytic Asymmetric Carbon-Carbon Bond Formation Using Alkenes as Alkylmetal Equivalents}

The development of new methods for carbon-carbon bond formation is a challenging topic at the heart of organic chemistry. Over the past ten years a number of methods for the catalytic asymmetric 1,4-addition of organometallic reagents such as Grignard, organozinc and organoaluminium reagents have been reported. However these reagents suffer from many limitations, including the need for cryogenic temperatures, which prevent their widespread use. Here we have developed a new asymmetric method: the copper-catalysed enantioselective 1,4addition of alkylzirconium compounds, generated in situ, from alkenes.

A general introduction into the formation of carbon-carbon bonds and catalytic asymmetric 1,4addition reactions is first given. We then focus our attention on hydrometallation reactions and their current use in the addition of alkenyl and alkyl groups in asymmetric 1,4-addition reactions. In Chapter two, we introduce the development of our methodology. We found that by using copper complex $(\boldsymbol{S}, \boldsymbol{S}, \boldsymbol{S})$-A, high enantioselectivities can be achieved (up to $96 \% e e$ ), in the presence of a broad range of functional groups which are often not compatible with comparable methods using pre-made organometallic reagents. The method gives good enantioselectivity at room temperature, in a wide range of solvents, using readily available alkenes (Scheme i).

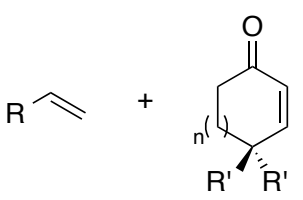

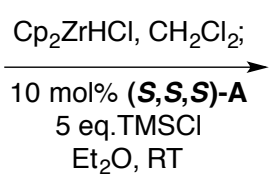

$\mathrm{Et}_{2} \mathrm{O}, \mathrm{RT}$

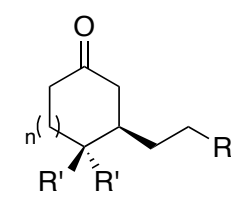

25 examples $19-74 \%$ yield $71-96 \%$ ee

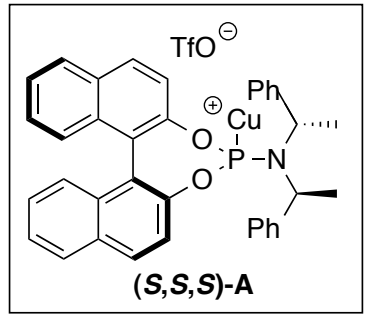

Scheme i: Chapter one results

Chapter three discusses the expansion of our method to the 1,4- and 1,6-addition to complex steroids. Modified conditions were then found to enable the addition to $\beta$-substituted enones, to form quaternary centres. This is followed by the successful addition to $\alpha, \beta$-unsaturated lactones, another difficult substrate class. All these results gave excellent selectivity.

In summary, we have developed a new reaction which offers an alternative to current methods reported in the literature. This robust reaction can tolerate a variety of functional groups and we hope that this will aid in the synthesis of important molecules. 


\section{Acknowledgements}

First and foremost I would like to thank my supervisor Dr Stephen Fletcher for his constant support and guidance throughout my DPhil. I feel very privileged to have been given the opportunity to help start up Steve's research group with him and have learnt so much since I started 4 years ago. I have enjoyed seeing the group develop and look forward to hearing about all the great work that will inevitably come from the group in the coming years. It has been an exciting and ever changing atmosphere to work in and I will miss it very much.

I would like to extend my gratitude to Dr Martin Smith and his group. They welcomed me into their group meetings and provided me with an intellectual and social environment when I first started at Oxford.

Since I have started, the Fletcher group has grown significantly. From the initial days on my own, to Phil, Tina, Sami and Dave joining me to make the "first generation" Fletcher group. We had a lot of laughs (and fondues...!). Since then the group grew to Andrew, Nancy and Ed, followed by the huge expansion when we gained postdocs Elle, Mireia and Manu, new DPhil students, Emeline, Hengzhi, Phil and Thomas, as well as part II students Simon and Nessa (and not forgetting all the visiting post docs and students), all who have made the group a crazy fun atmosphere to work (and party!) in. I would like to thank them all for making my time in the group so enjoyable. I would like to extend my thanks to Phil Roth, who has worked with me on the project and has also continued to provide me with chocolate and cheese to help me make it to the end of my DPhil. To Dr Elle Maciver (now Milosevic!) for being my coffee and wine (and occasionally running...) buddy and becoming a great friend in the process. Thanks to the Ed Anderson group for fun times, when sharing the lab \& in Vienna, a lot of friendships have been formed and I hope they continue!

I would like to thank all the CRL staff. Especially stores and workshop, who have put up with my endless questions when setting up the lab at the beginning. Thank you to all the NMR and Mass spectrometry staff for their assistance during my DPhil. Extended thanks goes to Dr Barbara Odell, who has helped with many NMR studies and to Dr Amber Thompson for X-ray crystallography data.

A massive thanks has to go to all the amazing friends I have made during my time in Oxford. Having to endure my DPhil without girly dinners, outrageous outbursts, hilarious stories, ring of fire, Maxwell antics and a whole lot more, would have been hard work! You have all been there for me during tough times and happy times and I couldn't have asked for better friends. Laura, 
Charley, Cat, Kylie, Smita and Liley I can't thank you enough... you have all become such great friends and I know the fun times will continue wherever we all are in the world!

Lots of love goes to my fellow Angels, Katie and Liley, you are my best friends and are always there to put me back on track. We have come a long way since our days in Bristol, a lot of laughs, tea, cheese, boys, chaos and fun has been had and I look forward to the same and more in the future. Thank you for everything!

Thank you to all my hockey friends at Oxford Hockey Club, you have helped me stay sane (and fit!) throughout my DPhil and have helped me escape the "oxford bubble". Thank you to all my friends outside of Oxford, I am so lucky to have such an extended set of friends all over the world. Even though I can be terrible with contact, you are always in my thoughts and thank you for always being there if I need you.

Additional gratitude has to go to everyone who has proof read my thesis, Katie, Laura, Mireia, Emeline and Andrew, I am so grateful for your input and to Smita who has proof read, listened to me rant, helped me focus and just been an amazing friend during this hellish time, thank you so much and bring on Belgium fun!

Special thanks and love goes to the person who has had to endure all the highs and lows of my DPhil. Rich, you have put up with so much the past 3 years and I am so grateful for everything you have done for me. You have always believed in me and I cannot thank you enough.

Finally I would like to extend my utmost love and gratitude to my family. Jess, you're a great sister, even if we don't always agree on everything (!), your always there for a chat and Mum and Dad, I wouldn't be here without your endless love and support. You have always been there for me, while growing up and throughout the whole of my studies. You have encouraged me in everything I do and I hope I can continue to make you proud. 


\section{Abbreviations}

\begin{tabular}{|c|c|}
\hline$\AA$ & Ångstroms \\
\hline ACA & asymmetric conjugate addition \\
\hline Ac & acetyl \\
\hline acac & acetylacetonate \\
\hline aq. & aqueous \\
\hline Ar & generic aromatic system \\
\hline$(9-B B N)_{2}$ & 9-borabicyclo[3.3.1]nonane dimer \\
\hline binap & 2,2'-bis(diphenylphosphino)-1,1'-binaphthyl \\
\hline BINOL & 1,1'-bi-2-naphthol \\
\hline Bn & benzyl \\
\hline Boc & tert-butoxycarbonyl \\
\hline $\mathrm{Bu}$ & butyl \\
\hline${ }^{\circ} \mathrm{C}$ & degrees celsius \\
\hline $\boldsymbol{c}$ & concentration \\
\hline cat. & catalytic/catalyst \\
\hline calcd & calculated \\
\hline $\operatorname{cod}$ & cycloctadiene \\
\hline conc. & concentration \\
\hline conv. & conversion \\
\hline Cp & cyclopentadienyl \\
\hline$C p^{*}$ & pentamethylcyclopentadienyl \\
\hline Cy & cyclohexyl \\
\hline d & days \\
\hline de & diastereoselectivity \\
\hline DCE & dichloroethane \\
\hline DIBAL-H & diisobutyl aluminium hydride \\
\hline DMAP & 4-dimethylaminopyridine \\
\hline DME & dimethoxyethane \\
\hline DMF & dimethylformamide \\
\hline DMSO & dimethylsulfoxide \\
\hline DPEN & 1,2-bis(2-hydroxyphenyl)ethylenediamine \\
\hline$d r$ & diastereomeric ratio \\
\hline DTBM & 3,5-di-tert-butyl-4-methoxyphenyl \\
\hline EDG & electron-donating group \\
\hline$e e$ & enantiomeric excess \\
\hline El & electron-impact ionization \\
\hline eq. & equivalents \\
\hline ESI & electrospray ionization \\
\hline Et & ethyl \\
\hline
\end{tabular}




\begin{tabular}{|c|c|}
\hline EWG & electron-withdrawing group \\
\hline g & grams \\
\hline h & hours \\
\hline HOMO & highest occupied molecular orbital \\
\hline HPLC & high-performance liquid chromatography \\
\hline HRMS & high-resolution mass spectrometry \\
\hline HSQC & heteronuclear single-quantum correlation spectroscopy \\
\hline $\mathrm{Hz}$ & hertz \\
\hline$i$ & iso \\
\hline IPA & iso-propanol \\
\hline IR & infra-red \\
\hline IUPAC & International Union of Pure and Applied Chemistry \\
\hline Josiphos & (Dicyclohexylphosphino)ethyl]-2-(diphenylphosphino)ferrocene \\
\hline K & kilo- \\
\hline $\mathbf{L}$ & litres \\
\hline$L^{*}$ & Ligand \\
\hline LA & Lewis acid \\
\hline LDA & lithium diisopropylamide \\
\hline LUMO & lowest unoccupied molecular orbital \\
\hline $\boldsymbol{\mu}$ & micro- \\
\hline m & milli- \\
\hline M & moles per litre \\
\hline$m$ & meta- \\
\hline m & metres \\
\hline Me & methyl \\
\hline $\min$ & minutes \\
\hline mol & moles \\
\hline MP & melting point \\
\hline MS & mass spectrometry \\
\hline Ms & methanesulfonyl \\
\hline $\mathbf{n}$ & generic integer \\
\hline$n-$ & normal \\
\hline N/A & not applicable \\
\hline NHC & $N$-heterocyclic carbene \\
\hline ND & not determined \\
\hline NMR & nuclear magnetic resonance \\
\hline nOe & nuclear Overhauser effect \\
\hline NOESY & nuclear Overhauser effect spectroscopy \\
\hline NR & no reaction \\
\hline 0 & ortho- \\
\hline $0 / n$ & over night \\
\hline
\end{tabular}




\begin{tabular}{|c|c|}
\hline$p$ & para- \\
\hline Piv & pivaloyl \\
\hline $\mathbf{P h}$ & phenyl \\
\hline ppm & parts per million \\
\hline $\operatorname{Pr}$ & propyl \\
\hline$p$-TSA & para-toluenesulfonic acid \\
\hline quant. & quantitative \\
\hline $\mathbf{R}$ & generic substituent \\
\hline recryst. & recrystallisation \\
\hline RT & room temperature \\
\hline $\mathbf{S}$ & seconds \\
\hline segphos & 5,5'-Bis(diphenylphosphino)-4,4'-bi-1,3-benzodioxole \\
\hline SM & starting material \\
\hline$t$ & tertiary \\
\hline TC & thiophene 2-carboxylate \\
\hline tert & tertiary \\
\hline $\mathbf{T}$ & temperature \\
\hline $\mathbf{t}$ & time \\
\hline Tol & $p$-tolyl \\
\hline TBS & tert-butyldimethylsilyl \\
\hline temp. & temperature \\
\hline TMS & trimethylsilyl \\
\hline Tf & trifluoromethanesulfonyl \\
\hline THF & tetrahydrofuran \\
\hline TLC & thin-layer chromatography \\
\hline TMEDA & tetramethylethylenediamine \\
\hline TMS & trimethylsilyl \\
\hline triflate & trifluoromethanesulfonate \\
\hline Ts & toluenesulfonyl \\
\hline TSAF & tris(dimethylamino)sulfonium difluorotrimethylsilicate \\
\hline UHP & urea-hydrogen peroxide adduct \\
\hline VS. & versus \\
\hline
\end{tabular}




\section{Contents}

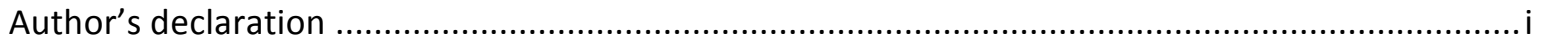

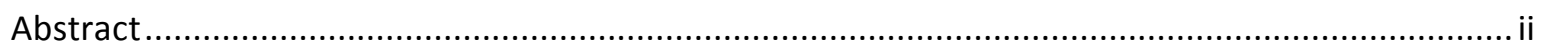

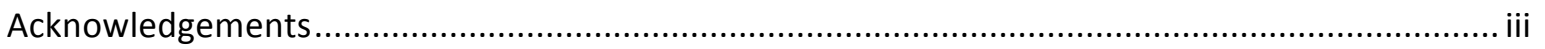

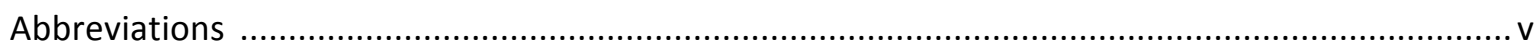

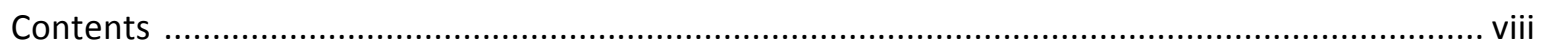

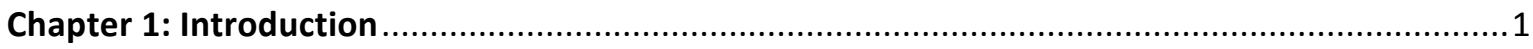

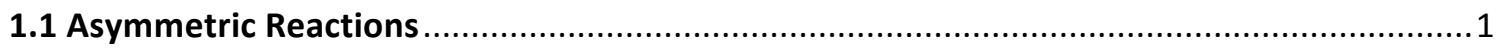

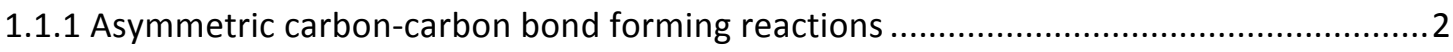

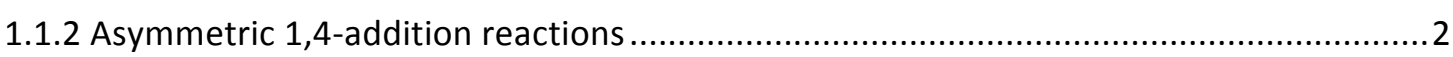

1.1.3 Copper-catalysed asymmetric 1,4-addition employing pre-made organometallic

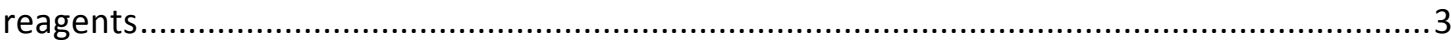

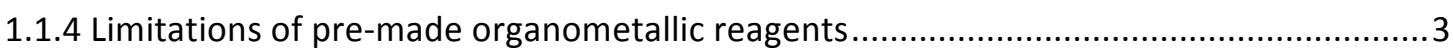

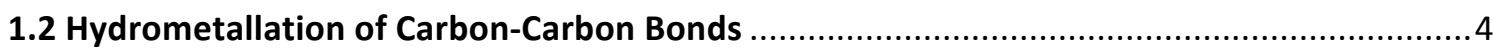

1.2.1 Tandem hydrometallation-asymmetric addition reactions starting from alkynes ............7

1.2.1.1 ACA initiated using nucleophiles generated by hydroboration ..................................

1.2.1.2 ACA initiated using nucleophiles generated by hydroalumination ...........................8

1.2.1.3 ACA initiated using nucleophiles generated by hydrosilylation ................................11

1.2.1.4 ACA initiated using zirconium nucleophiles ...........................................................14

1.2.2 Tandem hydrometallation-addition reactions starting from alkenes .............................17

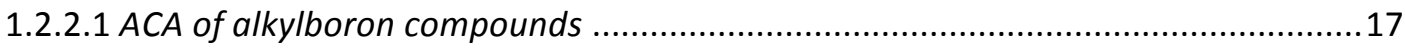

1.2.2.2 Addition of alkylzirconium compounds in 1,4-addition reactions ...........................19

Chapter 2: Development of a New Catalytic Asymmetric 1,4-Addition Reaction .........................22

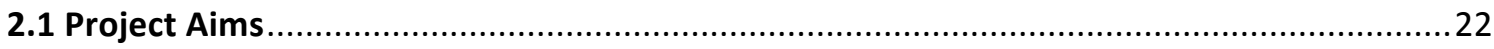

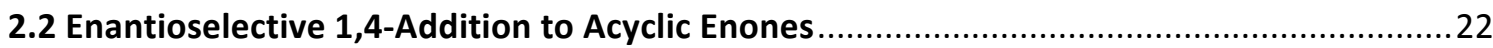

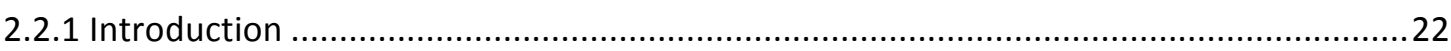

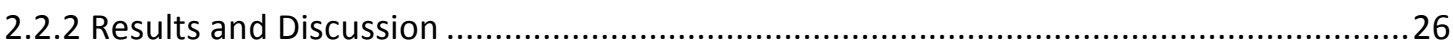

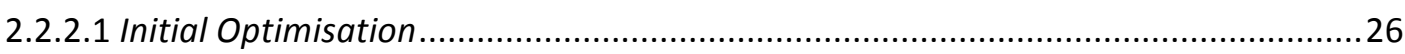

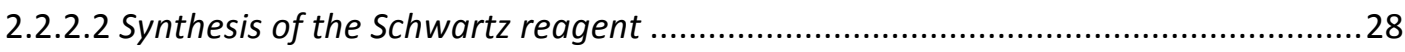

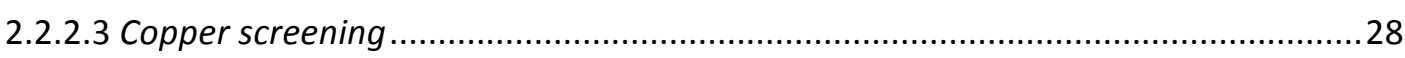

2.2.2.4 Synthesis of copper triflate benzene complex ..................................................... 30

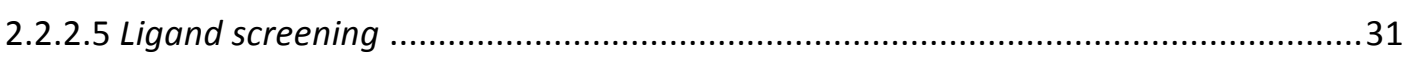

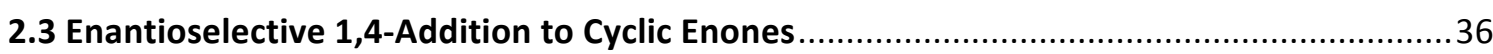

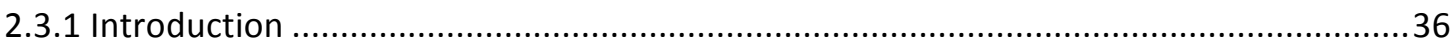

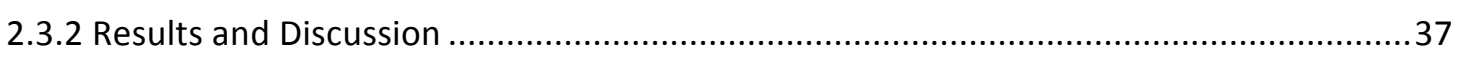

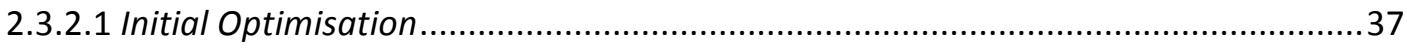

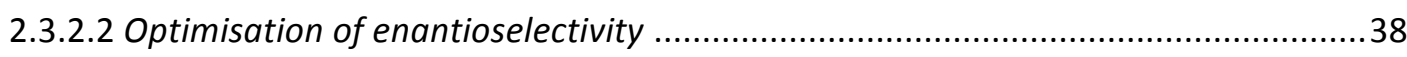

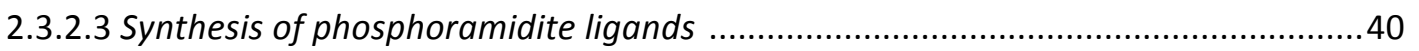

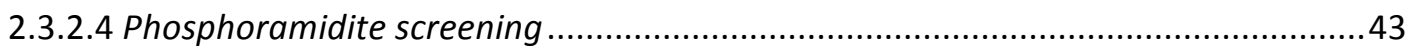

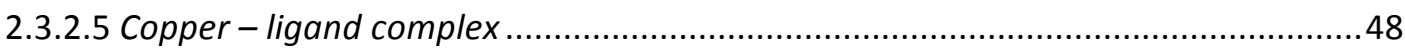

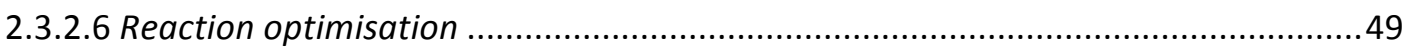




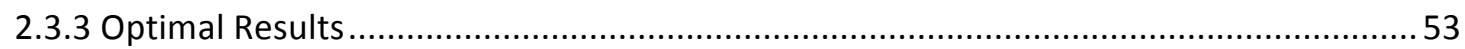

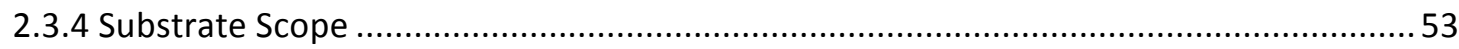

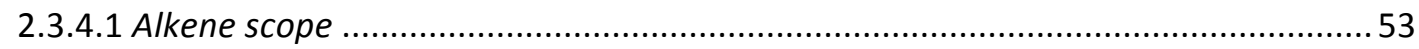

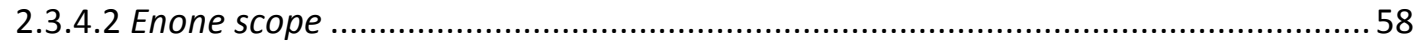

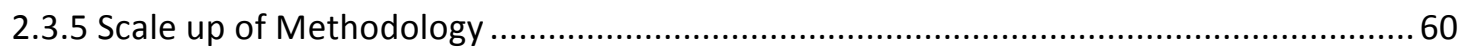

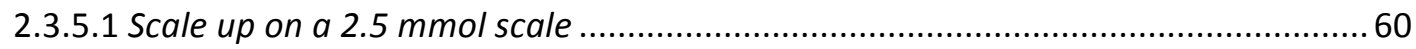

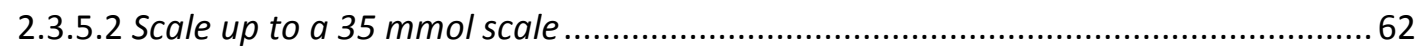

Chapter 3: Extending the Scope of Our 1,4-Addition Reaction........................................6 64

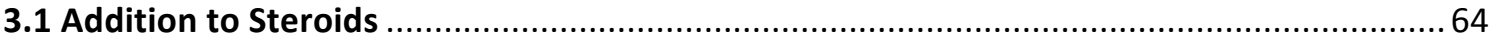

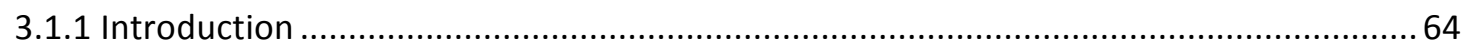

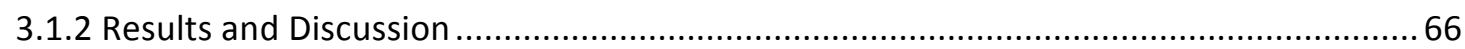

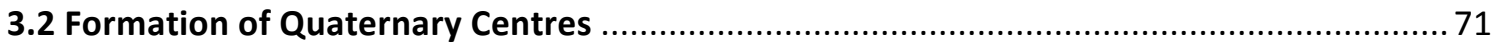

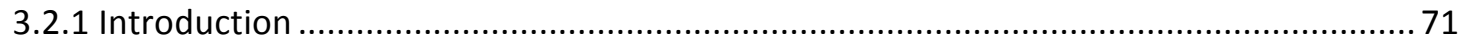

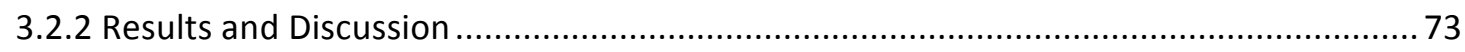

3.2.2.1 Phosphoramidite screening .................................................................... 73

3.2.2.2 Optimised conditions and substrate screen ................................................ 77

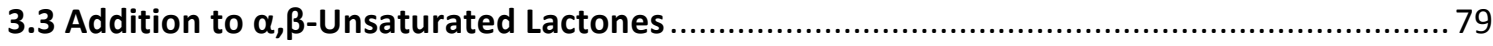

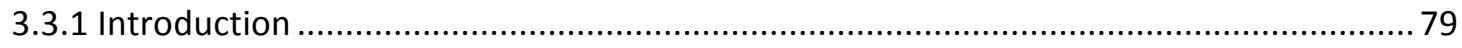

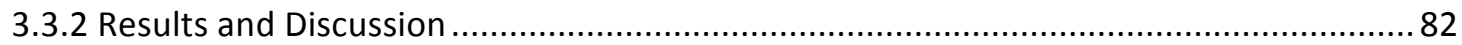

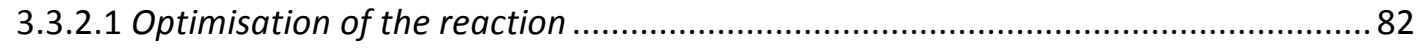

3.3.3 Substrate Scope ........................................................................................... 91

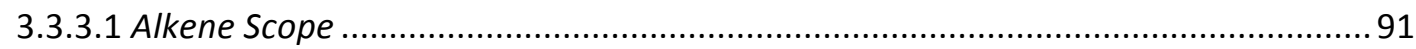

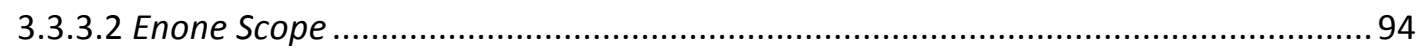

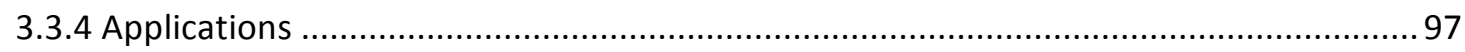

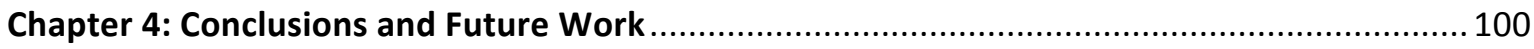

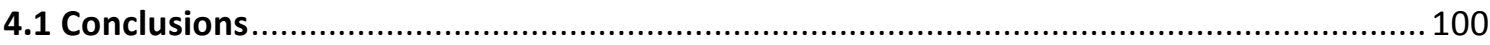

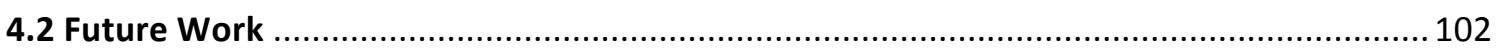

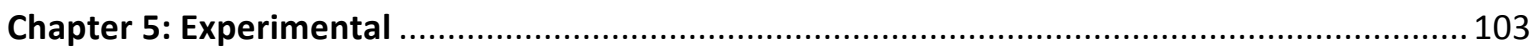

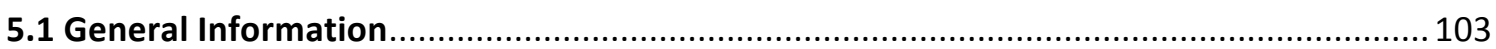

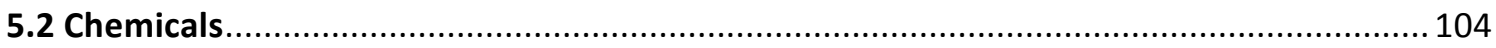

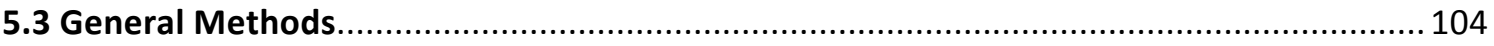

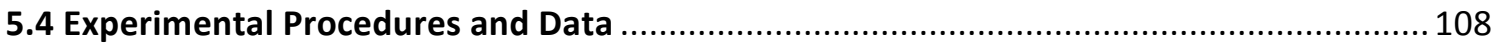

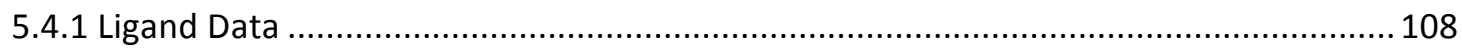

5.4.2 Copper - Ligand Complex Data .............................................................. 115

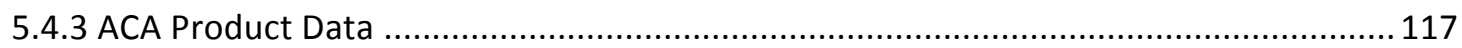

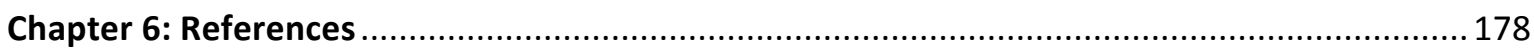

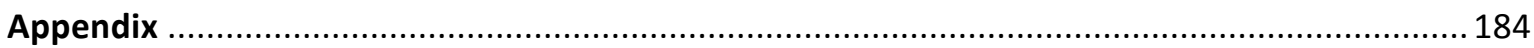




\section{Chapter 1: Introduction}

\subsection{Asymmetric Reactions}

Obtaining enantiomerically pure compounds is an important objective in organic synthesis. Natural products are often obtained as one enantiomer, and so it is desirable to develop methods that only give the natural enantiomer. Furthermore, many clinically significant medicines exhibit different biological activities for each enantiomer, underlying the importance of enantiomerically selective synthesis. ${ }^{1}$ Enantiomerically enriched organic materials have traditionally been synthesised by two principal methods: (i) synthesis with compounds that naturally occur as single enantiomers, often called the "chiral pool" or (ii) isolation of the desired isomer from a racemic mixture of enantiomers. These techniques typically involve the resolution of synthesised diastereomers, for example, by crystallisation. However, as half of the material is lost, the yields of such resolution processes cannot exceed $50 \%$ unless the undesired enantiomer can be recycled (Figure 1.1). ${ }^{2}$

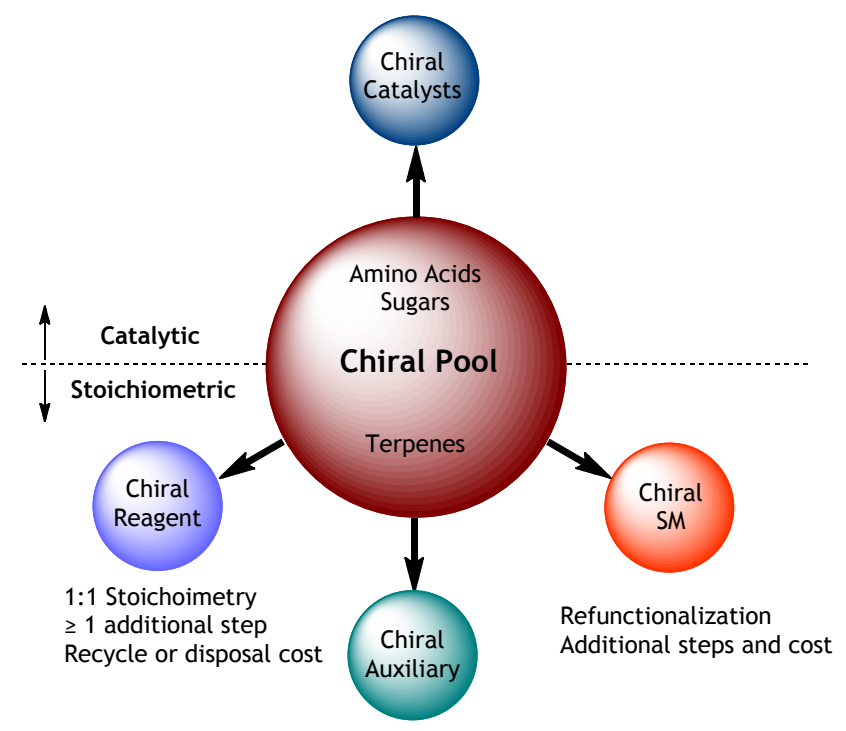

Figure 1.1: Routes for enantioselective synthesis

More recently, the transformation of pro-chiral starting materials into enantioenriched products, known as asymmetric synthesis, has become increasingly common. ${ }^{3}$ Early work involved using stoichiometric amounts of a chiral reagent, however, recent developments have successfully reduced this to catalytic amounts, known as asymmetric catalysis. ${ }^{4}$ 


\subsubsection{Asymmetric carbon-carbon bond forming reactions}

Carbon-carbon $(\mathrm{C}-\mathrm{C})$ bond forming reactions constitute approximately $11 \%$ of reactions employed in the pharmaceutical industry, making the formation of these bonds extremely important in both academic research and industry. ${ }^{5}$ In the latter, the majority of C-C bond forming reactions are palladium mediated cross-coupling reactions, ${ }^{5 a}$ whilst asymmetric formation of $\mathrm{C}-\mathrm{C}$ bonds is scarcely used. This issue was highlighted by Carey et al. (2006): "despite the huge academic effort invested in asymmetric $\mathrm{C}-\mathrm{C}$ bond forming reactions, they hardly appear in the list of reactions used in process development", ${ }^{6}$ and is still observed today. The principal factors limiting the industrial application of this method include: (i) the accessibility of suitable starting materials, (ii) poor tolerance of other functional groups; and (iii) procedures that are insufficiently robust to a variety of reaction conditions. ${ }^{5 a}$ Despite their limited use on an industrial scale, there exist a wide range of methods which enable catalytic asymmetric formation of $\mathrm{C}-\mathrm{C}$ bonds with excellent enantioselectivity, for example, $1,2-{ }^{7}$ and 1,4 -additions, ${ }^{8}$ allylic alkylations, ${ }^{8}$ Mannich reactions, ${ }^{9}$ carbometallation of alkenes, ${ }^{10}$ and $\mathrm{C}-\mathrm{H}$ insertions. ${ }^{11}$

\subsubsection{Asymmetric 1,4-addition reactions}

The asymmetric addition of carbon nucleophiles to $\alpha, \beta$-unsaturated carbonyl compounds represents a longstanding and crucial strategy in synthesis. ${ }^{12}$ The majority of early enantioselective methods obtained high selectivity using stoichiometric amounts of reagents, or a 'chiral auxiliary' (a removable, chiral modification to the starting material). ${ }^{13}$ The use of substoichiometric amounts of chiral reagents, generally in the form of a chiral catalyst, has revolutionised this field. ${ }^{14} \mathrm{~A}$ variety of soft and hard nucleophiles have been used in catalytic asymmetric 1,4-addition procedures; these involve the use of magnesium, zinc, boron or aluminium nucleophiles catalysed by lanthanides, ruthenium, iridium, nickel, palladium, rhodium or copper species. ${ }^{14}$ Such methods require the development of a suitable catalyst that is not only enantioselective, but also highly regioselective in order to avoid 1,2-addition, which competes with the 1,4-addition (Scheme 1.1). 


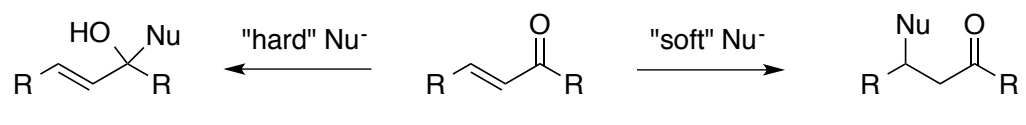

1,2-addition

1,4-addition

Scheme 1.1: Competing 1,2- vs. 1,4-addition

Hard nucleophiles can be transmetallated to soft nucleophilic copper species, which leads to preferential attack in the $\beta$-position on the enone, affording the desired 1,4-addition product.

\subsubsection{Copper-catalysed asymmetric 1,4-addition employing pre-made organometallic reagents}

The use of copper in the nucleophilic addition of organometallic reagents was first demonstrated by Gilman et al. in 1936. The authors reported the transmetallation of PhMgl with $\mathrm{CuCl}$ to yield a reactive phenylcopper species, which could be added to acylchlorides. ${ }^{15}$ Kharasch later demonstrated the use of $\mathrm{MeMgBr}$ and copper in a racemic conjugate addition to isophorone to yield racemic products. ${ }^{16}$ With the first catalytic asymmetric methods appearing 25 years ago, copper-catalysed 1,4-additions have since attracted a lot of attention, ${ }^{8}$ and multiple catalytic asymmetric procedures have been developed using Grignard, dialkylzinc and organoaluminium reagents, as highlighted in numerous reviews and book chapters. ${ }^{8,14,17}$

\subsubsection{Limitations of pre-made organometallic reagents}

Although exceptional results have been obtained in the 1,4-addition of organometallic reagents, challenges and opportunities still remain. Pre-made organometallic reagents often have limited accessibility, as few are commercially available. Furthermore, these reagents are not compatible with many functional groups.

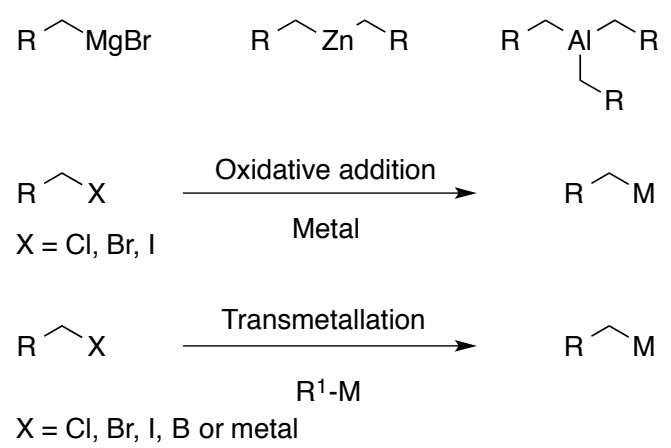

Scheme 1.2: Methods of obtaining pre-made organometallic reagents 
Grignard reagents, dialkyl zincs and alkylaluminiums are generally formed from their corresponding halides by direct oxidative insertion of a metal, or via transmetallation with a stoichiometric amount of a different pre-formed organometallic reagent (Scheme 1.2). Oxidative processes are limited in their functional group compatibility, while transmetallation contributes to excessive waste generation. Transmetallation uses multiple pre-formed organometallic reagents, giving rise to super-stoichiometric amounts of by-products, and require the use of multiple air and moisture sensitive materials, as they are very reactive by nature. ${ }^{18}$ This leads to high sensitivity to reaction conditions such as solvent, concentration, rate of addition, and/or presence of additives. Consequently, it is often necessary to perform the reaction at cryogenic temperatures to obtain high levels of selectivity. These requirements are often difficult to meet in industry and are a significant limitation of these methods.

A review by Howell highlighted the drawbacks of these methods. ${ }^{19}$ Organolithium, organoaluminium and dialkyl zinc reagents have not been used in large-scale asymmetric conjugate addition, although potentially scalable methods using dialkyl zinc reagents have been reported. ${ }^{19}$ Grignard reagents are preferred as they are less flammable; gram scale reactions have been described in the synthesis of extended polypropionate units. ${ }^{20,21}$ However, large-scale $(\geq 100$ g) asymmetric conjugate additions have not yet been reported. Therefore, it is important to develop a robust, scalable asymmetric conjugate addition method able to tolerate a range of functional groups and be integrated into the existing reaction set currently used by pharmaceutical companies. ${ }^{19}$

\subsection{Hydrometallation of Carbon-Carbon Bonds}

Hydrometallation is the process of adding a hydrogen and metal across alkenes, alkynes and related $\pi$-bonds. A thermodynamically favourable process due to the formation of a stronger sigma bond from the weaker $\pi$-bond, it involves the formation of an initial $\pi$-complex, followed by syn-addition of the metal to one end of the $\pi$-bond, and the hydrogen to the other. This proceeds 
via a four-centered transition state (Scheme 1.3). ${ }^{22},{ }^{23}$ Alkynes preferentially undergo hydrometallation over alkenes as they are linear sp hybridised compounds, thus they are highly reactive towards hydrometallation due to their greater electron density. Hydrometallation can be complicated by the presence of competing functional groups, such as carbonyls, as well as acidic protons and nucleophilic heteroatoms such as alcohols and amines. Moreover, the use of unsymmetrical alkenes can result in the production of multiple products, and hydrometallation of internal or bulky alkenes can be reversible or react at different rates, which can all complicate hydrometallation processes.

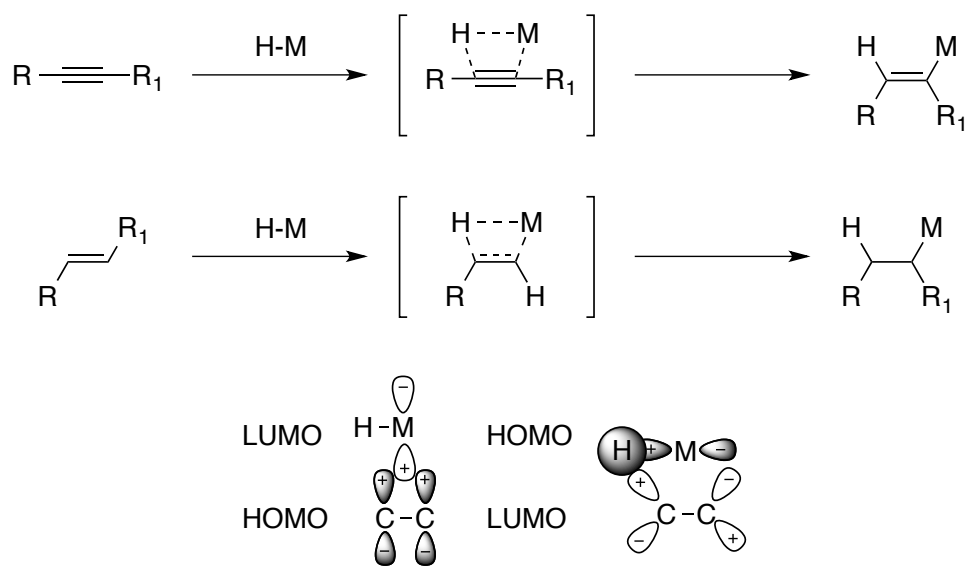

Scheme 1.3: Hydrometallation mechanism

Hydrometallation allows the use of simple starting materials to form reactive nucleophiles in situ, for use in further reactions. In asymmetric 1,4-addition reactions this provides an alternative way of preparing organometallic regents than those typically used. If the hydrometallation process were compatible with functional groups, this approach would also offer a method to obtain functionalised nucleophiles, which may then be useful for further elaboration of the products.

One of the most frequently used hydrometallation strategies involves the use of boron derivatives. Alkylborons can be synthesised in a highly chemo-, regio- and diastereoselective manner via hydroboration. Steric and electronic factors affect the rate of hydroboration: the steric bulk of the alkene is a key factor, with electron-rich terminal alkenes reacting the most rapidly. ${ }^{24}$ The resulting alkylborons can either undergo transmetallation to a different metal 
species, or be used directly in reactions including oxidations, cross-couplings and conjugate additions. ${ }^{24}$

Another attractive alternative is zirconium. In the lithosphere, zirconium is present at approximately the same abundance as carbon. It is one of the least expensive transition metals and is non-toxic thus making it an attractive option for the generation of hard nucleophiles. ${ }^{25}$ Initially used as polymerisation catalysts, organozirconium compounds have been widely used in organic synthesis since the early 1970's. Their frequent use has been attributed to the carbonzirconium bond's ability to transmetallate directly to form other carbon-metal bonds. Notable uses have included Negishi cross-coupling reactions and carboalumination reactions. ${ }^{26}$

In 1976 Schwartz and co-workers reported the use of zirconocene hydrochloride (Schwartz reagent) to hydrometallate alkenes (Figure 1.2) and convert them into more functionalised organic compounds under mild conditions. ${ }^{27}$ The polarisation of the carbon-zirconium bond is similar to that of Grignard reagents, however, in the Schwartz reagent the bulky cyclopentadienyl (Cp) groups prevent attack of many carbon electrophiles. As a result, such zirconium species have considerable functional group tolerance. ${ }^{28}$

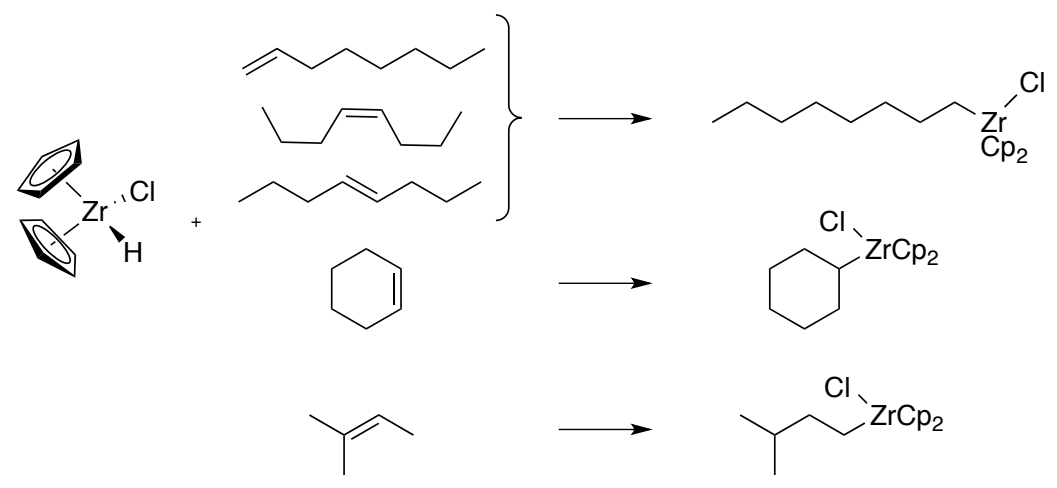

Figure 1.2: Hydrometallation with Schwartz reagent

In the case of internal alkenes, the terminal organozirconium species is obtained quantitatively by migration to the least sterically hindered position. This is driven by congestion from the bulky $\mathrm{Cp}$ rings, leading to $\beta$-hydride elimination/hydrometallation sequences to give the terminal alkylzirconocene (Scheme 1.4). Such a procedure is useful when a mixture of alkenes having the 
same length of carbon chain and varying double bond positions are present, as they will converge to the same product.

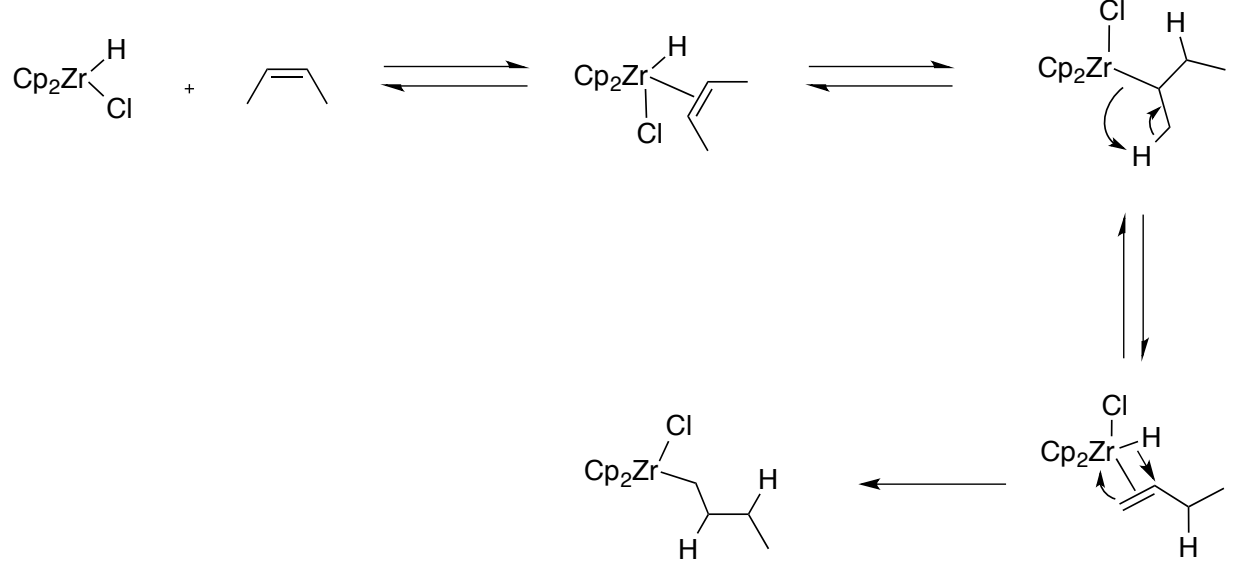

Scheme 1.4: Migration of C-Zr bond via $\beta$-hydride elimination

Despite the ability of organozirconiums to provide alkyl nucleophiles, their uses were limited until efficient transmetallation processes were developed. ${ }^{29}$ Alkylzirconium species are relatively stable, which allows a variety of transmetallation-addition processes to occur. The ability to transmetallate from zirconium to copper allows access to conjugate addition products, as further discussed below.

\subsubsection{Tandem hydrometallation-asymmetric addition reactions starting from alkynes}

The use of alkenyl nucleophiles in asymmetric conjugate addition (ACA) reactions have been reported for over 15 years, ${ }^{30}$ mostly using either rhodium- or copper-based catalysts. Rhodium catalysts were associated with the addition of aryl boronic acids, ${ }^{31}$ whereas copper catalysts were used to add alkyl organometallic reagents. ${ }^{30 c}$ The addition of alkenyl units (non-aromatic, linear $\mathrm{sp}^{2}$-hybridised nucleophiles) is valuable due to their presence in natural products, for example in prostaglandins, and also allows further functionalisation of the double bond.

\subsubsection{ACA initiated using nucleophiles generated by hydroboration}

Hayashi et al. first reported the addition of alkenyl boronic acids to enones in $1998 .{ }^{32}$ Due to the difficulties with preparation, isolation and purification of boronic acids, the authors developed a procedure using alkenyl-catecholboranes, which can be obtained directly from hydroboration of 
alkynes with catecholborane. ${ }^{33}$ The alkenyl-1,3,2-benzodioxaboroles were isolated before use in the 1,4-addition. Comparable results were obtained by performing the reaction in a one-pot sequence. However, it is necessary to remove unreacted materials from the hydroboration prior to the addition of the necessary reagents for the 1,4-addition (Scheme 1.5). ${ }^{33}$

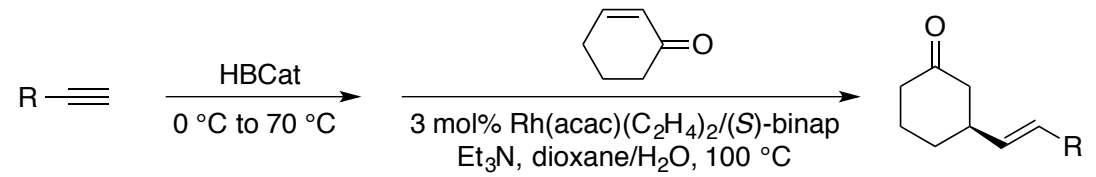

$$
\begin{aligned}
& \mathrm{R}=n-\mathrm{C}_{5} \mathrm{H}_{11} ; 85 \% \text { yield, } 95 \% \text { ee } \\
& \mathrm{R}=t-\mathrm{Bu} ; 75 \% \text { yield, } 92 \% \text { ee }
\end{aligned}
$$

\section{Scheme 1.5: Hayashi's one-pot procedure}

The use of alkenyl borons has been exploited in the synthesis of Ecklonialactones. ${ }^{34}$ Fürster et al. modified conditions previously developed by Carreira, ${ }^{35}$ and applied a 1,4 -addition to furanone as the first step (Scheme 1.6). A long reaction time and high temperature were required to hydrometallate phenylacetylene with pinacolborane. Subsequently, the addition step proceeded over 3 days.

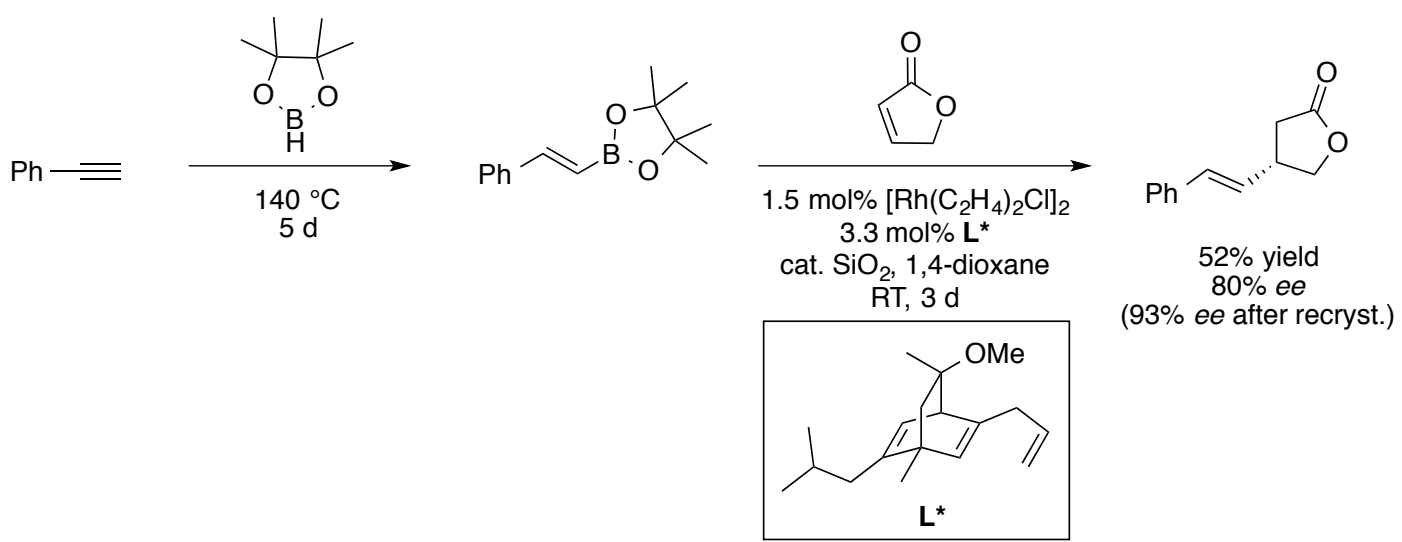

Scheme 1.6: Furster's first step towards the synthesis of Ecklonialactones

\subsubsection{ACA initiated using nucleophiles generated by hydroalumination}

In 2005, Alexakis and Woodward performed the first hydrometallation carboalumination with copper: a conjugate addition sequence with alkenylaluminiums. ${ }^{36} \mathrm{~A}$ combination of phenylacetylene and trimethyl aluminium catalysed by $\mathrm{Cp}_{2} \mathrm{ZrCl}_{2}$ resulted in the alkenylaluminium nucleophile, which lead to the 1,4-conjugate addition product upon addition of copper (Scheme 
1.7). Following this, Woodward et al. demonstrated that the use of modified aryl ferrophites could improve the ee to $85 \%$ (Scheme 1.8$).{ }^{37}$

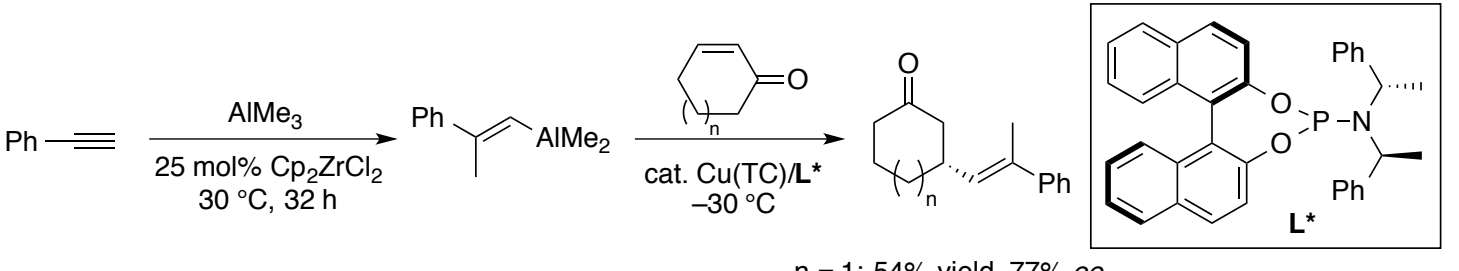

$$
\begin{aligned}
& \mathrm{n}=1 ; 54 \% \text { yield, } 77 \% \text { ee } \\
& n=2 ; 48 \% \text { yield, } 72 \% \text { ee }
\end{aligned}
$$

Scheme 1.7: Carboalumination ACA as reported by Alexakis and Woodward
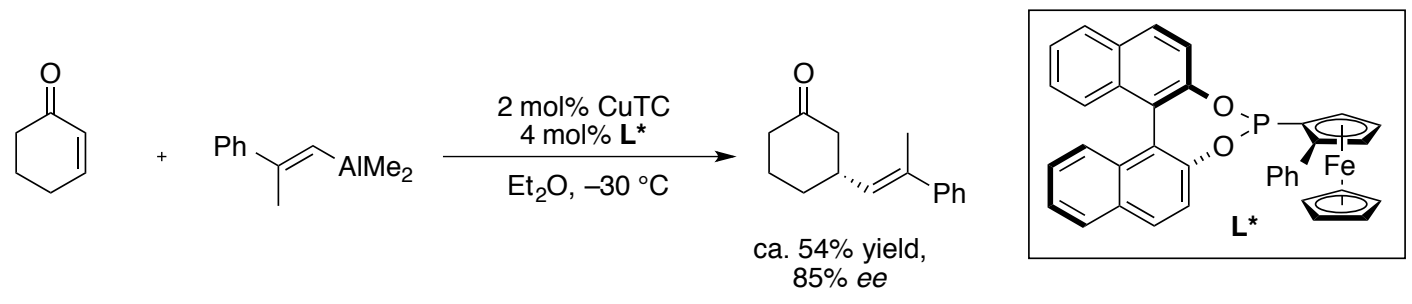

Scheme 1.8: Woodward's modified procedure

Alexakis et al. showed that alternatively, alkenylaluminium reagents could be obtained from alkynes using Zweifel conditions. ${ }^{38}$ Using DIBAL-H instead of trimethylaluminium significantly reduced the time taken to generate aluminium species from alkynes. These reagents were applied in 1,4-conjugate additions to $\beta$-substituted enones, to form quaternary centres (Scheme 1.9). ${ }^{39,40}$

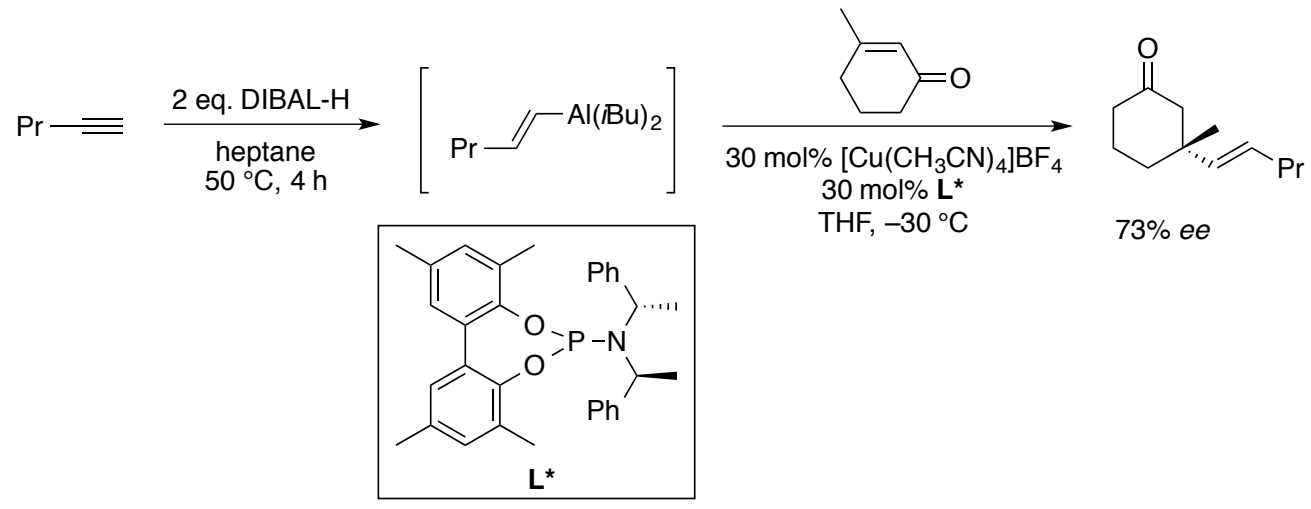

\section{Scheme 1.9: Alexakis's quaternary centre formation}

The same authors also demonstrated the use of chiral phosphanamine ligands to add a range of simple and more functionalised alkenylaluminiums to $\beta$-substituted enones (Scheme 1.10). Higher ee values were obtained, however, some substrates required further activation with 1 eq. $\mathrm{Me}_{3} \mathrm{Al}$ to prevent low conversions. ${ }^{41}$ 
<smiles>[R]C1=CC(=O)CCC1</smiles>

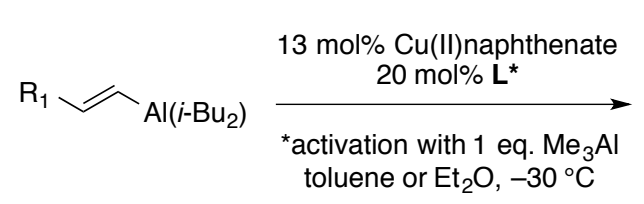<smiles>[R]C1(/C=C/Br)CCCC(=O)C1</smiles><smiles>C[C@]1(/C=C/[Al])CCCC(=O)C1</smiles><smiles>CCCCC=C[C@]1(C)CCCC(=O)C1</smiles>

91 yield $41 \%$ yield $^{*}$
$89 \%$ ee<smiles>CCCCC=C[C@]1(C)CCCCC(=O)C1</smiles><smiles>C[C@]1(/C=C/CCCCl)CCCC(=O)C1</smiles>

57 yield $^{*}$
$79 \%$ ee 62 yield
$76 \%$ ee

\section{Scheme 1.10: Alexakis's scope of quaternary centre formation}

Hoveyda et al. reported hydrometallation of alkynlsilanes with DIBAL-H to form silicon-substituted alkenylaluminium reagents. ${ }^{42}$ These resulted in high enantioselectivities in combination with a chiral-NHC complex. The method was used in the total synthesis of Riccardiphenol B (Scheme 1.11)..$^{42}$

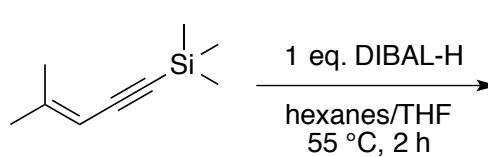
$55^{\circ} \mathrm{C}, 2 \mathrm{~h}$<smiles>COc1ccc(O)c(CC2=C(C)CCC[C@@]2(C)C=CC=C(C)C)c1</smiles>

Riccardiphenol B<smiles>CCCCCCCCCCCC(C)=CC=C(C)C</smiles><smiles>CC1=CC(=O)CCC1</smiles>
$\underset{2.5 \mathrm{~mol}_{2} \cdot \mathrm{L}^{*}}{5 \mathrm{~mol}_{2} \mathrm{O}}$

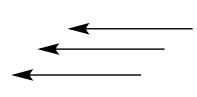

Scheme 1.11: Hoveyda's example and application in the total synthesis of Riccardiphenol B

These silicon-substituted alkenyl aluminiums have also been used in asymmetric allylic alkylations to form quaternary centres (Scheme 1.12). ${ }^{43}$ Recently, Woodward and Alexakis et al. obtained alkenylaluminiums ${ }^{44}$ using $\mathrm{HAICl}_{2} \cdot 2 \mathrm{THF}$ and catalytic $\mathrm{Cp}^{*}{ }_{2} \mathrm{ZrCl}_{2}$. Activation of this species with MeLi, followed by addition of a catalyst, resulted in highly enantioselective 1,4-addition products (Scheme 1.13). ${ }^{44}$ 


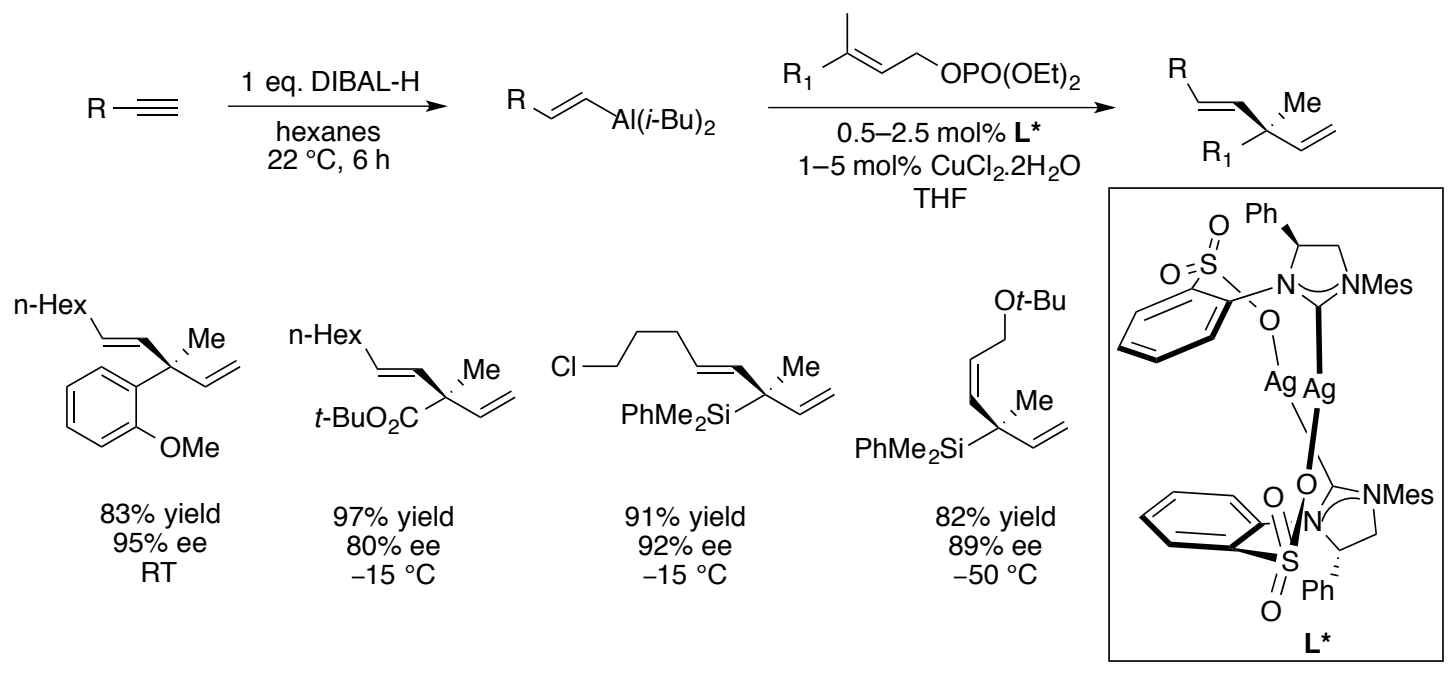

Scheme 1.12: Hoveyda's allylic alkylation

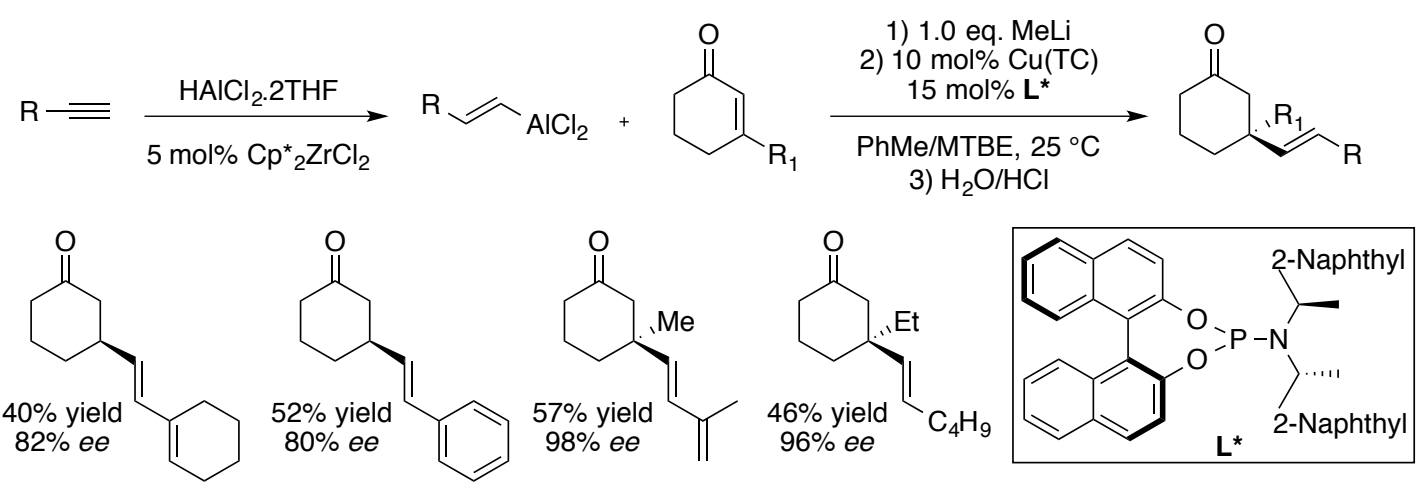

Scheme 1.13: Alexakis's and Woodward's improved methodology

\subsubsection{ACA initiated using nucleophiles generated by hydrosilylation}

\section{Rhodium catalysis}

Organosilicon reagents can be obtained by hydrosilylation of alkynes to form alkenylsilyl reagents, which can then be transmetallated and used in further reactions. Inoue et al. performed the first asymmetric 1,4 -addition using a silicon reagent. ${ }^{45}$ An alkenyl-trialkoxysilane was formed by rhodium-catalysed hydrosilylation with triethoxysilane. The alkenyl-trialkoxysilane then reacted with 2-cyclohexenone to afford the alkenyl conjugate addition products using a different chiralrhodium catalyst. High enantioselectivities were observed and, importantly, both cis and trans silanes were accessible with appropriate hydrometallation catalysts (Scheme 1.14). 


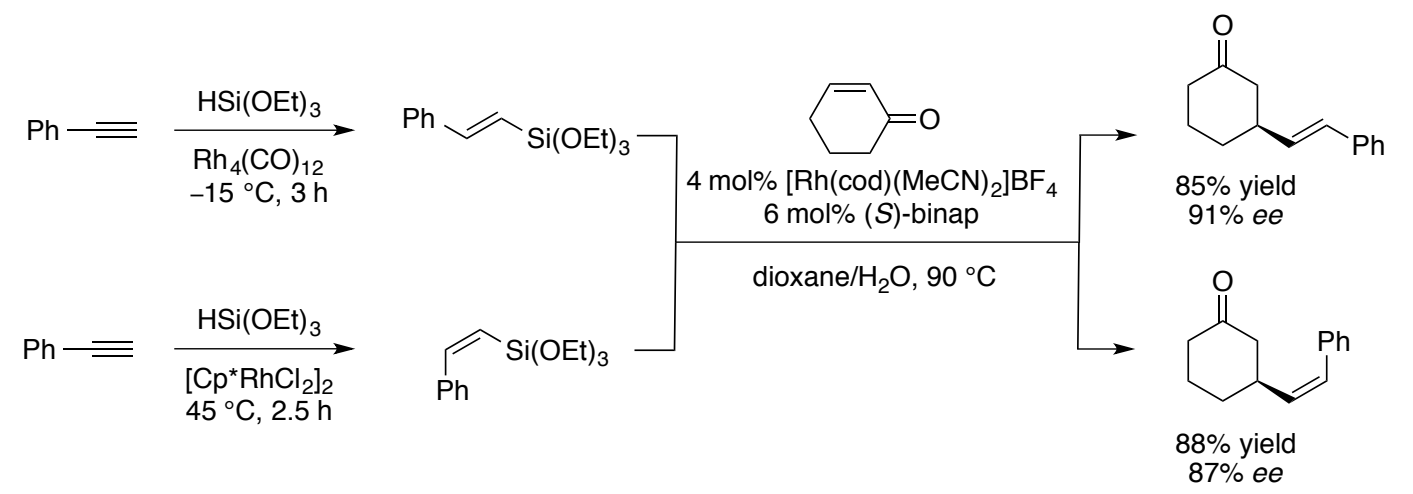

Scheme 1.14: Inoue's ACA using organosilicon reagents

Hayashi et al. developed a one-pot procedure using the same rhodium source for both the hydrosilylation and 1,4-addition. Reaction conditions were the same as those used by Inoue et al. ${ }^{45}$ however, catalyst loadings were decreased and the alkenyl substrate scope was extended (Scheme 1.15). ${ }^{46}$

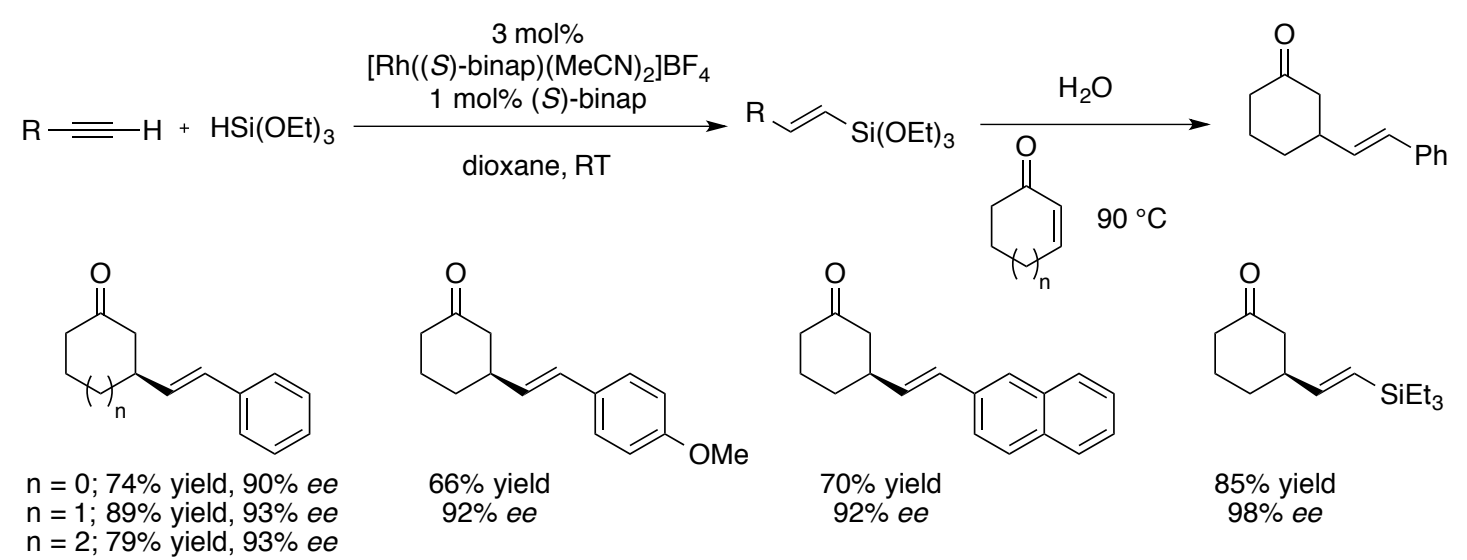

\section{Scheme 1.15: Hayashi's one-pot system}

A tetraorganosilicon species was developed by Hayashi et al. from the hydrosilylation of a stable and reusable silicon reagent. The tetraorganosilicon reagent was obtained in two overnight steps, and then subjected to platinum-catalysed hydrosilylation before use in 1,4-addition. However, only two examples were subjected to the asymmetric conditions (Scheme 1.16). ${ }^{47}$ The same conditions were applied to the 1,4-addition of alkenylsilane reagents to acyclic enones, indicating that steric bulk adjacent to the carbonyl is correlated to high enantioselectivity (Figure 1.3). ${ }^{48}$ 


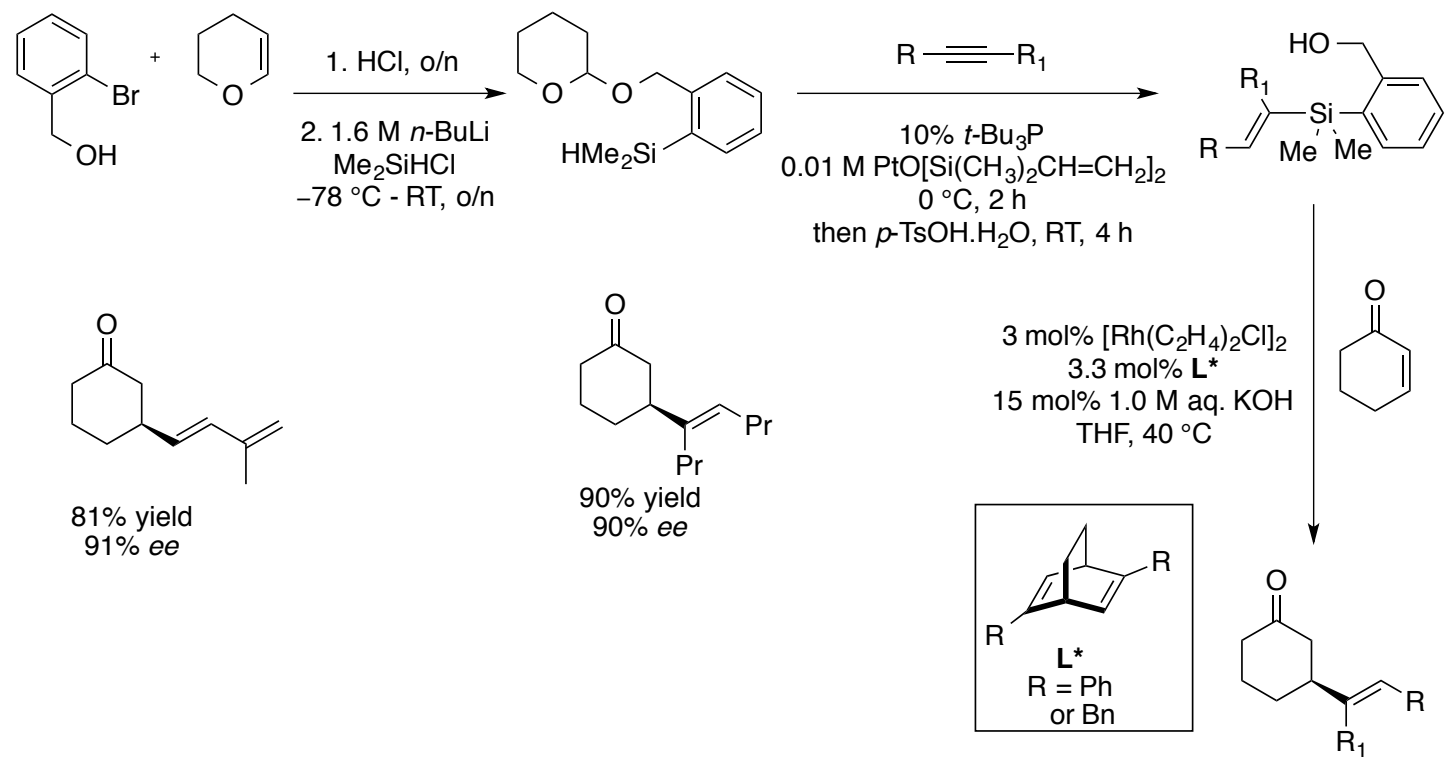

Scheme 1.16: Hayashi's procedure using tetraorganosilicon reagents<smiles>CCC[SiH2]C(/C=C/C=[V])CC(C)=O</smiles>

$91 \%$ yield $56 \%$ ee<smiles>CC=C(C)[C@H](CC(=O)c1ccccc1)[SiH2]CC</smiles>

$92 \%$ yield $91 \%$ ee<smiles>C/C=C(\C)C(CC(=O)CCCCC)[SiH2]C(C)C</smiles>

$98 \%$ yield $94 \%$ ee<smiles>CCCC=C(CCCC)C(CC(=O)c1ccccc1)[SiH2]CCC</smiles>

$92 \%$ yield $93 \%$ ee

Figure 1.3: Hayashi's extension to linear enones

\section{Copper catalysis}

The use of copper in tandem hydrosilylation-conjugate addition processes has thus far been limited. Hoveyda et al. reported the only example that employs alkenylsilicon reagents, where the alkyne underwent a two-step platinum-catalysed hydrosilylation with trichlorosilyl hydride, followed by reaction with $\mathrm{Na}_{2} \mathrm{SiF}_{6}$ to form a trifluoro-silane (Scheme 1.17 ). ${ }^{49}$ Only moderate enantioselectivities were obtained in the majority of cases. 


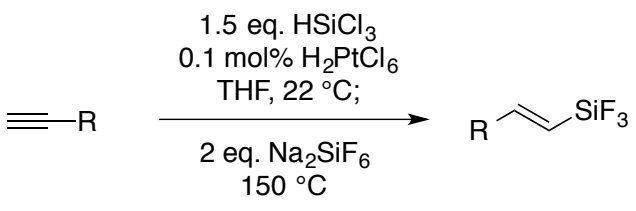<smiles>O=C1CCCC(/C=C/c2ccccc2)C1</smiles>

$\mathrm{n}=0-3$

$80-93 \%$ yield

$47-92 \%$ ee

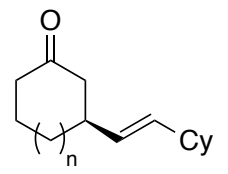

$\mathrm{n}=1-3$

$83-97 \%$ yield

$67 \%-77 \%$ ee
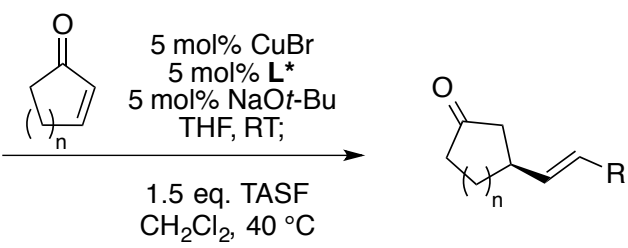

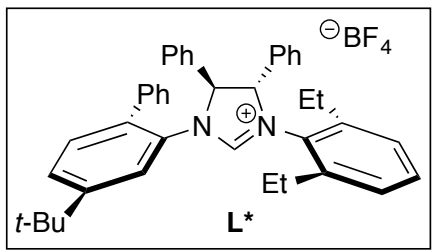

Scheme 1.17: Hoveyda's copper-catalysed example using alkenylsilicon reagents

\subsubsection{ACA initiated using zirconium nucleophiles}

Alkenylzirconium reagents are easily prepared by hydrozirconation of alkynes using the Schwartz reagent in THF at room temperature. Inoue et al. used the resulting alkenylzirconocene species in 1,4-conjugate additions catalysed by rhodium. ${ }^{50}$ Moderate to excellent enantioselectivities were obtained, under mild conditions, where the entire reaction is performed at room temperature (Scheme 1.18).

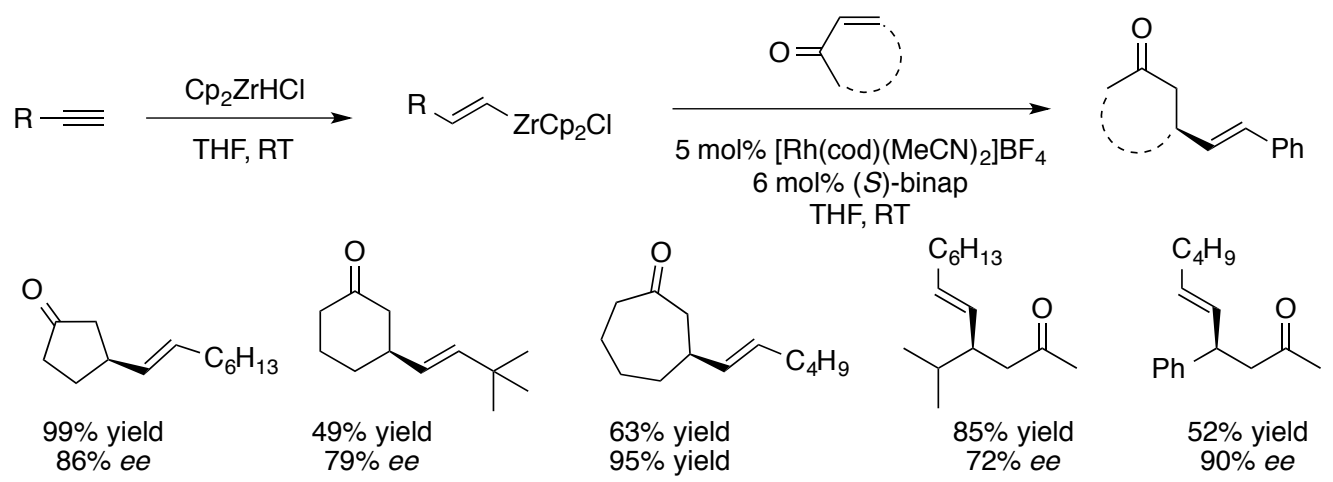

Scheme 1.18: Inoue's ACA using alkenylzirconium reagents

It was hypothesised that the reaction mechanism involved the generation of an alkenylrhodium species from the transmetallation of the alkenylzirconium reagent. Following this, addition of the rhodium species to the $\alpha, \beta$-unsaturated enone results in the production of an oxa- $\pi$-allylrhodium species. It is then able to react with another equivalent of alkenylzirconium reagent to give a zirconium enolate that is quenched upon workup (Scheme 1.19). ${ }^{50}$ 


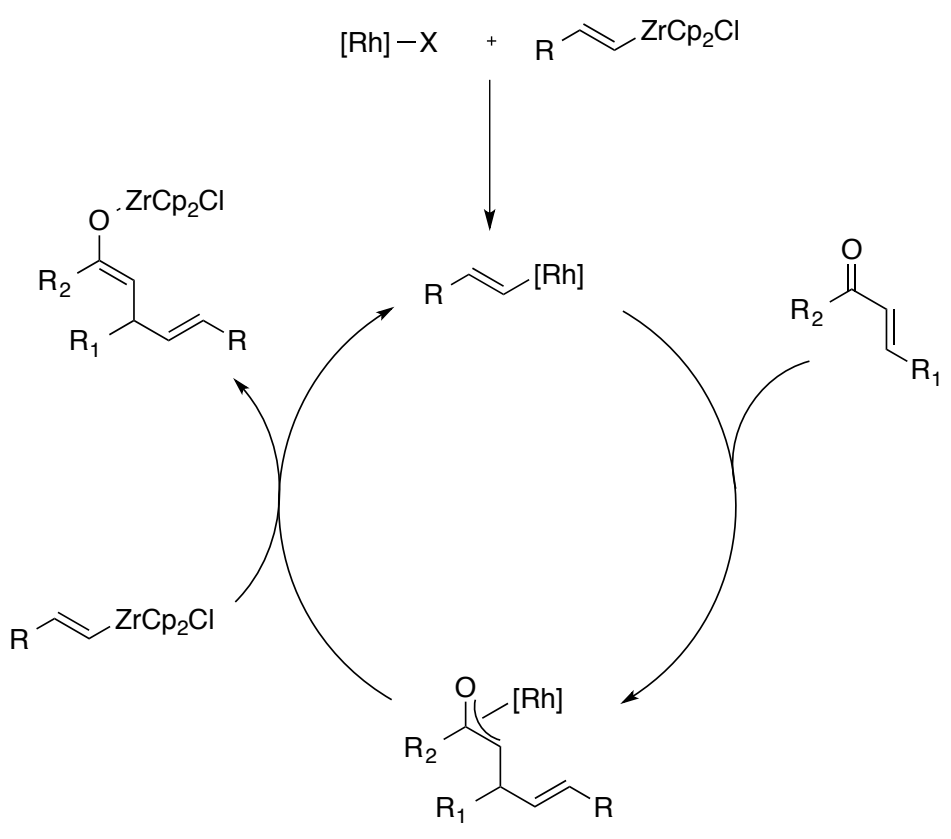

Scheme 1.19: Inoue's proposed mechanism

Based on Inoue's pioneering work ${ }^{50}$ Nicolaou et al. developed a three-component system, which involved the trapping of the resulting zirconium enolate with an aldehyde to give aldol products (Scheme 1.20). ${ }^{51}$ This method was applied to the synthesis of the spiro-unit of Vannusal A (Scheme 1.21). ${ }^{51}$

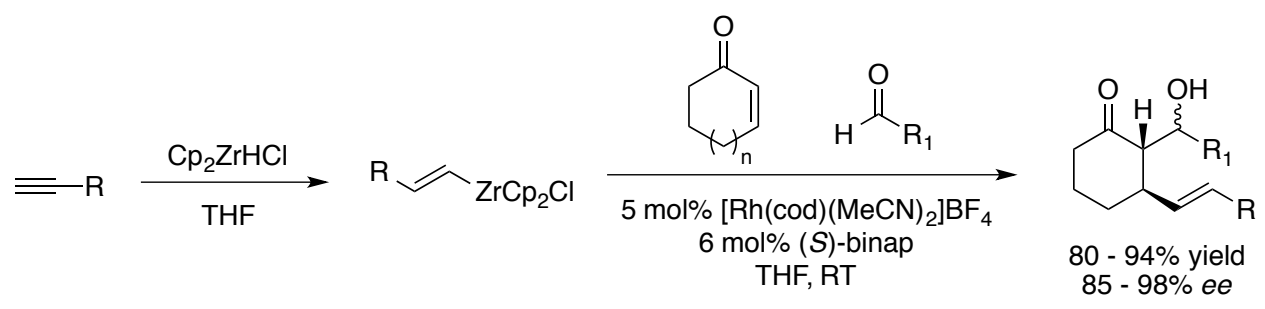

Scheme 1.20: Nicolaou's ACA-aldol tandem reaction 


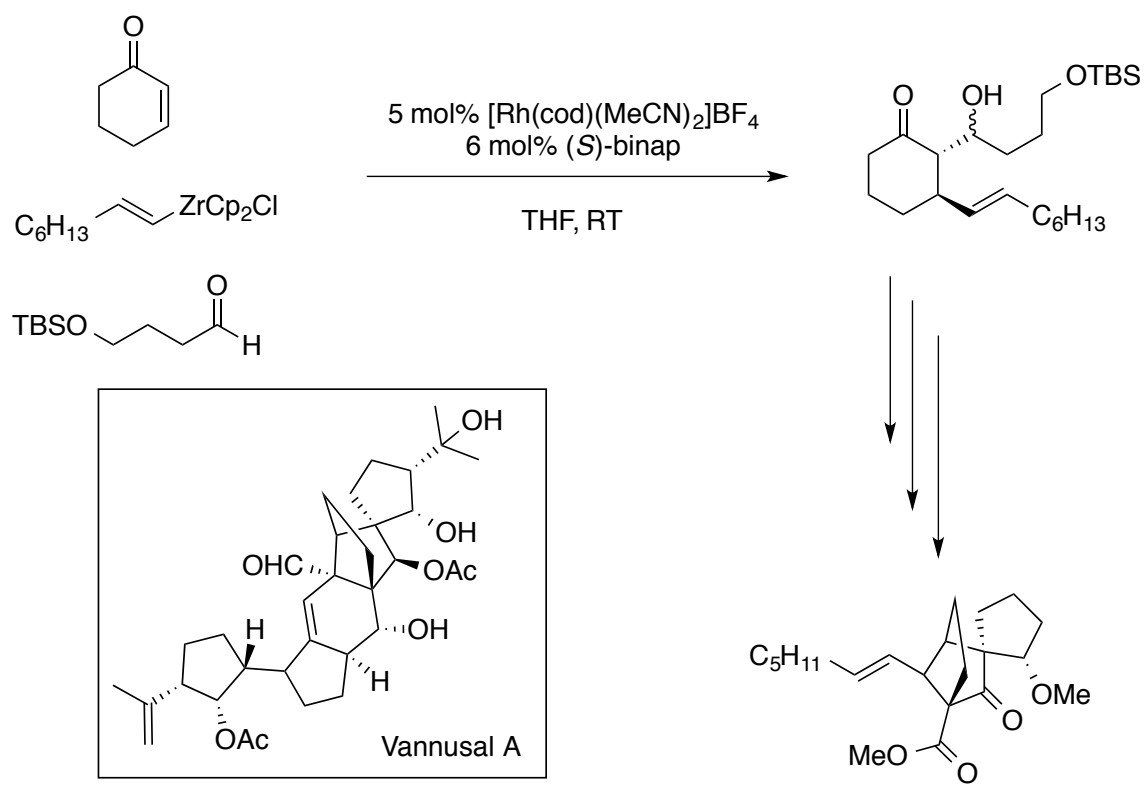

Scheme 1.21: Nicolaou's system applied towards the total synthesis of Vannusal A

More recently, Zezschwitz et al. reported the first tandem addition of alkenylzirconocenes followed by silyl trapping to give enol silanes. ${ }^{52}$ Treatment of the zirconium enolate with an excess of MeLi at $-78{ }^{\circ} \mathrm{C}$ resulted in a lithium enolate that is consequently trapped as a silane. The reaction is quenched with a urea-hydrogen peroxide adduct, which allows isolation of the enolsilane. In addition, the authors have reported a modified conjugate addition procedure, where uniformly high enantioselectivities are obtained (Scheme 1.22). This method was used in the total synthesis of Sarkomycin in $19 \%$ overall yield and $96 \%$ ee (Scheme 1.23$).{ }^{53}$

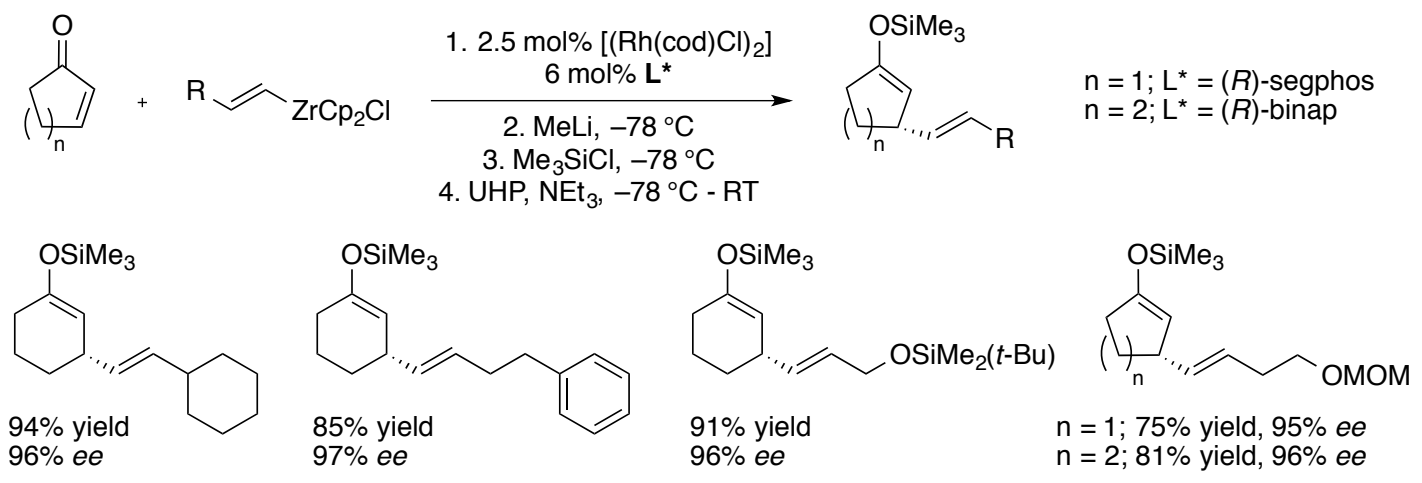

Scheme 1.22: Zezschwitz's silyl trapping. UHP = urea-hydrogen peroxide adduct 


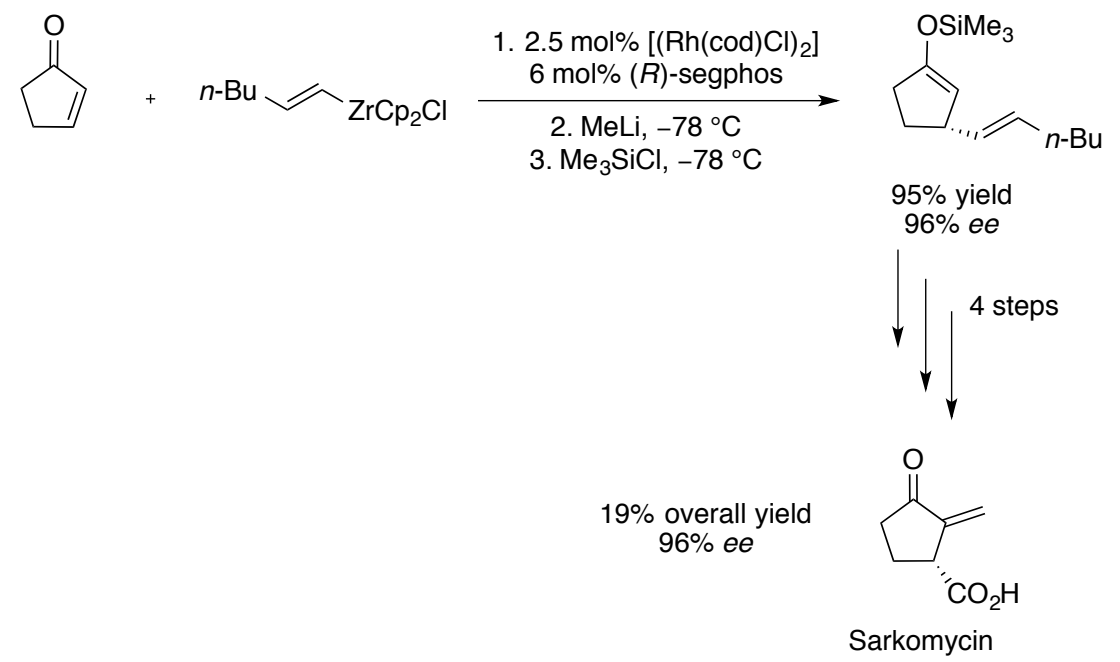

Scheme 1.23: Zezschwitz's total synthesis of Sarkomycin

\subsubsection{Tandem hydrometallation-addition reactions starting from alkenes}

1,4-Conjugate addition processes using organometallic reagents generated by the hydrometallation of alkenes have received substantially less attention than alkynes. This is partially due to the hydrometallation product undergoing $\beta$-hydride elimination to afford the starting alkene and free hydrometallating reagent. ${ }^{54}$ Such processes have only been recently reported, and involve hydrometallation of alkenes with the organoborane $(9-\mathrm{BBN})_{2}$ to produce an alkylboron reagent.

\subsubsection{ACA of alkylboron compounds}

The first catalytic asymmetric conjugate additions of alkylboron reagents were developed by Sawamura et $a l .{ }^{55}$ Addition of the alkylboron reagent (generated in mesitylene at $60{ }^{\circ} \mathrm{C}$ ) was catalysed by a combination of PhOK, which presumably activates the boron towards attack, copper chloride and a chiral NHC ligand at $30^{\circ} \mathrm{C}$ (Scheme 1.24). 


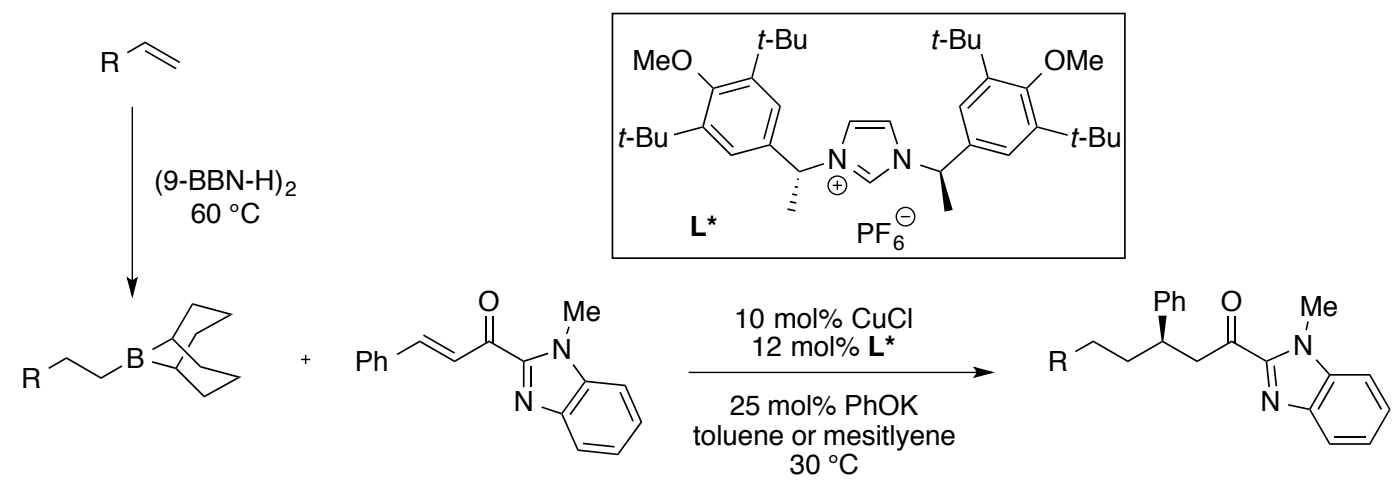

Scheme 1.24: Sawamura's ACA using alkylboron reagents

The reaction uses a variety of substrates rarely observed in conjugate addition reactions, such as substituted aromatic rings and esters (Figure 1.4)..$^{55}$ However, the method is currently limited to imidazol-2-yl $\alpha, \beta$-unsaturated ketones, thus extension to other electrophiles is desirable.

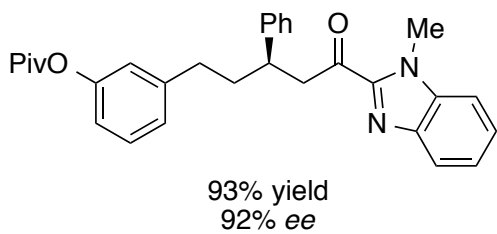<smiles>Cn1c(C(=O)C[C@H](CCc2ccc(Cl)cc2)c2ccccc2)nc2ccccc21</smiles><smiles>COc1ccc(CC[C@H](CC(=O)c2nc3ccccc3n2C)c2ccccc2)cc1OC</smiles><smiles>COC(=O)CC(C)(C)CC[C@H](CC(=O)c1nc2ccccc2n1C)c1ccccc1</smiles>

Figure 1.4: Sawamura's substrate scope

As well as the conjugate addition reaction, ${ }^{55}$ Sawamura et al. also developed the first successful catalytic asymmetric allylic alkylation with alkylboron reagents (Scheme 1.25$) \cdot{ }^{56}$ The method also involved hydrometallation with $(9-\mathrm{BBN})_{2}$ followed by copper-catalysed addition. In this case, MeOK was used to activate the boron towards attack, and the copper complex existed as a bulky bisphosphine ligand (di-t-butylmethoxyphenyl-segphos) and (CuOTf) $)_{2}$ PhMe. High enantioselectivities were obtained with a wide range of substituted allylic alkylation products at $15^{\circ} \mathrm{C}$. 

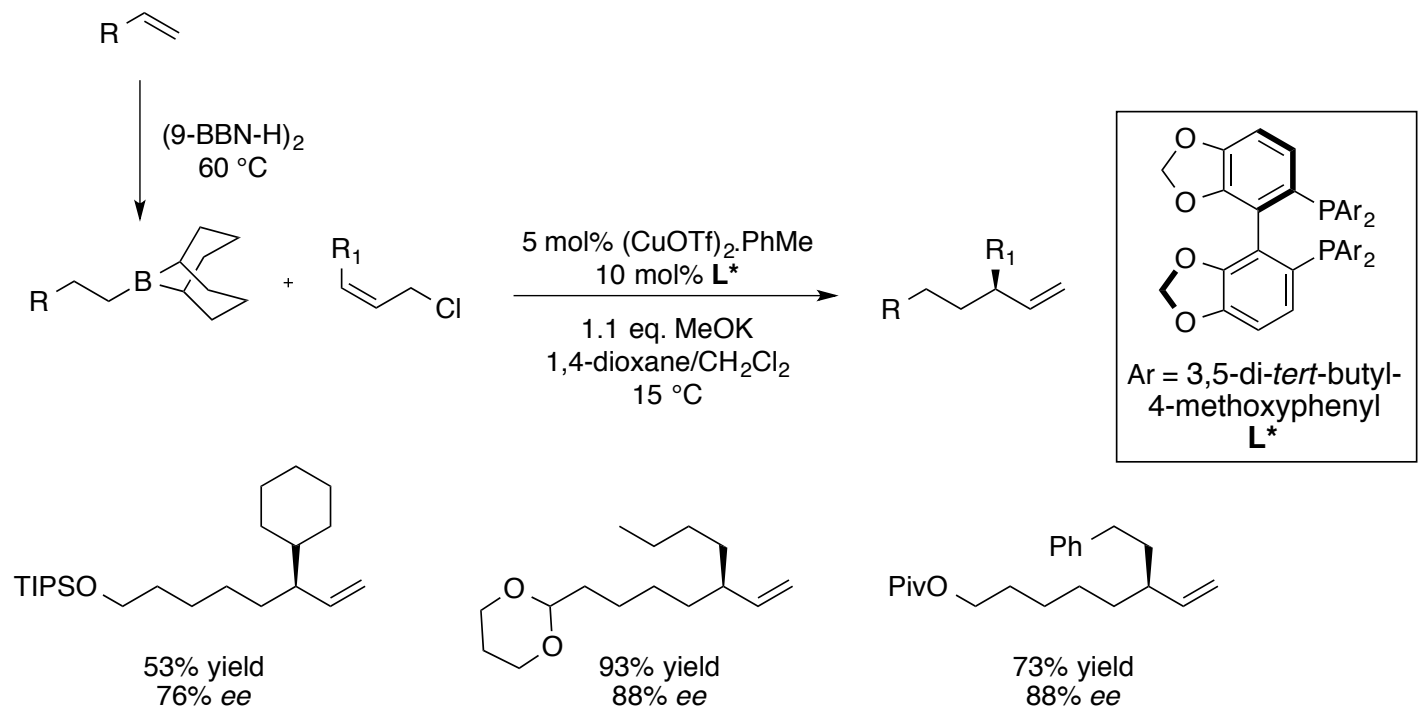

Scheme 1.25: Sawamura's asymmetric allylic alkylation using alkylboron reagents

\subsubsection{Addition of alkylzirconium compounds in 1,4-addition reactions}

Extensive development of procedures for hydrometallation of alkenes using the Schwartz reagent followed by a 1,4-conjugate addition reaction has been carried out by Wipf et al. ${ }^{57}$ However, most were limited to the formation of racemic products. Hydrometallation of 1-hexene with the Schwartz reagent in THF either at room temperature with sonication, or at $40^{\circ} \mathrm{C}$, gave the desired hexylzirconocene which was then combined with catalytic amounts of copper bromide dimethylsulfide. This results in addition to 2-cyclohexenone forming a new C-C bond (Scheme $1.26)^{57}$

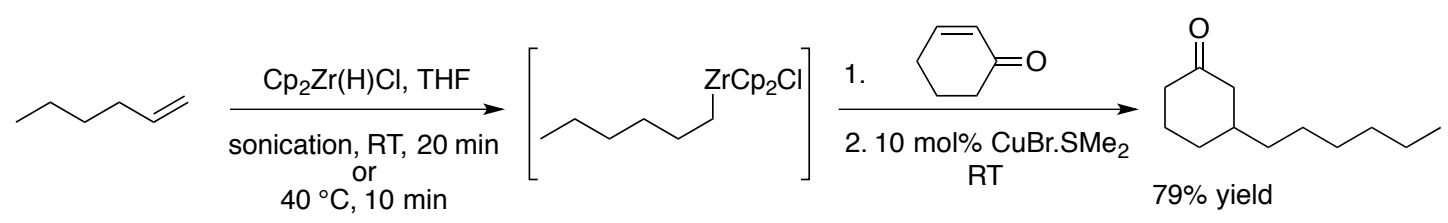

Scheme 1.26: Wipf's non-enantioselective conjugate addition using alkyl zirconocene

A wide variety of alkene-based nucleophiles could be added; difficulties would be observed when utilising a number of these functional groups in other methods for copper-catalysed 1,4-addition. For example, protected alcohols, alkyl groups containing acetals and sterically hindered groups could all be added (Scheme 1.27). ${ }^{58}$ 

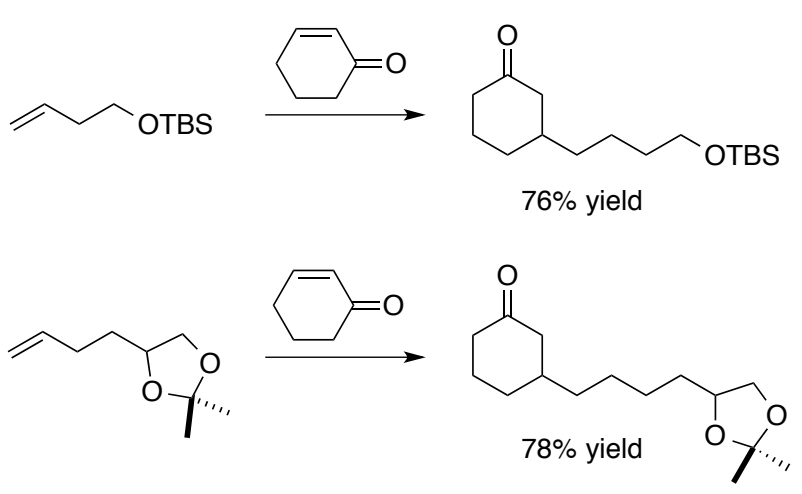
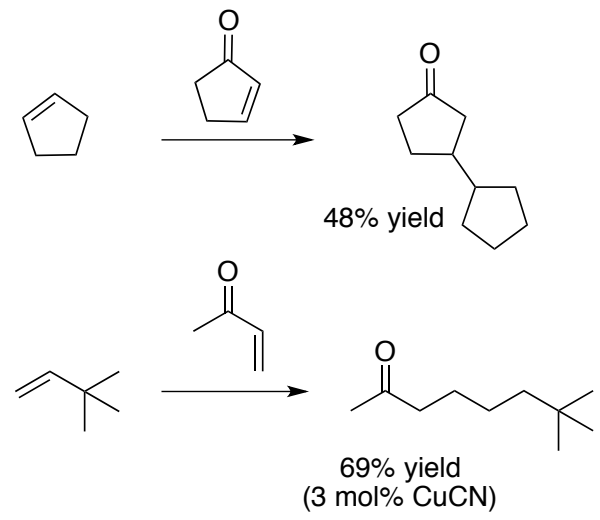

\section{Scheme 1.27: Wipf's examples}

${ }^{13} \mathrm{C}$ NMR analysis provided insight into the reaction mechanism (Scheme 1.28). ${ }^{58}$ Direct transmetallation of the alkylzirconocene to alkylcopper species was not observed, rather, it is likely that a short-lived alkylcopper-zirconium complex was slowly formed (i). It is hypothesised that in the presence of the enone, the zirconium complexes to the carbonyl moiety (ii), which facilitates the complexation of copper to the $\mathrm{C}=\mathrm{C}$ (iii). Subsequent transmetallation of the organic residue from zirconium to copper results in a copper(III) intermediate (v). The final step involved reductive elimination to give the zirconium enolate, and regeneration of the copper(I) species (vi). ${ }^{58}$

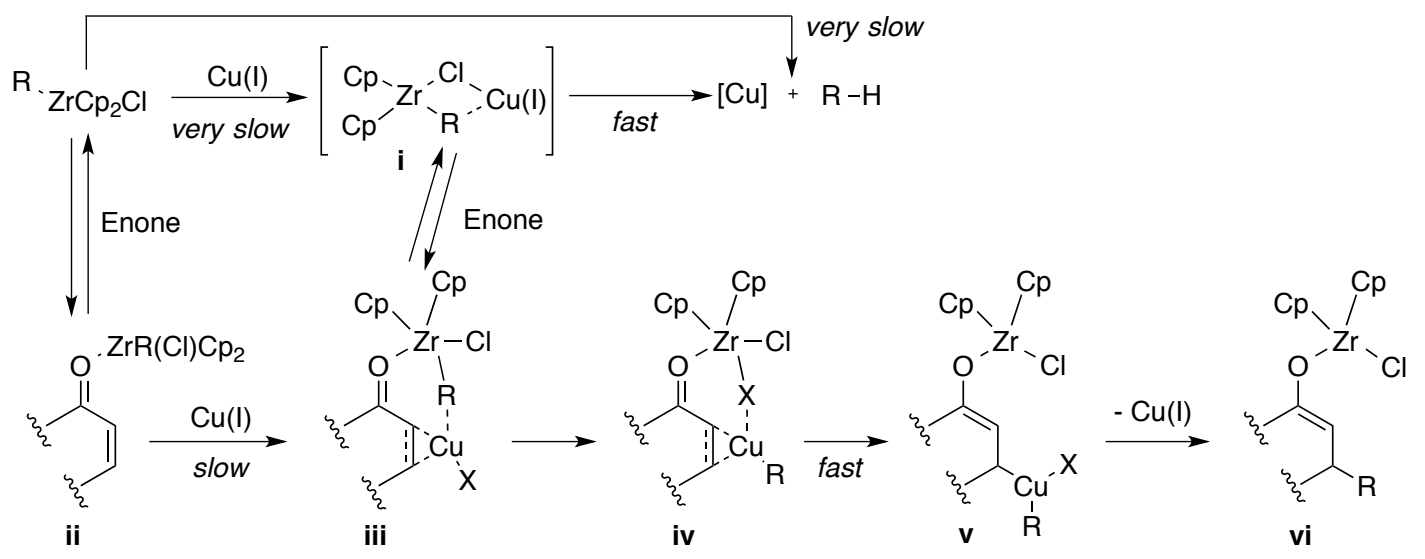

Scheme 1.28: Proposed mechanism of alkylzirconium 1,4-addition

Subsequently, Wipf et al. reported an asymmetric variation of the 1,4-addition, involving a diastereoselective conjugate addition onto a substrate bearing an Evans chiral auxiliary. This enabled a highly diastereoselective addition of a variety of functionalised alkenes (Scheme 1.29). ${ }^{59}$ 


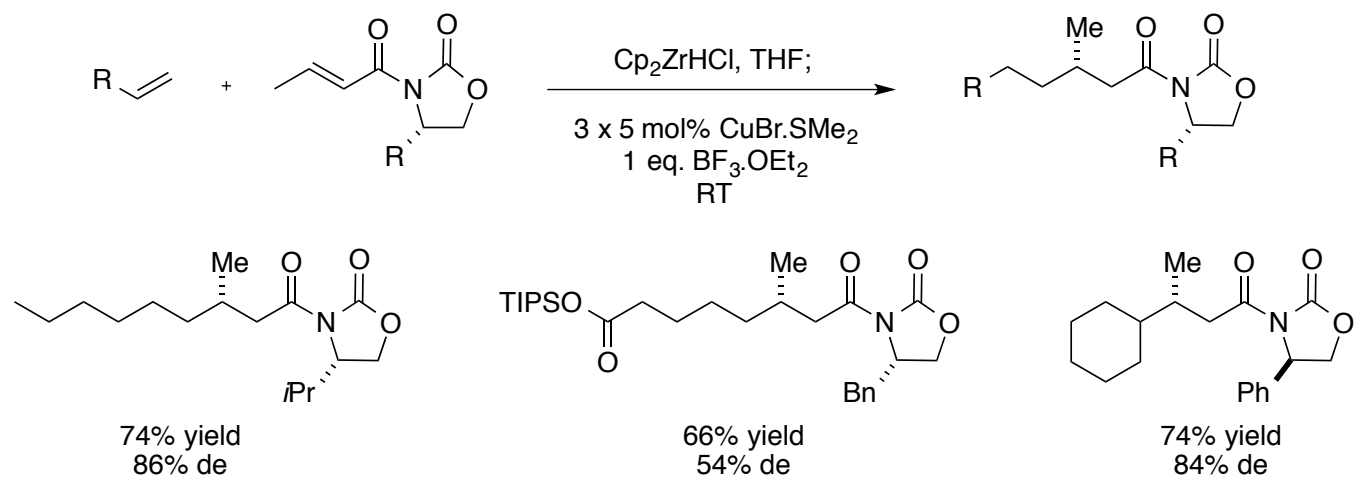

Scheme 1.29: Wipf's diastereoselective synthesis

There are limited reports of hydrometallated alkenes being used in enantioselective addition reactions. Given the wide availability of alkenes, if an effective route is developed, numerous possibilities for their use in C-C bond forming reactions are offered. In particular, the lack of a catalytic ACA reaction using alkylzirconium reagents warrants further research. By using knowledge gained from the development of modern catalytic ACA reactions using pre-made organometallics, there exist plentiful possibilities for the conjugate addition of alkylzirconium reagents to be explored. Thus the research in this thesis focuses on the development of new catalytic addition reactions initiated by the hydrozirconation of simple alkenes. 


\section{Chapter 2: Development of a New Catalytic Asymmetric 1,4-Addition Reaction}

In this chapter, we describe the development of the method used throughout this thesis. The aims of this project and the relevant literature on asymmetric 1,4-addition of alkyl metal species to linear $\alpha, \beta$-unsaturated enones are discussed. I will then go into detail on my own work: first I will discuss the development of an asymmetric 1,4-addition to linear $\alpha, \beta$-unsaturated enones. Following this previous work on 1,4-additions to cyclic enones is examined, followed by optimisation of a new system for these additions. The substrate scope and scale up of this new system are then described. Parts of this chapter have been previously published in Nature Chemistry, ${ }^{60}$ Chemical Communications, ${ }^{61}$ and Synthesis. ${ }^{62}$

\subsection{Project Aims}

The aim of this project was to develop new methods for asymmetric 1,4-conjugate addition reactions of alkyl nucleophiles to $\alpha, \beta$-unsaturated compounds. We aimed to develop effective and highly enantioselective methods that use alkenes as the equivalents to currently used organometallic reagents. Furthermore, scale-up of these procedures was explored in order to assess their potential use on a commercial scale. Current methods pose serious risks on industrial scales due to the reactive nature of the nucleophiles used and typically require cryogenic temperatures. ${ }^{19}$ Thus, the development of new methods to overcome these problems is highly desirable.

\subsection{Enantioselective 1,4-Addition to Acyclic Enones}

\subsubsection{Introduction}

1,4-Addition reactions to acyclic enones pose an important challenge in asymmetric catalysis. Acyclic enones are common building blocks for the synthesis of natural products and therefore the 1,4-addition to enone allows the incorporation of structural features present in a wide range of biologically active compounds. Copper-catalysed additions represent an important class of these reactions. These 1,4-additions generally involve a hard organometallic nucleophile, selected 
from dialkylzinc, Grignard or organoaluminium reagents. The use of copper significantly biases these reactions to proceed via the preferred 1,4-addition as opposed to the competing 1,2addition reaction, which would primarily occur in the presence of such nucleophiles in the absence of a copper catalyst. Copper-catalysed asymmetric additions are well known, and the challenges in obtaining high selectivity in additions to acyclic enones have been highlighted in recent reviews. ${ }^{8}$ The earliest approach was reported by van Koten et al., whom achieved up to $76 \%$ ee when using a chiral arenethiolatocopper(I) complex as the catalyst (Scheme 2.1$){ }^{63}$<smiles>CC(=O)/C=C/c1ccccc1</smiles><smiles>CCOCCOCCO</smiles><smiles>CC(=O)CC(C)c1ccccc1</smiles>

up to $97 \%$ yield up to $76 \%$ ee

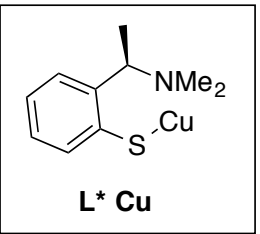

\section{Scheme 2.1: Early work by van Koten}

Sammakia et al. employed chiral ferrocenyl phosphine oxazoline ligands to obtain up to $81 \%$ ee in asymmetric 1,4 -additions, only a single example with an acyclic substrate was detailed. ${ }^{64}$ Notably, in 2002, Hoveyda et al. reported the use of chiral peptidic phosphine ligands in these reactions, obtaining high yields and excellent enantioselectivities of up to $95 \%$ (Scheme 2.2). ${ }^{65}$ Such processes employed the successful addition of diethylzinc reagents to a variety of acyclic enones.
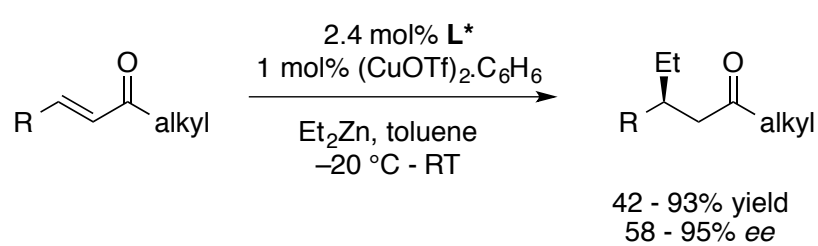

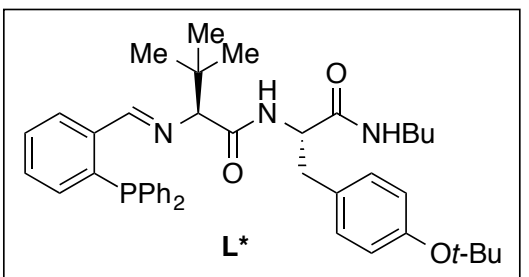

$\mathbf{L}^{*}$

Scheme 2.2: Hoveyda's results using peptidic phosphine ligands

The challenges associated with these additions have been well documented by Alexakis and coworkers, who clearly demonstrated that such additions are highly dependent on both the ligand and substrate structure. ${ }^{66}$ In 2004 , a significant breakthrough was made by Feringa et al., who described the use of Grignard nucleophiles in combination with a ferrocenyl diphosphine ligand. ${ }^{67}$ The authors showed it was possible to add a range of alkyl Grignard reagents with excellent 
enantioselectivities (Scheme 2.3).

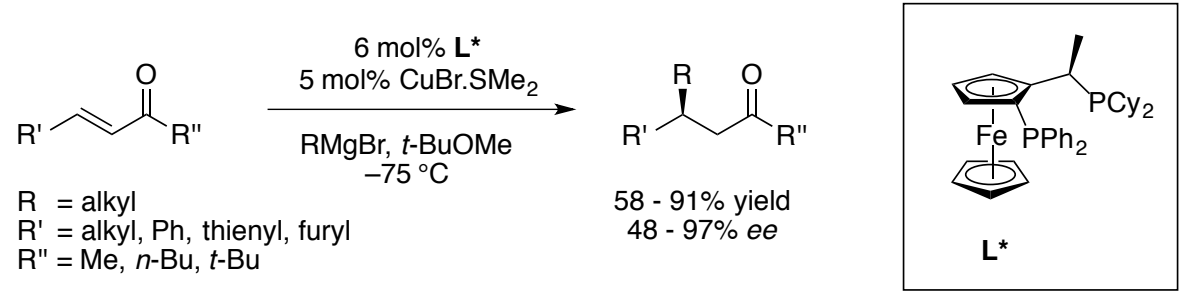

Scheme 2.3: Feringa's results using ferrocenyl diphosphine ligands

In 2006, Nakamura et al. reported the highly enantioselective addition of dimethyl- and diethylzinc reagents to varied acyclic enones when employing alanine-derived aminohydroxyphosphine ligands, with enantioselectivities exceeding $98 \%$ (Scheme 2.4$).{ }^{68}$

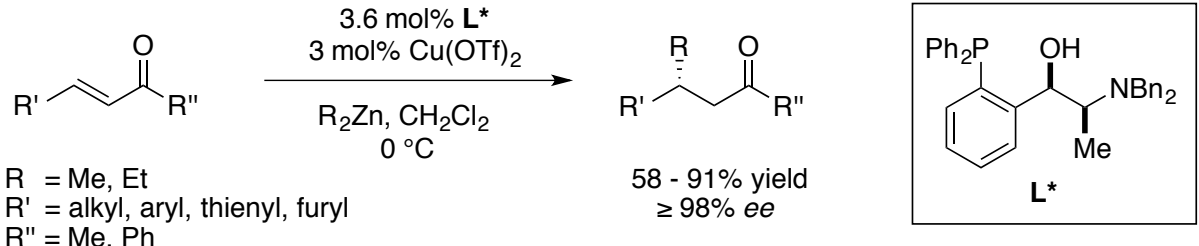

\section{Scheme 2.4: Nakamura's dialkylzinc addition}

The addition of pre-made organometallic reagents to acyclic enones continues to be an intensive area of research and as such, a number of developments have been made since this project began. In 2010, Štěpnička et al. showed that the addition of diethylzinc to rotationally restricted chalcone, using phosphanyl-ferrocenecarboxamide ligands with amino acid pendant groups gave enantioselectivities of $90 \%$, and full conversion within four hours. ${ }^{69}$ Similarly, Shibata et al. demonstrated the use of multinuclear BINOL derived catalysts to add diethylzinc to chalcone and its derivatives and achieved consistently excellent enantioselectivities and yields (Figure 2.1). ${ }^{70}$ 

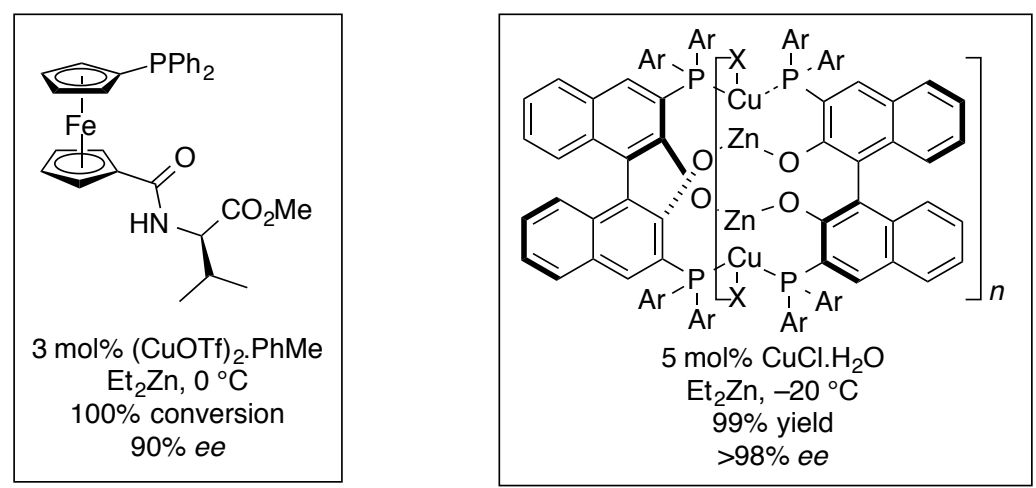

Figure 2.1: Štěpnička and Shibata's conditions for 1,4-addition to chalcone

There is continued focus on the addition of dialkylzinc reagents to acyclic enones. Sakaguchi et al. employed chiral NHC-azolium ligands with copper triflate benzene complex to gain enantioselectivities of up to $96 \%$ in up to $94 \%$ isolated yield. ${ }^{71}$ Mauduit et al. showed that diphenylphosphinoazomethinylate salts (DiPPAM) could be used as an alternative to the structurally similar peptidic ligands employed by Hoveyda (Scheme 2.2). ${ }^{65}$ Such ligands are more easily synthesised and provide the product in moderate to good yields, with enantioselectivities of up to $98 \% .^{72}$ Jurczak et al. investigated the use of sugar based monodentate phosphoramidite ligands, however, only obtained enantioselectivities up to $77 \%$ ee in additions to chalcone. ${ }^{73}$

In addition to Grignard and dialkylzinc reagents, highly reactive organoaluminium reagents have been used. ${ }^{8 a, c}$ Recently, Shibata et al. showed that the addition of trimethylaluminium to chalcone derived enones, catalysed by multinuclear complexes, resulted in enantioselectivities of up to $98 \%$ (Scheme 2.5).$^{74}$

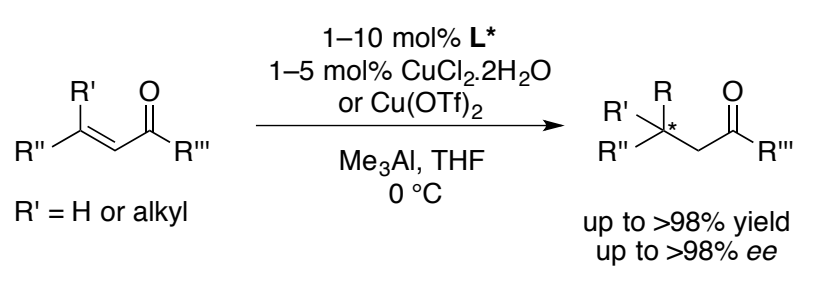

Scheme 2.5: Shibata's addition with trimethylaluminium

In summary, although excellent results have been obtained in a number of systems, all require the use of pre-made organometallic reagents, which can be difficult to handle. Furthermore, the 
outcome of these reactions is often highly sensitive to minor changes in conditions (solvent, temperature, rate of addition etc.). Thus, there is a need to develop new systems for asymmetric 1,4-addition reactions to acyclic enones, which do not rely on the use of pre-made organometallic reagents, and can be readily adapted to a variety of substrates.

\subsubsection{Results and Discussion}

\subsubsection{Initial optimisation}

The initial approach to this problem was to utilise knowledge gained from previous work on asymmetric copper-catalysed reactions involving organometallic reagents, to evaluate if the same conditions could be applied to alkylzirconium reagents. As the hydrometallation of alkenes with the Schwartz reagent followed by 1,4 -addition to yield racemic products had previously been described, ${ }^{57}$ the aim was to develop an asymmetric version of the reaction.

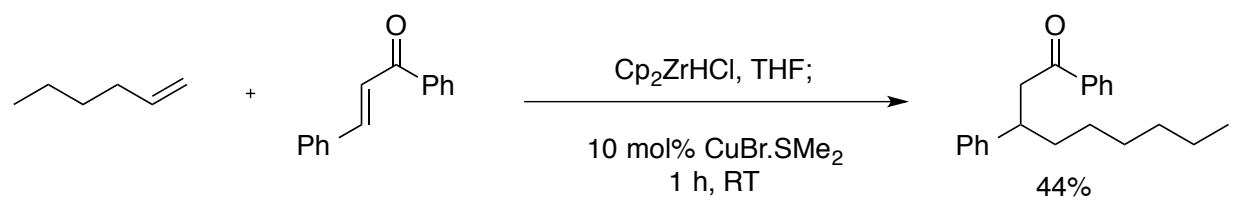

Scheme 2.6: Wipf's racemic addition

Our initial studies were carried out on the simple $\alpha, \beta$-unsaturated ketone, trans-4-phenyl-3buten-2-one. This enone was chosen as (i) it is readily available and (ii) contains a chromophore, which would enable determination of the enantioselectivity of the reaction by HPLC on a chiral non-racemic stationary phase. We decided to use 1-hexene, a simple, linear chain, unfunctionalised alkene as the coupling partner to minimise any electronic and steric interactions, which may bias the reaction. In addition, 1-hexene was used in the racemic 1,4 addition reactions of alkylzirconium species reported by Wipf et al. (Scheme 2.6). ${ }^{57,58}$

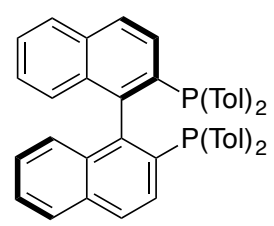

Tol-binap L1

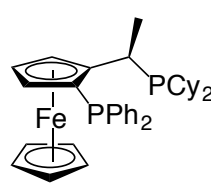

Josiphos L2

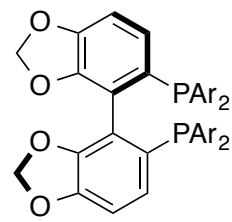

$\mathrm{Ar}=\mathrm{Ph} \mathbf{L} \mathbf{3}$ $\mathrm{Ar}=\mathrm{DTBM}$ L4

Figure 2.2: Initial screening ligands 
Tol-binap (L1) and JosiPhos (L2) were chosen as initial screening ligands (Figure 2.2); 1-hexene was hydrozirconated with the Schwartz reagent to form a hexylzirconocene species. Hydrometallation was complete when a clear homogeneous yellow solution was observed. This yellow reaction mixture was then transferred to a pre-stirred solution of $\mathrm{Cul}$ or $\mathrm{CuBr} . \mathrm{SMe}_{2}$ and the desired ligand. Continuous stirring of this mixture for at least one hour was carried out before addition of the enone. We were pleased to observe that in the majority of cases, the use of $\mathbf{L} \mathbf{1}$ and $\mathbf{L} \mathbf{2}$ in a variety of different solvents promoted the desired 1,4-addition (Table 2.1); however, only racemic products were obtained. Similarly, further attempts using the Segphos ligands L3 and L4 only resulted in racemic products; nevertheless such results indicated that reactivity could be achieved in our desired copper-catalysed system.

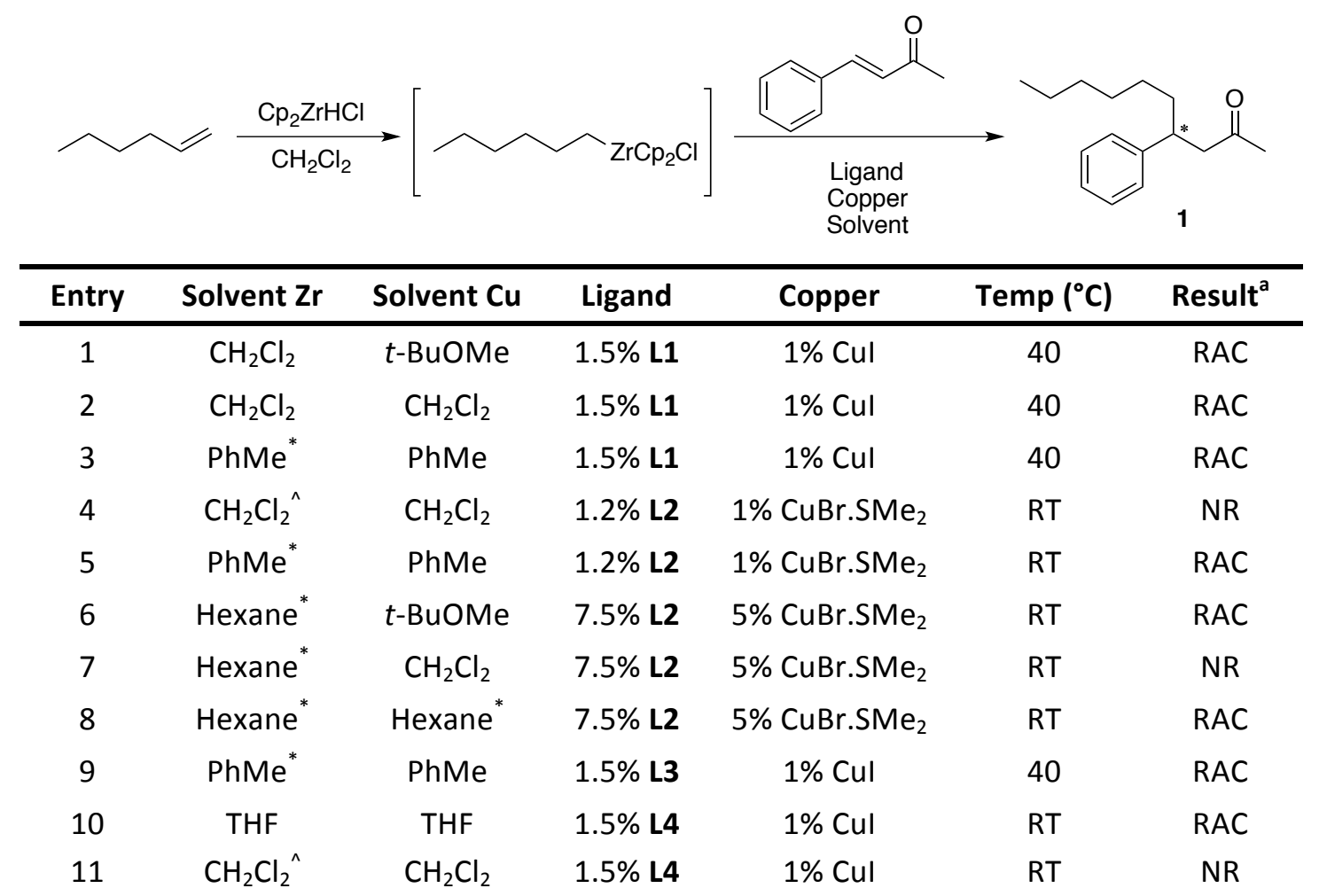

Conditions - 1-hexene (2.5 eq.), $\mathrm{Cp}_{2} \mathrm{ZrHCl}$ (2.0 eq.), trans-4-phenyl-3-buten-2-one (1.0 eq.). ${ }^{*} \mathrm{Hydrozirconation}$ in $\mathrm{CH}_{2} \mathrm{Cl}_{2}$ then the solvent removed by rotary evaporator under vacuum and replaced with noted solvent. ${ }^{\wedge}$ Hydrozirconation in THF then the solvent removed and replaced with noted solvent. a) ee determined by chiral HPLC. NR = no reaction.

Table 2.1: Initial screening 


\subsubsection{Synthesis of the Schwartz reagent}

During this initial screening, we found that commercially available Schwartz reagent was often suitable for use upon first purchase. However, the hydrozirconation reaction appeared to slow down over time and an insoluble white precipitate was often observed, rather than a homogeneous solution. Therefore, we found it was necessary to synthesise the Schwartz reagent using a procedure published by Buchwald and co-workers. ${ }^{75}$ This allowed storage of freshly prepared reagent in a Schlenk flask under argon, and more rigorous control of its exposure to air and moisture, thus improving reproducibility.

We have performed this preparation on up to $30 \mathrm{~g}$ of zirconocene dichloride, which consistently yielded high-quality reagent which hydrozirconated quickly. If stored in a proper manner (as mention above) this material also had a considerably longer shelf life than commercially available Schwartz reagent, therefore it was used in all further experiments detailed.

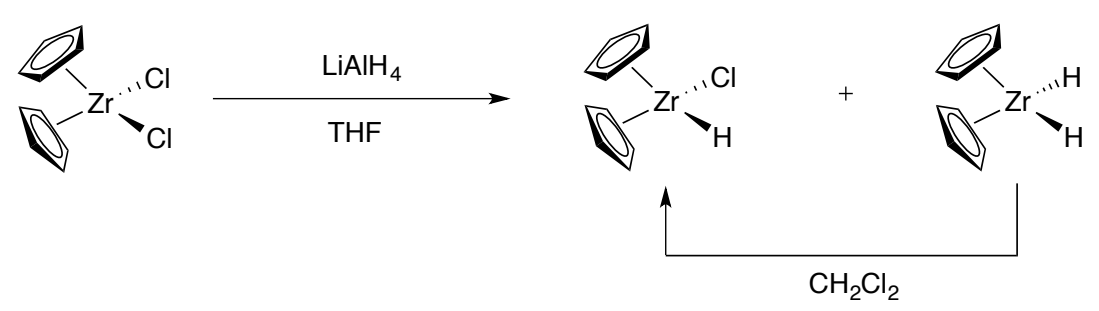

Scheme 2.7: Synthesis of the Schwartz reagent

\subsubsection{Copper screening}

Different copper sources were then examined in combination with L3 (chosen because it was readily available in the laboratory) in an attempt to obtain enantioselectivity in this reaction (Table 2.2). $\mathrm{CH}_{2} \mathrm{Cl}_{2}$ was used as the solvent for the hydrozirconation, as it appeared to increase the rate at this stage of the reaction. 


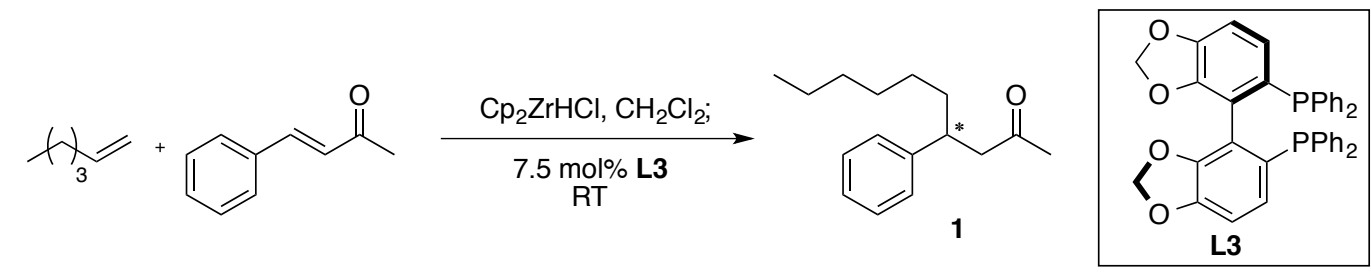

\begin{tabular}{|c|c|c|c|}
\hline Entry & Solvent $^{*}$ & Copper (mol\%) & Result $(\% \text { ee })^{a}$ \\
\hline 1 & PhMe & $5 \% \mathrm{CuBr}$ & NR \\
\hline 2 & PhMe & $5 \% \mathrm{CuCl}$ & RAC \\
\hline 3 & PhMe & $5 \% \mathrm{CuCN}$ & Complex mixture \\
\hline 4 & PhMe & $15 \% \mathrm{CuOAc}$ & RAC \\
\hline 5 & PhMe & $5 \%(\mathrm{CuOTf})_{2} \cdot \mathrm{C}_{6} \mathrm{H}_{6}$ & 7 \\
\hline 6 & PhMe & $7.5 \%(\mathrm{CuOTf})_{2} \cdot \mathrm{C}_{6} \mathrm{H}_{6}$ & 11 \\
\hline 7 & PhMe & $5 \% \mathrm{Cu}(\mathrm{OTf})_{2}$ & 3 \\
\hline 8 & $\mathrm{CH}_{2} \mathrm{Cl}_{2}$ & $15 \% \mathrm{CuBr} . \mathrm{SMe}_{2}$ & 3 \\
\hline 9 & $\mathrm{CH}_{2} \mathrm{Cl}_{2}$ & $15 \%\left[\left(\mathrm{CH}_{3} \mathrm{CN}\right)_{4} \mathrm{Cu}\right] \mathrm{PF}_{6}$ & 5 \\
\hline 10 & $\mathrm{CH}_{2} \mathrm{Cl}_{2}$ & $15 \% \mathrm{Cu}(\mathrm{TC})$ & 4 \\
\hline
\end{tabular}

Conditions - 1-hexene (2.5 eq.), $\mathrm{Cp}_{2} \mathrm{ZrHCl}$ (2.0 eq.), trans-4-phenyl-3-buten-2-one (1.0 eq.). ${ }^{*}$ Reactions involving PhMe involve the hydrozirconation being performed in $\mathrm{CH}_{2} \mathrm{Cl}_{2}$ then the solvent removed and replaced with toluene. a) ee determined by chiral HPLC. NR $=$ no reaction .

\section{Table 2.2: Screening different copper sources}

Initial screening with simple copper sources (entries $1-4$ ) yielded unpromising results until an excess of copper (compared to ligand) was used. By using a 1:0.75 ratio of copper to ligand (entry 5) we were able to achieve the first non-racemic result of $7 \%$ ee, however, further screening of copper sources provided limited improvements. In the case of diphosphine ligands, one copper atom is expected to be chelated between the two phosphine moieties, and these results suggested that this copper-binding mode was not effective at providing enantioselectivity. However, increasing the copper to diphosphine ligand ratio to $2: 1$ was found to improve enantioselectivity. This was particularly highlighted when copper triflate benzene complex was used with a 2:1 ratio of copper:ligand. $11 \%$ ee was obtained (entry 6$)$, suggesting that one copper for every phosphine moiety was necessary to obtain enantioselectivity. Using this optimal ratio, and L3 with copper triflate benzene complex, a variety of solvents were investigated (Table 2.3). 
$\mathrm{CH}_{2} \mathrm{Cl}_{2}$ gave the best result of $20 \%$ ee (entry 5) with no change in enantioselectivity when the temperature was lowered to $0^{\circ} \mathrm{C}$ (entry 6).

\begin{tabular}{|c|c|c|c|}
\hline & $\begin{array}{r}\mathrm{Cp}_{2} \mathrm{Z} \\
7.5 \\
7.5 \mathrm{~mol} \%\end{array}$ & & 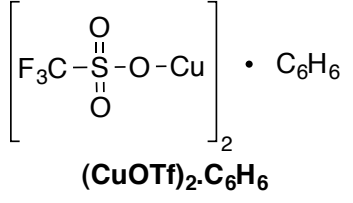 \\
\hline Entry & Solvent* & $\operatorname{Temp}\left({ }^{\circ} \mathrm{C}\right)$ & Result $(\% e e)^{a}$ \\
\hline 1 & PhMe & RT & 11 \\
\hline 2 & THF & RT & 7 \\
\hline 3 & $\mathrm{Et}_{2} \mathrm{O}$ & RT & 17 \\
\hline 4 & $t$-BuOMe & RT & 13 \\
\hline 5 & $\mathrm{CH}_{2} \mathrm{Cl}_{2}$ & RT & 20 \\
\hline 6 & $\mathrm{CH}_{2} \mathrm{Cl}_{2}$ & 0 & 20 \\
\hline
\end{tabular}

Conditions - 1-hexene (2.5 eq.), $\mathrm{Cp}_{2} \mathrm{ZrHCl}$ (2.0 eq.), trans-4-phenyl-3-buten-2-one (1.0 eq.) ${ }^{*}$ Hydrozirconation in $\mathrm{CH}_{2} \mathrm{Cl}_{2}$ then the solvent removed by rotary evaporator under vacuum and replaced with noted solvent. a) ee determined by chiral HPLC.

\section{Table 2.3: Solvent screen with copper triflate benzene complex}

\subsubsection{Synthesis of copper triflate benzene complex}

In order to obtain good results with copper triflate benzene complex, we found it necessary to synthesise the compound as opposed to purchase it from a commercial supplier. The commercial product is a brown/black solid, whereas freshly prepared copper triflate benzene complex is a white or off-white powder, which must be stored under argon in a Schlenk flask. The copper triflate benzene complex was synthesised on a regular basis, using a modified procedure by Solomon and co-workers: ${ }^{76}$ copper oxide was combined with triflic anhydride and heated in benzene under reflux until the red solution decolourised to a grey/brown colour (Scheme 2.8).

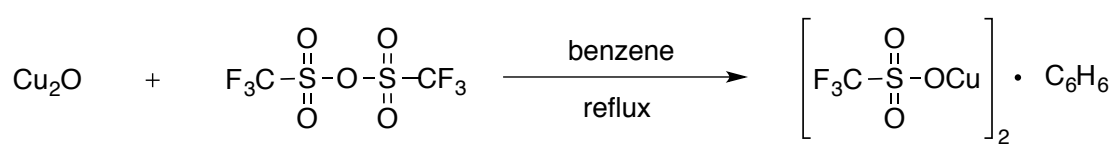

Scheme 2.8: Synthesis of copper triflate benzene complex 
The time taken for the solution to decolourise varied; this is attributed to the amount of adventitious triflic acid in the triflic anhydride. Upon addition of a catalytic amount of triflic acid, the reaction proceeded within one hour; far more rapidly than the uncatalysed reaction, which can take up to 24 hours. The white solid is air sensitive, and over time degraded to a green, and subsequently brown/black colour. On testing of the commercial and synthesised reagents, it was confirmed that commercial sources were of lesser quality unless used as soon as they were purchased (Table 2.4). It was necessary to store the complex under argon and weigh out the reagent very quickly to ensure the compound was not exposed to too much air. It was found that the compound was still effective if slightly yellow, however it was discarded when brown.

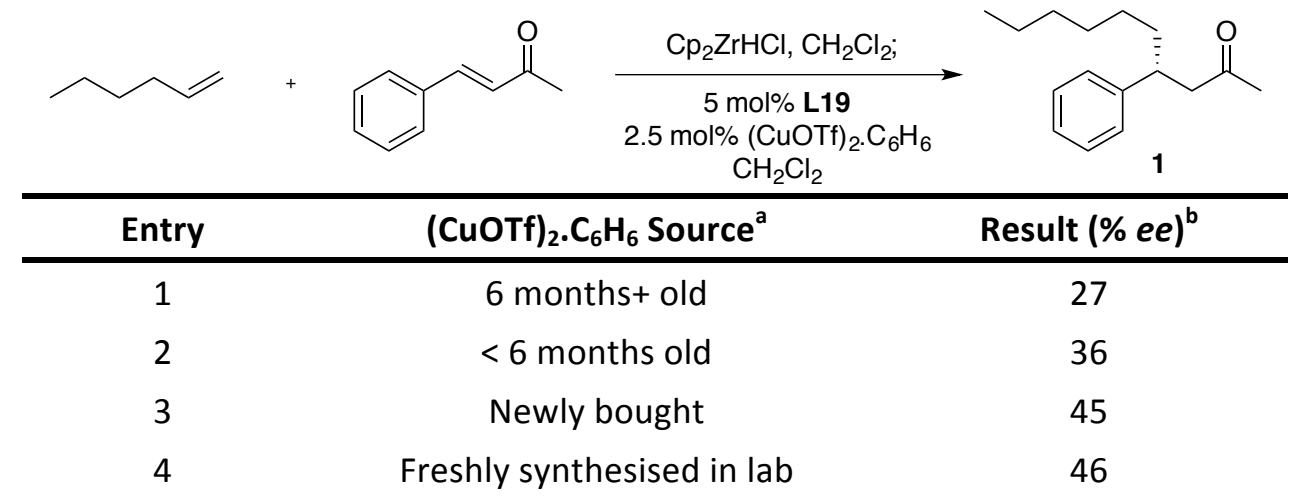

a) (CuOTf) $2 . \mathrm{C}_{6} \mathrm{H}_{6}$ purchased from Sigma Aldrich b) ee determined by chiral HPLC.

Table 2.4: Variation in quality of (CuOTf) ${ }_{2} \cdot \mathrm{C}_{6} \mathrm{H}_{6}$

\subsubsection{Ligand screening}

Using the current optimal conditions we found, we screened a variety of diphosphine and monophosphine ligands, as well as other potentially useful ligand classes. A number of the diphosphine ligands gave promising enantioselectivities: up to $24 \%$ ee was obtained with ligand $\mathbf{L 7}$ when a ratio of 2:1 copper to ligand was used (Figure 2.3). 


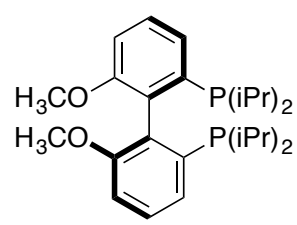

L5

Racemic

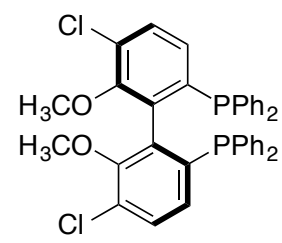

L8

$15 \%$ ee<smiles>Pc1ccccc1-c1c(-c2ccccc2)ccc2c1OCCO2</smiles>

$\stackrel{L 6}{18 \%}$ ee

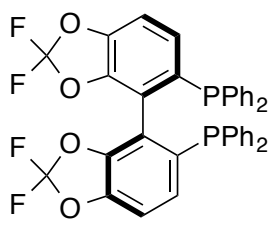

L9<smiles>COc1cc([Y20]#N)c(-c2c(OC)nc(OC)nc2OC)c(OC)c1</smiles>

L7

$24 \%$ ee

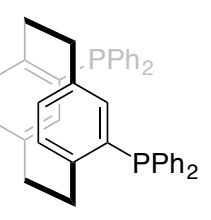

L10

Conditions -5 mol\% ligand and 5 mol\% (CuOTf) ${ }_{2} \cdot \mathrm{C}_{6} \mathrm{H}_{6}$ (1:2 ratio of ligand to copper).

Figure 2.3: Diphosphine ligands screened

A range of phosphorous-based ligand classes gave poor results when screened. For example, Quinap (L12), which is known to give good enantioselectivity in other asymmetric reactions, only resulted in $8 \%$ ee in our reaction (Figure 2.4).

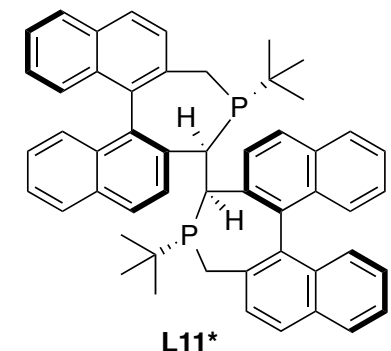

Racemic<smiles></smiles>

$\mathbf{L} 12$
$8 \%$ ee

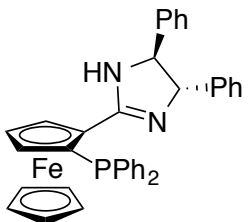

L13
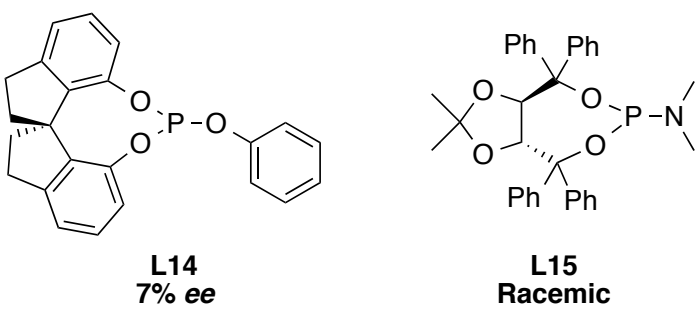

L15

Racemic

Conditions - 5 mol\% Ligand and 2.5 mol\% (CuOTf) ${ }_{2} \cdot C_{6} \mathrm{H}_{6}(1: 1$ ratio of ligand to copper) *5 mol\% Ligand and 5 mol\% $(\mathrm{CuOTf})_{2} \cdot \mathrm{C}_{6} \mathrm{H}_{6}(1: 2)$ was used in this case.

Figure 2.4: Miscellaneous ligands screened

In contrast, screening of phosphoramidite ligands (Figure 2.5) resulted in enantioselectivities of up to $45 \%$ ee (L19) when they were used in a 1:1 ratio with (CuOTf) ${ }_{2} \cdot \mathrm{C}_{6} \mathrm{H}_{6}$. Future screening efforts therefore focused on this ligand class. 


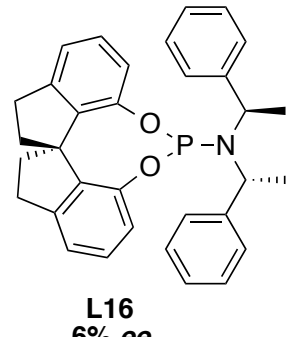

$6 \%$ ee

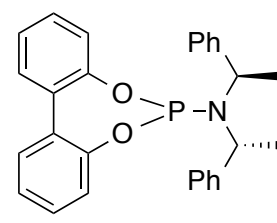

L17

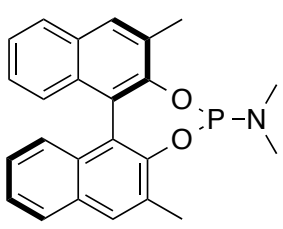

L18

Racemic

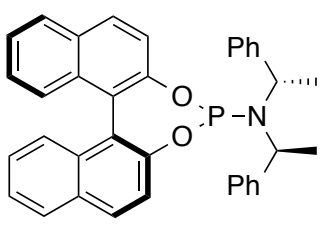

L19

Conditions -5 mol\% Ligand and 2.5 mol\% (CuOTf) $)_{2} \cdot C_{6} \mathrm{H}_{6}$ (1:1 ratio of ligand to copper).

Figure 2.5: A range of phosphoramidite ligands screened

\section{Phosphoramidite ligands}

Subsequently, different solvents were screened using the most successful ligand, L19. ${ }^{77}$ We found that upon screening, alkane and ethereal solvents gave poor results with $\mathrm{CH}_{2} \mathrm{Cl}_{2}$ remaining the best solvent for use (Table 2.5).

\begin{tabular}{ccc}
\hline Entry & Solvent & ${\text { Result (\% ee })^{\mathrm{a}}}^{\mathrm{C}}$ \\
\hline 1 & $\mathrm{CH}_{2} \mathrm{Cl}_{2}$ & 45 \\
2 & $\mathrm{Et}_{2} \mathrm{O}$ & 21 \\
3 & $t$-BuOMe & 25 \\
4 & Acetonitrile & $\mathrm{NR}$ \\
5 & DME & 30 \\
6 & Pentane & 17
\end{tabular}

Conditions - 1-hexene (2.5 eq.), $\mathrm{Cp}_{2} \mathrm{ZrHCl}$ (2.0 eq.), trans-4-phenyl-3-buten-2-one (1.0 eq.), (CuOTf) ${ }_{2} . \mathrm{C}_{6} \mathrm{H}_{6}$ (5 mol\%), Ligand $\mathbf{L 1 9}$ (5 mol\%). a) ee determined by chiral HPLC. NR $=$ no reaction

Table 2.5: Solvent screen with phosphoramidite L19

The ratio of ligand to copper was also re-investigated. We found that using $5 \mathrm{~mol} \%$ ligand and varying the copper ratio did not result in a clear correlation with enantioselectivity (Table 2.6). However, the experiment varied two significant parameters; ligand to copper ratio and also the copper loading. Therefore the experiments were repeated while controlling the copper loading (Table 2.7). This revealed a clear optimum ligand to copper ratio of 1:1 for a given copper loading (entry 4). 


\begin{tabular}{cccc}
\hline Entry & Ligand (mol\%) & Copper $(\mathbf{m o l} \%)$ & ${\text { Result }(\% \text { ee })^{\mathbf{a}}}^{\text {(m) }}$ \\
\hline 1 & 5 & 2.5 & 39 \\
2 & 5 & 5 & 45 \\
3 & 5 & 7.5 & 38 \\
4 & 5 & 10 & 45 \\
5 & 5 & 12.5 & 41
\end{tabular}

Conditions - 1-hexene (2.5 eq.), $\mathrm{Cp}_{2} \mathrm{ZrHCl}$ (2.0 eq.), trans-4-phenyl-3-buten-2-one (1.0 eq.), (CuOTf) $2 . \mathrm{C}_{6} \mathrm{H}_{6}$, Ligand L19 (5 mol\%). a) ee determined by chiral HPLC.

Table 2.6: Variation of the amount of copper

\begin{tabular}{cccc}
\hline Entry & Ligand (mol\%) & Copper (mol\%) & Result $(\% \text { ee })^{\text {a }}$ \\
\hline 1 & 1.25 & 5 & 18 \\
2 & 2.5 & 5 & 32 \\
3 & 3.75 & 5 & 35 \\
4 & 5 & 5 & $45^{\mathrm{b}}$ \\
5 & 6.25 & 5 & 35 \\
6 & 7.5 & 5 & 36 \\
7 & 10 & 5 & 36
\end{tabular}

Conditions - 1-hexene (2.5 eq.), $\mathrm{Cp}_{2} \mathrm{ZrHCl}$ (2.0 eq.), trans-4-phenyl-3-buten-2-one (1.0 eq.), (CuOTf) ${ }_{2} . \mathrm{C}_{6} \mathrm{H}_{6}$, Ligand L19 (5 mol\%). a) ee determined by chiral HPLC, b) data from table 2.6 entry 2.

Table 2.7: Variation of the amount of L19

Changes in enantioselectivity were investigated over time. The reaction was monitored over 48 hours using a 1:1 ligand to copper ratio and a 20 mol\% catalyst loading (Table 2.8). We were pleased to see that the enantioselectivity remained consistent throughout the reaction. The conversion was also measured and was found to stop at $85 \%$. In addition, when 20 mol\% ligand was used the enantioselectivity increased by ca. 10\% (55\% ee compared to $45 \%$ ee at 5 mol\%). 


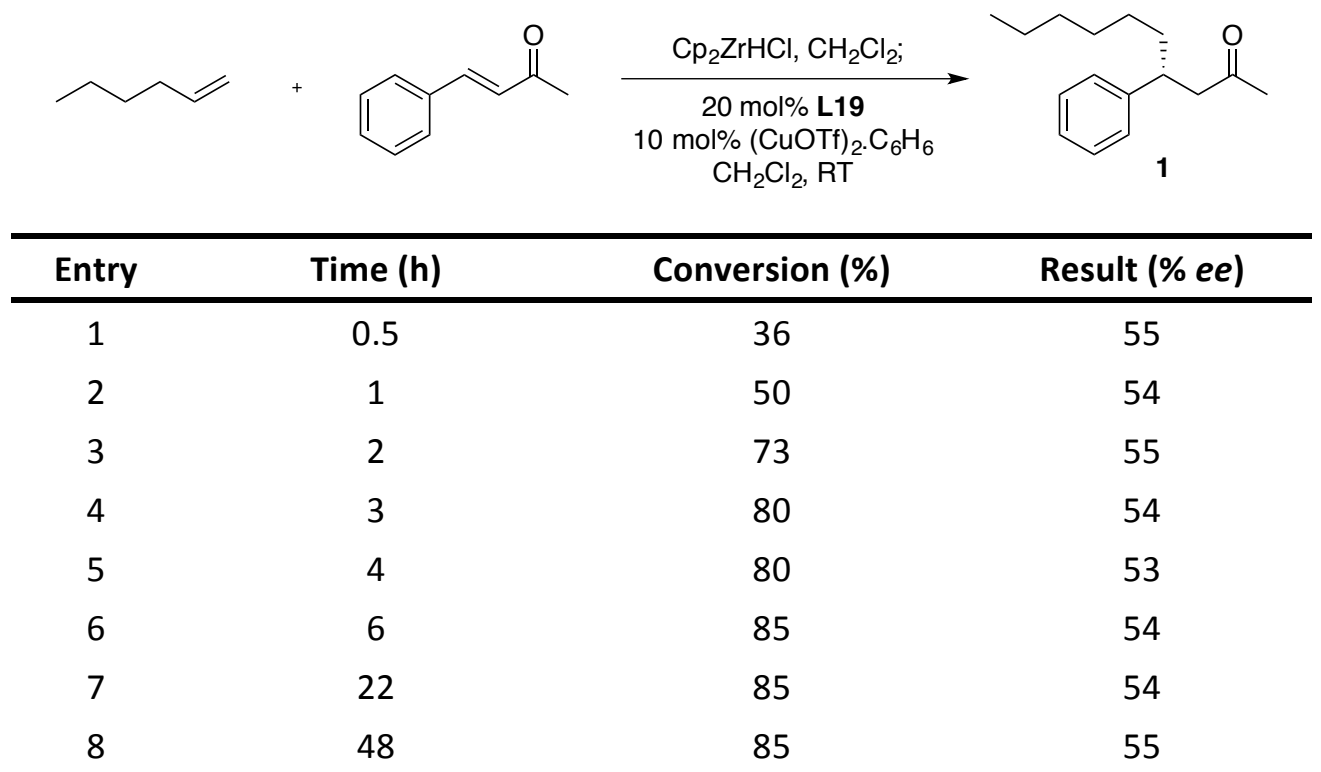

Conditions - 1-hexene (2.5 eq.), $\mathrm{Cp}_{2} \mathrm{ZrHCl}$ (2.0 eq.), trans-4-phenyl-3-buten-2-one (1.0 eq.). a) ee determined by chiral HPLC.

Table 2.8: Measuring enantioselectivity over time

Disappointingly, this suggested that this system was not optimal for the acyclic substrate. As previously discussed, $\alpha, \beta$-unsaturated acyclic ketones are known to be difficult substrates in asymmetric 1,4 -additions. This is likely due to the conformational flexibility in acyclic substrates, and their ability to undergo $s$-cis-s-trans interconversions (Figure 2.6). ${ }^{78}$

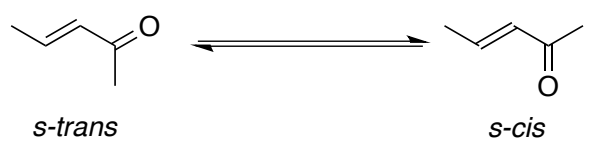

Figure 2.6: Interconversion of s-cis-s-trans

Utilising substrates with a fixed confirmation would eliminate this issue, which subsequently could lead to a highly enantioselective addition procedure. 2-Cyclohexenone has been extensively used as a substrate in asymmetric catalysis, and has a locked s-trans configuration. Thus we aim to develop a procedure suitable for cyclic substrates before continuing further investigations into the use of acyclic substrates. 


\subsection{Enantioselective 1,4-Addition to Cyclic Enones}

\subsubsection{Introduction}

After struggling to exceed $45 \%$ ee with the acyclic substrate, efforts were re-focused on the 1,4addition to cyclic substrates. As with the acyclic substrates, the majority of copper-catalysed conjugate addition reactions developed to cyclic enones involve the use of Grignard, dialkylzincs and organoaluminium reagents. The first Grignard reagents were used in asymmetric coppercatalysed conjugate additions in $1988^{79}$ and the first use of dialkylzinc reagents employing chiral phosphorous ligands was investigated by Alexakis et al. in $1993 .{ }^{80}$ Since this time, an extensive amount of work using dialkylzinc reagents has been carried out, and excellent enantioselectivities and yields have been achieved. ${ }^{8 a, c}$ In 1997, Feringa et al. obtained excellent enantioselectivities using phosphoramidite ligands and found phosphoramidite $L^{*}$ to be particularly effective (Scheme 2.9). ${ }^{77}$
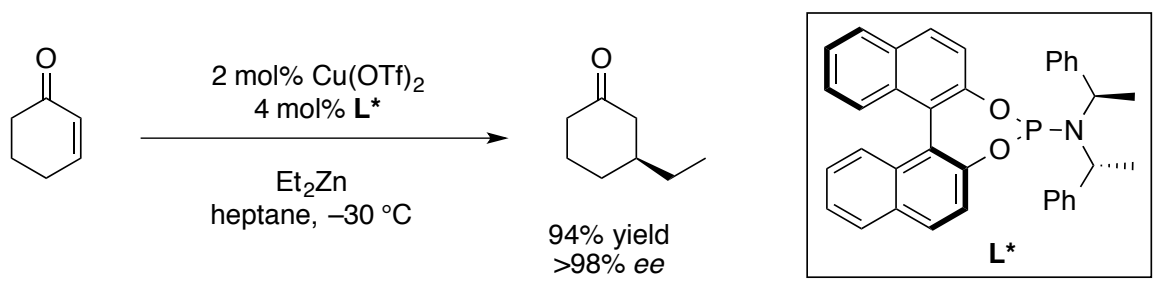

Scheme 2.9: Feringa's 1,4-addition to cyclic enones using phosphoramidite ligands

Pfaltz et al. showed that chiral binaphthyl phosphite ligands, bearing an oxazoline moiety, promoted good enantioselectivity with 5-, 6- and 7- membered cyclic enones (Scheme 2.10). ${ }^{81}$
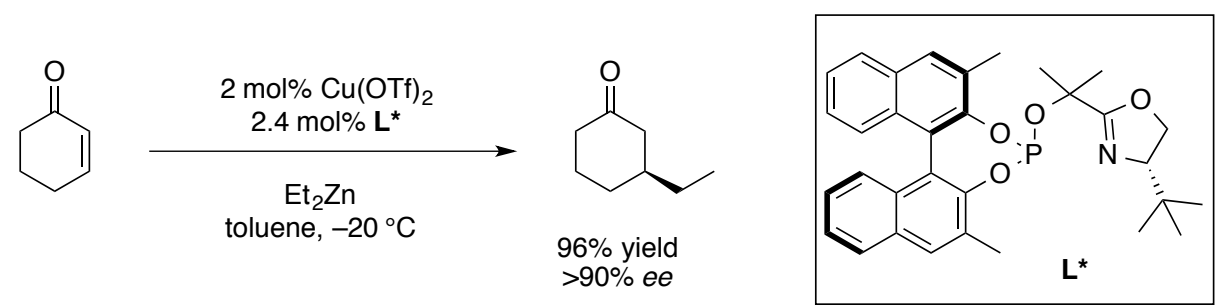

Scheme 2.10: Pfaltz's results using phosphite ligands

Hoveyda et al. used peptidic ligands in additions to cyclic enones; 5-, 6- and 7- membered enones were tolerated achieving excellent enantioselectivities and yields with a variety of dialklyzinc reagents (Scheme 2.11). ${ }^{82}$ 


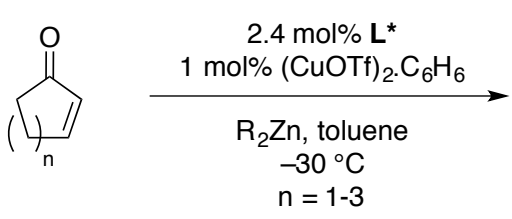

$$
\begin{gathered}
1 \mathrm{~mol} \%(\mathrm{CuOTf})_{2} \cdot \mathrm{C}_{6} \mathrm{H}_{6} \\
\underset{\mathrm{R}_{2} \mathrm{Zn}, \text { toluene }}{2.4 \mathrm{~mol} \%} \\
\begin{array}{c}
-30 \mathrm{C} \\
\mathrm{n}=1-3
\end{array}
\end{gathered}
$$

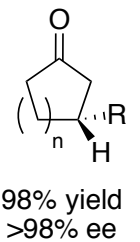

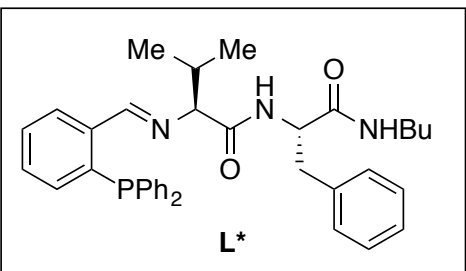

Scheme 2.11: Hoveyda's results using peptidic ligands

Thus, although excellent enantioselectivities were obtained with dialkylzinc reagents, only limited success was achieved with Grignard reagents until 2004, when Feringa et al. reported the use of ferrocenyl diphosphine ligands. The authors showed that up to $96 \%$ ee could be obtained in additions with ethyl magnesium bromide in diethyl ether at $0^{\circ} \mathrm{C}$ (Scheme 2.12)..$^{83}$

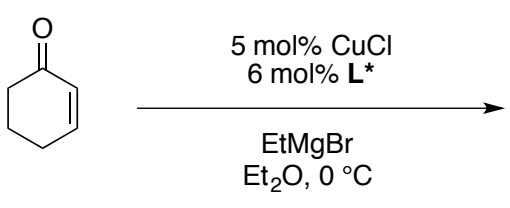

$$
\text { 95:5 1,4 vs. } 1,2 \text { addition }
$$$$
\begin{aligned}
& 69 \% \text { yield } \\
& 96 \% \text { ee }
\end{aligned}
$$

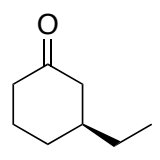

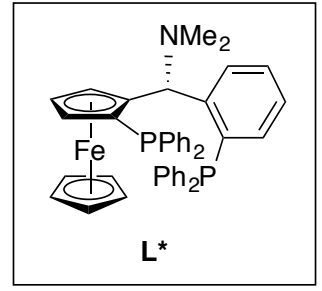

Scheme 2.12: Feringa's use of ferrocenyl ligands with Grignard reagents

Typically, the limitations faced in addition reactions to cyclic enones are similar to those faced in additions to acyclic enones. There are a number of difficulties associated with the need to use reactive pre-made organometallic reagents including, in most cases, a need for cryogenic temperatures and generally poor functional group tolerance, which are issues addressed in our research.

\subsubsection{Results and Discussion}

\subsubsection{Initial optimisation}

Although there was still motivation to study coupling reactions between simple building blocks, particularly commercially available materials, simple cyclic enones such as 2-cyclohexenone do not possess a useful chromophore for HPLC analysis. Therefore, upon addition of our simple alkene, 1-hexene, the ability to determine enantioselectivity would be a problem. This was resolved by employing a method from Alexakis whereby $(R, R)$-DPEN is used to form 
diastereomers (3). ${ }^{84}{ }^{13} \mathrm{C}$ NMR spectroscopy can then be used to determine the enantioselectivity; which enabled the use of the desired unfunctionalised straight chain alkene (Scheme 2.13).

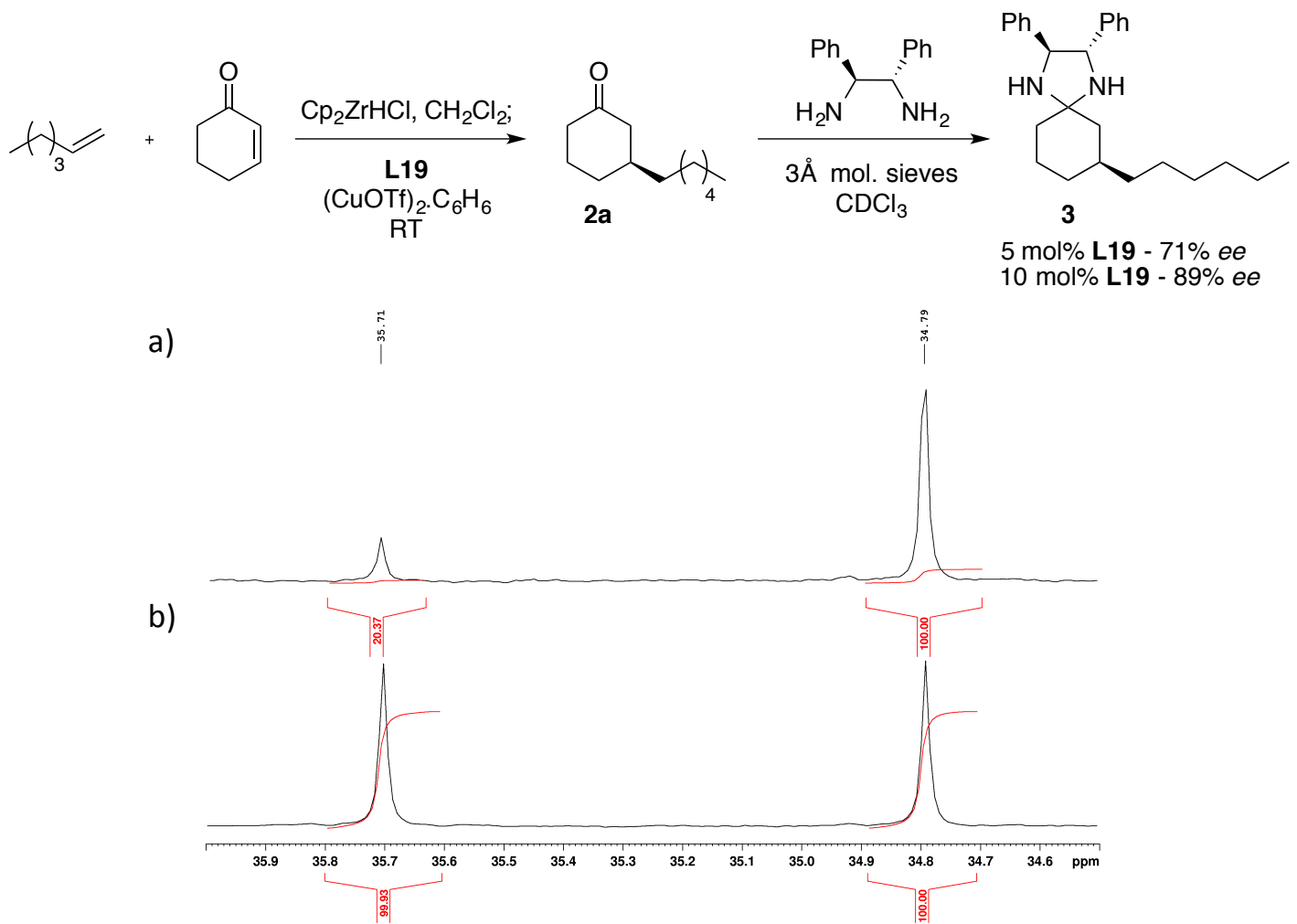

Scheme 2.13: ${ }^{13} \mathrm{C}$ NMR of derivatised ketone $2 \mathrm{a}$ : a) $71 \%$ ee, b) racemate

Optimal conditions from the acyclic system were employed and gave pleasing results. $71 \%$ ee was obtained when 5 mol\% of catalyst was used. Increasing the catalyst loading to 10 mol\% produced a highly acceptable $89 \%$ ee.

\subsubsection{Optimisation of enantioselectivity}

After successfully obtaining high enantioselectivity using a combination of phosphoramidite L19 and copper triflate benzene complex in the addition to 2-cyclohexenone at room temperature, efforts were made to further improve the enantioselectivity. A wide range of solvents was screened (Table 2.9) and remarkably in contrast to comparable asymmetric conjugate addition reactions, ${ }^{8}$ we found our reaction tolerated a variety of solvents while maintaining good levels of enantioselectivity. The majority of solvents screened resulted in greater than $75 \%$ ee with only benzene and bromobenzene giving lower levels of enantioselectivity (entries $7 \& 8$ ). We found 
our original solvent, $\mathrm{CH}_{2} \mathrm{Cl}_{2}$, continued to produce the best results, however, similar levels of selectivity were obtained with diglyme and 2-MeTHF (entries 1, 13 \& 16).
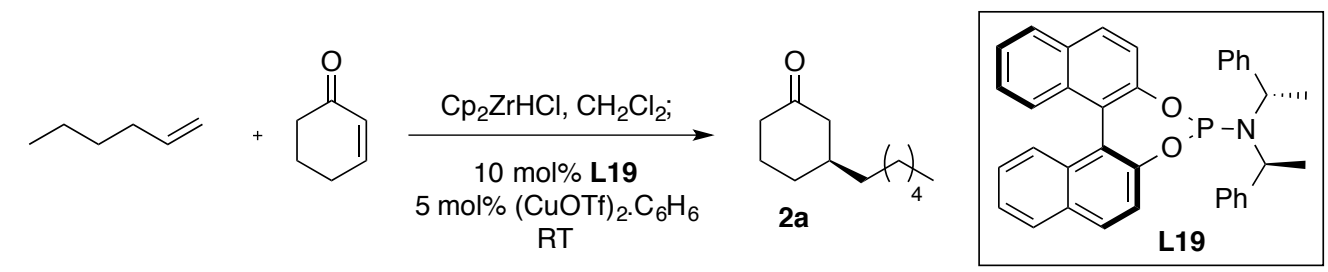

\begin{tabular}{ccc}
\hline Entry & Solvent & ${\text { Result }(\% e e)^{\mathrm{a}}}^{\mathrm{a}}$ \\
\hline 1 & $\mathrm{CH}_{2} \mathrm{Cl}_{2}$ & 89 \\
2 & $\mathrm{Et}_{2} \mathrm{O}$ & 85 \\
3 & $t$-BuOMe & 84 \\
4 & Toluene & 83 \\
5 & Hexane & 75 \\
6 & THF & 80 \\
7 & Benzene & 40 \\
8 & Bromobenzene & 50 \\
9 & 1,2-Dichloroethane & 75 \\
10 & $m$-Xylene & 75 \\
11 & Chloroform & 80 \\
12 & 1,4-Dioxane & 75 \\
13 & Diglyme & 89 \\
14 & Diisopropyl ether & 84 \\
15 & 2,2-Dimethoxypropane & 86 \\
16 & 2-MeTHF & 88
\end{tabular}

Conditions - 1-hexene (2.5 eq.), $\mathrm{Cp}_{2} \mathrm{ZrHCl}$ (2.0 eq.), 2-cyclohexenone (1.0 eq.). a) ee determined by derivatisation then ${ }^{13}$ CNMR.

Table 2.9: Solvent screen

Using $\mathrm{CH}_{2} \mathrm{Cl}_{2}$, diglyme, 2-MeTHF, and THF, further optimisation of the enantiomeric excess was carried out by varying the temperature (Table 2.10). We found that $\mathrm{CH}_{2} \mathrm{Cl}_{2}$ and diglyme gave optimal results at room temperature; however, in the case of THF, lowering the temperature to $5{ }^{\circ} \mathrm{C}$ produced $92 \%$ ee (entry 7 ), and with $2-\mathrm{MeTHF}$ at $10{ }^{\circ} \mathrm{C}$ gave our best enantioselectivity of $94 \%$ ee (entry 12). The ability to produce good results in a range of solvents and different temperatures is a huge advantage for these methods; different compounds can exhibit different solubility and reactivity profiles thus the ability to change reaction conditions without detrimental effects on enantioselectivity are very beneficial. 


\begin{tabular}{cccc}
\hline Entry & Solvent & Temperature $\left({ }^{\circ} \mathrm{C}\right)$ & Result $(\% \text { ee })^{\text {a }}$ \\
\hline 1 & $\mathrm{CH}_{2} \mathrm{Cl}_{2}$ & 0 & 68 \\
2 & $\mathrm{CH}_{2} \mathrm{Cl}_{2}$ & 5 & 83 \\
3 & $\mathrm{CH}_{2} \mathrm{Cl}_{2}$ & 10 & 78 \\
4 & $\mathrm{CH}_{2} \mathrm{Cl}_{2}$ & 30 & 84 \\
5 & $\mathrm{CH}_{2} \mathrm{Cl}_{2}$ & 40 & 73 \\
6 & $\mathrm{THF}$ & 0 & 40 \\
7 & $\mathrm{THF}$ & 5 & 92 \\
8 & $\mathrm{THF}$ & 10 & 56 \\
9 & $\mathrm{THF}$ & 30 & 79 \\
10 & $\mathrm{THF}$ & 40 & 71 \\
11 & $2-\mathrm{MeTHF}$ & 5 & 93 \\
12 & $2-\mathrm{MeTHF}$ & 10 & 94 \\
13 & $2-\mathrm{MeTHF}$ & 40 & 73 \\
14 & Diglyme & 0 & 14 \\
15 & Diglyme & 40 & 75
\end{tabular}

Conditions - 1-hexene (2.5 eq.), $\mathrm{Cp}_{2} \mathrm{ZrHCl}$ (2.0 eq.), 2-cyclohexenone (1.0 eq.), (CuOTf) ${ }_{2} . \mathrm{C}_{6} \mathrm{H}_{6}$ (5 mol\%), $\mathrm{L19}$ (10 mol\%). a) ee determined by derivatisation then ${ }^{13} \mathrm{C} N \mathrm{NM}$.

\section{Table 2.10: Temperature screen}

After observing successful conditions for the addition of 1-hexene to 2-cyclohexenone we decided to continue investigations with $\mathrm{CH}_{2} \mathrm{Cl}_{2}$ at room temperature. The decision was due to room temperature being the most useful and easily attained temperature in industry.

\subsubsection{Synthesis of phosphoramidite ligands}

Given the excellent observed results with phosphoramidite ligands, we turned our attention to the synthesis of these ligands. The need to synthesise the phosphoramidite ligands was not only due to the possibility of producing structurally different ligands, but also the cost of such ligands. L19 is commercially available at $f 198$ for $500 \mathrm{mg}$, therefore under screening conditions, this ligand was very expensive. The synthesis involved coupling a binaphthyl backbone, BINOL (4) and a secondary amine (5) (Scheme 2.14). 


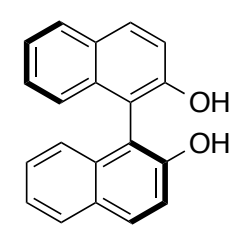

4

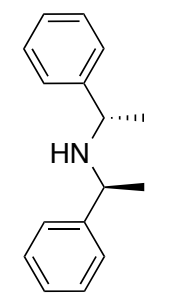

5

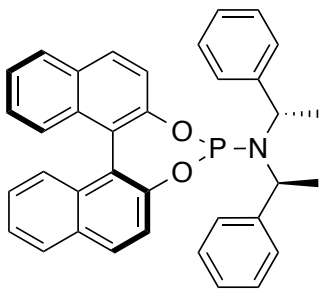

L19

Scheme 2.14: General synthesis of phosphoramidite ligands

The secondary amines required for the ligand synthesis were synthesised in two ways, depending on the number of stereocentres present in the amine product. Both methods begin by coupling a primary amine and a ketone. In order to obtain amine $\mathbf{5}$, where two stereocentres are present, a modified procedure of Alexakis et al. was followed where titanium(IV) isopropoxide is used to condense the amine 6 and ketone 7, followed by hydrogenation over $\mathrm{Pd} / \mathrm{C}$ and then recrystallisation to obtain the desired diastereomer (Scheme 2.15). ${ }^{85}$<smiles>C[C@H](N)c1ccccc1</smiles>

6<smiles>CC(=O)c1ccccc1</smiles>

7

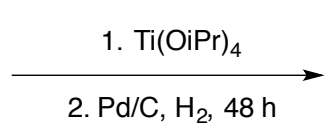

2. $\mathrm{Pd} / \mathrm{C}, \mathrm{H}_{2}, 48 \mathrm{~h}$<smiles>C[C@H](N[C@@H](C)c1ccccc1)c1ccccc1</smiles>

5

Scheme 2.15: Synthesis of the amine portion with two stereocentres

The second method is based on the procedures of RajanBabu and Davies ${ }^{86,87}$ and has been used for the synthesis of numerous amines containing one or zero stereocentres. It involves combining sodium triacetoxyborohydride with, for example, amine $\mathbf{8}$ and ketone $\mathbf{9}$, and results in the desired amine 10 in one step (Scheme 2.16).

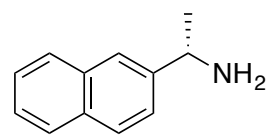

8<smiles>O=C1CCCC1</smiles>

9

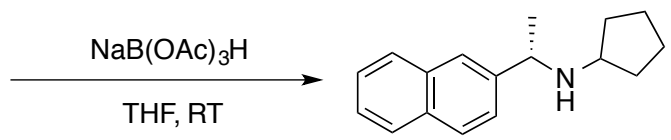

10

Scheme 2.16: Synthesis of the amine portion with one stereocentre

The second method was preferred due to its simplicity; there is no need to separate diastereomers, as there is only one stereocentre, which is fixed in the starting material. Once the secondary amines were obtained, it was then possible to use them in the synthesis of the desired ligands. These were obtained using two different methods. Feringa et al. reported the first 
method; $\mathrm{BINOL} 4$ was combined with $\mathrm{PCl}_{3}$ to form phosphorchloridite $\mathbf{1 1}$ before addition to a solution of the $n$-BuLi deprotonated secondary amine 12, and stirred overnight (Scheme 2.17). ${ }^{88}$<smiles>Oc1ccc2ccccc2c1-c1c(O)ccc2ccccc12</smiles>

4

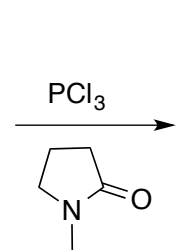<smiles>[O-]c1ccc2ccccc2c1-c1c(O)ccc2ccccc12</smiles>

11

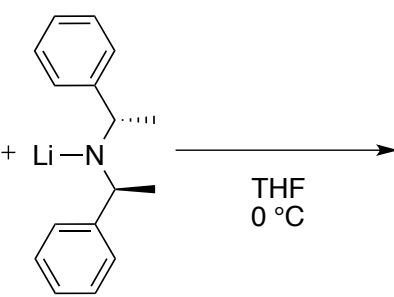

12

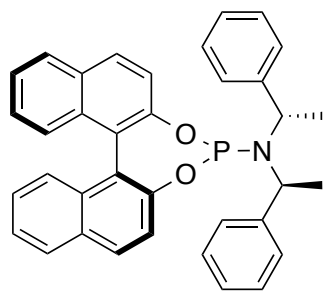

L19

Scheme 2.17: Feringa's method for synthesis of phosphoramidite L19

Although reliable, this method only obtained a maximum of $50 \%$ yield in our hands. In addition, two steps were required as the phosphorchloridite $\mathbf{1 1}$ must be isolated, before addition to the amine 12.

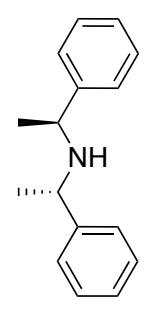

5

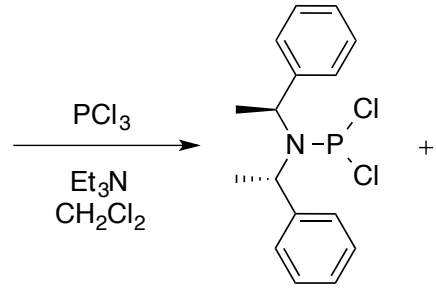

13

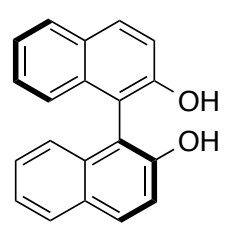

4

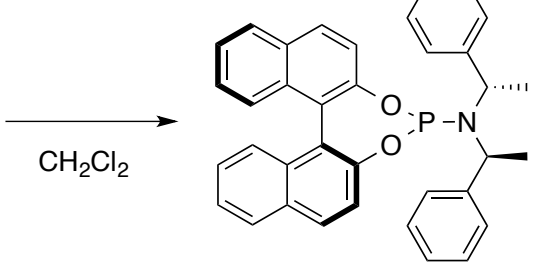

L19

Scheme 2.18: Trost's method for synthesis of phosphoramidite L19

The second method used was a modified procedure by Trost et al., where $\mathrm{PCl}_{3}$ was mixed with triethylamine before the addition of the amine 5 to give the nitrogen-phosphorus bond (13) in situ. Subsequently, BINOL 4 was added and the mixture stirred at room temperature overnight (Scheme 2.18). ${ }^{89}$ This method produced higher yields (70\%) and required only a single synthetic step. A number of ligands were synthesised using these methods, with some examples given below (Figure 2.7). 


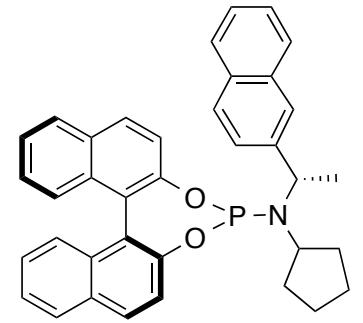

L20

$39 \%$ yield

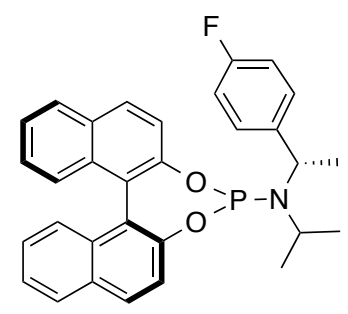

L23

$41 \%$ yield

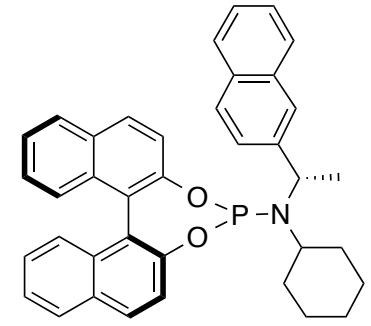

L21

$16 \%$ yield

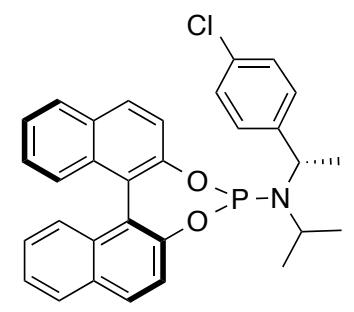

L24

$43 \%$ yield
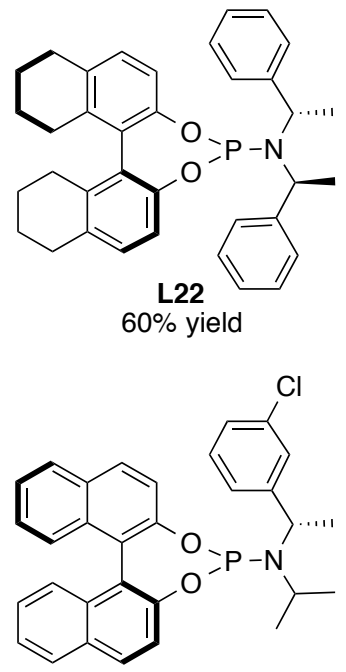

L25

$52 \%$ yield

Figure 2.7: Phosphoramidite ligands synthesised

\subsubsection{Phosphoramidite screening}

A number of screening reactions were undertaken using 1-hexene as the nucleophilic coupling partner, however, the requirement to derivatise and analyse these products by ${ }^{13} \mathrm{C} \mathrm{NMR}$ spectroscopy was time consuming. We rationalised that by changing the alkene to 4-phenyl-1butene, analysis of the products by HPLC could be carried out using a chiral non-racemic stationary phase. On trialling this alkene, it was found that using the previous conditions, $80 \% e e$ compared to $89 \%$ ee with 1-hexene, was achieved (Scheme 2.19). As this was successful, further screening reactions were carried out with 4-phenyl-1-butene.

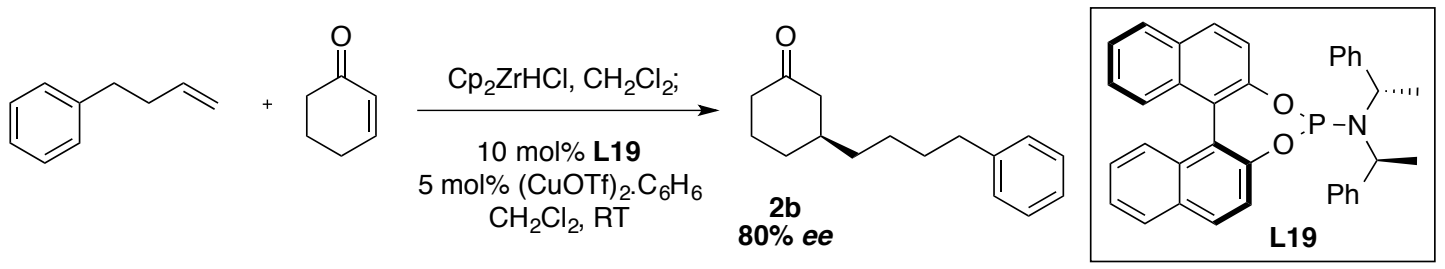

Scheme 2.19: Screening with 4-phenyl-1-butene

A variety of phosphoramidite ligands had thus been prepared, providing a reasonable opportunity to screen ligands. In total, over 60 different phosphoramidite ligands were screened (Appendix A2). A 1:1 copper to ligand ratio was used in all cases. Taking direction from phosphoramidite L19, 
it was first decided to vary the BINOL backbone; it was found that in general, adding or removing bulk from the backbone were detrimental to the enantioselectivity (Figure 2.8). Partial dehydrogenation of the BINOL backbone (L22) produced $68 \%$ ee, similar to that of adding a methyl group to the $3,3^{\prime}$ position ( $\mathbf{L} 28,65 \%$ ee). Adding any bulkier moieties to the $3,3^{\prime}$ position were found to decrease the enantioselectivity considerably (L29, 32\% ee); addition of $\mathrm{Br}$ atoms in the 3, 3' and 7, 7' position (L30 \& L31) gave slightly lower enantioselectivities ( 28 and $67 \%$ ee, respectively).<smiles>C[C@H](c1ccccc1)N([C@H](C)c1ccccc1)P1Oc2ccccc2O1</smiles><smiles>CC(c1ccccc1)N(Pc1ccccc1)[C@@H](C)c1ccccc1-c1ccccc1</smiles>
$\stackrel{\text { L26 }}{17 \% e e}$<smiles>Cc1cc2ccccc2c(-c2c(OP(P)N(Cc3ccccc3)C(C)c3ccccc3)c(C)cc3ccccc23)c1O</smiles>
$\stackrel{\text { L28 }}{65 \% e e}$<smiles>Oc1c(-c2ccccc2)cc2ccccc2c1-c1c(O)c(-c2ccccc2)cc2ccccc12</smiles>

$$
{ }_{32 \% e e}^{\mathbf{L} 29}
$$<smiles>CC(c1ccccc1)N(Pc1ccc2c(c1Oc1cccc3c1CCCC3)CCCC2)C(C)c1ccccc1</smiles>
L22 $68 \%$ ee<smiles>CC(c1ccccc1)N(P)C(C)c1ccccc1</smiles>
L30
$28 \%$ ee<smiles>CC(c1ccccc1)N(C(C)c1ccccc1)P(Oc1c(-c2ccccc2)c(-c2ccccc2)cc2ccccc12)c1ccccc1</smiles><smiles>CC(c1ccccc1)N([C@H](C)c1ccccc1)P(Oc1ccc2ccc(Br)cc2c1)c1cccc2ccc(Br)cc12</smiles>

$\stackrel{\text { L31 }}{67 \% e e}$

Figure 2.8: Variation of phosphoramidite backbone

With these results in hand, we decided that the original BINOL backbone would be maintained and the amine moiety changed. In this section, the ligands are described only by the amine moiety (Figure 2.9).

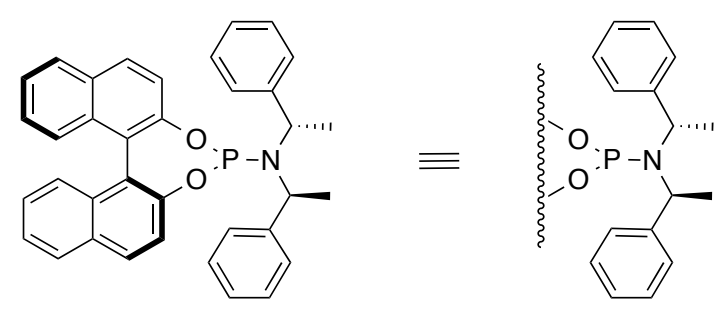

Figure 2.9: Abbreviated ligand

Slight alterations of the amine were initially tested; the mismatch (L32), $((S, R, R)$ instead of $(S, S$, S)), of L19 was found to give slightly lower enantioselectivity, suggesting that the matched ligand was better suited for our reaction. Thus, a series of matched ligands were synthesised, i.e. $(S, S, S)$ 
or $(R, R, R)$ and $(S, S)$ or $(R, R)$, this stereochemistry is the case for all the ligands shown below unless specified otherwise.
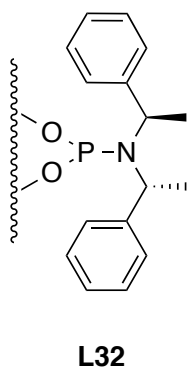

$(S, R, R)$

$74 \%$ ee

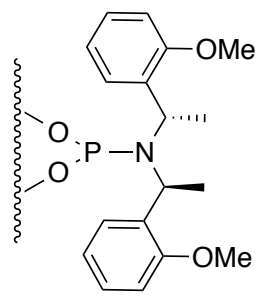

L33

$43 \%$ ee
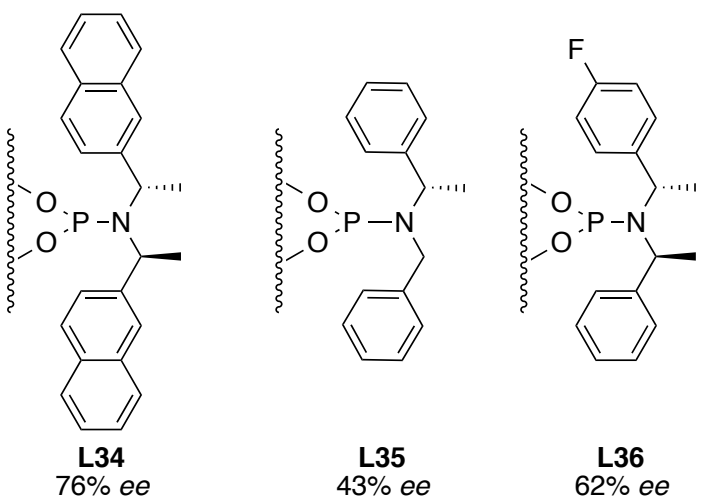

Figure 2.10: Alterations of amine from L19

From slight alterations to the amine (Figure 2.10) we found that adding methoxy groups at the ortho-position gave moderate enantioselectivity (L33, $43 \%$ ee), and changing the phenyl rings to 2-naphthyl groups gave 76\% ee (L34), which did not exceed previous results (Scheme 2.19). Small changes were observed to induce a major effect; for example, removing one of the two methyl groups from the amine lead to a significant decrease in enantioselectivity (L35, $43 \%$ ee). We next decided to remove any asymmetry from the amine, and use simple alkyl based units (Figure 2.11). In all cases, the enantioselectivity was lowered. However, a broad trend was observed with the size of the unit: smaller amines gave poor enantioselectivity, and similarly, if the size of the amine substituents was too large, enantioselectivity also decreased.

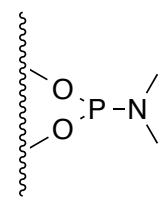

L37
$17 \%$ ee

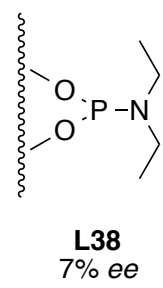

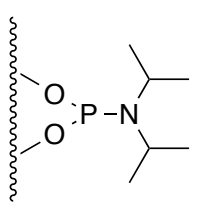

$\mathbf{L} 39$
$37 \%$ ee
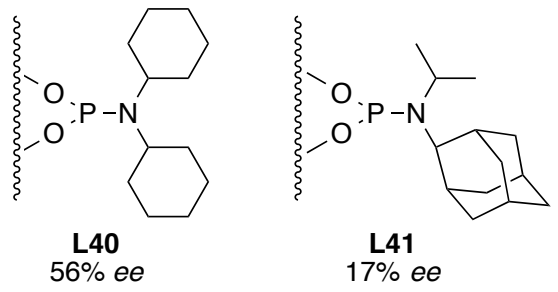

Figure 2.11: Ligands with no stereocentres on the amine

After the screening of a wide variety of ligands (synthesised by P. M. C. Roth), it was discovered that an amine with a 2-naphthyl group on one side and isopropyl on the other resulted in promising enantioselectivity (68\% ee) (L42 Figure 2.12). Such ligands were further investigated 
due to the relative ease of their synthesis. In this series of ligands, only one stereocentre was present, which removes the need to purify the desired diastereomer by recrystallisation.
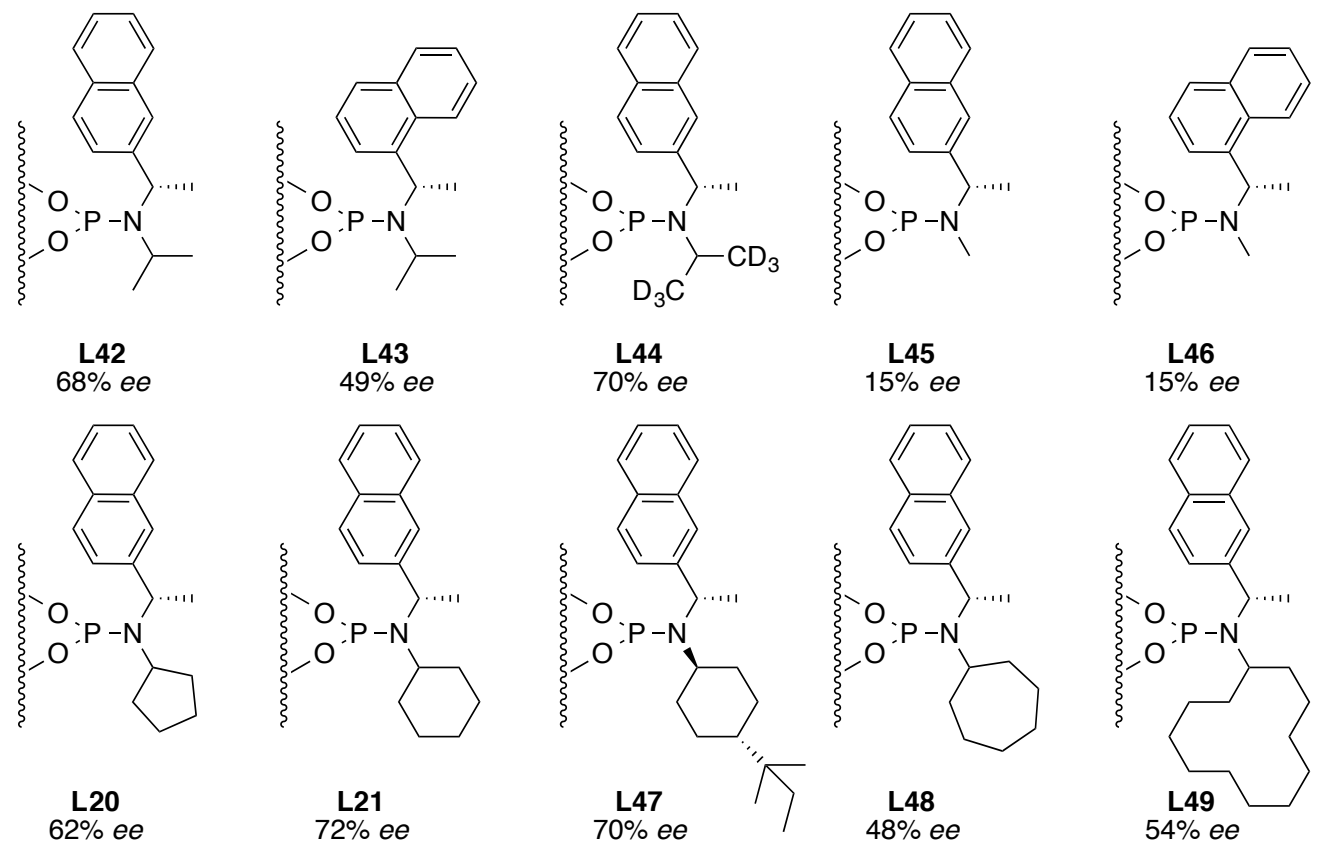

Figure 2.12: Ligands with a naphthyl- unit on the amine

With this promising enantioselectivity in hand, a variety of ligands of this type were tested (Figure 2.12); 1-naphthyl instead of 2-naphthyl gave poorer results, therefore 2-naphthyl was used in the majority of examples. When deuterated isopropyl (L44) instead of non-deuterated isopropyl was used, there was a slight increase in enantioselectivity. On changing the isopropyl group to a methyl group (L45 \& L46), the enantioselectivity decreased drastically. Different ring sizes were also tested in place of the isopropyl unit. Use of a cyclopentane ring (L20) gave $62 \%$ ee. Enantioselectivity was improved by increasing the ring size to cyclohexane (L21) (72\% ee), and no significant change was observed when bulk was added to the cyclohexane ring (L47). However, further increasing the ring size had a detrimental effect on the enantioselectivity (L48 \& L49). 

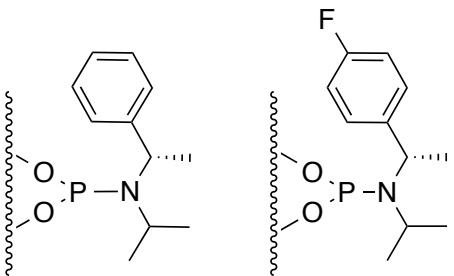

$$
\begin{gathered}
\text { L50 } \\
51 \% \text { ee }
\end{gathered}
$$

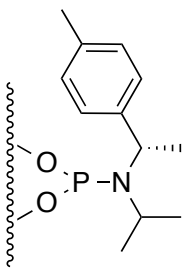

$\mathbf{L 5 2}$
$64 \%$ ee

$$
\begin{gathered}
\text { L23 } \\
60 \% \text { ee }
\end{gathered}
$$

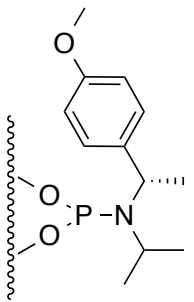

L53
$72 \%$ ee

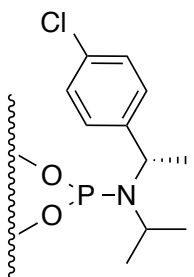

L24
$67 \%$ ee

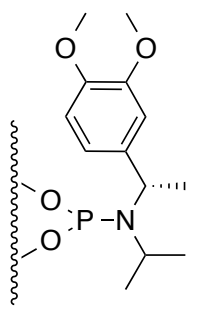

L54
$58 \%$ ee

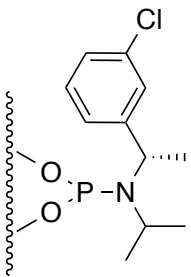

L25
$65 \%$

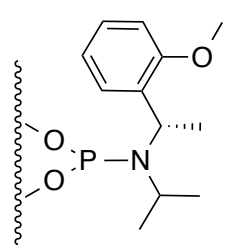

$\mathbf{L 5 5}$
$66 \%$ ee

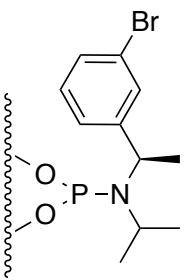

L51
$67 \%$

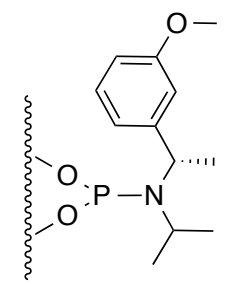

L56 $69 \%$ ee

Figure 2.13: Changing the electronics on the amine portion

The effect of substitution on the aromatic ring was then investigated (Figure 2.13). A variety of different groups were added to the amine. It was found that addition of halogen groups increased the enantioselectivity in comparison to the naked ligand (L50), from $51 \%$ ee to $60 \%$ ee with fluorine (L23), and up to 67\% ee by adding a chlorine or bromine group (L24 \& L51).

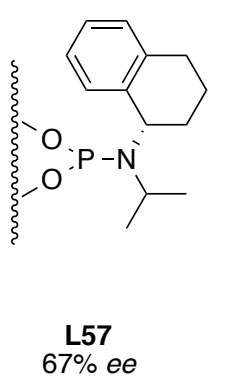

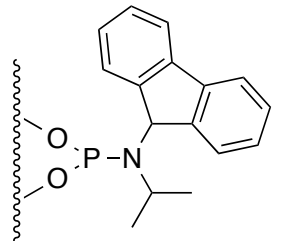
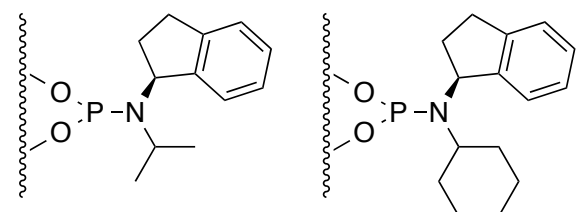

$$
\begin{aligned}
& \mathbf{L} 5 \mathbf{8} \\
& 53 \% \text { ee }
\end{aligned}
$$

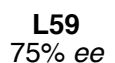

$$
\mathbf{L 6 0}
$$

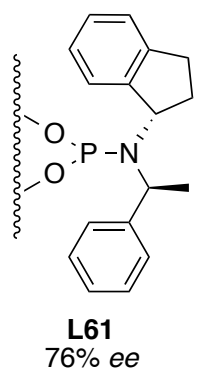

Figure 2.14: Bicyclic structures on the amine portion

Ligands were tested that had varying bicyclic structures were tested (Figure 2.14); these produced enantioselectivities of up to $76 \%$ ee (L61), however again did not exceed the ee of the original phosphoramidite ligand L19. Finally, phenyl-substituted ligands, which did not contain any stereogenic centres on the amine, were screened. Using these ligands was convenient, as the amine was easily prepared in one step by reductive amination. Furthermore, a chiral non-racemic amine was not required therefore the starting materials were inexpensive, and there was no requirement to separate diastereomers at any stage, making them easily accessible. It was found 
that having phenyl groups directly attached to the nitrogen atom gave a poor enantioselectivity of 31\% ee (L62), however, surprisingly, use of a geometrical isomer (L63) of original ligand L19 gave 78\% ee (Figure 2.15). As no increase in enantioselectivity was observed, the use of L19 was continued for further screening reactions as it gave the highest ee (80\%).
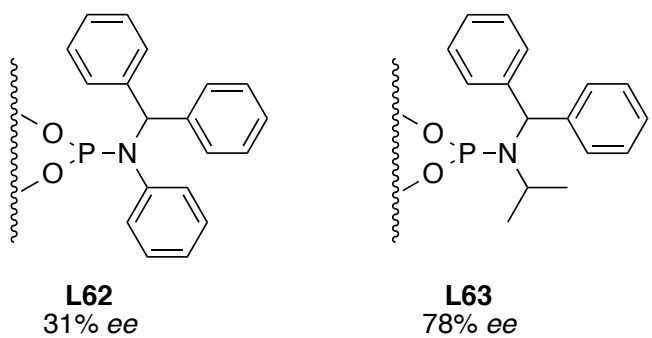

Figure 2.15: Phenyl substituted ligands

\subsubsection{Copper - ligand complex}

As previously mentioned, weighing out copper triflate benzene complex was particularly important, due to it being an air sensitive compound, if exposed to air for too long, significant decomposition of the copper triflate benzene complex was observed. With a preferred ligand in hand, the formation of a copper-ligand complex was investigated (Scheme 2.20). By simply combining a 2:1 ratio of $\mathbf{L 1 9}$ and copper triflate benzene complex in $\mathrm{CH}_{2} \mathrm{Cl}_{2}$ and stirring for one hour, a complex that could be isolated was obtained and subsequently characterised by removal of the solvent.

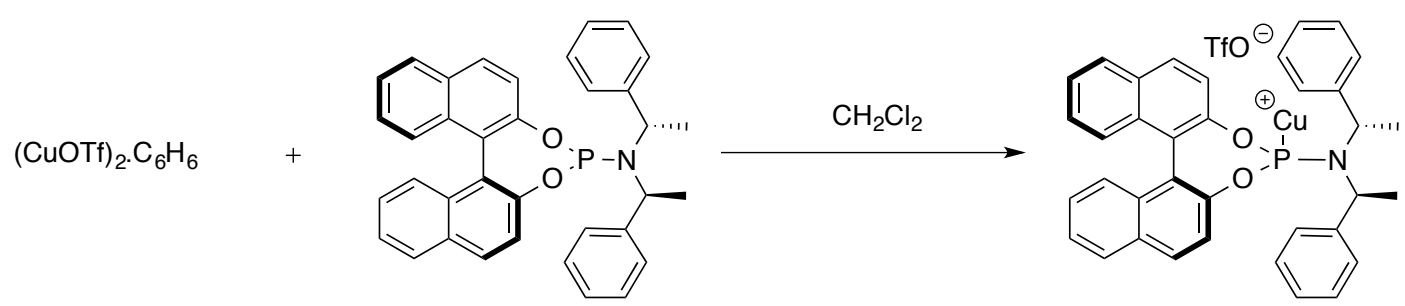

Scheme 2.20: Formation of copper-ligand complex $(S, S, S)-A$

Upon isolation of the complex, an air stable solid was formed which could be weighed and handled in air. Careful storage of the complex under argon allowed storage for months without noticeable decomposition. This enabled elimination of the one-hour pre-complexation of the 
copper and ligand, and ensured consistent results throughout the rest of these studies. Thus $(S, S, S)$-A was used from herein unless additional ligands were screened.

\subsubsection{Reaction optimisation}

In order to determine the best system for our reaction, additional screening reactions were carried out (Table 2.11). Using $(S, S, S)-A$, the concentration effect was studied. The reaction concentration used previously was $0.2 \mathrm{M}$ (entry 3 ), it was found that by decreasing or increasing the concentration was detrimental to the enantioselectivity (entries, 1, $2 \& 4$ ). In addition, the alkylzirconocene was added dropwise to the reaction mixture, however considerable erosion of the enantioselectivity was observed (entries $5 \& 6$ ).

\begin{tabular}{ccc} 
& Conditions & Result $(\% e e)^{\mathbf{a}}$ \\
\hline Entry & $0.06 \mathrm{M}$ & 68 \\
\hline 1 & $0.1 \mathrm{M}$ & 70 \\
2 & $0.2 \mathrm{M}$ & 80 \\
3 & $0.4 \mathrm{M}$ & 75 \\
4 & $\mathrm{Cp}_{2} \mathrm{ZrHCl}, \mathrm{CH}_{2} \mathrm{Cl}_{2} ;$ \\
5 & Alkylzirconocene added dropwise & 36 \\
6 & Alkylzirconocene added dropwise $\left(\mathrm{Et}_{2} \mathrm{O}\right)$ & 27
\end{tabular}

Conditions - 4-phenyl-1-butene (2.5 eq.), $\mathrm{Cp} 2 \mathrm{ZrHCl}$ (2.0 eq.), $\mathrm{CH}_{2} \mathrm{Cl}_{2} ;$ 2-cyclohexenone (1.0 eq.), (S,S,S)-A (10 mol\%), $\mathrm{CH}_{2} \mathrm{Cl}_{2}$ (unless otherwise stated), RT. a) ee determined by chiral HPLC.

\section{Table 2.11: Effect of concentration}

During optimisation, it became apparent that reaction yields were low and found we could only isolate between $20-30 \%$ of the product, despite full consumption of starting material being observed. It was therefore hypothesised that an additional reaction must occur with the product or starting material. It was difficult to identify the competing reaction from the ${ }^{1} \mathrm{H}$ NMR spectra of the crude reaction products as the peaks of the desired product and 'by product' were overlapped, making them hard to distinguish. Analysis of the products by GC-MS suggested that the by-product(s) resulted from further reaction of the desired product. Upon addition of the 
nucleophile, a zirconium enolate was formed, which persisted until work-up. This enolate acted as a nucleophile in an additional reaction with another equivalent of the starting enone, to form a dimer like product 14 (Scheme 2.21).

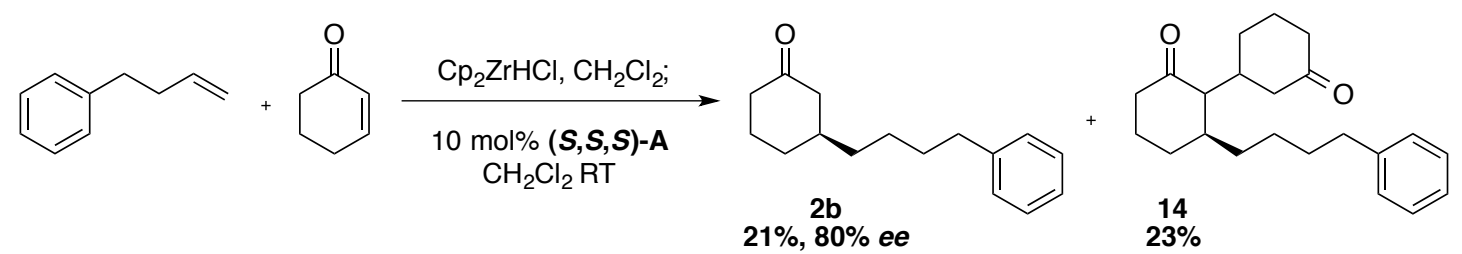

Scheme 2.21: Side reaction

By closely monitoring the reaction over time and isolating the product $\mathbf{2 b}$ and by-product $\mathbf{1 4}$ of the reaction in $\mathrm{CH}_{2} \mathrm{Cl}_{2}$, the rate of by-product formation was seen to be similar to the rate of formation of the desired product $\mathbf{2} \mathbf{b}$. However, this did not account for the entire mass balance of the reaction and we believed that the enolate from formation of the dimer-type product underwent further addition reactions to more starting enone, forming higher oligomers and/or a polymer which are not easily observed. The formation of oligomeric products is perhaps not surprising; similar oligomerisation has been observed in related reactions. ${ }^{90}$ Reingold et al. also showed that Brønsted acids can catalyse double 1,4-addition reactions ${ }^{91}$ and Cossy et al. demonstrated the use of bistrifluoromethanesulfonate to catalyse the reaction. ${ }^{92}$ There have also been reports of using resulting zirconium enolates to initiate the formation of more complex structures. ${ }^{93}$ Other research demonstrated that the zirconium enolate is prone to further reaction. De Clercq et al. showed it was possible to trap the resulting enolate in situ with formaldehyde, (Scheme 2.22$)^{94}$ resulting in an additional step, involving a retro aldol, to obtain the desired 1,4-addition product. ${ }^{95}$

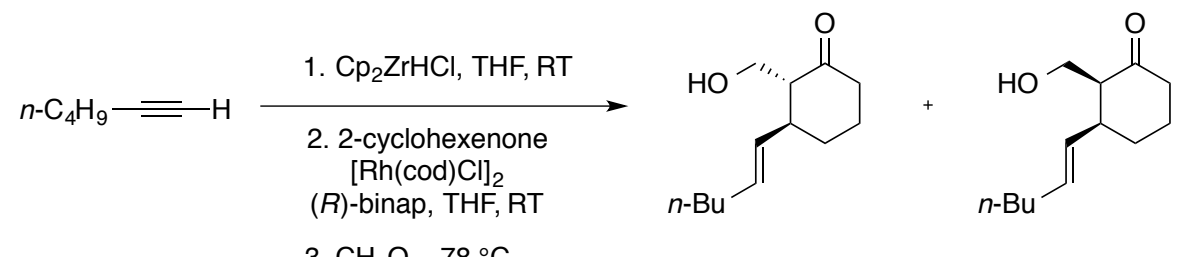

Scheme 2.22: Proof of reactive zirconium enolate 


\begin{tabular}{ccccc}
\hline Entry & Solvent & Yield (\%) $^{\mathbf{a}}$ & $\boldsymbol{e e ~ ( \% ) ~}$ & Byproduct Yield (\%) $^{\mathbf{a}}$ \\
\hline 1 & $\mathrm{CH}_{2} \mathrm{Cl}_{2}$ & 21 & 81 & 23 \\
2 & $\mathrm{Et}_{2} \mathrm{O}$ & 37 & 79 & 20 \\
3 & $\mathrm{THF}$ & 30 & 75 & 26 \\
4 & Toluene & 33 & 70 & 17 \\
5 & $\mathrm{Et}_{2} \mathrm{O} / 3$ eq. Schwartz & 31 & 80 & 24 \\
6 & $\mathrm{Et}_{2} \mathrm{O} / 4$ eq. Schwartz & 26 & 74 & 22
\end{tabular}

Conditions - 4-phenyl-1-butene (2.5 eq.), $\mathrm{Cp} 2 \mathrm{ZrHCl}$ (2.0 eq.), $\mathrm{CH}_{2} \mathrm{Cl}_{2} ;$ 2-cyclohexenone (1.0 eq.), (S,S,S)-A (10 mol\%), solvent, RT. a) Isolated yield b) ee determined by chiral HPLC.

Table 2.12: Varying solvent to optimise yield

The attempts to increase the yield of the product $\mathbf{2} \mathbf{b}$ by varying the solvent are detailed in Table 2.12. $\mathrm{Et}_{2} \mathrm{O}$, THF and Toluene gave slightly better yields than in $\mathrm{CH}_{2} \mathrm{Cl}_{2}$, with $\mathrm{Et}_{2} \mathrm{O}$ producing the highest yield at $37 \%$. Although the yield was insufficient, $\mathrm{Et}_{2} \mathrm{O}$ was used as solvent for further screening reactions as it gave comparable enantioselectivity and the highest yield. The amount of Schwartz reagent was also examined; it was found that the increased use of reagent correlated with lower yield; 2.0 eq. provided better results than 3.0 eq. or 4.0 eq. Screening was continued in order to determine the effect of a large number of additives. The aim was to either trap the enolate or block a binding site on the zirconium (Figure 2.16, where an enolate and ketone are bound to zirconium) with a sacrificial ketone or base.

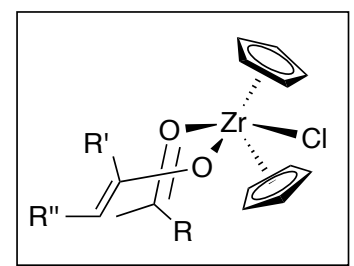

Figure 2.16: Enolate and ketone bound to zirconium

Many reagents were assessed for trapping the enolate (boronic esters, silyl reagents, metals etc.), however, no trapping product was isolated. However, it was observed that the use of 5.0 eq. TMSCl increased the enantioselectivity from $80 \%$ to $89 \%$. After finding a suitable increase in enantioselectivity albeit without an increase in the yield, we continued our screening of additives with 5.0 eq. of TMSCl (Table 2.13). 


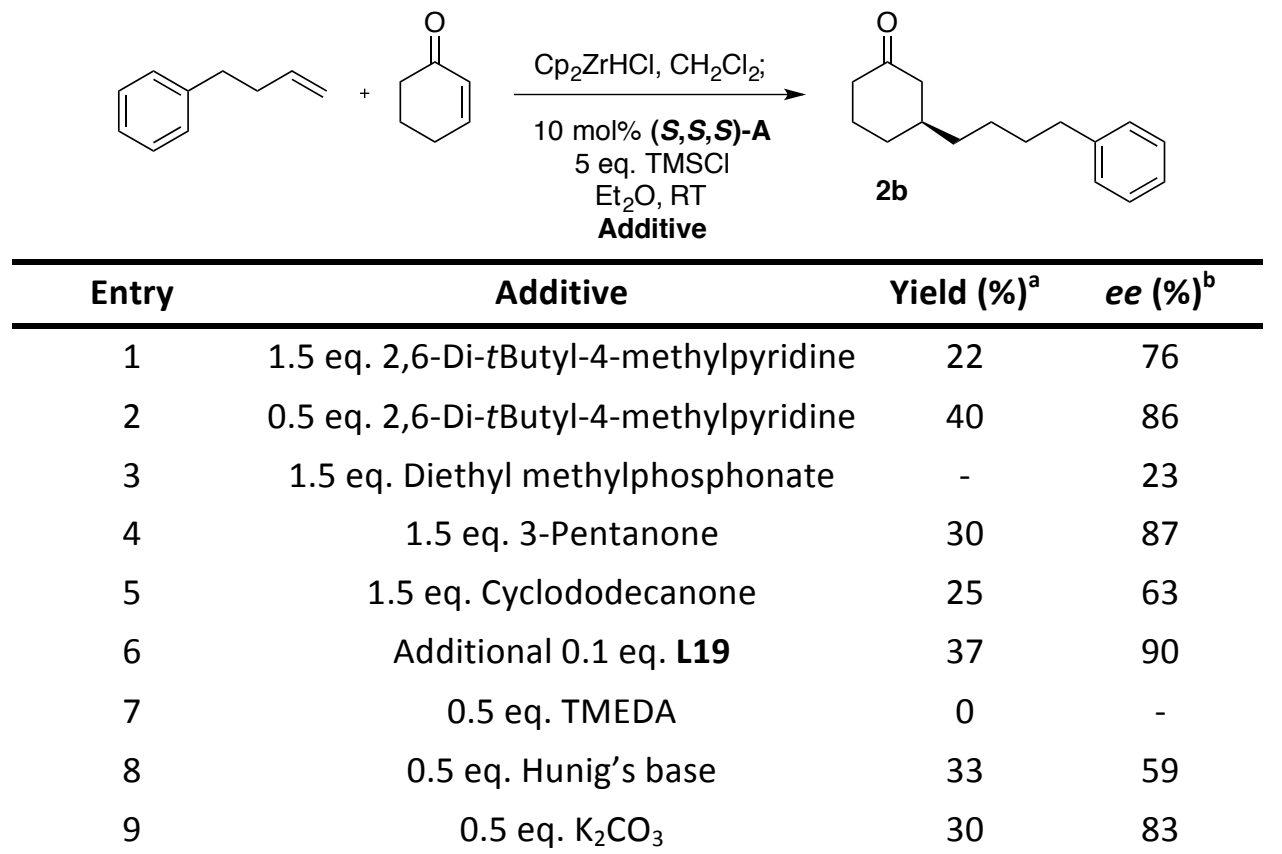

a) Isolated yield b) ee determined by chiral HPLC.

Table 2.13: Additive screen

With the aim to potentially occupy the vacant zirconium site with amines or ketones, even though the yields were not improved, the enantioselectivity remained relatively constant in the majority of reactions, which highlights the robustness of our reaction. Variation of the addition procedure and catalyst loadings were briefly examined (Table 2.14), it was observed that adding the enone dropwise, or pre-mixing the enone and $\mathrm{TMSCl}$ before addition of the copper catalyst and alkylzirconocene, lowered the ee and yield (entries $1 \& 2$ 2). Changing the catalyst loading to $1 \%$ catalyst eroded the ee, however an increase in yield was observed (entry 3). An increase to 20 mol\% catalyst gave comparable results to using 10 mol\% catalyst (entry 4).

\begin{tabular}{cccc}
\hline Entry & Conditions & Yield (\%) $^{\mathbf{a}}$ & ee (\%) \\
\hline 1 & Enone added dropwise & 18 & 78 \\
2 & Pre-mix enone and TMSCl & 22 & 85 \\
3 & 1 mol\% catalyst & 52 & 23 \\
4 & 20 mol\% catalyst & 36 & 90
\end{tabular}

Conditions - 4-phenyl-1-butene (2.5 eq.), $\mathrm{Cp} 2 \mathrm{ZrHCl}$ (2.0 eq.), $\mathrm{CH}_{2} \mathrm{Cl}_{2} ; 2$-cyclohexenone (1.0 eq.), (S,S,S)-A (10 mol\% unless otherwise stated), 5.0 eq. TMSCl, Et $t_{2} \mathrm{O}, \mathrm{RT}$. a) Isolated yield b) ee determined by chiral HPLC.

Table 2.14: Altering addition order and catalyst loadings 


\subsubsection{Optimal Results}

After completion of the screening reactions it was found that we could not exceed the variable yield for our 2 -cyclohexenone system past $59 \%$ with product $\mathbf{2} \mathbf{b}$. In an attempt to prevent the oligomerisation during the reaction, the sterics of the starting material were changed. When a cyclic enone with steric hindrance in the 4-position was employed, such as 4,4dimethylcyclohexenone, a significant increase in yield to $74 \%$ was obtained to give product 15 a while maintaining enantioselectivity (Scheme 2.23).

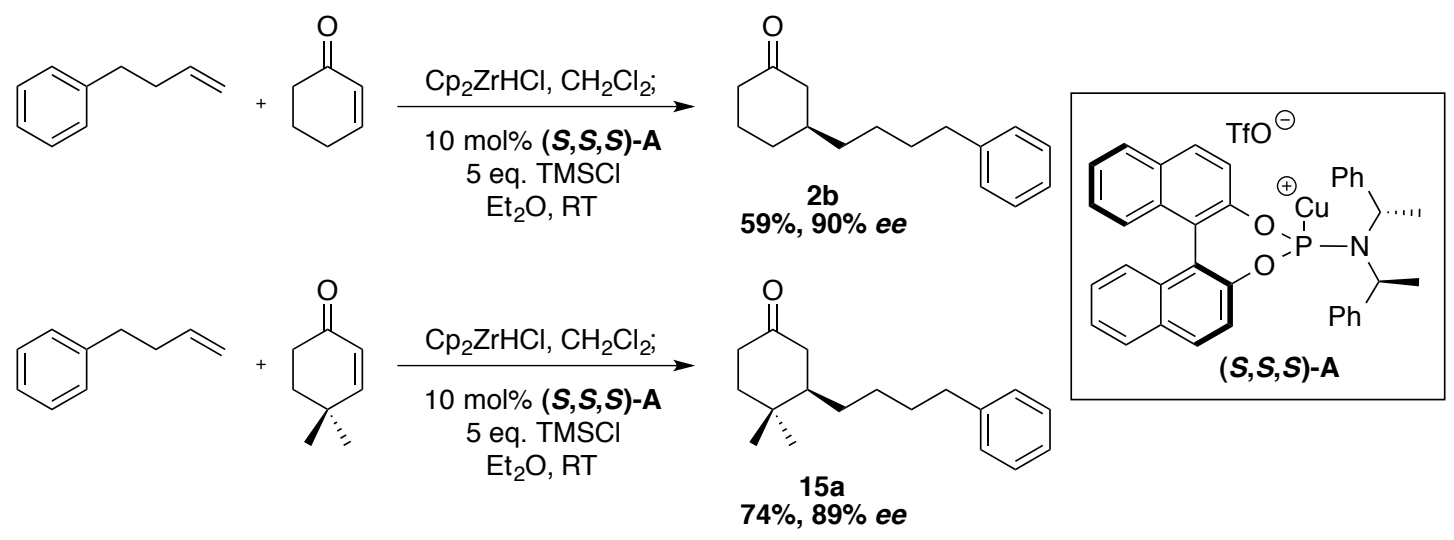

Scheme 2.23: Optimal results with cyclic substrates

\subsubsection{Substrate Scope}

\subsubsection{Alkene scope}

With optimised conditions in hand, attention was turned to substrate scope. To begin, relatively simple alkenes were used which gave good yields and excellent enantioselectivities (Table 2.15). The reactions were normally complete within 3 hours, yet some were arbitrarily left overnight without any effect to the resulting product. We found it was possible to add phenyl containing alkenes, 4-phenyl-1-butene and 6-phenyl-1-hexene (entries 1 \& 2), as well as short and long straight chain alkenes to produce good results (entries $3-5$ ). A sterically hindered alkene containing a bulky $t$-butyl group gave good ee (entry 6 ) and it was possible to add cyclic substrates with decent results (entries $7 \& 8$ ). In addition a doubly substituted symmetrical alkene could be added, however, 10 eq. was required to statistically favour the mono-hydrozirconation (entry 9). 


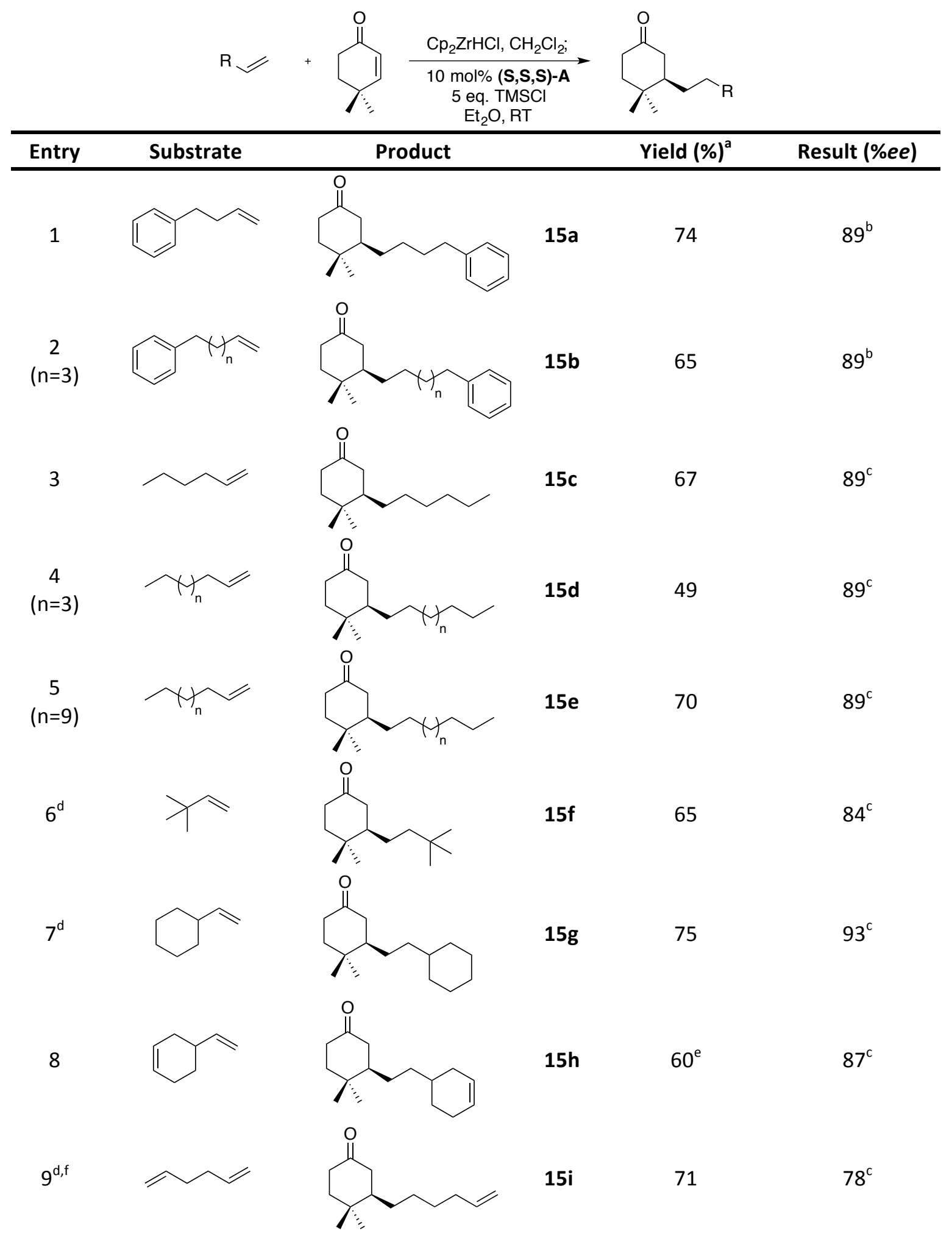

a) Isolated yield b) ee determined by chiral HPLC c) ee determined by derivatisation then ${ }^{13} \mathrm{C} N M R$ d) experiment conducted by P. M. C. Roth e) 1:1 diastereomeric mixture obtained f) 10 eq. of alkene was used

Table 2.15: Substrate screening 


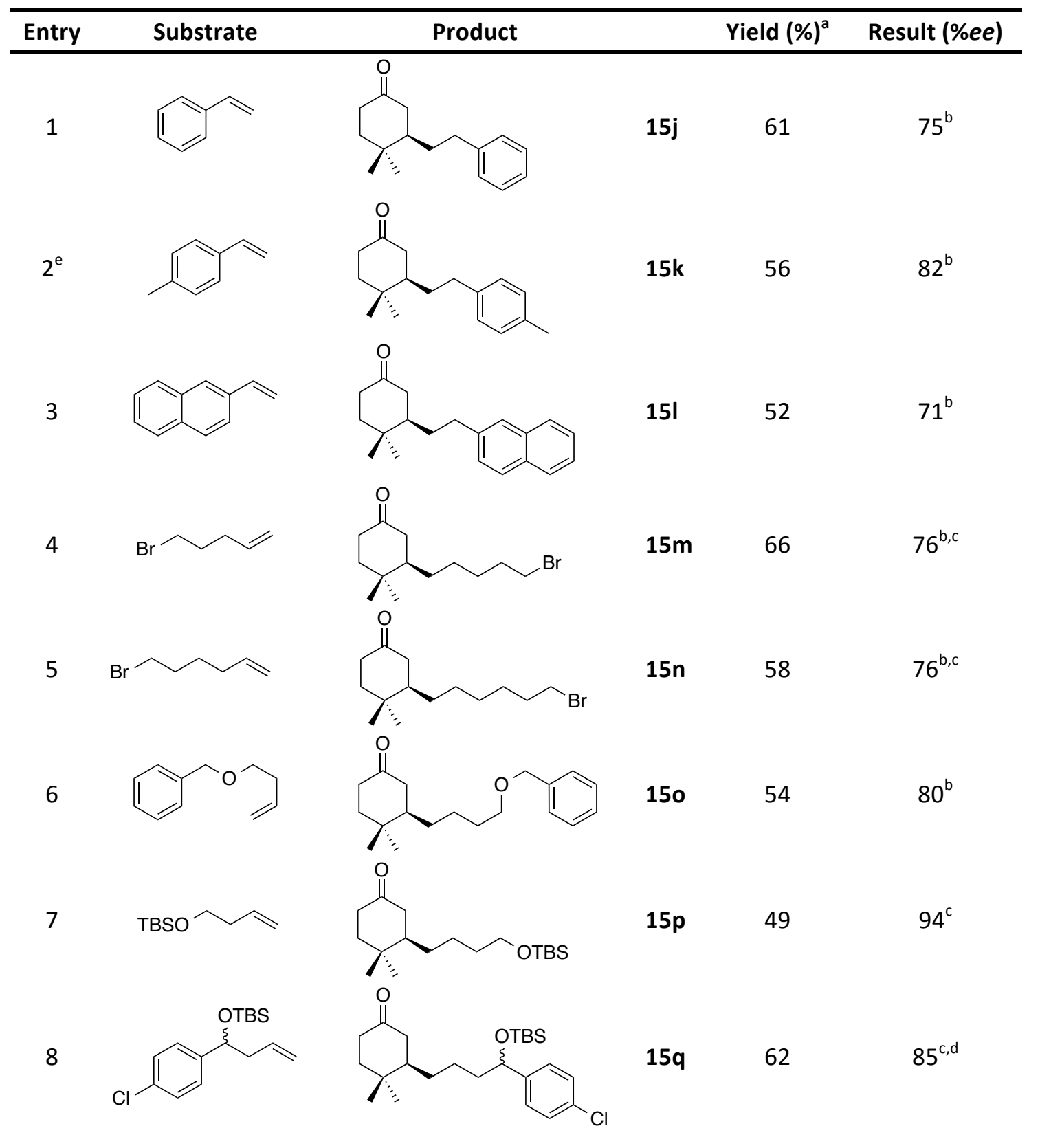

Conditions - alkene (2.5 eq.), $\mathrm{Cp}_{2} \mathrm{ZrHCl}$ (2.0 eq.), $\mathrm{CH}_{2} \mathrm{Cl}_{2} ; 4$,4-dimethyl-2-cyclohexenone (1.0 eq.), (S,S,S)-A (10 mol\%), 5.0 eq. TMSCl, Et $t_{2} \mathrm{O}, \mathrm{RT}$. a) Isolated yield b) ee determined by chiral $\mathrm{HPLC} C$ ) ee determined by derivatisation and $\left.{ }^{13} \mathrm{CNMR} d\right)$ ee determined by deprotection of the product with TBAF followed by HPLC e) experiment conducted by P. M. C. Roth.

Table 2.16: Substrate screening with functionalised alkenes

Given the success of the simple alkenes in our reaction, the use of more complex alkenes was explored. The reaction was able to tolerate alkenes with functional groups that pre-made organometallics employed in asymmetric 1,4-addition could not (Table 2.16), as well as alkyl bromine units, a functional group particularly incompatible with the formation of Grignard reagents (entries 4 \& 5). ${ }^{96}$ Styrenyl and naphthyl units could also be used, however slightly lower 
enantioselectivities were observed (entries 1 \& 3), however, appreciable enantioselectivity was obtained with a para-methyl styrenyl group (entry 2). Benzyl and silyl protected alcohols were also tolerated, especially when the tert-butyldimethylsilyl protecting group was used (entries 6 \& 7). In addition to reactive units, it was also possible to add alkenes with multiple functional groups, as in the case of entry 8 where both a protected alcohol and para-chlorophenyl substituent are present.

Interestingly, investigation of the use of the highly reactive allyl chloride gave good results (Scheme 2.24). Allyl chloride is a reactive electrophile, with a tendency to perform SN2 or SN2' reactions. Although low yield was obtained of product $15 r$, it was interesting to note that the expected by-product (15s) did not appear to be formed. Instead, cleavage of the $\mathrm{C}-\mathrm{Cl}$ bond occurred, resulting in the propyl addition product (15t).

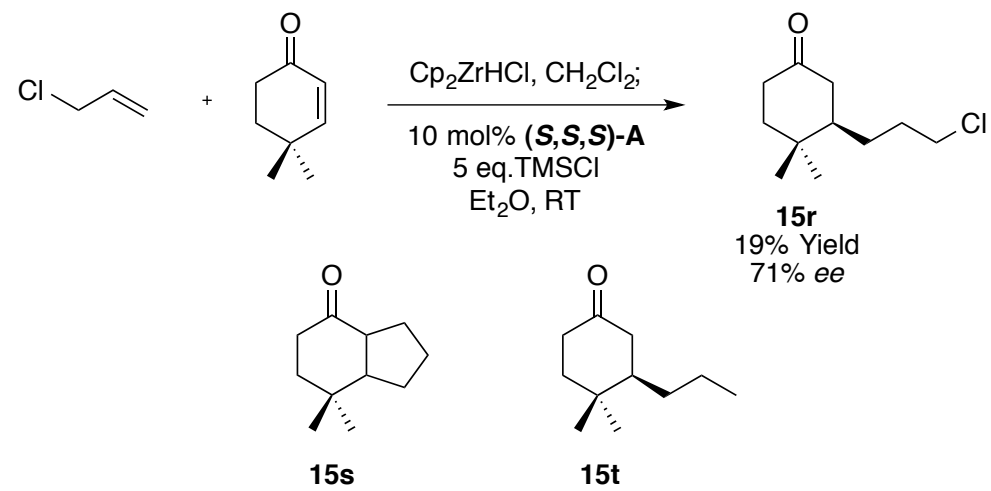

Scheme 2.24: Addition of allyl chloride

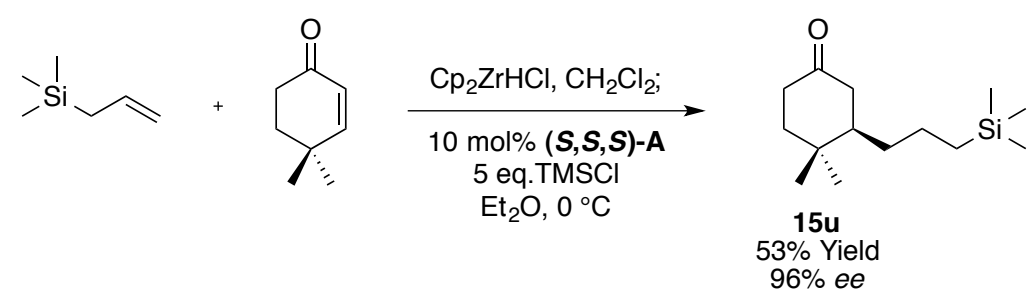

Scheme 2.25: Addition of allyl silane

Another reactive substrate tolerated was allyl silane. As this is a reactive nucleophile, we were pleased to see a $53 \%$ yield of $15 u$ and the highest enantioselectivity of $96 \%$ was obtained (Scheme 2.25). It was necessary to perform this reaction at $0{ }^{\circ} \mathrm{C}$ to gain the highest yield, as significant reduction of the starting enone to the allylic alcohol was observed. This may be due to: (i) 
reduction by free Schwartz reagent, which is regenerated during the reaction; and (ii) the reversibility of hydrozirconation when using electron rich alkenes, resulting in reversion back to alkene and free Schwartz reagent (Scheme 2.26).

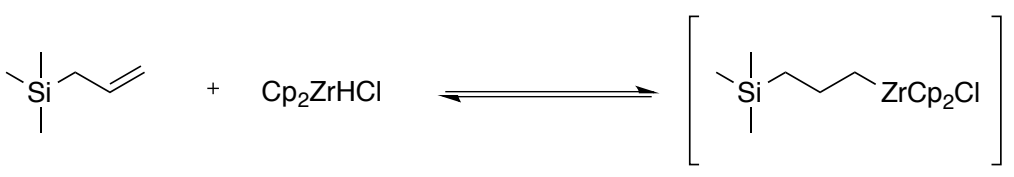

Scheme 2.26: Reversible addition of the Schwartz reagent

Moreover, we were also able to synthesise the natural product $\mathbf{2 c}$, a compound isolated from the New Zealand liverwort Balantiopsis rosea. ${ }^{97}$ This was achieved using commercially available starting materials in one step where 2-cyclohexenone and para-methoxystyrene were coupled to obtain a good yield and excellent enantioselectivity (Scheme 2.27).

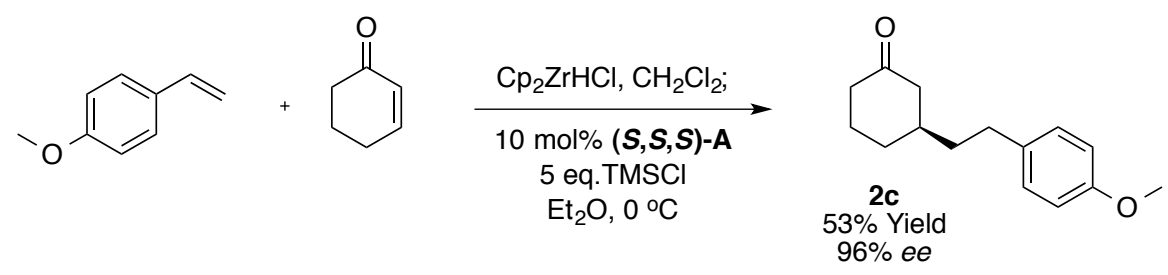

Scheme 2.27: One step synthesis of the natural product Balantiopsis rosea

During our studies we found that some alkenes were unsuccessful (Figure 2.17). Although the addition of allyl chloride gave product, the addition of the more reactive allyl bromide $\mathbf{1 6}$ lead to no reaction, presumably due to inherent reactivity of the allyl bromide. No product was formed in the case of alkenes $\mathbf{1 7}$ and $\mathbf{1 8}$ containing esters: instead it is believed that the Schwartz reagent reduced the ester instead of performing the hydrozirconation. ${ }^{27 b}$ Hydrozirconation of amines was attempted after reports in the literature of a successful hydrozirconation of alkene $\mathbf{1 9}$ followed by 1,2-addition to acyl chlorides. ${ }^{98}$ After attempts with a variety of amines, it was found that only the use of bulky amine 19 resulted in product, which was later found to be racemic. Epoxides were also briefly investigated; although the addition of expoxide $\mathbf{2 0}$ was successful, the product was racemic. Finally use of complex alkene $\mathbf{2 1}$, which had epoxide and protected alcohol functionality, was attempted. Despite the substrate being successfully hydrozirconated, only starting material 
was recovered.
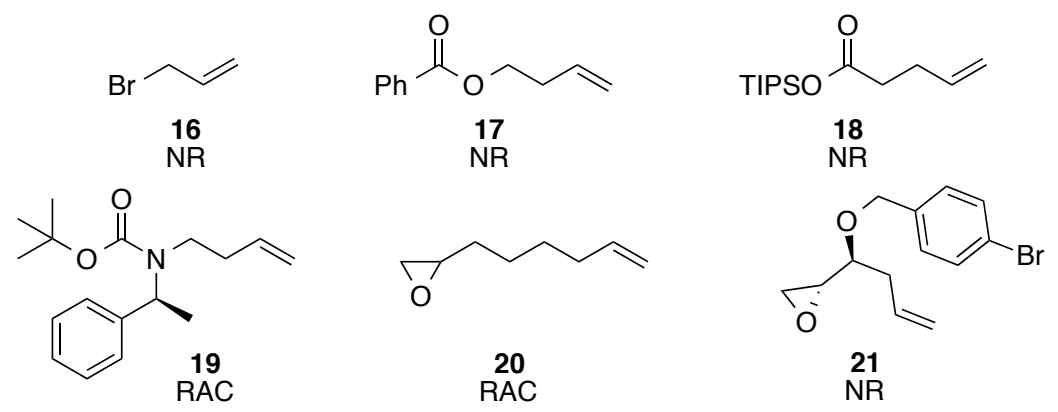

Figure 2.17: Unsuccessful alkenes

\subsubsection{Enone scope}

After screening of alkene substrates, the enone scope was examined (Table 2.17). Cyclopentenone was first tried, resulting in low yields at both room temperature and $0{ }^{\circ} \mathrm{C}$, however, an increase in the enantioselectivity was observed from $75 \%$ to $85 \%$ ee when the temperature was lowered (entries 1 \& 2). This substrate is well known to give poor yields in 1,4addition reactions, likely due to its flat structure. ${ }^{99}$ A 4,4-diphenyl substituted enone gave $c a$. the same yield (23a, 75\%) as the 4,4-dimethyl substrate, and excellent enantioselectivity (entry 3). Cycloheptenone was found to be an effective substrate under our reaction conditions (entry 4). In addition a moderate yield and enantioselectivity for a macrocyclic compound was obtained (entry 5). This initial result with a macrocyclic compound is positive and could be further optimised by varying, solvent, temperature etc. to suit a particular target synthesis. 


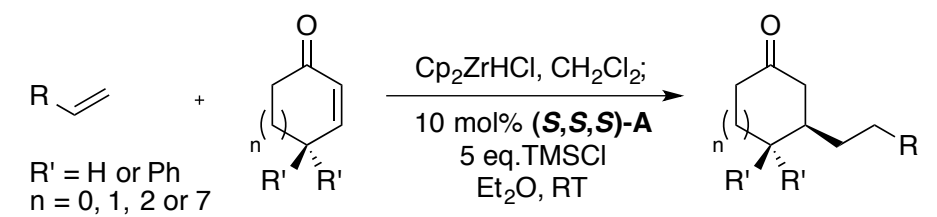

Entry Substrate

a) Isolated yield b) ee determined by chiral $H P L C$ c) reaction performed at $0{ }^{\circ} \mathrm{C} d$ ) absolute configuration unknown.

\section{Table 2.17: Enone scope}

Further attempts were made utilising other enones, however, poor enantioselectivities were obtained (Table 2.18). In addition, low conversions were observed in the majority of cases (entries $1,2 \& 5)$. Poor results may have been due to the deactivating nature of the phenyl ring in the semi-cyclic system (entry 1), or due to the bulky nature of product $\mathbf{2 7}$ (entry 2 ) to yield the poor diastereoselectivities and reactivities observed. In the case of products $\mathbf{2 8}$ and $\mathbf{2 9}$ decomposition of the products were observed and the lower enantioselectivities may be attributed to the flatter structure of the starting enones (entries $3 \& 4$ ). A linear substrate was also tested (entry 5), low enantioselectivity was obtained, which proved optimisation of these substrates is still required. In addition, it was observed to react preferentially in the 1,4-position over the sterically crowded 1,6-position. To enable high enantioselectivities, further optimisation with these substrates is required as our method does not appear to translate to these types of enones. Development of 
new phosphoramidite ligands is ongoing and may result in the use of one of these ligands to obtain the desired results.

Entry Substrate

Conditions - alkene, 4-phenyl-1-butene or 1-hexene, (2.5 eq.), $\mathrm{Cp}_{2} \mathrm{ZrHCl}$ (2.0 eq.), $\mathrm{CH}_{2} \mathrm{Cl}_{2}$; enone (1.0 eq.), (S,S,S)-A (10 mol\%), TMSCl (5.0 eq.), Et $t_{2} \mathrm{O}, \mathrm{RT}$. a) ee determined from crude mixture by chiral HPLC.

\section{Table 2.18: Poor enone results}

\subsubsection{Scale up of Methodology}

It is important for the scale up of this methodology to be feasible in order for the reaction to be suitable for industrial use. In a recent review on large-scale asymmetric conjugate addition reactions it was highlighted that "there are still challenges to be met in order to achieve large scale ACA with synthetically useful alkyl units" ${ }^{19}$

\subsubsection{Scale up on a $2.5 \mathrm{mmol}$ scale}

Reactions herein were performed on a $2.5 \mathrm{mmol}$ scale, and a variety of previously used alkenes and new alkenes were examined. Results gained in collaboration with $\mathrm{Dr}$ M. Sidera showed the addition of gaseous alkenes could be achieved (Table 2.19). These alkenes were hydrozirconated by bubbling a balloon of the relevant gas through the reaction mixture. 


Entry Substrate

Conditions - alkene (balloon), $\mathrm{Cp}_{2} \mathrm{ZrHCl}$ (2.0 eq.), $\mathrm{CH}_{2} \mathrm{Cl}_{2}$; enone (1.0 eq.), (R,R,R)-A (10 mol\%), TMSCl (5.0 eq.) Et 2 , RT. a) Reactions performed by $\operatorname{Dr}$ M. Sidera. b) Isolated yield c) ee determined by chiral HPLC d) ee determined by derivatisation then ${ }^{13} \mathrm{CNMR}$

Table 2.19: Addition of gaseous alkenes ${ }^{a}$

These simple gaseous alkenes were added obtaining good to excellent yields and consistently good enantioselectivities. It is possible to isolate up to $0.5 \mathrm{~g}$ of product very easily. Furthermore, it is also possible to scale up the reaction with more complex alkenes (Table 2.20), $0.33 \mathrm{~g}$ of the non-natural enantiomer of the natural product $(\mathbf{2 c})$ was isolated (entry 1 ), and a phenyl containing alkene to 5-, 6-, and 7-membered enones could be added (entries 2-4). The addition of a straight chain chloroalkyl unit gave excellent enantioselectivity (entry 5) and the addition of an alkene containing an internal alkyne was feasible where the hydrozirconation occurred exclusively at the terminal alkene and the internal alkyne was left untouched. We were also able to isolate $0.87 \mathrm{~g}$ of the multi-functional substrate (entry 7 ). 


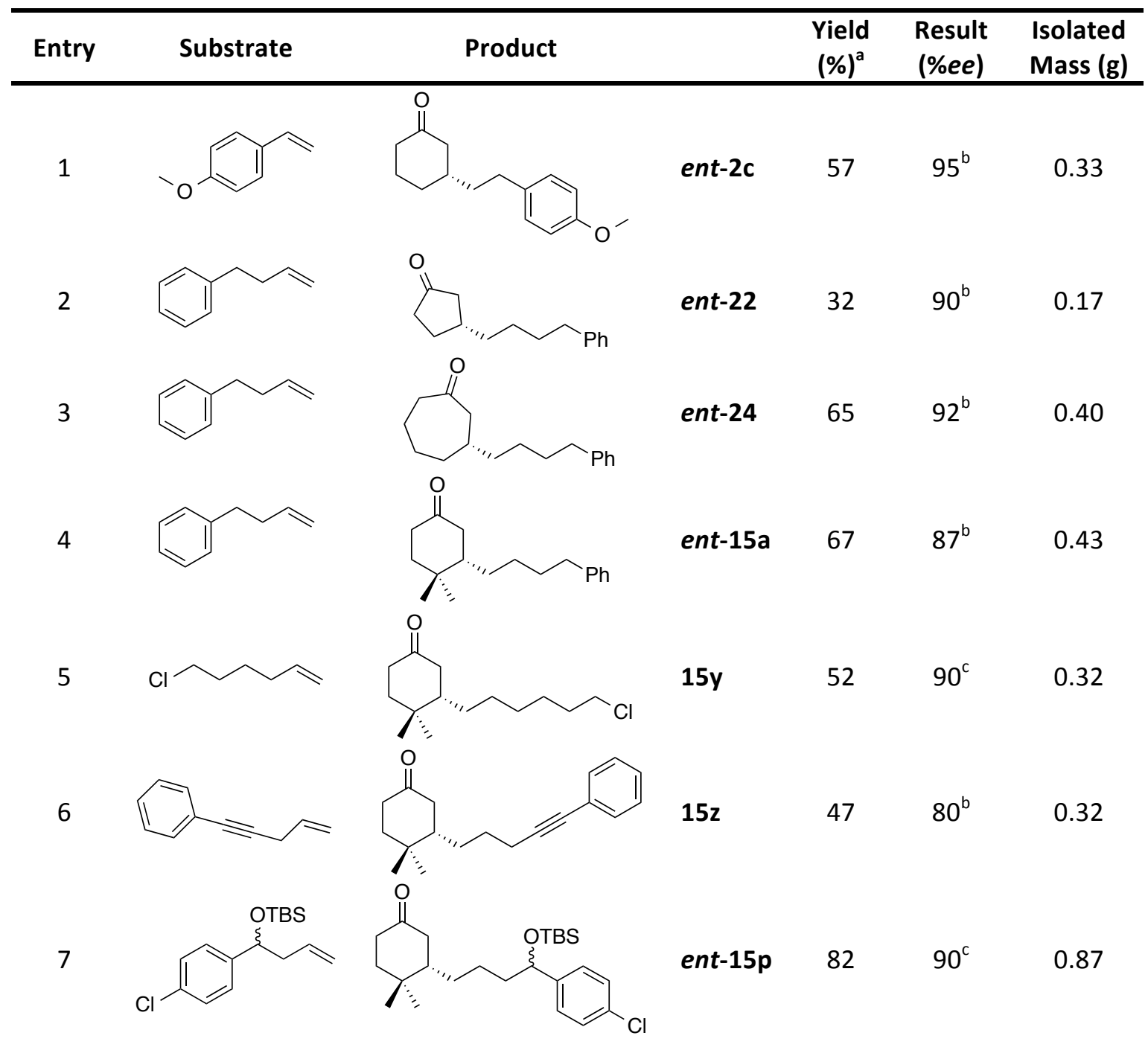

Conditions - alkene (2.5 eq.), $\mathrm{Cp}_{2} \mathrm{ZrHCl}$ (2.0 eq.), $\mathrm{CH}_{2} \mathrm{Cl}_{2}$; enone (1.0 eq.), (R,R,R)-A (10 mol\%), TMSCl (5.0 eq.), Et $t_{2} \mathrm{O}$. a) Isolated yield $b$ ) ee determined by chiral HPLC C) ee determined by derivatisation and ${ }^{13} C N M R$.

Table 2.20: Scale-up with functionalised alkenes

\subsubsection{Scale up to $35 \mathrm{mmol}$ scale}

The scale of these reactions was not limited to $2.5 \mathrm{mmol}$. The examination of the scale up of product $(+\mathbf{+}-\mathbf{2} \mathbf{b}$ on $5 \mathrm{mmol}, 10 \mathrm{mmol}$ and $35 \mathrm{mmol}$ scales showed that both the yield and enantioselectivity were maintained (Scheme 2.28). On a $35-\mathrm{mmol}$ scale, $4.82 \mathrm{~g}$ of product was isolated, highlighting the potential of this reaction to be used on an industrial scale. 

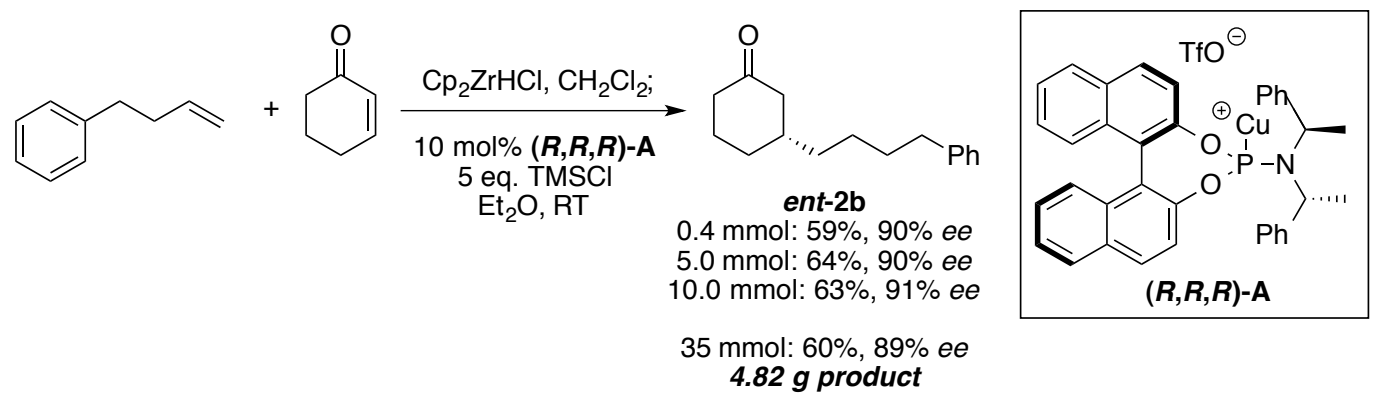

Scheme 2.28: Scale up results

In conclusion, we have developed a new asymmetric 1,4-addition reaction, which enables the hydrozirconation of readily available alkenes with the Schwartz reagent. The resulting alkylzirconocene complex can then be combined with copper catalyst $\mathbf{A}$ to give a highly enantioselective method for the formation of C-C bonds. 


\section{Chapter 3: Extending the Scope of Our 1,4-Addition Reaction}

In this chapter, first I am applying the methodology developed in chapter one to the 14- and 1,6addition to complex steroids. I will then go into detail about additional methodology that has been developed since the project started. I will briefly discuss the addition to $\beta$-substituted enones to form quaternary centres followed by a more in depth investigation into the addition of alkylzirconocenes to $\alpha, \beta$-unsaturated lactones, the optimisation procedure, substrate scope and application of the methodology. Parts of this chapter have been previously published in Chemical Communications, ${ }^{61}$ Angewandte Chemie, ${ }^{100}$ and Nature Protocols. ${ }^{101}$

\subsection{Addition to Steroids}

\subsubsection{Introduction}

After successfully developing reaction conditions for the 1,4-conjugate addition of alkylzirconium species to cyclic enones, we investigated the applicability of the conditions to alternative compounds. It was decided to apply the method to commercially available steroids. Steroids may be considered to be more challenging substrates as they contain multiple functionalities, which could potentially interfere with the conjugate addition process leading to poor yield and/or selectivity. The ability to functionalise steroids in a selective manner could be especially beneficial as these are valuable compounds, for example cholesteryl benzoate (Figure 3.1) was the first compound found to form chiral nematic liquid crystals. ${ }^{102}$

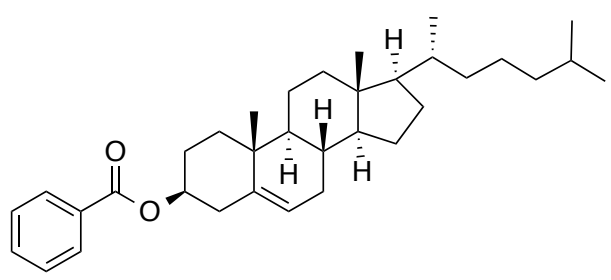

Figure 3.1: Cholesteryl benzoate

Fulvestrant (Faslodex), an estrogen receptor antagonist, is an established breast cancer drug that has been synthesised on a commercial scale. ${ }^{103}$ AstraZeneca's route to Fulvestrant involves a key copper-catalysed 1,6-conjugate addition of an alkyl Grignard reagent forming a new C-C bond at 
position 7 in the substrate (Scheme 3.1). ${ }^{104}$
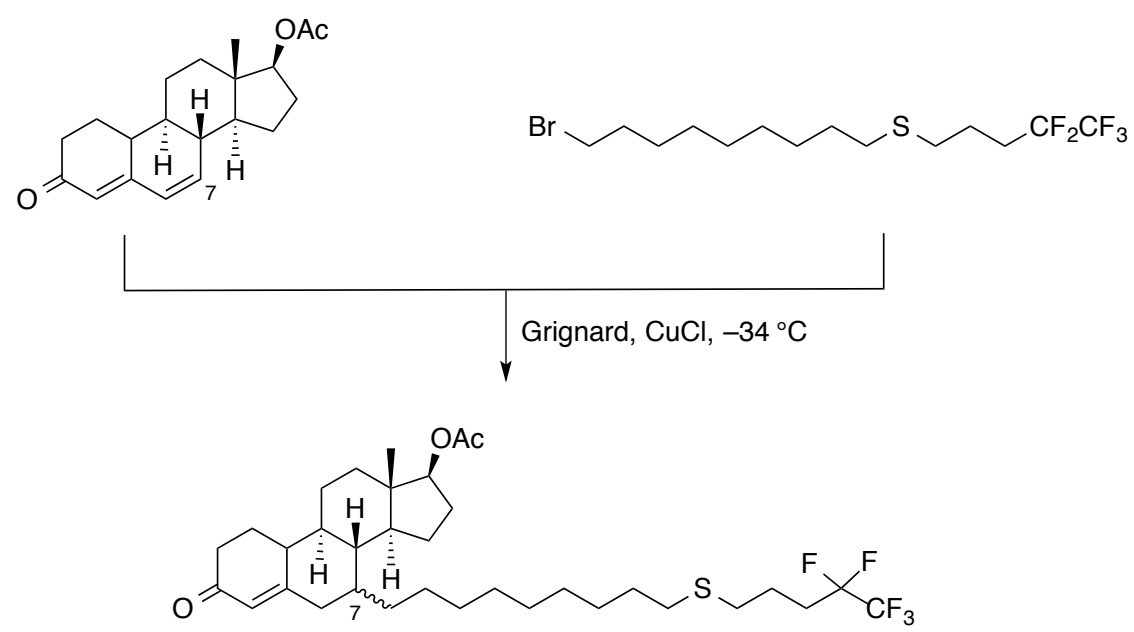

(mixture of $\mathrm{C} 7$ isomers)

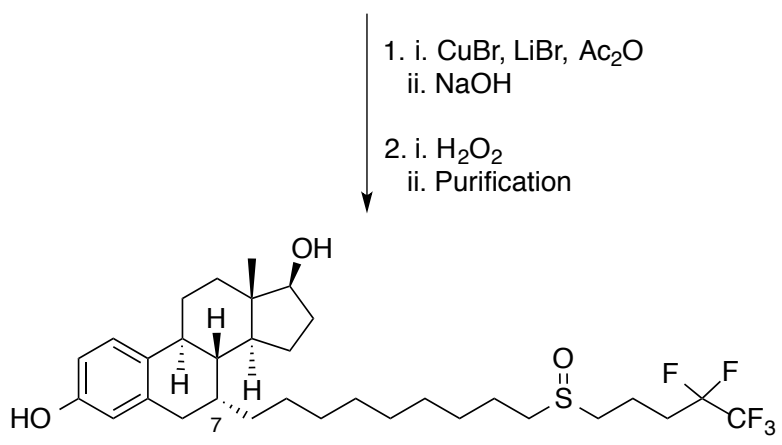

Scheme 3.1: Key Grignard addition step for the synthesis of Fulvestrant

These examples represent important compounds, and the ability to vary the products in a facile manner could be useful in the development of new effective drugs. Methods developed for the addition of carbon nucleophiles in 1,4- and 1,6-additions to steroid derivatives have involved the use of hard organometallic nucleophiles. ${ }^{105}$ In the case of 1,4-addition reactions, the combination of organoaluminium reagents and copper catalysts were restricted to the addition of methyl and ethyl groups. ${ }^{106}$ The use of a nickel catalyst allowed the addition of organoaluminium compounds in a 1,4-fashion to aryl groups. ${ }^{107}$ Aside from highly reactive organoaluminium reagents, copper bromide dimethyl sulfide catalysed addition has allowed addition of alkyl Grignard reagents. An example of a Grignard bearing a terminal alkene has been reported, and is shown below (Scheme 3.2). In this case, the alkene was subjected to further functionalisation in order to access steroids designed to act as tracers. These tracers were used as components in the development of a 
fluorescent polarisation assay for testosterone detection. ${ }^{108}$
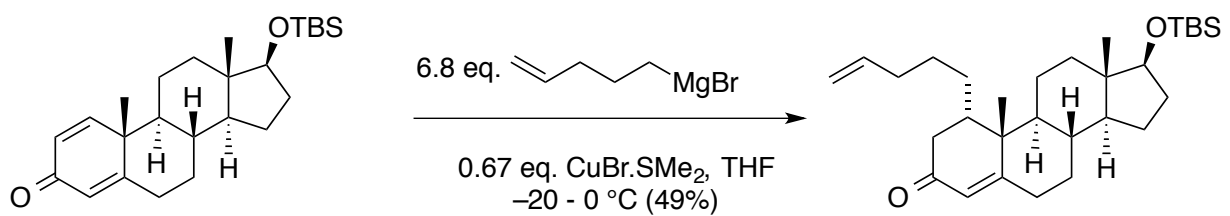

Scheme 3.2: Reddy's Grignard 1,4-addition to steroids

In the case of 1,6-addition to steroids, the use of simple Grignard reagents combined with copper chloride has been reported, ${ }^{109}$ as has addition of organolithium reagents generated in situ from $t$ BuLi and a phenylalkyl iodide. ${ }^{110}$ Katzenellenbogen et al. reported a method for copper iodide mediated addition of 4-pentenylmagnesium bromide to estradiol derivatives, albeit with a high excess of both copper and Grignard reagent (Scheme 3.3) ${ }^{111}$ The products of these reactions were used to access compounds for the study of the structure of an estrogen receptor.
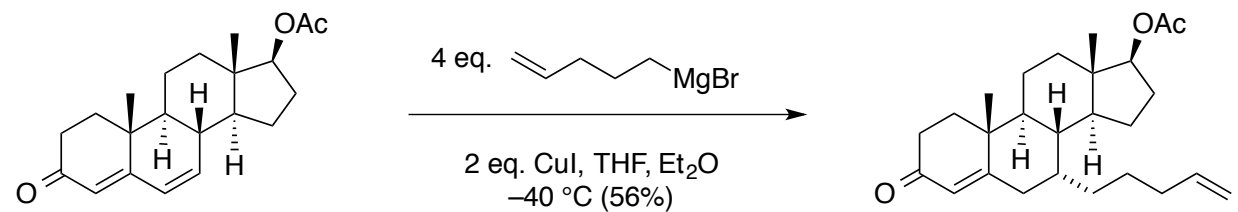

Scheme 3.3: Katzenellenbogen's Grignard 1,6-addition to steroids

As highlighted above, previous work on the 1,4- and 1,6-addition to steroid derivatives largely relied on using high excesses of reagents. Also, very few additions of functionalised nucleophiles have been reported, suggesting that the reported procedures are largely limited to relatively simple nucleophiles such as alkyl moieties.

\subsubsection{Results and Discussion}

The substrates we chose to study for the conjugate addition reaction were commercially available steroid derivatives. 1,4-Androstadiene-3,17-dione (31) is a cross conjugated dienone, in which two different 1,4-additon reactions are possible: 1,2-additions to both the dienone and ketone moieties, as well as a number of other possible reactions such as rearrangements and deprotonations. Canrenone (32) also contains considerable functionality: a sensitive lactone is 
present in the structure and the substrate could undergo 1,2-, 1,4-, and 1,6-addition reactions (Figure 3.2).

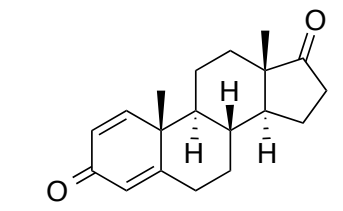

31

1,4-Androstadiene-3,17-dione

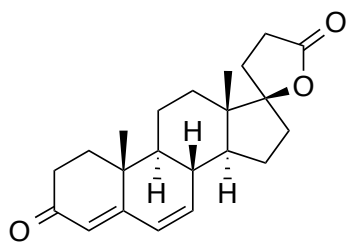

32

Figure 3.2: Steroids used in this study

Optimised conditions found in chapter two (for the addition of alkylzirconium species to cyclic enones) were applied for the addition to steroidal compounds. Simple alkene, 1-hexene, was initially used (Scheme 3.4).

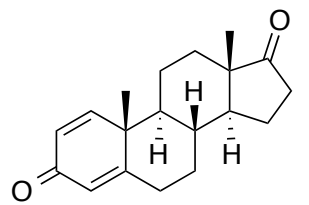

31

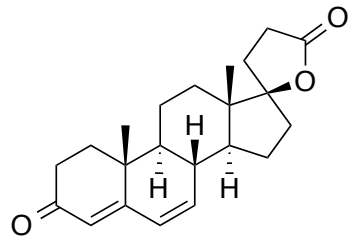

32

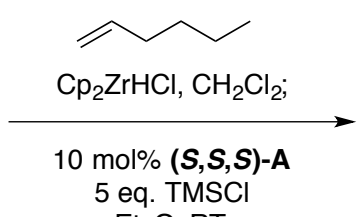

$\mathrm{Et}_{2} \mathrm{O}, \mathrm{RT}$

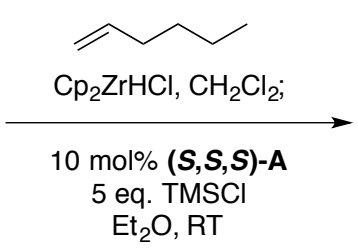

$\mathrm{Et}_{2} \mathrm{O}, \mathrm{RT}$

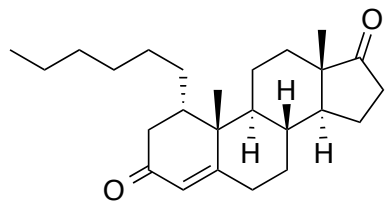

31a $50 \%+16 \% \beta$-isomer<smiles></smiles>

$59 \%+21 \% \beta$-isomer

Scheme 3.4: Results for the addition of 1-hexene

Gratifyingly, good yields of both the 1,4- and 1,6-addition products were observed on addition of 1-hexene to steroids $\mathbf{3 1}$ and $\mathbf{3 2}$. In the case of 31a, 1,4-addition to the less substituted carbon was observed exclusively. The crude ratio of diastereomers when using $(\boldsymbol{S}, \boldsymbol{S}, \boldsymbol{S})-\mathbf{A}$ was $\sim 3.5: 1$ and the favoured $\alpha$-isomer was isolated in $50 \%$ yield whilst the minor $\beta$-isomer was isolated in $16 \%$ yield. In comparison, when using $(\boldsymbol{R}, \boldsymbol{R}, \boldsymbol{R})$-A we found a small matched/mismatched effect, and the crude ratio of isomers was $\sim 5: 1$, still in favour of the $\alpha$-isomer 31a. We also tested the use of achiral copper sources to see if our complex was necessary for this reaction. Both $\mathrm{CuBr} . \mathrm{SMe}_{2}$ and 
$(\mathrm{CuOTf})_{2} \cdot \mathrm{C}_{6} \mathrm{H}_{6}$ resulted in an $\alpha: \beta$ ratio of $>8: 1$; yet in the case of using $\mathrm{CuBr}$. $\mathrm{SMe}_{2}$ the reaction proceeded no further after only $<25 \%$ conversion and in the case of $(\mathrm{CuOTf})_{2} \cdot \mathrm{C}_{6} \mathrm{H}_{6}$, a large number of impurities were observed limiting conversion to the desired product. In the addition of 1hexene to steroid $\mathbf{3 2}$ using $(S, S, S)$-A we found 1,6-addition was observed exclusively. Product 32a was formed with a crude 3.8:1 ratio of diastereomers in favour of the $\alpha$-isomer. This major isomer could be isolated in $59 \%$ yield and the minor $\beta$-isomer in $21 \%$ yield (Scheme 3.4 ). Here, use of $(\boldsymbol{R}, \boldsymbol{R}, \boldsymbol{R})-\mathbf{A}$ was found to reverse the sense of diastereoselectivity, so that a $\sim 1: 2, \alpha: \beta$ ratio was favoured.

After demonstrating that simple alkenes could undergo hydrometallation - addition reactions to steroidal compounds we wanted to examine the use of more structurally complex alkenes. The addition of 5-bromo-1-pentene under standard conditions occurred smoothly in the case of steroid $\mathbf{3 1}$ and we obtained an isolated yield of $60 \%$ of the $\alpha$-isomer $31 \mathrm{~b}$. In the case of steroid $\mathbf{3 2}$ 1,6-addition was observed but the yield was limited to 33\% of the $\alpha$-isomer $\mathbf{3 2 b}$ (Scheme 3.5).

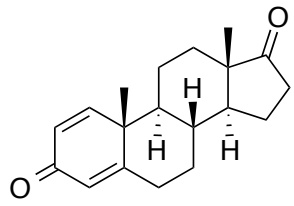

31

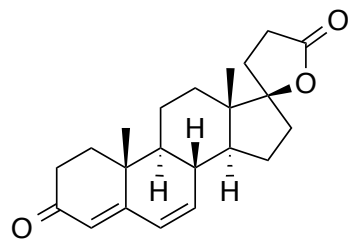

32

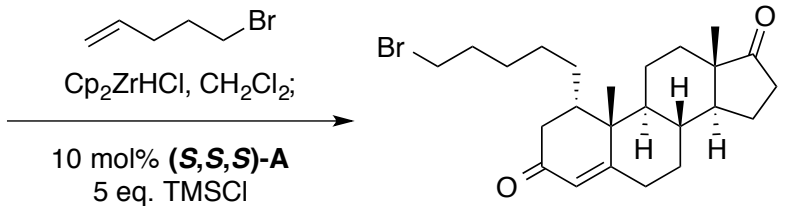

$\mathrm{Et}_{2} \mathrm{O}, \mathrm{RT}$

$60 \%+8 \% \beta$-isomer

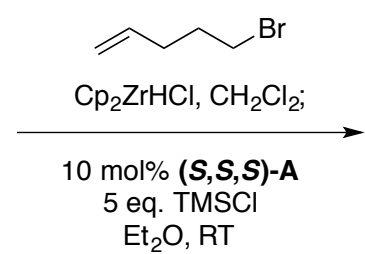

$\mathrm{Et}_{2} \mathrm{O}, \mathrm{RT}$

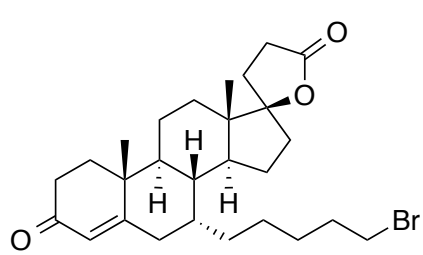

$32 b$

$33 \%+8 \% \beta$-isomer

Scheme 3.5: Results for the addition of 5-bromo-1-pentene

Additionally, we demonstrated that it was possible to add a reactive and complex benzyl chloride moiety to both steroids using the optimised procedure; in this case only moderate yields were obtained (Scheme 3.6). These results were promising and, although beyond the scope of work 
described in this chapter, suggest that these reactions could be optimised using other phosphoramidite-Cu complexes, or other ligands, including inexpensive achiral options, if desired.

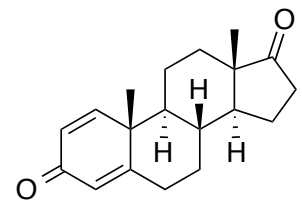

31

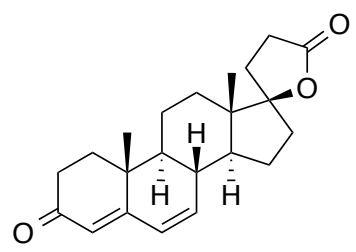

32

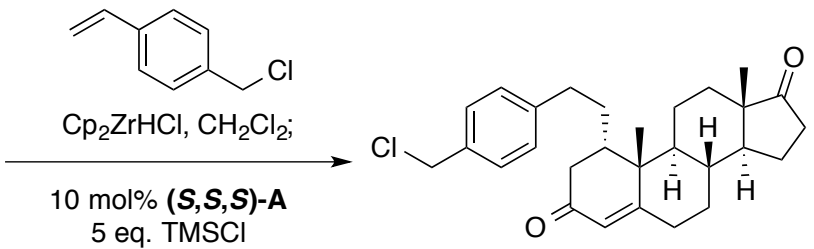

$31 \mathrm{c}$

$30 \%$ yield

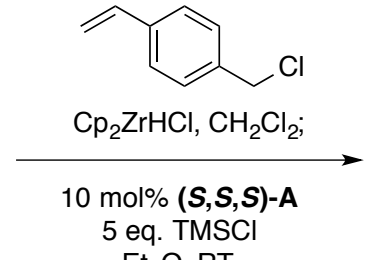

$\mathrm{Et}_{2} \mathrm{O}, \mathrm{RT}$

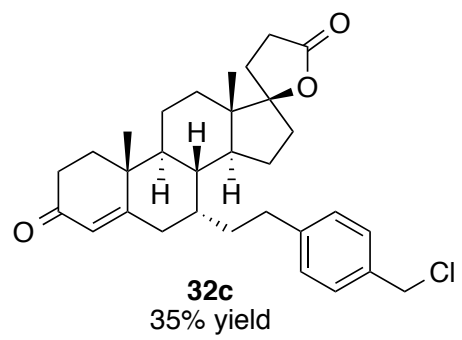

Scheme 3.6: Results for the addition of 4-vinylbenzyl chloride

The major product obtained from the 1,6-addition of 4-vinylbenzyl chloride to steroid $\mathbf{3 2}$ was confirmed through single crystal X-ray studies of 32c, which showed addition took place on the lower face (Figure 3.3).

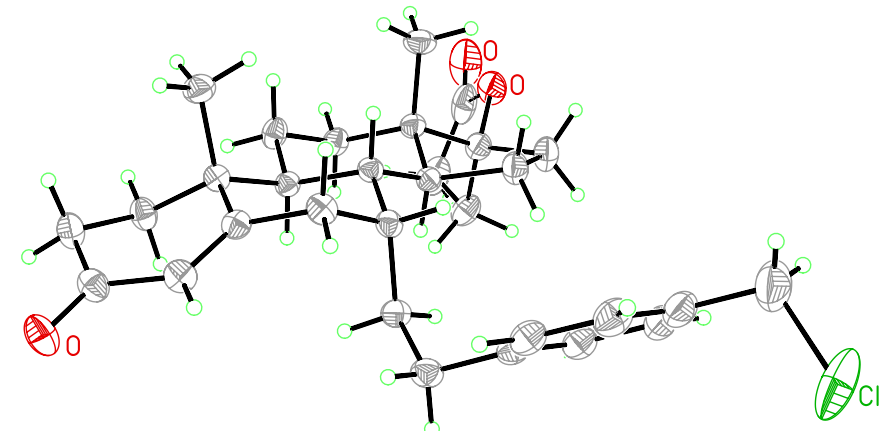

Figure 3.3: Crystal structure of steroid 32c

In addition, we showed the ability to add a styrene substituted with a para-boronic ester derivative, here we obtained a good yield (45\%) and only observed the $\alpha$-isomer $32 \mathrm{~d}$ (Scheme 3.7). 


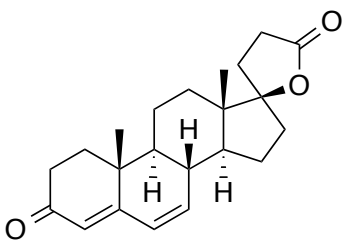

32

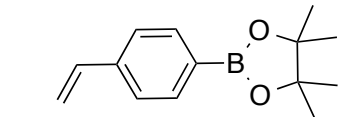

$\mathrm{Cp}_{2} \mathrm{ZrHCl}, \mathrm{CH}_{2} \mathrm{Cl}_{2}$;

$10 \mathrm{~mol} \%(\boldsymbol{S}, \mathbf{S}, \mathbf{S})-\mathrm{A}$ 5 eq. TMSCl $\mathrm{Et}_{2} \mathrm{O}, \mathrm{RT}$

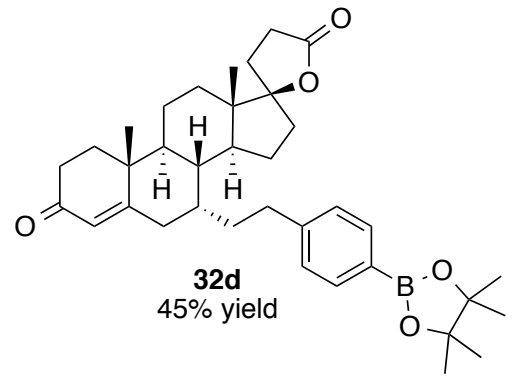

Scheme 3.7: Results for the addition of a para-boronic ester derivative

Promising results were obtained with the addition to steroids, we have shown we can add simple and more functionalised alkenes in moderate to good yields, using the system optimised for asymmetric addition to cyclic enones. We believe that this method could be very useful for future synthesis of highly functionalised steroids, which are important in medicine. Further optimisation of the conditions for these substrates, which are already chiral and non-racemic, may lead to higher yielding procedures. The stereochemical outcome of these reactions was largely substrate controlled and addition to the $\alpha$-face is preferred, although in the case of steroid $\mathbf{3 2}$ we have shown that it is possible to reverse the normally preferred stereochemical outcome (to $2: 1$ in favour of the $\beta$-anomer) by use of catalyst $(\boldsymbol{R}, \boldsymbol{R}, \boldsymbol{R})-\mathbf{A}$. 


\subsection{Formation of Quaternary Centres}

\subsubsection{Introduction}

The formation of all-carbon quaternary centres with control over the absolute stereochemistry is widely viewed as a challenging, yet important goal in organic synthesis. ${ }^{112}$ Extensive work on the copper-catalysed asymmetric addition of $n$-alkyl groups, primarily by Alexakis and Hoveyda, has been reported ${ }^{17 b}$ but there is still a lot of ongoing research in this area. Those reported involve the use of organoaluminium, dialkylzinc and Grignard reagents as nucleophiles. Earlier methods relied primarily on the use of the more reactive organoaluminium reagents; where the strong Lewis acidity of the aluminium species activates the relatively unreactive tertiary enone toward conjugate addition more so than magnesium or zinc-based reagents. The reactivity of tertiary enones is believed to be poorer due to the steric bulk around the $\beta$-carbon. In addition the methyl group is electron donating leaving the carbon atom less electrophilic.
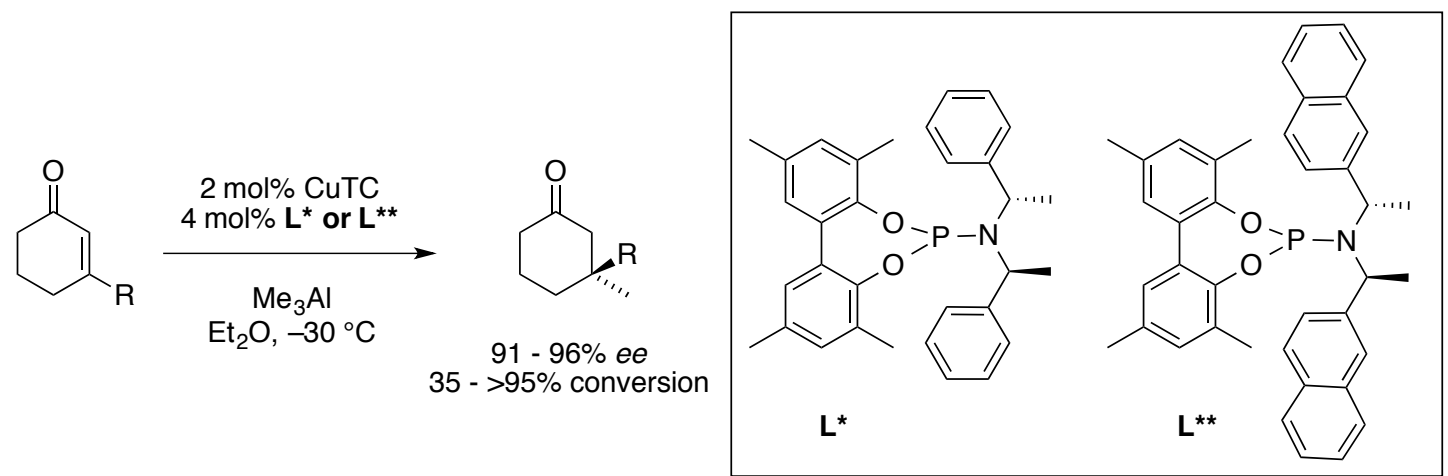

Scheme 3.8: Alexakis's addition to tertiary enones with trimethyl aluminium

Work by Alexakis et al. involved the addition of trimethyl aluminium to a variety of different $\beta$ substituted enones. ${ }^{113}$ Excellent enantioselectivities were achieved when the combination of copper thiophene carboxylate and a biphenol-based phosphoramidite ligand were used to catalyse the reaction (Scheme 3.8). A number of subsequent methods for the addition of organoaluminium reagents were developed $39,40,114$ but research became more focused on extending these methods for use with dialkylzinc and Grignard reagents, due to their greater accessibility. Hoveyda et al. first reported the addition of dialkylzinc reagents to 'doubly activated' 
tetrasubstituted olefins, where a cyclic enone contained an additional electron-withdrawing group. ${ }^{115}$ Presumably, the presence of an electron withdrawing $\mathrm{CO}_{2} \mathrm{Me}$ on the $\alpha$-carbon lead to a more reactive electrophile and hence allowed the conjugate addition to the $\beta$-substituted carbon to occur. Hoveyda et al. developed their method further, so that it did not require the use of an $\alpha$ substituted carbon by using NHC ligands instead of peptide-based ligands. ${ }^{116}$ It is assumed the formation of a chiral NHC-copper complex was what allowed highly enantioselective addition to both 6-and 7-membered $\beta$-substituted enones with a range of dialkylzinc reagents (Scheme 3.9).<smiles></smiles>

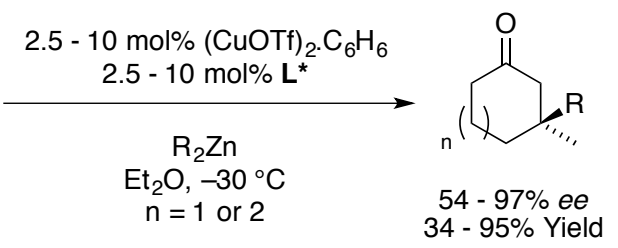

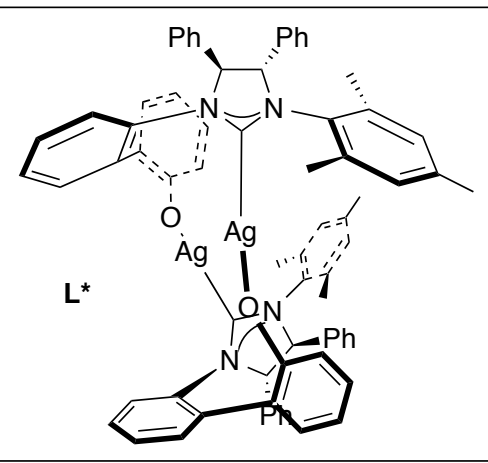

Scheme 3.9: Hoveyda's results using an NHC-copper complex

Since the development of NHC-complexes, Hoveyda has continued to expand the scope of dialkylzinc additions to 5 -membered enones as well as activated $\beta$-substituted enones. ${ }^{117}$ During this time, methods that allow Grignard reagents to be used were also being developed, primarily by Alexakis. He found using alkoxy-group bearing NHC ligands produced the most promising enantioselectivities (Scheme 3.10). ${ }^{118}$<smiles>CC1=CC(=O)CCC1</smiles>
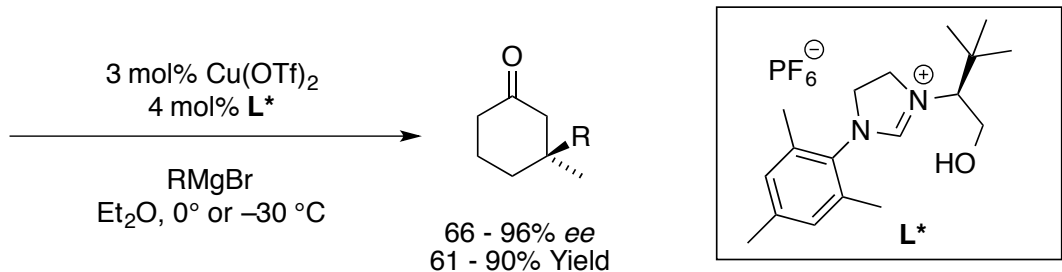

Scheme 3.10: Alexakis's results using Grignard reagents combined with NHC ligands

Tomioka et al. developed a similar NHC-catalysed system with Grignard reagents, but in this case only moderate enantioselectivities were obtained. ${ }^{119}$ Alexakis et al. continued their research to develop a second-generation method using alkoxy-NHC ligands, and extended its use to 
polyconjugated systems. ${ }^{120}$ The applicability of this method was demonstrated in the first step of the enantioselective total syntheses of (-)-neovibsanin $G$ and (-)-14-epi-neovibsanin $G$, including scale-up of the reaction to $12 \mathrm{~g} \cdot{ }^{121}$ General limitations of these systems are similar to those mentioned previously; there are practical and safety issues associated with the use of organometallic reagents and it is necessary to perform these reactions at cryogenic temperatures. The development of new methodologies for these reactions is highly desirable, and so attempts to extend our method ${ }^{60}$ to the highly enantioselective synthesis of quaternary stereocentres was carried out.

\subsubsection{Results and Discussion}

Taking the optimised conditions for the formation of tertiary centres, which involved $(S, S, S)$-A combined with 5 eq. $\mathrm{TMSCl}$ in $\mathrm{Et}_{2} \mathrm{O}$ at room temperature, a $\beta$-substituted system was examined (Scheme 3.11).

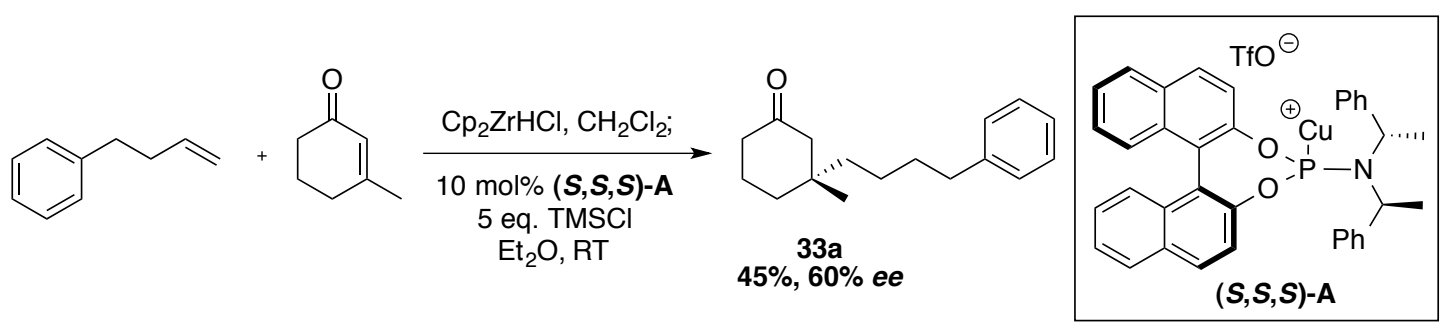

Scheme 3.11: Initial result using previously optimised conditions from chapter two

We were pleased to see that addition of 4-phenyl-1-butene to 3-methyl-cyclohexenone occurred with a moderate yield of $45 \%$, and $60 \%$ ee. Although these conditions were not directly suitable for addition to $\beta$-substituted enones in very high enantioselectivity, this result suggested that with some modification we could optimise the conditions to obtain a highly enantioselective system.

\subsubsection{Phosphoramidite screening}

A large library of phosphoramidite ligands (synthesised by P. M. C Roth) was screened to see if a promising result with a different phosphoramidite ligand could be obtained. The conditions used were the basic conditions we have used to screen ligands previously, using no additives and 
keeping the solvent $\left(\mathrm{CH}_{2} \mathrm{Cl}_{2}\right)$ the same throughout. Initial screening of the ligand backbone showed the current binaphthol backbone was optimal, and any alterations to this structure lead to a decrease in enantioselectivity (Figure 3.4).
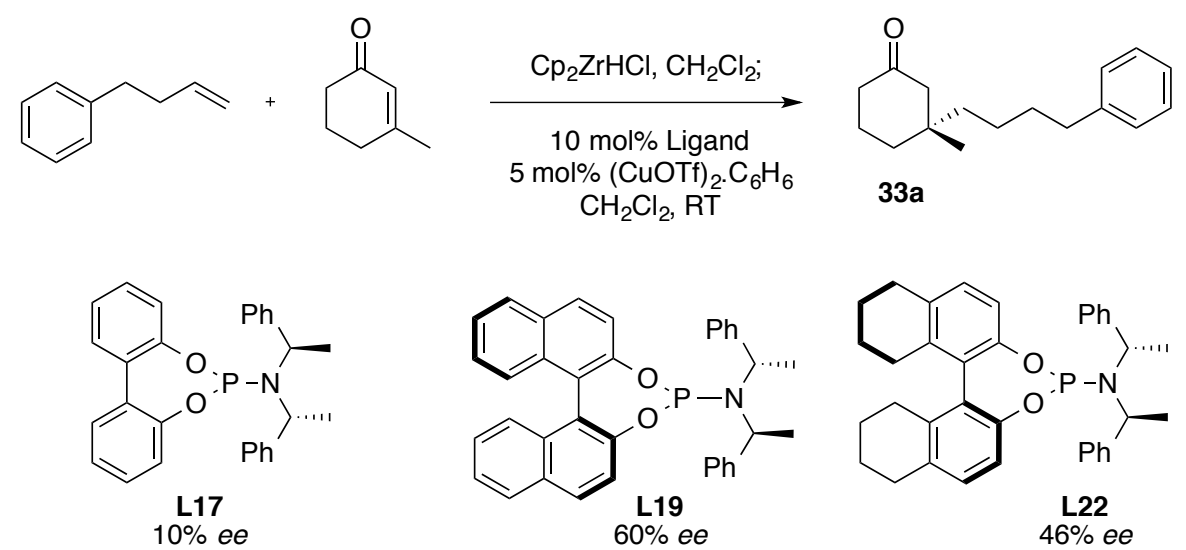<smiles>CC(c1ccccc1)N(P)C(C)c1ccccc1Oc1ccc2c(c1-c1c(O)ccc3c1CCCC3)CCCC2</smiles><smiles></smiles>

L28 $25 \%$ ee<smiles>CC(c1ccccc1)N(C(=O)c1cccc(Oc2ccc3ccc(Br)cc3c2-c2cccc(Br)c2)c1)[C@@H](C)c1ccccc1</smiles>

$$
\text { L31 }
$$$$
54 \% \text { ee }
$$

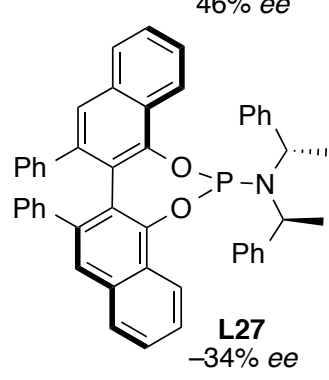

Figure 3.4: Results when changing the backbone of phosphoramidite ligands

Screening continued with ligands featuring different amine moieties; from herein only the amine was shown, where the BINOL backbone has been omitted the stereochemistry was "matched" [(i.e. $(S, S, S)$ or $(R, R, R)$ and $(S, S)$ or $(R, R)]$ unless otherwise stated. We found the "mis-matched" of L19 (L32 $(S, R, R)$ instead of $(S, S, S))$ resulted in improved enantioselectivity $(69 \%$ ee), whereas Alexakis's methoxy-substituted phosphoramidite L33 $(48 \%$ ee) gave a decreased result. The naphthyl-variant of the dimethyl biphenyl amine gave a lower $47 \%$ ee (Figure 3.5 ). Small alterations to L19 by removing a methyl group (L35) or adding a single fluorine atom (L36) also reduced the enantioselectivity. It was also seen that reducing the size and complexity of the amine gave a lower enantioselectivity (Figure 3.6). 

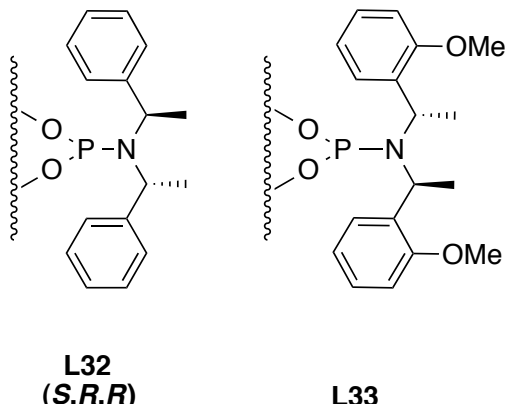

$(S, R, R)$

$69 \%$ ee

L33

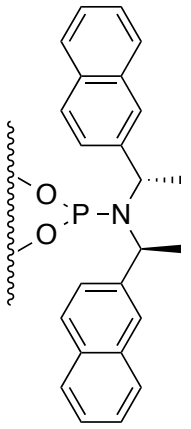

L34
$47 \%$ ee
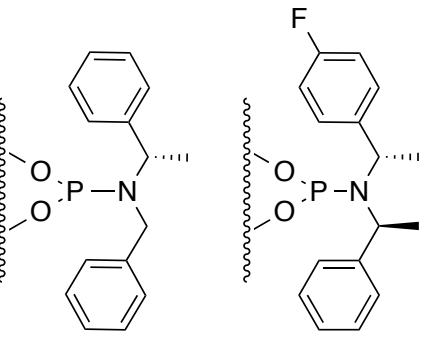

L35
$46 \% e e$

L36

$31 \%$ ee

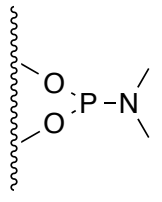

L37
$33 \%$ ee

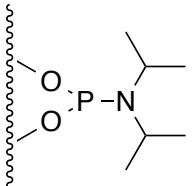

L39
$47 \%$ ee

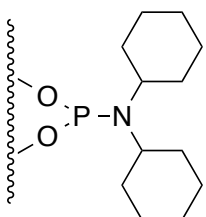

L40

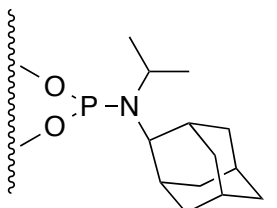

L41
$32 \%$ ee

Figure 3.6: Results with removing the stereocentres on the amine

A range of unsymmetrical 1- and 2-naphthyl-based amines was screened where the bulk on the one side of the amine was altered (Figure 3.7). No increase in enantioselectivity was observed; the best result was obtained with L47, which bears a bulky cyclohexane ring resulting in $62 \%$ ee.

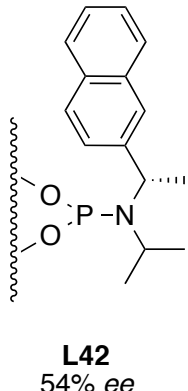

$54 \%$ ee

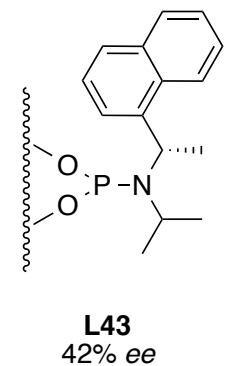

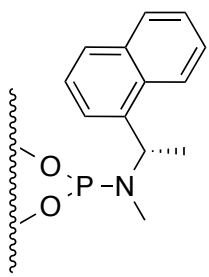

$\mathbf{L} 46$
$27 \%$ ee

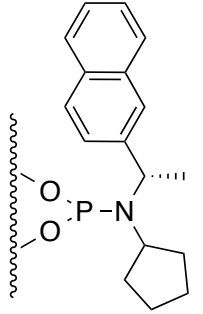

$\mathrm{L} 20$

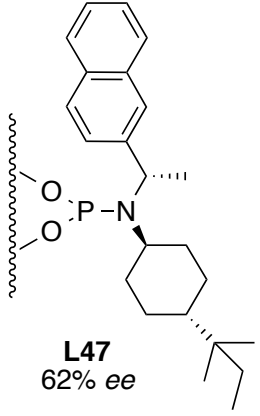

Figure 3.7: Results with changing the sterics on the amine

a-Methyl benzyl ligands, which had different electronic and steric properties, were screened. The results showed, in this case, that electronic properties did not significantly affect the enantioselectivity, and enantioselectivities between $40-56 \%$ ee were obtained (Figure 3.8). 

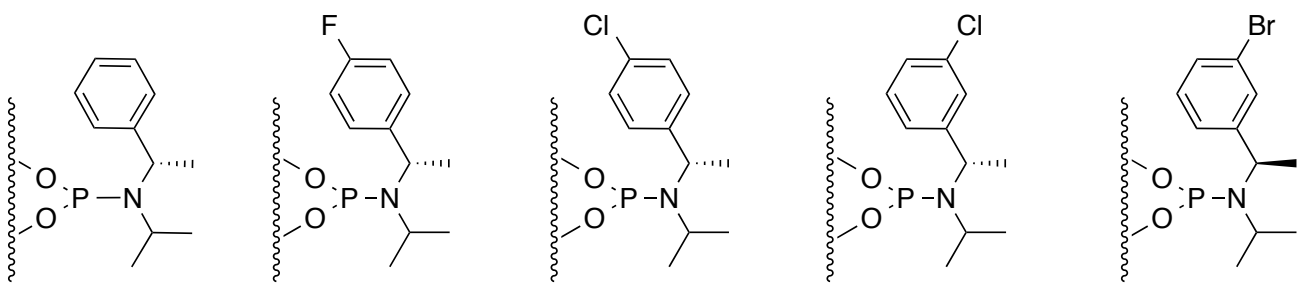

$\begin{array}{cc}\text { L50 } & \text { L23 } \\ 45 \% \text { ee } & 47 \% \text { ee }\end{array}$

L24
$47 \%$ ee

$\stackrel{\mathbf{L 2 5}}{53 \% e e}$

L51
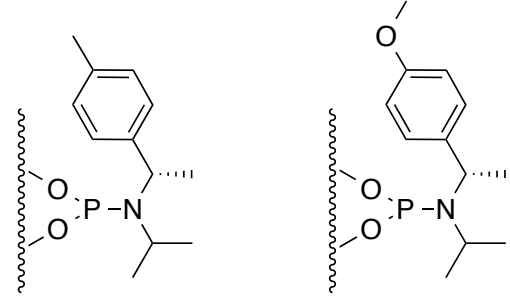

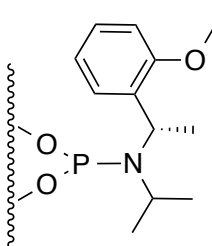

L55

$40 \%$ ee

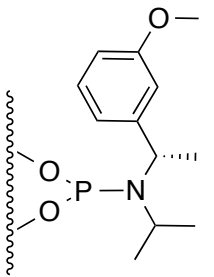

L56

$58 \%$ ee

Figure 3.8: Results with changing the electronics on the amine

An assortment of ligands bearing bicyclic amines was examined including ligands that do not have any stereogenic elements on the amine moiety (L62 \& L63). Similar results were obtained; an increased enantioselectivity was observed with L57 (67\%), while the remaining bicyclic amines gave enantioselectivities around 60\% ee, except for L62, which produced only $48 \%$ ee (Figure 3.9). After examining our library of phosphoramidite ligands, a significant increase in enantioselectivity was not observed; this suggested that further screening reactions were needed in order to find an optimal system for this reaction.
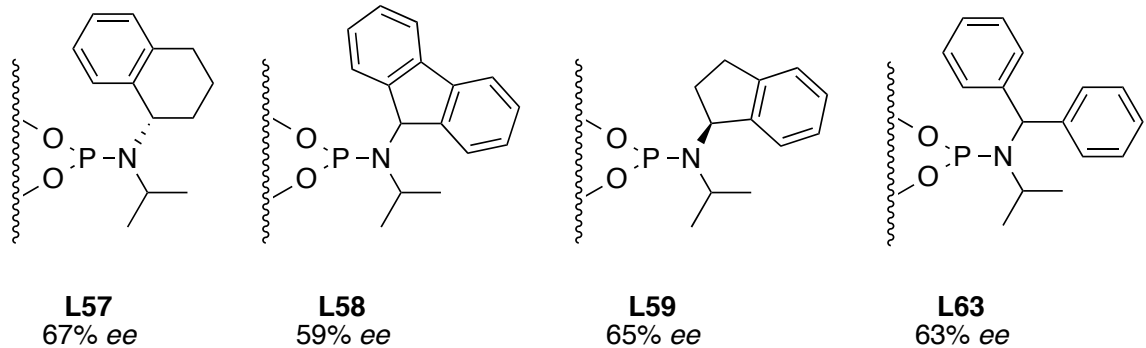

$\mathbf{L 5 9}$
$65 \%$

L63

\% ee

$59 \%$ ee

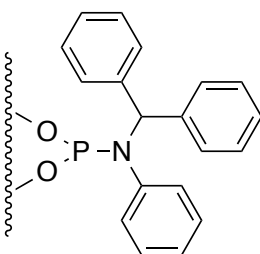

L62

$48 \%$ ee

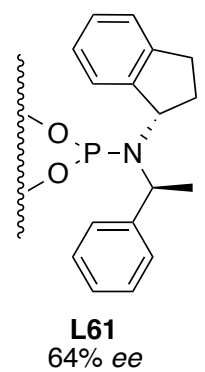

Figure 3.9: Results when using bicyclic amines 


\subsubsection{Optimised conditions and substrate screen}

At this point the project was taken over by other members of the group, $\mathrm{Dr} \mathrm{M}$. Sidera and P. M. C Roth, and the reaction was developed further. ${ }^{122}$ Here, a brief overview of the optimised conditions obtained, as well as the substrate scope is described. Promising and easily assessable ligands were screened with a variety of copper sources (Table 3.1). It was found that using the triflimide anion in $\mathrm{CH}_{2} \mathrm{Cl}_{2}$ with ligands $\mathbf{L} 32$ and $\mathbf{L 6 3}, 78$ and $92 \%$ ee respectively was obtained (entries 1 \& 2). Upon solvent screening it was found that L32 was optimal in toluene and resulted in $91 \%$ ee (entry 5) and for $\mathbf{L 6 3}$ the use of $t$-BuOMe gave $94 \%$ ee (entry 6).

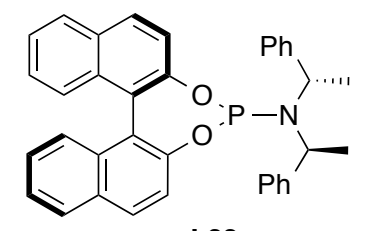

L32

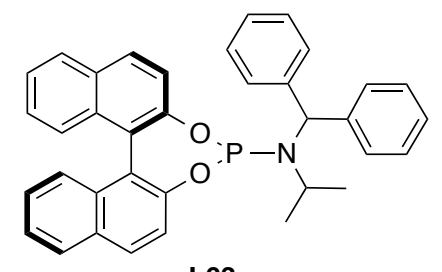

L63

\begin{tabular}{ccccc}
\hline Entry $^{\text {a }}$ & Solvent & Copper & Ligand & Result $(\boldsymbol{e e ~ \% ) ~}$ \\
\hline 1 & $\mathrm{CH}_{2} \mathrm{Cl}_{2}$ & $\mathrm{CuCl}+\mathrm{AgNTf}_{2}$ & $\mathbf{L 3 2}$ & 78 \\
2 & $\mathrm{CH}_{2} \mathrm{Cl}_{2}$ & $\mathrm{CuCl}+\mathrm{AgSbF}_{6}$ & $\mathbf{L 3 2}$ & 73 \\
3 & $\mathrm{CH}_{2} \mathrm{Cl}_{2}$ & $\mathrm{CuCl}+\mathrm{AgNTf}_{2}$ & $\mathbf{L 6 3}$ & 92 \\
4 & $\mathrm{CH}_{2} \mathrm{Cl}_{2}$ & $\mathrm{CuCl}+\mathrm{AgSbF}_{6}$ & $\mathbf{L 6 3}$ & 41 \\
5 & Toluene & $\mathrm{CuCl}+\mathrm{AgNTf}_{2}$ & $\mathbf{L 3 2}$ & 91 \\
6 & $t$-BuOMe & $\mathrm{CuCl}+\mathrm{AgNTf}_{2}$ & $\mathbf{L 6 3}$ & 94
\end{tabular}

Conditions - 4-phenyl-1-butene (2.5 eq.), $\mathrm{Cp} \mathrm{Z}_{2} \mathrm{ZrHCl}$ (2.0 eq.), $\mathrm{CH}_{2} \mathrm{Cl}_{2} ; 3-$ methyl-2-cyclohexenone (1.0 eq.), ligand (10 mol\%), copper (10 mol\%), solvent, RT. a) Reactions performed by Dr M. Sidera and P. M. C. Roth b) ee determined by chiral HPLC.

Table 3.1: Various results varying the ligand and copper source

After optimal conditions were found, the substrate scope of both alkene and enone was studied. Below a few examples of the excellent enantioselectivities obtained are shown (Table 3.2). The reaction tolerated a wide range of simple alkenes (entries $1 \& 2$ ) as well as sterically hindered alkenes (entry 3). Alkenes containing bromine substituents as well as a silicon-protected alcohol were both tolerated with good to excellent enantioselectivities (entries $4 \& 5$ ). The reactive allyl silane produced excellent enantioselectivity (entry 6). 5-membered enones were seen to give 
consistently lower enantioselectivities on the addition of 4-phenyl-1-butene (entry 7), however, 7membered enones resulted in a good ee with the addition of 1-hexene. ${ }^{123}$

Entry

Conditions - alkene (2.5 eq.), $\mathrm{Cp}_{2} \mathrm{ZrHCl}$ (2.0 eq.), $\mathrm{CH}_{2} \mathrm{Cl}_{2}$; enone (1.0 eq.). a) Isolated yield b) ee determined by chiral HPLC c) ee determined by derivatisation and ${ }^{13}$ C NMR d) synthesised by Dr M. Sidera or P. M. C. Roth.

Table 3.2: Select examples for the addition to tertiary enones

In conclusion, we have developed a highly enantioselective procedure in which we can add a range of simple and functionalised alkenes under mild conditions to form quaternary centres. Scale-up of the reaction was also possible, isolating up to $1.5 \mathrm{~g}$ of product 33a in high yield and enantioselectivity. 


\subsection{Addition to $\alpha, \beta$-Unsaturated Lactones}

\subsubsection{Introduction}

$\alpha, \beta$-Unsaturated lactones are important building blocks in complex molecule synthesis; the ability to access these lactones in enantiomerically enriched form is very important. We were interested in applying our methodology to the synthesis of $\beta$-substituted lactones. Our attention towards the addition of carbon nucleophiles to $\alpha, \beta$-unsaturated lactones was due to the relative deficiency of direct highly asymmetric conjugate addition procedures in comparison to the work reported with enones. The importance of obtaining these functionalised products is due to their relative abundance in natural products, for example in sesquiterpene lactones, ${ }^{124}$ macrolides $^{125}$ and diterpenoid type backbones ${ }^{126}$ which exhibit important biological activity (Figure 3.10).

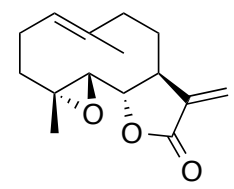

Parthenolide

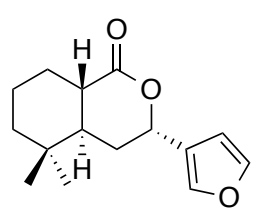

Ricciocarpin A

Sesquiterpene Lactones<smiles>CC(=O)OC1C[C@H](C(C)=O)[C@]2(C)CC[C@H]3C(=O)O[C@H](c4ccoc4)C[C@H]3[C@H]2C1=O</smiles>

Salvinorin A

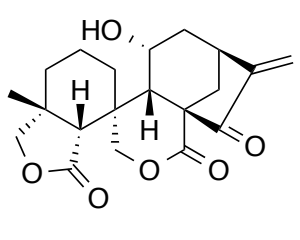

Ludongnin $\mathrm{A}$

Diterpenoid type

Figure 3.10: Lactones found in natural products

Spescha et al. first reported work in this area in 1993, where he extended his work on the 1,4addition to enones from addition of butylmagnesium chloride to 5,6-dihydropyran-2-one. ${ }^{127}$ The use of a copper-thiosugar based catalyst gave the product in only $10 \%$ ee, and $54 \%$ yield. Many groups have since attempted to develop an asymmetric 1,4 -addition to $\alpha, \beta$-unsaturated lactones with varying success. The two main classes of nucleophiles used were Grignard and dialkylzinc reagents. Tomioka et al. showed the use of Grignard reagents combined with copper iodide and a diphosphine derived pyrrolidine resulted in good enantioselectivity and yields, however, high catalyst loadings were required (32 mol\%) (Scheme 3.12). ${ }^{128}$ Schmalz et al. reported the addition of ethylmagnesium bromide when using a chiral phosphine-phosphite ligand derived from TADDOL and obtained up to $71 \% e e .{ }^{99 b}$ 
<smiles>O=C1C=CCCO1</smiles>

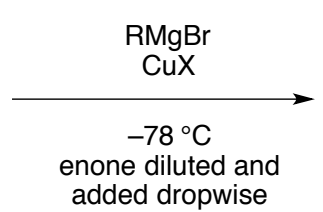

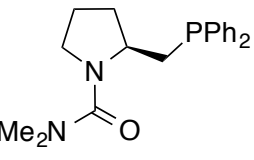

Tomioka up to $70 \%$ Yield up to $90 \%$ ee

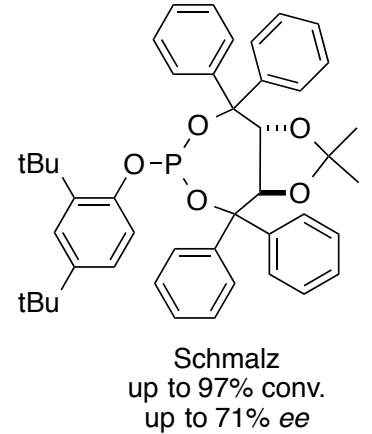

Scheme 3.12: Tomioka and Schmalz's results with Grignard reagents

Chan et al. first used dialkylzinc reagents in these additions, where the system used a chiral diphosphite-copper catalyst. ${ }^{129}$ Similarly Reetz ${ }^{130}$ employed diethylzinc reagents combined with a chiral diphosphonite ligand, and Mauduit ${ }^{131}$ showed the use of NHC ligands (Scheme 3.13). Despite high enantioselectivities obtained in these systems only conversions were reported and not isolated yields. In addition, limited nucleophile scopes have been reported with the majority of examples involving only addition of an ethyl nucleophile.

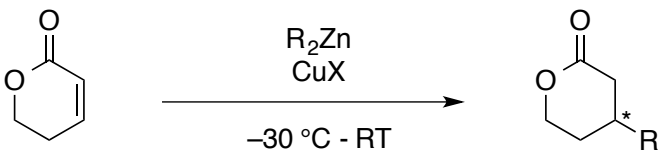

$\mathrm{R}=\mathrm{Me}, \mathrm{Et}$

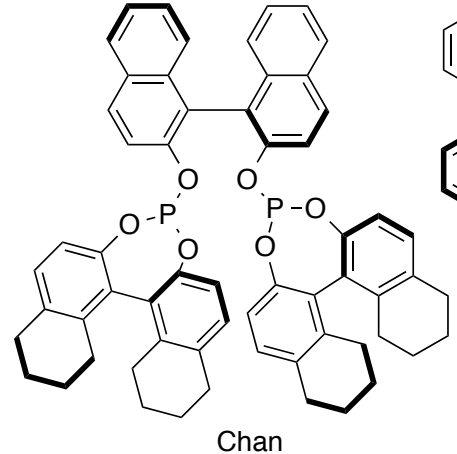

up to $100 \%$ conv. up to $98 \%$ ee

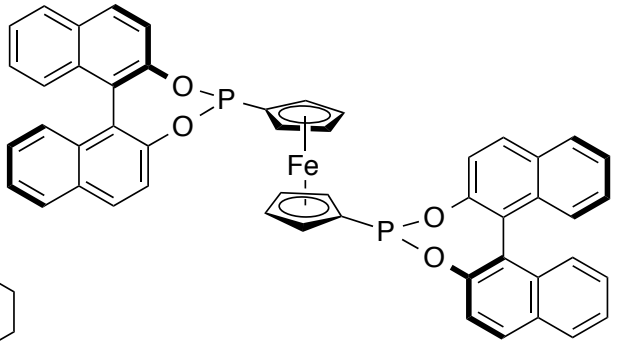

Reetz up to $100 \%$ conv. up to $88 \%$ ee

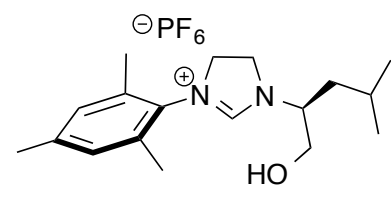

Mauduit

up to $99 \%$ GC yield up to $72 \%$ ee

Scheme 3.13: Chan, Reetz and Mauduit's results with dialkylzinc reagents

Hoveyda et al. reported a more thorough investigation into the 1,4-addition to $\alpha, \beta$-unsaturated lactones. ${ }^{95}$ The author's isolated products derived from trapping the resulting enolate with an aldehyde to give an aldol-type product, which then required a retroaldol reaction to give the desired 1,4-addition product. This was necessary, as the resulting enolates are very reactive. The problem was attributed with previously reported procedures due to: "adventitious ketene 
formation or intermolecular Michael addition ${ }^{\prime \prime 55}$ which decreased the isolated yield of the products. By following this trapping procedure Hoveyda was able to isolate the products with excellent enantioselectivities and yields; they also showed that the reaction conditions were applied to 5- and 7-benzosubstituted rings (Scheme 3.14).

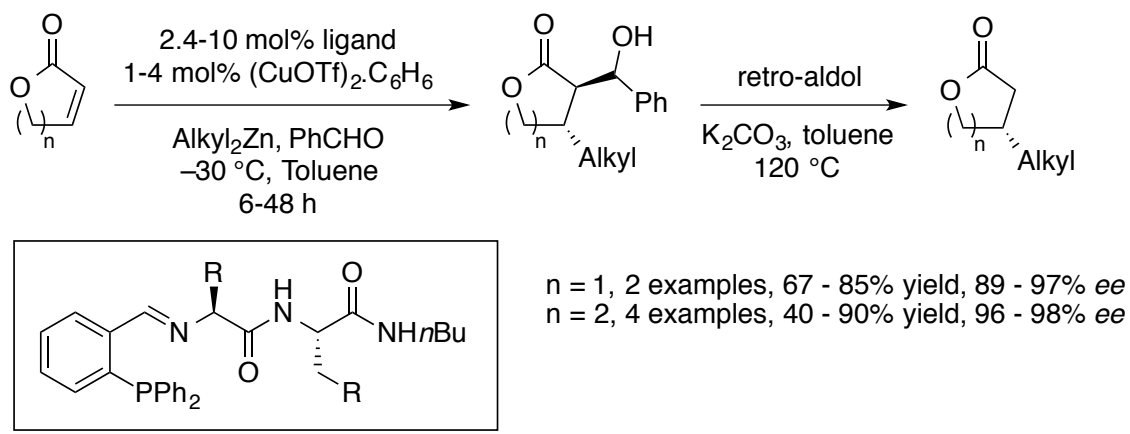

Scheme 3.14: Hoveyda's results requiring trapping of the enolate

Recent work illustrated challenges in this field are still apparent, Feringa et al. demonstrated the addition of Grignard reagents combined with ferrocenyl diphosphine ligand resulted in excellent enantioselectivities and yields. ${ }^{132}$ The authors avoided trapping the intermediate enolate, however, in order to achieve this, low temperatures $\left(-72^{\circ} \mathrm{C}\right)$ were required as well as pseudoinfinite dilution conditions, where a dilute solution of 5,6-dihydropyran-2-one was slowly added to the reaction mixture (Scheme 3.15).
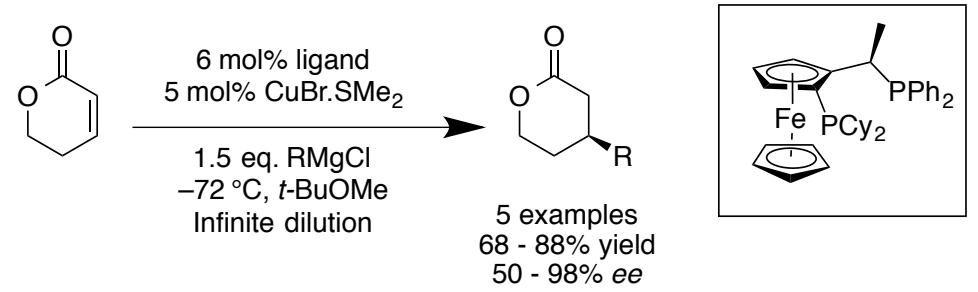

Scheme 3.15: Feringa's results requiring infinite dilutions

Developing a generally useful procedure for these additions that does not require any special reaction conditions or subsequent steps to liberate the desired product is sought after. The ability to add functionalised nucleophiles represents a highly desirable goal - as shown by the previous literature asymmetric additions are generally limited to very simple alkyl units. Šebesta et al. recently highlighted the difficulties associated with these reactions, they used a ferrocene phosphane carbene based catalyst to add ethyl Grignard reagent in good regio and 
enantioselectivity, however, even though high conversions are reported, lower yields are isolated $(47 \%) .{ }^{133}$ The authors suggested, due to little detection of side products, the reason for the low yield was due to product decomposition on work-up. They support this by showing the possibility of being able to successfully intercept the intermediate enolate with an electrophile, such as an imine or aldehyde, where higher yields were achieved than the conjugate addition reaction itself. $^{133}$

There is still a lot of work to be done in developing a catalytic asymmetric addition of alkylnucleophiles to $\alpha, \beta$-unsaturated lactones, particularly when this reaction is compared to the successes achieved in addition to carbocyclic substrates. Our aim here was to develop a generally useful method for addition of alkylzirconium reagents generated in situ from alkenes which does not require any special reaction conditions, additional steps, or cryogenic temperatures.

\subsubsection{Results and Discussion}

This project was jointly worked on with Dr E. E. Maciver. The optimisation of the reaction was performed together. The compounds synthesised by Dr E. E. Maciver have been noted in the tables.

\subsubsection{Optimisation of the reaction}

Due to the previously illustrated limitations in the addition of alkyl units to $\alpha, \beta$-unsaturated lactones, it was a challenge to see if our methodology would be applicable to substrates other than unsaturated carbonyl compounds. We took our previously developed conditions for cyclic enones and used 5,6-dihydropyran-2-one as an addition partner. 4-Phenyl-1-butene was hydrozirconated with the Schwartz reagent, the resulting alkylzirconocene reagent was added to a solution of $(S, S, S)$-A. 5,6-dihydropyran-2-one was then added followed by TMSCl and the resulting mixture was stirred at room temperature (Scheme 3.16). 


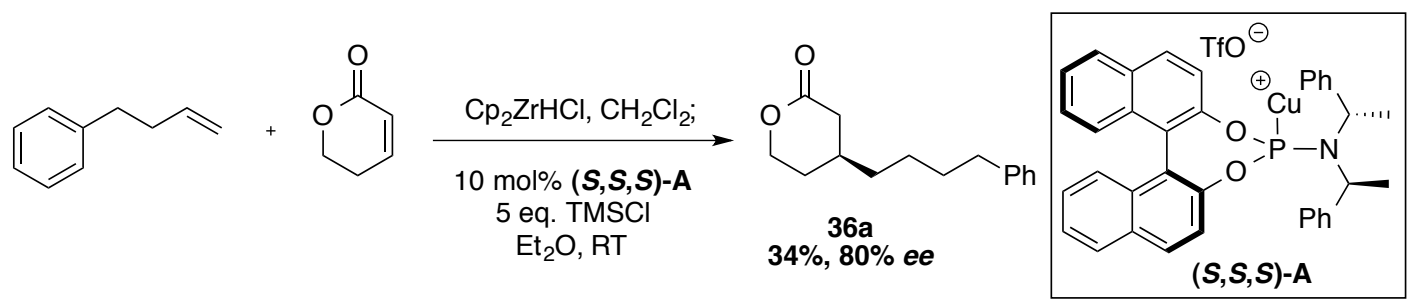

Scheme 3.16: Initial result using previously optimised conditions from chapter two

We were pleased to see that our conditions gave promising enantioselectivity $(80 \% e e)$ and moderate yield. This suggested that with some modification of the method the enantioselectivity and yield could be improved. Using a similar approach to that used elsewhere in this Thesis, we first changed the phosphoramidite ligand, varying both the backbone and amine moieties (Figure 3.11). The reaction conditions involved use of copper triflate benzene complex as the copper source and 5 eq. $\mathrm{TMSCl}$ as an additive in diethylether. We found that changing the backbone from dinaphthyl to diphenyl eroded the enantioselectivity, as well as the stereochemical mismatched ligand (L32) of L19, but to a much lesser extent (55\%). Other BINOL-modifications examined included a ligand with methyl groups added to the 3 and 3' positions L28 (75\% ee) and spirobackbone L16 (72\% ee), both had little effect on the enantioselectivity. No improvements were observed when modifying the backbone, which lead to ligands where the amine portion was varied. It was found that a mono-fluorinated version (L36) of L19 decreased the ee to $61 \%$, and similar results were obtained with $\mathbf{L 6 0}$, which bares a bicyclic amine structure $(57 \%$ ee). 2-Naphthyl isopropyl ligand (L42) resulted in comparable results to ligand L19, yet altering this structure from an isopropyl to a cyclohexyl group (L21) decreased the enantioselectivity to $48 \%$. Substituted benzyl groups showed little effect, only decreasing the enantioselectivity slightly. 


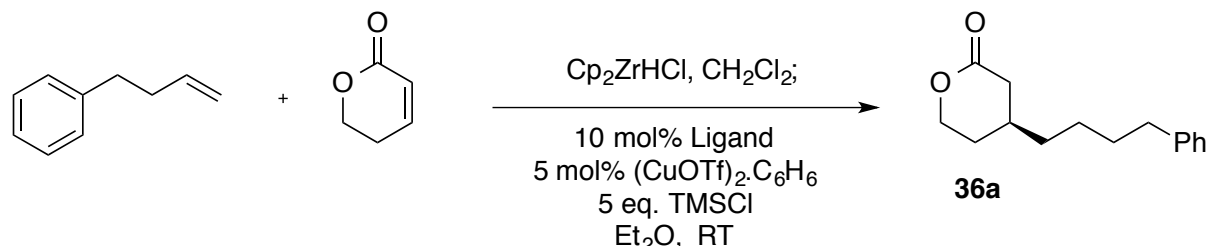<smiles>CC(c1ccccc1)N(Cc1ccccc1)Pc1ccccc1-c1ccccc1</smiles>

L17

$1 \%$ ee<smiles></smiles>

L16<smiles>CC(C)N(P)[C@H](C)Cc1cc2ccccc2cc1Oc1ccc2ccccc2c1-c1c(O)ccc2ccccc12</smiles>

L42 $79 \%$ ee<smiles>CC(c1ccccc1)N(C(C)c1ccccc1)C(C)C(O)c1ccc2ccccc2c1-c1c(O)ccc2ccccc12</smiles><smiles>CC(c1ccccc1)N(C(C)c1ccccc1)P(Oc1c(-c2ccccc2)cc2ccccc2c1-c1ccccc1)c1ccccc1</smiles>

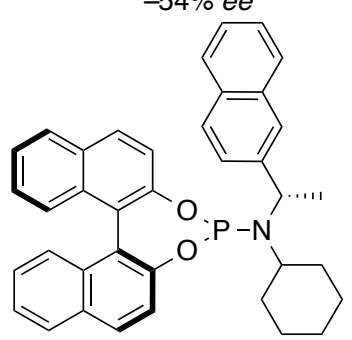

L21 $48 \%$ ee<smiles>CC(c1ccccc1)N(P)C(C)c1ccccc1Oc1ccc2c(c1-c1c(O)ccc3c1CCCC3)CCCC2</smiles>
年e<smiles>Cc1cc2ccccc2c(-c2ccccc2)c1OC1=c2c(c(C)cc3ccccc23)=C1O</smiles>
$75 \%$ ee<smiles>CC(c1ccc(F)cc1)N(PP)C(C)c1ccc(Oc2ccc3ccccc3c2-c2c(O)ccc3ccccc23)cc1</smiles><smiles>Oc1ccc2ccccc2c1-c1c(O)ccc2ccccc12</smiles>

$\stackrel{\mathbf{L 6 0}}{57 \% \text { ee }}$<smiles>CC(C)N(PP)[C@@H](C)c1ccc(Cl)cc1Oc1ccc2ccccc2c1-c1c(O)ccc2ccccc12</smiles>

$\mathbf{L} 24$
$67 \% e e$

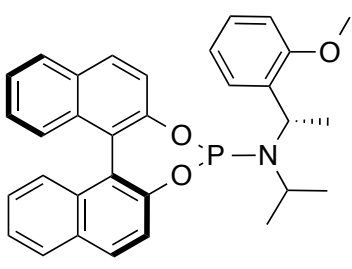

L55

Figure 3.11: Ligand screening results with (CuOTf) $)_{2} \cdot \mathrm{C}_{6} \mathrm{H}_{6}$

Very similar results were obtained between ligands $\mathbf{L} \mathbf{1 9}$ and $\mathbf{L 4 2}$, and so we decided to investigate the use of L42 further and different conditions were screened (Table 3.3). Removal of the TMSCI with $\mathrm{Et}_{2} \mathrm{O}$ drastically decreased the ee to $3 \%$ (entry 1 ), however in the case of $\mathrm{CH}_{2} \mathrm{Cl}_{2}$ the reaction proceeded better without TMSCl (entries $2 \& 3$ ). Varying the solvents resulted in no improvement of enantioselectivity (entries $4-6$ ). The number of equivalents of TMSCl was then studied; we found that using 2 eq. or 10 eq. lowered the enantioselectivity (entries $7-9$ ). Changing the temperature of the reaction showed a comparable result of $81 \%$ ee at $5{ }^{\circ} \mathrm{C}$ and a decrease to $70 \%$ ee at $10{ }^{\circ} \mathrm{C}$ (entries $10 \& 11$ ). Upon variation of the equivalents of the ligand used, we found by using an excess of ligand (15 mol\%) in relation to copper (10 mol\%) no appreciable increase in enantioselectivity was observed (entry 12), in comparison, when using a decreased amount of 
ligand, in relation to copper, (7.5 and 5 mol\%) the enantioselectivity decreased (entries 13 \& 14), suggesting a 1:1 copper to ligand ratio was optimal in this reaction.

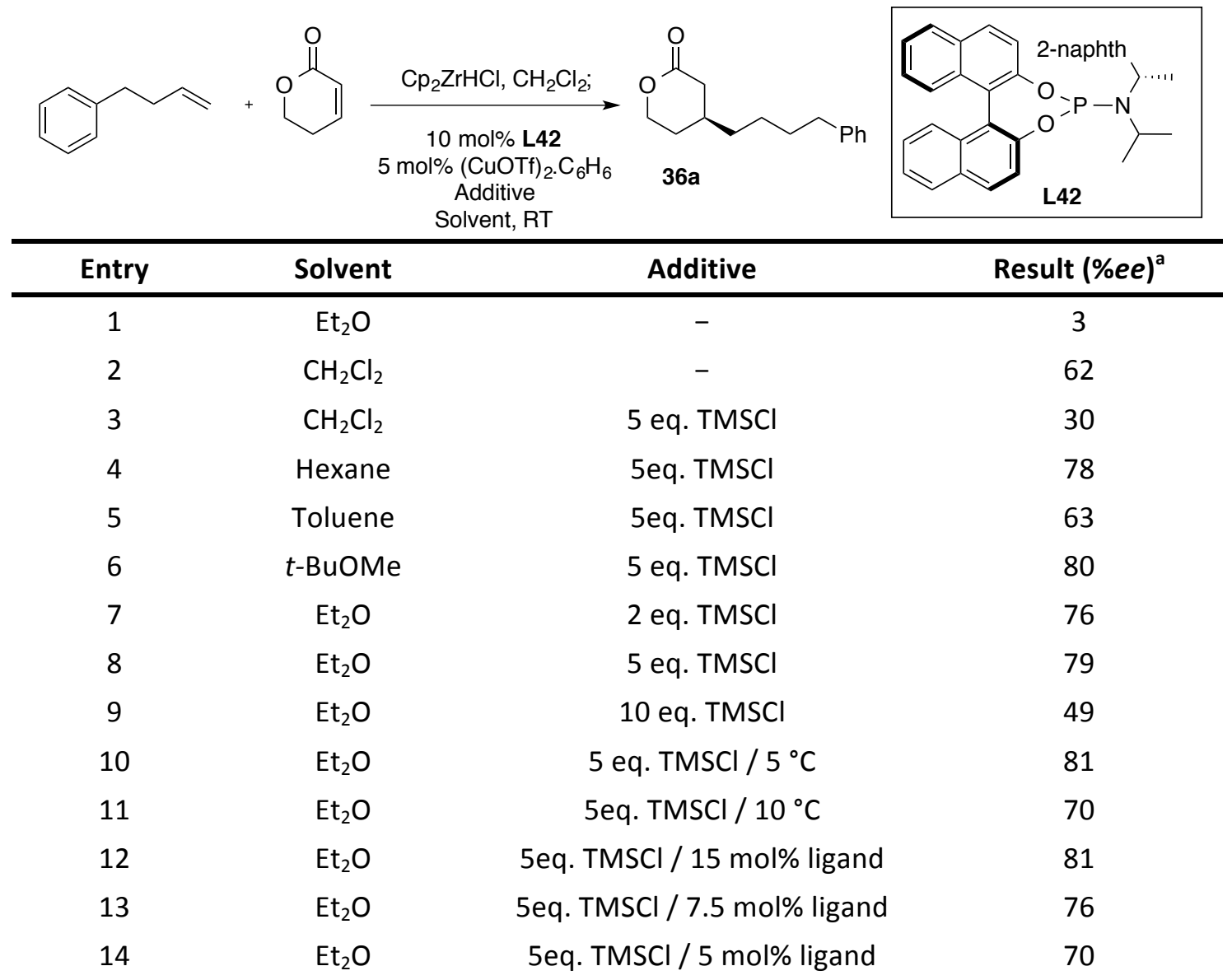

Conditions - 4-phenyl-1-butene (2.5 eq.), $\mathrm{Cp} 2 \mathrm{ZrHCl}$ (2.0 eq.), $\mathrm{CH}_{2} \mathrm{Cl}_{2} ;$ 5,6-dihydropyran-2-one (1.0 eq.). a) ee determined by chiral HPLC.

Table 3.3: Varying additive and solvent used

After no significant increase in enantioselectivity, we decided to screen different copper sources (Table 3.4). Variations of the copper-triflate source were tested, where different coordinating nitrile groups (MeCN, $i$-PrCN or $t$-BuCN) were present, however, lower enantioselectivities were seen (entries $1-4)$. Different anions were then screened using the method of silver salt - anion exchange, in $\mathrm{Et}_{2} \mathrm{O}$ with $\mathrm{TMSCl}$, enantioselectivities were very poor (entries $5-8$ ), however when changing the solvent to $\mathrm{CH}_{2} \mathrm{Cl}_{2}$ and excluding the $\mathrm{TMSCl}$ the enantioselectivities improved significantly in some cases. Poor enantioselectivities were seen for CuOTs, CuOMs and $\mathrm{CuONO}_{2}$ (entries 10, 11 and 13), however improved enantioselectivities were seen for the use of $\mathrm{CuN}(\mathrm{Tf})_{2}$ and $\mathrm{CuClO}_{4}$ (84 and $82 \%$ ee, respectively). Different solvents were then tested with the copper 
triflimide reagent, and lower enantioselectivities were observed when using $\mathrm{Et}_{2} \mathrm{O}, \mathrm{THF}$ and toluene (entries $15-17)$.

\begin{tabular}{|c|c|c|c|c|}
\hline Entry & Copper & Solvent & Additive & Result $(\% e e)^{a}$ \\
\hline 1 & $(\mathrm{MeCN})_{4} \mathrm{CuOTf}$ & $\mathrm{CH}_{2} \mathrm{Cl}_{2}$ & 5 eq. TMSCl & 67 \\
\hline 2 & $(\mathrm{MeCN})_{2} \mathrm{CuOTf}$ & $\mathrm{CH}_{2} \mathrm{Cl}_{2}$ & 5 eq. TMSCl & 71 \\
\hline 3 & $(i \operatorname{PrCN})_{2} \mathrm{CuOTf}$ & $\mathrm{CH}_{2} \mathrm{Cl}_{2}$ & 5 eq. TMSCl & 62 \\
\hline 4 & $(t \mathrm{BuCN})_{2} \mathrm{CuOTf}$ & $\mathrm{CH}_{2} \mathrm{Cl}_{2}$ & 5 eq. TMSCl & 74 \\
\hline 5 & $\mathrm{CuCl}+\mathrm{AgN}(\mathrm{Tf})_{2}$ & $\mathrm{Et}_{2} \mathrm{O}$ & 5 eq. TMSCl & 36 \\
\hline 6 & $\mathrm{CuCl}+\mathrm{AgO}(\mathrm{Ts})$ & $\mathrm{Et}_{2} \mathrm{O}$ & 5 eq. TMSCl & RAC \\
\hline 7 & $\mathrm{CuCl}+\mathrm{AgBF}_{4}$ & $\mathrm{Et}_{2} \mathrm{O}$ & 5 eq. TMSCl & 5 \\
\hline 8 & $\mathrm{CuCl}+\mathrm{AgPF}_{6}$ & $\mathrm{Et}_{2} \mathrm{O}$ & 5 eq. TMSCl & 4 \\
\hline 9 & $\mathrm{CuCl}+\mathrm{AgClO}_{4}$ & $\mathrm{CH}_{2} \mathrm{Cl}_{2}$ & 5 eq. TMSCl & 42 \\
\hline 10 & $\mathrm{CuCl}+\mathrm{AgO}(\mathrm{Ts})$ & $\mathrm{CH}_{2} \mathrm{Cl}_{2}$ & - & 3 \\
\hline 11 & $\mathrm{CuCl}+\mathrm{AgO}(\mathrm{Ms})$ & $\mathrm{CH}_{2} \mathrm{Cl}_{2}$ & - & 4 \\
\hline 12 & $\mathrm{CuCl}+\mathrm{AgN}(\mathrm{Tf})_{2}$ & $\mathrm{CH}_{2} \mathrm{Cl}_{2}$ & - & 84 \\
\hline 13 & $\mathrm{CuCl}+\mathrm{AgNO}_{3}$ & $\mathrm{CH}_{2} \mathrm{Cl}_{2}$ & - & 7 \\
\hline 14 & $\mathrm{CuCl}+\mathrm{AgClO}_{4}$ & $\mathrm{CH}_{2} \mathrm{Cl}_{2}$ & - & 82 \\
\hline 15 & $\mathrm{CuCl}+\mathrm{AgN}(\mathrm{Tf})_{2}$ & $\mathrm{Et}_{2} \mathrm{O}$ & - & 70 \\
\hline 16 & $\mathrm{CuCl}+\mathrm{AgN}(\mathrm{Tf})_{2}$ & THF & - & 75 \\
\hline 17 & $\mathrm{CuCl}+\mathrm{AgN}(\mathrm{Tf})_{2}$ & Toluene & - & 74 \\
\hline
\end{tabular}

Conditions - 4-phenyl-1-butene (2.5 eq.), $\mathrm{Cp} 2 \mathrm{ZrHCl}$ (2.0 eq.), $\mathrm{CH}_{2} \mathrm{Cl}_{2} ;$ 5,6-dihydropyran-2-one (1.0 eq.), $\mathrm{L} 42$ (10 mol\%), copper (10 mol\%), solvent, RT a) ee determined by chiral HPLC.

Table 3.4: Screening copper sources

Different ligands were screened with the perchlorate and triflimide anions. First the triflimide anion was screened (Figure 3.12); we found when combining CuN(Tf $)_{2}$ with L19 we improved the enantioselectivity to $90 \%$. Using the hydrogenated backbone (L22) or 3, 3'-substituted ligands (L28 \& L30) resulted in 87\%, 72 and $66 \%$ ee respectively and a comparable enantioselectivity of 90\% was obtained with the spiro-backbone ligand (L16). It was found when making alterations to the amine moiety that subtle variations, such as removing a methyl group from the amine (L35), gave significantly reduced enantioselectivity (36\%), fluoro-substituted L36 gave good enantioselectivity (82\%) yet an ortho-methoxy-substituted group (L33) gave only $39 \%$ ee. 
Chlorine-substituted (L24) produced only a moderate enantioselectivity (69\%), however, 93\% ee was obtained when using an isomer of L19, L63.

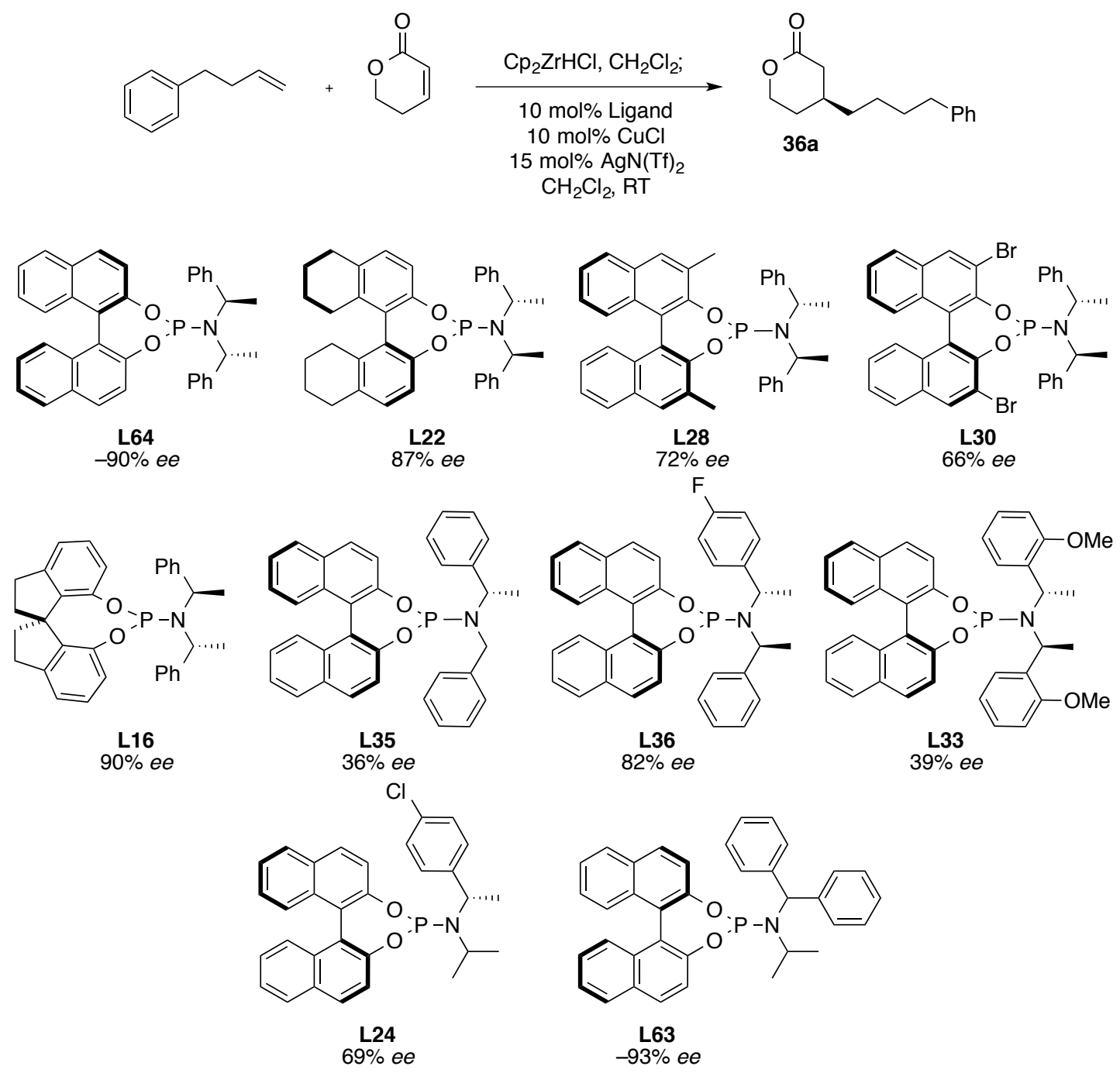

Figure 3.12: Ligand screening results with $\mathrm{CuN}(\mathrm{Tf})_{2}$

Ligands were then screened with the perchlorate anion (Figure 3.13), very similar results were obtained when compared to the triflimide anion. These experiments lead to two sets of conditions where the best enantioselectivity was obtained with L63 (93\% ee) using either triflimide or perchlorate anion. 

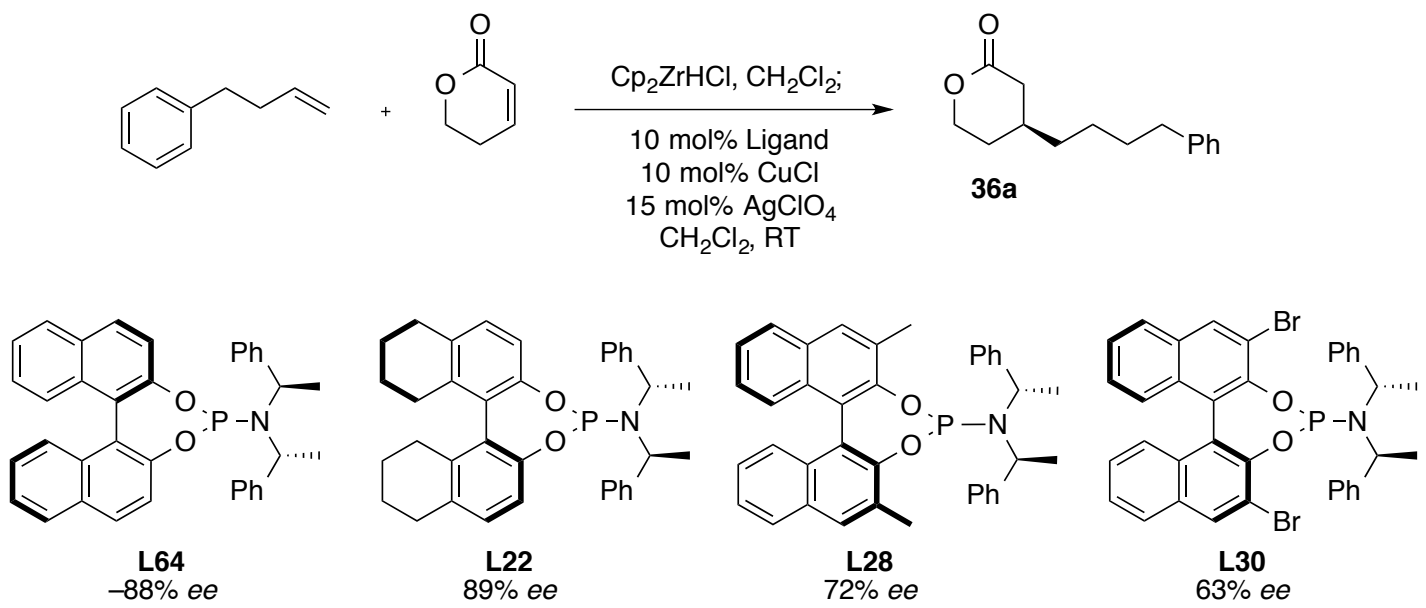

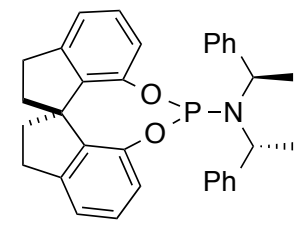

$\stackrel{\text { L16 }}{\sim 91 \% \text { ee }}$
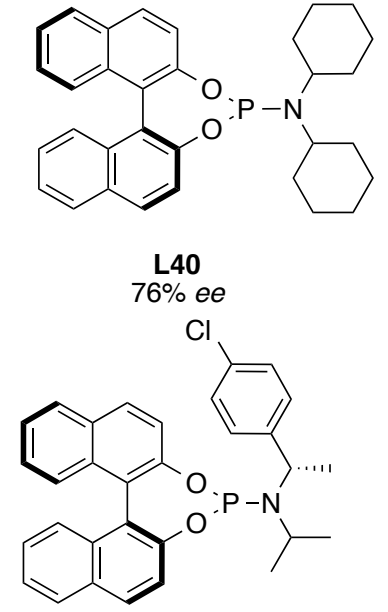

L24
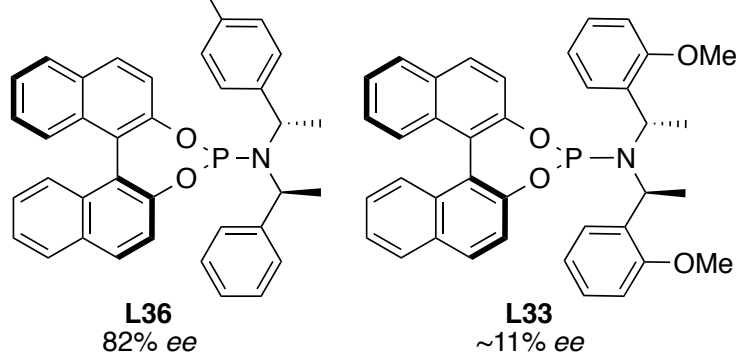

Figure 3.13: Ligand screening results with $\mathrm{CuClO}_{4}$

Taking the current optimal ligand (L63) we were disappointed to find that upon isolating the product, the yields were less than $10 \%$ with both the triflimide and perchlorate anions, even though full conversion was observed within two hours. This was not completely unforeseen; as mentioned previously, the same problem was documented in previous research, where further enolate addition and decomposition on work-up was suggested. ${ }^{95,133}$ Different anions, solvents and additives were tested with $\mathbf{L 6 3}$ in an attempt to solve the problem (Table 3.5); TMSCl was added to the reaction, as expected, the enantioselectivity decreased (entries $1,2 \& 4)$, changing the solvent of the perchlorate anion (entry 5) to $\mathrm{Et}_{2} \mathrm{O}$ resulted in good enantioselectivity, however a poor yield. Changing the anion to the bulky $\left[\mathrm{BAr}_{4}^{\mathrm{F}}\right]$ anion gave good enantioselectivity but the product was barely detectable by HPLC or TLC, suggesting that the reactivity was poor (entries 7 \& 
8). Different additives, $\mathrm{ZrCl}_{4}$ and 18-crown-6 were added; it was found that addition of $\mathrm{ZrCl}_{4}$ gave racemic products (entries $9 \& 10$ ) and using 18-crown-6 gave an excellent result of $94 \%$ ee, yet a poor isolated yield ( $\sim 30 \%)$. At this point we tested $(\mathrm{CuOTf})_{2} \cdot \mathrm{C}_{6} \mathrm{H}_{6}$ with $\mathbf{L 6 3}$ and were pleased to see a high enantioselectivity when using this combination (entry 12) and upon isolation we obtained $89 \%$ yield. No significant increase in selectivity was seen at $0{ }^{\circ} \mathrm{C}$, so we proceeded to keep reaction conditions at room temperature (entry 13).

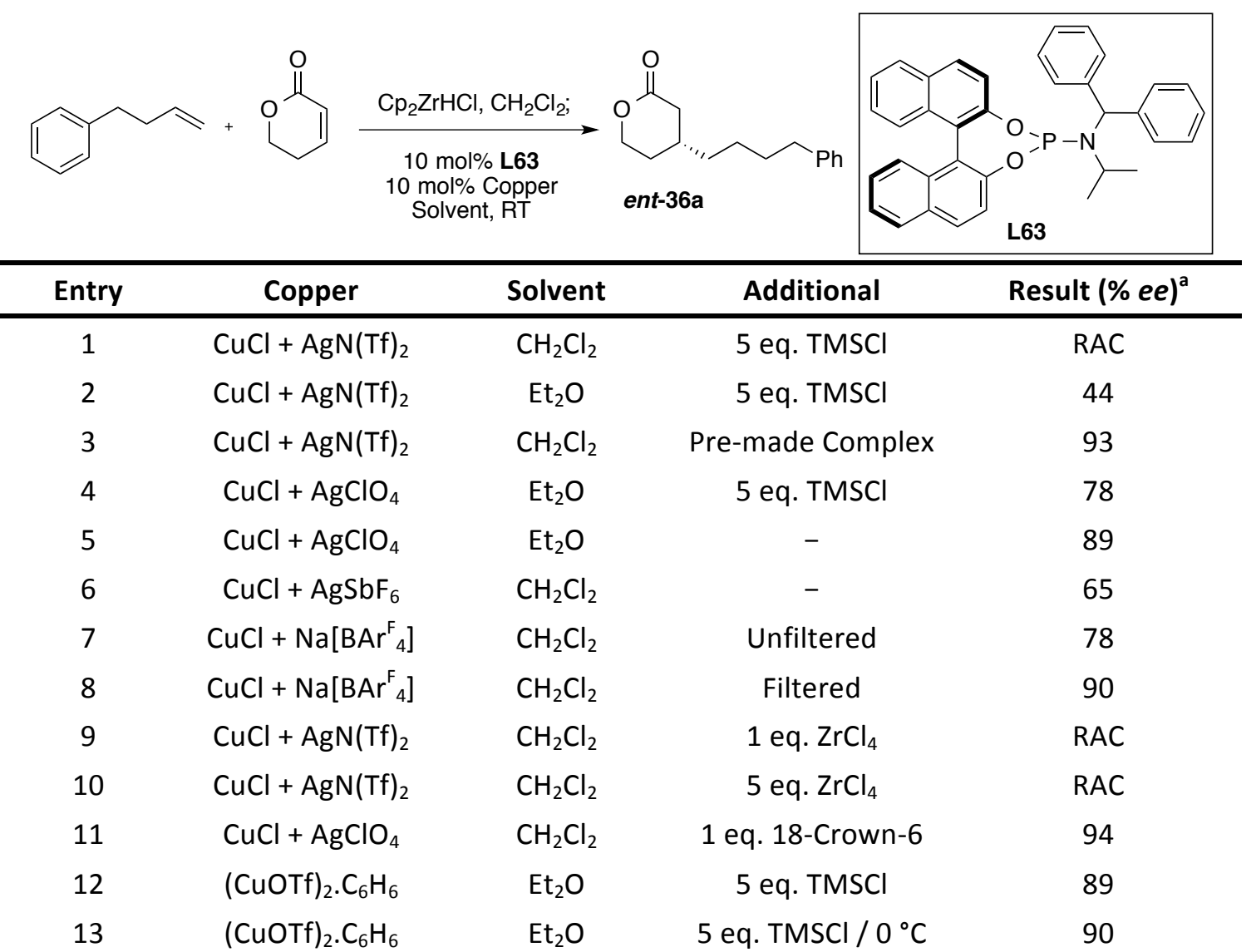

Conditions - 4-phenyl-1-butene (2.5 eq.), $\mathrm{Cp}_{2} \mathrm{ZrHCl}$ (2.0 eq.), $\mathrm{CH}_{2} \mathrm{Cl}_{2} ;$ 5,6-dihydropyran-2-one (1.0 eq.). a) ee determined by chiral HPLC.

\section{Table 3.5: Screening copper sources}

In an attempt to further increase the ee we screened different solvents with (CuOTf) $)_{2} \cdot \mathrm{C}_{6} \mathrm{H}_{6}$ and L63. At this point we chose to complex the copper triflate benzene complex with the ligand to form complex $(\boldsymbol{R})$-B, which is an air stable material. From screening different solvents, we found the best result was obtained using $\mathrm{Et}_{2} \mathrm{O}$ with the presence of $\mathrm{TMSCl}$ (entry 2, 89\% ee). In the case of $\mathrm{CH}_{2} \mathrm{Cl}_{2}$, use of TMSCl gave poorer enantioselectivity, yet up to $91 \%$ ee was observed without 
TMSCl (entry 3). In the case of THF, when combined with TMSCl an appreciable enantioselectivity was obtained $(79 \%$, entry 6$)$, yet a poor result was obtained when $\mathrm{TMSCl}$ was excluded $(42 \%$, entry 5). Better results were obtained with 2-MeTHF instead of THF, with higher ee observed when TMSCl was added (entries $7 \& 8$ ). When $t$-BuOMe was used $88 \%$ ee was obtained with $\mathrm{TMSCl}$ and only $70 \%$ ee without TMSCl. The reverse trend was seen when using dichloroethane, an excellent enantioselectivity was obtained without the use of $\operatorname{TMSCl}(92 \%$, entry 11$)$ and a poorer result seen when using $\mathrm{TMSCl}$ (entry 12).

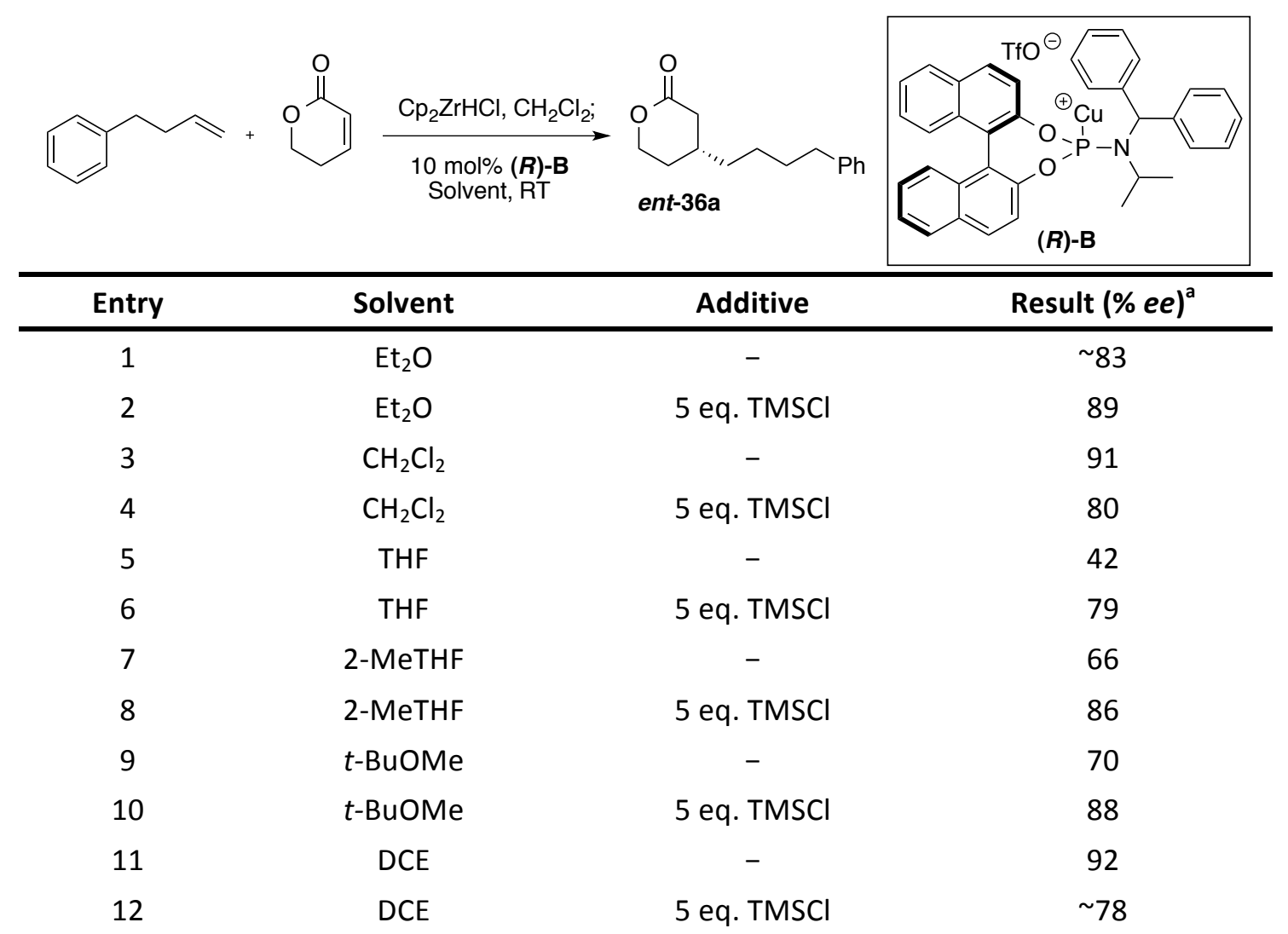

Conditions - 4-phenyl-1-butene (2.5 eq.), $\mathrm{Cp}_{2} \mathrm{ZrHCl}$ (2.0 eq.), $\mathrm{CH}_{2} \mathrm{Cl}_{2} ;$ 5,6-dihydropyran-2-one (1.0 eq.). a) ee determined by chiral HPLC.

Table 3.6: Screening solvents

Even though some results gave slightly elevated enantioselectivities compared to the use of $\mathrm{Et}_{2} \mathrm{O}$ (entries 3 \& 11), full conversion was not observed and the yields of the reactions, as analysed by crude ${ }^{1} \mathrm{H}$ NMR spectroscopy, were judged to be poor, leading us to continue using the combination of $\mathrm{Et}_{2} \mathrm{O}$ and $\mathrm{TMSCl}$. 


\subsubsection{Substrate Scope}

\subsubsection{Alkene scope}

With our optimised conditions we wanted to see if the reaction tolerated other substrates, however, first we required a method to determine the enantioselectivity of the substrates, which did not contain a chromophore. We found the derivatisation with DPEN (Chapter 2, Scheme 2.13) was unsuccessful; hence an alternative method was required to derivatise these substrates. Instead, the lactone was ring-opened with a primary benzyl amine using $\mathrm{AlMe}_{3}$ to form a secondary amide, which could then be analysed by HPLC to determine the enantioselectivity. This proved to be successful, and experimentally simple, and so was the method used for all substrates that did not contain a chromophore (Scheme 3.17).
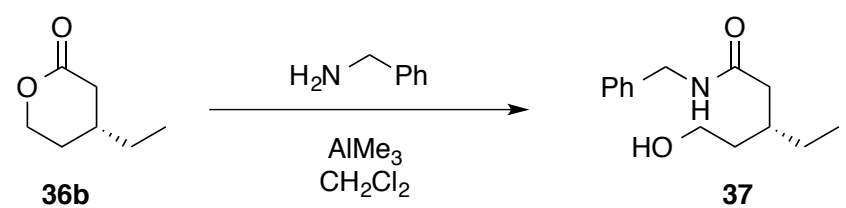

Scheme 3.17: Derivatisation of cyclic lactones

Simple alkyl substrates were tested in the asymmetric addition to $\alpha, \beta$-unsaturated lactones, and the enantioselectivity remained high throughout (Table 3.7). Straight chain alkyl groups gave moderate to good yields and excellent enantioselectivities (entries $1-4$ ), in addition bulkier alkyl groups such as cyclohexyl groups and $t$-butyl groups gave good results (entries $5 \& 6$ ). 


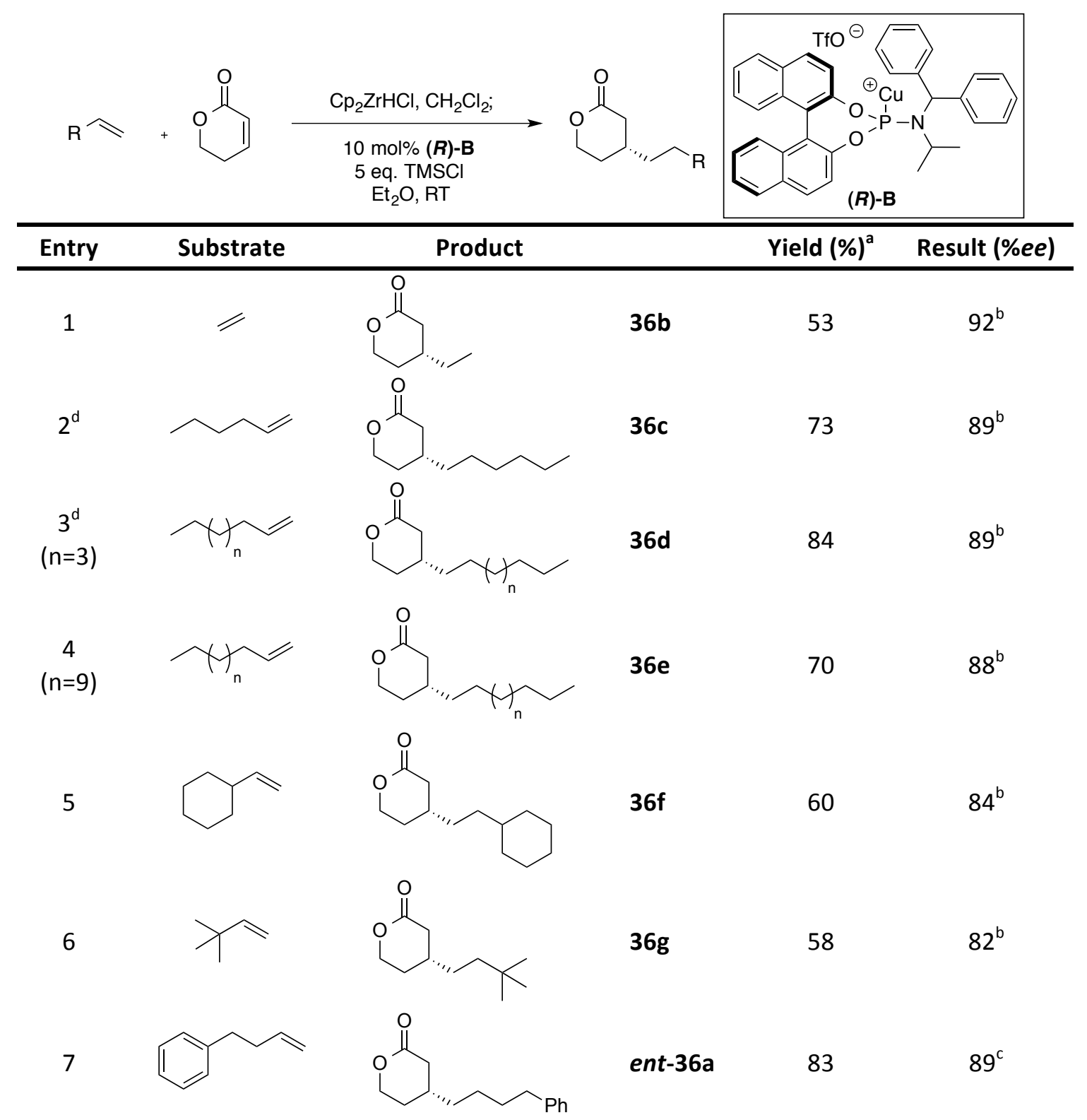

a) Isolated yield b) ee determined by chiral HPLC c) ee determined by derivatisation then chiral HPLC d) reactions performed by Dr. E. E. Maciver.

Table 3.7: Substrate screening

Expansion to more complex alkenes was studied; we found excellent enantioselectivities with a range of functionalised alkenes were obtained (Table 3.8). An alkene bearing an internal alkene, was left unreacted, obtaining moderate yield and good enantioselectivity (entry 1). Many different ethers and silyl-protected alcohols were reacted, all resulting in consistently good yields and enantioselectivities (entries $2-5$ ). In addition, excellent results were produced when para- 
bromo styrene (entry 6), a reactive allyl silane (entry 7) and multi-functionalised alkenes were added.

Entry

Conditions - 4-phenyl-1-butene (2.5 eq.), $\mathrm{Cp} 2 \mathrm{ZrHCl}$ (2.0 eq.), $\mathrm{CH}_{2} \mathrm{Cl}_{2} ;$ 5,6-dihydropyran-2-one (1.0 eq.), (R)-B (10 mol\%), $T M S C l\left(5.0\right.$ eq.), Et ${ }_{2} O, R T$. a) Isolated yield b) ee determined by chiral HPLC C) ee determined by derivatisation then chiral HPLC d) reactions performed by Dr E. E. Maciver.

\section{Table 3.8: Substrate screening}

A crystal structure of lactone $\mathbf{3 6} \mathbf{j}$ was obtained and confirmed the stereochemistry of the 6membered enones, which followed the same confirmation as that reported by Hoveyda. ${ }^{95}$ 


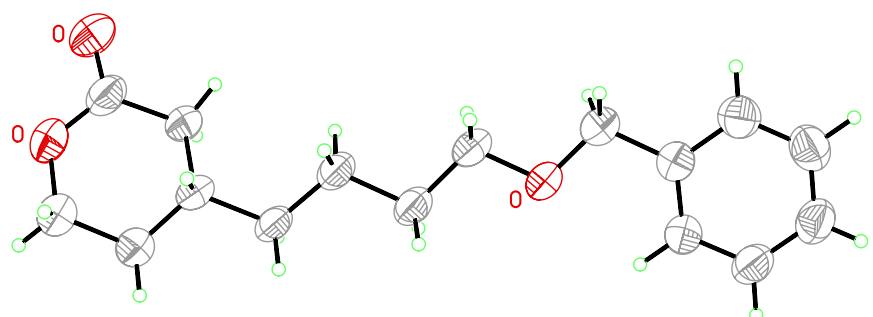

Figure 3.14: Crystal structure of lactone 36j

Despite a large range of successful alkenes screened, some were more successful than others. It was found that addition of styrenyl alkenes gave poor yields, $30 \%$ product was isolated on a number of occasions (Figure 3.15). Some attempts at optimisation of these substrates, by cooling to $0{ }^{\circ} \mathrm{C}$ and/or adding 5,6-dihydropyran-2-one dropwise did not improve the yield or enantioselectivity significantly. Due to the low yielding nature of these substrates, we decided to pursue them no further. However, future development of phosphoramidite ligands or a new copper catalyst system may result in improved results for these substrates.

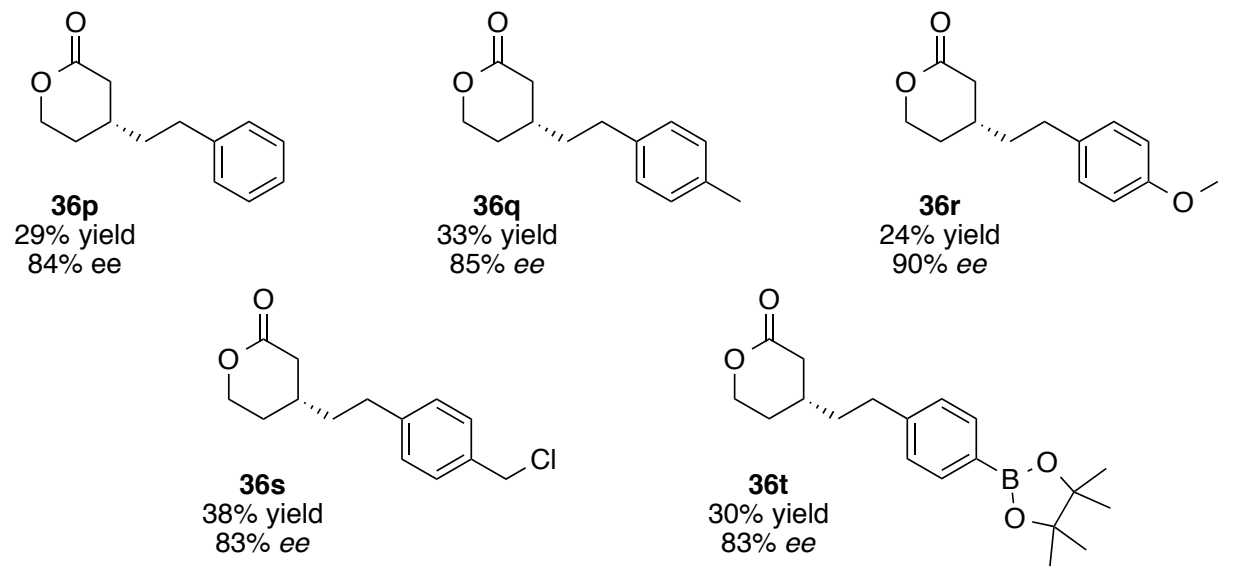

Figure 3.15: Addition of styrenyl alkenes

\subsubsection{Enone scope}

The next aim was to extend the scope of the lactones used, however this was more problematic than initially thought, the substrates were not commercially available, and the synthesis of these $\alpha, \beta$-unsaturated lactones did not involve simple steps. 
<smiles></smiles><smiles>CC1(C)C=CC(=O)O1</smiles>

38<smiles>CC1=CC(=O)OCC1</smiles>

40<smiles>O=C1C=CCCCO1</smiles>

41<smiles>O=C1C=CCc2ccccc2O1</smiles>

42

Figure 3.16: $\alpha, \beta$-Unsaturated lactones screened

The successful synthesis of lactones $\mathbf{3 9}, \mathbf{4 0}$ and $\mathbf{4 2}$ proceeded via the same route, which involved the nucleophilic addition of an alcohol to an acyl chloride followed by elimination to give the desired ester product. The resulting product undergoes ring-closing metathesis to give the desired cyclic $\alpha, \beta$-unsaturated lactone (Scheme 3.18). ${ }^{134}$

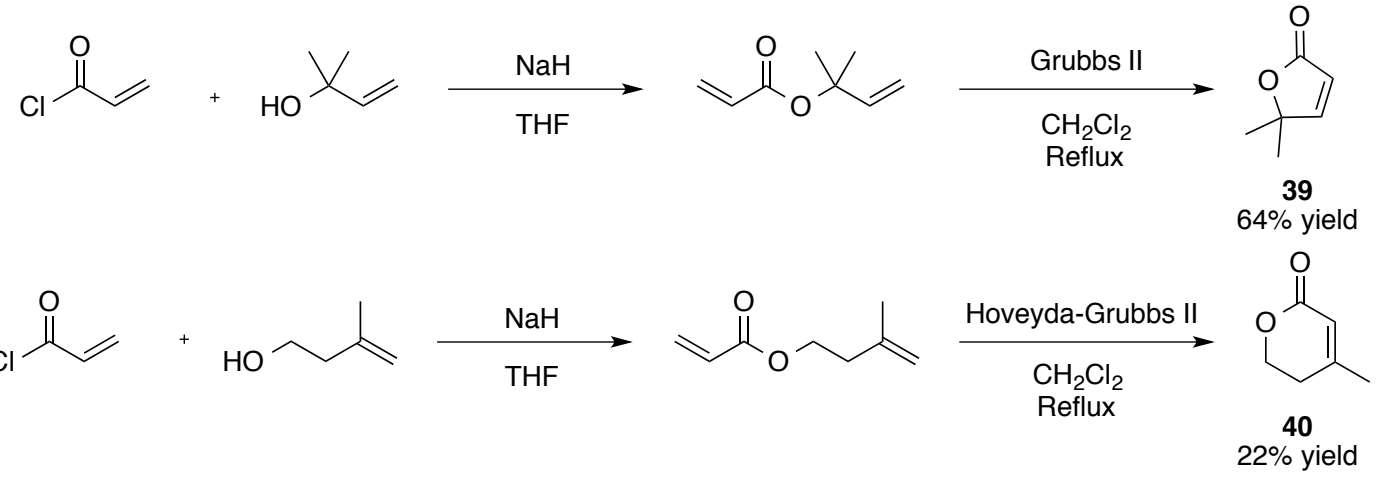<smiles>C=CC(=O)Cl</smiles><smiles>C=CCc1ccccc1O</smiles><smiles>C=CCc1ccccc1OC(=O)C=C</smiles>

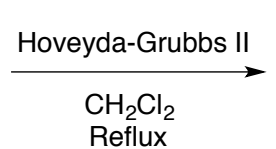<smiles>O=C1C=CCc2ccccc2O1</smiles>
$29 \%$ yield

Scheme 3.18: Synthesis of $\alpha, \beta$-unsaturated lactones

During attempts to obtain 7-membered lactone $\mathbf{4 1}$, the desired product was not obtained but a macrocycle of the product (Figure 3.17).<smiles>O=C1/C=C/CCCOC(=O)/C=C/CCCO1</smiles>

Figure 3.17: Macrocycle of the ring closing 
This was additionally observed in the synthesis of lactone $\mathbf{4 2}$, however, some desired product was isolated, albeit in low yields. In order to obtain lactone $\mathbf{4 1}$ the $\varepsilon$-caprolactone was taken and a selenoxide elimination reaction was performed to obtain the starting reagent (Scheme 3.19). ${ }^{135}$
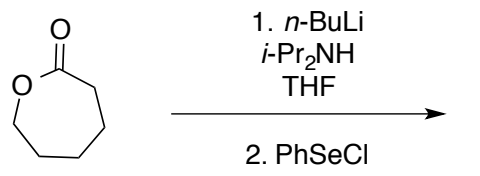

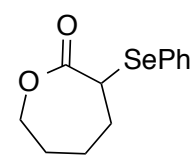

$27 \%$ yield
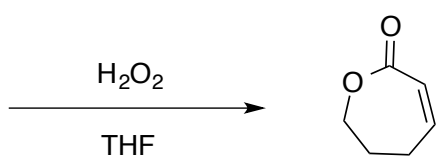

41 $82 \%$ yield

Scheme 3.19: Synthesis of 7-membered $\alpha, \beta$-unsaturated lactone

Having obtained a variety 5-, 6- and 7- membered $\alpha, \beta$-unsaturated lactones they were examined using the optimised reaction conditions (Figure 3.16). Upon attempts at reacting lactone $\mathbf{3 8}$ complete consumption of starting material was seen, however, no product was isolated. This was in direct contrast to the results obtained when using lactones $\mathbf{3 9}$ and $\mathbf{4 0}$ where no reaction was observed. In these cases the starting materials proved to be very stable to the reaction conditions and only remaining starting material by TLC and ${ }^{1} \mathrm{H}$ NMR spectroscopy was seen. In comparison, 7-membered lactone $\mathbf{4 1}$ reacted remarkably well, different functionalised alkenes were added in good to excellent yields and high enantioselectivity (41a and $\mathbf{4 1 b}$ ). It was also possible to add to lactone 42 in good yield and enantioselectivity (42a) (Figure 3.18). In addition, a crystal structure of lactone 41b was obtained, confirming the stereochemistry is the same as the 6-membered lactones.

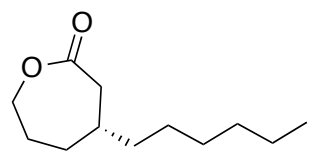

$41 a$ 95\% Yield $93 \%$ ee

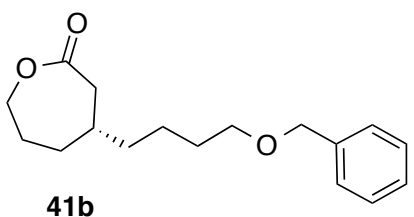

41b $90 \%$ ee

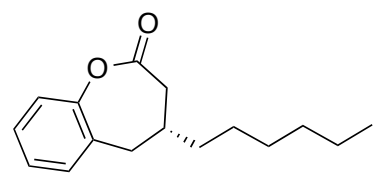

$42 a$

$63 \%$ Yield

$88 \%$ ee

Figure 3.18: Successful results

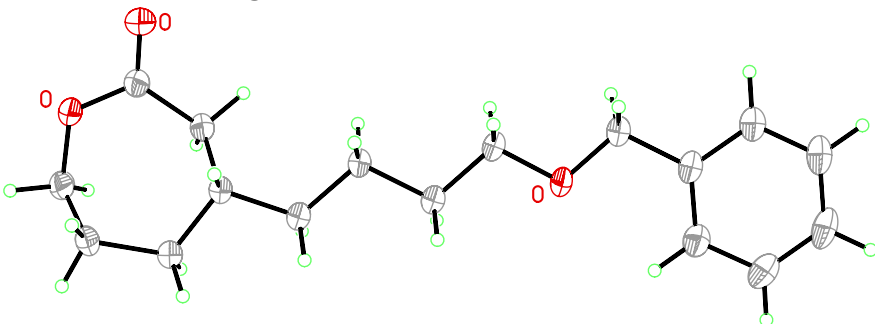

Figure 3.19: Crystal structure of lactone 41b 


\subsubsection{Applications}

We wanted to test the applicability of the reaction methodology; the reaction was scaled up to a $4.0 \mathrm{mmol}$ scale. $0.87 \mathrm{~g}$ ( $76 \%$ yield) of product $36 \mathrm{l}$ was isolated while maintaining high enantioselectivity (90\%) without the need for any special procedures or cryogenic temperatures (Scheme 3.20).

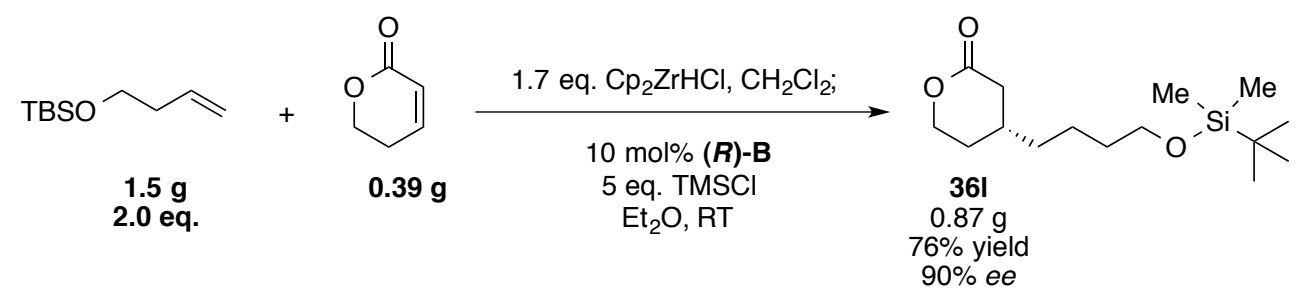

Scheme 3.20: Scale-up result

In addition, the products of the reaction were derivatised with a complex amine. Addition of an amine to lactone ent-36a by $\mathrm{Me}_{3} \mathrm{Al}$-mediated ring opening formed amide 43 in almost quantitative yield (98\%) without loss of the enantiomeric excess (89\%) (Scheme 3.21).
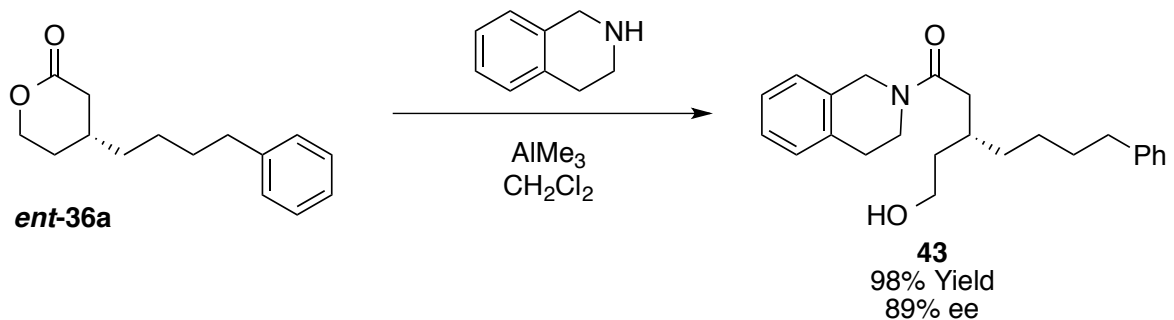

Scheme 3.21: Ring opening with a complex amine

A compound previously used in natural product synthesis was prepared (Scheme 3.22). Racemic 44 is a late-stage intermediate in the syntheses of rac-Onikulactone ${ }^{136}$ and racMitsugashiwalactone. ${ }^{137}$ The only asymmetric syntheses of mitsugashiwalactone were reported from the chiral pool ${ }^{138}$ and enantiomerically resolved materials, ${ }^{139}$ but not from material where asymmetry was introduced from a catalytic asymmetric approach. Access to $\mathbf{4 4}$ was a more demanding test of the method than we had seen so far, it was hypothesised that an appropriately functionalised lactone could be converted to $\mathbf{4 4}$ by dehydrative condensation, but the stability of the lactone ring and the stereochemical integrity of tertiary carbon centre during cyclisation were questionable. 


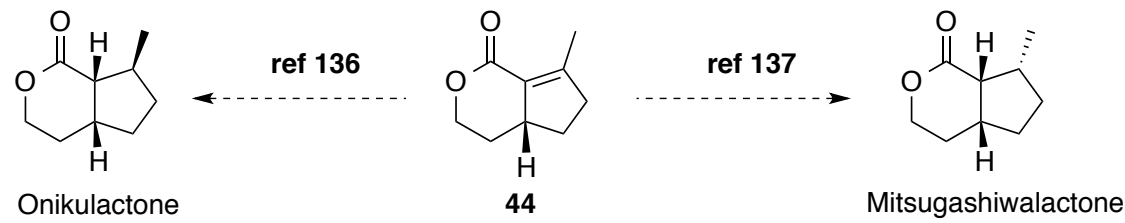

Scheme 3.22: Natural product synthesis from intermediate 44

To synthesise $\mathbf{4 4}$, the hydrometallation - asymmetric conjugate addition of alkenes $\mathbf{4 5}$ and $\mathbf{4 6}$ to 5,6-dihydropyran-2-one was studied (Table 3.9). Using acetal-protected 45, the 1,4-addition was observed in modest yield (41\%) and in 79\% ee (entry 1). By cooling the reaction vessel to $0{ }^{\circ} \mathrm{C}$ the enantioselectivity was increased to $85 \%$, but at the expense of reaction yield (entry 2 ). The use of conjugated diene $\mathbf{4 6}$ showed excellent chemoselectivity between the mono and disubstituted olefins and gave $\mathbf{3 6 v}$ with high enantioselectivity (entry $3,85 \%$ ee) at room temperature. Although the yield of $36 \mathrm{v}$ was low on our standard scale, $0.40 \mathrm{mmol}$ (entry $3,45 \%$ ), the formation of 36v occurred smoothly, and the yield was much improved when the reaction was performed on a 6.0 mmol scale (entry $4,78 \%$ yield, $83 \% e e$ ). Using isoprene 46 as a starting material is also attractive because it is readily available and inexpensive, whilst $\mathbf{4 5}$ is prepared in multiple steps.

\begin{tabular}{|c|c|c|c|c|c|c|}
\hline Entry & Substrate & Product & & Notes & Yield (\%) ${ }^{a}$ & Result (\%ee) \\
\hline 1 & & & & $\mathrm{RT}, 0.4 \mathrm{mmol}$ & 41 & 79 \\
\hline 2 & & & & $0{ }^{\circ} \mathrm{C}, 0.4 \mathrm{mmol}$ & 28 & 85 \\
\hline 3 & & & & $\mathrm{RT}, 0.4 \mathrm{mmol}$ & 45 & 85 \\
\hline 4 & & & & $\mathrm{RT}, 6.0 \mathrm{mmol}$ & 78 & 83 \\
\hline
\end{tabular}

Conditions - alkene (2.5 eq.), $\mathrm{Cp}_{2} \mathrm{ZrHCl}$ (2.0 eq.), $\mathrm{CH}_{2} \mathrm{Cl}_{2} ;$ 5,6-dihydropyran-2-one (1.0 eq.), (R)-B (10 mol\%), TMSCl (5.0 eq.), $E t_{2} \mathrm{O}$. a) Isolated yield b) ee determined by derivatisation then chiral HPLC d) reactions performed by $\operatorname{Dr} E$. $E$. Maciver.

Table 3.9: ACA of alkenes 45 and $46^{d}$

Once lactone 36v was obtained, cleavage of the 1,1-disubstituted-olefin functionality by ozonolysis gave $\mathbf{4 7}$ quantitatively, and cyclisation to $\mathbf{4 4}$ was accomplished using tosylic acid under Dean-Stark conditions ( $81 \%$ yield from 36v) with no detectable loss of stereochemistry during any of these operations (Scheme 3.23). In conclusion, we have shown the highly enantioselective 1,4- 
addition of alkylzirconocenes to $\alpha, \beta$-unsaturated lactones and further extended this procedure to the application of a variety of useful compounds.
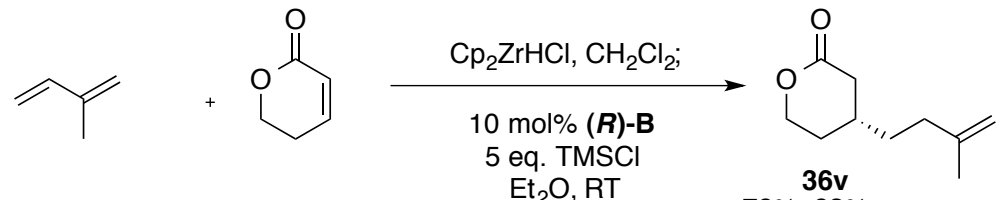

$78 \%, 83 \%$ ee

1. $\mathrm{O}_{3}, \mathrm{CH}_{2} \mathrm{Cl}_{2}$

$\mathrm{MeOH}$

2. $\mathrm{Me}_{2} \mathrm{~S}$

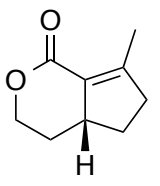

44

$81 \%$ over 2 steps

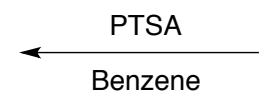<smiles>CC(=O)CC[C@H]1CCOC(=O)C1</smiles>

47 $83 \%$ ee

Scheme 3.23: Synthesis of intermediate 44 


\section{Chapter 4: Conclusions and Future Work}

\subsection{Conclusions}

The aim of this project was to develop a new catalytic asymmetric conjugate addition reaction using alkenes as equivalents to pre-made organometallic reagents. These reagents are incompatible with a range of functional groups; often only simple alkyl groups can be added. In addition, special conditions, such as cryogenic temperatures and slow addition procedures are required, and the reactions are sensitive to small changes in reaction conditions.

We succeeded in developing a system for the asymmetric 1,4-addition of a range of alkylzirconium nucleophiles to cyclic enones. Alkylzirconium reagents are generated in situ from the Schwartz reagent and alkenes. Both simple and functionalised alkenes were used to give a wide range of products. Our reaction proceeds at room temperature and tolerates a range of reaction conditions.
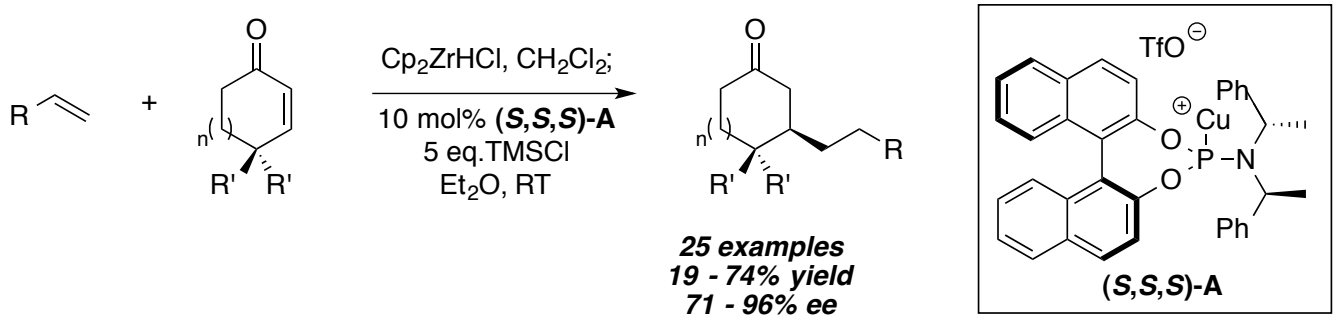

Scheme 4.1: Addition to cyclic enones

High enantioselectivities are observed when using copper complex $(\boldsymbol{S}, \boldsymbol{S}, \boldsymbol{S})-\mathbf{A}$, which was developed during this work. We have published these results ${ }^{60}$ (Scheme 4.1 ) and used the method in the 1,4- and 1,6- addition to complex steroids. ${ }^{61}$ In addition, we scale-up these reactions to show the robustness of our method. ${ }^{62}$ We extended our methodology to more challenging substrates, in the addition of alkylzirconium reagents to $\beta$-substituted enones to form the sterically crowded quaternary centre. We published a successful system, obtaining high yields and enantioselectivities with 21 examples (Scheme 4.2). ${ }^{100,101}$ 

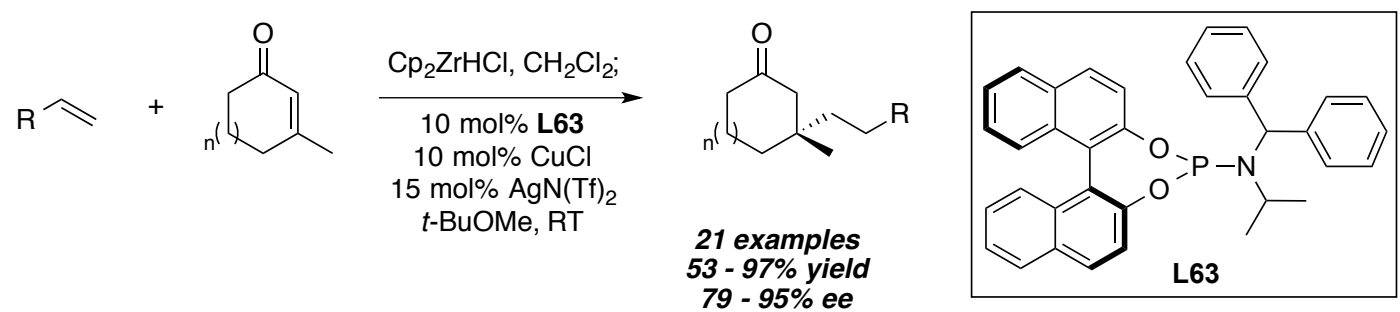

Scheme 4.2: Formation of quaternary centres

Our final and most recent work, has been carried out in the 1,4 -addition to $\alpha, \beta$-unsaturated lactones. Even though these substrates visually look similar to cyclic enones, they have inherently different properties and are known to be difficult substrates. We have succeeded in developing a general system to add to these substrates, however it is currently limited to 6- and 7-membered lactones with issues faced with addition to 5 -membered rings.

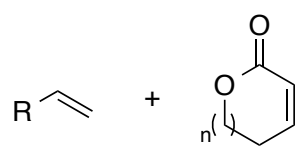<smiles>O=C1CC(CCP)CCO1</smiles>

20 examples
$45-95 \%$ yield $82-93 \%$ ee

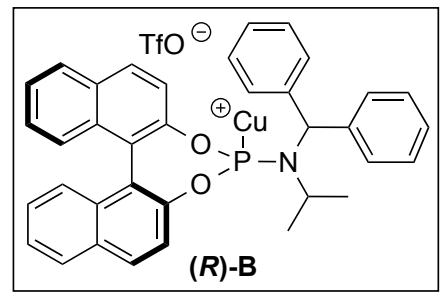

\section{Scheme 4.3: Addition to $\alpha, \beta$-unsaturated lactones}

We have submitted this work for publication showing the possibility to add to $\alpha, \beta$-unsaturated lactones without any trapping procedures or infinitely dilute conditions. 20 examples have been shown with high enantioselectivities (Scheme 4.3).

Overall, we have developed a new highly asymmetric catalytic methodology, which allows us to form new carbon-carbon bonds. Our reaction allows us to add functional groups that have previously not been tolerated in these types of reactions. No special addition procedures are required and the majority of the reactions are performed at room temperature. This makes the method robust and has the potential to be used in an industrial setting. We hope that the development of this methodology will prove to be a complementary approach to currently developed methods. 


\subsection{Future work}

This is a new project, which has opened to many doors, leading to a large amount of possibilities in the future. Initially future work would involve developing the 1,4-addition to linear substrates. Enantioselectivities of up to $45 \%$ ee have been obtained with ligand L19 (Scheme 4.4), however we have not surpassed this result.

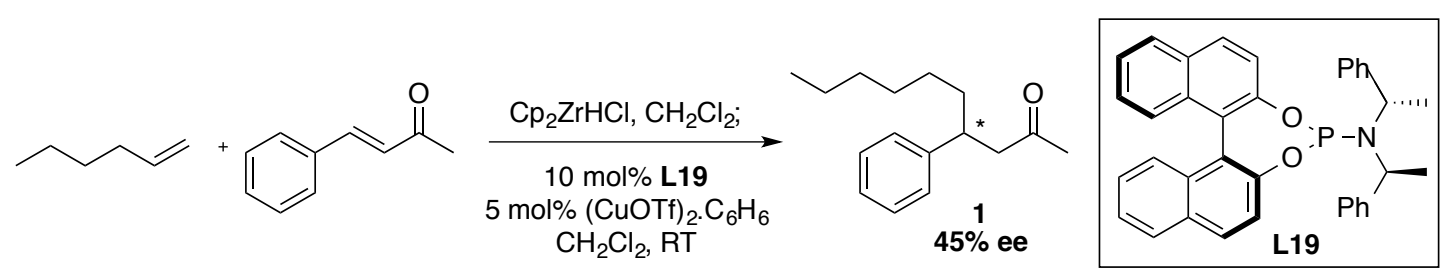

Scheme 4.4: Current best result with acyclic enones

Research into the addition to different carbonyl 1,4-acceptors such as esters, thioesters and amides as well as other classes of 1,4-acceptors, for example nitriles and sulfones would be of interest. Many different phosphoramidite ligands have been synthesised within the group, which may lead to their use in these reactions as well as investigating different ligand classes. These could also be applied to different reactions such as allylic alkylations and 1,2-additions. Lowering the catalytic loading from 10 mol\% would improve the cost efficiency of the reaction as well as a longer term goal of using catalytic amounts of zirconium. The mechanism of these reactions is poorly understood. Performing NMR studies on the reaction will help us understand the way the reaction proceeds. Knowledge of the way the reaction proceeds would give us an insight into how selectivity is obtained, widening the ability of tailoring our reactions to obtain the best possible outcome. Alkenes containing internal double bonds are of great interest. Hydrozirconation of these bonds leads to migration of the zirconium to the terminal position by a number of $\beta$-hydride eliminations due to the steric bulk of the Schwartz reagent. 


\section{Chapter 5: Experimental}

\subsection{General Information}

Procedures using oxygen- and/or moisture-sensitive materials were performed with anhydrous solvents (vide infra) under an atmosphere of anhydrous argon in flame-dried flasks, using standard Schlenk techniques. Analytical thin-layer chromatography was performed on pre-coated glass-backed plates (Silica Gel $60 \mathrm{~F}_{254}$; Merck), and visualised using a combination of UV light (254 $\mathrm{nm}$ ) and aqueous ceric ammonium molybdate (CAM), aqueous basic potassium permanganate stains or vanillin solution. Flash column chromatography was carried out using Apollo Scientific silica gel $60(0.040-0.063 \mathrm{~nm})$, Merck $60 \AA$ silica gel, VWR $(40-63 \mu \mathrm{m})$ silica gel and Sigma Aldrich silica gel. Pressure was applied at the column head via hand bellows or a flow of nitrogen with the solvent system used in parentheses.

Cooling of reaction mixtures to $0{ }^{\circ} \mathrm{C}$ was achieved using an ice-water bath. Other temperatures were obtained using a Julabo FT902 immersion cooler.

Unless stated otherwise, solution NMR spectra were recorded at room temperature; ${ }^{1} \mathrm{H}$ and ${ }^{13} \mathrm{C}$ nuclear magnetic resonance experiments were carried out using Bruker DPX-200 (200/50 MHz), DPX-400 (400/100 MHz), DQX-400 (400/100 MHz) or AVC-500 (500/125 MHz) spectrometers. Chemical shifts are reported in ppm from the residual solvent peak. Chemical shifts $(\delta)$ are given in ppm and coupling constants $(J)$ are quoted in Hertz $(\mathrm{Hz})$. Resonances are described as $s$ (singlet), br. S (broad singlet), d (doublet), t (triplet), q (quartet), quin (quintet) and m (multiplet). Labels $\mathrm{H}$ and $\mathrm{H}^{\prime}$ refer to diastereotopic protons attached to the same carbon and impart no stereochemical information. Assignments were made with the assistance of gCOSY, DEPT-135, gHSQC and gHMBC or gHMQC NMR spectra.

Numbering and names of structures accompanying reported data is non-IUPAC, and solely for reference.

Low-resolution mass spectra were recorded using a Walters LCT premier XE. High-resolution mass spectra (EI and ESI) were recorded using a Bruker MicroTOF spectrometer by the internal service at the University of Oxford.

Chiral HPLC separations were achieved using an Agilent 1230 Infinity series normal phase HPLC unit and HP Chemstation software. Chiralpak ${ }^{\circledR}$ columns $(250 \times 4.6 \mathrm{~mm})$, fitted with matching Chiralpak ${ }^{\circledR}$ Guard Cartridges $(10 \times 4 \mathrm{~mm})$, were used as specified in the text. Solvents used were of HPLC grade (Fisher Scientific, Sigma Aldrich or Rathburn); all eluent systems were isocratic. 
Infrared measurements (neat, thin film) were carried out using a Bruker Tensor 27 FT-IR with internal calibration in the range $4000-600 \mathrm{~cm}^{-1}$.

Optical rotations were recorded using a Perkin-Elmer 241 Polarimeter. CD spectra were recorded using an Applied Photophysics ChiraScan CD/Fluorometer.

\subsection{Chemicals}

Dry THF, $\mathrm{CH}_{2} \mathrm{Cl}_{2}, \mathrm{Et}_{2} \mathrm{O}$, toluene, benzene, hexane, pentane, $\mathrm{DME}$, acetonitrile and 1,2dichloroethane were collected fresh from an mBraun SPS-800 solvent purification system having been passed through anhydrous alumina columns. Dry tert-butyl methyl ether and 2-Me-THF were purchased from Acros with an AcroSeal ${ }^{\circledR}$. All other dry solvents used were dried over $3 \AA$ molecular sieves and stored under argon. All other solvents were used as purchased from Sigma Aldrich, Rathburn or Fisher Scientific.

Unless stated otherwise, commercially available reagents were purchased from Sigma-Aldrich, Fisher Scientific, Apollo Scientific, Acros Organics, Strem Chemicals, Alfa Aesar or TCI UK and were used without purification. Petroleum ether refers to light petroleum boiling in the range $40-60{ }^{\circ} \mathrm{C}$. TMSCl, styrene, allyl chloride and 4,4-dimethyl-2-cyclohexenone were distilled before use and stored in Schlenk flasks under an argon atmosphere. Deuterated solvents were purchased from Sigma-Aldrich $\left(\mathrm{CD}_{2} \mathrm{Cl}_{2}, \mathrm{CDCl}_{3}\right)$. Schwartz reagent was prepared according to the literature procedure ${ }^{75}$ from $\mathrm{Cp}_{2} \mathrm{ZrCl}_{2}$ provided by Alfa Aesar or Strem Chemicals. (CuOTf) $2 . \mathrm{C}_{6} \mathrm{H}_{6}$ was synthesised using a modified literature procedure ${ }^{76}$ and carefully maintained under an inert atmosphere. (CuOTf) ${ }_{2} \cdot \mathrm{C}_{6} \mathrm{H}_{6}$ should be a white or off-white powder, not green or brown. 2vinylnaphthalene was recrystallised from hexane before use. Starting amines ${ }^{86,87}$ and phosphoramidite ligands ${ }^{88,89}$ were synthesised according to the literature procedures.

\subsection{General Methods}

The compounds reported here are the result of my own work. However due to some work involving collaboration, compound data synthesised by other group members has been added to provide a complete set of results. P. M. C. Roth synthesised compounds: $\mathbf{1 5 f}, \mathbf{1 5 g}$ and 15i. Dr E. E. Maciver synthesised compounds: 36c, 36d, 36i, 36j, 36k, 36I, 36m, 36n, 41a, 41b, 36u, 36v.

\section{Synthesis of copper triflate benzene complex}

(CuOTf) ${ }_{2} \cdot \mathrm{C}_{6} \mathrm{H}_{6}$ is prepared from a modification of the procedure reported by Salomon and $\mathrm{Kochi}^{76}$ as described here: Copper (I) oxide ( $1.50 \mathrm{~g}, 10.5 \mathrm{mmol}, 1.0$ eq.) was added to a flame-dried 100 $\mathrm{mL}$ one-neck round bottomed flask fitted with a condenser and septum under argon. Benzene (30 
$\mathrm{mL}$ ) was added and the red suspension was stirred. Trifluoromethanesulfonic anhydride (2.6 $\mathrm{mL}$, $15.7 \mathrm{mmol}, 1.5$ eq.) was added dropwise via syringe over about $1 \mathrm{~min}$ from the top of the condenser and washed down with a small amount $(\sim 3 \mathrm{~mL})$ of additional benzene. The reaction is then heated to reflux until the red solution is decolourised to a brown/grey solution. This can take a few hours or may need to be left overnight. (Note: it is believed that a small quantity of trifluoromethanesulfonic acid in the trifluomethanesulfonic anhydride helps the reaction to proceed. Older $\mathrm{Tf}_{2} \mathrm{O}$ is likley to contain a greater amount of $\mathrm{TfOH}$, which often led the reaction to proceed faster. We have found that addition of a small amount $(<0.1 \mathrm{~mL})$ of $\mathrm{TfOH}$ promotes decolourisation within a few hours). The resulting hot suspension is filtered through a sintered Schlenk filter (pore size 4) - completely under argon - to a new flame dried Schlenk flask. The resulting grey filtrate is cooled and a white precipitate forms. Once cool the Schlenk filter and used round bottom flask are removed under a high flow of argon and replaced with a rubber septum. The benzene is then removed using a syringe and the resulting solid is washed with benzene $\left(2 \times 10 \mathrm{~mL}\right.$, to ensure removal of the excess $\left.\mathrm{Tf}_{2} \mathrm{O}\right)$. The remaining solvent is then removed under high vacuum to afford (CuOTf) $)_{2} \cdot \mathrm{C}_{6} \mathrm{H}_{6}$ as a white to off-white powder. Commercially available (CuOTf) ${ }_{2} \cdot \mathrm{C}_{6} \mathrm{H}_{6}$ is often green or brown in colour: The use green or brown (CuOTf) ${ }_{2} \cdot \mathrm{C}_{6} \mathrm{H}_{6}$ will result in products with lower levels of enantiomeric excess.

\section{Synthesis of phosphorochloridite}

Freshly distilled $\mathrm{PCl}_{3}(5 \mathrm{~mL})$ was added to a purged flask containing $(S)-2,2^{\prime}$-BINOL $(1.88 \mathrm{~g}, 6.57$ mmol) followed by a few drops of $\mathrm{N}$-methyl-2-pyrrolidinone. The reaction was heated to reflux. After $2 \mathrm{~h}$ the solution was cooled to room temperature before removing the excess $\mathrm{PCl}_{3}$ by the addition of dry toluene $(5 \mathrm{~mL})$ and then removing the solvent in vacuo, this was repeated multiple times, to afford a pale yellow foamy wax.

\section{Synthesis of racemates}

Using a modified procedure of Wipf et al., ${ }^{57} \mathrm{Cp}_{2} \mathrm{ZrHCl}$ (206 mg, $0.80 \mathrm{mmol}, 2.0$ eq.) was added to a stirred, room temperature, solution of alkene $\left(1.0 \mathrm{mmol}, 2.5\right.$ eq.) in $\mathrm{CH}_{2} \mathrm{Cl}_{2}(2.0 \mathrm{~mL})$ under an argon atmosphere. After stirring for about $40 \mathrm{~min}, \mathrm{CuBr} . \mathrm{Me}_{2} \mathrm{~S}(82 \mathrm{mg}, 0.40 \mathrm{mmol} 1.0$ eq.), was added to the reaction mixture and the resulting black mixture was allowed to stir for an additional $10 \mathrm{~min}$ before the $\alpha, \beta$-unsaturated compound ( $0.40 \mathrm{mmol}, 1.0$ eq.) was added via syringe. Stirring at room temperature was continued arbitrarily for $15 \mathrm{~h}$ before the reaction was quenched by the addition of $\mathrm{Et}_{2} \mathrm{O}\left(c a 3 \mathrm{~mL}\right.$ ) and then $\mathrm{NH}_{4} \mathrm{Cl}(1 \mathrm{M}$ aq., $c a 1.5 \mathrm{~mL})$. The mixture was partitioned between water and $\mathrm{Et}_{2} \mathrm{O}$ and the aqueous phase extracted with $\mathrm{Et}_{2} \mathrm{O}(3 \times 10 \mathrm{~mL})$. The combined organic phase was washed with $\mathrm{NaHCO}_{3}$ (aq. sat., ca $10 \mathrm{~mL}$ ), dried $\left(\mathrm{Na}_{2} \mathrm{SO}_{4}\right)$, filtered and 
concentrated in vacuo to give an oil. Flash column chromatography of the residue (EtOAc:petrol; $\mathrm{SiO}_{2}$ ) gave the desired 1,4-addition product.

\section{Catalytic asymmetric conjugate addition with copper-ligand complex formed in situ:}

Copper source $(0.040 \mathrm{mmol}, 0.1$ eq.) and the phosphoramidite ligand $(0.040 \mathrm{mmol}, 0.1$ eq. $)$ were dissolved in the reaction solvent $(1.0 \mathrm{~mL})$ under an argon atmosphere and allowed to stir for $1 \mathrm{~h}$ at room temperature. In another flask, $\mathrm{Cp}_{2} \mathrm{ZrHCl}(206 \mathrm{mg}, 0.80 \mathrm{mmol}, 2.0$ eq.) was added to a stirred, room temperature, solution of alkene (1.0 mmol, 2.5 eq.) in $\mathrm{CH}_{2} \mathrm{Cl}_{2}(0.20 \mathrm{~mL})$ under an argon atmosphere. After stirring for about $40 \mathrm{~min}$, the resulting clear yellow solution was transferred via syringe over about $1 \mathrm{~min}$ to the stirred solution containing the copper and ligand under an argon atmosphere. The resulting dark mixture was allowed to stir for an additional 10 min before $\alpha, \beta$-unsaturated compound $(0.40 \mathrm{mmol}, 1.0$ eq.) was added dropwise via syringe. The reaction was stirred between $3-5 \mathrm{~h}$ and was quenched by the addition of $\mathrm{Et}_{2} \mathrm{O}$ ( $c a 3 \mathrm{~mL}$ ) and then $\mathrm{NH}_{4} \mathrm{Cl}$ (1 M aq., ca $1.5 \mathrm{~mL}$ ). The mixture was partitioned between water and $\mathrm{Et}_{2} \mathrm{O}$ and the aqueous phase extracted with $\mathrm{Et}_{2} \mathrm{O}(3 \times 10 \mathrm{~mL})$. The combined organic phase was washed with $\mathrm{NaHCO}_{3}$ (aq. sat., $\mathrm{ca} 10 \mathrm{~mL}$ ), dried $\left(\mathrm{Na}_{2} \mathrm{SO}_{4}\right)$, filtered and concentrated in vacuo to give an oil. Flash column chromatography of the residue (EtOAc:petrol; $\mathrm{SiO}_{2}$ ) gave the desired product.

\section{Catalytic asymmetric conjugate addition with copper-ligand complex formed in situ with silver} salts:

$\mathrm{CuCl}(3.6 \mathrm{mg}, 0.040 \mathrm{mmol}, 0.10$ eq.) and the phosphoramidite ligand $(0.040 \mathrm{mmol}, 0.10$ eq.) were dissolved in the reaction solvent $(2.0 \mathrm{~mL})$ under an argon atmosphere and allowed to stir for $1 \mathrm{~h}$ at room temperature. Then the silver salt $(0.060 \mathrm{mmol}, 0.15$ eq.) was added and the suspension was stirred for another $15 \mathrm{~min}$. In another flask, $\mathrm{Cp}_{2} \mathrm{ZrHCl}(206 \mathrm{mg}, 0.80 \mathrm{mmol}, 2.0$ eq.) was added to a stirred, room temperature, solution of alkene $\left(1.0 \mathrm{mmol}, 2.5\right.$ eq.) in $\mathrm{CH}_{2} \mathrm{Cl}_{2}(0.40 \mathrm{~mL})$ under an argon atmosphere. After stirring for $15 \mathrm{~min}$, the stirred solution containing the copper and ligand was transferred and filtered using a syringe filter to the hydrozirconation solution. The resulting black mixture was allowed to stir for an additional $10 \mathrm{~min}$ before the $\alpha, \beta$-unsaturated compound ( $0.40 \mathrm{mmol}, 1.0$ eq.) was added dropwise via syringe. Stirring continued for 3-5 $\mathrm{h}$ before the reaction was quenched by the addition of $\mathrm{Et}_{2} \mathrm{O}(c a 3 \mathrm{~mL})$ and then $\mathrm{NH}_{4} \mathrm{Cl}(1 \mathrm{M}$ aq., $c a$ $1.5 \mathrm{~mL}$ ). The mixture was partitioned between the aqueous and $\mathrm{Et}_{2} \mathrm{O}$ layers and the aqueous phase extracted with $\mathrm{Et}_{2} \mathrm{O}(3 \times 10 \mathrm{~mL})$. The combined organic phase was washed with $\mathrm{NaHCO}_{3}$ (aq. sat., $c a 10 \mathrm{~mL}$ ), dried $\left(\mathrm{Na}_{2} \mathrm{SO}_{4}\right)$, filtered and concentrated in vacuo to give an oil. Flash column chromatography (EtOAc:petrol; $\mathrm{SiO}_{2}$ ) of the residue gave the desired product. 


\section{Catalytic asymmetric conjugate addition with pre-made complex:}

$\mathrm{Cp}_{2} \mathrm{ZrHCl}$ (206 mg, $0.80 \mathrm{mmol}, 2.0$ eq.) was added to a stirred, room temperature, solution of alkene (1.0 mmol, 2.5 eq.) in $\mathrm{CH}_{2} \mathrm{Cl}_{2}(0.40 \mathrm{~mL})$ under an argon atmosphere. After stirring for about $40 \mathrm{~min}$, the resulting clear yellow solution was transferred via syringe over about $1 \mathrm{~min}$ to a clear colourless stirred solution of copper-ligand complex $(0.040 \mathrm{mmol}, 0.10$ eq.) in solvent $(2.0 \mathrm{~mL})$ under an argon atmosphere. The resulting dark mixture was allowed to stir for an additional 10 min before the $\alpha, \beta$-unsaturated compound ( $0.40 \mathrm{mmol}, 1.0$ eq.) was added dropwise via syringe. The reaction was stirred for 3-5 h. The reaction was quenched by the addition of $\mathrm{Et}_{2} \mathrm{O}(c a 3 \mathrm{~mL})$ and then $\mathrm{NH}_{4} \mathrm{Cl}$ (1 M. aq., ca $1.5 \mathrm{~mL}$ ). The mixture was partitioned between water and $\mathrm{Et}_{2} \mathrm{O}$ and the aqueous phase extracted with $\mathrm{Et}_{2} \mathrm{O}(3 \times 10 \mathrm{~mL})$. The combined organic phase was washed with $\mathrm{NaHCO}_{3}$ (aq. sat., ca $10 \mathrm{~mL}$ ), dried $\left(\mathrm{Na}_{2} \mathrm{SO}_{4}\right)$, filtered and concentrated in vacuo to give an oil. Flash column chromatography of the residue (EtOAc:petrol; $\mathrm{SiO}_{2}$ ) gave the desired compound.

\section{Derivatisation of cyclic ketones:}

Using the procedure from Alexakis and coworkers: ${ }^{84}$ Crude material from the 1,4-addition was transferred to a vial with $\mathrm{CDCl}_{3}$ and $3 \AA$ molecular sieves. (+)-( $(R, R)-1,2$-diphenylethylenediamine $((+)-(R, R)$-DPEN ca 1.2-2.0 eq.) was added and the vial was shaken and allowed to stand for at least $1 \mathrm{~h}$ before the mixture was filtered through a glass pipette containing a small cotton plug and transferred to a NMR tube. ${ }^{13} \mathrm{C}$ NMR spectroscopy (500 MHz, 1024 scans) and comparison with racemic material was used to determine the enantiomeric excess.

\section{Derivatisation of cyclic lactones}

Compounds lacking a suitable chromaphore were derivatised via ring opening with benzylamine before HPLC analysis using the following procedure. $\mathrm{AlMe}_{3}$ (1.1 eq.) was added dropwise to a stirring solution of the desired substrate (1.0 eq.) and benzylamine (2.0 eq.) in $\mathrm{CH}_{2} \mathrm{Cl}_{2}(0.1 \mathrm{M})$ at 0 ${ }^{\circ} \mathrm{C}$. The resulting solution was allowed to warm to $\mathrm{RT}$ overnight before quenching with $\mathrm{HCl}(1 \mathrm{M}$, aq.). The aqueous layer was extracted with $\mathrm{CH}_{2} \mathrm{Cl}_{2}$, dried over $\mathrm{MgSO}_{4}$, filtered and concentrated in vacuo. The resulting oil was dissolved in a minimum amount of EtOAc/ $\mathrm{CH}_{2} \mathrm{Cl}_{2}$ and filtered through a pad of silica (eluent EtOAc:NEt 3 , 99:1). The filtrate was concentrated in vacuo and used directly for HPLC analysis. 


\subsection{Experimental Procedures and Data}

\subsubsection{Ligand Data}

Cyclopentyl-((S)-1-(naphthalen-2-yl)ethyl)-(S)-dinaphtho[2,1-d :1',2'-f][1,3,2]dioxaphosphepin4-amine (L20)

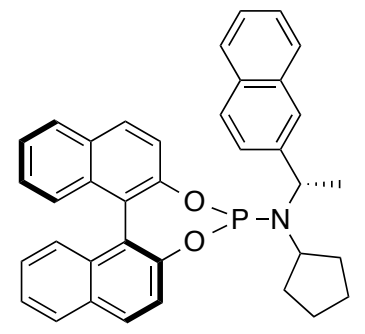

$\mathrm{NaB}(\mathrm{OAc})_{3} \mathrm{H}(0.30 \mathrm{~g}, 1.4 \mathrm{mmol}, 2.2$ eq.) and cyclopentanone $(0.09 \mathrm{~mL}, 1.0 \mathrm{mmol}, 1.6$ eq.) were added to a stirred solution (S)-1-(2-naphthyl)ethylamine $(0.11 \mathrm{~g}, 0.63 \mathrm{mmol}, 1.0$ eq.) in THF (3 mL) at room temperature. The reaction mixture was stirred for $24 \mathrm{~h}$ before a saturated aqueous solution of $\mathrm{NaHCO}_{3}(10 \mathrm{~mL})$ was added and stirring continued for 15 min. $\mathrm{Et}_{2} \mathrm{O}(c a 10 \mathrm{~mL}$ ) was added and the aqueous phase was separated and extracted with $\mathrm{Et}_{2} \mathrm{O}(3 \times 10 \mathrm{~mL})$. The combined organic phases were washed with brine $(20 \mathrm{~mL})$, dried $\left(\mathrm{Na}_{2} \mathrm{SO}_{4}\right)$, filtered and concentrated in vacuo. The resulting amine was dissolved in THF $(3.0 \mathrm{~mL})$ and the solution was cooled to $0{ }^{\circ} \mathrm{C}$. BuLi (1.2 $\mathrm{M}$ solution in hexane, $0.46 \mathrm{~mL}, 0.56 \mathrm{mmol}, 1.1 \mathrm{eq}$.) was added dropwise over about $5 \mathrm{~min}$ to the stirred and cooled solution. A solution of phosphorochloridite (177 mg, $0.51 \mathrm{mmol}, 1.0 \mathrm{eq}$.) in THF $(2.0 \mathrm{~mL})$ was added and the resulting mixture stirred for $10 \mathrm{~min}$ at $0{ }^{\circ} \mathrm{C}$. The reaction was allowed to warm to room temperature and stirred for $18 \mathrm{~h}$. $\mathrm{Et}_{2} \mathrm{O}(c a 10 \mathrm{~mL})$ was added and the reaction mixture was stirred for another $20 \mathrm{~min}$. The reaction mixture was filtered through a layer of celite sitting on a very thin layer of silica and washed with $\mathrm{Et}_{2} \mathrm{O}(10 \mathrm{~mL})$. The filtrate was concentrated in vacuo. $\mathrm{Et}_{2} \mathrm{O}(10 \mathrm{~mL})$ was added to precipitate impurities, and the impurities were removed by filtration through celite and a very thin layer of silica. The solid was washed with $\mathrm{Et}_{2} \mathrm{O}$ $(10 \mathrm{~mL})$ and the combined filtrate was dried $\left(\mathrm{Na}_{2} \mathrm{SO}_{4}\right)$, filtered and concentrated in vacuo to afford ligand $\mathbf{L 2 0}$ as an off white foam. Purification by flash column chromatography (3:97; $\mathrm{Et}_{2} \mathrm{O}$ :petrol; $\mathrm{SiO}_{2}$ ) gave the desired phosphoramidite $(108 \mathrm{mg}, 0.20 \mathrm{mmol}, 39 \%)$ as a white foam.

${ }^{1}{ }^{H}$ NMR $\left(400 \mathrm{MHz}, \mathrm{CDCl}_{3}\right) \delta_{\mathrm{H}} / \mathrm{ppm} 1.38-1.47(\mathrm{~m}, 1 \mathrm{H}), 1.48-1.67(\mathrm{~m}, 6 \mathrm{H}), 1.70(\mathrm{~d}, J=6.8 \mathrm{~Hz}, 3$ H), $1.83-1.95(m, J=3.6 \mathrm{~Hz}, 1 \mathrm{H}), 3.23-3.39(\mathrm{~m}, 1 \mathrm{H}), 4.56-4.68(\mathrm{~m}, 1 \mathrm{H}), 7.15(\mathrm{~s}, 2 \mathrm{H}), 7.28-$ 7.44 (br. s, 7 H), $7.45-7.52$ (m, 1 H), 7.55 (s, 1 H), 7.79 (dt, J = 8.4, 17.1 Hz, 2 H), 7.90 (s, 3 H), 8.00 (d, $J=8.7 \mathrm{~Hz}, 1 \mathrm{H}$ ).

${ }^{13} \mathrm{C}$ NMR $\left(125 \mathrm{MHz}, \mathrm{CDCl}_{3}\right) \delta_{\mathrm{c}} / \mathrm{ppm} 22.6,23.2,23.7,32.7$ (d, $\left.J=10.2 \mathrm{~Hz}\right), 34.3,53.7,57.1,122.2$, $122.4(\mathrm{~d}, J=1.7 \mathrm{~Hz}, 2 \mathrm{C}), 124.1,124.1,124.3,124.7,125.3,125.7,125.8,125.9,126.0,126.0$, 
127.1, 127.1, 127.6, 127.8, 128.0 (2 C), 128.1, 128.3, 129.4, 130.3, 130.39, 131.3, 132.3, 132.6, $132.8,133.3,150.0,150.4(\mathrm{~d}, J=8.5 \mathrm{~Hz})$.

${ }^{31}$ P NMR (200 MHz, $\left.\mathrm{CDCl}_{3}\right) \delta_{\mathrm{p}} / \mathrm{ppm} 152.19$ (s, $\left.1 \mathrm{P}\right)$.

HRMS (EI) $\mathrm{m} / \mathrm{z}$ calcd for $\mathrm{C}_{37} \mathrm{H}_{32} \mathrm{NO}_{2} \mathrm{P}[\mathrm{M}]^{+}:$553.2171, found: 553.2175 .

$[\alpha]_{589}^{20}=-28.99\left(\mathrm{c} 0.45, \mathrm{CHCl}_{3}\right)$.

IR $\left(v_{\max } / \mathrm{cm}^{-1}\right): 749,820,948,1230,1431,1506,1619,2360,2960$.

\section{(11bS)-N-cyclohexyl-N-((S)-1-(naphthalen-2-yl)ethyl)-(S)-dinaphtho[2,1-d:1',2'} f] $[1,3,2]$ dioxaphosphepin-4-amine (L21)

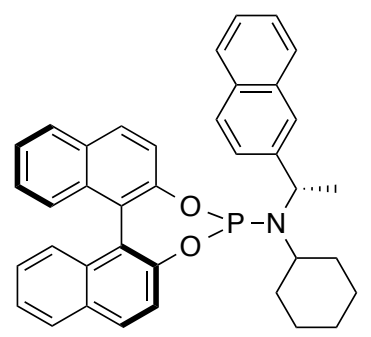

$\mathrm{NaB}(\mathrm{OAc})_{3} \mathrm{H}(0.30 \mathrm{~g}, 1.4 \mathrm{mmol}, 2.2$ eq.) and cyclohexanone $(0.10 \mathrm{~mL}, 0.95 \mathrm{mmol}, 1.5$ eq.) were added to a stirred solution (S)-1-(2-naphthyl)ethylamine $(0.11 \mathrm{~g}, 0.63 \mathrm{mmol}, 1.0 \mathrm{eq}$ ) $)$ in THF (3.0 $\mathrm{mL}$ ) at room temperature. The reaction mixture was stirred for $24 \mathrm{~h}$ before a saturated aqueous solution of $\mathrm{NaHCO}_{3}(10 \mathrm{~mL})$ was added and stirring continued for 15 min. $\mathrm{Et}_{2} \mathrm{O}(\mathrm{ca} 10 \mathrm{~mL}$ ) was added and the aqueous phase was separated and extracted with $\mathrm{Et}_{2} \mathrm{O}(3 \times 10 \mathrm{~mL})$. The combined organic phases were washed with brine $(20 \mathrm{~mL})$, dried $\left(\mathrm{Na}_{2} \mathrm{SO}_{4}\right)$, filtered and concentrated in vacuo. The resulting amine was dissolved in THF $(3.0 \mathrm{~mL})$ and the solution was cooled to $0{ }^{\circ} \mathrm{C}$. BuLi (1.2 $\mathrm{M}$ solution in hexane, $0.47 \mathrm{~mL}, 0.56 \mathrm{mmol}, 1.1$ eq.) was added dropwise over about $5 \mathrm{~min}$ to the stirred and cooled solution. A solution of phosphorochloridite (180 mg, $0.51 \mathrm{mmol}, 1.0 \mathrm{eq}$.) in THF $(2.0 \mathrm{~mL})$ was added and the resulting mixture stirred for $10 \mathrm{~min}$ at $0{ }^{\circ} \mathrm{C}$. The reaction was allowed to warm to room temperature and stirred for $18 \mathrm{~h}$. $\mathrm{Et}_{2} \mathrm{O}(c a 10 \mathrm{~mL}$ ) was added and the reaction mixture was stirred for another $20 \mathrm{~min}$. The reaction mixture was filtered through a layer of celite sitting on a very thin layer of silica and washed with $\mathrm{Et}_{2} \mathrm{O}(10 \mathrm{~mL})$. The filtrate was concentrated in vacuo. $\mathrm{Et}_{2} \mathrm{O}(10 \mathrm{~mL})$ was added to precipitate impurities, and the impurities were removed by filtration through celite and a very thin layer of silica. The solid was washed with $\mathrm{Et}_{2} \mathrm{O}$ $(10 \mathrm{~mL})$ and the combined filtrate was dried $\left(\mathrm{Na}_{2} \mathrm{SO}_{4}\right)$, filtered and concentrated in vacuo to afford ligand L21 as an off white foam. Purification by flash column chromatography (2:98 $\mathrm{Et}_{2} \mathrm{O}$ :petrol; $\mathrm{SiO}_{2}$ ) gave the desired phosphoramidite ( $46 \mathrm{mg}, 0.08 \mathrm{mmol}, 16 \%$ ) as a white foam. 
${ }^{1} \mathrm{H}$ NMR $\left(500 \mathrm{MHz}, \mathrm{CDCl}_{3}\right) \delta_{\mathrm{H}} / \mathrm{ppm} 0.56(\mathrm{~d}, J=10.7 \mathrm{~Hz}, 1 \mathrm{H}), 0.77-1.01(\mathrm{~m}, 2 \mathrm{H}), 1.31-1.72(\mathrm{~m}, 6$ H), 1.83 (br. s, 3 H), 1.94 (br. s, 1 H), 2.84 (br s, 1 H), 4.75 (br. s, 1 H), 7.28 (d, J = 5.7 Hz, 2 H), 7.34 $7.66(m, 8 H), 7.77(d, J=7.3 \mathrm{~Hz}, 1 \mathrm{H}), 7.81-8.13(\mathrm{~m}, 8 \mathrm{H})$.

${ }^{13} \mathrm{C}$ NMR $\left(125 \mathrm{MHz}, \mathrm{CDCl}_{3}\right) \delta_{\mathrm{c}} / \mathrm{ppm} 24.8(\mathrm{~d}, J=17.6 \mathrm{~Hz}), 25.9,26.7(2 \mathrm{C}), 34.6(\mathrm{~d}, J=5.5 \mathrm{~Hz}), 35.4$, $54.4(\mathrm{~d}, J=16.6 \mathrm{~Hz}), 56.1$ (d, $J=5.5 \mathrm{~Hz}), 122.1,122.5,122.9,124.6(\mathrm{~d}, J=5.5 \mathrm{~Hz}), 124.8,125.2$, $125.8,126.2,126.4,126.5,126.5$ (2 C), 127.6, 127.6, 128.1, 128.3, 128.5, 128.6, 128.8, 130.0, $130.8,130.9,131.8,132.9,133.2,133.4,133.9,143.5,150.5,151.0$ (d, $J=7.4 \mathrm{~Hz})$.

${ }^{31} \mathrm{P}$ NMR $\left(200 \mathrm{MHz}, \mathrm{CDCl}_{3}\right) \delta_{\mathrm{p}} / \mathrm{ppm} 152.43(\mathrm{~s}, 1 \mathrm{P})$.

HRMS (EI) $m / z$ calcd for $\mathrm{C}_{38} \mathrm{H}_{34} \mathrm{NO}_{2} \mathrm{P}[\mathrm{M}]^{+}:$567.2327, found: 567.2317 .

$[\alpha]^{20}{ }_{589}=-6.11\left(c 1.03, \mathrm{CHCl}_{3}\right)$.

IR $\left(v_{\max } / \mathrm{cm}^{-1}\right): 624,746,789,819,946,1229,1328,1590,2160,2928,3302$.

(11bS)-N,N-bis((S)-1-phenylethyl)-(S)-8,9,10,11,12,13,14,15-octahydrodinaphtho[2,1-d:1',2'$f][1,3,2]$ dioxaphosphepin-4-amine (L22)

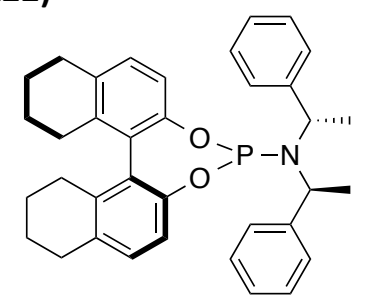

Triethylamine $(0.71 \mathrm{~mL}, 5.1 \mathrm{mmol}, 5.0$ eq.) was added dropwise to a stirred ice-cooled solution of $\mathrm{PCl}_{3}\left(0.09 \mathrm{~mL}, 1.0 \mathrm{mmol}, 1.0\right.$ eq.) in $\mathrm{CH}_{2} \mathrm{Cl}_{2}(4 \mathrm{~mL})$. The ice bath was removed and the solution left to warm to room temperature before (-)-bis[(S)-1-phenylethyl]amine $(0.23 \mathrm{~mL}, 1.0 \mathrm{mmol}, 1.0$ eq.) was added to the stirring solution. After $5 \mathrm{~h}(S)-(-)-5,5^{\prime}, 6,6^{\prime}, 7,7^{\prime}, 8,8^{\prime}$-octahydro-1,1'-bi-2-naphthol ( $300 \mathrm{mg}, 1.0 \mathrm{mmol}, 1.0$ eq.) was added to the suspension and the subsequent mixture was left to stir for an additional $15 \mathrm{~h}$. The solution was then filtered on a small pad of silica and celite ${ }^{\circledR}$ and rinsed with $\mathrm{CH}_{2} \mathrm{Cl}_{2}(\sim 15 \mathrm{~mL})$. The resulting solution was concentrated under reduced pressure to afford a yellow residue. After flash column chromatography (84:15:1; Petrol: $\mathrm{CH}_{2} \mathrm{Cl}_{2}: \mathrm{Et}_{3} \mathrm{~N} ; \mathrm{SiO}_{2}$ ), ligand L22 was obtained as a crystalline white solid (335 mg, $0.61 \mathrm{mmol}, 60 \%$ ).

${ }^{1} \mathrm{H}$ NMR $\left(500 \mathrm{MHz}, \mathrm{CDCl}_{3}\right) \delta_{\mathrm{H}} / \mathrm{ppm} 1.47-1.61(\mathrm{~m}, 2 \mathrm{H}), 1.66(\mathrm{~s}, 6 \mathrm{H}), 1.73-1.87(\mathrm{~m}, 6 \mathrm{H}), 2.23(\mathrm{dt}$, $J=16.5,5.6 \mathrm{~Hz}, 1 \mathrm{H}), 2.31(\mathrm{dt}, J=16.1,6.3 \mathrm{~Hz}, 1 \mathrm{H}), 2.58-2.92(\mathrm{~m}, 6 \mathrm{H}), 4.33-4.44(\mathrm{~m}, 2 \mathrm{H}), 6.83$ (dd, $J=33.4,8.2 \mathrm{~Hz}, 2 \mathrm{H}), 7.10(q, J=8.3 \mathrm{~Hz}, 3 \mathrm{H}), 7.13-7.23(\mathrm{~m}, 10 \mathrm{H})$.

${ }^{13} \mathrm{C}$ NMR $\left(125 \mathrm{MHz}, \mathrm{CDCl}_{3}\right) \delta_{\mathrm{C}} / \mathrm{ppm} 23.0,23.16,23.20$ (2 C), 23.3, 23.4, 28.1, 28.4, 29.5, 29.7, 54.8, 54.9, $119.3(2 \mathrm{C}), 119.5$ (d, J = 3.0 Hz), 126.9 (2 C), 127.5 (d, J = 2.3 Hz), 128.1 (4 C), $128.5(2 \mathrm{C})$, $128.5(2 \mathrm{C}), 129.3,129.9,132.8,134.5(\mathrm{~d}, J=1.8 \mathrm{~Hz}), 137.8(\mathrm{~d}, J=1.2 \mathrm{~Hz}), 138.5(\mathrm{~d}, J=2.1 \mathrm{~Hz})$, 
143.9 (2 C), 149.2 (d, J = 8.3 Hz), 149.6.

${ }^{31}$ P NMR $\left(200 \mathrm{MHz}, \mathrm{CDCl}_{3}\right) \delta_{\mathrm{P}} / \mathrm{ppm} 146.99$ (s, $\left.1 \mathrm{P}\right)$.

HRMS (EI) $m / z$ calcd for $\mathrm{C}_{36} \mathrm{H}_{37} \mathrm{NO}_{2} \mathrm{P}[\mathrm{M}-\mathrm{H}]^{+}: 546.2562$, found: 546.0087 .

$[\alpha]^{20}{ }_{589}=-95.16\left(c 1.24, \mathrm{CHCl}_{3}\right)$.

IR $\left(v_{\max } / \mathrm{cm}^{-1}\right):$ 698, 763, 940, 1201, 1494, 2340, 2933.

(11bS)-N-((S)-1-(4-fluorophenyl)ethyl)-N-isopropyl-(S)-dinaphtho[2,1-d:1',2'$f][1,3,2]$ dioxaphosphepin-4-amine (L23)

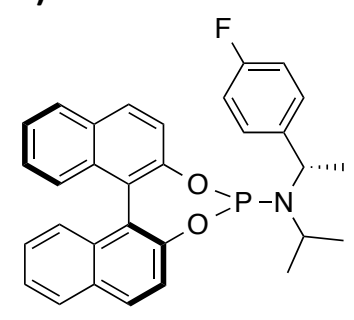

$\mathrm{NaB}(\mathrm{OAc})_{3} \mathrm{H}(0.83 \mathrm{~g}, 4.3 \mathrm{mmol}, 2.0$ eq.) and acetone $(0.25 \mathrm{~mL}, 4.3 \mathrm{mmol}, 2.0$ eq.) were added to a stirred solution (S)-1-(4-fluorophenyl)ethanamine $(0.23 \mathrm{~mL}, 1.7 \mathrm{mmol}, 1.0$ eq.) at room temperature. The reaction mixture was stirred for $24 \mathrm{~h}$ before a saturated aqueous solution of $\mathrm{NaHCO}_{3}(10 \mathrm{~mL})$ was added and stirring continuously for $15 \mathrm{~min}$. $\mathrm{Et}_{2} \mathrm{O}(\mathrm{ca} 20 \mathrm{~mL}$ ) was added and the aqueous phase was separated and extracted with $\mathrm{Et}_{2} \mathrm{O}(3 \times 20 \mathrm{~mL})$. The combined organic phases were washed with brine $(20 \mathrm{~mL})$, dried $\left(\mathrm{Na}_{2} \mathrm{SO}_{4}\right)$, filtered and concentrated in vacuo.

Triethylamine ( $0.77 \mathrm{~mL}, 5.5 \mathrm{mmol}, 5.0$ eq.) was added dropwise to a stirred ice-cooled solution of $\mathrm{PCl}_{3}\left(0.10 \mathrm{~mL}, 1.1 \mathrm{mmol}, 1.0\right.$ eq.) in $\mathrm{CH}_{2} \mathrm{Cl}_{2}(3 \mathrm{~mL})$. The ice bath was removed and the solution left to warm to room temperature before (S)- $N$-(1-(4-fluorophenyl)ethyl)propan-2-amine (200 mg, $1.1 \mathrm{mmol}, 1.0$ eq.) was added to the stirring solution. After $5 \mathrm{~h}(S)$-binaphthol (315 mg, $1.1 \mathrm{mmol}$, 1.0 eq.) was added to the suspension and the subsequent mixture was left to stir for an additional $15 \mathrm{~h}$. The solution was then filtered on a small pad of silica and celite ${ }^{\circledR}$ and rinsed with $\mathrm{CH}_{2} \mathrm{Cl}_{2}$ ( $15 \mathrm{~mL}$ ). The resulting solution was concentrated under reduced pressure to afford a yellow residue. After flash column chromatography (84:15:1; Petrol: $\mathrm{CH}_{2} \mathrm{Cl}_{2}: \mathrm{Et}_{3} \mathrm{~N} ; \mathrm{SiO}_{2}$ ), ligand $\mathbf{L} 23$ was obtained as a crystalline white solid (226 mg, $0.46 \mathrm{mmol}, 41 \%)$.

${ }^{1} \mathrm{H}$ NMR $\left(500 \mathrm{MHz}, \mathrm{CDCl}_{3}\right) \delta_{\mathrm{H}} / \mathrm{ppm} 1.01(\mathrm{~d}, J=6.9 \mathrm{~Hz}, 3 \mathrm{H}), 1.20(\mathrm{~d}, J=6.6 \mathrm{~Hz}, 3 \mathrm{H}), 1.76(\mathrm{dd}, J=$ 7.2, $0.3 \mathrm{~Hz}, 3 \mathrm{H}), 3.34-3.45(\mathrm{~m}, 1 \mathrm{H}), 4.50-4.61(\mathrm{~m}, 1 \mathrm{H}), 7.11$ - $7.20(\mathrm{~m}, 2 \mathrm{H}), 7.27$ - $7.34(\mathrm{~m}, 2 \mathrm{H})$, 7.34 - $7.40(\mathrm{~m}, 2 \mathrm{H}), 7.42$ - $7.51(\mathrm{~m}, 3 \mathrm{H}), 7.56-7.66(\mathrm{~m}, 3 \mathrm{H}), 7.86$ (dd, $J=8.8,0.2 \mathrm{~Hz}, 1 \mathrm{H}), 7.93$ (d, $J=8.5 \mathrm{~Hz}, 1 \mathrm{H}), 7.98(\mathrm{~d}, J=8.1 \mathrm{~Hz}, 1 \mathrm{H}), 8.04(\mathrm{~d}, J=8.8 \mathrm{~Hz}, 1 \mathrm{H})$.

${ }^{13} \mathrm{C}$ NMR $\left(125 \mathrm{MHz}, \mathrm{CDCl}_{3}\right) \delta_{\mathrm{c}} / \mathrm{ppm} 23.6(\mathrm{~d}, J=5.1 \mathrm{~Hz}), 24.3(\mathrm{~d}, J=4.4 \mathrm{~Hz}), 25.1(\mathrm{~d}, J=19.0 \mathrm{~Hz})$, $47.0(\mathrm{~d}, J=4.4 \mathrm{~Hz}), 52.8(\mathrm{~d}, J=19.2 \mathrm{~Hz}), 115.4,115.5,122.1(\mathrm{~d}, J=2.5 \mathrm{~Hz}), 122.5,122.8(\mathrm{~d}, J=2.1$ 
$\mathrm{Hz}), 124.5(\mathrm{~d}, J=5.5 \mathrm{~Hz}), 124.8,125.2,126.3,126.4,127.5,127.6,128.6,128.7,129.0(\mathrm{~d}, J=3.0$

Hz), $129.1(d, J=3.1 \mathrm{~Hz}), 129.5,129.9,130.7,130.9,131.8,133.1(\mathrm{~d}, J=1.2 \mathrm{~Hz}), 133.3(\mathrm{~d}, J=1.8$

$\mathrm{Hz}), 141.8,150.3,150.8$ (d, J = 7.6 Hz).

${ }^{31} \mathrm{P}$ NMR (200 MHz, $\left.\mathrm{CDCl}_{3}\right) \delta_{\mathrm{p}} / \mathrm{ppm} 151.77$ (s, $\left.1 \mathrm{P}\right)$.

${ }^{19}$ F NMR $\left(400 \mathrm{MHz}, \mathrm{CDCl}_{3}\right) \delta_{\mathrm{F}} / \mathrm{ppm}-116.65(\mathrm{~s}, 1 \mathrm{~F})$.

HRMS (EI) $m / z$ calcd for $\mathrm{C}_{31} \mathrm{H}_{27} \mathrm{FNO}_{2} \mathrm{P}[\mathrm{M}]^{+}: 495.1764$, found: 495.1882 .

$[\alpha]^{20}{ }_{589}=+196.44\left(c 1.24, \mathrm{CHCl}_{3}\right)$.

IR $\left(v_{\max } / \mathrm{cm}^{-1}\right):$ 750, 822, 947, 1231, 1591, 2360, 2973.

(11bS)-N-((S)-1-(4-chlorophenyl)ethyl)-N-isopropyl-(S)-dinaphtho[2,1-d:1',2'$f][1,3,2]$ dioxaphosphepin-4-amine (L24)

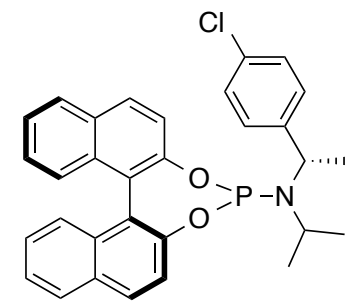

$\mathrm{NaB}(\mathrm{OAc})_{3} \mathrm{H}(0.64 \mathrm{~g}, 3.0 \mathrm{mmol}, 1.5$ eq.) and acetone $(0.29 \mathrm{~mL}, 4.0 \mathrm{mmol}, 2.0$ eq.) were added to a stirred solution (S)-4-chloro- $\alpha$-methylbenzylamine $(0.28 \mathrm{~mL}, 2.0 \mathrm{mmol}, 1.0$ eq.) in THF (10 mL) at room temperature. The reaction mixture was stirred for $24 \mathrm{~h}$ before a saturated aqueous solution of $\mathrm{NaHCO}_{3}(10 \mathrm{~mL})$ was added and stirring continued for $15 \mathrm{~min}$. $\mathrm{Et}_{2} \mathrm{O}(\mathrm{ca} 10 \mathrm{~mL})$ was added and the aqueous phase was separated and extracted with $\mathrm{Et}_{2} \mathrm{O}(3 \times 10 \mathrm{~mL})$. The combined organic phases were washed with brine $(20 \mathrm{~mL})$, dried $\left(\mathrm{Na}_{2} \mathrm{SO}_{4}\right)$, filtered and concentrated in vacuo. Triethylamine ( $0.71 \mathrm{~mL}, 5.1 \mathrm{mmol}, 5.0$ eq.) was added dropwise to a stirred ice-cooled solution of $\mathrm{PCl}_{3}$ (0.09 mL, $1.0 \mathrm{mmol}, 1.0$ eq.) in $\mathrm{CH}_{2} \mathrm{Cl}_{2}(4 \mathrm{~mL})$. The ice bath was removed and the solution left to warm to room temperature before $(S)-N$-(1-(4-chlorophenyl)ethyl)propan-2-amine (200 $\mathrm{mg}$, $1.0 \mathrm{mmol}, 1.0$ eq.) was added to the stirring solution. After $5 \mathrm{~h}(S)$-binaphthol $(289 \mathrm{mg}, 1.0 \mathrm{mmol}$, 1.0 eq.) was added to the suspension and the subsequent mixture was left to stir for an additional $15 \mathrm{~h}$. The solution was then filtered on a small pad of silica and celite ${ }^{\circledR}$ and rinsed with $\mathrm{CH}_{2} \mathrm{Cl}_{2}$ ( $15 \mathrm{~mL}$ ). The resulting solution was concentrated under reduced pressure to afford a yellow residue. After flash column chromatography (84:15:1; Petrol: $\mathrm{CH}_{2} \mathrm{Cl}_{2}: \mathrm{Et}{ }_{3} \mathrm{~N} ; \mathrm{SiO}_{2}$ ), ligand $\mathbf{L 2 4}$ was obtained as a crystalline white solid ( $220 \mathrm{mg}, 0.43 \mathrm{mmol}, 43 \%$ ).

${ }^{1} \mathbf{H}$ NMR $\left(500 \mathrm{MHz}, \mathrm{CDCl}_{3}\right) \delta_{\mathrm{H}} / \mathrm{ppm} 1.00(\mathrm{~d}, J=6.8 \mathrm{~Hz}, 3 \mathrm{H}), 1.20(\mathrm{~d}, J=6.6 \mathrm{~Hz}, 3 \mathrm{H}), 1.75(\mathrm{~d}$, $J=7.2 \mathrm{~Hz}, 3 \mathrm{H}), 3.33-3.44(\mathrm{~m}, 1 \mathrm{H}), 4.54(\mathrm{dq}, J=14.0,7.1 \mathrm{~Hz}, 1 \mathrm{H}), 7.27-7.34(\mathrm{~m}, 2 \mathrm{H}), 7.34-7.39$ $(\mathrm{m}, 2 \mathrm{H}), 7.41-7.50(\mathrm{~m}, 5 \mathrm{H}), 7.59(\mathrm{~m}, J=8.5 \mathrm{~Hz}, 3 \mathrm{H}), 7.86(\mathrm{~d}, J=8.8 \mathrm{~Hz}, 1 \mathrm{H}), 7.93(\mathrm{~d}, J=8.5 \mathrm{~Hz}, 1$ 
H), $7.98(\mathrm{~d}, J=8.3 \mathrm{~Hz}, 1 \mathrm{H}), 8.04(\mathrm{~d}, J=8.7 \mathrm{~Hz}, 1 \mathrm{H})$.

${ }^{13} \mathrm{C}$ NMR $\left(125 \mathrm{MHz}, \mathrm{CDCl}_{3}\right) \delta_{\mathrm{c}} / \mathrm{ppm} 23.4(\mathrm{~d}, J=5.1 \mathrm{~Hz}), 24.1(\mathrm{~d}, J=4.4 \mathrm{~Hz}), 24.8(\mathrm{~d}, J=18.5 \mathrm{~Hz}$ ), $46.8(\mathrm{~d}, J=4.6 \mathrm{~Hz}), 52.8(\mathrm{~d}, J=19.0 \mathrm{~Hz}), 121.9(\mathrm{~d}, J=2.3 \mathrm{~Hz}), 122.3,122.5$ (d, J = 2.1 Hz), 124.2 (d, J = $5.3 \mathrm{~Hz}$ ), 124.6, 125.0, 126.1, 126.2, 127.3, 127.4, 128.4, 128.5, 128.6 (2 C), 128.7, 128.7, 129.7 (d, $J=0.5 \mathrm{~Hz}), 130.5,130.7,131.6(\mathrm{~d}, J=0.9 \mathrm{~Hz}), 132.6,132.9(\mathrm{~d}, J=1.4 \mathrm{~Hz}), 133.1(\mathrm{~d}, J=2.1 \mathrm{~Hz}$ ), $144.5(\mathrm{~d}, J=0.7 \mathrm{~Hz}), 150.1,150.5(\mathrm{~d}, J=7.6 \mathrm{~Hz})$.

${ }^{31}$ P NMR (200 MHz, $\left.\mathrm{CDCl}_{3}\right) \delta_{\mathrm{p}} / \mathrm{ppm} 151.50$ (s, $\left.1 \mathrm{P}\right)$.

HRMS (EI) $m / z$ calcd for $\mathrm{C}_{31} \mathrm{H}_{27} \mathrm{CINO}_{2} \mathrm{P}[\mathrm{M}]^{+}: 511.1468$, found: 511.1542 .

$[\alpha]^{20}{ }_{589}=+104.67\left(c 1.20, \mathrm{CHCl}_{3}\right)$.

IR $\left(v_{\max } / \mathrm{cm}^{-1}\right):$ 749, 821, 949, 1328, 1619, 2360, 2972.

\section{(11bS)-N-((S)-1-(3-chlorophenyl)ethyl)-N-isopropyl-(S)-dinaphtho[2,1-d:1',2'-}

\section{$f][1,3,2]$ dioxaphosphepin-4-amine (L25)}

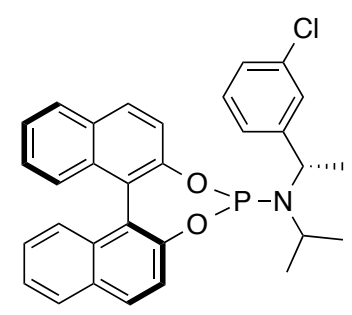

$\mathrm{NaB}(\mathrm{OAc})_{3} \mathrm{H}(0.64 \mathrm{~g}, 3.0 \mathrm{mmol}, 1.5 \mathrm{eq}$.$) and acetone (0.29 \mathrm{~mL}, 4.0 \mathrm{mmol}, 2.0$ eq.) were added to a stirred solution (S)-3-chloro- $\alpha$-methylbenzylamine $(0.28 \mathrm{~mL}, 2.0 \mathrm{mmol}, 1.0$ eq.) in THF (10 mL) at room temperature. The reaction mixture was stirred for $24 \mathrm{~h}$ before a saturated aqueous solution of $\mathrm{NaHCO}_{3}(10 \mathrm{~mL})$ was added and stirring continued for $15 \mathrm{~min}$. $\mathrm{Et}_{2} \mathrm{O}(\mathrm{ca} 10 \mathrm{~mL})$ was added and the aqueous phase was separated and extracted with $\mathrm{Et}_{2} \mathrm{O}(3 \times 10 \mathrm{~mL})$. The combined organic phases were washed with brine $(20 \mathrm{~mL})$, dried $\left(\mathrm{Na}_{2} \mathrm{SO}_{4}\right)$, filtered and concentrated in vacuo.

Triethylamine $(0.71 \mathrm{~mL}, 5.1 \mathrm{mmol}, 5.0$ eq.) was added dropwise to a stirred ice-cooled solution of $\mathrm{PCl}_{3}\left(0.09 \mathrm{~mL}, 1.0 \mathrm{mmol}, 1.0\right.$ eq.) in $\mathrm{CH}_{2} \mathrm{Cl}_{2}(5 \mathrm{~mL})$. The ice bath was removed and the solution left to warm to room temperature before (S)- $N$-(1-(3-chlorophenyl)ethyl)propan-2-amine (200 mg, $1.0 \mathrm{mmol}, 1.0$ eq.) was added to the stirring solution. After $5 \mathrm{~h}(S)$-binaphthol ( $289 \mathrm{mg}, 1.0 \mathrm{mmol}$, 1.0 eq.) was added to the suspension and the subsequent mixture was left to stir for an additional $15 \mathrm{~h}$. The solution was then filtered on a small pad of silica and celite ${ }^{\circledR}$ and rinsed with $\mathrm{CH}_{2} \mathrm{Cl}_{2}$ ( $15 \mathrm{~mL}$ ). The resulting solution was concentrated under reduced pressure to afford a yellow residue. After flash column chromatography (84:15:1; Petrol: $\mathrm{CH}_{2} \mathrm{Cl}_{2}: \mathrm{Et}_{3} \mathrm{~N} ; \mathrm{SiO}_{2}$ ), ligand $\mathbf{L 2 5}$ was obtained as a crystalline white solid ( $266 \mathrm{mg}, 0.52 \mathrm{mmol}, 52 \%)$. 
${ }^{1} \mathrm{H}$ NMR $\left(500 \mathrm{MHz}, \mathrm{CDCl}_{3}\right) \delta_{\mathrm{H}} / \mathrm{ppm} 0.96(\mathrm{~d}, J=6.9 \mathrm{~Hz}, 3 \mathrm{H}), 1.16(\mathrm{~d}, J=6.6 \mathrm{~Hz}, 3 \mathrm{H}), 1.72(\mathrm{~d}, J=7.2$ $\mathrm{Hz}, 3 \mathrm{H}), 3.36(\mathrm{dq}, J=13.5,6.8 \mathrm{~Hz}, 1 \mathrm{H}), 4.49(\mathrm{dq}, J=14.0,7.1 \mathrm{~Hz}, 1 \mathrm{H}), 7.22-7.39(\mathrm{~m}, 6 \mathrm{H}), 7.39-$ $7.45(\mathrm{~m}, 3 \mathrm{H}), 7.50(\mathrm{~d}, J=7.7 \mathrm{~Hz}, 1 \mathrm{H}), 7.55(\mathrm{~d}, J=8.8 \mathrm{~Hz}, 1 \mathrm{H}), 7.62(\mathrm{~m}, J=2.1,2.1 \mathrm{~Hz}, 1 \mathrm{H}), 7.83(\mathrm{~d}$, $J=8.8 \mathrm{~Hz}, 1 \mathrm{H}), 7.89(\mathrm{~d}, J=7.8 \mathrm{~Hz}, 1 \mathrm{H}), 7.93(\mathrm{~d}, J=8.2 \mathrm{~Hz}, 1 \mathrm{H}), 7.99(\mathrm{~d}, J=8.7 \mathrm{~Hz}, 1 \mathrm{H})$.

${ }^{13} \mathrm{C}$ NMR $\left(125 \mathrm{MHz}, \mathrm{CDCl}_{3}\right) \delta_{\mathrm{c}} / \mathrm{ppm} 23.6(\mathrm{~d}, J=5.5 \mathrm{~Hz}), 24.3(\mathrm{~d}, J=4.6 \mathrm{~Hz}), 24.8(\mathrm{~d}, J=19.4 \mathrm{~Hz})$, $47.1(\mathrm{~d}, J=5.5 \mathrm{~Hz}$ ), 53.1 (d, $J=19.4 \mathrm{~Hz}), 122.1,122.5,122.7,124.5(\mathrm{~d}, J=5.5 \mathrm{~Hz}), 124.8,125.2$, $125.6(d, J=2.8 \mathrm{~Hz}), 126.3,126.5,127.2,127.55,127.59,127.8,128.68,128.74,130.01,130.04$, $130.8,131.0,131.8,133.1,133.3$ (d, J = $1.9 \mathrm{~Hz}), 134.7,148.4,150.3,150.7$ (d, $J=7.4 \mathrm{~Hz}$ ).

${ }^{31}$ P NMR (200 MHz, $\left.\mathrm{CDCl}_{3}\right) \delta_{\mathrm{p}} / \mathrm{ppm} 151.46(\mathrm{~s}, 1 \mathrm{P})$.

HRMS (EI) $m / z$ calcd for $\mathrm{C}_{31} \mathrm{H}_{27} \mathrm{ClNO}_{2} \mathrm{P}[\mathrm{M}]^{+}: 511.1468$, found: 511.1548 .

$[\alpha]_{589}^{20}=+153.52\left(c 0.88, \mathrm{CHCl}_{3}\right)$.

IR $\left(v_{\max } / \mathrm{cm}^{-1}\right):$ 749, 821, 1232, 1506, 2340, 2974.

\section{$N$-Benzhydryl-N-isopropyl-(S)-dinaphtho[2,1-d:1',2'-f][1,3,2]dioxaphosphepin-4-amine (L63)}

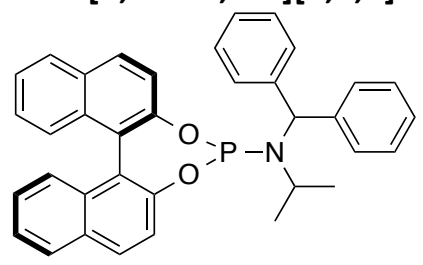

Triethylamine (3.7 mL, $26.6 \mathrm{mmol}, 5.0$ eq.) was added dropwise over about $5 \mathrm{~min}$ to a stirred, cooled (water / ice bath) solution of $\mathrm{PCl}_{3}\left(0.47 \mathrm{~mL}, 5.33 \mathrm{mmol}, 1.0\right.$ eq.) in $\mathrm{CH}_{2} \mathrm{Cl}_{2}(40 \mathrm{~mL})$. The ice bath was removed and the solution left to warm to room temperature before $\mathrm{N}$ benzhydrylpropan-2-amine (1.2 g, $5.3 \mathrm{mmol}, 1.0$ eq.) was added to the stirring solution. $(R)$ Binaphthol (1.52 g, $5.3 \mathrm{mmol}, 1.0 \mathrm{eq}$.) was added to the suspension after $5 \mathrm{~h}$ and the subsequent mixture was left stirring overnight. The solution was then filtered over a small silica $(3 \mathrm{~mm})$ and celite $(1.5 \mathrm{~mm})$ pad and then washed with $\mathrm{CH}_{2} \mathrm{Cl}_{2}$. After removing the solvent in vacuo, flash column chromatography of the yellow residue $\left(78: 17: 1 ;\right.$ Petrol/ $\left.\mathrm{CH}_{2} \mathrm{Cl}_{2} / \mathrm{Et}_{3} \mathrm{~N} ; \mathrm{SiO}_{2}\right)$ gave ligand $\mathbf{L 6 3}$ (2.21 g, $4.1 \mathrm{mmol}, 77 \%)$ as a white solid.

${ }^{1} \mathrm{H}$ NMR $\left(500 \mathrm{MHz}, \mathrm{CDCl}_{3}\right) \delta_{\mathrm{H}} / \mathrm{ppm} 1.06(\mathrm{~d}, J=6.6 \mathrm{~Hz}, 3 \mathrm{H}), 1.18(\mathrm{~d}, J=6.1 \mathrm{~Hz}, 3 \mathrm{H}), 3.68(\mathrm{dq}, J=$ 6.1 \& $17.0 \mathrm{~Hz}, 1 \mathrm{H}), 5.80(\mathrm{~d}, J=17.0 \mathrm{~Hz}, 1 \mathrm{H}), 7.23-7.28(\mathrm{~m}, 1 \mathrm{H}), 7.28-7.46(\mathrm{~m}, 11 \mathrm{H}), 7.50(\mathrm{q}, J=$ $7.6 \mathrm{~Hz}, 4 \mathrm{H}), 7.58(\mathrm{~d}, J=7.6 \mathrm{~Hz}, 2 \mathrm{H}), 7.86(\mathrm{~d}, J=8.8 \mathrm{~Hz}, 1 \mathrm{H}), 7.94(\mathrm{~d}, J=7.6 \mathrm{~Hz}, 2 \mathrm{H}), 7.98(\mathrm{~d}, J=$ $8.8 \mathrm{~Hz}, 1 \mathrm{H})$.

${ }^{13} \mathrm{C}$ NMR $\left(125 \mathrm{MHz}, \mathrm{CDCl}_{3}\right) \delta_{\mathrm{c}} / \mathrm{ppm} 23.0,23.2,46.8,60.6,121.6,122.1,122.3,124.0,124.3,124.6$, 125.8, 125.9, 127.0, 127.0 (2 C), 127.1, 128.1 (2 C), 128.2 (2 C), 128.2, 128.3 (2 C), 128.7 (2 C), 129.0 (2 C), 129.3, 130.1, 130.4, 131.3, 132.7, 143.3, 143.5, 149.1, 150.4 . 
${ }^{31} \mathrm{P}$ NMR $\left(200 \mathrm{MHz} \mathrm{CDCl}_{3}\right) \delta_{\mathrm{P}} / \mathrm{ppm} 150.64$ (s, $\left.1 \mathrm{P}\right)$.

HMRS (EI) $\mathrm{m} / \mathrm{z}$ calcd for $\mathrm{C}_{36} \mathrm{H}_{30} \mathrm{NO}_{2} \mathrm{P}[\mathrm{M}]^{+}: 539.2014$, found: 539.2018 .

$[\alpha]^{20}{ }_{589}=-175.88\left(c\right.$ 1.06, $\left.\mathrm{CHCl}_{3}\right)$.

IR $\left(v_{\max } / \mathbf{c m}^{-1}\right): 747,982,1250,1590,3060$.

\subsubsection{Copper - Ligand Complex Data}

Preparation of copper-ligand complex $(S, S, S)-A$

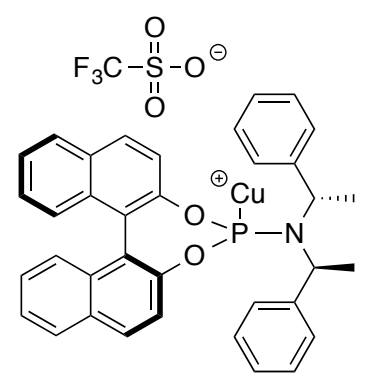

To a flame dried Schlenk flask containing (CuOTf) ${ }_{2} . \mathrm{C}_{6} \mathrm{H}_{6}(239 \mathrm{mg}, 0.48 \mathrm{mmol}, 0.5$ eq.) at room temperature under an argon atmosphere was added the phosphoramidite ligand $(513 \mathrm{mg}, 0.95$ mmol, 1.0 eq.) and then $\mathrm{CH}_{2} \mathrm{Cl}_{2}(10 \mathrm{~mL})$. This mixture was stirred for $1 \mathrm{~h}$ before the resulting clear colourless solution was cannula filtrated into another Schlenk flask. The solvent was then gently removed by use of an oil-pump vacuum (with liquid nitrogen trapping). The resulting off-white solid was dried for at least one extra hour under oil-pump vacuum before storing the catalyst complex under argon.

${ }^{1} \mathrm{H}$ NMR $\left(500 \mathrm{MHz}, \mathrm{CD}_{2} \mathrm{Cl}_{2}\right) \delta_{\mathrm{H}} / \mathrm{ppm} 1.89(\mathrm{~d}, J=6.7 \mathrm{~Hz}, 6 \mathrm{H}) 4.59-4.71(\mathrm{~m}, 2 \mathrm{H}) 7.19-7.44(\mathrm{~m}, 6 \mathrm{H})$ $7.45-7.61(\mathrm{~m}, 10 \mathrm{H}) 7.69(\mathrm{~d}, J=8.8 \mathrm{~Hz}, 2 \mathrm{H}) 7.94-8.07(\mathrm{~m}, 3 \mathrm{H}) 8.14(\mathrm{~d}, J=8.8 \mathrm{~Hz}, 1 \mathrm{H})$

${ }^{13} \mathrm{C}$ NMR $\left(125 \mathrm{MHz}, \mathrm{CD}_{2} \mathrm{Cl}_{2}\right.$ ) $\delta_{\mathrm{C}} / \mathrm{ppm} 22.0$ (2 C), 53.8 (2C under solvent peak), 121.1, 121.2, 122.1, 123.60, 123.64, 125.90, 125.97, 126.9, 127.1, 127.2, 127.7, 127.8 (4 C), 128.5 (2 C), 128.7, 129.0, 129.6 (4 C), 131.2, 131.5, 132.5, 132.80, 133.0, 141.37, 141.41, 148.2 (d, $J=3.9 \mathrm{~Hz}), 149.4$ (d, $J=$ $14.2 \mathrm{~Hz})$.

${ }^{31}$ P NMR (200 MHz, $\left.\mathrm{CD}_{2} \mathrm{Cl}_{2}\right) \delta_{\mathrm{P}} / \mathrm{ppm} 124.1$ (br. s, $\left.1 \mathrm{P}\right)$.

${ }^{19}$ F NMR $\left(500 \mathrm{MHz}, \mathrm{CD}_{2} \mathrm{Cl}_{2}\right) \delta_{\mathrm{F}} / \mathrm{ppm}-78.2(\mathrm{~s}, 1 \mathrm{~F})$.

HRMS (EI) $\mathrm{m} / \mathrm{z}$ calcd for $\mathrm{C}_{36} \mathrm{H}_{30} \mathrm{CuNO}_{2} \mathrm{P}\left[\mathrm{M}-\mathrm{CF}_{3} \mathrm{O}_{3} \mathrm{~S}\right]^{+}: 602.1310$, found: 602.1319. Also found: $\mathrm{CuL}_{2}$ cald for $\mathrm{C}_{72} \mathrm{H}_{60} \mathrm{CuN}_{2} \mathrm{O}_{4} \mathrm{P}_{2}[\mathrm{M}]^{+}:$: 1141.3324, found: 1141.3325 .

IR $\left(v_{\max } / \mathrm{cm}^{-1}\right): 633,670,748,827,949,1025,1223$.

$[\alpha]^{20}{ }_{589}=+51.87\left(\mathrm{c} 0.62, \mathrm{CHCl}_{3}\right)$. 


\section{Preparation of copper-ligand complex $(R)$-B}

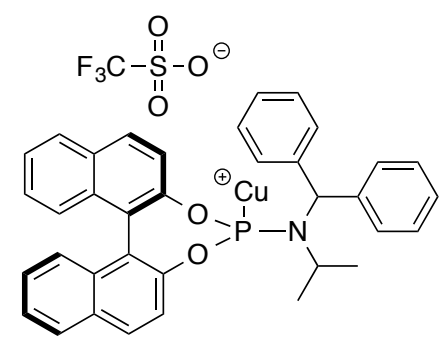

To a flame dried Schlenk flask containing (CuOTf) ${ }_{2} \cdot \mathrm{C}_{6} \mathrm{H}_{6}(239 \mathrm{mg}, 0.48 \mathrm{mmol}, 0.5$ eq.) at room temperature under an argon atmosphere was added the phosphoramidite ligand (513 mg, 0.95 mmol, 1.0 eq.) and then $\mathrm{CH}_{2} \mathrm{Cl}_{2}(10 \mathrm{~mL})$. This mixture was stirred for $1 \mathrm{~h}$ before the resulting clear colourless solution was filtrated through a syringe filter into another Schlenk flask. The solvent was then gently removed by use of an oil-pump vacuum (with liquid nitrogen trapping). The resulting off-white solid was dried for at least one extra hour under oil-pump vacuum before storing the catalyst complex under argon.

${ }^{1} \mathrm{H}$ NMR $\left(500 \mathrm{MHz}, \mathrm{CD}_{2} \mathrm{Cl}_{2}\right) \delta_{\mathrm{H}} / \mathrm{ppm} 8.06(\mathrm{~d}, J=9.1 \mathrm{~Hz}, 1 \mathrm{H}), 8.01(\mathrm{~d}, J=8.8 \mathrm{~Hz}, 1 \mathrm{H}), 7.97(\mathrm{t}, J=8.7$ $\mathrm{Hz}, 2 \mathrm{H}), 7.48$ - $7.58(\mathrm{~m}, 9 \mathrm{H}), 7.44-7.47(\mathrm{~m}, 1 \mathrm{H}), 7.35$ - $7.44(\mathrm{~m}, 5 \mathrm{H}), 7.30$ - $7.35(\mathrm{~m}, 1 \mathrm{H}), 7.26(\mathrm{~d}$, $J=3.8 \mathrm{~Hz}, 2 \mathrm{H}$ ), $5.83(\mathrm{~d}, J=22.0 \mathrm{~Hz}, 1 \mathrm{H}$ ), $3.49-3.53(\mathrm{~m}, 1 \mathrm{H}), 1.06$ (apparent t, $J=6.1 \mathrm{~Hz}, 6 \mathrm{H}$ ).

${ }^{13} \mathrm{C}$ NMR $\left(125 \mathrm{MHz}, \mathrm{CD}_{2} \mathrm{Cl}_{2}\right) \delta_{\mathrm{C}} / \mathrm{ppm} 149.0,148.2,142.0,133.0,132.6,131.9,131.3,130.5$ (2 C), 130.3 (2 C), 129.2 (2 C), 129.0 (2 C), 128.8 (2 C), 128.1 (2 C), 127.6 (2 C), 127.3 (2 C), 127.2, 127.2, $126.2,126.1,123.7,121.8,121.6,121.0,61.4,61.2,22.8,22.5$.

${ }^{31} \mathrm{P}$ NMR $\left(200 \mathrm{MHz}, \mathrm{CD}_{2} \mathrm{Cl}_{2}\right) \delta_{\mathrm{p}} / \mathrm{ppm} 123.14$ (br. s, $\left.1 \mathrm{P}\right)$.

${ }^{19} \mathrm{~F} \mathrm{NMR}\left(380 \mathrm{MHz}, \mathrm{CD}_{2} \mathrm{Cl}_{2}\right) \delta_{\mathrm{F}} / \mathrm{ppm}-76.20(\mathrm{~s}, 1 \mathrm{~F})$.

HRMS (EI) $\mathrm{m} / \mathrm{z}$ calcd for $\mathrm{C}_{36} \mathrm{H}_{30} \mathrm{CUNO}_{2} \mathrm{P}\left[\mathrm{M}-\mathrm{CF}_{3} \mathrm{O}_{3} \mathrm{~S}\right]^{+}: 602.1310$, found: 602.1321 .

$[\alpha]^{20}{ }_{589}=-97.63\left(c 1.31, \mathrm{CHCl}_{3}\right)$.

IR $\left(v_{\max } / \mathrm{cm}^{-1}\right): 1217,1228,1367,1739,2970$. 


\subsubsection{ACA Product Data}

$(+)-(R)-4-P h e n y l d e c a n-2-o n e(1)$

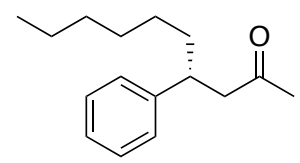

$\mathrm{Cp}_{2} \mathrm{ZrHCl}$ (206 mg, $0.80 \mathrm{mmol}, 2.0$ eq.) was added to a stirred, room temperature, solution of 1-hexene (0.13 mL, $1.0 \mathrm{mmol}, 2.5$ eq.) in $\mathrm{CH}_{2} \mathrm{Cl}_{2}(0.40 \mathrm{~mL})$ under an argon atmosphere. After stirring for $20 \mathrm{~min}$, the resulting clear yellow solution was transferred via syringe over about $1 \mathrm{~min}$ to a clear colourless stirred solution of $(S, S, S)-A\left(15.0 \mathrm{mg}, 0.040 \mathrm{mmol}, 0.05\right.$ eq.) in $\mathrm{CH}_{2} \mathrm{Cl}_{2}(2.0 \mathrm{~mL}$ ) under an argon atmosphere. The resulting dark mixture was allowed to stir for an additional 10 min before trans-4-phenyl-3-buten-2-one $(58 \mathrm{mg}, 0.40 \mathrm{mmol}, 1.0 \mathrm{eq}$.) was added. Stirring at room temperature was continued for $2.5 \mathrm{~h}$ before the reaction was quenched by the addition of $\mathrm{Et}_{2} \mathrm{O}$ (ca $3 \mathrm{~mL}$ ) and then $\mathrm{NH}_{4} \mathrm{Cl}(1 \mathrm{M}$ aq., $c a 1.5 \mathrm{~mL}$ ). The mixture was partitioned between water and $\mathrm{Et}_{2} \mathrm{O}$ and the aqueous phase extracted with $\mathrm{Et}_{2} \mathrm{O}(3 \times 10 \mathrm{~mL})$. The combined organic phase was washed with $\mathrm{NaHCO}_{3}$ (aq. sat., ca $10 \mathrm{~mL}$ ), dried $\left(\mathrm{Na}_{2} \mathrm{SO}_{4}\right)$, filtered and concentrated in vacuo. Flash column chromatography of the yellow residue (3:97; EtOAc:petrol; $\mathrm{SiO}_{2}$ ) gave $(+)-(R)-4-$ phenyldecan-2-one ( $28.2 \mathrm{mg}, 0.12 \mathrm{mmol}, 31 \%$ ) as a yellow oil.

HPLC analysis indicated an enantiomeric excess of $45 \%$ [Chiralpak ${ }^{\circledR} \mathrm{IB}$; flow: $1.5 \mathrm{~mL} / \mathrm{min}$; hexane/iPrOH: 99:1; $\lambda=210 \mathrm{~nm}$; major enantiomer, $t_{R}=4.35 \mathrm{~min}$; minor enantiomer, $t_{R}=4.66 \mathrm{~min}$ ].

Characterisation data were consistent with those reported in the literature for the corresponding compound: ${ }^{1} \mathrm{H},{ }^{13} \mathrm{C}, \mathrm{MS}^{140}$

${ }^{1} \mathrm{H}$ NMR $\left(400 \mathrm{MHz}, \mathrm{CDCl}_{3}\right) \delta_{\mathrm{H}} / \mathrm{ppm} 0.85(\mathrm{t}, J=6.8 \mathrm{~Hz}, 3 \mathrm{H}), 1.05-1.30(\mathrm{~m}, 8 \mathrm{H}), 1.57(\mathrm{~m}, 2 \mathrm{H}), 2.02$ (s, $3 \mathrm{H}), 2.72(\mathrm{dd}, J=13.3,2.2 \mathrm{~Hz}, 2 \mathrm{H}), 3.1(\mathrm{~m}, 1 \mathrm{H}), 7.19(\mathrm{~m}, 3 \mathrm{H}), 7.29(\mathrm{~m}, 2 \mathrm{H})$.

${ }^{13} \mathrm{C}$ NMR $\left(100 \mathrm{MHz}, \mathrm{CDCl}_{3}\right) \delta_{\mathrm{C}} / \mathrm{ppm} 14.0,22.6,27.3,29.2,30.7,31.7,36.5,41.3,51.0,126.3,127.5$ (2 C), 128.4 (2 C), 144.6, 208.1.

MS (ESI) $m / z[\mathrm{M}+\mathrm{Na}]^{+}: 255.2(100)$.

$[\alpha]^{20}{ }_{589}=+8.01\left(c \quad 1.22, \mathrm{CHCl}_{3}\right)$.

IR ( $\left.v_{\max } / \mathrm{cm}^{-1}\right):$ 700, 1159, 1356, 1454, 1718, 2925.

Absolute configuration assigned by analolgy. ${ }^{140}$ 


\section{(-)-(S)-3-Hexylcyclohexanone (2a)}

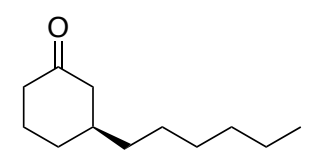

$\mathrm{Cp}_{2} \mathrm{ZrHCl}$ (206 mg, $0.80 \mathrm{mmol}, 2.0$ eq.) was added to a stirred, room temperature, solution of 1-hexene (0.13 mL, $1.0 \mathrm{mmol}, 2.5$ eq.) in $\mathrm{CH}_{2} \mathrm{Cl}_{2}(0.40 \mathrm{~mL})$ under an argon atmosphere. After stirring for $20 \mathrm{~min}$, the resulting clear yellow solution was transferred via syringe over about $1 \mathrm{~min}$ to a clear colourless stirred solution of $(S, S, S)-A\left(30.0 \mathrm{mg}, 0.040 \mathrm{mmol}, 0.10\right.$ eq.) in $\mathrm{Et}_{2} \mathrm{O}(2.0 \mathrm{~mL})$ under an argon atmosphere. The resulting dark mixture was allowed to stir for an additional 10 min before 2-cyclohexenone ( $39 \mu \mathrm{L}, 0.40 \mathrm{mmol}, 1.0$ eq.) and then $\mathrm{TMSCl}(0.25 \mathrm{~mL}, 2.0 \mathrm{mmol}, 5.0$ eq.) were added dropwise via syringe. Stirring at room temperature was continued for $2.5 \mathrm{~h}$ before the reaction was quenched by the addition of $\mathrm{Et}_{2} \mathrm{O}(\mathrm{ca} 3 \mathrm{~mL})$ and then $\mathrm{NH}_{4} \mathrm{Cl}(1 \mathrm{M}$ aq., $c a$ $1.5 \mathrm{~mL}$ ). The mixture was partitioned between water and $\mathrm{Et}_{2} \mathrm{O}$ and the aqueous phase extracted with $\mathrm{Et}_{2} \mathrm{O}(3 \times 10 \mathrm{~mL})$. The combined organic phase was washed with $\mathrm{NaHCO}_{3}$ (aq. sat., ca $\left.10 \mathrm{~mL}\right)$, dried $\left(\mathrm{Na}_{2} \mathrm{SO}_{4}\right)$, filtered and concentrated in vacuo. Flash column chromatography of the yellow residue (1:19; EtOAc:petrol; $\mathrm{SiO}_{2}$ ) gave (-)-(S)-3-hexylcyclohexanone (44 mg, $\left.0.24 \mathrm{mmol}, 59 \%\right)$ as a colourless oil. Enantiomeric excess (93\% ee) was determined by integration of the diastereomeric mixture of the corresponding (+)-(R,R)-DPEN derivative by ${ }^{13} \mathrm{C}$ NMR spectroscopic analysis.

Characterisation data were consistent with those reported in the literature for the corresponding racemic compound: ${ }^{1} \mathrm{H},{ }^{13} \mathrm{C}, \mathrm{IR}, \mathrm{MS} .{ }^{57}$

${ }^{1} \mathrm{H}$ NMR $\left(400 \mathrm{MHz}, \mathrm{CDCl}_{3}\right) \delta_{\mathrm{H}} / \mathrm{ppm} 0.88(\mathrm{t}, J=7.2 \mathrm{~Hz}, 3 \mathrm{H}), 1.25-1.40(\mathrm{~m}, 11 \mathrm{H}), 1.58-1.72(\mathrm{~m}, 2 \mathrm{H})$, 1.85-1.95 (m, $1 \mathrm{H}), 1.96-2.1(\mathrm{~m}, 2 \mathrm{H}), 2.2-2.3(\mathrm{~m}, 1 \mathrm{H}), 2.32-2.47(\mathrm{~m}, 2 \mathrm{H})$.

${ }^{13} \mathrm{C}$ NMR $\left(100 \mathrm{MHz}, \mathrm{CDCl}_{3}\right) \delta_{\mathrm{c}} / \mathrm{ppm} 14.1,22.6,25.3,26.6,29.3,31.3,31.8,36.6,39.1,41.5,48.2$, 212.2.

HRMS (EI) $m / z$ calcd for $\mathrm{C}_{12} \mathrm{H}_{22} \mathrm{O}[\mathrm{M}]^{+}:$182.1671, found: 182.1663 .

$[\alpha]^{24}{ }_{589}=-10.0\left(c 1.00, \mathrm{CHCl}_{3}\right)$.

IR $\left(\mathbf{v}_{\max } / \mathrm{cm}^{-1}\right):$ 1422, 1717, 2925.

CD: $\Theta(\gamma)=-9.04(293 \mathrm{~nm}),\left(\mathrm{c}=0.70 \mathrm{mg} / \mathrm{ml}\right.$ in $\left.\mathrm{CH}_{3} \mathrm{CN}\right)$.

Absolute configuration assigned by analysis of the optical rotation and circular dichroism spectra. ${ }^{141}$ 


\section{(-)-(S)-3-(4-Phenylbutyl)cyclohexanone (2b)}

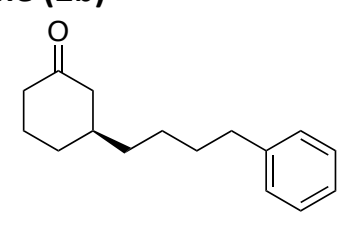

$\mathrm{Cp}_{2} \mathrm{ZrHCl}$ (206 mg, $0.80 \mathrm{mmol}, 2.0$ eq.) was added to a stirred, room temperature, solution of 4-phenyl-1-butene $\left(0.15 \mathrm{~mL}, 1.0 \mathrm{mmol}, 2.5\right.$ eq.) in $\mathrm{CH}_{2} \mathrm{Cl}_{2}(0.4 \mathrm{~mL})$ under an argon atmosphere. After stirring for $15 \mathrm{~min}$, the resulting yellow clear solution was transferred via syringe over about $1 \mathrm{~min}$ to a clear colourless stirred solution of $(S, S, S)-A\left(30.0 \mathrm{mg}, 0.040 \mathrm{mmol}, 0.10\right.$ eq.) in $\mathrm{Et}_{2} \mathrm{O}(2.0$ $\mathrm{mL}$ ) under an argon atmosphere. The resulting dark mixture was allowed to stir for an additional $10 \mathrm{~min}$ before 2-cyclohexenone ( $39 \mu \mathrm{L}, 0.40 \mathrm{mmol}, 1.0$ eq.) and then TMSCl $(0.25 \mathrm{~mL}, 2.0 \mathrm{mmol}$, 5.0 eq.) were added dropwise via syringe. Stirring at room temperature was continued for 150 min before the reaction was quenched by the addition of $\mathrm{Et}_{2} \mathrm{O}(\mathrm{ca} 3 \mathrm{~mL})$ and then $\mathrm{NH}_{4} \mathrm{Cl}(1 \mathrm{M}$ aq., ca $1.5 \mathrm{~mL}$ ). The mixture was partitioned between the aqueous and $\mathrm{Et}_{2} \mathrm{O}$ layers and the aqueous phase extracted with $\mathrm{Et}_{2} \mathrm{O}(3 \times 10 \mathrm{~mL})$. The combined organic phase was washed with $\mathrm{NaHCO}_{3}$ (aq. sat., ca $10 \mathrm{~mL}$ ), dried $\left(\mathrm{Na}_{2} \mathrm{SO}_{4}\right)$, filtered and concentrated in vacuo. Flash column chromatography of the yellow residue (1:19; EtOAc:petrol; $\left.\mathrm{SiO}_{2}\right)$ gave (-)-(S)-3-(4phenylbutyl)cyclohexanone ( $54.3 \mathrm{mg}, 0.24 \mathrm{mmol}, 59 \%$ ) as a colourless oil.

HPLC analysis indicated an enantiomeric excess of $90 \%$ [Chiralpak ${ }^{\circledR} \mathrm{IC}$; flow: $1.5 \mathrm{~mL} / \mathrm{min}$; hexane/iPrOH: $95: 5 ; \lambda=210 \mathrm{~nm}$; minor enantiomer, $t_{R}=8.99 \mathrm{~min}$; major enantiomer, $t_{R}=9.58 \mathrm{~min}$ ].

${ }^{1} \mathrm{H}$ NMR $\left(400 \mathrm{MHz}, \mathrm{CDCl}_{3}\right) \delta_{\mathrm{H}} / \mathrm{ppm} 1.26-1.45(\mathrm{~m}, 5 \mathrm{H}), 1.55-1.70(\mathrm{~m}, 3 \mathrm{H}), 1.71-1.83(\mathrm{~m}, 1 \mathrm{H})$, 1.84 - $1.94(\mathrm{~m}, 1 \mathrm{H}), 1.95-2.09(\mathrm{~m}, 2 \mathrm{H}), 2.21-2.31(\mathrm{~m}, 1 \mathrm{H}), 2.32$ - $2.39(\mathrm{~m}, 1 \mathrm{H}), 2.39$ - $2.47(\mathrm{~m}, 1$ H), $2.61(t, J=7.6 \mathrm{~Hz}, 2 \mathrm{H}), 7.13-7.23(\mathrm{~m}, 3 \mathrm{H}), 7.25-7.33(\mathrm{~m}, 2 \mathrm{H})$.

${ }^{13} \mathrm{C}$ NMR $\left(100 \mathrm{MHz}, \mathrm{CDCl}_{3}\right) \delta_{\mathrm{C}} / \mathrm{ppm} 25.3,26.3,31.3,31.4,35.8,36.4,39.0,41.5,48.2,125.6,128.1$ (2 C), 128.3 (2 C), 142.5, 212.0.

HRMS (EI) $\mathrm{m} / \mathrm{z}$ calcd for $\mathrm{C}_{20} \mathrm{H}_{24} \mathrm{O}[\mathrm{M}]^{+}: 230.1671$, found: 230.1675 .

$[\alpha]^{24}{ }_{589}=-11.96\left(c 1.52, \mathrm{CHCl}_{3}\right)$.

IR $\left(v_{\max } / \mathrm{cm}^{-1}\right):$ 700, 749, 1453, 1711, 2856, 2929.

CD: $\Theta(\gamma)=-7.94(293.0 \mathrm{~nm}),\left(\mathrm{c}=0.41 \mathrm{mg} / \mathrm{ml}\right.$ in $\left.\mathrm{CH}_{3} \mathrm{CN}\right)$.

Absolute configuration assigned by analysis of optical rotation and circular dichroism spectra. ${ }^{141}$

Scale up of the reaction was performed involving $\mathrm{Cp}_{2} \mathrm{ZrHCl}$ (970 mg, $3.8 \mathrm{mmol}, 1.5$ eq.) and 4-phenyl-1-butene (0.64 mL, $4.3 \mathrm{mmol}, 1.7$ eq.) in $\mathrm{CH}_{2} \mathrm{Cl}_{2}(1.9 \mathrm{~mL})$ The resulting alkylzirconocene was added to a solution of $(\boldsymbol{R}, \boldsymbol{R}, \boldsymbol{R})-\mathbf{A}\left(188 \mathrm{mg}, 0.25 \mathrm{mmol}, 0.10\right.$ eq.) in $\mathrm{Et}_{2} \mathrm{O}$ (13 mL) followed by 2- 
cyclohexenone ( $0.24 \mathrm{~mL}, 2.5 \mathrm{mmol}, 1.0$ eq.) then TMSCl (1.6 mL, $12.5 \mathrm{mmol}, 5.0$ eq.). (+)-(R)-3-(4phenylbutyl)cyclohexanone (1.46 g, $6.34 \mathrm{mmol}, 63 \%)$ was obtained as a colourless oil.

HPLC analysis indicated an enantiomeric excess of $91 \%$ [Chiralpak ${ }^{\circledR} \mathrm{IC}$; flow: $1.5 \mathrm{~mL} / \mathrm{min}$; hexane/iPrOH: 99:1; $\lambda=210 \mathrm{~nm}$; major enantiomer, $t_{R}=19.9 \mathrm{~min}$; minor enantiomer, $\left.\mathrm{t}_{\mathrm{R}}=21.8 \mathrm{~min}\right]$. $[\alpha]^{20}{ }_{589}=+5.92\left(c 1.30, \mathrm{CHCl}_{3}\right)$.

\section{(-)-(R)-4,4-Dimethyl-3-(4-phenylbutyl)cyclohexanone (15a)}

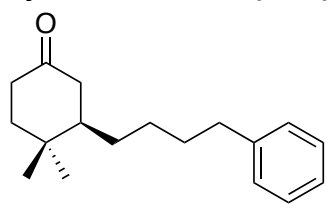

$\mathrm{Cp}_{2} \mathrm{ZrHCl}$ (206 mg, $0.80 \mathrm{mmol}, 2.0$ eq.) was added to a stirred, room temperature, solution of 4-phenyl-1-butene $\left(0.15 \mathrm{~mL}, 1.0 \mathrm{mmol}, 2.5\right.$ eq.) in $\mathrm{CH}_{2} \mathrm{Cl}_{2}(0.40 \mathrm{~mL})$ under an argon atmosphere. After stirring for $30 \mathrm{~min}$ at room temperature, $\mathrm{CH}_{2} \mathrm{Cl}_{2}(0.40 \mathrm{~mL})$ was added. The resulting clear yellow solution was transferred via syringe over about $1 \mathrm{~min}$ to a clear colourless stirred solution of $(\mathbf{S}, \mathbf{S}, \mathbf{S})-\mathrm{A}$ (30.0 mg, $0.040 \mathrm{mmol}, 0.10$ eq.) in $\mathrm{Et}_{2} \mathrm{O}(2.0 \mathrm{~mL})$ under an argon atmosphere. The resulting dark mixture was allowed to stir for $10 \mathrm{~min}$ before 4,4-dimethyl-2-cyclohexenone (53 $\mu \mathrm{L}$, $0.40 \mathrm{mmol}, 1.0$ eq.) and then $\mathrm{TMSCl}(0.25 \mathrm{~mL}, 2.0 \mathrm{mmol}, 5.0$ eq.) were added dropwise via syringe. Stirring at room temperature was continued for $3 \mathrm{~h}$ before the reaction was quenched by the addition of $\mathrm{Et}_{2} \mathrm{O}\left(c a 3 \mathrm{~mL}\right.$ ) and then $\mathrm{NH}_{4} \mathrm{Cl}(1 \mathrm{M}$ aq., $c a 1.5 \mathrm{~mL}$ ). The mixture was partitioned between water and $\mathrm{Et}_{2} \mathrm{O}$ and the aqueous phase was extracted with $\mathrm{Et}_{2} \mathrm{O}(3 \times 10 \mathrm{~mL})$. The combined organic phase was washed with $\mathrm{NaHCO}_{3}$ (aq. sat., ca $10 \mathrm{~mL}$ ), dried $\left(\mathrm{Na}_{2} \mathrm{SO}_{4}\right)$, filtered and concentrated in vacuo. Flash column chromatography of the yellow residue (1:19; EtOAc:petrol; $\mathrm{SiO}_{2}$ ) gave (-)-(R)-4,4-dimethyl-3-(4-phenylbutyl)cyclohexanone $(76.7 \mathrm{mg}, 0.30$ mmol, 74\%) as colourless crystals.

HPLC analysis indicated an enantiomeric excess of $89 \%$ [Chiralpak ${ }^{\circledR}$ IB; flow: $1.0 \mathrm{~mL} / \mathrm{min}$; hexane/iPrOH: 99.4:0.6; $\lambda=210 \mathrm{~nm}$; minor enantiomer, $t_{R}=10.14 \mathrm{~min}$; major enantiomer, $t_{R}=10.63 \mathrm{~min}$ ]. ${ }^{1} \mathrm{H}$ NMR $\left(400 \mathrm{MHz}, \mathrm{CDCl}_{3}\right) \delta_{\mathrm{H}} / \mathrm{ppm} 1.00(\mathrm{~s}, 3 \mathrm{H}), 1.03(\mathrm{~s}, 3 \mathrm{H}), 1.04-1.11(\mathrm{~m}, 1 \mathrm{H}), 1.12-1.25(\mathrm{~m}, 1$ H), 1.36 - $1.52(\mathrm{~m}, 2 \mathrm{H}), 1.52-1.66(\mathrm{~m}, 4 \mathrm{H}), 1.68$ - $1.77(\mathrm{~m}, 1 \mathrm{H}), 1.97-2.11(\mathrm{~m}, 1 \mathrm{H}), 2.21$ - 2.33 (m, $1 \mathrm{H}), 2.35$ - $2.48(\mathrm{~m}, 2 \mathrm{H}), 2.56-2.65(\mathrm{~m}, 2 \mathrm{H}), 7.14$ - $7.22(\mathrm{~m}, 3 \mathrm{H}), 7.24-7.34(\mathrm{~m}, 2 \mathrm{H})$.

${ }^{13} \mathrm{C}$ NMR $\left(100 \mathrm{MHz}, \mathrm{CDCl}_{3}\right) \delta_{\mathrm{H}} / \mathrm{ppm} 19.5,27.1,28.7,30.4,31.5,32.8,35.9,38.3,40.5,42.9,46.7$, $125.7,128.3$ (2 C), 128.4 (2 C), 142.5, 212.30.

HRMS (EI) m/z calcd for $\mathrm{C}_{18} \mathrm{H}_{26} \mathrm{O}: 258.1984$, found: 258.1979 .

$[\alpha]^{24}{ }_{589}=-17.79\left(\mathrm{c} 0.73, \mathrm{CHCl}_{3}\right)$. 
IR $\left(\mathbf{v}_{\max } / \mathrm{cm}^{-1}\right):$ 698, 1711, 2858, 2932.

$\mathrm{CD}: \Theta(\gamma)=-2.23(295.5 \mathrm{~nm}),\left(\mathrm{c}=0.57 \mathrm{mg} / \mathrm{ml}\right.$ in $\left.\mathrm{CH}_{3} \mathrm{CN}\right)$.

M. P. $27-29^{\circ} \mathrm{C}$.

Absolute configuration assigned by analysis of optical rotation and circular dichroism spectra. ${ }^{141}$

Scale up of the reaction was performed involving $\mathrm{Cp}_{2} \mathrm{ZrHCl}(970 \mathrm{mg}, 3.8 \mathrm{mmol}, 1.5$ eq.) and 4-phenyl-1-butene $\left(0.64 \mathrm{~mL}, 4.3 \mathrm{mmol}, 1.7\right.$ eq.) in $\mathrm{CH}_{2} \mathrm{Cl}_{2}(1.9 \mathrm{~mL})$. The resulting alkylzirconocene was added to a solution of $(\boldsymbol{R}, \boldsymbol{R}, \boldsymbol{R})-\mathbf{A}\left(188 \mathrm{mg}, 0.25 \mathrm{mmol}, 0.10\right.$ eq.) in $\mathrm{Et}_{2} \mathrm{O}(13 \mathrm{~mL})$ followed by 4,4-dimethyl-2-cyclohexenone ( $0.33 \mathrm{~mL}, 2.5 \mathrm{mmol}, 1.0$ eq.) then TMSCl (1.6 mL, $12.5 \mathrm{mmol}, 5.0$ eq.). (+)-(S)-3-(4-phenylbutyl)-4,4-dimethylcyclohexanone (431 mg, $1.67 \mathrm{mmol}, 67 \%$ ) was obtained as a white solid.

HPLC analysis indicated an enantiomeric excess of $87 \%$ [Chiralpak ${ }^{\circledR} \mathrm{IB}$; flow: $1.0 \mathrm{~mL} / \mathrm{min}$; hexane/iPrOH: 99.4:0.6; $\lambda=210 \mathrm{~nm}$; major enantiomer, $t_{R}=10.99 \mathrm{~min}$; minor enantiomer, $t_{R}=11.95 \mathrm{~min}$ ]. $[\alpha]^{20}{ }_{589}=+17.09\left(c 1.17, \mathrm{CHCl}_{3}\right)$.

\section{(-)-(R)-4,4-Dimethyl-3-(6-phenylhexyl)cyclohexanone (15b)}

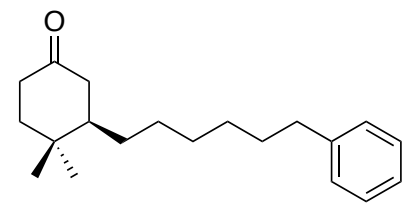

$\mathrm{Cp}_{2} \mathrm{ZrHCl}$ (206 mg, $0.80 \mathrm{mmol}, 2.0$ eq.) was added to a stirred, room temperature, solution of 6-phenyl-1-hexene $\left(0.18 \mathrm{~mL}, 1.0 \mathrm{mmol}, 2.5\right.$ eq.) in $\mathrm{CH}_{2} \mathrm{Cl}_{2}(0.40 \mathrm{~mL})$ under an argon atmosphere. After stirring for $50 \mathrm{~min}$ the resulting clear yellow solution was transferred via syringe over about $1 \mathrm{~min}$ to a clear colourless stirred solution of $(S, S, S)-A\left(30.0 \mathrm{mg}, 0.040 \mathrm{mmol}, 0.10\right.$ eq.) in $\mathrm{Et}_{2} \mathrm{O}(2.0$ $\mathrm{mL}$ ) under an argon atmosphere. The resulting dark mixture was allowed to stir for an additional $10 \mathrm{~min}$ before 4,4-dimethyl-2-cyclohexenone $(53 \mu \mathrm{L}, 0.40 \mathrm{mmol}, 1.0$ eq.) and then TMSCl $(0.25$ $\mathrm{mL}, 2.0 \mathrm{mmol}, 5.0$ eq.) were added dropwise via syringe. Stirring at room temperature was continued for $15 \mathrm{~h}$ before the reaction was quenched by the addition of $\mathrm{Et}_{2} \mathrm{O}\left(\mathrm{ca} 3 \mathrm{~mL}\right.$ ) and $\mathrm{NH}_{4} \mathrm{Cl}$ (1 $\mathrm{M}$ aq., $c a 1.5 \mathrm{~mL}$ ). The mixture was partitioned between water and $\mathrm{Et}_{2} \mathrm{O}$ and the aqueous phase extracted with $\mathrm{Et}_{2} \mathrm{O}(3 \times 10 \mathrm{~mL})$. The combined organic phase was washed with $\mathrm{NaHCO}_{3}$ (sat. aq., ca $10 \mathrm{~mL})$, dried $\left(\mathrm{Na}_{2} \mathrm{SO}_{4}\right)$, filtered and concentrated in vacuo. Flash column chromatography of the yellow residue (1:19; EtOAc:petrol; $\left.\mathrm{SiO}_{2}\right)$ gave (-)-(R)-4,4-dimethyl-3-(6phenylhexyl)cyclohexanone ( $75 \mathrm{mg}, 0.26 \mathrm{mmol}, 65 \%)$ as a colourless oil. 
HPLC analysis indicated an enantiomeric excess of $89 \%$ [Chiralpak ${ }^{\circledR} \mathrm{IC}$; flow: $1.5 \mathrm{~mL} / \mathrm{min}$; hexane/iPrOH: 99:1; $\lambda=210 \mathrm{~nm}$; minor enantiomer, $t_{R}=13.68 \mathrm{~min}$; major enantiomer, $t_{R}=14.45 \mathrm{~min}$ ].

${ }^{1} \mathrm{H}$ NMR $\left(400 \mathrm{MHz}, \mathrm{CDCl}_{3}\right) \delta_{\mathrm{H}} / \mathrm{ppm} 0.99(\mathrm{~s}, 3 \mathrm{H}), 1.02(\mathrm{~s}, 3 \mathrm{H}), 1.04-1.43(\mathrm{~m}, 7 \mathrm{H}), 1.43-1.67(\mathrm{~m}, 5$ H), 1.67 - $1.76(\mathrm{~m}, 1 \mathrm{H}), 2.04(\mathrm{dd}, J=14.4,12.4 \mathrm{~Hz}, 1 \mathrm{H}), 2.23-2.32(\mathrm{~m}, 1 \mathrm{H}), 2.36-2.47(\mathrm{~m}, 2 \mathrm{H})$, $2.61(t, J=7.7 \mathrm{~Hz}, 2 \mathrm{H}), 7.15-7.22(\mathrm{~m}, 3 \mathrm{H}), 7.25-7.32(\mathrm{~m}, 2 \mathrm{H})$.

${ }^{13} \mathrm{C}$ NMR $\left(100 \mathrm{MHz}, \mathrm{CDCl}_{3}\right) \delta_{\mathrm{C}} / \mathrm{ppm} 19.5,27.4,28.7,29.2,29.6,30.5,31.4,32.9,35.9,38.3,40.5$, $42.9,46.8,125.9,128.2$ (2 C), 128.4 (2 C), 142.8, 212.4.

HRMS (EI) $m / z$ calcd for $\mathrm{C}_{20} \mathrm{H}_{30} \mathrm{O}[\mathrm{M}]^{+}:$:286.2297, found: 286.2296 .

$[\alpha]^{24}{ }_{589}=-19.6\left(c 0.88, \mathrm{CHCl}_{3}\right)$.

IR $\left(v_{\max } / \mathrm{cm}^{-1}\right):$ 699, 1453, 1714, 2929.

Absolute configuration assigned by optical rotation. ${ }^{141}$

\section{(-)-(R)-3-Hexyl-4,4-dimethylcyclohexanone (15c)}

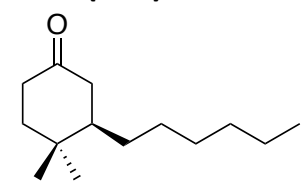

$\mathrm{Cp}_{2} \mathrm{ZrHCl}$ (206 mg, $0.80 \mathrm{mmol}, 2.0$ eq.) was added to a stirred, room temperature, solution of 1-hexene (0.13 mL, $1.00 \mathrm{mmol}, 2.5$ eq.) in $\mathrm{CH}_{2} \mathrm{Cl}_{2}(0.40 \mathrm{~mL})$ under an argon atmosphere. After stirring for $20 \mathrm{~min}$, the resulting clear yellow solution was transferred via syringe over about $1 \mathrm{~min}$ to a clear colourless stirred solution of $(S, S, S)-A\left(30.0 \mathrm{mg}, 0.040 \mathrm{mmol}, 0.10\right.$ eq.) in $\mathrm{Et}_{2} \mathrm{O}(2.0 \mathrm{~mL})$ under an argon atmosphere. The resulting dark mixture was allowed to stir for an additional 10 min before 4,4-dimethyl-2-cyclohexenone ( $53 \mu \mathrm{L}, 0.40 \mathrm{mmol}, 1.0$ eq.) and then $\mathrm{TMSCl}(0.25 \mathrm{~mL}$, $2.0 \mathrm{mmol}, 5.0$ eq.) were added dropwise via syringe. Stirring at room temperature was continued arbitrarily for $15 \mathrm{~h}$ before the reaction was quenched by the addition of $\mathrm{Et}_{2} \mathrm{O}$ (ca $3 \mathrm{~mL}$ ) and $\mathrm{NH}_{4} \mathrm{Cl}$ (1 $\mathrm{M}$ aq., $c a 1.5 \mathrm{~mL}$ ). The mixture was partitioned between water and $\mathrm{Et}_{2} \mathrm{O}$ and the aqueous phase extracted with $\mathrm{Et}_{2} \mathrm{O}(3 \times 10 \mathrm{~mL})$. The combined organic phase was washed with $\mathrm{NaHCO}_{3}$ (sat. aq., ca $10 \mathrm{~mL})$, dried $\left(\mathrm{Na}_{2} \mathrm{SO}_{4}\right)$, filtered and concentrated in vacuo. Flash column chromatography of the yellow residue (3:97; EtOAc:petrol; $\mathrm{SiO}_{2}$ ) gave (-)-(R)-3-hexyl-4,4-dimethylcyclohexanone (57 $\mathrm{mg}, 0.27 \mathrm{mmol}, 67 \%)$ as a colourless oil.

Enantiomeric excess ( $89 \%$ ee) was determined by integration of the diastereomeric mixture of the corresponding $(+)-(R, R)$-DPEN derivative by ${ }^{13} \mathrm{C}$ NMR spectroscopic analysis.

${ }^{1} \mathrm{H}$ NMR $\left(400 \mathrm{MHz}, \mathrm{CDCl}_{3}\right) \delta_{\mathrm{H}} / \mathrm{ppm} 0.88(\mathrm{t}, J=6.9 \mathrm{~Hz}, 3 \mathrm{H}), 0.99(\mathrm{~s}, 3 \mathrm{H}), 1.02(\mathrm{~s}, 3 \mathrm{H}), 1.04-1.42$ (m, 9 H), $1.43-1.57(\mathrm{~m}, 2 \mathrm{H}), 1.56-1.66(\mathrm{~m}, 1 \mathrm{H}), 1.67-1.75(\mathrm{~m}, 1 \mathrm{H}), 2.04$ (ddd, $J=14.7,12.1,0.9$ 
$\mathrm{Hz}, 1 \mathrm{H}), 2.22-2.31(\mathrm{~m}, 1 \mathrm{H}), 2.35-2.46(\mathrm{~m}, 2 \mathrm{H})$.

${ }^{13} \mathrm{C}$ NMR $\left(100 \mathrm{MHz}, \mathrm{CDCl}_{3}\right) \delta_{\mathrm{c}} / \mathrm{ppm} 14.1,19.5,22.6,27.5,28.7,29.4,30.6,31.8,32.8,38.3,40.5$, $42.9,46.8,212.4$.

HRMS (EI) $\mathrm{m} / \mathrm{z}$ calcd for $\mathrm{C}_{14} \mathrm{H}_{26} \mathrm{O}[\mathrm{M}]^{+}: 210.1984$, found: 210.1982 .

$[\alpha]^{24}{ }_{589}=-20.1\left(c 1.03, \mathrm{CHCl}_{3}\right)$.

IR $\left(\mathbf{v}_{\max } / \mathrm{cm}^{-1}\right): 1467,1715,2928$.

Absolute configuration assigned by optical rotation. ${ }^{141}$

\section{(-)-(R)-4,4-Dimethyl-3-octylcyclohexanone (15d)}

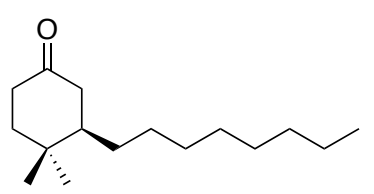

$\mathrm{Cp}_{2} \mathrm{ZrHCl}$ (206 mg, $0.80 \mathrm{mmol}, 2.0$ eq.) was added to a stirred, room temperature, solution of 1-octene $\left(0.16 \mathrm{~mL}, 1.0 \mathrm{mmol}, 2.5\right.$ eq.) in $\mathrm{CH}_{2} \mathrm{Cl}_{2}(0.40 \mathrm{~mL})$ under an argon atmosphere. After stirring for $15 \mathrm{~min}$, the resulting clear yellow solution was transferred via syringe over about $1 \mathrm{~min}$ to a clear colourless stirred solution of $(S, S, S)-A\left(30.0 \mathrm{mg}, 0.040 \mathrm{mmol}, 0.10\right.$ eq.) in $\mathrm{Et}_{2} \mathrm{O}(2.0 \mathrm{~mL})$ under an argon atmosphere. The resulting dark mixture was allowed to stir for an additional 10 min before 4,4-dimethyl-2-cyclohexenone (53 $\mu \mathrm{L}, 0.40 \mathrm{mmol}, 1.0$ eq.) and then $\mathrm{TMSCl}(0.25 \mathrm{~mL}$, $2.0 \mathrm{mmol}, 5.0$ eq.) were added dropwise via syringe. Stirring at room temperature was continued arbitrarily for $15 \mathrm{~h}$ before the reaction was quenched by the addition of $\mathrm{Et}_{2} \mathrm{O}(\mathrm{ca} 3 \mathrm{~mL})$ and $\mathrm{NH}_{4} \mathrm{Cl}$ (1 $\mathrm{M}$ aq., $c a 1.5 \mathrm{~mL}$ ). The mixture was partitioned between water and $\mathrm{Et}_{2} \mathrm{O}$ and the aqueous phase extracted with $\mathrm{Et}_{2} \mathrm{O}(3 \times 10 \mathrm{~mL})$. The combined organic phase was washed with $\mathrm{NaHCO}_{3}$ (sat. aq., ca $10 \mathrm{~mL})$, dried $\left(\mathrm{Na}_{2} \mathrm{SO}_{4}\right)$, filtered and concentrated in vacuo. Flash column chromatography of the yellow residue (3:97; EtOAc:petrol; $\mathrm{SiO}_{2}$ ) gave (-)-(R)-4,4-dimethyl-3-octylcyclohexanone (46 $\mathrm{mg}, 0.19 \mathrm{mmol}, 49 \%)$ as a colourless oil.

Enantiomeric excess ( $89 \%$ ee) was determined by integration of the diastereomeric mixture of the corresponding (+)-(R,R)-DPEN derivative by ${ }^{13} \mathrm{C}$ NMR spectroscopic analysis.

${ }^{1} \mathrm{H}$ NMR $\left(400 \mathrm{MHz}, \mathrm{CDCl}_{3}\right) \delta_{\mathrm{H}} / \mathrm{ppm} 0.88(\mathrm{t}, J=6.8 \mathrm{~Hz}, 3 \mathrm{H}), 0.99(\mathrm{~s}, 3 \mathrm{H}), 1.02(\mathrm{~s}, 3 \mathrm{H}), 1.04-1.42$ $(\mathrm{m}, 13 \mathrm{H}), 1.42-1.75(\mathrm{~m}, 4 \mathrm{H}), 2.04(\mathrm{ddd}, J=14.6,11.9,0.6 \mathrm{~Hz}, 1 \mathrm{H}), 2.22-2.31(\mathrm{~m}, 1 \mathrm{H}), 2.35-$ $2.47(m, 2 \mathrm{H})$.

${ }^{13} \mathrm{C}$ NMR $\left(100 \mathrm{MHz}, \mathrm{CDCl}_{3}\right) \delta_{\mathrm{c}} / \mathrm{ppm} 14.1,19.5,22.7,27.5,28.7,29.3,29.5,29.7,30.6,31.9,32.8$, $38.3,40.5,42.9,46.8,212.4$.

HRMS (EI) $m / z$ calcd for $\mathrm{C}_{16} \mathrm{H}_{30} \mathrm{O}[\mathrm{M}]^{+}:$238.2297, found: 238.2292 . 
$[\alpha]^{24}{ }_{589}=-17.1\left(c\right.$ 1.07, $\left.\mathrm{CHCl}_{3}\right)$.

IR $\left(v_{\max } / \mathrm{cm}^{-1}\right):$ 1467, 1716, 2924.

Absolute configuration assigned by optical rotation. ${ }^{141}$

\section{(-)-(R)-4,4-Dimethyl-3-tetradecylcyclohexanone (15e)}

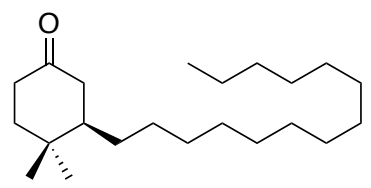

$\mathrm{Cp}_{2} \mathrm{ZrHCl}$ (206 mg, $0.80 \mathrm{mmol}, 2.0$ eq.) was added to a stirred, room temperature, solution of 1-tetradecene ( $0.25 \mathrm{~mL}, 1.0 \mathrm{mmol}, 2.5$ eq.) in $\mathrm{CH}_{2} \mathrm{Cl}_{2}(0.40 \mathrm{~mL})$ under an argon atmosphere. After stirring for $20 \mathrm{~min}$, the resulting clear yellow solution was transferred via syringe over about $1 \mathrm{~min}$ to a clear colourless stirred solution of $(S, S, S)-A\left(30.0 \mathrm{mg}, 0.040 \mathrm{mmol}, 0.10\right.$ eq.) in $\mathrm{Et}_{2} \mathrm{O}(2.0 \mathrm{~mL})$ under an argon atmosphere. The resulting dark mixture was allowed to stir for an additional 10 min before 4,4-dimethyl-2-cyclohexenone (53 $\mu \mathrm{L}, 0.40 \mathrm{mmol}, 1.0$ eq.) and then TMSCl $(0.25 \mathrm{~mL}$, $2.0 \mathrm{mmol}, 5.0$ eq.) were added dropwise via syringe. Stirring at room temperature was continued arbitrarily for $15 \mathrm{~h}$ before the reaction was quenched by the addition of $\mathrm{Et}_{2} \mathrm{O}\left(\mathrm{ca} 3 \mathrm{~mL}\right.$ ) and $\mathrm{NH}_{4} \mathrm{Cl}$ (1 $\mathrm{M}$ aq., $c a 1.5 \mathrm{~mL}$ ). The mixture was partitioned between water and $\mathrm{Et}_{2} \mathrm{O}$ and the aqueous phase extracted with $\mathrm{Et}_{2} \mathrm{O}(3 \times 10 \mathrm{~mL})$. The combined organic phase was washed with $\mathrm{NaHCO}_{3}$ (sat. aq., ca $10 \mathrm{~mL})$, dried $\left(\mathrm{Na}_{2} \mathrm{SO}_{4}\right)$, filtered and concentrated in vacuo. Flash column chromatography of the yellow residue (3:97; EtOAc:petrol; $\mathrm{SiO}_{2}$ ) gave (-)-(R)-4,4-dimethyl-3-tetradecylcyclohexanone (46 mg, $0.19 \mathrm{mmol}, 49 \%$ ) as a colourless oil.

Enantiomeric excess ( $89 \%$ ee) was determined by integration of the diastereomeric mixture of the corresponding $(+)-(R, R)$-DPEN derivative by ${ }^{13} \mathrm{C}$ NMR spectroscopic analysis.

${ }^{1} \mathrm{H}$ NMR $\left(400 \mathrm{MHz}, \mathrm{CDCl}_{3}\right) \delta_{\mathrm{H}} / \mathrm{ppm} 0.89(\mathrm{t}, J=6.8 \mathrm{~Hz}, 3 \mathrm{H}), 0.99(\mathrm{~s}, 3 \mathrm{H}), 1.03(\mathrm{~s}, 3 \mathrm{H}), 1.16-1.42$ (m, $25 \mathrm{H}), 1.43-1.65(\mathrm{~m}, 3 \mathrm{H}), 1.67-1.75(\mathrm{~m}, 1 \mathrm{H}), 2.04$ (app. dd, J = 14.4, $12.5 \mathrm{~Hz}, 1 \mathrm{H}$ ), 2.23 $2.31(m, 1 H), 2.36-2.47(m, 2 H)$.

${ }^{13} \mathrm{C}$ NMR $\left(100 \mathrm{MHz}, \mathrm{CDCl}_{3}\right) \delta_{\mathrm{C}} / \mathrm{ppm} 14.1,19.5,22.7,27.5,28.7,29.4,29.57,29.61,29.65$ (5 C), $29.74,30.6,31.9,32.8,38.3,40.5,42.9,46.8,212.5$.

HRMS (EI) $m / z$ calcd for $\mathrm{C}_{22} \mathrm{H}_{42} \mathrm{O}[\mathrm{M}]^{+}: 322.3236$, found: 322.3241 .

$[\alpha]^{24}{ }_{589}=-18.5\left(c 1.03, \mathrm{CHCl}_{3}\right)$.

IR $\left(\boldsymbol{v}_{\max } / \mathrm{cm}^{-1}\right):$ 1466, 1716, 2922.

Absolute configuration assigned by optical rotation. ${ }^{141}$ 


\section{(-)-(R)-3-(3,3-Dimethylbutyl)-4,4-dimethylcyclohexanone (15f)}

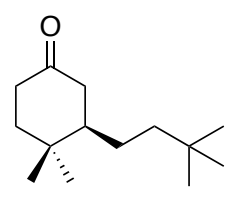

$\mathrm{Cp}_{2} \mathrm{ZrHCl}$ (206 mg, $0.80 \mathrm{mmol}, 2.0$ eq.) was added to a stirred, room temperature, solution of 3,3-dimethylbut-1-ene (0.13 mL, $1.0 \mathrm{mmol}, 2.5$ eq.) in $\mathrm{CH}_{2} \mathrm{Cl}_{2}(0.40 \mathrm{~mL})$ under an argon atmosphere. After stirring for $60 \mathrm{~min}$ at room temperature, $\mathrm{CH}_{2} \mathrm{Cl}_{2}(0.20 \mathrm{~mL})$ was added and the reaction mixture was heated to $30{ }^{\circ} \mathrm{C}$ for $40 \mathrm{~min}$ and then allowed to cool to room temperature. The resulting clear yellow solution was transferred via syringe over about $1 \mathrm{~min}$ to a clear colourless stirred solution of $(S, S, S)-A\left(30.0 \mathrm{mg}, 0.040 \mathrm{mmol}, 0.10\right.$ eq.) in $\mathrm{Et}_{2} \mathrm{O}(2.0 \mathrm{~mL})$ under an argon atmosphere. The resulting dark mixture was allowed to stir for an additional $10 \mathrm{~min}$ before 4,4-dimethyl-2-cyclohexenone ( $53 \mu \mathrm{L}, 0.40 \mathrm{mmol}, 1.0$ eq.) and then TMSCl $(0.25 \mathrm{~mL}, 2.0 \mathrm{mmol}$, 5.0 eq.) were added dropwise via syringe. Stirring at room temperature was continued for $18 \mathrm{~h}$ before the reaction was quenched by the addition of $\mathrm{Et}_{2} \mathrm{O}(\mathrm{ca} 3 \mathrm{~mL})$ and then $\mathrm{NH}_{4} \mathrm{Cl}(1 \mathrm{M}$ aq., $c a$ $1.5 \mathrm{~mL}$ ). The mixture was partitioned between water and $\mathrm{Et}_{2} \mathrm{O}$ and the aqueous phase extracted with $\mathrm{Et}_{2} \mathrm{O}(3 \times 10 \mathrm{~mL})$. The combined organic phase was washed with $\mathrm{NaHCO}_{3}$ (aq. sat., ca $10 \mathrm{~mL}$ ), dried $\left(\mathrm{Na}_{2} \mathrm{SO}_{4}\right)$, filtered and concentrated in vacuo. Flash column chromatography of the yellow residue (3:97; EtOAc:petrol; $\mathrm{SiO}_{2}$ ) gave (-)-(R)-3-(3,3-dimethylbutyl)-4,4-dimethylcyclohexanone (54 $\mathrm{mg}, 0.26 \mathrm{mmol}, 65 \%$ ) as a colourless oil.

Enantiomeric excess ( $84 \%$ ee) was determined by integration of the diastereomeric mixture of the corresponding $(+)-(R, R)$-DPEN derivative by ${ }^{13} \mathrm{C}$ NMR spectroscopic analysis.

${ }^{1} \mathrm{H}$ NMR $\left(400 \mathrm{MHz}, \mathrm{CDCl}_{3}\right) \delta_{\mathrm{H}} / \mathrm{ppm} 0.85(\mathrm{~s}, 9 \mathrm{H}), 0.89-0.97(\mathrm{~m}, 2 \mathrm{H}), 0.99(\mathrm{~s}, 3 \mathrm{H}), 1.02(\mathrm{~s}, 3 \mathrm{H})$, $1.20-1.30(m, 1 H), 1.32-1.42(m, 1 H), 1.48-1.62(m, 2 H), 1.65-1.74(m, 1 H), 1.98-2.07(m, 1$ H), $2.21-2.29(m, 1 H), 2.33-2.46(m, 2 H)$.

${ }^{13} \mathrm{C}$ NMR $\left(100 \mathrm{MHz}, \mathrm{CDCl}_{3}\right) \delta_{\mathrm{C}} / \mathrm{ppm} 19.5,25.3,28.7,29.24$ (3 C), 30.3, 33.0, 38.2, 40.4, 42.1, 43.0, $47.8,212.2$.

HRMS (EI) $\mathrm{m} / \mathrm{z}$ calcd for $\mathrm{C}_{14} \mathrm{H}_{26} \mathrm{O}[\mathrm{M}]^{+}:$:210.1984, found: 210.1986 .

$[\alpha]^{24}{ }_{589}=-23.0\left(c 0.52, \mathrm{CHCl}_{3}\right)$.

IR $\left(v_{\max } / \mathrm{cm}^{-1}\right):$ 1390, 1469, 1715, 2866, 2954.

Absolute configuration assigned by optical rotation. ${ }^{141}$ 


\section{(-)-(R)-3-(2-Cyclohexylethyl)-4,4-dimethylcyclohexanone (15g)}

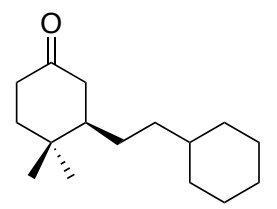

$\mathrm{Cp}_{2} \mathrm{ZrHCl}$ (206 mg, $0.80 \mathrm{mmol}, 2.0$ eq.) was added to a stirred, room temperature, solution of vinylcyclohexane $\left(0.14 \mathrm{~mL}, 1.0 \mathrm{mmol}, 2.5\right.$ eq.) in $\mathrm{CH}_{2} \mathrm{Cl}_{2}(0.40 \mathrm{~mL})$ under an argon atmosphere. After stirring for $15 \mathrm{~min}$, the resulting clear yellow solution was transferred via syringe over about $1 \mathrm{~min}$ to a clear colourless stirred solution of $(\boldsymbol{S}, S, S)-\mathrm{A}\left(30.0 \mathrm{mg}, 0.040 \mathrm{mmol}, 0.10 \mathrm{eq}\right.$.) in $\mathrm{Et}_{2} \mathrm{O}(2.0$ $\mathrm{mL}$ ) under an argon atmosphere. The resulting dark mixture was allowed to stir for an additional 20 min before 4,4-dimethyl-2-cyclohexenone $(53 \mu \mathrm{L}, 0.40 \mathrm{mmol}, 1.0$ eq.) and then $\mathrm{TMSCl}(0.25$ $\mathrm{mL}, 2.0 \mathrm{mmol}, 5.0$ eq.) were added dropwise via syringe. Stirring at room temperature was continued arbitrarily for $18 \mathrm{~h}$ before the reaction was quenched by the addition of $\mathrm{Et}_{2} \mathrm{O}(\mathrm{ca} 3 \mathrm{~mL}$ ) and $\mathrm{NH}_{4} \mathrm{Cl}\left(1 \mathrm{M}\right.$ aq., ca $1.5 \mathrm{~mL}$ ). The mixture was partitioned between water and $\mathrm{Et}_{2} \mathrm{O}$ and the aqueous phase extracted with $\mathrm{Et}_{2} \mathrm{O}(3 \times 10 \mathrm{~mL})$. The combined organic phase was washed with $\mathrm{NaHCO}_{3}\left(1 \mathrm{M}\right.$ aq., ca $10 \mathrm{~mL}$ ), dried $\left(\mathrm{Na}_{2} \mathrm{SO}_{4}\right)$, filtered and concentrated in vacuo. Flash column chromatography of the yellow residue (3:97; EtOAc:petrol; $\left.\mathrm{SiO}_{2}\right)$ gave (-)-(R)-3-(2cyclohexylethyl)-4,4-dimethylcyclohexanone $(72.0 \mathrm{mg}, 0.30 \mathrm{mmol}, 75 \%)$ as a colourless oil.

Enantiomeric excess $(93 \%$ ee) was determined by integration of the diastereomeric mixture of the corresponding (+)-(R,R)-DPEN derivative by ${ }^{13} \mathrm{C}$ NMR spectroscopic analysis.

${ }^{1} \mathrm{H}$ NMR $\left(400 \mathrm{MHz}, \mathrm{CDCl}_{3}\right) \delta_{\mathrm{H}} / \mathrm{ppm} 0.70-0.96(\mathrm{~m}, 3 \mathrm{H}), 0.97(\mathrm{~s}, 3 \mathrm{H}), 1.01(\mathrm{~s}, 3 \mathrm{H}), 1.08-1.27(\mathrm{~m}$, $5 \mathrm{H}), 1.36$ - $1.46(\mathrm{~m}, 1 \mathrm{H}), 1.50$ - $1.59(\mathrm{~m}, 2 \mathrm{H}), 1.60$ - $1.74(\mathrm{~m}, 6 \mathrm{H}), 1.96-2.06(\mathrm{~m}, 1 \mathrm{H}), 2.20-2.31$ $(m, 1 \mathrm{H}), 2.33-2.47(\mathrm{~m}, 2 \mathrm{H})$.

${ }^{13} \mathrm{C}$ NMR $\left(100 \mathrm{MHz}, \mathrm{CDCl}_{3}\right) \delta_{\mathrm{H}} / \mathrm{ppm} 19.4,26.3,26.3,26.6,27.7,28.7,32.9,33.7,35.3,37.7,37.8$, $38.3,40.5,42.9,47.0,212.2$.

HRMS (EI) $\mathrm{m} / \mathrm{z}$ calcd for $\mathrm{C}_{16} \mathrm{H}_{28} \mathrm{O}[\mathrm{M}]^{+}: 236.2140$, found: 236.2149 .

$[\alpha]^{24}{ }_{589}=-17.2\left(\mathrm{c} 0.47, \mathrm{CHCl}_{3}\right)$.

IR $\left(v_{\max } / \mathrm{cm}^{-1}\right): 1458,1714,2926$.

Absolute configuration assigned by optical rotation. ${ }^{141}$ 


\section{(-)-(3R)-3-(2-(Cyclohex-3-enyl)ethyl)-4,4-dimethylcyclohexanone (15h)}

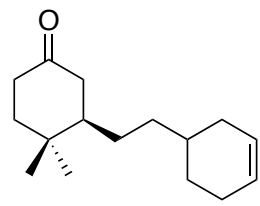

$\mathrm{Cp}_{2} \mathrm{ZrHCl}$ (206 mg, $0.80 \mathrm{mmol}, 2.0$ eq.) was added to a stirred, room temperature, solution of 4-vinyl-1-cyclohexene (0.13 mL, $1.0 \mathrm{mmol}, 2.5$ eq.) in $\mathrm{CH}_{2} \mathrm{Cl}_{2}(0.40 \mathrm{~mL})$ under an argon atmosphere. After stirring for $25 \mathrm{~min}$, the resulting clear yellow solution was transferred via syringe over about $1 \mathrm{~min}$ to a clear colourless stirred solution of $(S, S, S)-A(30.0 \mathrm{mg}, 0.040 \mathrm{mmol}$, 0.10 eq.) in $\mathrm{Et}_{2} \mathrm{O}(2.0 \mathrm{~mL})$ under an argon atmosphere. The resulting dark mixture was allowed to stir for an additional $10 \mathrm{~min}$ before 4,4-dimethyl-2-cyclohexenone (53 $\mu \mathrm{L}, 0.40 \mathrm{mmol}, 1.0$ eq.) and then $\mathrm{TMSCl}(0.25 \mathrm{~mL}, 2.0 \mathrm{mmol}, 5.0$ eq.) were added dropwise via syringe. Stirring at room temperature was continued for $15 \mathrm{~h}$ before the reaction was quenched by the addition of $\mathrm{Et}_{2} \mathrm{O}$ ( $c a$ $3 \mathrm{~mL}$ ) and $\mathrm{NH}_{4} \mathrm{Cl}(1 \mathrm{M}$ aq., $c a 1.5 \mathrm{~mL})$. The mixture was partitioned between water and $\mathrm{Et}_{2} \mathrm{O}$ and the aqueous phase extracted with $\mathrm{Et}_{2} \mathrm{O}(3 \times 10 \mathrm{~mL})$. The combined organic phase was washed with $\mathrm{NaHCO}_{3}$ (sat. aq., ca $10 \mathrm{~mL}$ ), dried $\left(\mathrm{Na}_{2} \mathrm{SO}_{4}\right)$, filtered and concentrated in vacuo. Flash column chromatography of the yellow residue (3:97; EtOAc:petrol; $\mathrm{SiO}_{2}$ ) gave a 1:1 mixture of diastereomers of (-)-(3R)-3-(2-(cyclohex-3-enyl)ethyl)-4,4-dimethylcyclohexanone (57 mg, 0.24 $\mathrm{mmol}, 60 \%)$ as a yellow oil.

Each of the diastereomers was found to have an enantiomeric excess of $87 \%$ ee, as determined by integration of the corresponding (+)-(R,R)-DPEN derivatives followed by ${ }^{13} \mathrm{C} N \mathrm{NR}$ spectroscopic analysis.

The 1:1 diastereomeric mixture contained:

${ }^{1} \mathrm{H}$ NMR $\left(400 \mathrm{MHz}, \mathrm{CDCl}_{3}\right) \delta_{\mathrm{H}} / \mathrm{ppm} 1.00(\mathrm{~s}, 3 \mathrm{H}), 1.04(\mathrm{~s}, 3 \mathrm{H}), 1.06-1.38(\mathrm{~m}, 4 \mathrm{H}), 1.42-1.55(\mathrm{~m}, 2$ H), 1.55 - $1.78(m, 5$ H), 1.98 - $2.14(m, 4$ H), 2.23 - $2.32(m, 1$ H), 2.36 - $2.49(m, 2$ H), 5.60 - 5.70 (m, $2 \mathrm{H})$.

${ }^{13} \mathrm{C}$ NMR $\left(100 \mathrm{MHz}, \mathrm{CDCl}_{3}\right) \delta_{\mathrm{c}} / \mathrm{ppm} 19.5,19.6,25.1,25.2,27.7,27.8,28.5,28.7$ (2 C), 29.3, 31.6, 32.2, 32.9 (2 C), 33.4, 33.6, 34.4, 34.5, 38.3 (2 C), 40.46, 40.49, 42.9, 43.0, 46.9, 47.1, 126.3, 126.5, $127.0,127.1,212.3(2 \mathrm{C})$.

HRMS (EI) $m / z$ calcd for $\mathrm{C}_{16} \mathrm{H}_{26} \mathrm{O}[\mathrm{M}]^{+}:$234.1984, found: 234.1985 .

$[\alpha]^{24}{ }_{589}=-24.2\left(c 0.84, \mathrm{CHCl}_{3}\right)$.

IR $\left(v_{\max } / \mathbf{c m}^{-1}\right):$ 654, 1454, 1714, 2915.

Absolute configuration assigned by optical rotation. ${ }^{141}$ 


\section{(-)-(R)-3-(Hex-5-en-1-yl)-4,4-dimethylcyclohexanone (15i)}

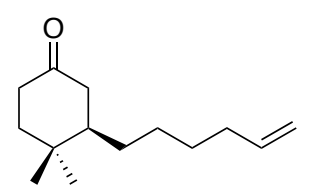

$\mathrm{Cp}_{2} \mathrm{ZrHCl}$ (206 mg, $0.80 \mathrm{mmol}, 2.0$ eq.) was added to a stirred, room temperature, solution of 1,5-hexadiene ( $0.48 \mathrm{~mL}, 4.0 \mathrm{mmol}, 10.0$ eq.) in $\mathrm{CH}_{2} \mathrm{Cl}_{2}(0.40 \mathrm{~mL})$ under an argon atmosphere. After stirring for $2 \mathrm{~h}$ at room temperature, $\mathrm{CH}_{2} \mathrm{Cl}_{2}(0.40 \mathrm{~mL})$ was added. After stirring for an additional $100 \mathrm{~min}$, the resulting clear yellow solution was transferred via syringe over about $1 \mathrm{~min}$ to a clear colourless stirred solution of $(S, S, S)-A\left(30.0 \mathrm{mg}, 0.040 \mathrm{mmol}, 0.10\right.$ eq.) in $\mathrm{Et}_{2} \mathrm{O}(2.0 \mathrm{~mL})$ under an argon atmosphere. The resulting dark mixture was allowed to stir for an additional 20 min before 4,4-dimethyl-2-cyclohexenone (53 $\mu \mathrm{L}, 0.40 \mathrm{mmol}, 1.0$ eq.) and then TMSCl $(0.25 \mathrm{~mL}, 2.0 \mathrm{mmol}$, 5.0 eq.) were added dropwise via syringe. Stirring at room temperature was continued arbitrarily for $20 \mathrm{~h}$ before the reaction was quenched by the addition of $\mathrm{Et}_{2} \mathrm{O}(\mathrm{ca} 3 \mathrm{~mL})$ and then $\mathrm{NH}_{4} \mathrm{Cl}(1 \mathrm{M}$ aq., $c a 1.5 \mathrm{~mL}$ ). The mixture was partitioned between water and $\mathrm{Et}_{2} \mathrm{O}$ and the aqueous phase extracted with $\mathrm{Et}_{2} \mathrm{O}(3 \times 10 \mathrm{~mL})$. The combined organic phase was washed with $\mathrm{NaHCO}_{3}$ (aq. sat., ca $10 \mathrm{~mL})$, dried $\left(\mathrm{Na}_{2} \mathrm{SO}_{4}\right)$, filtered and concentrated in vacuo. Flash column chromatography of the yellow residue (3:97; EtOAc:petrol; $\left.\mathrm{SiO}_{2}\right)$ gave $(-)-(R)-3-($ hex-5-en-1-yl)-4,4dimethylcyclohexanone (60 $\mathrm{mg}, 0.29 \mathrm{mmol}, 71 \%$ ) as a colourless oil.

Enantiomeric excess ( $78 \%$ ee) was determined by integration of the diastereomeric mixture of the corresponding (+)-(R,R)-DPEN derivative by ${ }^{13} \mathrm{C}$ NMR spectroscopic analysis.

${ }^{1} \mathrm{H}$ NMR $\left(400 \mathrm{MHz}, \mathrm{CDCl}_{3}\right) \delta_{\mathrm{H}} / \mathrm{ppm} 0.99(\mathrm{~s}, 3 \mathrm{H}), 1.03(\mathrm{~s}, 3 \mathrm{H}), 1.06-1.26(\mathrm{~m}, 2 \mathrm{H}), 1.30-1.43(\mathrm{~m}$, $2 \mathrm{H}), 1.44$ - $1.52(\mathrm{~m}, 1 \mathrm{H}), 1.52-1.66(\mathrm{~m}, 2 \mathrm{H}), 1.67$ - $1.77(\mathrm{~m}, 1 \mathrm{H}), 1.97-2.10(\mathrm{~m}, 3 \mathrm{H}), 2.21$ - 2.32 (m, $1 \mathrm{H}), 2.34-2.49(\mathrm{~m}, 2 \mathrm{H}), 4.89-5.07$ (m, $1 \mathrm{H}), 5.79$ (ddt, J=17.0, 10.3, 6.7, Hz, $1 \mathrm{H}$ ).

${ }^{13} \mathrm{C}$ NMR $\left(100 \mathrm{MHz}, \mathrm{CDCl}_{3}\right) \delta_{\mathrm{c}} / \mathrm{ppm} 19.5,26.9,28.7,28.9,30.4,32.8,33.7,38.3,40.5,42.9,46.8$, $114.4,138.8,212.4$.

HRMS (EI) m/z calcd for $\mathrm{C}_{14} \mathrm{H}_{24} \mathrm{O}: 208.1827$, found: 208.1832 .

$[\alpha]^{24}{ }_{589}=-23.17\left(c 0.72, \mathrm{CHCl}_{3}\right)$.

IR $\left(v_{\max } / \mathrm{cm}^{-1}\right):$ 908, 1464, 1714, 2858, 2929.

Absolute configuration assigned by optical rotation. ${ }^{141}$ 


\section{(-)-(R)-4,4-Dimethyl-3-(2-phenylethyl)cyclohexanone (15j)}

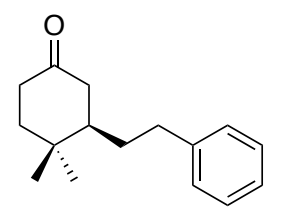

$\mathrm{Cp}_{2} \mathrm{ZrHCl}$ (206 mg, $0.80 \mathrm{mmol}, 2.0$ eq.) was added to a stirred, room temperature, solution of styrene $\left(0.11 \mathrm{~mL}, 1.0 \mathrm{mmol}, 2.5\right.$ eq.) in $\mathrm{CH}_{2} \mathrm{Cl}_{2}(0.40 \mathrm{~mL})$ under an argon atmosphere. After stirring for $15 \mathrm{~min}$ the resulting clear dark orange solution was transferred via syringe over about $1 \mathrm{~min}$ to a clear colourless stirred solution of $(\boldsymbol{S}, S, S)-A\left(30.0 \mathrm{mg}, 0.040 \mathrm{mmol}, 0.10\right.$ eq.) in $\mathrm{Et}_{2} \mathrm{O}(2.0 \mathrm{~mL})$ under an argon atmosphere. The resulting dark mixture was allowed to stir for an additional 10 min before 4,4-dimethyl-2-cyclohexenone (53 $\mu \mathrm{L}, 0.40 \mathrm{mmol}, 1.0$ eq.) and then TMSCl $(0.25 \mathrm{~mL}$, $2.0 \mathrm{mmol}, 5.0$ eq.) were added dropwise via syringe. Stirring at room temperature was continued for $15 \mathrm{~h}$ before the reaction was quenched by the addition of $\mathrm{Et}_{2} \mathrm{O}(\mathrm{ca} 3 \mathrm{~mL})$ and $\mathrm{NH}_{4} \mathrm{Cl}(1 \mathrm{M}$ aq., ca $1.5 \mathrm{~mL}$ ). The mixture was partitioned between water and $\mathrm{Et}_{2} \mathrm{O}$ and the aqueous phase extracted with $\mathrm{Et}_{2} \mathrm{O}(3 \times 10 \mathrm{~mL})$. The combined organic phase was washed with $\mathrm{NaHCO}_{3}$ (sat. aq., ca $10 \mathrm{~mL})$, dried $\left(\mathrm{Na}_{2} \mathrm{SO}_{4}\right)$, filtered and concentrated in vacuo. Flash column chromatography of the yellow residue (1:19; EtOAc:petrol; $\left.\mathrm{SiO}_{2}\right)$ gave (-)-(R)-4,4-dimethyl-3-(2phenylethyl)cyclohexanone (38 $\mathrm{mg}, 0.17 \mathrm{mmol}, 61 \%$ ) as a yellow oil.

HPLC analysis indicated an enantiomeric excess of $75 \%$ [Chiralpak ${ }^{\circledR} \mathrm{IC}$; flow: $1.5 \mathrm{~mL} / \mathrm{min}$; hexane/iPrOH: 99:1; $\lambda=210 \mathrm{~nm}$; minor enantiomer, $t_{R}=15.86 \mathrm{~min}$; major enantiomer, $\left.t_{R}=16.81 \mathrm{~min}\right]$.

${ }^{1} \mathrm{H}$ NMR $\left(400 \mathrm{MHz}, \mathrm{CDCl}_{3}\right) \delta_{\mathrm{H}} / \mathrm{ppm} 1.01(\mathrm{~s}, 3 \mathrm{H}), 1.01(\mathrm{~s}, 3 \mathrm{H}), 1.35(\mathrm{dtd}, J=13.4,10.6,10.6,4.8 \mathrm{~Hz}$, $1 \mathrm{H}), 1.52$ - 1.65 (m, $2 \mathrm{H}), 1.67$ - 1.75 (m, $1 \mathrm{H}$ ), 1.91 (dddd, $J=13.4,10.6,6.8,2.3 \mathrm{~Hz}, 1 \mathrm{H}$ ), 2.15 (ddd, $J=14.9,12.0,0.9 \mathrm{~Hz}, 1 \mathrm{H}$ ), $2.25-2.33(\mathrm{~m}, 1 \mathrm{H}), 2.38-2.49(\mathrm{~m}, 2 \mathrm{H}), 2.55$ (ddd, $J=14.9,4.4$, $2.1 \mathrm{~Hz}, 1 \mathrm{H}$ ), 2.73 (ddd, $J=13.9,10.6,5.1 \mathrm{~Hz}, 1 \mathrm{H}), 7.15-7.23(\mathrm{~m}, 3 \mathrm{H}), 7.27-7.32(\mathrm{~m}, 2 \mathrm{H})$.

${ }^{13} \mathrm{C}$ NMR $\left(100 \mathrm{MHz}, \mathrm{CDCl}_{3}\right) \delta_{\mathrm{c}} / \mathrm{ppm} 19.6,28.7,32.7,32.9,33.8,38.3,40.3,42.8,46.3,125.9,128.3$ (2 C), 128.4 (2 C), 142.1, 211.9.

HRMS (EI) $m / z$ calcd for $\mathrm{C}_{16} \mathrm{H}_{22} \mathrm{O}[\mathrm{M}]^{+}: 230.1671$, found: 230.1677 .

$[\alpha]^{24}{ }_{589}=-20.5\left(c 0.84, \mathrm{CHCl}_{3}\right)$.

IR $\left(\mathbf{v}_{\max } / \mathrm{cm}^{-1}\right): 1453,1712,2958$.

Absolute configuration assigned by optical rotation. ${ }^{141}$ 


\section{(-)-(R)-4,4-Dimethyl-3-(4-methylphenethyl)cyclohexanone (15k)}

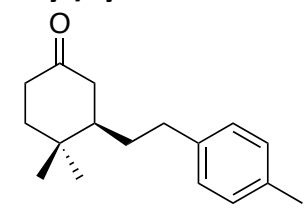

$\mathrm{Cp}_{2} \mathrm{ZrHCl}$ (206 mg, $0.80 \mathrm{mmol}, 2.0$ eq.) was added to a stirred, room temperature, solution of 1-methyl-4-vinylbenzene $\left(0.14 \mathrm{~mL}, 1.0 \mathrm{mmol}, 2.5\right.$ eq.) in $\mathrm{CH}_{2} \mathrm{Cl}_{2}(0.40 \mathrm{~mL})$ under an argon atmosphere. The resulting clear orange solution was transferred via syringe over about $1 \mathrm{~min}$ to a clear colourless stirred solution of $(S, S, S)-A\left(30.0 \mathrm{mg}, 0.040 \mathrm{mmol}, 0.10\right.$ eq.) in $\mathrm{Et}_{2} \mathrm{O}(2.0 \mathrm{~mL})$ under an argon atmosphere. The resulting dark mixture was allowed to stir for 10 min before 4,4-dimethyl-2-cyclohexenone (53 $\mathrm{LL}, 0.40 \mathrm{mmol}, 1.0$ eq.) and then TMSCl $(0.25 \mathrm{~mL}, 2.0 \mathrm{mmol}$, 5.0 eq.) were added dropwise via syringe. Stirring at room temperature was continued for $18 \mathrm{~h}$ before the reaction was quenched by the addition of $\mathrm{Et}_{2} \mathrm{O}(\mathrm{ca} 3 \mathrm{~mL})$ and then $\mathrm{NH}_{4} \mathrm{Cl}(1 \mathrm{M}$ aq., ca $1.5 \mathrm{~mL}$ ). The mixture was partitioned between water and $\mathrm{Et}_{2} \mathrm{O}$ and the aqueous phase was extracted with $\mathrm{Et}_{2} \mathrm{O}(3 \times 10 \mathrm{~mL})$. The combined organic phase was washed with $\mathrm{NaHCO}_{3}$ (aq. sat., ca $10 \mathrm{~mL})$, dried $\left(\mathrm{Na}_{2} \mathrm{SO}_{4}\right)$, filtered and concentrated in vacuo. Flash column chromatography of the yellow residue (1:19; EtOAc:petrol; $\left.\mathrm{SiO}_{2}\right)$ gave (-)-(R)-4,4-dimethyl-3-(4methylphenethyl)cyclohexanone (55 mg, $0.23 \mathrm{mmol}, 56 \%)$ as a colourless oil.

HPLC analysis indicated an enantiomeric excess of $82 \%$ [Chiralpak ${ }^{\circledast} \mathrm{IB}$; flow: $1.0 \mathrm{~mL} / \mathrm{min}$; hexane/iPrOH: 99.4:0.6; $\lambda=210 \mathrm{~nm}$; minor enantiomer, $t_{R}=11.10 \mathrm{~min}$; major enantiomer, $t_{R}=12.10 \mathrm{~min}$ ]. ${ }^{1} \mathrm{H}$ NMR $\left(500 \mathrm{MHz}, \mathrm{CDCl}_{3}\right) \delta_{\mathrm{H}} / \mathrm{ppm} 1.00(\mathrm{~s}, 3 \mathrm{H}), 1.02(\mathrm{~s}, 3 \mathrm{H}), 1.29-1.37(\mathrm{~m}, 1 \mathrm{H}), 1.52-1.64(\mathrm{~m}, 2$ H), 1.68 - 1.74 (m, 1 H), 1.88 (dddd, $J=13.2,10.6,6.9,2.4$ Hz, 1 H), 2.14 (dd, $J=14.0,12.0 \mathrm{~Hz}, 1 \mathrm{H}$ ), $2.24-2.31(\mathrm{~m}, 1 \mathrm{H}), 2.32(\mathrm{~s}, 3 \mathrm{H}), 2.35-2.47(\mathrm{~m}, 2 \mathrm{H}), 2.54$ (ddd, J=15.0, 4.0, $2.0 \mathrm{~Hz}, 1 \mathrm{H}), 2.66$ $2.73(m, 1 H), 7.04-7.08(m, 2 H), 7.09-7.13(m, 2 H)$.

${ }^{13} \mathrm{C}$ NMR $\left(125 \mathrm{MHz}, \mathrm{CDCl}_{3}\right) \delta_{\mathrm{c}} / \mathrm{ppm} 19.5,21.0,28.6,32.8,32.9,33.3,38.3,40.3,42.8,46.2,128.2$ (2C), $129.1(2 \mathrm{C}), 135.3,139.0,212.0$.

HRMS (ESI) $\mathrm{m} / \mathrm{z}$ calcd for $\mathrm{C}_{17} \mathrm{H}_{24} \mathrm{NaO}[\mathrm{M}+\mathrm{Na}]^{+}: 267.1722$, found: 267.1719 .

$[\alpha]^{20}{ }_{589}=-15.12\left(\mathrm{c} 0.85, \mathrm{CHCl}_{3}\right)$.

IR $\left(v_{\max } / \mathrm{cm}^{-1}\right): 808,1040,1717,2924$.

Absolute configuration assigned by optical rotation. ${ }^{141}$ 


\section{$(-)-(R)-4,4-D i m e t h y l-3-(2-($ naphthalen-2-yl)ethyl)cyclohexanone (15I)}

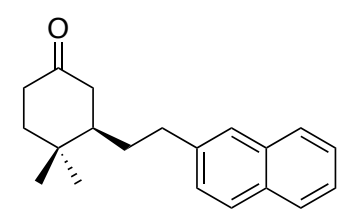

$\mathrm{Cp}_{2} \mathrm{ZrHCl}$ (206 mg, $0.80 \mathrm{mmol}, 2.0$ eq.) was added to a stirred, room temperature, solution of 2-vinylnaphthalene (160 mg, $1.0 \mathrm{mmol}, 2.5$ eq.) in $\mathrm{CH}_{2} \mathrm{Cl}_{2}(0.40 \mathrm{~mL})$ under an argon atmosphere. After stirring for $30 \mathrm{~min}$, the resulting dark red clear solution was transferred via syringe over about $1 \mathrm{~min}$ to a clear colourless stirred solution of $(S, S, S)-A(30.0 \mathrm{mg}, 0.040 \mathrm{mmol}, 0.10$ eq.) in $\mathrm{Et}_{2} \mathrm{O}(2.0 \mathrm{~mL})$ under an argon atmosphere. The resulting dark mixture was allowed to stir for an additional $10 \mathrm{~min}$ before 4,4-dimethyl-2-cyclohexenone (53 $\mu \mathrm{L}, 0.40 \mathrm{mmol}, 1.0 \mathrm{eq}$.) and then TMSCl $(0.25 \mathrm{~mL}, 2.0 \mathrm{mmol}, 5.0$ eq.) were added dropwise via syringe. Stirring at room temperature was arbitrarily continued for $15 \mathrm{~h}$ before the reaction was quenched by the addition of $\mathrm{Et}_{2} \mathrm{O}(c a 3 \mathrm{~mL})$ and then $\mathrm{NH}_{4} \mathrm{Cl}(1 \mathrm{M}$ aq., $c a 1.5 \mathrm{~mL})$. The mixture was partitioned between water and $\mathrm{Et}_{2} \mathrm{O}$ and the aqueous phase extracted with $\mathrm{Et}_{2} \mathrm{O}(3 \times 10 \mathrm{~mL})$. The combined organic phase was washed with $\mathrm{NaHCO}_{3}$ (aq. sat., ca $10 \mathrm{~mL}$ ), dried $\left(\mathrm{Na}_{2} \mathrm{SO}_{4}\right.$ ), filtered and concentrated in vacuo. Flash column chromatography of the yellow residue (3:97; EtOAc:petrol; $\mathrm{SiO}_{2}$ ) gave $(-)-(R)-4,4-$ dimethyl-3-(2-(naphthalen-2-yl)ethyl)cyclohexanone $(59 \mathrm{mg}, 0.21 \mathrm{mmol}, 52 \%)$ as a colourless oil. HPLC analysis indicated an enantiomeric excess of $71 \%$ [Chiralpak ${ }^{\circledR} \mathrm{IC}$; flow: $1.0 \mathrm{~mL} / \mathrm{min}$; hexane/iPrOH: $90: 10 ; \lambda=210 \mathrm{~nm}$; minor enantiomer, $t_{R}=12.50 \mathrm{~min}$; major enantiomer, $t_{R}=13.30 \mathrm{~min}$ ].

${ }^{1} \mathrm{H}$ NMR $\left(500 \mathrm{MHz}, \mathrm{CDCl}_{3}\right) \delta_{\mathrm{H}} / \mathrm{ppm} 1.02(\mathrm{~s}, 6 \mathrm{H}), 1.38-1.50(\mathrm{~m}, 1 \mathrm{H}), 1.54-1.64(\mathrm{~m}, 2 \mathrm{H}), 1.67-$ $1.76(m, 1 H), 1.94-2.05(m, 1 H), 2.13-2.24(m, 1 H), 2.25-2.35(m, 1 H), 2.38-2.49(m, 1 H)$, 2.55 - 2.67 (m, 2 H), $2.87-2.94$ (m, 1 H), 7.33 (dd, J = 8.5, 1.6 Hz, 1 H), $7.41-7.51$ (m, 2 H), 7.62 (s, $1 \mathrm{H}), 7.76-7.87(\mathrm{~m}, 3 \mathrm{H})$.

${ }^{13} \mathrm{C}$ NMR $\left(125 \mathrm{MHz}, \mathrm{CDCl}_{3}\right) \delta_{\mathrm{C}} / \mathrm{ppm} 19.6,28.6,32.5,32.8,33.8,38.2,40.2,42.8,46.1,125.2$, $125.9,126.3,127.1,127.4,127.6,128.0,132.0,133.6,139.5,211.9$.

HRMS (EI) $\mathrm{m} / \mathrm{z}$ calcd for $\mathrm{C}_{20} \mathrm{H}_{24} \mathrm{O}[\mathrm{M}]^{+}:$: 280.1827, found: 280.1827 .

$[\alpha]^{24}{ }_{589}=-1.23\left(\right.$ c $\left.0.32, \mathrm{CHCl}_{3}\right)$.

IR $\left(v_{\max } / \mathbf{c m}^{-1}\right): 747,913,1714,2862,2958$.

Absolute configuration assigned by optical rotation. ${ }^{141}$ 


\section{$(-)-(R)-3-(5-B r o m o p e n t y l)-4,4-d i m e t h y l c y c l o h e x a n o n e ~(15 m)$}

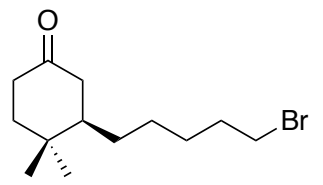

$\mathrm{Cp}_{2} \mathrm{ZrHCl}$ (206 mg, $0.80 \mathrm{mmol}, 2.0$ eq.) was added to a stirred, room temperature, solution of 5-bromo-1-pentene $\left(0.12 \mathrm{~mL}, 1.0 \mathrm{mmol}, 2.5\right.$ eq.) in $\mathrm{CH}_{2} \mathrm{Cl}_{2}(0.40 \mathrm{~mL})$ under an argon atmosphere. After stirring for $15 \mathrm{~min}$, the resulting clear yellow solution was transferred via syringe over about $1 \mathrm{~min}$ to a clear colourless stirred solution of $(S, S, S)-A\left(30.0 \mathrm{mg}, 0.040 \mathrm{mmol}, 0.10\right.$ eq.) in $\mathrm{Et}_{2} \mathrm{O}(2.0$ $\mathrm{mL}$ ) under an argon atmosphere. The resulting dark mixture was allowed to stir for an additional $10 \mathrm{~min}$ before 4,4-dimethyl-2-cyclohexenone $(53 \mu \mathrm{L}, 0.40 \mathrm{mmol}, 1.0$ eq.) and then TMSCl $(0.25$ $\mathrm{mL}, 2.0 \mathrm{mmol}$, 5.0 eq.) were added dropwise via syringe. Stirring at room temperature was continued for $15 \mathrm{~h}$ before the reaction was quenched by the addition of $\mathrm{Et}_{2} \mathrm{O}$ (ca $3 \mathrm{~mL}$ ) and $\mathrm{NH}_{4} \mathrm{Cl}$ (1 M aq., $c a 1.5 \mathrm{~mL}$ ). The mixture was partitioned between water and $\mathrm{Et}_{2} \mathrm{O}$ and the aqueous phase extracted with $\mathrm{Et}_{2} \mathrm{O}(3 \times 10 \mathrm{~mL})$. The combined organic phase was washed with $\mathrm{NaHCO}_{3}$ (sat. aq., ca $10 \mathrm{~mL})$, dried $\left(\mathrm{Na}_{2} \mathrm{SO}_{4}\right)$, filtered and concentrated in vacuo. Flash column chromatography of the yellow residue (5:95; EtOAc:petrol; $\left.\mathrm{SiO}_{2}\right)$ gave (-)-(R)-3-(5-bromopentyl)-4,4dimethylcyclohexanone $(76 \mathrm{mg}, 0.28 \mathrm{mmol}, 66 \%$ ) as a colourless oil.

Enantiomeric excess $(76 \%$ ee) was determined by two methods: 1) Integration of the diastereomeric mixture of the corresponding $(+)-(R, R)$-DPEN derivative by ${ }^{13} \mathrm{C} N \mathrm{NM}$ spectroscopic analysis, and 2) HPLC analysis: [Chiralpak ${ }^{\circledR} \mathrm{IA}$; flow: $1.0 \mathrm{~mL} / \mathrm{min}$; hexane/i-PrOH: 99:1; $\lambda=210 \mathrm{~nm}$; minor enantiomer, $t_{R}=7.51 \mathrm{~min}$; major enantiomer, $\left.t_{R}=8.00 \mathrm{~min}\right]$.

${ }^{1} \mathrm{H}$ NMR $\left(400 \mathrm{MHz}, \mathrm{CDCl}_{3}\right) \delta_{\mathrm{H}} / \mathrm{ppm} 1.00(\mathrm{~s}, 3 \mathrm{H}), 1.02-1.05(\mathrm{~m}, 3 \mathrm{H}), 1.05-1.27(\mathrm{~m}, 2 \mathrm{H}), 1.33-$ $1.66(\mathrm{~m}, 6 \mathrm{H}), 1.67-1.76(\mathrm{~m}, 1 \mathrm{H}), 1.79-1.92(\mathrm{~m}, 2 \mathrm{H}), 2.05$ (ddd, J = 14.6, 12.0, $1.0 \mathrm{~Hz}, 1 \mathrm{H}$ ), 2.23 $2.32(\mathrm{~m}, 1 \mathrm{H}), 2.36-2.47(\mathrm{~m}, 2 \mathrm{H}), 3.41(\mathrm{t}, J=6.8 \mathrm{~Hz}, 2 \mathrm{H})$.

${ }^{13} \mathrm{C}$ NMR $\left(100 \mathrm{MHz}, \mathrm{CDCl}_{3}\right) \delta_{\mathrm{c}} / \mathrm{ppm} 19.5,26.7,28.3,28.7,30.4,32.7,32.9,33.8,38.3,40.4,42.9$, $46.7,212.2$.

HRMS (EI) $\mathrm{m} / \mathrm{z}$ calcd for $\mathrm{C}_{13} \mathrm{H}_{23} \mathrm{OBr}[\mathrm{M}]^{+}:$274.0932, found: 274.0940 .

$[\alpha]^{24}{ }_{589}=-24.1\left(c 1.04, \mathrm{CHCl}_{3}\right)$.

IR $\left(v_{\max } / \mathbf{c m}^{-1}\right):$ 1464, 1713, 2934.

Absolute configuration assigned by optical rotation. ${ }^{141}$ 


\section{$(-)-(R)-3-(6-B r o m o h e x y l)-4,4-d i m e t h y l c y c l o h e x a n o n e ~(15 n)$}

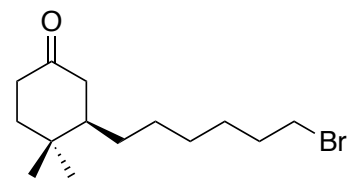

$\mathrm{Cp}_{2} \mathrm{ZrHCl}$ (206 mg, $0.80 \mathrm{mmol}, 2.0$ eq.) was added to a stirred, room temperature, solution of 6-bromo-1-hexene $\left(0.13 \mathrm{~mL}, 1.0 \mathrm{mmol}, 2.5\right.$ eq.) in $\mathrm{CH}_{2} \mathrm{Cl}_{2}(0.40 \mathrm{~mL})$ under an argon atmosphere. After stirring for $25 \mathrm{~min}$, the resulting clear yellow solution was transferred via syringe over about $1 \mathrm{~min}$ to a clear colourless stirred solution of $(\boldsymbol{S}, S, S)-A\left(30.0 \mathrm{mg}, 0.040 \mathrm{mmol}, 0.10\right.$ eq.) in $\mathrm{Et}_{2} \mathrm{O}$ $(2.0 \mathrm{~mL})$ under an argon atmosphere. The resulting dark mixture was allowed to stir for an additional $10 \mathrm{~min}$ before 4,4-dimethyl-2-cyclohexenone ( $53 \mu \mathrm{L}, 0.40 \mathrm{mmol}, 1.0$ eq.) and then TMSCl $(0.25 \mathrm{~mL}, 2.0 \mathrm{mmol}, 5.0$ eq.) were added dropwise, via syringe. Stirring at room temperature was continued for $15 \mathrm{~h}$ before the reaction was quenched by the addition of $\mathrm{Et}_{2} \mathrm{O}$ ( $c a$ $3 \mathrm{~mL}$ ) and $\mathrm{NH}_{4} \mathrm{Cl}$ (1 M aq., $c a 1.5 \mathrm{~mL}$ ). The mixture was partitioned between water and $\mathrm{Et}_{2} \mathrm{O}$ and the aqueous phase extracted with $\mathrm{Et}_{2} \mathrm{O}(3 \times 10 \mathrm{~mL})$. The combined organic phase was washed with $\mathrm{NaHCO}_{3}$ (sat. aq., ca $10 \mathrm{~mL}$ ), dried $\left(\mathrm{Na}_{2} \mathrm{SO}_{4}\right)$, filtered and concentrated in vacuo. Flash column chromatography of the yellow residue (1:19; EtOAc:petrol; $\mathrm{SiO}_{2}$ ) gave (-)-(R)-3-(6-bromohexyl)4,4-dimethylcyclohexanone (67 mg, $0.23 \mathrm{mmol}, 58 \%$ ) as a colourless oil.

Enantiomeric excess $(76 \%$ ee) was determined by two methods: 1) Integration of the diastereomeric mixture of the corresponding $(+)-(R, R)$-DPEN derivative by ${ }^{13} \mathrm{C} N \mathrm{NR}$ spectroscopic analysis, and 2) HPLC analysis: [Chiralpak ${ }^{\circledR} \mathrm{IA}$; flow: $1.0 \mathrm{~mL} / \mathrm{min}$; hexane/i-PrOH: 99:1; $\lambda=210 \mathrm{~nm}$; minor enantiomer, $t_{R}=8.85 \mathrm{~min}$; major enantiomer, $\left.t_{R}=9.29 \mathrm{~min}\right]$.

${ }^{1} \mathrm{H}$ NMR $\left(400 \mathrm{MHz}, \mathrm{CDCl}_{3}\right) \delta_{\mathrm{H}} / \mathrm{ppm} 1.00(\mathrm{~s}, 3 \mathrm{H}), 1.03(\mathrm{~s}, 3 \mathrm{H}), 1.06-1.66(\mathrm{~m}, 10 \mathrm{H}), 1.67-1.77(\mathrm{~m}$, $1 \mathrm{H}$ ), 1.85 (quin, $J=7.1 \mathrm{~Hz}, 2 \mathrm{H}$ ), 2.05 (ddd, $J=14.7,12.2,1.0 \mathrm{~Hz}, 1 \mathrm{H}$ ), $2.23-2.32(\mathrm{~m}, 1 \mathrm{H}), 2.36-$ $2.47(\mathrm{~m}, 2 \mathrm{H}), 3.41(\mathrm{t}, J=6.8 \mathrm{~Hz}, 2 \mathrm{H})$.

${ }^{13} \mathrm{C}$ NMR $\left(100 \mathrm{MHz}, \mathrm{CDCl}_{3}\right) \delta_{\mathrm{c}} / \mathrm{ppm} 19.5,27.3,28.0,28.7,28.8,30.4,32.7,32.8,33.9,38.3,40.4$, $42.9,46.8,212.3$.

HRMS (EI) $\mathrm{m} / \mathrm{z}$ calcd for $\mathrm{C}_{14} \mathrm{H}_{25} \mathrm{OBr}[\mathrm{M}]^{+}: 288.1089$, found: 288.1086 .

$[\alpha]^{24}{ }_{589}=-15.4\left(c 0.96, \mathrm{CHCl}_{3}\right)$.

IR $\left(\mathbf{v}_{\max } / \mathrm{cm}^{-1}\right): 1465,1713,2931$.

Absolute configuration assigned by optical rotation. ${ }^{141}$ 
(-)-(R)-3-(4-(Benzyloxybutyl))-4,4-dimethylcyclohexanone (150)

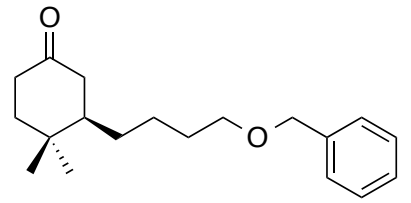

$\mathrm{Cp}_{2} \mathrm{ZrHCl}$ (206 mg, $0.80 \mathrm{mmol}, 2.0$ eq.) was added to a stirred, room temperature, solution of ((but-3-en-1-yloxy)methyl) benzene $\left(0.17 \mathrm{~mL}, 1.0 \mathrm{mmol}, 2.5\right.$ eq.) in $\mathrm{CH}_{2} \mathrm{Cl}_{2}(0.40 \mathrm{~mL})$ under an argon atmosphere. After stirring for $25 \mathrm{~min}$, the resulting clear yellow solution was transferred via syringe over about $1 \mathrm{~min}$ to a clear colourless stirred solution of $(S, S, S)-A(30.0 \mathrm{mg}, 0.040 \mathrm{mmol}$, 0.10 eq.) in $\mathrm{Et}_{2} \mathrm{O}(2.0 \mathrm{~mL})$ under an argon atmosphere. The resulting dark mixture was allowed to stir for an additional $10 \mathrm{~min}$ before 4,4-dimethyl-2-cyclohexenone (53 $\mu \mathrm{L}, 0.40 \mathrm{mmol}, 1.0$ eq.) and then $\mathrm{TMSCl}(0.25 \mathrm{~mL}, 2.0 \mathrm{mmol}, 5.0$ eq.) were added dropwise via syringe. Stirring at room temperature was continued arbitrarily for $15 \mathrm{~h}$ before the reaction was quenched by the addition of $\mathrm{Et}_{2} \mathrm{O}\left(c a 3 \mathrm{~mL}\right.$ ) and $\mathrm{NH}_{4} \mathrm{Cl}(1 \mathrm{M}$ aq., $c a 1.5 \mathrm{~mL}$ ). The mixture was partitioned between water and $\mathrm{Et}_{2} \mathrm{O}$ and the aqueous phase extracted with $\mathrm{Et}_{2} \mathrm{O}(3 \times 10 \mathrm{~mL})$. The combined organic phase was washed with $\mathrm{NaHCO}_{3}$ (sat. aq., ca $10 \mathrm{~mL}$ ), dried $\left(\mathrm{Na}_{2} \mathrm{SO}_{4}\right)$, filtered and concentrated in vacuo. Flash column chromatography of the yellow residue (5:95; EtOAc:petrol; $\left.\mathrm{SiO}_{2}\right)$ gave $(-)-(R)-3-(4-$ (benzyloxybutyl))-4,4-dimethylcyclohexanone (62 mg, $0.21 \mathrm{mmol}, 54 \%$ ) as a colourless oil.

HPLC analysis indicated an enantiomeric excess of $80 \%$ [Chiralpak ${ }^{\circledR} \mathrm{IC}$; flow: $1.0 \mathrm{~mL} / \mathrm{min}$; hexane/iPrOH: 90:10; $\lambda=210 \mathrm{~nm}$; minor enantiomer, $t_{R}=14.42 \mathrm{~min}$; major enantiomer, $t_{R}=15.40 \mathrm{~min}$ ].

${ }^{1} \mathrm{H}$ NMR $\left(400 \mathrm{MHz}, \mathrm{CDCl}_{3}\right) \delta_{\mathrm{H}} / \mathrm{ppm} 0.98(\mathrm{~s}, 3 \mathrm{H}) 1.00-1.03(\mathrm{~m}, 3 \mathrm{H}) 1.04-1.33(\mathrm{~m}, 2 \mathrm{H}) 1.39-1.75$ (m, 7 H) 2.04 (ddd, $J=14.7,12.2,0.8$ Hz, 1 H) $2.26(\mathrm{~s}, 1 \mathrm{H}) 2.34-2.47(\mathrm{~m}, 2 \mathrm{H}) 3.46(\mathrm{t}, J=6.4 \mathrm{~Hz}, 2$ H) $4.47-4.53(\mathrm{~m}, 2 \mathrm{H}) 7.24-7.39(\mathrm{~m}, 5 \mathrm{H})$.

${ }^{13} \mathrm{C}$ NMR $\left(100 \mathrm{MHz}, \mathrm{CDCl}_{3}\right) \delta_{\mathrm{c}} / \mathrm{ppm} 19.5,24.2,28.7,29.9,30.4,32.8,38.3,40.5,42.9,46.8,70.2$, 72.9, 127.5, 127.6 (2 C), 128.4 (2 C), 138.6, 212.2.

HRMS (EI) $\mathrm{m} / z$ calcd for $\mathrm{C}_{19} \mathrm{H}_{28} \mathrm{O}_{2}[\mathrm{M}]^{+}:$: 288.2089, found: 288.2088 .

$[\alpha]^{24}{ }_{589}=-17.2\left(\mathrm{c} 0.83, \mathrm{CHCl}_{3}\right)$.

IR $\left(\mathbf{v}_{\max } / \mathrm{cm}^{-1}\right): 1100,1713,2936$.

Absolute configuration assigned by optical rotation. ${ }^{141}$ 


\section{(-)-(R)-3-(4-((tert-Butyldimethylsilyl)oxy)butyl))-4,4-dimethylcyclohexanone (15p)}

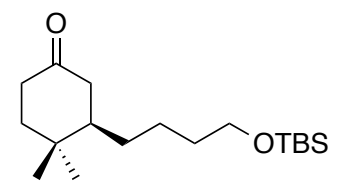

$\mathrm{Cp}_{2} \mathrm{ZrHCl}$ (206 mg, $0.80 \mathrm{mmol}, 2.0$ eq.) was added to a stirred, room temperature, solution of 4-[(tert-butyldimethylsilyl)oxy]-1-butene $\left(0.22 \mathrm{~mL}, 1.0 \mathrm{mmol}, 2.5\right.$ eq.) in $\mathrm{CH}_{2} \mathrm{Cl}_{2}(0.40 \mathrm{~mL})$ under an argon atmosphere. After stirring for $40 \mathrm{~min}$, the resulting clear yellow solution was transferred via syringe over about $1 \mathrm{~min}$ to a clear colourless stirred solution of $(\boldsymbol{S}, \mathbf{S}, \mathbf{S})-\mathrm{A}(30.0 \mathrm{mg}, 0.040 \mathrm{mmol}$, 0.10 eq.) in $\mathrm{Et}_{2} \mathrm{O}(2.0 \mathrm{~mL})$ under an argon atmosphere. The resulting dark mixture was allowed to stir for an additional $10 \mathrm{~min}$ before 4,4-dimethyl-2-cyclohexenone ( $53 \mu \mathrm{L}, 0.40 \mathrm{mmol}, 1.0$ eq.) and then $\mathrm{TMSCl}(0.25 \mathrm{~mL}, 2.0 \mathrm{mmol}, 5.0$ eq.) were added dropwise via syringe. Stirring at room temperature was continued arbitrarily for $15 \mathrm{~h}$ before the reaction was quenched by the addition of $\mathrm{Et}_{2} \mathrm{O}\left(c a 3 \mathrm{~mL}\right.$ ) and $\mathrm{NH}_{4} \mathrm{Cl}(1 \mathrm{M}$ aq., $c a 1.5 \mathrm{~mL})$. The mixture was partitioned between water and $\mathrm{Et}_{2} \mathrm{O}$ and the aqueous phase extracted with $\mathrm{Et}_{2} \mathrm{O}(3 \times 10 \mathrm{~mL})$. The combined organic phase was washed with $\mathrm{NaHCO}_{3}$ (sat. aq., $\mathrm{ca} 10 \mathrm{~mL}$ ), dried $\left(\mathrm{Na}_{2} \mathrm{SO}_{4}\right)$, filtered and concentrated in vacuo. Flash column chromatography of the yellow residue (1:24; EtOAc:petrol; $\left.\mathrm{SiO}_{2}\right)$ gave (-)-(R)-3-(4-((tertbutyldimethylsilyl)oxy)butyl))-4,4-dimethylcyclohexanone (61 mg, $0.20 \mathrm{mmol}, 49 \%$ ) as a colourless oil.

Enantiomeric excess $\left(94 \%\right.$ ee) was determined by ${ }^{13} \mathrm{C}$ NMR spectroscopic analysis of the diastereomeric mixture of the corresponding $(+)-(R, R)$-DPEN derivative.

${ }^{1} \mathrm{H}$ NMR $\left(400 \mathrm{MHz}, \mathrm{CDCl}_{3}\right) \delta_{\mathrm{H}} / \mathrm{ppm} 0.05(\mathrm{~s}, 6 \mathrm{H}), 0.89(\mathrm{~s}, 9 \mathrm{H}), 0.99(\mathrm{~s}, 3 \mathrm{H}), 1.03(\mathrm{~s}, 3 \mathrm{H}), 1.05-1.35$ (m, 3 H), $1.36-1.66(m, 5$ H), $1.67-1.76(m, 1$ H), 2.05 (ddd, $J=14.6,12.1,1.0$ Hz, 1 H), $2.23-2.32$ $(m, 1 \mathrm{H}), 2.36-2.47(\mathrm{~m}, 2 \mathrm{H}), 3.60(\mathrm{t}, J=6.3 \mathrm{~Hz}, 2 \mathrm{H})$.

${ }^{13} \mathrm{C}$ NMR $\left(100 \mathrm{MHz}, \mathrm{CDCl}_{3}\right) \delta_{\mathrm{C}} / \mathrm{ppm}-5.3$ (2 C), 18.4, 19.5, 23.8, 26.0 (3 C), 28.7, 30.4, 32.8, 32.9, $38.3,40.5,42.9,46.8,63.0,212.3$.

HRMS (EI) $\mathrm{m} / \mathrm{z}$ calcd for $\mathrm{C}_{14} \mathrm{H}_{27} \mathrm{O}_{2} \mathrm{Si}\left[\mathrm{M}-\mathrm{C}_{4} \mathrm{H}_{9}\right]^{+}: 255.1780$, found: 255.1775 .

$[\alpha]^{24}{ }_{589}=-16.8\left(c 0.56, \mathrm{CHCl}_{3}\right)$.

IR $\left(\mathbf{v}_{\max } / \mathrm{cm}^{-1}\right): 1253,1716,2930$.

Absolute configuration assigned by optical rotation. ${ }^{141}$ 


\section{(-)-(3-R)-3-(4-((tert-Butyldimethylsilyl)oxy)-4-(4-chlorophenyl)butyl)-4,4-}

dimethylcyclohexanone (15q)

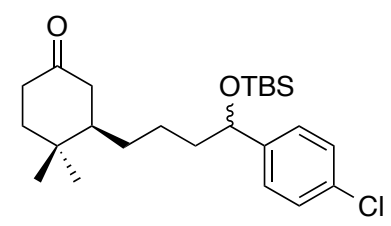

$\mathrm{Cp}_{2} \mathrm{ZrHCl}$ (206 mg, $0.80 \mathrm{mmol}, 2.0$ eq.) was added to a stirred, room temperature, solution of tert-butyl((1-(4-chlorophenyl)but-3-en-1-yl)oxy)dimethylsilane $(0.23 \mathrm{~mL}, 1.0 \mathrm{mmol}, 2.5$ eq.) in $\mathrm{CH}_{2} \mathrm{Cl}_{2}(0.45 \mathrm{~mL})$ under an argon atmosphere. The resulting clear yellow solution was transferred via syringe over about $1 \mathrm{~min}$ to a clear colourless stirred solution of $(\boldsymbol{S}, \boldsymbol{S}, \boldsymbol{S})-\mathrm{A}(30.0 \mathrm{mg}, 0.040$ $\mathrm{mmol}, 0.10$ eq.) in $\mathrm{Et}_{2} \mathrm{O}(2.0 \mathrm{~mL})$ under an argon atmosphere. The resulting dark mixture was allowed to stir for $10 \mathrm{~min}$ before 4,4-dimethyl-2-cyclohexenone (53 $\mu \mathrm{L}, 0.40 \mathrm{mmol}, 1.0$ eq.) and then $\mathrm{TMSCl}(0.25 \mathrm{~mL}, 2.0 \mathrm{mmol}, 5.0$ eq.) were added dropwise via syringe. Stirring at room temperature was continued for $5 \mathrm{~h}$ before the reaction was quenched by the addition of $\mathrm{Et}_{2} \mathrm{O}$ ( $c a$ $3 \mathrm{~mL}$ ) and then $\mathrm{NH}_{4} \mathrm{Cl}\left(1 \mathrm{M}\right.$ aq., $c a 1.5 \mathrm{~mL}$ ). The mixture was partitioned between water and $\mathrm{Et}_{2} \mathrm{O}$ and the aqueous phase was extracted with $\mathrm{Et}_{2} \mathrm{O}(3 \times 10 \mathrm{~mL})$. The combined organic phase was washed with $\mathrm{NaHCO}_{3}$ (aq. sat., ca $10 \mathrm{~mL}$ ), dried $\left(\mathrm{Na}_{2} \mathrm{SO}_{4}\right)$, filtered and concentrated in vacuo. Flash column chromatography of the yellow residue (1:33; EtOAc:petrol; $\left.\mathrm{SiO}_{2}\right)$ gave (-)-(3R)-3-(4-((tertbutyldimethylsilyl)oxy)-4-(4-chlorophenyl)butyl)-4,4-dimethylcyclohexanone (107 mg, 0.25 mmol, $63 \%)$ as a colourless oil.

Enantiomeric excess ( $85 \%$ ee) was determined by integration of the diastereomeric mixture of the corresponding $(+)-(R, R)$-DPEN derivative by ${ }^{13} \mathrm{C}$ NMR spectroscopic analysis.

The enantiomeric excess was also determined by HLPC on a chiral non-racemic stationary phase of the corresponding deprotected alcohol. The $d r$ and ee ratios are assumed to be the same for the silyl and deprotected alcohol. The silyl protecting group was removed by addition of TBAF $3 \mathrm{H}_{2} \mathrm{O}$ (1 $\mathrm{M}$ in THF, 2 eq.) to a solution of the silyl ether in THF (0.8 $\mathrm{M}$ solution) and stirring overnight at room temperature. Filtration of the crude mixture over silica gel afforded the deprotected alcohol quantitatively. HPLC analysis indicated a 1:1 diastereomeric ratio and an enantiomeric excess of $85 \%$ [Chiralpak ${ }^{\circledR}$ AY-H; flow: $1.2 \mathrm{~mL} / \mathrm{min}$; hexane/i-PrOH: 92:8; $\lambda=210 \mathrm{~nm}$; minor enantiomer, $t_{R}=19.38 \mathrm{~min} \& 34.00 \mathrm{~min}$; major enantiomer, $\left.t_{R}=16.56 \mathrm{~min} \& 25.21 \mathrm{~min}\right]$.

${ }^{1} \mathrm{H}$ NMR $\left(400 \mathrm{MHz}, \mathrm{CDCl}_{3}\right) \delta_{\mathrm{H}} / \mathrm{ppm}-0.15(\mathrm{~s}, 3 \mathrm{H}), 0.02(\mathrm{~s}, 3 \mathrm{H}), 0.88(\mathrm{~s}, 9 \mathrm{H}), 0.97(\mathrm{~s}, 3 \mathrm{H}), 1.00(\mathrm{~d}$, $J=2.78 \mathrm{~Hz}, 3 \mathrm{H}), 1.08-1.24(\mathrm{~m}, 1 \mathrm{H}), 1.25-1.40(\mathrm{~m}, 1 \mathrm{H}), 1.40-1.55(\mathrm{~m}, 3 \mathrm{H}), 1.56-1.63(\mathrm{~m}, 2 \mathrm{H})$, $1.63-1.74(\mathrm{~m}, 2 \mathrm{H}), 2.01(\mathrm{t}, J=13.4 \mathrm{~Hz} 1 \mathrm{H}), 2.21-2.30(\mathrm{~m}, 1 \mathrm{H}), 2.33-2.46(\mathrm{~m}, 2 \mathrm{H}), 4.59$ (q, $J=6.15 \mathrm{~Hz}, 1 \mathrm{H}), 7.18-7.24(\mathrm{~m}, 2 \mathrm{H}), 7.25-7.30(\mathrm{~m}, 2 \mathrm{H})$. 
${ }^{13} \mathrm{C}$ NMR $\left(100 \mathrm{MHz}, \mathrm{CDCl}_{3}\right) \delta_{\mathrm{c}} / \mathrm{ppm}-5.0,-4.7,18.1,19.4,23.3,25.8$ (3 C), 28.7, 30.5, 32.8, 38.2, $40.4,41.0,42.8,46.7,74.2,127.1$ (2 C), 128.2 (2 C), 132.4, 144.2, 212.2.

HRMS (ESI) $\mathrm{m} / \mathrm{z}$ calcd for $\mathrm{C}_{24} \mathrm{H}_{39} \mathrm{ClO}_{2} \mathrm{SiNa}[\mathrm{M}+\mathrm{Na}]^{+}: 445.2300$, found: 445.2301 .

$[\alpha]^{20}{ }_{589}=-13.17\left(c 1.02, \mathrm{CHCl}_{3}\right)$.

IR $\left(\mathbf{v}_{\max } / \mathbf{c m}^{-1}\right):$ 859, 1089, 1715, 2954.

Absolute configuration assigned by optical rotation. ${ }^{141}$

Scale up of the reaction was performed involving $\mathrm{Cp}_{2} \mathrm{ZrHCl}(970 \mathrm{mg}, 3.8 \mathrm{mmol}, 1.5$ eq.) and tert-butyl((1-(4-chlorophenyl)but-3-en-1-yl)oxy)dimethylsilane (1.26 g, $4.3 \mathrm{mmol}, 1.7$ eq.) in $\mathrm{CH}_{2} \mathrm{Cl}_{2}(1.9 \mathrm{~mL})$. The resulting alkylzirconocene was added to a solution of $(\boldsymbol{R}, \boldsymbol{R}, \boldsymbol{R})-\mathbf{A}(188 \mathrm{mg}, 0.25$ mmol, 0.10 eq.) in $\mathrm{Et}_{2} \mathrm{O}(13 \mathrm{~mL})$ followed by 4,4-dimethyl-2-cyclohexenone $(0.33 \mathrm{~mL}, 2.5 \mathrm{mmol}$, 1.0 eq.) then $\mathrm{TMSCl}(1.6 \mathrm{~mL}, 12.5 \mathrm{mmol}, 5.0$ eq.). (+)-(3S)-3-(4-((tert-butyldimethylsilyl)oxy)-4-(4chlorophenyl)butyl)-4,4-dimethylcyclohexanone (867 mg, $2.05 \mathrm{mmol}, 82 \%)$ was obtained as a colourless oil. Enantiomeric excess ( $90 \%$ ee) was determined by integration of the diastereomeric mixture of the corresponding (+)-(R,R)-DPEN derivative by ${ }^{13} C$ NMR spectroscopic analysis.

$[\alpha]^{20}{ }_{589}=+7.31\left(c 1.04, \mathrm{CHCl}_{3}\right)$.

\section{(-)-(R)-3-(3-Chloropropyl)-4,4-dimethylcyclohexanone (15r)}<smiles>CC1CCC(=O)CC1CCCCl</smiles>

$\mathrm{Cp}_{2} \mathrm{ZrHCl}$ (206 mg, $0.80 \mathrm{mmol}, 2.0$ eq.) was added to a stirred, room temperature, solution of allyl chloride $\left(0.08 \mathrm{~mL}, 1.0 \mathrm{mmol}, 2.5\right.$ eq.) in $\mathrm{CH}_{2} \mathrm{Cl}_{2}(0.40 \mathrm{~mL})$ under an argon atmosphere. After stirring for $10 \mathrm{~min}$, the resulting clear yellow solution was transferred via syringe over about $1 \mathrm{~min}$ to a clear colourless stirred solution of $(S, S, S)-A\left(30.0 \mathrm{mg}, 0.040 \mathrm{mmol}, 0.10\right.$ eq.) in $\mathrm{Et}_{2} \mathrm{O}$ (2.0 mL) under an argon atmosphere. The resulting dark mixture was allowed to stir for an additional 10 min before 4,4-dimethyl-2-cyclohexenone ( $53 \mu \mathrm{L}, 0.40 \mathrm{mmol}, 1.0$ eq.) and then $\mathrm{TMSCl}(0.25 \mathrm{~mL}$, $2.0 \mathrm{mmol}, 5.0$ eq.) were added dropwise via syringe. Stirring at room temperature was continued for $15 \mathrm{~h}$ before the reaction was quenched by the addition of $\mathrm{Et}_{2} \mathrm{O}(\mathrm{ca} 3 \mathrm{~mL})$ and $\mathrm{NH}_{4} \mathrm{Cl}(1 \mathrm{M}$ aq., $c a 1.5 \mathrm{~mL}$ ). The mixture was partitioned between water and $\mathrm{Et}_{2} \mathrm{O}$ and the aqueous phase extracted with $\mathrm{Et}_{2} \mathrm{O}(3 \times 10 \mathrm{~mL})$. The combined organic phase was washed with $\mathrm{NaHCO}_{3}$ (sat. aq., ca $10 \mathrm{~mL})$, dried $\left(\mathrm{Na}_{2} \mathrm{SO}_{4}\right)$, filtered and concentrated in vacuo. Flash column chromatography of the yellow residue $\left(10: 90 ; \quad \mathrm{Et}_{2} \mathrm{O}\right.$ :petrol; $\left.\mathrm{SiO}_{2}\right)$ gave (-)-(R)-3-chloropropyl-4,4dimethylcyclohexanone (15 mg, $0.08 \mathrm{mmol}, 19 \%)$ as a clear, yellow oil. 
Enantiomeric excess $\left(71 \%\right.$ ee) was determined by ${ }^{13} \mathrm{C}$ NMR spectroscopic analysis of the corresponding $(+)-(R, R)$-DPEN derivatives.

${ }^{1} \mathrm{H}$ NMR $\left(400 \mathrm{MHz}, \mathrm{CDCl}_{3}\right) \delta_{\mathrm{H}} / \mathrm{ppm} 1.02(\mathrm{~s}, 3 \mathrm{H}), 1.06(\mathrm{~s}, 3 \mathrm{H}), 1.15(\mathrm{dtd}, J=12.7,10.4,10.4,4.3 \mathrm{~Hz}$, $1 \mathrm{H}), 1.47$ - $1.56(\mathrm{~m}, 1 \mathrm{H}), 1.56$ - 1.68 (m, 2 H), 1.69 - 1.92 (m, 3 H), 2.09 (ddd, J = 14.7, 12.2, $0.8 \mathrm{~Hz}$, $1 \mathrm{H}), 2.25-2.34(\mathrm{~m}, 1 \mathrm{H}), 2.36-2.48(\mathrm{~m}, 2 \mathrm{H}), 3.48-3.59(\mathrm{~m}, 2 \mathrm{H})$.

${ }^{13} \mathrm{C}$ NMR $\left(125 \mathrm{MHz}, \mathrm{CDCl}_{3}\right) \delta_{\mathrm{c}} / \mathrm{ppm} 19.4,27.9,28.7,30.5,32.9,38.2,40.3,42.8,45.0,46.3,211.7$.

HRMS (EI) $m / z$ calcd for $\mathrm{C}_{11} \mathrm{H}_{19} \mathrm{OCl}[\mathrm{M}]^{+}:$202.1124, found: 202.1131 .

$[\alpha]^{24}{ }_{589}=-7.6\left(c 0.48, \mathrm{CHCl}_{3}\right)$.

IR $\left(\mathbf{v}_{\max } / \mathrm{cm}^{-1}\right): 1463,1713,2959$.

Absolute configuration assigned by optical rotation. ${ }^{141}$

\section{(-)-(R)-4,4-Dimethyl-3-(2-(trimethylsilyl)ethyl)cyclohexanone (15u)}

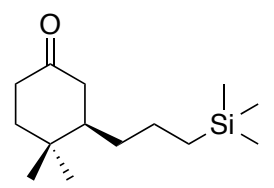

$\mathrm{Cp}_{2} \mathrm{ZrHCl}$ (206 mg, $0.80 \mathrm{mmol}, 2.0$ eq.) was added to a stirred, room temperature, solution of allyltrimethylsilane $\left(0.13 \mathrm{~mL}, 1.0 \mathrm{mmol}, 2.5\right.$ eq.) in $\mathrm{CH}_{2} \mathrm{Cl}_{2}(0.40 \mathrm{~mL})$ under an argon atmosphere. After stirring for $10 \mathrm{~min}$, the resulting clear orange solution was transferred via syringe over about $1 \mathrm{~min}$ to a clear colourless stirred solution of $(S, S, S)-A\left(30.0 \mathrm{mg}, 0.040 \mathrm{mmol}, 0.10\right.$ eq.) in $\mathrm{Et}_{2} \mathrm{O}(2.0$ $\mathrm{mL}$ ) at $0{ }^{\circ} \mathrm{C}$ under an argon atmosphere. The resulting dark mixture was allowed to stir for an additional $10 \mathrm{~min}$ before 4,4-dimethyl-2-cyclohexenone (53 $\mu \mathrm{L}, 0.40 \mathrm{mmol}, 1.0$ eq.) and then TMSCl $\left(0.25 \mathrm{~mL}, 2.0 \mathrm{mmol}, 5.0\right.$ eq.) were added dropwise via syringe. Stirring at $0{ }^{\circ} \mathrm{C}$ was continued for $4 \mathrm{~h}$ before the reaction was quenched by the addition of $\mathrm{Et}_{2} \mathrm{O}(\mathrm{ca} 3 \mathrm{~mL}$ ) and then $\mathrm{NH}_{4} \mathrm{Cl}\left(1 \mathrm{M}\right.$ aq., $1.5 \mathrm{~mL}$ ). The mixture was partitioned between water and $\mathrm{Et}_{2} \mathrm{O}$ and the aqueous phase extracted with $\mathrm{Et}_{2} \mathrm{O}(3 \times 10 \mathrm{~mL})$. The combined organic phase was washed with $\mathrm{NaHCO}_{3}$ (aq. sat., ca $10 \mathrm{~mL}$ ), dried $\left(\mathrm{Na}_{2} \mathrm{SO}_{4}\right)$, filtered and concentrated in vacuo. Flash column chromatography of the yellow residue (3:97; EtOAc:petrol; $\mathrm{SiO}_{2}$ ) gave (-)-(R)-4,4-dimethyl-3-(2(trimethylsilyl)ethyl)cyclohexanone $(50.6 \mathrm{mg}, 0.21 \mathrm{mmol}, 53 \%)$ as a yellow oil.

Enantiomeric excess ( $96 \%$ ee) was determined by integration of the diastereomeric mixture of the corresponding $(+)-(R, R)$-DPEN derivative by ${ }^{13} \mathrm{C}$ NMR spectroscopic analysis.

${ }^{1} \mathrm{H}$ NMR $\left(400 \mathrm{MHz}, \mathrm{CDCl}_{3}\right) \delta_{\mathrm{H}} / \mathrm{ppm}-0.03(\mathrm{~s}, 9 \mathrm{H}), 0.34-0.44(\mathrm{~m}, 1 \mathrm{H}), 0.46-0.57(\mathrm{~m}, 1 \mathrm{H}), 0.99$ (s, $3 \mathrm{H}), 1.02(\mathrm{~s}, 3 \mathrm{H}), 1.04-1.17(\mathrm{~m}, 2 \mathrm{H}), 1.33-1.45(\mathrm{~m}, 1 \mathrm{H}), 1.46-1.57(\mathrm{~m}, 2 \mathrm{H}), 1.57-1.66(\mathrm{~m}, 1$ H), $1.67-1.76(\mathrm{~m}, 1 \mathrm{H}), 2.05(\mathrm{dd}, J=14.7,12.3 \mathrm{~Hz}, 1 \mathrm{H}), 2.22-2.32(\mathrm{~m}, 1 \mathrm{H}), 2.35-2.47(\mathrm{~m}, 2 \mathrm{H})$. 
${ }^{13} \mathrm{C}$ NMR $\left(100 \mathrm{MHz}, \mathrm{CDCl}_{3}\right) \delta_{\mathrm{c}} / \mathrm{ppm}-1.7$ (3 C), 16.8, 19.5, 21.9, 28.7, 32.8, 34.6, 38.3, 40.5, 42.9, 46.6, 212.5 .

HRMS (EI) m/z calcd for $\mathrm{C}_{14} \mathrm{H}_{28} \mathrm{OSi}[\mathrm{M}]^{+}: 240.1909$, found: 240.1914 .

$[\alpha]^{20}{ }_{589}=-25.51\left(c 1.07, \mathrm{CHCl}_{3}\right)$.

IR $\left(v_{\max } / \mathrm{cm}^{-1}\right): 835,1715.2341,2360,2860,2954$.

Absolute configuration assigned by optical rotation. ${ }^{141}$

\section{(-)-(S)-3-(4-Methoxyphenethyl)cyclohexanone (2c)}

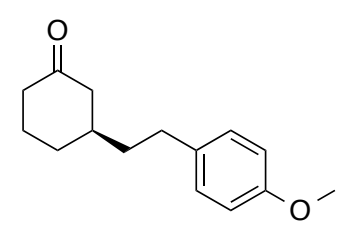

(CuOTf) ${ }_{2} . \mathrm{C}_{6} \mathrm{H}_{6}(10.1 \mathrm{mg}, 0.020 \mathrm{mmol}, 0.05$ eq.) and the phosphoramidite ligand 19 ( $21.6 \mathrm{mg}, 0.040$ mmol, 0.10 eq.) were dissolved in $\mathrm{Et}_{2} \mathrm{O}(2.0 \mathrm{~mL})$ under an argon atmosphere and allowed to stir for $1 \mathrm{~h}$ at room temperature before cooling to $0{ }^{\circ} \mathrm{C}$. In another flask, $\mathrm{Cp}_{2} \mathrm{ZrHCl}(206.0 \mathrm{mg}, 0.80$ $\mathrm{mmol}, 2.0$ eq.) was added to a stirred, room temperature, solution of 4-methoxystyrene $(0.13 \mathrm{~mL}$, $1.0 \mathrm{mmol}, 2.5$ eq.) in $\mathrm{CH}_{2} \mathrm{Cl}_{2}(0.40 \mathrm{~mL})$ under an argon atmosphere. After stirring for $15 \mathrm{~min}$, the resulting clear red/orange solution was transferred via syringe over about $1 \mathrm{~min}$ to the stirred solution containing the copper and ligand under an argon atmosphere at $0{ }^{\circ} \mathrm{C}$. The resulting black mixture was allowed to stir for an additional $10 \mathrm{~min}$ before 2-cyclohexenone $(39 \mu \mathrm{L}, 0.40 \mathrm{mmol}$, 1.0 eq.) and then $\mathrm{TMSCl}(0.26 \mathrm{~mL}, 2.0 \mathrm{mmol}, 5.0$ eq.) were added dropwise via syringe. Stirring continued at $0{ }^{\circ} \mathrm{C}$ for $6 \mathrm{~h}$ before the reaction was quenched by the addition of $\mathrm{Et}_{2} \mathrm{O}(c a 3 \mathrm{~mL})$ and then $\mathrm{NH}_{4} \mathrm{Cl}\left(1 \mathrm{M}\right.$ aq., $c a 1.5 \mathrm{~mL}$ ). The mixture was partitioned between the aqueous and $\mathrm{Et}_{2} \mathrm{O}$ layers and the aqueous phase extracted with $\mathrm{Et}_{2} \mathrm{O}(3 \times 10 \mathrm{~mL})$. The combined organic phase was washed with $\mathrm{NaHCO}_{3}$ (aq. sat., $\mathrm{ca} 10 \mathrm{~mL}$ ), dried $\left(\mathrm{Na}_{2} \mathrm{SO}_{4}\right)$, filtered and concentrated in vacuo. Flash column chromatography of the yellow residue $\left(5: 95 \rightarrow\right.$ 1:9 EtOAc/petrol; $\left.\mathrm{SiO}_{2}\right)$ gave (S)-3-(4methoxyphenethyl)cyclohexanone (49 mg, $0.21 \mathrm{mmol}, 53 \%$ ) as a colourless oil.

HPLC analysis indicated an enantiomeric excess of 95\% [Chiralpak ${ }^{\circledR}$ IB; flow: $1 \mathrm{~mL} / \mathrm{min}$; hexane/iPrOH: $98: 2 ; \lambda=210 \mathrm{~nm}$; minor enantiomer, $t_{R}=11.74 \mathrm{~min}$; major enantiomer, $t_{R}=12.49 \mathrm{~min}$ ]

Data in accordance with published literature. ${ }^{97}$

${ }^{1} \mathrm{H}$ NMR $\left(400 \mathrm{MHz}, \mathrm{CDCl}_{3}\right) \delta_{\mathrm{H}} / \mathrm{ppm} 1.32$ - $1.45(\mathrm{~m}, 1 \mathrm{H}), 1.55$ - $1.73(\mathrm{~m}, 3 \mathrm{H}), 1.75-1.87(\mathrm{~m}, 1 \mathrm{H})$, $1.91-2.00(\mathrm{~m}, 1 \mathrm{H}), 2.01-2.11(\mathrm{~m}, 2 \mathrm{H}), 2.28$ (dddd, $J=14.2,12.0,6.0,1.0 \mathrm{~Hz}, 1 \mathrm{H}), 2.33-2.41(\mathrm{~m}$, $1 \mathrm{H}$ ), 2.49 (ddt, $J=13.8,4.0,1.9,1.9 \mathrm{~Hz}, 1 \mathrm{H}), 2.58(\mathrm{t}, J=8.0 \mathrm{~Hz}, 2 \mathrm{H}), 3.79(\mathrm{~s}, 3 \mathrm{H}), 6.83(\mathrm{~d}, J=8.7$ $\mathrm{Hz}, 2 \mathrm{H}), 7.09(\mathrm{~d}, J=8.7 \mathrm{~Hz}, 2 \mathrm{H})$. 
${ }^{13} \mathrm{C}$ NMR $\left(100 \mathrm{MHz}, \mathrm{CDCl}_{3}\right) \delta_{\mathrm{c}} / \mathrm{ppm} 25.1,31.2,32.0,38.4,38.6,41.5,48.0,55.2,113.8$ (2 C), 129.1

(2 C), 134.0, 157.7, 211.8 .

HRMS (ESI) $\mathrm{m} / \mathrm{z}$ calcd for $\mathrm{C}_{15} \mathrm{H}_{20} \mathrm{NaO}_{2}[\mathrm{M}+\mathrm{Na}]^{+}: 255.1356$ found: 255.1349 .

$[\alpha]^{20}{ }_{589}=-19.8\left(c 0.43, \mathrm{CHCl}_{3}\right)$.

IR $\left(\mathbf{v}_{\max } / \mathrm{cm}^{-1}\right): 1034,1245,1512,1710,2933$.

CD: $\Theta(\gamma)=-2.94(290 \mathrm{~nm}),(c=0.17 \mathrm{mg} / \mathrm{ml}$ in EtOH $)$.

Absolute configuration assigned by analysis of optical rotation and circular dichroism spectra. ${ }^{141}$

Scale up of the reaction was performed involving $\mathrm{Cp}_{2} \mathrm{ZrHCl}(970 \mathrm{mg}, 3.8 \mathrm{mmol}, 1.5$ eq.) and 4-methoxystyrene $\left(0.57 \mathrm{~mL}, 4.3 \mathrm{mmol}, 1.7\right.$ eq.) in $\mathrm{CH}_{2} \mathrm{Cl}_{2}(1.9 \mathrm{~mL})$. The resulting alkylzirconocene was added to a solution of $(\boldsymbol{R}, \boldsymbol{R}, \boldsymbol{R})-\mathbf{A}\left(188 \mathrm{mg}, 0.25 \mathrm{mmol}, 0.10\right.$ eq.) in $\mathrm{Et}_{2} \mathrm{O}(13 \mathrm{~mL})$ followed by 2cyclohexenone $(0.24 \mathrm{~mL}, 2.5 \mathrm{mmol}, 1.0$ eq.) then $\mathrm{TMSCl}(1.6 \mathrm{~mL}, 12.5 \mathrm{mmol}, 5.0$ eq.). (+)-(R)-3-(4-methoxyphenethyl)cyclohexanone $(329 \mathrm{mg}, 1.42 \mathrm{mmol}, 57 \%)$ was obtained as a colourless oil. HPLC analysis indicated an enantiomeric excess of 95\% [Chiralpak ${ }^{\circledast}$ IB; flow: 1 $\mathrm{mL} / \mathrm{min}$; hexane/i-PrOH: 98:2; $\lambda=210 \mathrm{~nm}$; major enantiomer, $\mathrm{t}_{\mathrm{R}}=11.23 \mathrm{~min}$; minor enantiomer, $\left.t_{R}=12.48 \mathrm{~min}\right]$.

$[\alpha]^{20}{ }_{589}=+8.84\left(c 1.12, \mathrm{CHCl}_{3}\right)$.

\section{(-)-(S)-3-(4-Phenylbutyl)cyclopentanone (22)}

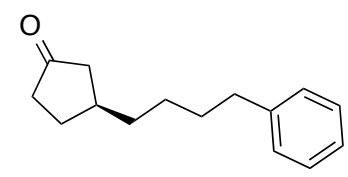

$\mathrm{Cp}_{2} \mathrm{ZrHCl}$ (103 mg, $0.40 \mathrm{mmol}, 2.0$ eq.) was added to a stirred, room temperature, solution of 4-phenyl-1-butene $\left(0.08 \mathrm{~mL}, 0.5 \mathrm{mmol}, 2.5\right.$ eq.) in $\mathrm{CH}_{2} \mathrm{Cl}_{2}(0.20 \mathrm{~mL})$ under an argon atmosphere. After stirring for $15 \mathrm{~min}$, the resulting yellow clear solution was transferred via syringe over about $1 \mathrm{~min}$ to a clear colourless stirred solution of $(S, S, S)-A\left(15.0 \mathrm{mg}, 0.020 \mathrm{mmol}, 0.10\right.$ eq.) in $\mathrm{Et}_{2} \mathrm{O}$ $(1.0 \mathrm{~mL})$ under an argon atmosphere. The resulting dark mixture was cooled to $0{ }^{\circ} \mathrm{C}$ before cyclopentenone ( $17 \mu \mathrm{L}, 0.20 \mathrm{mmol}, 1.0$ eq.) and then $\mathrm{TMSCl}(0.13 \mathrm{~mL}, 1.0 \mathrm{mmol}, 5.0$ eq.) were added dropwise via syringe. Stirring at $0{ }^{\circ} \mathrm{C}$ was continued for $2 \mathrm{~h}$ before the reaction was quenched by the addition of $\mathrm{Et}_{2} \mathrm{O}(c a 3 \mathrm{~mL})$ and then $\mathrm{NH}_{4} \mathrm{Cl}(1 \mathrm{M}$ aq., $c a 1.5 \mathrm{~mL})$. The mixture was partitioned between the aqueous and $\mathrm{Et}_{2} \mathrm{O}$ layers and the aqueous phase extracted with $\mathrm{Et}_{2} \mathrm{O}(3 \times$ $10 \mathrm{~mL}$ ). The combined organic phase was washed with $\mathrm{NaHCO}_{3}$ (aq. sat., $\mathrm{ca} 10 \mathrm{~mL}$ ), dried $\left(\mathrm{Na}_{2} \mathrm{SO}_{4}\right)$, filtered and concentrated in vacuo. Flash column chromatography of the yellow residue 
(1:19; EtOAc:petrol; $\mathrm{SiO}_{2}$ ) gave (-)-(S)-3-(4-phenylbutyl)cyclopentanone $(11.7 \mathrm{mg}, 0.055 \mathrm{mmol}$, $29 \%)$ as a colourless oil.

HPLC analysis indicated an enantiomeric excess of $85 \%$ [Chiralpak ${ }^{\circledR} \mathrm{IC}$; flow: $1.5 \mathrm{~mL} / \mathrm{min}$; hexane/iPrOH: 99:1; $\lambda=210 \mathrm{~nm}$; minor enantiomer, $\mathrm{t}_{\mathrm{R}}=22.85 \mathrm{~min}$; major enantiomer, $\mathrm{t}_{\mathrm{R}}=24.22 \mathrm{~min}$ ].

${ }^{1} \mathrm{H}$ NMR $\left(400 \mathrm{MHz}, \mathrm{CDCl}_{3}\right) \delta_{\mathrm{H}} / \mathrm{ppm} 1.31-1.56(\mathrm{~m}, 5 \mathrm{H}), 1.65$ (dt, $J=15.2,7.5 \mathrm{~Hz}, 2 \mathrm{H}$ ), 1.79 (app. $\mathrm{dd}, J=18.0,9.6 \mathrm{~Hz}, 1 \mathrm{H}), 2.07-2.22(\mathrm{~m}, 3 \mathrm{H}) 2.23-2.44(\mathrm{~m}, 2 \mathrm{H}), 2.63(\mathrm{t}, J=7.3 \mathrm{~Hz}, 2 \mathrm{H}), 7.15-$ $7.22(m, 3 H), 7.27-7.33(m, 2 H)$.

${ }^{13} \mathrm{C}$ NMR $\left(100 \mathrm{MHz}, \mathrm{CDCl}_{3}\right) \delta_{\mathrm{H}} / \mathrm{ppm} 27.5,29.5,31.5,35.5,35.8,37.1,38.5,45.3,125.7,128.3$ (2 C), 128.4 (2 C), 142.5, 219.9.

HRMS (ESI) $\mathrm{m} / \mathrm{z}$ calcd for $\mathrm{C}_{15} \mathrm{H}_{20} \mathrm{ONa}[\mathrm{M}+\mathrm{Na}]^{+}: 239.1406$, found: 239.1404 .

$[\alpha]^{20}{ }_{589}=-70.54\left(c 0.53, \mathrm{CHCl}_{3}\right)$.

IR $\left(v_{\max } / \mathbf{c m}^{-1}\right):$ 699, 1158, 1740, 2927.

Absolute configuration assigned by optical rotation. ${ }^{141}$

Scale up of the reaction was performed involving $\mathrm{Cp}_{2} \mathrm{ZrHCl}(970 \mathrm{mg}, 3.8 \mathrm{mmol}, 1.5$ eq.) and 4-phenyl-1-butene $\left(0.64 \mathrm{~mL}, 4.3 \mathrm{mmol}, 1.7\right.$ eq.) in $\mathrm{CH}_{2} \mathrm{Cl}_{2}(1.9 \mathrm{~mL})$. The resulting alkylzirconocene was added to a solution of $(\boldsymbol{R}, \boldsymbol{R}, \boldsymbol{R})-\mathbf{A}\left(188 \mathrm{mg}, 0.25 \mathrm{mmol}, 0.10\right.$ eq.) in $\mathrm{Et}_{2} \mathrm{O}(13 \mathrm{~mL})$ followed by cyclopentenone ( $0.20 \mathrm{~mL}, 2.5 \mathrm{mmol}, 1.0$ eq.) then TMSCl (1.6 mL, $12.5 \mathrm{mmol}, 5.0$ eq.). (+)-(R)-3-(4phenylbutyl)cyclopentanone (170 mg, $0.79 \mathrm{mmol}, 32 \%$ ) was obtained as a yellow oil. HPLC analysis indicated an enantiomeric excess of $90 \%$ [Chiralpak ${ }^{\circledR} \mathrm{IC}$; flow: $1.5 \mathrm{~mL} / \mathrm{min}$; hexane/iPrOH: 99:1; $\lambda=210 \mathrm{~nm}$; major enantiomer, $t_{R}=23.15 \mathrm{~min}$; minor enantiomer, $t_{R}=25.15 \mathrm{~min}$ ]. $[\alpha]^{20}{ }_{589}=+76.73\left(c 1.07, \mathrm{CHCl}_{3}\right)$.

\section{$(-)-(R)-3-H e x y l-4,4-d i p h e n y l c y c l o h e x a n o n e ~(23 a)$}

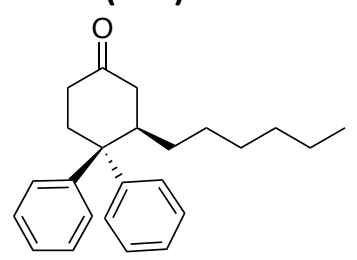

$\mathrm{Cp}_{2} \mathrm{ZrHCl}$ (206 mg, $0.80 \mathrm{mmol}, 2.0$ eq.) was added to a stirred, room temperature, solution of 1-hexene $\left(0.13 \mathrm{~mL}, 1.0 \mathrm{mmol}, 2.5\right.$ eq.) in $\mathrm{CH}_{2} \mathrm{Cl}_{2}(0.40 \mathrm{~mL})$ under an argon atmosphere. The resulting clear yellow solution was transferred via syringe over about $1 \mathrm{~min}$ to a clear colourless stirred solution of $(S, S, S)-A\left(30.0 \mathrm{mg}, 0.040 \mathrm{mmol}, 0.10\right.$ eq.) in $\mathrm{Et}_{2} \mathrm{O}(2.0 \mathrm{~mL})$ under an argon atmosphere. The resulting dark mixture was allowed to stir for 10 min before 4,4-diphenyl-2cyclohexenone (100 mg, $0.40 \mathrm{mmol}, 1.0$ eq.) and then $\mathrm{TMSCl}(0.25 \mathrm{~mL}, 2.0 \mathrm{mmol}, 5.0$ eq.) were 
added dropwise via syringe. Stirring at room temperature was continued arbitrarily for $18 \mathrm{~h}$ before the reaction was quenched by the addition of $\mathrm{Et}_{2} \mathrm{O}(c a 3 \mathrm{~mL})$ and then $\mathrm{NH}_{4} \mathrm{Cl}(1 \mathrm{M}$ aq., $c a$ $1.5 \mathrm{~mL}$ ). The mixture was partitioned between water and $\mathrm{Et}_{2} \mathrm{O}$ and the aqueous phase was extracted with $\mathrm{Et}_{2} \mathrm{O}(3 \times 10 \mathrm{~mL})$. The combined organic phase was washed with $\mathrm{NaHCO}_{3}$ (aq. sat., ca $10 \mathrm{~mL})$, dried $\left(\mathrm{Na}_{2} \mathrm{SO}_{4}\right)$, filtered and concentrated in vacuo. Flash column chromatography of the yellow residue (1:49; EtOAc:petrol; $\mathrm{SiO}_{2}$ ) gave (-)-(R)-3-hexyl-4,4-diphenylcyclohexanone (101 $\mathrm{mg}, 0.30 \mathrm{mmol}, 75 \%)$ as a colourless solid.

HPLC analysis indicated an enantiomeric excess of $90 \%$ [Chiralpak ${ }^{\circledR} \mathrm{IB}$; flow: $1.0 \mathrm{~mL} / \mathrm{min}$; hexane/iPrOH: 95:5; $\lambda=210 \mathrm{~nm}$; major enantiomer, $t_{R}=5.77 \mathrm{~min}$; minor enantiomer, $t_{R}=6.34 \mathrm{~min}$ ].

${ }^{1} \mathrm{H}$ NMR $\left(500 \mathrm{MHz}, \mathrm{CDCl}_{3}\right) \delta_{\mathrm{H}} / \mathrm{ppm} 0.83(\mathrm{t}, J=7.3 \mathrm{~Hz}, 3 \mathrm{H}), 1.02-1.16(\mathrm{~m}, 7 \mathrm{H}), 1.17-1.26(\mathrm{~m}, 2$ H), $1.36-1.48(\mathrm{~m}, 1 \mathrm{H}), 2.27-2.35(\mathrm{~m}, 1 \mathrm{H}), 2.36-2.43(\mathrm{~m}, 1 \mathrm{H}), 2.49(\mathrm{dt}, J=14.7,2.8 \mathrm{~Hz}, 1 \mathrm{H})$, $2.62(\mathrm{td}, J=13.6,4.4 \mathrm{~Hz}, 1 \mathrm{H}), 2.81(\mathrm{dd}, J=14.8,5.0 \mathrm{~Hz}, 1 \mathrm{H}), 2.93-3.00(\mathrm{~m}, 1 \mathrm{H}), 3.04(\mathrm{br} . \mathrm{s}, 1 \mathrm{H})$, 7.09 - $7.16(\mathrm{~m}, 1 \mathrm{H}), 7.17$ - $7.23(\mathrm{~m}, 1 \mathrm{H}), 7.24-7.31(\mathrm{~m}, 4 \mathrm{H}), 7.36(\mathrm{t}, J=7.9 \mathrm{~Hz}, 2 \mathrm{H}), 7.53$ (d, $J=$ $7.6 \mathrm{~Hz}, 2 \mathrm{H})$.

${ }^{13} \mathrm{C}$ NMR $\left(125 \mathrm{MHz}, \mathrm{CDCl}_{3}\right) \delta_{\mathrm{C}} / \mathrm{ppm} 14.0,22.5,28.0,29.0,29.5,30.0,31.5,38.3,42.2,43.0,48.7$, 125.7, 126.1, 126.4 (2 C), 126.6 (2 C), 128.3 (2 C), 128.9 (2 C), 145.0, 146.6, 211.6.

HRMS (ESI) $m / z$ calcd for $\mathrm{C}_{24} \mathrm{H}_{30} \mathrm{NaO}[\mathrm{M}+\mathrm{Na}]^{+}: 357.2189$, found: 357.2186 .

$[\alpha]^{20}{ }_{589}=-194.11\left(c 1.02, \mathrm{CHCl}_{3}\right)$.

IR $\left(v_{\max } / \mathrm{cm}^{-1}\right):$ 701, 1460, 1713, 2388, 2927.

Melting Point: $61-63^{\circ} \mathrm{C}$

Absolute configuration assigned by optical rotation. ${ }^{141}$

\section{(-)-(S)-3-(4-Phenylbutyl)cycloheptanone (24)}

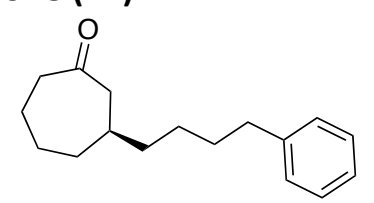

$\mathrm{Cp}_{2} \mathrm{ZrHCl}$ (206 mg, $0.80 \mathrm{mmol}, 2.0$ eq.) was added to a stirred, room temperature, solution of 4-phenyl-1-butene $\left(0.15 \mathrm{~mL}, 1.0 \mathrm{mmol}, 2.5\right.$ eq.) in $\mathrm{CH}_{2} \mathrm{Cl}_{2}(0.40 \mathrm{~mL})$ under an argon atmosphere. After stirring for $15 \mathrm{~min}$, the resulting yellow clear solution was transferred via syringe over about $1 \mathrm{~min}$ to a clear colourless stirred solution of $(S, S, S)-A\left(30.0 \mathrm{mg}, 0.040 \mathrm{mmol}, 0.10\right.$ eq.) in $\mathrm{Et}_{2} \mathrm{O}$ $(2.0 \mathrm{~mL})$ under an argon atmosphere. The resulting dark mixture was allowed to stir for an additional $10 \mathrm{~min}$ before cycloheptenone $(56 \mu \mathrm{L}, 0.40 \mathrm{mmol}, 1.0$ eq.) and then $\mathrm{TMSCl}(0.25 \mathrm{~mL}$, $2.0 \mathrm{mmol}, 5.0$ eq.) were added dropwise via syringe. Stirring at room temperature was continued for $3 \mathrm{~h}$ before the reaction was quenched by the addition of $\mathrm{Et}_{2} \mathrm{O}(\mathrm{ca} 3 \mathrm{~mL})$ and then $\mathrm{NH}_{4} \mathrm{Cl}(1 \mathrm{M}$ 
aq., $c a 1.5 \mathrm{~mL}$ ). The mixture was partitioned between the aqueous and $\mathrm{Et}_{2} \mathrm{O}$ layers and the aqueous phase extracted with $\mathrm{Et}_{2} \mathrm{O}(3 \times 10 \mathrm{~mL})$. The combined organic phase was washed with $\mathrm{NaHCO}_{3}$ (aq. sat., ca $10 \mathrm{~mL}$ ), dried $\left(\mathrm{Na}_{2} \mathrm{SO}_{4}\right)$, filtered and concentrated in vacuo. Flash column chromatography of the yellow residue (1:33; EtOAc:petrol; $\left.\mathrm{SiO}_{2}\right)$ gave (-)-(S)-3-(4phenylbutyl)cycloheptanone $(72.0 \mathrm{mg}, 0.29 \mathrm{mmol}, 73 \%)$ as a light yellow oil.

HPLC analysis indicated an enantiomeric excess of $91 \%$ [Chiralpak ${ }^{\circledR} \mathrm{IB}$; flow: $1.5 \mathrm{~mL} / \mathrm{min}$; hexane/iPrOH: 99.4:0.6; $\lambda=210 \mathrm{~nm}$; minor enantiomer, $t_{R}=9.56 \mathrm{~min}$; major enantiomer, $\left.t_{R}=10.07 \mathrm{~min}\right]$.

${ }^{1} \mathrm{H}$ NMR $\left(400 \mathrm{MHz}, \mathrm{CDCl}_{3}\right) \delta_{\mathrm{H}} / \mathrm{ppm} 1.19-1.51(\mathrm{~m}, 6 \mathrm{H}), 1.52$ - $1.74(\mathrm{~m}, 4 \mathrm{H}), 1.80$ - $1.98(\mathrm{~m}, 3 \mathrm{H})$, $2.34-2.43$ (app. dd, J = 10.4, 14.2 Hz, 1 H) $2.43-2.52(\mathrm{~m}, 3 \mathrm{H}) 2.62(\mathrm{t}, J=7.60 \mathrm{~Hz}, 2 \mathrm{H}) 7.14-7.22$ (m, 3 H) $7.25-7.33(m, 2 H)$.

${ }^{13} \mathrm{C} \mathrm{NMR}\left(100 \mathrm{MHz}, \mathrm{CDCl}_{3}\right) \delta_{\mathrm{H}} / \mathrm{ppm} 24.4,26.6,28.5,31.5,35.9,36.0,36.9,37.1,43.91,49.93$, $125.7,128.3(2 \mathrm{C}), 128.4(2 \mathrm{C}), 142.6,214.6$.

HRMS (ESI) $\mathrm{m} / \mathrm{z}$ calcd for $\mathrm{C}_{17} \mathrm{H}_{24} \mathrm{ONa}[\mathrm{M}+\mathrm{Na}]^{+}: 267.1719$, found: 267.1717 .

$[\alpha]^{20}{ }_{589}=-28.57\left(c 1.06, \mathrm{CHCl}_{3}\right)$.

IR $\left(v_{\max } / \mathrm{cm}^{-1}\right):$ 699, 1453, 1699, 2855, 2927.

Absolute configuration assigned by optical rotation. ${ }^{141}$

Scale up of the reaction was performed involving $\mathrm{Cp}_{2} \mathrm{ZrHCl}(970 \mathrm{mg}, 3.8 \mathrm{mmol}, 1.5$ eq.) and 4-phenyl-1-butene $\left(0.64 \mathrm{~mL}, 4.3 \mathrm{mmol}, 1.7\right.$ eq.) in $\mathrm{CH}_{2} \mathrm{Cl}_{2}(1.9 \mathrm{~mL})$. The resulting alkylzirconocene was added to a solution of $(\boldsymbol{R}, \boldsymbol{R}, \boldsymbol{R})-\mathrm{A}\left(188 \mathrm{mg}, 0.25 \mathrm{mmol}, 0.10\right.$ eq.) in $\mathrm{Et}_{2} \mathrm{O}(13 \mathrm{~mL})$ followed by cycloheptenone (0.28 mL, $2.5 \mathrm{mmol}, 1.0$ eq.) then TMSCl (1.6 mL, $12.5 \mathrm{mmol}, 5.0$ eq.). (+)-(R)-3-(4-phenylbutyl)cycloheptanone (397.1 mg, $1.62 \mathrm{mmol}, 65 \%)$ was obtained as a light yellow oil. HPLC analysis indicated an enantiomeric excess of 92\% [Chiralpak ${ }^{\otimes}$ IB; flow: 1.5 $\mathrm{mL} / \mathrm{min}$; hexane/i-PrOH: 99.4:0.6; $\lambda=210 \mathrm{~nm}$; major enantiomer, $t_{R}=10.95 \mathrm{~min}$; minor enantiomer, $\left.\mathrm{t}_{\mathrm{R}}=12.29 \mathrm{~min}\right]$.

$[\alpha]^{20}{ }_{589}=+29.28\left(c 1.18, \mathrm{CHCl}_{3}\right)$. 


\section{(-)-3-(4-Phenylbutyl)cyclododecanone (25)}

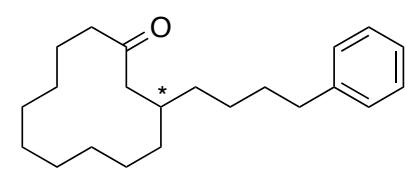

$\mathrm{Cp}_{2} \mathrm{ZrHCl}$ (103 $\mathrm{mg}, 0.40 \mathrm{mmol}, 2.0$ eq.) was added to a stirred, room temperature, solution of 4-phenyl-1-butene $\left(0.08 \mathrm{~mL}, 0.5 \mathrm{mmol}, 2.5\right.$ eq.) in $\mathrm{CH}_{2} \mathrm{Cl}_{2}(0.20 \mathrm{~mL})$ under an argon atmosphere. After stirring for $15 \mathrm{~min}$, the resulting yellow clear solution was transferred via syringe over about $1 \mathrm{~min}$ to a clear colourless stirred solution of $(S, S, S)-A\left(15.0 \mathrm{mg}, 0.020 \mathrm{mmol}, 0.10\right.$ eq.) in $\mathrm{Et}_{2} \mathrm{O}$ $(1.0 \mathrm{~mL})$ under an argon atmosphere. The resulting dark mixture was allowed to stir for an additional $10 \mathrm{~min}$ before trans-cyclododecenone $(36 \mathrm{mg}, 0.20 \mathrm{mmol}, 1.0$ eq.) and then TMSCl ( $0.13 \mathrm{~mL}, 1.0 \mathrm{mmol}, 5.0$ eq.) were added dropwise via syringe. Stirring at room temperature was continued for $2 \mathrm{~h}$ before the reaction was quenched by the addition of $\mathrm{Et}_{2} \mathrm{O}(\mathrm{ca} 3 \mathrm{~mL}$ ) and then $\mathrm{NH}_{4} \mathrm{Cl}$ (1 M aq., ca $1.5 \mathrm{~mL}$ ). The mixture was partitioned between the aqueous and $\mathrm{Et}_{2} \mathrm{O}$ layers and the aqueous phase extracted with $\mathrm{Et}_{2} \mathrm{O}(3 \times 10 \mathrm{~mL})$. The combined organic phase was washed with $\mathrm{NaHCO}_{3}$ (aq. sat., ca $10 \mathrm{~mL}$ ), dried $\left(\mathrm{Na}_{2} \mathrm{SO}_{4}\right)$, filtered and concentrated in vacuo. Flash column chromatography of the yellow residue (1:99 EtOAc/petrol; $\left.\mathrm{SiO}_{2}\right)$ gave (-)-3-(4phenylbutyl)cyclododecanone (30.7 $\mathrm{mg}, 0.098 \mathrm{mmol}, 49 \%$ ) as a light yellow oil.

HPLC analysis indicated an enantiomeric excess of $60 \%$ [Chiralpak ${ }^{\circledR} \mathrm{IC}$; flow: $1.0 \mathrm{~mL} / \mathrm{min}$; hexane/iPrOH: 95:5; $\lambda=210 \mathrm{~nm}$; major enantiomer, $t_{R}=6.14 \mathrm{~min}$; minor enantiomer, $t_{R}=6.66 \mathrm{~min}$ ].

${ }^{1} \mathrm{H}$ NMR $\left(400 \mathrm{MHz}, \mathrm{CDCl}_{3}\right) \delta_{\mathrm{H}} / \mathrm{ppm} 1.13-1.56(\mathrm{~m}, 19 \mathrm{H}), 1.56-1.70(\mathrm{~m}, 2 \mathrm{H}), 1.81-1.96(\mathrm{~m}, 1 \mathrm{H})$, $1.98-2.11(\mathrm{~m}, 1 \mathrm{H}), 2.24-2.44(\mathrm{~m}, 3 \mathrm{H}), 2.55$ (ddd, $J=15.3,11.5,3.5 \mathrm{~Hz}, 1 \mathrm{H}), 2.62(\mathrm{t}, J=7.7 \mathrm{~Hz}, 2$ $H), 7.15-7.23(m, 3 H), 7.26-7.34(m, 2 H)$.

${ }^{13} \mathrm{C} \mathrm{NMR}\left(100 \mathrm{MHz}, \mathrm{CDCl}_{3}\right) \delta_{\mathrm{H}} / \mathrm{ppm} 21.7,22.3,22.5,24.1,24.6,24.7,25.0,26.8,29.3,31.6,33.0$, $33.9,35.9,40.7,46.6,125.6,128.2$ (2 C), 128.4 (2 C), 142.7, 212.6.

HRMS (ESI) $\mathrm{m} / \mathrm{z}$ calcd for $\mathrm{C}_{22} \mathrm{H}_{34} \mathrm{ONa}[\mathrm{M}+\mathrm{Na}]^{+}: 337.2502$, found: 337.2498 .

$[\alpha]^{20}{ }_{589}=-2.47\left(c 0.66, \mathrm{CHCl}_{3}\right)$.

IR $\left(v_{\max } / \mathrm{cm}^{-1}\right):$ 698, 1496, 1707, 2929.

Absolute configuration unknown. 


\section{(+)-(S)-3-(6-Chlorohexyl)-4,4-dimethylcyclohexanone (15y)}

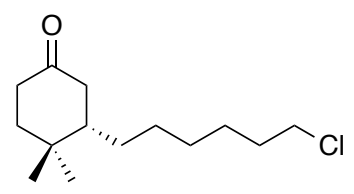

$\mathrm{Cp}_{2} \mathrm{ZrHCl}$ (970 mg, $3.8 \mathrm{mmol}, 1.5$ eq.) was added to a stirred, room temperature, solution of 6-chloro-1-hexene (0.56 mL, $4.3 \mathrm{mmol}, 1.7$ eq.) in $\mathrm{CH}_{2} \mathrm{Cl}_{2}(1.9 \mathrm{~mL})$ under an argon atmosphere. After stirring for $30 \mathrm{~min}$ at room temperature, the resulting clear yellow solution was transferred via syringe over about $1 \mathrm{~min}$ to a clear colourless stirred solution of $(\boldsymbol{R}, \boldsymbol{R}, \boldsymbol{R})-\mathbf{A}(188.0 \mathrm{mg}, 0.25$ mmol, 0.10 eq.) in $\mathrm{Et}_{2} \mathrm{O}(12.5 \mathrm{~mL})$ under an argon atmosphere. The resulting dark mixture was allowed to stir for $10 \mathrm{~min}$ before 4,4-dimethyl-2-cyclohexenone (0.33 mL, $2.5 \mathrm{mmol}, 1.0$ eq.) and then $\mathrm{TMSCl}(1.6 \mathrm{~mL}, 12.5 \mathrm{mmol}, 5.0$ eq.) were added dropwise via syringe. Stirring at room temperature was continued arbitrarily overnight before the reaction was quenched by the addition of $\mathrm{Et}_{2} \mathrm{O}\left(c a 13 \mathrm{~mL}\right.$ ) and then $\mathrm{NH}_{4} \mathrm{Cl}(1 \mathrm{M}$ aq., $c a 5 \mathrm{~mL}$ ). The mixture was partitioned between water and $\mathrm{Et}_{2} \mathrm{O}$ and the aqueous phase was extracted with $\mathrm{Et}_{2} \mathrm{O}(3 \times 20 \mathrm{~mL})$. The combined organic phase was washed with $\mathrm{NaHCO}_{3}$ (aq. sat., ca $20 \mathrm{~mL}$ ), dried $\left(\mathrm{MgSO}_{4}\right)$, filtered and concentrated in vacuo. Flash column chromatography of the yellow residue $(35: 65 \rightarrow 1: 1$; $\mathrm{CH}_{2} \mathrm{Cl}_{2}$ :petrol; $\mathrm{SiO}_{2}$ ) gave (+)-(S)-3-(6-chlorohexyl)-4,4-dimethylcyclohexanone (315.5 mg, 1.29 $\mathrm{mmol}, 52 \%)$ as an oil.

Enantiomeric excess ( $90 \%$ ee) was determined by integration of the diastereomeric mixture of the corresponding (+)-(R,R)-DPEN derivative by ${ }^{13} \mathrm{C}$ NMR spectroscopic analysis.

${ }^{1} \mathrm{H}$ NMR $\left(400 \mathrm{MHz}, \mathrm{CDCl}_{3}\right) \delta_{\mathrm{H}} / \mathrm{ppm} 0.98(\mathrm{~s}, 3 \mathrm{H}), 1.01(\mathrm{~s}, 3 \mathrm{H}), 1.07-1.18(\mathrm{~m}, 1 \mathrm{H}), 1.18-1.45(\mathrm{~m}$, $6 \mathrm{H}), 1.45-1.55(\mathrm{~m}, 2 \mathrm{H}), 1.59(\mathrm{dd}, J=13.2,4.9 \mathrm{~Hz}, 1 \mathrm{H}), 1.65-1.71(\mathrm{~m}, 1 \mathrm{H}), 1.72-1.80(\mathrm{~m}, 2 \mathrm{H})$, $2.03(\mathrm{dd}, J=14.5,12.3 \mathrm{~Hz}, 1 \mathrm{H}), 2.20-2.29(\mathrm{~m}, 1 \mathrm{H}), 2.32-2.46(\mathrm{~m}, 2 \mathrm{H}), 3.51(\mathrm{t}, J=6.7 \mathrm{~Hz}, 2 \mathrm{H})$.

${ }^{13} \mathrm{C}$ NMR $\left(100 \mathrm{MHz}, \mathrm{CDCl}_{3}\right) \delta_{\mathrm{H}} / \mathrm{ppm} 19.5,26.8,27.3,28.7,29.0,30.4,32.5,32.9,38.3,40.5,42.9$, $45.1,46.8,212.2$.

HRMS (ESI) $\mathrm{m} / \mathrm{z}$ calcd for $\mathrm{C}_{14} \mathrm{H}_{25} \mathrm{CINaO}[\mathrm{M}+\mathrm{Na}]^{+}: 267.1486$ found: 267.1503 .

$[\alpha]^{20}{ }_{589}=+14.63\left(c 1.08, \mathrm{CHCl}_{3}\right)$.

IR $\left(\mathbf{v}_{\max } / \mathrm{cm}^{-1}\right): 1213,1229,1366,1739,2970$.

Absolute configuration assigned by optical rotation. ${ }^{141}$ 
(+)-(S)-3-(5-Phenylpent-4-yn-1-yl)-4,4-dimethyl-cyclohexanone (15z)

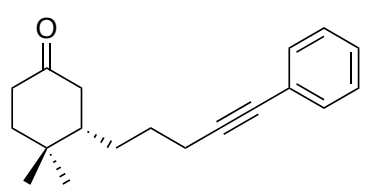

$\mathrm{Cp}_{2} \mathrm{ZrHCl}$ (970 mg, $3.8 \mathrm{mmol}, 1.5$ eq.) was added to a stirred, room temperature, solution of pent-4-en-1-yn-1-ylbenzene (0.65 mL, $4.25 \mathrm{mmol}, 1.7$ eq.) in $\mathrm{CH}_{2} \mathrm{Cl}_{2}(1.9 \mathrm{~mL})$ under an argon atmosphere The reaction flask was placed in a preheated bath at $40{ }^{\circ} \mathrm{C}$ and after stirring at this temperature for $10 \mathrm{~min}$ the flask was removed and the reaction mixture allowed to cool to room temperature. The resulting clear dark red solution was transferred via syringe over about $1 \mathrm{~min}$ to a clear colourless stirred solution of $(\boldsymbol{R}, \boldsymbol{R}, \boldsymbol{R})-\mathbf{A}\left(188.0 \mathrm{mg}, 0.25 \mathrm{mmol}, 0.10\right.$ eq.) in $\mathrm{Et}_{2} \mathrm{O}(12.5 \mathrm{~mL})$ under an argon atmosphere. The resulting dark mixture was allowed to stir for 10 min before 4,4-dimethyl-2-cyclohexenone ( $0.33 \mathrm{~mL}, 2.5 \mathrm{mmol}, 1.0$ eq.) and then TMSCl (1.6 mL, $12.5 \mathrm{mmol}$, 5.0 eq.) were added dropwise via syringe. Stirring at room temperature was continued arbitrarily overnight before the reaction was quenched by the addition of $\mathrm{Et}_{2} \mathrm{O}(\mathrm{ca} 13 \mathrm{~mL})$ and then $\mathrm{NH}_{4} \mathrm{Cl}(1$ $\mathrm{M}$ aq., ca $5 \mathrm{~mL}$ ). The mixture was partitioned between water and $\mathrm{Et}_{2} \mathrm{O}$ and the aqueous phase was extracted with $\mathrm{Et}_{2} \mathrm{O}(3 \times 20 \mathrm{~mL})$. The combined organic phase was washed with $\mathrm{NaHCO}_{3}$ (aq. sat., ca $20 \mathrm{~mL})$, dried $\left(\mathrm{MgSO}_{4}\right)$, filtered and concentrated in vacuo. Flash column chromatography of the yellow residue (3:97 $\rightarrow$ 5:95; EtOAc:petrol; $\mathrm{SiO}_{2}$ ) gave (+)-(S)-4,4-dimethyl-3-(5-phenylpent-4yn-1-yl)cyclohexanone (315 mg, $1.17 \mathrm{mmol}, 47 \%)$ as a colourless oil.

HPLC analysis indicated an enantiomeric excess of $80 \%$ [Chiralpak ${ }^{\circledast}$ AY-H; flow: $1.2 \mathrm{~mL} / \mathrm{min}$; hexane/i-PrOH: 96:4; $\lambda=254 \mathrm{~nm}$; major enantiomer, $\mathrm{t}_{\mathrm{R}}=11.13 \mathrm{~min}$; minor enantiomer, $\mathrm{t}_{\mathrm{R}}=16.50$ $\min ]$.

${ }^{1} \mathrm{H}$ NMR $\left(500 \mathrm{MHz}, \mathrm{CDCl}_{3}\right) \delta_{\mathrm{H}} / \mathrm{ppm} 1.03(\mathrm{~s}, 3 \mathrm{H}), 1.07(\mathrm{~s}, 3 \mathrm{H}), 1.13-1.28(\mathrm{~m}, 1 \mathrm{H}), 1.46$ (ddq, $J=12.9,9.9,6.4 \mathrm{~Hz}, 1 \mathrm{H}), 1.51-1.59(\mathrm{~m}, 1 \mathrm{H}), 1.63(\mathrm{dd}, J=13.2,4.7 \mathrm{~Hz}, 1 \mathrm{H}), 1.66-1.76(\mathrm{~m}, 2 \mathrm{H})$, $1.76-1.83(\mathrm{~m}, 1 \mathrm{H}), 2.10$ (ddd, $J=14.8,12.1,0.8 \mathrm{~Hz}, 1 \mathrm{H}), 2.25-2.33(\mathrm{~m}, 1 \mathrm{H}), 2.37-2.44(\mathrm{~m}, 3 \mathrm{H})$, $2.44-2.48(m, 1 H), 7.27-7.31(m, 3 H), 7.36-7.42(m, 2 H)$.

${ }^{13} \mathrm{C}$ NMR $\left(125 \mathrm{MHz}, \mathrm{CDCl}_{3}\right) \delta_{\mathrm{C}} / \mathrm{ppm} 19.4,19.5,26.6,28.7,29.8,32.9,38.3,40.4,42.9,46.4,81.0$, 89.7, 123.9, 127.5, 128.2 (2 C), 131.5 (2 C), 212.0.

HRMS (ESI) $\mathrm{m} / \mathrm{z}$ calcd for $\mathrm{C}_{19} \mathrm{H}_{24} \mathrm{NaO}[\mathrm{M}+\mathrm{Na}]^{+}: 291.1719$, found: 291.1721.

$[\alpha]^{20}{ }_{589}=+20.91$ (c 1.10, $\left.\mathrm{CHCl}_{3}\right)$.

IR $\left(\mathbf{v}_{\max } / \mathbf{c m}^{-1}\right):$ 692, 757, 1367, 1490, 1713, 2955.

Absolute configuration assigned by optical rotation. ${ }^{141}$ 


\section{(+)-1 $\alpha$-Hexyl-4-androstene-3,17-dione (31a)}

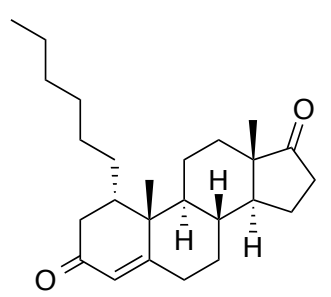

(CuOTf) ${ }_{2} . \mathrm{C}_{6} \mathrm{H}_{6}$ (10.1 mg, $0.02 \mathrm{mmol}, 0.05$ eq.) and the phosphoramidite ligand 19 (21.6 mg, 0.040 mmol, 0.10 eq.) were dissolved in $\mathrm{Et}_{2} \mathrm{O}(2.0 \mathrm{~mL})$ under an argon atmosphere and allowed to stir for $1 \mathrm{~h}$ at room temperature. In another flask, $\mathrm{Cp}_{2} \mathrm{ZrHCl}(206.0 \mathrm{mg}, 0.80 \mathrm{mmol}, 2.0$ eq.) was added to a stirred, room temperature, solution of 1-hexene $\left(0.13 \mathrm{~mL}, 1.0 \mathrm{mmol}, 2.5\right.$ eq.) in $\mathrm{CH}_{2} \mathrm{Cl}_{2}(0.40$ $\mathrm{mL}$ ) under an argon atmosphere. After stirring for $40 \mathrm{~min}$, the resulting clear yellow solution was transferred via syringe over about $1 \mathrm{~min}$ to the stirred solution containing the copper and ligand under an argon atmosphere. The resulting dark mixture was allowed to stir for an additional 10 min before 1,4-androstadiene-3,17-dione (114 mg, $0.40 \mathrm{mmol}, 1.0$ eq.) and TMSCl (0.26 mL, 2.0 $\mathrm{mmol}, 5.0$ eq.) were added sequentially dropwise via syringe. Stirring at room temperature was continued arbitrarily for $15 \mathrm{~h}$ before the reaction was quenched by the addition of $\mathrm{Et}_{2} \mathrm{O}$ ( $c a 3 \mathrm{~mL}$ ) and $\mathrm{NH}_{4} \mathrm{Cl}$ (1 M aq., ca $1.5 \mathrm{~mL}$ ). The mixture was partitioned between the aqueous and $\mathrm{Et}_{2} \mathrm{O}$ layers and the former phase was extracted with $\mathrm{Et}_{2} \mathrm{O}(3 \times 10 \mathrm{~mL})$. The combined organic phase was washed with $\mathrm{NaHCO}_{3}$ (sat. aq., ca $10 \mathrm{~mL}$ ), dried $\left(\mathrm{Na}_{2} \mathrm{SO}_{4}\right)$, filtered and concentrated in vacuo to give an oil.

Diastereomeric ratio of the crude product ( 3.5:1) was determined by integration of ${ }^{1} \mathrm{H}$ NMR spectra. Flash column chromatography of the yellow residue (5:30:65; EtOAc: $\mathrm{CH}_{2} \mathrm{Cl}_{2}$ :petrol; $\mathrm{SiO}_{2}$ ) gave first the major diastereomer ( $4 \alpha)(+)$-1 $\alpha$-hexyl-4-androstene-3,17-dione (75 mg, $0.20 \mathrm{mmol}$,

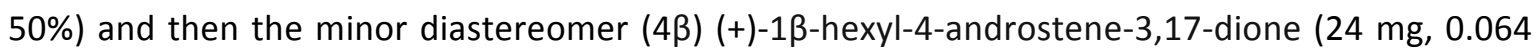
$\mathrm{mmol}, 16 \%)$.

${ }^{1} \mathrm{H}$ NMR $\left(500 \mathrm{MHz}, \mathrm{CDCl}_{3}\right) \delta_{\mathrm{H}} / \mathrm{ppm} 0.86(\mathrm{t}, J=7.0 \mathrm{~Hz}, 3 \mathrm{H}), 0.93(\mathrm{~s}, 3 \mathrm{H}), 1.03-1.31(\mathrm{~m}, 10 \mathrm{H}), 1.32$ (s, $3 \mathrm{H}), 1.33$ - $1.64(\mathrm{~m}, 7 \mathrm{H}), 1.75(\mathrm{qd}, J=10.9,3.3 \mathrm{~Hz}, 1 \mathrm{H}), 1.86(\mathrm{dt}, J=12.9,3.1 \mathrm{~Hz}, 1 \mathrm{H}), 1.89$ $1.96(\mathrm{~m}, 2 \mathrm{H}), 1.96-2.04(\mathrm{~m}, 1 \mathrm{H}), 2.11(\mathrm{dt}, J=17.6,9.0 \mathrm{~Hz}, 1 \mathrm{H}), 2.33-2.53(\mathrm{~m}, 4 \mathrm{H}), 2.61(\mathrm{dd}, J=$ 16.6, $4.5 \mathrm{~Hz}, 1 \mathrm{H}), 5.71(\mathrm{~s}, 1 \mathrm{H})$.

${ }^{13} \mathrm{C}$ NMR $\left(100 \mathrm{MHz}, \mathrm{CDCl}_{3}\right) \delta_{\mathrm{c}} / \mathrm{ppm} 13.7,14.1,19.5,20.5,21.7,22.6,27.1,27.4,29.4,29.8,31.2$, $31.7,32.7,35.0,35.7,37.9,41.7,41.8,46.3,47.5,51.0,124.0,167.8,199.0,220.5$.

HRMS (ESI) $\mathrm{m} / \mathrm{z}$ calcd for $\mathrm{C}_{25} \mathrm{H}_{38} \mathrm{NaO}_{2}[\mathrm{M}+\mathrm{Na}]^{+}: 393.2764$, found: 393.2748 .

$[\alpha]^{20}{ }_{589}=+140.9\left(c 1.12, \mathrm{CHCl}_{3}\right)$.

IR $\left(v_{\max } / \mathrm{cm}^{-1}\right):$ 1455, 1671, 1738, 2926. 


\section{(+)-1ß-Hexyl-4-androstene-3,17-dione}

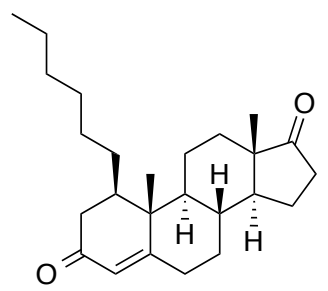

${ }^{1} \mathbf{H}$ NMR $\left(400 \mathrm{MHz}, \mathrm{CDCl}_{3}\right) \delta_{\mathrm{H}} / \mathrm{ppm} 0.87(\mathrm{t}, J=6.8 \mathrm{~Hz}, 3 \mathrm{H}), 0.91(\mathrm{~s}, 3 \mathrm{H}), 1.06-1.15(\mathrm{~m}, 2 \mathrm{H}), 1.16$ (s, $3 \mathrm{H}), 1.19$ - $1.43(\mathrm{~m}, 11 \mathrm{H}), 1.46$ - $1.64(\mathrm{~m}, 3 \mathrm{H}), 1.65$ - 1.77 (m, $1 \mathrm{H}), 1.81$ - 2.00 (m, $4 \mathrm{H}), 2.01$ 2.16 (m, 2 H), $2.20-2.31$ (m, 2 H), $2.40-2.55$ (m, 3 H), 5.79 (br. s, 1 H).

${ }^{13} \mathrm{C}$ NMR $\left(100 \mathrm{MHz}, \mathrm{CDCl}_{3}\right) \delta_{\mathrm{C}} / \mathrm{ppm} 13.8,14.1,16.3,21.9,22.3,22.6,27.8,29.5,30.1,31.4,31.7$, $33.1,33.8,35.5,35.7,38.6,40.1,43.2,47.6,50.9,53.4,123.0,170.7,199.2,220.3$.

HRMS (ESI) $m / z$ calcd for $\mathrm{C}_{25} \mathrm{H}_{38} \mathrm{NaO}_{2}[\mathrm{M}+\mathrm{Na}]^{+}: 393.2764$ found: 393.2753 .

$[\alpha]_{589}^{20}=+14.7\left(c 1.05, \mathrm{CHCl}_{3}\right)$.

IR ( $\left.\mathbf{v}_{\max } / \mathbf{c m}^{-1}\right):$ 1454, 1673, 1738, 2926.

(+)-7 $\alpha$-Hexyl-17 $\alpha$-hydroxy-3-oxo-pregn-4-ene-21-carboxylic acid $p$-lactone (32a)

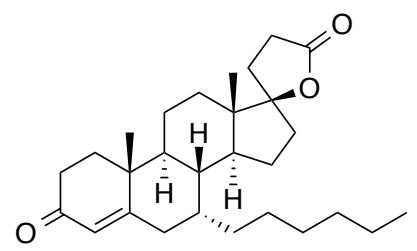

$(\mathrm{CuOTf})_{2} \cdot\left(\mathrm{C}_{6} \mathrm{H}_{6}\right)(10.1 \mathrm{mg}, 0.02 \mathrm{mmol}, 0.05$ eq.) and the phosphoramidite ligand 19 (21.6 mg, 0.040 mmol, 0.10 eq.) were dissolved in $\mathrm{Et}_{2} \mathrm{O}(2.0 \mathrm{~mL})$ under an argon atmosphere and allowed to stir for $1 \mathrm{~h}$ at room temperature. In another flask, $\mathrm{Cp}_{2} \mathrm{ZrHCl}(206.0 \mathrm{mg}, 0.80 \mathrm{mmol}, 2.0$ eq.) was added to a stirred, room temperature, solution of 1-hexene $\left(0.13 \mathrm{~mL}, 1.0 \mathrm{mmol}, 2.5\right.$ eq.) in $\mathrm{CH}_{2} \mathrm{Cl}_{2}(0.40$ $\mathrm{mL}$ ) under an argon atmosphere. After stirring for $40 \mathrm{~min}$, the resulting clear yellow solution was transferred via syringe over about $1 \mathrm{~min}$ to the stirred solution containing the copper and ligand under an argon atmosphere. The resulting dark mixture was allowed to stir for an additional 10 min before canrenone $(136.0 \mathrm{mg}, 0.40 \mathrm{mmol}, 1.0$ eq.) and $\mathrm{TMSCl}(0.26 \mathrm{~mL}, 2.0 \mathrm{mmol}, 5.0$ eq.) were added sequentially dropwise via syringe. Stirring at room temperature was continued arbitrarily for $15 \mathrm{~h}$ before the reaction was quenched by the addition of $\mathrm{Et}_{2} \mathrm{O}(\mathrm{ca} 3 \mathrm{~mL})$ and $\mathrm{NH}_{4} \mathrm{Cl}$ (1 $\mathrm{M}$ aq., ca $1.5 \mathrm{~mL}$ ). The mixture was partitioned between the aqueous and $\mathrm{Et}_{2} \mathrm{O}$ layers and the former phase was extracted with $\mathrm{Et}_{2} \mathrm{O}(3 \times 10 \mathrm{~mL})$. The combined organic phase was washed with $\mathrm{NaHCO}_{3}$ (sat. aq., ca $10 \mathrm{~mL}$ ), dried $\left(\mathrm{Na}_{2} \mathrm{SO}_{4}\right)$, filtered and concentrated in vacuo to give an oil. 
Diastereomeric ratio of the crude product ( 4.8:1) was determined by integration of ${ }^{1} \mathrm{H}$ NMR spectra. Flash column chromatography of the yellow residue (30:70; EtOAc:petrol; $\mathrm{SiO}_{2}$ ) gave first the major diastereomer (7 $\alpha)(+)-7 \alpha$-hexyl-17 $\alpha$-hydroxy-3-oxo-pregn-4-ene-21-carboxylic acid $y$ -

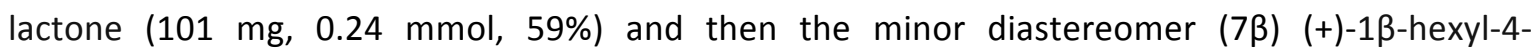
androstene-3,17-dione (35 mg, $0.082 \mathrm{mmol}, 21 \%)$.

${ }^{1}{ }_{\mathrm{H}} \mathrm{NMR}\left(500 \mathrm{MHz}, \mathrm{CDCl}_{3}\right) \delta_{\mathrm{H}} / \mathrm{ppm} 0.89(\mathrm{t}, J=7.1 \mathrm{~Hz}, 3 \mathrm{H}), 0.99(\mathrm{~s}, 3 \mathrm{H}), 1.04-1.20(\mathrm{~m}, 5 \mathrm{H}), 1.22$ $(\mathrm{s}, 3 \mathrm{H}), 1.24$ - $1.53(\mathrm{~m}, 11 \mathrm{H}), 1.55$ - $1.76(\mathrm{~m}, 5 \mathrm{H}), 1.77$ - $1.89(\mathrm{~m}, 2 \mathrm{H}), 1.90$ - $1.98(\mathrm{~m}, 1 \mathrm{H}), 2.03$ $2.10(m, 1 \mathrm{H}), 2.22$ - $2.31(\mathrm{~m}, 1 \mathrm{H}), 2.32-2.45(\mathrm{~m}, 4 \mathrm{H}), 2.47-2.62(\mathrm{~m}, 2 \mathrm{H}), 5.74(\mathrm{~s}, 1 \mathrm{H})$.

${ }^{13} \mathrm{C}$ NMR $\left(100 \mathrm{MHz}, \mathrm{CDCl}_{3}\right) \delta_{\mathrm{c}} / \mathrm{ppm} 14.1,14.4,18.1,20.7,22.4,22.6,25.2,27.3,29.3,29.5,31.2$, $31.5,31.9,34.0,35.3,36.0,36.1,36.6,38.6,39.3,44.8,45.6,46.9,95.8,125.9,169.5,176.7$, 199.1.

HRMS (ESI) $\mathrm{m} / \mathrm{z}$ calcd for $\mathrm{C}_{28} \mathrm{H}_{42} \mathrm{NaO}_{3}[\mathrm{M}+\mathrm{Na}]^{+}: 449.3026$ found: 449.3017.

$[\alpha]^{20}{ }_{589}=+37.3\left(c 1.00, \mathrm{CHCl}_{3}\right)$.

IR $\left(v_{\max } / \mathrm{cm}^{-1}\right):$ 1192, 1669, 1769, 2927.

Absolute configuration assigned by analogy to compound 32c.

(+)-7ß-Hexyl-17 $\alpha$-hydroxy-3-oxo-pregn-4-ene-21-carboxylic acid $\psi$-lactone

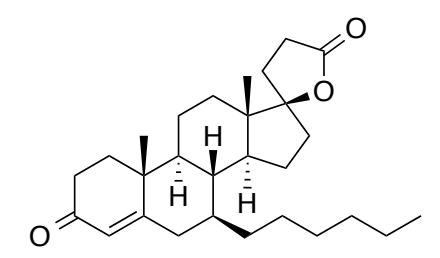

${ }^{1} \mathrm{H}$ NMR $\left(500 \mathrm{MHz}, \mathrm{CDCl}_{3}\right) \delta_{\mathrm{H}} / \mathrm{ppm} 0.90(\mathrm{t}, J=6.9 \mathrm{~Hz}, 3 \mathrm{H}), 0.92-0.99(\mathrm{~m}, 1 \mathrm{H}), 1.01(\mathrm{~s}, 3 \mathrm{H}), 1.18$ (s, $3 \mathrm{H}), 1.23-1.51(\mathrm{~m}, 13 \mathrm{H}), 1.57-1.84(\mathrm{~m}, 7 \mathrm{H}), 1.86-2.00(\mathrm{~m}, 2 \mathrm{H}), 2.05(\mathrm{dt}, J=13.3,4.3 \mathrm{~Hz}, 1$ H), 2.18 - $2.34(\mathrm{~m}, 3 \mathrm{H}), 2.34-2.46(\mathrm{~m}, 3 \mathrm{H}), 2.46-2.62$ (m, $2 \mathrm{H}), 5.73$ (br. $\mathrm{s}, 1 \mathrm{H})$.

${ }^{13} \mathrm{C}$ NMR $\left(100 \mathrm{MHz}, \mathrm{CDCl}_{3}\right) \delta_{\mathrm{C}} / \mathrm{ppm} 14.1,15.1,17.5,20.8,22.7,26.2,26.3,29.4,29.7,31.2,31.5$, $31.8,34.0,35.2,35.7,35.9,38.4,39.1,40.1,43.4,46.9,49.6,53.9,95.0,122.9,170.8,176.8$, 199.4.

HRMS (ESI) $\mathrm{m} / \mathrm{z}$ calcd for $\mathrm{C}_{28} \mathrm{H}_{42} \mathrm{NaO}_{3}[\mathrm{M}+\mathrm{Na}]^{+}: 449.3026$ found: 449.3009 .

$[\alpha]^{20}{ }_{589}=+34.6\left(\mathrm{c} 0.55, \mathrm{CHCl}_{3}\right)$.

IR $\left(v_{\max } / \mathbf{c m}^{-1}\right): 1174,1675,1770,2925$. 


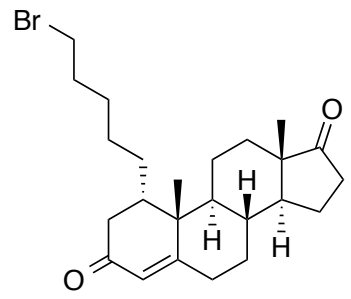

$\mathrm{Cp}_{2} \mathrm{ZrHCl}$ (206 mg, $0.80 \mathrm{mmol}, 2.0$ eq.) was added to a stirred, room temperature, solution of 5-bromo-1-pentene (0.12 mL, $1.0 \mathrm{mmol}, 2.5$ eq.) in $\mathrm{CH}_{2} \mathrm{Cl}_{2}(0.40 \mathrm{~mL})$ under an argon atmosphere. After stirring for about $15 \mathrm{~min}$, the resulting clear yellow solution was transferred via syringe over about $1 \mathrm{~min}$ to a clear colourless stirred solution of $(S, S, S)-A(30.0 \mathrm{mg}, 0.040 \mathrm{mmol}, 0.10$ eq.) in $\mathrm{Et}_{2} \mathrm{O}(2.0 \mathrm{~mL})$ under an argon atmosphere. The resulting dark mixture was allowed to stir for an additional $10 \mathrm{~min}$ before 1,4-androstadiene-3,17-dione (114 mg, $0.40 \mathrm{mmol}, 1.0$ eq.) and TMSCl (0.26 mL, $2.0 \mathrm{mmol}, 5.0$ eq.) were added sequentially dropwise via syringe. Stirring at room temperature was continued arbitrarily for $15 \mathrm{~h}$ before the reaction was quenched by the addition of $\mathrm{Et}_{2} \mathrm{O}(c a 3 \mathrm{~mL})$ and $\mathrm{NH}_{4} \mathrm{Cl}(1 \mathrm{M}$ aq., $c a 1.5 \mathrm{~mL}$ ). The mixture was partitioned between the aqueous and $\mathrm{Et}_{2} \mathrm{O}$ layers and the former phase was extracted with $\mathrm{Et}_{2} \mathrm{O}(3 \times 10 \mathrm{~mL})$. The combined organic phase was washed with $\mathrm{NaHCO}_{3}$ (sat. aq., ca $10 \mathrm{~mL}$ ), dried $\left(\mathrm{Na}_{2} \mathrm{SO}_{4}\right)$, filtered and concentrated in vacuo to give an oil.

Diastereomeric ratio of the crude product $(\sim 4.8: 1)$ was determined by integration of ${ }^{1} \mathrm{H}$ NMR spectra. Flash column chromatography of the yellow residue (10:30:60; $\mathrm{EtOAc}: \mathrm{CH}_{2} \mathrm{Cl}_{2}$ :petrol; $\mathrm{SiO}_{2}$ ) gave first the major diastereomer ( $5 \alpha)(+)$-1 $\alpha$-(5-bromopentyl)-4-androstene-3,17-dione (107 mg, $0.24 \mathrm{mmol}, 60 \%)$ and then the minor diastereomer $(5 \beta)(+)-1 \beta$-(5-bromopentyl)-4-androstene3,17-dione (14 mg, $0.032 \mathrm{mmol}, 8 \%$ ).

${ }^{1} \mathrm{H}$ NMR $\left(400 \mathrm{MHz}, \mathrm{CDCl}_{3}\right) \delta_{\mathrm{H}} / \mathrm{ppm} 0.92(\mathrm{~s}, 3 \mathrm{H}), 1.01-1.28(\mathrm{~m}, 4 \mathrm{H}), 1.31(\mathrm{~s}, 3 \mathrm{H}), 1.36-1.66(\mathrm{~m}, 9$ H), $1.69-2.04(\mathrm{~m}, 7 \mathrm{H}), 2.11(\mathrm{dt}, J=19.2,9.0 \mathrm{~Hz}, 1 \mathrm{H}), 2.32-2.54(\mathrm{~m}, 4 \mathrm{H}), 2.63(\mathrm{dd}, J=16.5,4.4$ $\mathrm{Hz}, 1 \mathrm{H}), 3.38$ (t, J = 6.7 Hz, $2 \mathrm{H}$ ), 5.71 (br. s, $1 \mathrm{H}$ ).

${ }^{13} \mathrm{C}$ NMR $\left(100 \mathrm{MHz}, \mathrm{CDCl}_{3}\right) \delta_{\mathrm{c}} / \mathrm{ppm} 13.7,19.6,20.5,21.8,26.6,27.1,28.4,29.8,31.2,32.6,32.7$, $33.8,35.0,35.7,37.9,41.7,41.8,46.3,47.5,51.0,124.0,167.6,198.8,220.3$.

HRMS (ESI) $\mathrm{m} / \mathrm{z}$ calcd for $\mathrm{C}_{24} \mathrm{H}_{35} \mathrm{BrNaO}_{2}[\mathrm{M}+\mathrm{Na}]^{+}: 457.1713$ found: 457.1705 .

$[\alpha]^{20}{ }_{589}=+122.4\left(c 1.055, \mathrm{CHCl}_{3}\right)$.

IR $\left(v_{\max } / \mathrm{cm}^{-1}\right):$ 703, 1051, 1669, 1737, 2934. 
(+)- $\alpha$-(5-Bromopentyl)-17 $\alpha$-hydroxy-3-oxo-pregn-4-ene-21-carboxylic acid $p$-lactone (32b)

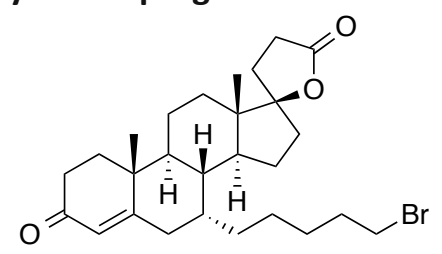

$\mathrm{Cp}_{2} \mathrm{ZrHCl}$ (206 mg, $0.80 \mathrm{mmol}, 2.0$ eq.) was added to a stirred, room temperature, solution of 5-bromo-1-pentene (0.12 mL, $1.0 \mathrm{mmol}, 2.5$ eq.) in $\mathrm{CH}_{2} \mathrm{Cl}_{2}(0.40 \mathrm{~mL})$ under an argon atmosphere. After stirring for about $15 \mathrm{~min}$, the resulting clear yellow solution was transferred via syringe over about $1 \mathrm{~min}$ to a clear colourless stirred solution of $(\boldsymbol{S}, S, S)-A(30.0 \mathrm{mg}, 0.040 \mathrm{mmol}, 0.10$ eq.) in $\mathrm{Et}_{2} \mathrm{O}(2.0 \mathrm{~mL})$ under an argon atmosphere. The resulting dark mixture was allowed to stir for an additional $10 \mathrm{~min}$ before canrenone $(136 \mathrm{mg}, 0.40 \mathrm{mmol}, 1.0$ eq.) and TMSCl (0.26 mL, $2.0 \mathrm{mmol}$, 5.0 eq.) were added sequentially dropwise via syringe. Stirring at room temperature was continued arbitrarily for $15 \mathrm{~h}$ before the reaction was quenched by the addition of $\mathrm{Et}_{2} \mathrm{O}(\mathrm{ca} 3 \mathrm{~mL})$ and $\mathrm{NH}_{4} \mathrm{Cl}$ (1 M aq., ca $1.5 \mathrm{~mL}$ ). The mixture was partitioned between the aqueous and $\mathrm{Et}_{2} \mathrm{O}$ layers and the former phase was extracted with $\mathrm{Et}_{2} \mathrm{O}(3 \times 10 \mathrm{~mL})$. The combined organic phase was washed with $\mathrm{NaHCO}_{3}$ (sat. aq., ca $10 \mathrm{~mL}$ ), dried $\left(\mathrm{Na}_{2} \mathrm{SO}_{4}\right)$, filtered and concentrated in vacuo to give an oil. Flash column chromatography of the yellow residue (30:70; EtOAc:petrol; $\mathrm{SiO}_{2}$ ) gave first the major diastereomer $(8 \alpha)(+)-7 \alpha-(5-b r o m o p e n t y l)-17 \alpha$-hydroxy-3-oxo-pregn-4-ene21-carboxylic acid $\gamma$-lactone $(64 \mathrm{mg}, 0.13 \mathrm{mmol}, 33 \%)$ and then the minor diastereomer (8ß) (+)-1ß-(5-bromopentyl)-4-androstene-3,17-dione (14 mg, $0.028 \mathrm{mmol}, 7 \%)$.

${ }^{1} \mathrm{H}$ NMR $\left(400 \mathrm{MHz}, \mathrm{CDCl}_{3}\right) \delta_{\mathrm{H}} / \mathrm{ppm} 0.98(\mathrm{~s}, 3 \mathrm{H}), 1.08-1.19(\mathrm{~m}, 3 \mathrm{H}), 1.21(\mathrm{~s}, 3 \mathrm{H}), 1.27-1.52(\mathrm{~m}, 7$ H), $1.53-1.89(m, 10 H), 1.91-2.00(m, 1 H), 2.02-2.11(m, 1 H), 2.20-2.48(m, 6 H), 2.48-2.63$ (m, $2 \mathrm{H}), 3.40(\mathrm{t}, J=6.7 \mathrm{~Hz}, 2 \mathrm{H}), 5.72$ (br. s, $1 \mathrm{H})$.

${ }^{13} \mathrm{C}$ NMR $\left(100 \mathrm{MHz}, \mathrm{CDCl}_{3}\right) \delta_{\mathrm{C}} / \mathrm{ppm} 14.4,18.1,20.7,22.4,25.1,26.5,28.4,29.3,31.2,31.5,32.7$, 33.95, 33.99, 35.3, 36.0 (2 C), 36.5, 38.6, 39.3, 44.8, 45.6, 46.9, 95.8, 125.9, 169.3, 176.7, 199.1.

HRMS (ESI) $m / z$ calcd for $\mathrm{C}_{27} \mathrm{H}_{39} \mathrm{BrNaO}_{3}[\mathrm{M}+\mathrm{Na}]^{+}: 513.1975$ found: 513.1961 .

$[\alpha]_{589}^{20}=+32.1\left(c 0.80, \mathrm{CHCl}_{3}\right)$.

IR ( $\left.\mathbf{v}_{\max } / \mathrm{cm}^{-1}\right):$ 728, 913, 1663, 1767, 2939.

Absolute configuration assigned by analogy to compound $\mathbf{3 2 c}$. 


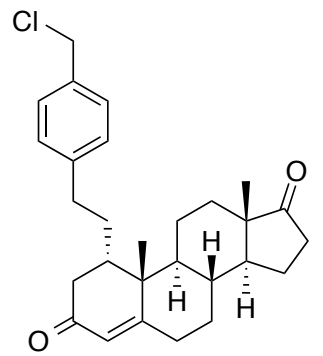

$\mathrm{Cp}_{2} \mathrm{ZrHCl}$ (206 mg, $0.80 \mathrm{mmol}, 2.0$ eq.) was added to a stirred, room temperature, solution of 4-vinylbenzyl chloride (0.14 mL, $1.0 \mathrm{mmol}, 2.5$ eq.) in $\mathrm{CH}_{2} \mathrm{Cl}_{2}(0.40 \mathrm{~mL})$ under an argon atmosphere. After stirring for about $15 \mathrm{~min}$, the resulting clear yellow solution was transferred via syringe over about $1 \mathrm{~min}$ to a clear colourless stirred solution of $(\boldsymbol{S}, S, S)-\mathbf{A}(30.0 \mathrm{mg}, 0.040 \mathrm{mmol}$, 0.10 eq.) in $\mathrm{Et}_{2} \mathrm{O}(2.0 \mathrm{~mL})$ under an argon atmosphere. The resulting dark mixture was allowed to stir for an additional $10 \mathrm{~min}$ before 1,4-androstadiene-3,17-dione (114 mg, $0.40 \mathrm{mmol}, 1.0$ eq.) and $\mathrm{TMSCl}(0.26 \mathrm{~mL}, 2.0 \mathrm{mmol}, 5.0$ eq.) were added sequentially dropwise via syringe. Stirring at room temperature was continued arbitrarily for $15 \mathrm{~h}$ before the reaction was quenched by the addition of $\mathrm{Et}_{2} \mathrm{O}(c a 3 \mathrm{~mL})$ and $\mathrm{NH}_{4} \mathrm{Cl}(1 \mathrm{M}$ aq., $c a 1.5 \mathrm{~mL})$. The mixture was partitioned between the aqueous and $\mathrm{Et}_{2} \mathrm{O}$ layers and the former phase was extracted with $\mathrm{Et}_{2} \mathrm{O}(3 \times 10 \mathrm{~mL})$. The combined organic phase was washed with $\mathrm{NaHCO}_{3}$ (sat. aq., ca $10 \mathrm{~mL}$ ), dried $\left(\mathrm{Na}_{2} \mathrm{SO}_{4}\right)$, filtered and concentrated in vacuo to give an oil. Flash column chromatography of the yellow residue $\left(5: 35: 60 \rightarrow 10: 30: 60 ; \mathrm{EtOAc}: \mathrm{CH}_{2} \mathrm{Cl}_{2}:\right.$ petrol; $\left.\mathrm{SiO}_{2}\right)$ gave major diastereomer $(6 \alpha)(+)-1 \alpha-(4-$ (chloromethyl)phenethyl))-4-androstene-3,17-dione $(54 \mathrm{mg}, 0.12 \mathrm{mmol}, 30 \%)$, minor diastereomer (+)-13-(4-(chloromethyl)phenethyl))-4-androstene-3,17-dione was not seen.

${ }^{1} \mathrm{H}$ NMR $\left(400 \mathrm{MHz}, \mathrm{CDCl}_{3}\right) \delta_{\mathrm{H}} / \mathrm{ppm} 0.90(\mathrm{~s}, 3 \mathrm{H}), 0.98-1.28(\mathrm{~m}, 3 \mathrm{H}), 1.29(\mathrm{~s}, 3 \mathrm{H}), 1.33-1.43(\mathrm{~m}, 3$ H), $1.43-1.64(\mathrm{~m}, 2 \mathrm{H}), 1.64-1.77(\mathrm{~m}, 2 \mathrm{H}), 1.81(\mathrm{dt}, J=12.9,3.1 \mathrm{~Hz}, 1 \mathrm{H}), 1.86-2.03(\mathrm{~m}, 3 \mathrm{H})$, $2.09(\mathrm{dt}, J=19.2,9.0 \mathrm{~Hz}, 1 \mathrm{H}), 2.32-2.52(\mathrm{~m}, 4 \mathrm{H}), 2.57(\mathrm{dd}, J=16.6,1.6 \mathrm{~Hz}, 1 \mathrm{H}), 2.68(\mathrm{dd}, J=$ 16.6, 3.3 Hz, 1 H), $2.73-2.82(m, 1 \mathrm{H}), 4.56$ (br. s, $2 \mathrm{H}), 5.74$ (br. s, $1 \mathrm{H}), 7.12(\mathrm{~d}, J=8.2 \mathrm{~Hz}, 2 \mathrm{H}$ ), $7.29(\mathrm{~d}, J=8.2 \mathrm{~Hz}, 2 \mathrm{H})$.

${ }^{13} \mathrm{C}$ NMR $\left(100 \mathrm{MHz}, \mathrm{CDCl}_{3}\right) \delta_{\mathrm{C}} / \mathrm{ppm} 13.7,19.4,20.4,21.7,29.4,29.8,31.1,32.6,33.2,35.0,35.7$, 37.9, 41.1, 41.8, 46.1, 46.4, 47.5, 51.0, 124.0, 128.7 (2 C), 128.8 (2 C), 135.2, 142.2, 167.6, 198.5, 220.2 .

HRMS (ESI) $\mathrm{m} / \mathrm{z}$ calcd for $\mathrm{C}_{28} \mathrm{H}_{35} \mathrm{ClNaO}_{2}[\mathrm{M}+\mathrm{Na}]^{+}: 461.2218$ found: 461.2204 .

$[\alpha]^{20}{ }_{589}=+125.0\left(\mathrm{c} \mathrm{0.28}, \mathrm{CHCl}_{3}\right)$.

IR $\left(v_{\max } / \mathbf{c m}^{-1}\right):$ 730, 1667, 1737, 2934. 


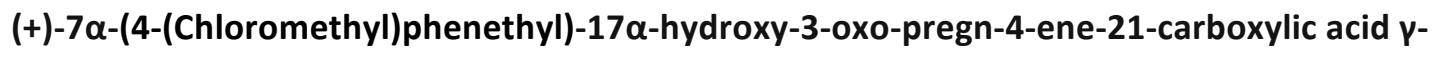
lactone (32c)

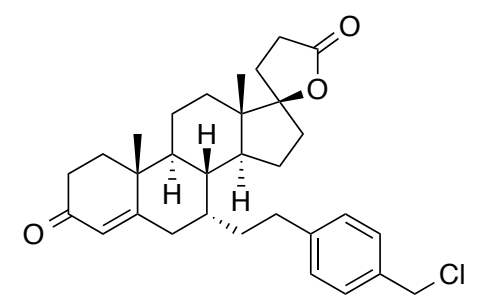

$\mathrm{Cp}_{2} \mathrm{ZrHCl}$ (206 mg, $0.80 \mathrm{mmol}, 2.0$ eq.) was added to a stirred, room temperature, solution of 4-vinylbenzyl chloride $\left(0.14 \mathrm{~mL}, 1.0 \mathrm{mmol}, 2.5\right.$ eq.) in $\mathrm{CH}_{2} \mathrm{Cl}_{2}(0.40 \mathrm{~mL})$ under an argon atmosphere. After stirring for about $15 \mathrm{~min}$, the resulting clear yellow solution was transferred via syringe over about $1 \mathrm{~min}$ to a clear colourless stirred solution of $(\boldsymbol{S}, S, S)-\mathrm{A}(30.0 \mathrm{mg}, 0.040 \mathrm{mmol}$, 0.10 eq.) in $\mathrm{Et}_{2} \mathrm{O}(2.0 \mathrm{~mL})$ under an argon atmosphere. The resulting dark mixture was allowed to stir for an additional $10 \mathrm{~min}$ before canrenone $(136 \mathrm{mg}, 0.40 \mathrm{mmol}, 1.0$ eq.) and TMSCl $(0.26 \mathrm{~mL}$, $2.0 \mathrm{mmol}, 5.0$ eq.) were added sequentially dropwise via syringe. Stirring at room temperature was continued arbitrarily for $15 \mathrm{~h}$ before the reaction was quenched by the addition of $\mathrm{Et}_{2} \mathrm{O}$ ( $\mathrm{ca} 3$ $\mathrm{mL}$ ) and $\mathrm{NH}_{4} \mathrm{Cl}$ (1 M aq., ca $1.5 \mathrm{~mL}$ ). The mixture was partitioned between the aqueous and $\mathrm{Et}_{2} \mathrm{O}$ layers and the former phase was extracted with $\mathrm{Et}_{2} \mathrm{O}(3 \times 10 \mathrm{~mL})$. The combined organic phase was washed with $\mathrm{NaHCO}_{3}$ (sat. aq., ca $10 \mathrm{~mL}$ ), dried $\left(\mathrm{Na}_{2} \mathrm{SO}_{4}\right)$, filtered and concentrated in vacuo to give an oil. Flash column chromatography of the yellow residue (35:65; EtOAc:petrol; $\mathrm{SiO}_{2}$ )

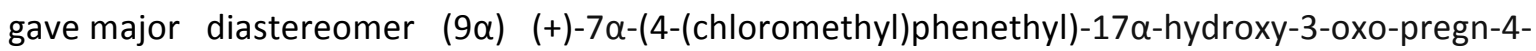
ene-21-carboxylic acid $\gamma$-lactone $(68 \mathrm{mg}, 0.14 \mathrm{mmol}, 35 \%)$, minor diastereomer (+)-7 $\beta-(4-$ (chloromethyl)phenethyl)-17 $\alpha$-hydroxy-3-oxo-pregn-4-ene-21-carboxylic acid $\gamma$-lactone was not seen.

${ }^{1} \mathrm{H}$ NMR $\left(400 \mathrm{MHz}, \mathrm{CDCl}_{3}\right) \delta_{\mathrm{H}} / \mathrm{ppm} 0.97(\mathrm{~s}, 3 \mathrm{H}), 1.10-1.21(\mathrm{~m}, 1 \mathrm{H}), 1.24$ (br. s, $\left.3 \mathrm{H}\right), 1.27-1.85$ $(m, 13 H), 1.86-1.94(m, 1 H), 2.01-2.12(m, 1 H), 2.16-2.27(m, 1 H), 2.28-2.60(m, 8 H), 2.69-$ $2.79(\mathrm{~m}, 1 \mathrm{H}), 4.56(\mathrm{~s}, 2 \mathrm{H}), 5.82($ br. $s, 1 \mathrm{H}), 7.13(\mathrm{~d}, J=8.1 \mathrm{~Hz}, 2 \mathrm{H}), 7.30(\mathrm{~d}, J=8.1 \mathrm{~Hz}, 2 \mathrm{H})$.

${ }^{13} \mathrm{C}$ NMR $\left(100 \mathrm{MHz} \mathrm{CDCl}_{3}\right) \delta_{\mathrm{c}} / \mathrm{ppm} 14.3,18.2,20.7,22.2,27.7,29.2,31.2,31.4,33.3,34.0,35.3$, $35.7,36.0,36.4,38.6,39.3,44.7,45.6,46.1,47.0,95.7,126.0,128.77$ (2 C), 128.81 (2 C), 135.3, $142.5,169.1,176.7,199.0$.

HRMS (ESI) $m / z$ calcd for $\mathrm{C}_{31} \mathrm{H}_{39} \mathrm{ClNaO}_{3}[\mathrm{M}+\mathrm{Na}]^{+}: 517.2480$ found: 517.2463 .

$[\alpha]^{20}{ }_{589}=+64.0\left(\mathrm{c} 0.88, \mathrm{CHCl}_{3}\right)$.

IR $\left(v_{\max } / \mathbf{c m}^{-1}\right):$ 727, 1663, 1765, 2945.

Absolute configuration assigned by X-ray crystal structure (See Appendix A3). 
(+)-7 $\alpha$-(4-(4,4,5,5-Tetramethyl-1,3,2-dioxaborolan-2-yl)phenethyl)-17 $\alpha$-hydroxy-3-oxo-pregn-4ene-21-carboxylic acid $y$-lactone (32d)

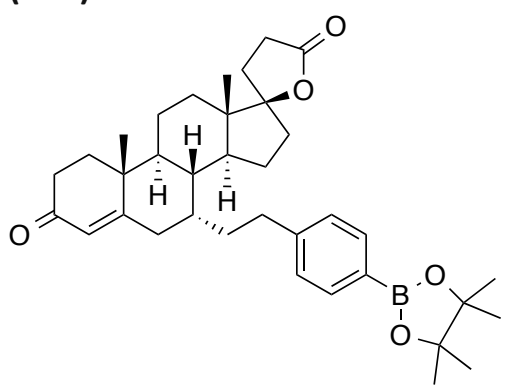

$\mathrm{Cp}_{2} \mathrm{ZrHCl}$ (206 mg, $0.80 \mathrm{mmol}, 2.0$ eq.) was added to a stirred, room temperature, solution of 4-vinylphenylpinacol-boronic ester $\left(230 \mathrm{mg}, 1.0 \mathrm{mmol}, 2.5\right.$ eq.) in $\mathrm{CH}_{2} \mathrm{Cl}_{2}(0.40 \mathrm{~mL})$ under an argon atmosphere. After stirring for about $15 \mathrm{~min}$, the resulting clear yellow solution was transferred via syringe over about $1 \mathrm{~min}$ to a clear colourless stirred solution of $(\boldsymbol{S}, \boldsymbol{S}, \boldsymbol{S})-\mathrm{A}(30.0 \mathrm{mg}, 0.040 \mathrm{mmol}$, 0.10 eq.) in $\mathrm{Et}_{2} \mathrm{O}(2.0 \mathrm{~mL})$ under an argon atmosphere. The resulting dark mixture was allowed to stir for an additional $10 \mathrm{~min}$ before canrenone $(136 \mathrm{mg}, 0.40 \mathrm{mmol}, 1.0$ eq.) and TMSCl (0.26 mL, $2.0 \mathrm{mmol}, 5.0$ eq.) were added sequentially dropwise via syringe. Stirring at room temperature was continued arbitrarily for $15 \mathrm{~h}$ before the reaction was quenched by the addition of $\mathrm{Et}_{2} \mathrm{O}$ ( $c a 3$ $\mathrm{mL}$ ) and $\mathrm{NH}_{4} \mathrm{Cl}$ (1 M aq., ca $1.5 \mathrm{~mL}$ ). The mixture was partitioned between the aqueous and $\mathrm{Et}_{2} \mathrm{O}$ layers and the former phase was extracted with $\mathrm{Et}_{2} \mathrm{O}(3 \times 10 \mathrm{~mL})$. The combined organic phase was washed with $\mathrm{NaHCO}_{3}$ (sat. aq., ca $10 \mathrm{~mL}$ ), dried $\left(\mathrm{Na}_{2} \mathrm{SO}_{4}\right)$, filtered and concentrated in vacuo to give an oil. Flash column chromatography of the yellow residue (30:70; EtOAc:petrol; $\mathrm{SiO}_{2}$ )

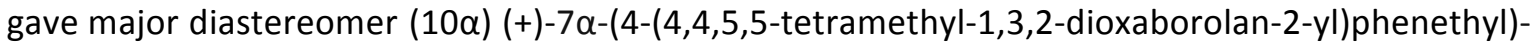
17a-hydroxy-3-oxo-pregn-4-ene-21-carboxylic acid $y$-lactone (104 mg, $0.18 \mathrm{mmol}, 45 \%)$, minor diastereomer (+)-7 $\beta$-(4-(4,4,5,5-tetramethyl-1,3,2-dioxaborolan-2-yl)phenethyl)-17 $\alpha$-hydroxy-3oxo-pregn-4-ene-21-carboxylic acid $\gamma$-lactone was not seen.

${ }^{1} \mathrm{H}$ NMR (400 MHz, CDCl $) \delta_{\mathrm{H}} / \mathrm{ppm} 0.97(\mathrm{~s}, 3 \mathrm{H}), 1.23(\mathrm{~s}, 3 \mathrm{H}), 1.25-1.33(\mathrm{~m}, 4 \mathrm{H}), 1.35$ (br. s, 12 H), 1.38 - $1.56(\mathrm{~m}, 6 \mathrm{H}), 1.60-1.94(\mathrm{~m}, 6 \mathrm{H}), 2.04-2.12(\mathrm{~m}, 1 \mathrm{H}), 2.31-2.58(\mathrm{~m}, 8 \mathrm{H}), 2.73-2.83$ $(\mathrm{m}, 1 \mathrm{H}), 5.84(\mathrm{~s}, 1 \mathrm{H}), 7.16(\mathrm{~d}, J=7.9 \mathrm{~Hz}, 2 \mathrm{H}), 7.74(\mathrm{~d}, J=7.9 \mathrm{~Hz}, 2 \mathrm{H})$.

${ }^{13} \mathrm{C}$ NMR $\left(100 \mathrm{MHz}, \mathrm{CDCl}_{3}\right) \delta_{\mathrm{C}} / \mathrm{ppm} 14.3,18.1,20.7,22.2,24.8$ (5 C), 27.5, 29.3, 31.2, 31.4, 33.7, 34.0, 35.3, 35.6, 36.0, 36.4, 38.6, 39.2, 44.7, 45.6, 46.9, 83.7 (2 C), 95.8, 126.0, 127.9 (2 C), 135.0 (2 C), 145.4, 169.2, 176.8, 199.1 .

HRMS (ESI) $\mathrm{m} / \mathrm{z}$ calcd for $\mathrm{C}_{36} \mathrm{H}_{49} \mathrm{BNaO}_{5}[\mathrm{M}+\mathrm{Na}]^{+}: 595.3565$ found: 595.3562 .

$[\alpha]^{20}{ }_{589}=+50.4\left(c 0.51, \mathrm{CHCl}_{3}\right)$.

IR $\left(v_{\max } / \mathbf{c m}^{-1}\right):$ 1360, 1666, 1769, 2976.

Absolute configuration assigned by analogy to compound 32c. 


\section{(-)-(R)-3-Methyl-3-(4-phenylbutyl)cyclohexanone (33a)}

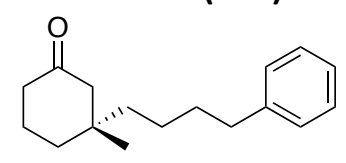

$\mathrm{CuCl}(3.6 \mathrm{mg}, 0.04 \mathrm{mmol}, 0.10$ eq.) and the phosphoramidite ligand $63(21.6 \mathrm{mg}, 0.040 \mathrm{mmol}, 0.10$ eq.) were dissolved in $t$-BuOMe $(2.0 \mathrm{~mL})$ under an argon atmosphere and the resulting mixture allowed to stir at room temperature. After 1 hour, $\operatorname{AgNTf}_{2}(23.2 \mathrm{mg}, 0.060 \mathrm{mmol}, 0.15$ eq.) was added and the suspension was stirred for another $15 \mathrm{~min}$. In another flask, $\mathrm{Cp}_{2} \mathrm{ZrHCl}(206 \mathrm{mg}, 0.80$ mmol, 2.0 eq.) was added to a stirred, room temperature, solution of 4-phenyl-1-butene (0.15 $\mathrm{mL}, 1.0 \mathrm{mmol}, 2.5$ eq.) in $\mathrm{CH}_{2} \mathrm{Cl}_{2}(0.40 \mathrm{~mL})$ under an argon atmosphere. After stirring for $15 \mathrm{~min}$, the stirred solution containing the copper and ligand was transferred and filtered using a syringe filter to the clear yellow solution containing the alkene/zirconium mixture. The resulting black mixture was allowed to stir for an additional $10 \mathrm{~min}$ before 3-methyl-2-cyclohexenone (45 $\mu \mathrm{L}$, $0.40 \mathrm{mmol}, 1.0$ eq.) was added dropwise via syringe. Stirring was continued for about $12 \mathrm{~h}$ before the reaction was quenched by the addition of $\mathrm{Et}_{2} \mathrm{O}(c a 3 \mathrm{~mL})$ and then $\mathrm{NH}_{4} \mathrm{Cl}(1 \mathrm{M}$ aq., $c a 1.5 \mathrm{~mL})$. The mixture was partitioned between the aqueous and $\mathrm{Et}_{2} \mathrm{O}$ layers and the aqueous phase extracted with $\mathrm{Et}_{2} \mathrm{O}(3 \times 10 \mathrm{~mL})$. The combined organic phase was washed with $\mathrm{NaHCO}_{3}$ (aq. sat., ca $10 \mathrm{~mL})$, dried $\left(\mathrm{Na}_{2} \mathrm{SO}_{4}\right)$, filtered and concentrated in vacuo. Flash column chromatography of the yellow residue (1:9; EtOAc:petrol; $\mathrm{SiO}_{2}$ ) gave (-)-(R)-3-Methyl-3-(4-phenylbutyl)cyclohexanone (65 mg, $0.27 \mathrm{mmol}, 66 \%$ ) as a colourless oil.

HPLC analysis indicated an enantiomeric excess of $94 \%$ [Chiralpak ${ }^{\circledR}$ IC; flow: $1 \mathrm{~mL} / \mathrm{min}$; hexane/iPrOH: 98:2; $\lambda=210 \mathrm{~nm}$; major enantiomer, $t_{R}=16.8 \mathrm{~min}$; minor enantiomer, $\left.t_{R}=17.9 \mathrm{~min}\right]$.

${ }^{1} \mathrm{H}$ NMR $\left(400 \mathrm{MHz}, \mathrm{CDCl}_{3}\right) \delta_{\mathrm{H}} / \mathrm{ppm} 0.82(\mathrm{~s}, 3 \mathrm{H}), 1.21(\mathrm{~m}, 4 \mathrm{H}), 1.39-1.61(\mathrm{~m}, 4 \mathrm{H}), 1.76(\mathrm{~m}, 2 \mathrm{H})$, $1.97-2.05(\mathrm{~m}, 1 \mathrm{H}), 2.06-2.13(\mathrm{~m}, 1 \mathrm{H}), 2.19(\mathrm{t}, J=6.8 \mathrm{~Hz}, 2 \mathrm{H}), 2.53$ (br. $t, J=6.8,6.8 \mathrm{~Hz}, 2 \mathrm{H}$ ), $7.04-7.12(m, 3 H), 7.16-7.23(m, 2 H)$.

${ }^{13} \mathrm{C}$ NMR $\left(100 \mathrm{MHz}, \mathrm{CDCl}_{3}\right) \delta_{\mathrm{c}} / \mathrm{ppm} 22.1,23.0,25.0,32.0,35.8,35.8,38.5,40.9,41.4,53.7,125.6$, $128.2(2 \mathrm{C}), 128.3(2 \mathrm{C}), 142.5,212.4$.

HRMS (ESI) $\mathrm{m} / \mathrm{z}$ calcd for $\mathrm{C}_{17} \mathrm{H}_{24} \mathrm{NaO}[\mathrm{M}+\mathrm{Na}]^{+}: 267.1719$, found: 267.1715 .

$[\alpha]^{20}{ }_{589}=-2.8\left(c 0.92, \mathrm{CHCl}_{3}\right)$.

IR $\left(v_{\max } / \mathrm{cm}^{-1}\right):$ 776, 836, 1088, 1252, 1713, 2857, 2932.

Absolute configuration assigned by analogy. ${ }^{40}$ 


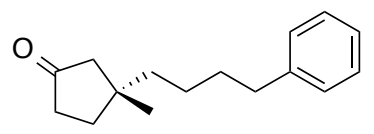

$\mathrm{CuCl}(1.8 \mathrm{mg}, 0.02 \mathrm{mmol}, 0.10$ eq.) and phosphoramidite ligand 63 (18.8 mg, $0.020 \mathrm{mmol}, 0.10$ eq.) were dissolved in $t$-BuOMe $(1.0 \mathrm{~mL})$ under an argon atmosphere and allowed to stir for $1 \mathrm{~h}$ at room temperature. $\operatorname{AgNTf}_{2}(8.5 \mathrm{mg}, 0.022 \mathrm{mmol}, 0.11$ eq.) was added and the suspension was stirred for another $15 \mathrm{~min}$. In another flask, $\mathrm{Cp}_{2} \mathrm{ZrHCl}(309 \mathrm{mg}, 1.60 \mathrm{mmol}, 3.0$ eq.) was added to a stirred, room temperature, solution of hex-4-en-1-ylbenzene $\left(0.3 \mathrm{~mL}, 2.0 \mathrm{mmol}, 5.0\right.$ eq.) in $\mathrm{CH}_{2} \mathrm{Cl}_{2}$ $(0.80 \mathrm{~mL})$ under an argon atmosphere. After stirring for $15 \mathrm{~min}$, the stirred solution containing the copper and ligand was transferred and filtered using a syringe filter to the clear yellow solution. The resulting black mixture was cooled with an ice bath allowed to stir for an additional 20 min before 3-methyl-2-cyclopentanone (20 $\mu \mathrm{L}, 0.20 \mathrm{mmol}, 1.0$ eq.) was added dropwise via syringe. Stirring continued $12 \mathrm{~h}$ leaving the ice melts so the reaction mixture slowly warmed up. The reaction was quenched by the addition of $\mathrm{Et}_{2} \mathrm{O}\left(c a 3 \mathrm{~mL}\right.$ ) and then $\mathrm{NH}_{4} \mathrm{Cl}(1 \mathrm{M}$ aq., $c a 1.5 \mathrm{~mL}$ ). The mixture was partitioned between the aqueous and $\mathrm{Et}_{2} \mathrm{O}$ layers and the aqueous phase extracted with $\mathrm{Et}_{2} \mathrm{O}(3 \times 10 \mathrm{~mL})$. The combined organic phase was washed with $\mathrm{NaHCO}_{3}$ (aq. sat., ca $\left.10 \mathrm{~mL}\right)$, dried $\left(\mathrm{Na}_{2} \mathrm{SO}_{4}\right)$, filtered and concentrated in vacuo. Flash column chromatography of the yellow residue (1:9; EtOAc:petrol; $\mathrm{SiO}_{2}$ ) gave (-)-(R)-3-Methyl-3-(4-phenylbutyl)cyclopentanone (25.8 mg, $0.11 \mathrm{mmol}, 56 \%)$ as a colourless oil.

HPLC analysis indicated an enantiomeric excess of $65 \%$ [Chiralpak ${ }^{\circledR} \mathrm{AY}-\mathrm{H}$; flow: $1 \mathrm{~mL} / \mathrm{min}$; hexane/i-PrOH: 95:5; $\lambda=210 \mathrm{~nm}$; major enantiomer, $t_{R}=9.50 \mathrm{~min}$; minor enantiomer, $t_{R}=8.60$ $\min ]$.

${ }^{1} \mathrm{H}$ NMR $\left(500 \mathrm{MHz}, \mathrm{CDCl}_{3}\right) \delta_{\mathrm{H}} / \mathrm{ppm} 1.05(\mathrm{~s}, 3 \mathrm{H}), 1.26-1.47(\mathrm{~m}, 4 \mathrm{H}), 1.64(\mathrm{dt}, J=15.1,7.4 \mathrm{~Hz}, 2 \mathrm{H})$, $1.73-1.85(\mathrm{~m}, 2 \mathrm{H}), 1.98-2.05(\mathrm{~m}, 1 \mathrm{H}), 2.05-2.12(\mathrm{~m}, 1 \mathrm{H}), 2.26-2.32(\mathrm{~m}, 2 \mathrm{H}), 2.64(\mathrm{t}, J=7.6$ $\mathrm{Hz}, 2 \mathrm{H}), 7.13-7.22(\mathrm{~m}, 3 \mathrm{H}), 7.25-7.33(\mathrm{~m}, 2 \mathrm{H})$.

${ }^{13} \mathrm{C}$ NMR $\left(125 \mathrm{MHz}, \mathrm{CDCl}_{3}\right) \delta_{\mathrm{C}} / \mathrm{ppm} 24.4,25.0,32.1,35.2,35.8,36.8,39.5,41.6,52.2,125.7$, $128.28(2 \mathrm{C}), 128.32(2 \mathrm{C}), 142.5,220.2$.

HRMS (ESI) $\mathrm{m} / \mathrm{z}$ calcd for $\mathrm{C}_{16} \mathrm{H}_{22} \mathrm{NaO}[\mathrm{M}+\mathrm{Na}]^{+}: 253.1563$ found: 253.1562 .

$[\alpha]^{20}{ }_{589}=+22.64\left(\mathrm{c} 0.53, \mathrm{CHCl}_{3}\right)$.

IR (ATR) v (cm $\left.{ }^{-1}\right):$ 699, 1454, 1496, 1711, 2930.

Absolute configuration assigned by analogy. ${ }^{40}$ 


\section{(-)-(R)-4-(4-Phenylbutyl)tetrahydro-2H-pyran-2-one (ent-36a)}

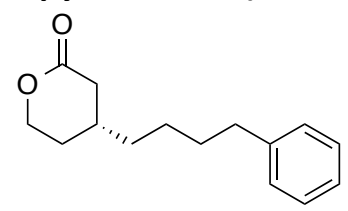

$\mathrm{Cp}_{2} \mathrm{ZrHCl}$ (206 mg, $0.80 \mathrm{mmol}, 2.0$ eq.) was added to a stirred, room temperature, solution of 4-phenyl-1-butene $\left(0.15 \mathrm{~mL}, 1.0 \mathrm{mmol}, 2.5\right.$ eq.) in $\mathrm{CH}_{2} \mathrm{Cl}_{2}(0.40 \mathrm{~mL})$ under an argon atmosphere. After stirring for $30 \mathrm{~min}$ at room temperature, the resulting clear yellow solution was transferred via syringe over about $1 \mathrm{~min}$ to a clear colourless stirred solution of $(\boldsymbol{R})-\mathbf{B}(30.0 \mathrm{mg}, 0.040 \mathrm{mmol}$, 0.10 eq.) in $\mathrm{Et}_{2} \mathrm{O}(2.0 \mathrm{~mL})$ under an argon atmosphere. The resulting dark mixture was allowed to stir for $10 \mathrm{~min}$ before 5,6-dihydro-2H-pyran-2-one ( $34 \mu \mathrm{L}, 0.40 \mathrm{mmol}, 1.0$ eq.) and then TMSCl ( $0.25 \mathrm{~mL}, 2.0 \mathrm{mmol}, 5.0$ eq.) were added via syringe. Stirring at room temperature was continued for $4 \mathrm{~h}$ before the reaction was quenched by the addition of $\mathrm{Et}_{2} \mathrm{O}(\mathrm{ca} 3 \mathrm{~mL})$ and then $\mathrm{NH}_{4} \mathrm{Cl}(1 \mathrm{M}$ aq., $c a 1.5 \mathrm{~mL}$ ). The mixture was partitioned between water and $\mathrm{Et}_{2} \mathrm{O}$ and the aqueous phase was extracted with $\mathrm{Et}_{2} \mathrm{O}(3 \times 10 \mathrm{~mL})$. The combined organic phase was washed with $\mathrm{NaHCO}_{3}$ (aq. sat., ca $10 \mathrm{~mL})$, dried $\left(\mathrm{MgSO}_{4}\right)$, filtered and concentrated in vacuo. Flash column chromatography of the yellow residue (1:9; EtOAc:petrol; $\mathrm{SiO}_{2}$ ) gave (-)-(R)-4-(4-phenylbutyl)tetrahydro-2H-pyran-2one (77 $\mathrm{mg}, 0.33 \mathrm{mmol}, 83 \%$ ) as a yellow oil.

HPLC analysis indicated an enantiomeric excess of $89 \%$ [Chiralpak ${ }^{\circledR} \mathrm{IA}$; flow: $1.0 \mathrm{~mL} / \mathrm{min}$; hexane/i-PrOH: 99.2:0.8; $\lambda=210 \mathrm{~nm}$; major enantiomer, $\mathrm{t}_{\mathrm{R}}=38.05 \mathrm{~min}$; minor enantiomer, $\mathrm{t}_{\mathrm{R}}=$ $45.48 \mathrm{~min}]$.

${ }^{1} \mathrm{H}$ NMR $\left(400 \mathrm{MHz}, \mathrm{CDCl}_{3}\right) \delta_{\mathrm{H}} / \mathrm{ppm}: 1.23-1.35(\mathrm{~m}, 4 \mathrm{H}), 1.37-1.49(\mathrm{~m}, 1 \mathrm{H}), 1.51-1.63(\mathrm{~m}, 1 \mathrm{H}), 1.80-$ $1.93(\mathrm{~m}, 1 \mathrm{H}), 2.05(\mathrm{dd}, J=17.2,9.9 \mathrm{~Hz}, 1 \mathrm{H}), 2.55(\mathrm{t}, J=7.5 \mathrm{~Hz}, 2 \mathrm{H}), 2.60$ (ddd, $J=17.2,5.6,1.6 \mathrm{~Hz}$, $1 \mathrm{H}), 4.16(\mathrm{td}, J=10.6,3.7 \mathrm{~Hz}, 1 \mathrm{H}), 4.28-4.36(\mathrm{~m}, 1 \mathrm{H}), 7.07-7.15(\mathrm{~m}, 3 \mathrm{H}), 7.17-7.24(\mathrm{~m}, 1 \mathrm{H})$.

${ }^{13} \mathrm{C}$ NMR $\left(100 \mathrm{MHz}, \mathrm{CDCl}_{3}\right) \delta_{\mathrm{c}} / \mathrm{ppm}: 26.0,28.9,31.3,31.5,35.8,36.1,36.6,68.5,125.8,128.4$ (4 C), $142.3,171.4$.

HRMS (EI) $m / z$ calcd for $\mathrm{C}_{15} \mathrm{H}_{20} \mathrm{NaO}_{2}[\mathrm{M}+\mathrm{Na}]^{+}: 255.1356$, found: 255.1358 .

$[\alpha]_{589}^{20}:-1.2\left(c 1.00, \mathrm{CHCl}_{3}\right)$.

IR $\left(v_{\max } / \mathrm{cm}^{-1}\right): 1717,3024$.

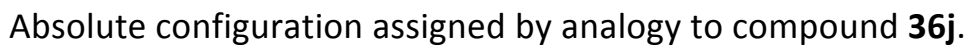


$(+)-(R)-4-E t h y l t e t r a h y d r o-2 H-p y r a n-2-o n e(36 b)$

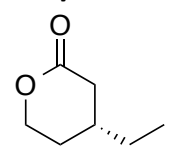

Ethylene was bubbled through a stirred, room temperature suspension of $\mathrm{Cp}_{2} \mathrm{ZrHCl}$ ( $206 \mathrm{mg}, 0.80$ mmol, 2.0 eq.) in $\mathrm{CH}_{2} \mathrm{Cl}_{2}(1.0 \mathrm{~mL}$ ) for $1 \mathrm{~min}$. After $1 \mathrm{~min}$ the reaction was stirred for $15 \mathrm{~min}$ under an ethylene atmosphere using a balloon. In another flask the $(R)$-B $(30.0 \mathrm{mg}, 0.040 \mathrm{mmol}, 0.10$ eq.) was dissolved in $\mathrm{Et}_{2} \mathrm{O}(2 \mathrm{~mL})$ and transferred via syringe to the resulting clear yellow solution ensuring the reaction is kept under an ethylene atmosphere. The resulting dark mixture was allowed to stir for an additional $10 \mathrm{~min}$ before 5,6-dihydro-2H-pyran-2-one (34 $\mu \mathrm{l}, 0.40 \mathrm{mmol}, 1.0$ eq.) and then $\mathrm{TMSCl}(0.25 \mathrm{~mL}, 2.0 \mathrm{mmol}, 5.0$ eq.) were added dropwise via syringe. The reaction was stirred for $3 \mathrm{~h}$ before the reaction was quenched by the addition of $\mathrm{Et}_{2} \mathrm{O}(\mathrm{ca} 3 \mathrm{~mL}$ ) and then $\mathrm{NH}_{4} \mathrm{Cl}$ (1 M. aq., ca $1.5 \mathrm{~mL}$ ). The mixture was partitioned between water and $\mathrm{Et}_{2} \mathrm{O}$ and the aqueous phase extracted with $\mathrm{Et}_{2} \mathrm{O}(3 \times 10 \mathrm{~mL})$. The combined organic phase was washed with $\mathrm{NaHCO}_{3}$ (aq. sat., ca $10 \mathrm{~mL}$ ), dried $\left(\mathrm{MgSO}_{4}\right)$, filtered and concentrated in vacuo, ensuring the vacuum does not go below 200 mbar due to the product being volatile. Flash column chromatography of the residue (25:75; $t$-BuOMe:petrol; $\left.\mathrm{SiO}_{2}\right)$ gave $(+)-(R)$-4-ethyltetrahydro- $2 \mathrm{H}$ pyran-2-one ( $27.2 \mathrm{mg}, 0.21 \mathrm{mmol}, 53 \%$ ) as a colourless oil.

HPLC analysis of the amide derivative of 36b (see general procedure for details) indicated an enantiomeric excess of $92 \%$ [Chiralpak ${ }^{\circledR}$ IC; flow: $1.0 \mathrm{~mL} / \mathrm{min}$; hexane/i-PrOH: 80:20; $\lambda=210 \mathrm{~nm}$; major enantiomer, $t_{R}=10.50 \mathrm{~min}$; minor enantiomer, $\left.t_{R}=12.05 \mathrm{~min}\right]$.

Data in accordance with published literature. ${ }^{95}$

${ }^{1} \mathrm{H}$ NMR $\left(400 \mathrm{MHz}, \mathrm{CDCl}_{3}\right) \delta_{\mathrm{H}} / \mathrm{ppm} 0.94(\mathrm{t}, J=7.5 \mathrm{~Hz}, 3 \mathrm{H}), 1.34-1.45(\mathrm{~m}, 2 \mathrm{H}), 1.46-1.59(\mathrm{~m}, 1$ H), $1.81-2.02(m, 2 H), 2.09-2.21(m, 1 H), 2.65-2.75(m, 1 H), 4.19-4.32(m, 1 H), 4.37-4.46$ $(\mathrm{m}, 1 \mathrm{H})$.

${ }^{13} \mathrm{C} \mathrm{NMR}\left(100 \mathrm{MHz}, \mathrm{CDCl}_{3}\right) \delta_{\mathrm{c}} / \mathrm{ppm} 10.9,28.5,28.9,33.1,36.3,68.6,171.6$.

MS (ESI) $m / z[\mathrm{M}+\mathrm{Na}]^{+}: 151.1(100)$.

$[\alpha]^{20}{ }_{589}=+20.63\left(c 0.63, \mathrm{CHCl}_{3}\right)$.

IR $\left(v_{\max } / \mathrm{cm}^{-1}\right): 1071,1223,1255,1735,2963$.

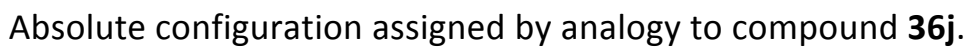




\section{$(+)-(R)-4-H e x y l t e t r a h y d r o-2 H-p y r a n-2-o n e(36 c)$}

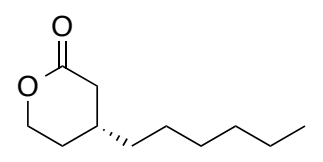

$\mathrm{Cp}_{2} \mathrm{ZrHCl}$ (206 mg, $0.80 \mathrm{mmol}, 2.0$ eq.) was added to a stirred, room temperature, solution of 1-hexene (0.13 mL, $1.0 \mathrm{mmol}, 2.5$ eq.) in $\mathrm{CH}_{2} \mathrm{Cl}_{2}(0.40 \mathrm{~mL})$ under an argon atmosphere. After stirring for $20 \mathrm{~min}$, the resulting clear yellow solution was transferred via syringe over about $1 \mathrm{~min}$ to a clear colourless stirred solution of $(\boldsymbol{R})-\mathbf{B}\left(30.0 \mathrm{mg}, 0.040 \mathrm{mmol}, 0.10\right.$ eq.) in $\mathrm{Et}_{2} \mathrm{O}(2.0 \mathrm{~mL})$ under an argon atmosphere. The resulting dark mixture was allowed to stir for an additional 10 min before 5,6-dihydro-2H-pyran-2-one ( $34 \mu \mathrm{L}, 0.40 \mathrm{mmol}, 1.0$ eq.) and then TMSCl (0.25 mL, 2.0 mmol, 5.0 eq.) were added via syringe. Stirring at room temperature was continued for $2 \mathrm{~h}$ before the reaction was quenched by the addition of $\mathrm{Et}_{2} \mathrm{O}(c a 3 \mathrm{~mL})$ and then $\mathrm{NH}_{4} \mathrm{Cl}(1 \mathrm{M}$ aq., $c a 1.5 \mathrm{~mL})$. The mixture was partitioned between water and $\mathrm{Et}_{2} \mathrm{O}$ and the aqueous phase extracted with $\mathrm{Et}_{2} \mathrm{O}$ $\left(3 \times 10 \mathrm{~mL}\right.$ ). The combined organic phase was washed with $\mathrm{NaHCO}_{3}$ (aq. sat., $c a 10 \mathrm{~mL}$ ), dried $\left(\mathrm{MgSO}_{4}\right)$, filtered and concentrated in vacuo. Flash column chromatography of the yellow residue (1:9; EtOAc:petrol; $\mathrm{SiO}_{2}$ ) gave (+)-(R)- 4-hexyltetrahydro-2H-pyran-2-one (54 mg, $0.29 \mathrm{mmol}, 73 \%$ ) as a yellow oil.

HPLC analysis of the amide derivative of 36c (see general procedure for details) indicated an enantiomeric excess of $89 \%$ [Chiralpak ${ }^{\circledR}$ IC; flow: $1.0 \mathrm{~mL} / \mathrm{min}$; hexane/i-PrOH: 90:10; $\lambda=210 \mathrm{~nm}$; major enantiomer, $t_{R}=25.28 \mathrm{~min}$; minor enantiomer, $\left.t_{R}=31.20 \mathrm{~min}\right]$.

${ }^{1} \mathrm{H}$ NMR $\left(400 \mathrm{MHz}, \mathrm{CDCl}_{3}\right) \delta_{\mathrm{H}} / \mathrm{ppm} 0.82(\mathrm{t}, J=7.1 \mathrm{~Hz}, 3 \mathrm{H}), 1.15-1.35(\mathrm{~m}, 10 \mathrm{H}), 1.39-1.51(\mathrm{~m}, 1 \mathrm{H})$, 1.83-1.95 (m, 2H) $2.05(\mathrm{dd}, J=17.1,10.3 \mathrm{~Hz}, 1 \mathrm{H}), 2.62(\mathrm{ddd}, J=17.4,5.9,1.7 \mathrm{~Hz}, 1 \mathrm{H}), 4.19(\mathrm{td}, J=$ $10.83 .4 \mathrm{~Hz}, 1 \mathrm{H}), 4.35(\mathrm{dt}, J=11.3,3.9 \mathrm{~Hz}, 1 \mathrm{H})$.

${ }^{13} \mathrm{C}$ NMR $\left(100 \mathrm{MHz}, \mathrm{CDCl}_{3}\right) \delta_{\mathrm{c}} / \mathrm{ppm} 14.1,22.6,26.4,28.9,29.1,31.5,31.7,36.2,36.6,68.6,171.6$

HRMS (EI) $m / z$ calcd for $\mathrm{C}_{11} \mathrm{H}_{20} \mathrm{NaO}_{2}[\mathrm{M}+\mathrm{Na}]^{+}: 207.1356$, found: 207.1360

$[\alpha]^{20}{ }_{589}:+20.5\left(c 0.60, \mathrm{CHCl}_{3}\right)$.

IR $\left(v_{\max } / \mathrm{cm}^{-1}\right):$ 1558, 1737, 2855, 2924.

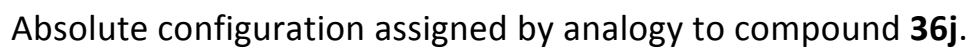




\section{$(+)-(R)-4-O c t y l t e t r a h y d r o-2 H-p y r a n-2-o n e(36 d)$}

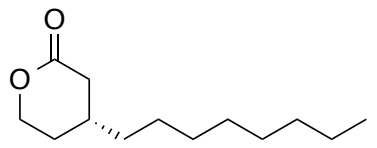

$\mathrm{Cp}_{2} \mathrm{ZrHCl}$ (206 mg, $0.80 \mathrm{mmol}, 2.0$ eq.) was added to a stirred, room temperature, solution of 1-octene $\left(0.16 \mathrm{~mL}, 1.0 \mathrm{mmol}, 2.5\right.$ eq.) in $\mathrm{CH}_{2} \mathrm{Cl}_{2}(0.40 \mathrm{~mL})$ under an argon atmosphere. After stirring for $15 \mathrm{~min}$, the resulting clear yellow solution was transferred via syringe over about $1 \mathrm{~min}$ to a clear colourless stirred solution of $(\boldsymbol{R})-\mathbf{B}\left(30.0 \mathrm{mg}, 0.040 \mathrm{mmol}, 0.10\right.$ eq.) in $\mathrm{Et}_{2} \mathrm{O}(2.0 \mathrm{~mL})$ under an argon atmosphere. The resulting dark mixture was allowed to stir for an additional 10 min before 5,6-dihydro-2H-pyran-2-one ( $34 \mu \mathrm{L}, 0.40 \mathrm{mmol}, 1.0$ eq.) and then TMSCl $(0.25 \mathrm{~mL}, 2.0$ $\mathrm{mmol}, 5.0$ eq.) were added dropwise via syringe. Stirring at room temperature was continued for $4 \mathrm{~h}$ before the reaction was quenched by the addition of $\mathrm{Et}_{2} \mathrm{O}(c a 3 \mathrm{~mL})$ and $\mathrm{NH}_{4} \mathrm{Cl}(1 \mathrm{M}$ aq., $c a 1.5$ $\mathrm{mL}$ ). The mixture was partitioned between water and $\mathrm{Et}_{2} \mathrm{O}$ and the aqueous phase extracted with $\mathrm{Et}_{2} \mathrm{O}(3 \times 10 \mathrm{~mL})$. The combined organic phase was washed with $\mathrm{NaHCO}_{3}$ (sat. aq., $c a 10 \mathrm{~mL}$ ), dried $\left(\mathrm{MgSO}_{4}\right)$, filtered and concentrated in vacuo. Flash column chromatography of the yellow residue (1:9; EtOAc:petrol; $\mathrm{SiO}_{2}$ ) gave (+)-(R)- 4-octyltetrahydro-2H-pyran-2-one (71 mg, $\left.0.34 \mathrm{mmol}, 84 \%\right)$ as a yellow oil.

HPLC analysis of the amide derivative of $\mathbf{3 6 \mathbf { d }}$ (see general procedure for details) indicated an enantiomeric excess of $89 \%$ [Chiralpak ${ }^{\circledR} \mathrm{IC}$; flow: $1.0 \mathrm{~mL} / \mathrm{min}$; hexane/i-PrOH: $90: 10 ; \lambda=210 \mathrm{~nm}$; major enantiomer, $t_{R}=23.20 \mathrm{~min}$; minor enantiomer, $\left.t_{R}=27.99 \mathrm{~min}\right]$.

${ }^{1} \mathrm{H}$ NMR $\left(400 \mathrm{MHz}, \mathrm{CDCl}_{3}\right) \delta_{\mathrm{H}} / \mathrm{ppm}: 0.71(\mathrm{t}, J=6.9 \mathrm{~Hz}, 3 \mathrm{H}), 1.03-1.22(\mathrm{~m}, 15 \mathrm{H}), 1.29-1.41(\mathrm{~m}, 1 \mathrm{H})$, 1.70-1.85 (m, 2H), 1.97 (dd, $J=17.4,10.3 \mathrm{~Hz}, 1 \mathrm{H}$ ), 2.52 (ddd, $J=17.4,5.9,2.0 \mathrm{~Hz}, 1 \mathrm{H}$ ), 4.08 (td, $J=10.8,3.4 \mathrm{~Hz}, 1 \mathrm{H}), 4.24(\mathrm{dt}, J=11.3,4.6 \mathrm{~Hz}, 1 \mathrm{H})$.

${ }^{13} \mathrm{C}$ NMR $\left(100 \mathrm{MHz}, \mathrm{CDCl}_{3}\right) \delta_{\mathrm{c}} / \mathrm{ppm}: 14.1,22.7,26.4,28.9,29.2,29.5,29.5,31.4,31.9,36.2,36.6$, 68.6, 171.6.

HRMS (EI) $m / z$ calcd for $\mathrm{C}_{13} \mathrm{H}_{24} \mathrm{NaO}_{2}[\mathrm{M}+\mathrm{Na}]^{+}: 235.1669$, found: 235.1678 .

$[\alpha]_{589}^{20}:+14.6\left(c 1.05, \mathrm{CHCl}_{3}\right)$.

IR $\left(v_{\max } / \mathrm{cm}^{-1}\right):$ 1465, 1738, 2854, 2923.

Absolute configuration assigned by analogy to compound $\mathbf{3 6 \mathbf { j }}$. 
(+)-(R)-4-Dodecyltetrahydro-2H-pyran-2-one (36e)

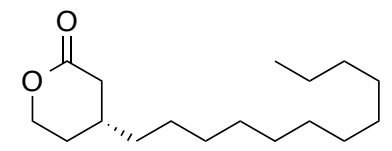

$\mathrm{Cp}_{2} \mathrm{ZrHCl}$ (206 mg, $0.80 \mathrm{mmol}, 2.0$ eq.) was added to a stirred, room temperature, solution of 1-dodecene $\left(0.17 \mathrm{~mL}, 1.0 \mathrm{mmol}, 2.5\right.$ eq.) in $\mathrm{CH}_{2} \mathrm{Cl}_{2}(0.40 \mathrm{~mL})$ under an argon atmosphere. After stirring for about $30 \mathrm{~min}$, the resulting clear yellow solution was transferred via syringe over about $1 \mathrm{~min}$ to a clear colourless stirred solution of $(\boldsymbol{R})-\mathbf{B}\left(30.0 \mathrm{mg}, 0.040 \mathrm{mmol}, 0.10 \mathrm{eq}\right.$.) in $\mathrm{Et}_{2} \mathrm{O}$ $(2.0 \mathrm{~mL})$ under an argon atmosphere. The resulting dark mixture was allowed to stir for an additional $10 \mathrm{~min}$ before 5,6-dihydro-2H-pyran-2-one ( $34 \mu \mathrm{l}, 0.40 \mathrm{mmol}, 1.0 \mathrm{eq}$.) and then TMSCl (0.25 mL, $2.0 \mathrm{mmol}, 5.0$ eq.) were added dropwise via syringe. The reaction was stirred for $3 \mathrm{~h}$ before the reaction was quenched by the addition of $\mathrm{Et}_{2} \mathrm{O}(c a 3 \mathrm{~mL})$ and then $\mathrm{NH}_{4} \mathrm{Cl}(1 \mathrm{M}$. aq., $c a$ $1.5 \mathrm{~mL}$ ). The mixture was partitioned between water and $\mathrm{Et}_{2} \mathrm{O}$ and the aqueous phase extracted with $\mathrm{Et}_{2} \mathrm{O}(3 \times 10 \mathrm{~mL})$. The combined organic phase was washed with $\mathrm{NaHCO}_{3}$ (aq. sat., ca $\left.10 \mathrm{~mL}\right)$, dried $\left(\mathrm{MgSO}_{4}\right)$, filtered and concentrated in vacuo. Flash column chromatography of the yellow residue (25:75; $\mathrm{Et}_{2} \mathrm{O}$ :petrol; $\mathrm{SiO}_{2}$ ) gave (+)-(R)-4-dodecyltetrahydro-2H-pyran-2-one $(75.1 \mathrm{mg}, 0.28$ $\mathrm{mmol}, 70 \%$ ) as light yellow solid.

HPLC analysis of the amide derivative of 36e (see general procedure for details) indicated an enantiomeric excess of $88 \%$ [Chiralpak ${ }^{\oplus}$ IC; flow: $1.5 \mathrm{~mL} / \mathrm{min}$; hexane/i-PrOH: $85: 15 ; \lambda=210 \mathrm{~nm}$; major enantiomer, $t_{R}=11.14 \mathrm{~min}$; minor enantiomer, $\left.t_{R}=12.93 \mathrm{~min}\right]$.

${ }^{1} \mathrm{H}$ NMR $\left(400 \mathrm{MHz}, \mathrm{CDCl}_{3}\right) \delta_{\mathrm{H}} / \mathrm{ppm} 0.71(\mathrm{t}, J=6.8 \mathrm{~Hz}, 3 \mathrm{H}), 1.02-1.21(\mathrm{~m}, 22 \mathrm{H}), 1.28-1.41(\mathrm{~m}, 1$ H), 1.72 - 1.84 (m, 2 H), 1.97 (dd, J = 17.3, 10.0 Hz, 1 H), 2.52 (ddd, J = 17.3, 5.6, $1.5 \mathrm{~Hz}, 1 \mathrm{H}$ ), 4.04 $4.13(\mathrm{~m}, 1 \mathrm{H}), 4.19-4.29(\mathrm{~m}, 1 \mathrm{H})$.

${ }^{13} \mathrm{C}$ NMR $\left(100 \mathrm{MHz}, \mathrm{CDCl}_{3}\right) \delta_{\mathrm{c}} / \mathrm{ppm} 14.1,22.7,26.4,28.9,29.4,29.5$ (2 C), 29.59, 29.64 (2 C), 29.7, $31.5,31.9,36.2,36.6,68.6,171.5$.

HRMS (ESI) $\mathrm{m} / \mathrm{z}$ calcd for $\mathrm{C}_{17} \mathrm{H}_{32} \mathrm{O}_{2} \mathrm{Na}[\mathrm{M}+\mathrm{Na}]^{+}:$291.2295, found: 291.2284.

$[\alpha]^{20}{ }_{589}=+15.51\left(c 0.49, \mathrm{CHCl}_{3}\right)$.

IR $\left(v_{\max } / \mathbf{c m}^{-1}\right): 1087,1237,1251,1470,1733,2918$.

MP $40-42^{\circ} \mathrm{C}$.

Absolute configuration assigned by analogy to compound $\mathbf{3 6 \mathbf { j }}$. 


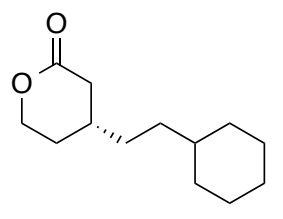

$\mathrm{Cp}_{2} \mathrm{ZrHCl}$ (206 mg, $0.80 \mathrm{mmol}, 2.0$ eq.) was added to a stirred, room temperature, solution of vinylcyclohexane $\left(0.14 \mathrm{~mL}, 1.0 \mathrm{mmol}, 2.5\right.$ eq.) in $\mathrm{CH}_{2} \mathrm{Cl}_{2}(0.40 \mathrm{~mL})$ under an argon atmosphere. After stirring for about $30 \mathrm{~min}$, the resulting clear yellow solution was transferred via syringe over about $1 \mathrm{~min}$ to a clear colourless stirred solution of $(\boldsymbol{R})-\mathrm{B}\left(30.0 \mathrm{mg}, 0.040 \mathrm{mmol}, 0.10\right.$ eq.) in $\mathrm{Et}_{2} \mathrm{O}$ $(2.0 \mathrm{~mL})$ under an argon atmosphere. The resulting dark mixture was allowed to stir for an additional $10 \mathrm{~min}$ before 5,6-dihydro-2H-pyran-2-one ( $34 \mu \mathrm{L}, 0.40 \mathrm{mmol}, 1.0$ eq.) and then TMSCl ( $0.25 \mathrm{~mL}, 2.0 \mathrm{mmol}, 5.0$ eq.) were added dropwise via syringe. The reaction was stirred for $3 \mathrm{~h}$ before the reaction was quenched by the addition of $\mathrm{Et}_{2} \mathrm{O}(c a 3 \mathrm{~mL})$ and then $\mathrm{NH}_{4} \mathrm{Cl}(1 \mathrm{M}$. aq., $c a$ $1.5 \mathrm{~mL}$ ). The mixture was partitioned between water and $\mathrm{Et}_{2} \mathrm{O}$ and the aqueous phase extracted with $\mathrm{Et}_{2} \mathrm{O}(3 \times 10 \mathrm{~mL})$. The combined organic phase was washed with $\mathrm{NaHCO}_{3}$ (aq. sat., $c a 10 \mathrm{~mL}$ ), dried $\left(\mathrm{MgSO}_{4}\right)$, filtered and concentrated in vacuo. Flash column chromatography of the yellow residue (30:70; $\mathrm{Et}_{2} \mathrm{O}$ :petrol; $\mathrm{SiO}_{2}$ ) gave $(+)-(R)-4$-(2-cyclohexylethyl)tetrahydro-2H-pyran-2-one $(50.2 \mathrm{mg}, 0.24 \mathrm{mmol}, 60 \%)$ as an off white solid.

HPLC analysis of the amide derivative of $\mathbf{3 6 f}$ (see general procedure for details) indicated an enantiomeric excess of $84 \%$ [Chiralpak ${ }^{\circledR} \mathrm{IC}$; flow: $1.0 \mathrm{~mL} / \mathrm{min}$; hexane/i-PrOH: 85:15; $\lambda=210 \mathrm{~nm}$; major enantiomer, $t_{R}=15.37 \mathrm{~min}$; minor enantiomer, $\left.t_{R}=18.56 \mathrm{~min}\right]$.

${ }^{1} \mathrm{H}$ NMR $\left(400 \mathrm{MHz}, \mathrm{CDCl}_{3}\right) \delta_{\mathrm{H}} / \mathrm{ppm} 0.70-0.89(\mathrm{~m}, 2 \mathrm{H}), 1.03-1.22(\mathrm{~m}, 6 \mathrm{H}), 1.22-1.36(\mathrm{~m}, 2 \mathrm{H})$, $1.38-1.52(\mathrm{~m}, 1 \mathrm{H}), 1.53-1.71(\mathrm{~m}, 5 \mathrm{H}), 1.77-1.94(\mathrm{~m}, 2 \mathrm{H}), 2.07(\mathrm{dd}, J=17.4,10.1 \mathrm{~Hz}, 1 \mathrm{H}), 2.62$ (ddd, $J=17.3,5.8,1.7 \mathrm{~Hz}, 1 \mathrm{H}$ ), 4.18 (ddd, $J=11.4,10.4,3.7 \mathrm{~Hz}, 1 \mathrm{H}$ ), $4.30-4.38(\mathrm{~m}, 1 \mathrm{H}$ ).

${ }^{13} \mathrm{C}$ NMR $\left(100 \mathrm{MHz}, \mathrm{CDCl}_{3}\right) \delta_{\mathrm{C}} / \mathrm{ppm} 26.3$ (2 C), 26.6, 29.0, 31.8, 33.3 (2 C), 33.5, 34.1, 36.7, 37.6, $68.6,171.5$.

HRMS (ESI) $\mathrm{m} / \mathrm{z}$ calcd for $\mathrm{C}_{13} \mathrm{H}_{22} \mathrm{O}_{2} \mathrm{Na}[\mathrm{M}+\mathrm{Na}]^{+}: 233.1512$, found: 233.1506 .

$[\alpha]^{20}{ }_{589}=+14.83\left(\mathrm{c} 0.72, \mathrm{CHCl}_{3}\right)$.

IR $\left(v_{\max } / \mathbf{c m}^{-1}\right):$ 1088, 1253, 1448, 1739, 2850, 2920.

MP $36-38^{\circ} \mathrm{C}$.

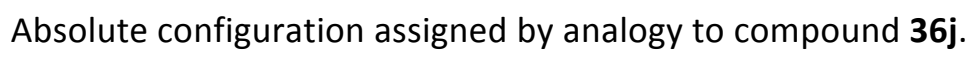


(+)-(R)-4-(3,3-Dimethylbutyl)tetrahydro-2H-pyran-2-one (36g)

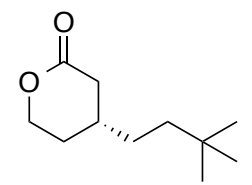

$\mathrm{Cp}_{2} \mathrm{ZrHCl}$ (206 mg, $0.80 \mathrm{mmol}, 2.0$ eq.) was added to a stirred, room temperature, solution of 3,3-dimethylbut-1-ene (0.13 mL, $1.0 \mathrm{mmol}, 2.5$ eq.) in $\mathrm{CH}_{2} \mathrm{Cl}_{2}(0.40 \mathrm{~mL})$ under an argon atmosphere. After stirring for about $30 \mathrm{~min}$, the resulting clear yellow solution was transferred via syringe over about $1 \mathrm{~min}$ to a clear colourless stirred solution of $(\boldsymbol{R})-\mathbf{B}(30.0 \mathrm{mg}, 0.040 \mathrm{mmol}, 0.10$ eq.) in $\mathrm{Et}_{2} \mathrm{O}(2.0 \mathrm{~mL})$ under an argon atmosphere. The resulting dark mixture was allowed to stir for an additional $10 \mathrm{~min}$ before 5,6-dihydro-2H-pyran-2-one (34 $\mu \mathrm{l}, 0.40 \mathrm{mmol}, 1.0$ eq.) and then TMSCl (0.25 mL, $2.0 \mathrm{mmol}, 5.0$ eq.) were added dropwise via syringe. The reaction was stirred for $3 \mathrm{~h}$ before the reaction was quenched by the addition of $\mathrm{Et}_{2} \mathrm{O}(c a 3 \mathrm{~mL})$ and then $\mathrm{NH}_{4} \mathrm{Cl}(1 \mathrm{M}$. aq., $c a 1.5 \mathrm{~mL}$ ). The mixture was partitioned between water and $\mathrm{Et}_{2} \mathrm{O}$ and the aqueous phase extracted with $\mathrm{Et}_{2} \mathrm{O}(3 \times 10 \mathrm{~mL})$. The combined organic phase was washed with $\mathrm{NaHCO}_{3}$ (aq. sat., ca $10 \mathrm{~mL})$, dried $\left(\mathrm{MgSO}_{4}\right)$, filtered and concentrated in vacuo. Flash column chromatography of the yellow residue (10:90; EtOAc:petrol; $\left.\mathrm{SiO}_{2}\right)$ gave $(+)-(R)-4-(3,3$-dimethylbutyl)tetrahydro-2Hpyran-2-one (43.1 mg, $0.23 \mathrm{mmol}, 58 \%$ ) as a light yellow oil.

HPLC analysis of the amide derivative of $\mathbf{3 6 g}$ (see general procedure for details) indicated an enantiomeric excess of $82 \%$ [Chiralpak ${ }^{\circledast} \mathrm{IC}$; flow: $1.0 \mathrm{~mL} / \mathrm{min}$; hexane/i-PrOH: 90:10; $\lambda=210 \mathrm{~nm}$; major enantiomer, $t_{R}=22.24 \mathrm{~min}$; minor enantiomer, $\left.t_{R}=25.42 \mathrm{~min}\right]$.

${ }^{1} \mathrm{H}$ NMR $\left(400 \mathrm{MHz}, \mathrm{CDCl}_{3}\right) \delta_{\mathrm{H}} / \mathrm{ppm} 0.80-0.83(\mathrm{~m}, 9 \mathrm{H}), 1.08-1.30(\mathrm{~m}, 4 \mathrm{H}), 1.39-1.51(\mathrm{~m}, 1 \mathrm{H})$, $1.75-1.94(\mathrm{~m}, 2 \mathrm{H}), 2.03-2.13(\mathrm{~m}, 1 \mathrm{H}), 2.64$ (ddd, J = 17.3, 5.9, $1.6 \mathrm{~Hz}, 1 \mathrm{H}), 4.18$ (ddd, $J=11.5$, 10.5, 3.7 Hz, $1 \mathrm{H}), 4.31-4.38(\mathrm{~m}, 1 \mathrm{H})$.

${ }^{13} \mathrm{C}$ NMR $\left(100 \mathrm{MHz}, \mathrm{CDCl}_{3}\right) \delta_{\mathrm{C}} / \mathrm{ppm} 29.0,29.3$ (3 C), 30.1, 31.1, 32.2, 36.7, 40.8, 68.5, 171.6.

HRMS (ESI) $\mathrm{m} / \mathrm{z}$ calcd for $\mathrm{C}_{11} \mathrm{H}_{20} \mathrm{O}_{2} \mathrm{Na}[\mathrm{M}+\mathrm{Na}]^{+}: 207.1356$, found: 207.1347.

$[\alpha]^{20}{ }_{589}=+10.63\left(\mathrm{c} 0.95, \mathrm{CHCl}_{3}\right)$.

IR $\left(v_{\max } / \mathrm{cm}^{-1}\right):$ 1089, 1223, 1254, 1401, 1740, 2953.

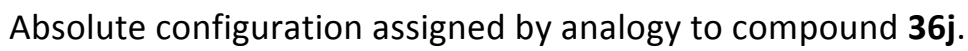




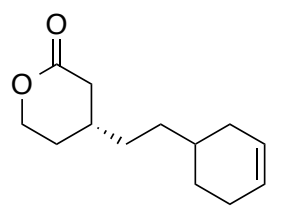

$\mathrm{Cp}_{2} \mathrm{ZrHCl}$ (206 mg, $0.80 \mathrm{mmol}, 2.0$ eq.) was added to a stirred, room temperature, solution of 4vinyl-1-cyclohexene $\left(0.13 \mathrm{~mL}, 1.0 \mathrm{mmol}, 2.5\right.$ eq.) in $\mathrm{CH}_{2} \mathrm{Cl}_{2}(0.40 \mathrm{~mL})$ under an argon atmosphere. After stirring for about $30 \mathrm{~min}$, the resulting clear yellow solution was transferred via syringe over about $1 \mathrm{~min}$ to a clear colourless stirred solution of $(\boldsymbol{R})-\mathbf{B}\left(30.0 \mathrm{mg}, 0.040 \mathrm{mmol}, 0.10\right.$ eq.) in $\mathrm{Et}_{2} \mathrm{O}$ $(2.0 \mathrm{~mL})$ under an argon atmosphere. The resulting dark mixture was allowed to stir for an additional $10 \mathrm{~min}$ before 5,6-dihydro-2H-pyran-2-one ( $34 \mu \mathrm{l}, 0.40 \mathrm{mmol}, 1.0$ eq.) and then TMSCl ( $0.25 \mathrm{~mL}, 2.0 \mathrm{mmol}, 5.0$ eq.) were added dropwise via syringe. The reaction was stirred for $3 \mathrm{~h}$ (TLC control (EtOAc/Petrol 4:6)) before the reaction was quenched by the addition of $\mathrm{Et}_{2} \mathrm{O}$ ( $c a 3$ $\mathrm{mL}$ ) and then $\mathrm{NH}_{4} \mathrm{Cl}\left(1 \mathrm{M}\right.$. aq., ca $1.5 \mathrm{~mL}$ ). The mixture was partitioned between water and $\mathrm{Et}_{2} \mathrm{O}$ and the aqueous phase extracted with $\mathrm{Et}_{2} \mathrm{O}(3 \times 10 \mathrm{~mL})$. The combined organic phase was washed with $\mathrm{NaHCO}_{3}$ (aq. sat., ca $10 \mathrm{~mL}$ ), dried $\left(\mathrm{MgSO}_{4}\right)$, filtered and concentrated in vacuo. Flash column chromatography of the yellow residue (30:70, $\mathrm{Et}_{2} \mathrm{O}$ :petrol; $\left.\mathrm{SiO}_{2}\right)$ gave $(+)-(R)-4-(2$-(cyclohex-3-en1-yl)ethyl)tetrahydro-2 $\mathrm{H}$-pyran-2-one $(38.2 \mathrm{mg}, 0.18 \mathrm{mmol}, 45 \%)$ as an off white solid as a $1: 1$ mixture of diastereomers.

HPLC analysis of the amide derivative of $\mathbf{3 6 h}$ (see general procedure for details) indicated an enantiomeric excess of $85 \%$ [Chiralpak ${ }^{\circledR} \mathrm{AY}-\mathrm{H}$; flow: $1.0 \mathrm{~mL} / \mathrm{min}$; hexane/i-PrOH: 90:10; $\lambda=210$ $\mathrm{nm}$; major enantiomer, $t_{R}=31.52 \mathrm{~min} \& 34.11 \mathrm{~min}$; minor enantiomer, $t_{R}=53.06 \mathrm{~min} \& 59.99$ $\min ]$.

${ }^{1} \mathrm{H}$ NMR $\left(400 \mathrm{MHz}, \mathrm{CDCl}_{3}\right) \delta_{\mathrm{H}} / \mathrm{ppm} 1.09-1.38(\mathrm{~m}, 5 \mathrm{H}), 1.38-1.52(\mathrm{~m}, 2 \mathrm{H}), 1.52-1.72(\mathrm{~m}, 2 \mathrm{H})$, $1.80-2.15(\mathrm{~m}, 6 \mathrm{H}), 2.58-2.69(\mathrm{~m}, 1 \mathrm{H}), 4.19(\mathrm{ddd}, J=11.4,10.5,3.6 \mathrm{~Hz}, 1 \mathrm{H}), 4.30-4.41(\mathrm{~m}, 1 \mathrm{H})$, $5.52-5.67(\mathrm{~m}, 2 \mathrm{H})$.

${ }^{13} \mathrm{C}$ NMR $\left(100 \mathrm{MHz}, \mathrm{CDCl}_{3}\right) \delta_{\mathrm{c}} / \mathrm{ppm} 25.1,28.8,29.0,31.76,31.83,33.3,33.47,33.52,36.7,68.5$, $126.3,127.1,171.5$.

HRMS (ESI) $\mathrm{m} / \mathrm{z}$ calcd for $\mathrm{C}_{13} \mathrm{H}_{20} \mathrm{O}_{2} \mathrm{Na}[\mathrm{M}+\mathrm{Na}]^{+}:$231.1356, found: 231.1350 .

$[\alpha]^{20}{ }_{589}=+14.81\left(c 0.27, \mathrm{CHCl}_{3}\right)$.

IR $\left(v_{\max } / \mathbf{c m}^{-1}\right):$ 1072, 1220, 1253, 1738, 2359, 2914.

MP $40-42^{\circ} \mathrm{C}$.

Absolute configuration assigned by analogy to compound $\mathbf{3 6 j}$. 


\section{$(+)-(R)-4-(3-P h e n o x y p r o p y l) t e t r a h y d r o-2 H-p y r a n-2-o n e(36 i)$}

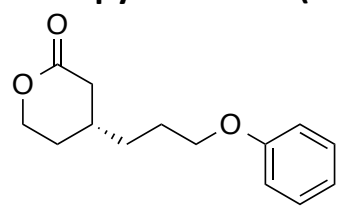

$\mathrm{Cp}_{2} \mathrm{ZrHCl}$ (206 mg, $0.80 \mathrm{mmol}, 2.0$ eq.) was added to a stirred, room temperature, solution of allyphenylether (135 mg, $1.0 \mathrm{mmol}, 2.5$ eq.) in $\mathrm{CH}_{2} \mathrm{Cl}_{2}(0.40 \mathrm{~mL})$ under an argon atmosphere. After stirring for $30 \mathrm{~min}$ at room temperature, the resulting clear yellow solution was transferred via syringe over about $1 \mathrm{~min}$ to a clear colourless stirred solution of $(\boldsymbol{R})$-B $(30.0 \mathrm{mg}, 0.040 \mathrm{mmol}$, 0.10 eq.) in $\mathrm{Et}_{2} \mathrm{O}(2.0 \mathrm{~mL})$ under an argon atmosphere. The resulting dark mixture was allowed to stir for $10 \mathrm{~min}$ before 5,6-dihydro-2H-pyran-2-one (34 $\mu \mathrm{L}, 0.40 \mathrm{mmol}, 1.0$ eq.) and then TMSCl $(0.25 \mathrm{~mL}, 2.0 \mathrm{mmol}, 5.0$ eq.) were added via syringe. Stirring at room temperature was continued for $2 \mathrm{~h}$ before the reaction was quenched by the addition of $\mathrm{Et}_{2} \mathrm{O}(\mathrm{ca} 3 \mathrm{~mL})$ and then $\mathrm{NH}_{4} \mathrm{Cl}(1 \mathrm{M}$ aq., $c a 1.5 \mathrm{~mL}$ ). The mixture was partitioned between water and $\mathrm{Et}_{2} \mathrm{O}$ and the aqueous phase was extracted with $\mathrm{Et}_{2} \mathrm{O}(3 \times 10 \mathrm{~mL})$. The combined organic phase was washed with $\mathrm{NaHCO}_{3}$ (aq. sat., ca $10 \mathrm{~mL})$, dried $\left(\mathrm{MgSO}_{4}\right)$, filtered and concentrated in vacuo. Flash column chromatography of the yellow residue (1:6; EtOAc:petrol; $\mathrm{SiO}_{2}$ ) gave $(+)-(R)-4$-(3-phenoxypropyl)tetrahydro-2H-pyran2-one (68 $\mathrm{mg}, 0.29 \mathrm{mmol}, 73 \%$ ) as a yellow oil.

HPLC analysis indicated an enantiomeric excess of $89 \%$ [Chiralpak ${ }^{\circledR}$ IA; flow: $1.5 \mathrm{~mL} / \mathrm{min}$; hexane/i-PrOH: 99:1; $\lambda=210 \mathrm{~nm}$; major enantiomer, $t_{R}=95.50 \mathrm{~min}$; minor enantiomer, $t_{R}=$ $110.73 \mathrm{~min}]$.

${ }^{1} \mathbf{H}$ NMR $\left(400 \mathrm{MHz}, \mathrm{CDCl}_{3}\right) \delta_{\mathrm{H}} / \mathrm{ppm}: 1.32-1.45(\mathrm{~m}, 3 \mathrm{H}), 1.59-1.70(\mathrm{~m}, 2 \mathrm{H}), 1.77-1.93(\mathrm{~m}, 2 \mathrm{H}), 2.01$ (dd, $J=17.1,10.3 \mathrm{~Hz}, 1 \mathrm{H}$ ), 2.56 (ddd, $J=17.1,5.6,1.5 \mathrm{~Hz}, 1 \mathrm{H}$ ), $3.79(\mathrm{t}, J=6.1 \mathrm{~Hz}, 2 \mathrm{H}$ ), 4.09 (td, $J=$ $11.3,3.9 \mathrm{~Hz}, 1 \mathrm{H}), 4.25(\mathrm{ddd}, J=11.5,4.7,3.9 \mathrm{~Hz}, 1 \mathrm{H}), 6.71(\mathrm{dq}, J=8.6,1.1 \mathrm{~Hz}, 2 \mathrm{H}), 6.77(\mathrm{td}, J=7.3$, $1.1 \mathrm{~Hz}, 1 \mathrm{H}), 7.08-7.14(\mathrm{~m}, 2 \mathrm{H})$.

${ }^{13} \mathrm{C}$ NMR $\left(100 \mathrm{MHz}, \mathrm{CDCl}_{3}\right) \delta_{\mathrm{C}} / \mathrm{ppm}: 26.2,28.8,31.3,32.7,36.5,67.3,68.4,114.3$ (2 C), 120.7, 129.4 (2 C), 158.7, 171.1.

HRMS (EI) $m / z$ calcd for $\mathrm{C}_{14} \mathrm{H}_{18} \mathrm{NaO}_{3}[\mathrm{M}+\mathrm{Na}]^{+}: 257.1148$, found: 257.1149 .

$[\alpha]^{20}{ }_{589}:+11.2\left(c 1.15, \mathrm{CHCl}_{3}\right)$.

IR $\left(v_{\max } / \mathrm{cm}^{-1}\right):$ 1599, 1732, 2872, 2942.

Absolute configuration assigned by analogy to compound $\mathbf{3 6 \mathbf { j }}$. 
(+)-(R)-4-(4-(Benzyloxy)butyl)tetrahydro-2H-pyran-2-one (36j)

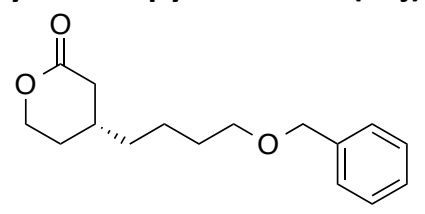

$\mathrm{Cp}_{2} \mathrm{ZrHCl}$ (206 mg, $0.80 \mathrm{mmol}, 2.0$ eq.) was added to a stirred, room temperature, solution of ((but-3-en-1-yloxy)methyl)benzene (162 mg, $1.0 \mathrm{mmol}, 2.5$ eq.) in $\mathrm{CH}_{2} \mathrm{Cl}_{2}(0.40 \mathrm{~mL})$ under an argon atmosphere. After stirring for $30 \mathrm{~min}$ at room temperature, the resulting clear yellow solution was transferred via syringe over about $1 \mathrm{~min}$ to a clear colourless stirred solution of $(\boldsymbol{R})$-B (30.0 mg, $0.040 \mathrm{mmol}, 0.10$ eq.) in $\mathrm{Et}_{2} \mathrm{O}(2.0 \mathrm{~mL})$ under an argon atmosphere. The resulting dark mixture was allowed to stir for $10 \mathrm{~min}$ before 5,6-dihydro-2H-pyran-2-one (34 $\mu \mathrm{L}, 0.40 \mathrm{mmol}, 1.0$ eq.) and then $\mathrm{TMSCl}(0.25 \mathrm{~mL}, 2.0 \mathrm{mmol}, 5.0$ eq.) were added via syringe. Stirring at room temperature was continued for $2.5 \mathrm{~h}$ before the reaction was quenched by the addition of $\mathrm{Et}_{2} \mathrm{O}$ (ca $3 \mathrm{~mL}$ ) and then $\mathrm{NH}_{4} \mathrm{Cl}(1 \mathrm{M}$ aq., $c a 1.5 \mathrm{~mL}$ ). The mixture was partitioned between water and $\mathrm{Et}_{2} \mathrm{O}$ and the aqueous phase was extracted with $\mathrm{Et}_{2} \mathrm{O}(3 \times 10 \mathrm{~mL})$. The combined organic phase was washed with $\mathrm{NaHCO}_{3}$ (aq. sat., ca $10 \mathrm{~mL}$ ), dried $\left(\mathrm{MgSO}_{4}\right)$, filtered and concentrated in vacuo. Flash column chromatography of the yellow residue (1:6; EtOAc:petrol; $\left.\mathrm{SiO}_{2}\right)$ gave $(+)-(R)-4-(4-$ (benzyloxy)butyl)tetrahydro-2H-pyran-2-one (69 mg, $0.26 \mathrm{mmol}, 66 \%$ ) as a white solid.

HPLC analysis indicated an enantiomeric excess of $90 \%$ [Chiralpak ${ }^{\circledR} \mathrm{IB}$; flow: $1.0 \mathrm{~mL} / \mathrm{min}$; hexane/iPrOH: 85:15; $\lambda=210 \mathrm{~nm}$; major enantiomer, $t_{R}=19.26 \mathrm{~min}$; minor enantiomer, $t_{R}=23.74 \mathrm{~min}$ ].

${ }^{1} \mathrm{H}$ NMR $\left(400 \mathrm{MHz}, \mathrm{CDCl}_{3}\right) \delta_{\mathrm{H}} / \mathrm{ppm}: 1.32-1.49(\mathrm{~m}, 4 \mathrm{H}), 1.50-1.58(\mathrm{~m}, 1 \mathrm{H}), 1.64(\mathrm{dt}, J=14.7,6.4 \mathrm{~Hz}$, $2 \mathrm{H}), 1.90-2.04(\mathrm{~m}, 2 \mathrm{H}), 2.15(\mathrm{dd}, J=17.4,10.0 \mathrm{~Hz}, 1 \mathrm{H}), 2.71$ (ddd, $J=17.4,5.9,1.7 \mathrm{~Hz}, 1 \mathrm{H}), 3.49(\mathrm{t}$, $J=6.4 \mathrm{~Hz}, 2 \mathrm{H}), 4.26(\mathrm{td}, J=10.5,3.7 \mathrm{~Hz}, 1 \mathrm{H}), 4.42(\mathrm{ddd}, J=11.3,4.9,3.7 \mathrm{~Hz}, 1 \mathrm{H}), 4.52(\mathrm{~s}, 2 \mathrm{H}), 7.27-$ $7.42(m, 5 H)$.

${ }^{13} \mathrm{C}$ NMR $\left(100 \mathrm{MHz}, \mathrm{CDCl}_{3}\right) \delta_{\mathrm{c}} / \mathrm{ppm}: 23.1,28.8,29.6,31.4,35.9,36.5,68.5,69.9,72.9,127.5$, 127.6

(2 C), 128.3 (2 C), 138.4, 171.3.

HRMS (EI) $m / z$ calcd for $\mathrm{C}_{16} \mathrm{H}_{22} \mathrm{NaO}_{3}[\mathrm{M}+\mathrm{Na}]^{+}: 285.1461$, found: 285.1451 .

$[\alpha]_{589}^{20}:+9.8\left(c 0.90, \mathrm{CHCl}_{3}\right)$.

IR $\left(v_{\max } / \mathrm{cm}^{-1}\right):$ 1496, 1733, 2801, 2856, 2934.

Absolute configuration assigned by X-ray crystallography (see Appendix A4). 


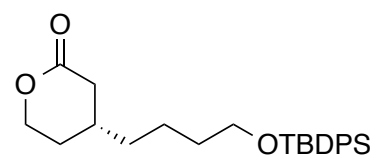

$\mathrm{Cp}_{2} \mathrm{ZrHCl}$ (206 mg, $0.80 \mathrm{mmol}, 2.0$ eq.) was added to a stirred, room temperature, solution of (but-3-en-1-yloxy)(tert-butyl)diphenylsilane (311 mg, $1.0 \mathrm{mmol}, 2.5$ eq.) in $\mathrm{CH}_{2} \mathrm{Cl}_{2}(0.40 \mathrm{~mL}$ ) under an argon atmosphere. After stirring for $30 \mathrm{~min}$ at room temperature, the resulting clear yellow solution was transferred via syringe over about 1 min to a clear colourless stirred solution of $(\boldsymbol{R})$-B (30.0 mg, $0.040 \mathrm{mmol}, 0.10$ eq.) in $\mathrm{Et}_{2} \mathrm{O}(2.0 \mathrm{~mL})$ under an argon atmosphere. The resulting dark mixture was allowed to stir for $10 \mathrm{~min}$ before 5,6-dihydro-2H-pyran-2-one (34 $\mu \mathrm{L}, 0.40 \mathrm{mmol}, 1.0$ eq.) and then $\mathrm{TMSCl}(0.25 \mathrm{~mL}, 2.0 \mathrm{mmol}, 5.0$ eq.) were added via syringe. Stirring at room temperature was continued for $3 \mathrm{~h}$ before the reaction was quenched by the addition of $\mathrm{Et}_{2} \mathrm{O}$ ( $c a$ $3 \mathrm{~mL}$ ) and then $\mathrm{NH}_{4} \mathrm{Cl}\left(1 \mathrm{M}\right.$ aq., $c a 1.5 \mathrm{~mL}$ ). The mixture was partitioned between water and $\mathrm{Et}_{2} \mathrm{O}$ and the aqueous phase was extracted with $\mathrm{Et}_{2} \mathrm{O}(3 \times 10 \mathrm{~mL})$. The combined organic phase was washed with $\mathrm{NaHCO}_{3}$ (aq. sat., ca $10 \mathrm{~mL}$ ), dried $\left(\mathrm{MgSO}_{4}\right)$, filtered and concentrated in vacuo. Flash column chromatography of the yellow residue (1:9; EtOAc:petrol; $\left.\mathrm{SiO}_{2}\right)$ gave $(+)-(R)-4-(4-(($ tertbutyldiphenylsilyl)oxy)butyl)tetrahydro-2H-pyran-2-one $(120 \mathrm{mg}, 0.35 \mathrm{mmol}, 88 \%)$ as a pale yellow oil.

HPLC analysis of the amide derivative of 36k (see general procedure for details) indicated an enantiomeric excess of $89 \%$ [Chiralpak ${ }^{\circledast} \mathrm{IC}$; flow: $1.0 \mathrm{~mL} / \mathrm{min}$; hexane/i-PrOH: 90:10; $\lambda=210 \mathrm{~nm}$; major enantiomer, $t_{R}=19.52 \mathrm{~min}$; minor enantiomer, $\left.t_{R}=22.10 \mathrm{~min}\right]$.

${ }^{1} \mathrm{H}$ NMR $\left(400 \mathrm{MHz}, \mathrm{CDCl}_{3}\right) \delta_{\mathrm{H}} / \mathrm{ppm}: 1.06(\mathrm{~s}, 9 \mathrm{H}), 1.25-1.65(\mathrm{~m}, 8 \mathrm{H}), 1.85-1.99(\mathrm{~m}, 2 \mathrm{H}), 2.11$ (dd, $J=17.4,10.0 \mathrm{~Hz}, 1 \mathrm{H}), 2.67(\mathrm{ddd}, J=17.4,5.6,1.7 \mathrm{~Hz}, 1 \mathrm{H}), 3.67(\mathrm{t}, J=6.4 \mathrm{~Hz}, 2 \mathrm{H}), 4.25(\mathrm{td}, J=10.5$, 3.7 Hz, $1 \mathrm{H}), 4.41(\mathrm{dt}, J=11.3,4.4 \mathrm{~Hz}, 1 \mathrm{H}), 7.34-7.47(\mathrm{~m}, 6 \mathrm{H}), 7.63-7.71(\mathrm{~m}, 4 \mathrm{H})$.

${ }^{13} \mathrm{C}$ NMR $\left(100 \mathrm{MHz}, \mathrm{CDCl}_{3}\right) \delta_{\mathrm{c}} / \mathrm{ppm}: 19.2,22.7,26.9$ (3 C), 28.9, 31.5, 32.3, 35.9, 36.6, 63.5, 68.5, 127.6 (4 C), 129.6 (2 C), 133.9 (2 C), 135.5 (4 C), 171.4.

HRMS (EI) $\mathrm{m} / z$ calcd for $\mathrm{C}_{25} \mathrm{H}_{34} \mathrm{NaO}_{3} \mathrm{Si}[\mathrm{M}+\mathrm{Na}]^{+}: 433.2169$, found: 433.2176 .

$[\alpha]_{589}^{20}:+3.2\left(c 0.50, \mathrm{CHCl}_{3}\right)$.

IR $\left(v_{\max } / \mathbf{c m}^{-1}\right): 1738,2857,2931$.

Absolute configuration assigned by analogy to compound $\mathbf{3 6 j}$. 


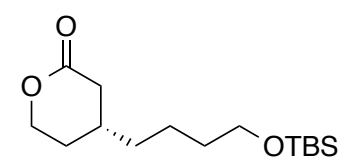

$\mathrm{Cp}_{2} \mathrm{ZrHCl}$ (206 mg, $0.80 \mathrm{mmol}, 2.0$ eq.) was added to a stirred, room temperature, solution of but-3-en-1-yl(tert-butyl)dimethylsilane (186 mg, $1.0 \mathrm{mmol}, 2.5$ eq.) in $\mathrm{CH}_{2} \mathrm{Cl}_{2}(0.40 \mathrm{~mL}$ ) under an argon atmosphere. After stirring for $30 \mathrm{~min}$ at room temperature, the resulting clear yellow solution was transferred via syringe over about $1 \mathrm{~min}$ to a clear colourless stirred solution of $(\boldsymbol{R})$-B (30.0 mg, $0.040 \mathrm{mmol}, 0.10$ eq.) in $\mathrm{Et}_{2} \mathrm{O}(2.0 \mathrm{~mL})$ under an argon atmosphere. The resulting dark mixture was allowed to stir for $10 \mathrm{~min}$ before 5,6-dihydro-2H-pyran-2-one (34 $\mu \mathrm{L}, 0.40 \mathrm{mmol}, 1.0$ eq.) and then $\mathrm{TMSCl}(0.25 \mathrm{~mL}, 2.0 \mathrm{mmol}, 5.0$ eq.) were added via syringe. Stirring at room temperature was continued for $4 \mathrm{~h}$ before the reaction was quenched by the addition of $\mathrm{Et}_{2} \mathrm{O}$ ( $c a$ $3 \mathrm{~mL}$ ) and then $\mathrm{NH}_{4} \mathrm{Cl}\left(1 \mathrm{M}\right.$ aq., $c a 1.5 \mathrm{~mL}$ ). The mixture was partitioned between water and $\mathrm{Et}_{2} \mathrm{O}$ and the aqueous phase was extracted with $\mathrm{Et}_{2} \mathrm{O}(3 \times 10 \mathrm{~mL})$. The combined organic phase was washed with $\mathrm{NaHCO}_{3}$ (aq. sat., $\mathrm{ca} 10 \mathrm{~mL}$ ), dried $\left(\mathrm{MgSO}_{4}\right)$, filtered and concentrated in vacuo. Flash column chromatography of the yellow residue (1:9; EtOAc:petrol; $\left.\mathrm{SiO}_{2}\right)$ gave (+)-(R)-4-(4-((tertbutyldimethylsilyl)oxy)butyl)tetrahydro-2H-pyran-2-one (95 $\mathrm{mg}, 0.33 \mathrm{mmol}, 83 \%$ ) as a colourless oil.

HPLC analysis of the amide derivative of $\mathbf{3 6 I}$ (see general procedure for details) indicated an enantiomeric excess of $89 \%$ [Chiralpak ${ }^{\circledR}$ IC; flow: $1.0 \mathrm{~mL} / \mathrm{min}$; hexane/i-PrOH: 90:10; $\lambda=210 \mathrm{~nm}$; major enantiomer, $t_{R}=17.96 \mathrm{~min}$; minor enantiomer, $\left.t_{R}=20.46 \mathrm{~min}\right]$.

${ }^{1} \mathrm{H}$ NMR $\left(400 \mathrm{MHz}, \mathrm{CDCl}_{3}\right) \delta_{\mathrm{H}} / \mathrm{ppm}: 0.05(\mathrm{~s}, 6 \mathrm{H}), 0.89(\mathrm{~s}, 9 \mathrm{H}), 1.35-1.41(\mathrm{~m}, 4 \mathrm{H}), 1.48-1.56(\mathrm{~m}, 3 \mathrm{H})$, 1.89-2.03 (m, 2H), $2.14(\mathrm{dd}, J=17.1,9.8 \mathrm{~Hz}, 1 \mathrm{H}), 2.70(\mathrm{ddd}, J=17.1,5.6,1.5 \mathrm{~Hz}, 1 \mathrm{H}), 3.61(\mathrm{t}, J=$ $6.1 \mathrm{~Hz}, 2 \mathrm{H}), 4.26(\mathrm{td}, J=10.8,3.7 \mathrm{~Hz}, 1 \mathrm{H}), 4.42(\mathrm{dt}, J=11.5,4.4 \mathrm{~Hz}, 1 \mathrm{H})$.

${ }^{13} \mathrm{C}$ NMR $\left(100 \mathrm{MHz}, \mathrm{CDCl}_{3}\right) \delta_{\mathrm{c}} / \mathrm{ppm}:-5.3$ (2 C), 18.3, 22.7, 25.9 (3 C), 28.9, 31.5, 32.6, 35.9, 36.6, $62.8,68.5,171.4$.

HRMS (EI) $\mathrm{m} / \mathrm{z}$ calcd for $\mathrm{C}_{15} \mathrm{H}_{30} \mathrm{NaO}_{3} \mathrm{Si}[\mathrm{M}+\mathrm{Na}]^{+}:$309.1856, found: 309.1845 .

$[\alpha]_{589}^{20}:+4.2\left(c 1.15, \mathrm{CHCl}_{3}\right)$.

IR $\left(v_{\max } / \mathrm{cm}^{-1}\right): 1472,1740,2857,2900,2929$.

Absolute configuration assigned by analogy to compound $\mathbf{3 6 \mathbf { j }}$. 


\section{$(+)-(R)-4-(4-B r o m o p h e n e t h y l) t e t r a h y d r o-2 H-p y r a n-2-o n e(36 m)$}

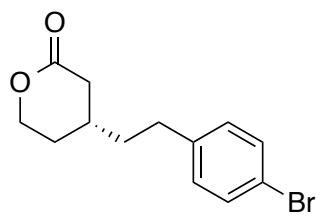

$\mathrm{Cp}_{2} \mathrm{ZrHCl}$ (206 $\mathrm{mg}, 0.80 \mathrm{mmol}, 2.0$ eq.) was added to a stirred, room temperature, solution of 4-bromostyrene $\left(0.13 \mathrm{~mL}, 1.0 \mathrm{mmol}, 2.5\right.$ eq.) in $\mathrm{CH}_{2} \mathrm{Cl}_{2}(0.40 \mathrm{~mL})$ under an argon atmosphere. After stirring for $30 \mathrm{~min}$ at room temperature, the resulting clear yellow solution was transferred via syringe over about $1 \mathrm{~min}$ to a clear colourless stirred solution of $(\boldsymbol{R})-\mathbf{B}(30.0 \mathrm{mg}, 0.040 \mathrm{mmol}$, 0.10 eq.) in $\mathrm{Et}_{2} \mathrm{O}(2.0 \mathrm{~mL})$ under an argon atmosphere. The resulting dark mixture was allowed to stir for $10 \mathrm{~min}$ before 5,6-dihydro-2H-pyran-2-one ( $34 \mu \mathrm{L}, 0.40 \mathrm{mmol}, 1.0$ eq.) and then TMSCl ( $0.25 \mathrm{~mL}, 2.0 \mathrm{mmol}, 5.0$ eq.) were added via syringe. Stirring at room temperature was continued for $4 \mathrm{~h}$ before the reaction was quenched by the addition of $\mathrm{Et}_{2} \mathrm{O}(c a 3 \mathrm{~mL})$ and then $\mathrm{NH}_{4} \mathrm{Cl}(1 \mathrm{M}$ aq., $c a 1.5 \mathrm{~mL}$ ). The mixture was partitioned between water and $\mathrm{Et}_{2} \mathrm{O}$ and the aqueous phase was extracted with $\mathrm{Et}_{2} \mathrm{O}(3 \times 10 \mathrm{~mL})$. The combined organic phase was washed with $\mathrm{NaHCO}_{3}$ (aq. sat., ca $10 \mathrm{~mL})$, dried $\left(\mathrm{MgSO}_{4}\right)$, filtered and concentrated in vacuo. Flash column chromatography of the yellow residue (1:9; EtOAc:petrol; $\mathrm{SiO}_{2}$ ) gave $(+)-(R)-4$-(4-bromophenethyl)tetrahydro-2Hpyran-2-one (70 $\mathrm{mg}, 0.24 \mathrm{mmol}, 61 \%$ ) as a yellow oil.

HPLC analysis indicated an enantiomeric excess of $88 \%$ [Chiralpak ${ }^{\circledR} \mathrm{IB}$; flow: $1.0 \mathrm{~mL} / \mathrm{min}$; hexane/iPrOH: 90:10; $\lambda=210 \mathrm{~nm}$; major enantiomer, $t_{R}=30.19 \mathrm{~min}$; minor enantiomer, $t_{R}=34.73 \mathrm{~min}$ ].

${ }^{1} \mathrm{H}$ NMR $\left(400 \mathrm{MHz}, \mathrm{CDCl}_{3}\right) \delta_{\mathrm{H}} / \mathrm{ppm}: 1.49-1.70(\mathrm{~m}, 3 \mathrm{H}), 1.90-2.03(\mathrm{~m}, 2 \mathrm{H}), 2.19(\mathrm{dd}, J=17.4,10.0$ $\mathrm{Hz}, 1 \mathrm{H}), 2.61(\mathrm{t}, J=7.8 \mathrm{~Hz}, 2 \mathrm{H}), 2.72(\mathrm{ddd}, J=17.2,5.9,1.7 \mathrm{~Hz}, 1 \mathrm{H}), 4.24(\mathrm{td}, J=11.3,3.4 \mathrm{~Hz}, 1 \mathrm{H})$, 4.41 (ddd, $J=11.3,4.9,3.9 \mathrm{~Hz}, 1 \mathrm{H}), 7.04(\mathrm{~d}, J=8.3 \mathrm{~Hz}, 2 \mathrm{H}), 7.40(\mathrm{~d}, J=8.3 \mathrm{~Hz}, 2 \mathrm{H}$ ).

${ }^{13} \mathrm{C}$ NMR $\left(100 \mathrm{MHz}, \mathrm{CDCl}_{3}\right) \delta_{\mathrm{c}} / \mathrm{ppm}: 28.7,30.7,32.0,36.3,37.6,68.3,119.8,129.9$ (2 C), 131.5 (2 C), 140.1, 170.9.

HRMS (EI) $m / z$ calcd for $\mathrm{C}_{13} \mathrm{H}_{15} \mathrm{BrNaO}_{2}[\mathrm{M}+\mathrm{Na}]^{+}: 305.0148$, found: 305.0137.

$[\alpha]^{20}{ }_{589}:+19.6\left(\right.$ c 2.25, $\left.\mathrm{CHCl}_{3}\right)$.

IR $\left(v_{\max } / \mathrm{cm}^{-1}\right):$ 1488, 1731, 2858, 2918.

Absolute configuration assigned by analogy to compound $\mathbf{3 6 \mathbf { j }}$. 
(+)-(R)-4-(3-(Trimethylsilyl)propyl)tetrahydro-2H-pyran-2-one (36n)<smiles>C[Si](C)(C)CCCC1CCOC(=O)C1</smiles>

$\mathrm{Cp}_{2} \mathrm{ZrHCl}$ (206 mg, $0.80 \mathrm{mmol}, 2.0$ eq.) was added to a stirred, room temperature, solution of allyl silane (0.16 mL, $1.0 \mathrm{mmol}, 2.5$ eq.) in $\mathrm{CH}_{2} \mathrm{Cl}_{2}(0.40 \mathrm{~mL})$ under an argon atmosphere. After stirring for $30 \mathrm{~min}$ at room temperature, the resulting clear yellow solution was transferred via syringe over about $1 \mathrm{~min}$ to a clear colourless stirred solution of $(\boldsymbol{R})$-B $(30.0 \mathrm{mg}, 0.040 \mathrm{mmol}, 0.10 \mathrm{eq}$.) in $\mathrm{Et}_{2} \mathrm{O}(2.0 \mathrm{~mL})$ under an argon atmosphere. The resulting dark mixture was allowed to stir for 10 min before 5,6-dihydro-2H-pyran-2-one ( $34 \mu \mathrm{L}, 0.40 \mathrm{mmol}, 1.0$ eq.) and then TMSCl (0.25 mL, 2.0 $\mathrm{mmol}, 5.0$ eq.) were added via syringe. Stirring at room temperature was continued for $3.5 \mathrm{~h}$ before the reaction was quenched by the addition of $\mathrm{Et}_{2} \mathrm{O}(c a 3 \mathrm{~mL})$ and then $\mathrm{NH}_{4} \mathrm{Cl}(1 \mathrm{M}$ aq., $c a$ $1.5 \mathrm{~mL}$ ). The mixture was partitioned between water and $\mathrm{Et}_{2} \mathrm{O}$ and the aqueous phase was extracted with $\mathrm{Et}_{2} \mathrm{O}(3 \times 10 \mathrm{~mL})$. The combined organic phase was washed with $\mathrm{NaHCO}_{3}$ (aq. sat., ca $10 \mathrm{~mL})$, dried $\left(\mathrm{MgSO}_{4}\right)$, filtered and concentrated in vacuo. Flash column chromatography of the yellow residue (1:9; EtOAc:petrol; $\mathrm{SiO}_{2}$ ) gave $(+)-(R)-4-(3-($ trimethylsilyl)propyl)tetrahydro-2Hpyran-2-one (61 $\mathrm{mg}, 0.28 \mathrm{mmol}, 71 \%$ ) as a pale yellow oil.

HPLC analysis of the amide derivative of $\mathbf{3 6 n}$ (see general procedure for details) indicated an enantiomeric excess of 92\% [Chiralpak ${ }^{\circledR} \mathrm{IC}$; flow: $1.0 \mathrm{~mL} / \mathrm{min}$; hexane/i-PrOH: 90:10; $\lambda=210 \mathrm{~nm}$; major enantiomer, $t_{R}=19.69 \mathrm{~min}$; minor enantiomer, $\left.t_{R}=22.90 \mathrm{~min}\right]$.

${ }^{1} \mathrm{H}$ NMR $\left(400 \mathrm{MHz}, \mathrm{CDCl}_{3}\right) \delta_{\mathrm{H}} / \mathrm{ppm}:-0.02(\mathrm{~s}, 9 \mathrm{H}), 0.45-0.51(\mathrm{~m}, 2 \mathrm{H}), 1.28-1.41(\mathrm{~m}, 3 \mathrm{H}), 1.48-1.57$ $(\mathrm{m}, 1 \mathrm{H}), 1.89-2.02(\mathrm{~m}, 2 \mathrm{H}), 2.14(\mathrm{dd}, J=17.4,10.3 \mathrm{~Hz}, 1 \mathrm{H}), 2.69(\mathrm{ddd}, J=17.4,5.6,1.7 \mathrm{~Hz}, 1 \mathrm{H})$, $4.26(\mathrm{td}, J=11.3,3.7 \mathrm{~Hz}, 1 \mathrm{H}), 4.41$ (ddd, $J=11.3,4.9,4.2 \mathrm{~Hz}, 1 \mathrm{H})$.

${ }^{13} \mathrm{C}$ NMR $\left(100 \mathrm{MHz}, \mathrm{CDCl}_{3}\right) \delta_{\mathrm{c}} / \mathrm{ppm}:-1.7$ (3 C), 16.5, 20.8, 28.9, 31.1, 36.5, 40.0, 68.6, 171.5.

HRMS (EI) $m / z$ calcd for $\mathrm{C}_{11} \mathrm{H}_{22} \mathrm{NaO}_{2} \mathrm{Si}[\mathrm{M}+\mathrm{Na}]^{+}: 237.1281$, found: 237.1286 .

$[\alpha]_{589}^{20}:+16.4\left(c 0.50, \mathrm{CHCl}_{3}\right)$.

IR $\left(v_{\max } / \mathrm{cm}^{-1}\right): 1653,1740,2858,2918,2952$.

Absolute configuration assigned by analogy to compound $\mathbf{3 6 \mathbf { j }}$. 
(+)-(R)-4-(-(4-((tert-butyldimethylsilyl)oxy)-4-(4-chlorophenyl)butyl)-tetrahydropyran-2-one (360)

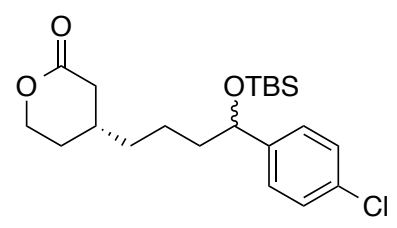

$\mathrm{Cp}_{2} \mathrm{ZrHCl}$ (206 mg, $0.80 \mathrm{mmol}, 2.0$ eq.) was added to a stirred, room temperature, solution of tert-butyl((1-(4-chlorophenyl)but-3-en-1-yl)oxy)dimethylsilane $(297 \mathrm{mg}, 1.0 \mathrm{mmol}, 2.5 \mathrm{eq}$.) in $\mathrm{CH}_{2} \mathrm{Cl}_{2}(0.40 \mathrm{~mL})$ under an argon atmosphere. After stirring for about $30 \mathrm{~min}$, the resulting clear yellow solution was transferred via syringe over about $1 \mathrm{~min}$ to a clear colourless stirred solution of $(\boldsymbol{R})$-B $\left(30.0 \mathrm{mg}, 0.040 \mathrm{mmol}, 0.10\right.$ eq.) in $\mathrm{Et}_{2} \mathrm{O}(2.0 \mathrm{~mL})$ under an argon atmosphere. The resulting dark mixture was allowed to stir for an additional $10 \mathrm{~min}$ before 5,6-dihydro-2H-pyran-2one (34 $\mu \mathrm{l}, 0.40 \mathrm{mmol}, 1.0$ eq.) and then $\mathrm{TMSCl}(0.25 \mathrm{~mL}, 2.0 \mathrm{mmol}, 5.0$ eq.) were added dropwise via syringe. The reaction was stirred for $6 \mathrm{~h}$ before the reaction was quenched by the addition of $\mathrm{Et}_{2} \mathrm{O}\left(c a 3 \mathrm{~mL}\right.$ ) and then $\mathrm{NH}_{4} \mathrm{Cl}(1 \mathrm{M}$. aq., $c a 1.5 \mathrm{~mL}$ ). The mixture was partitioned between water and $\mathrm{Et}_{2} \mathrm{O}$ and the aqueous phase extracted with $\mathrm{Et}_{2} \mathrm{O}(3 \times 10 \mathrm{~mL})$. The combined organic phase was washed with $\mathrm{NaHCO}_{3}$ (aq. sat., ca $10 \mathrm{~mL}$ ), dried $\left(\mathrm{MgSO}_{4}\right)$, filtered and concentrated in vacuo. Flash column chromatography of the yellow residue (15:85; EtOAc:petrol; $\left.\mathrm{SiO}_{2}\right)$ gave (+)-(R)-4-(-(4((tert-butyldimethylsilyl)oxy)-4-(4-chlorophenyl)butyl)-tetrahydropyran-2-one (103.5 mg, 0.26 mmol, $65 \%) *$ as a yellow oil. *contaminated with a small amount $(\sim 5 \%)$ of alcohol by-product.

To measure the enantiomeric excess, 360 was treated with TBAF (1.5 eq.) in THF and the resulting alcohol oxidised to the ketone (removing the diastereomers) with the Dess-Martin reagent.

HPLC analysis of the crude product indicated an enantiomeric excess of $88 \%$ [Chiralpak ${ }^{\circledR}$ IA; flow: $1.0 \mathrm{~mL} / \mathrm{min}$; hexane/i-PrOH: 75: 25; $\lambda=210 \mathrm{~nm}$; major enantiomer, $t_{R}=15.94 \mathrm{~min}$; minor enantiomer, $\left.t_{R}=18.23 \mathrm{~min}\right]$.

${ }^{1} \mathrm{H}$ NMR $\left(400 \mathrm{MHz}, \mathrm{CDCl}_{3}\right) \delta_{\mathrm{H}} / \mathrm{ppm}-0.14(\mathrm{~s}, 3 \mathrm{H}), 0.02(\mathrm{~s}, 3 \mathrm{H}), 0.88(\mathrm{~s}, 9 \mathrm{H}), 1.18-1.76(\mathrm{~m}, 6 \mathrm{H})$, $1.83-2.01(\mathrm{~m}, 2 \mathrm{H}), 2.11(\mathrm{dd}, J=17.4,10.0 \mathrm{~Hz}, 1 \mathrm{H}), 2.60-2.72(\mathrm{~m}, 1 \mathrm{H}), 4.23(\mathrm{td}, J=11.1,3.6 \mathrm{~Hz}$, $1 \mathrm{H}), 4.39(\mathrm{dt}, J=11.4,4.2 \mathrm{~Hz}, 1 \mathrm{H}), 4.62(\mathrm{dd}, J=7.3,4.9 \mathrm{~Hz}, 1 \mathrm{H}), 7.18-7.31(\mathrm{~m}, 5 \mathrm{H})$.

${ }^{13} \mathrm{C}$ NMR $\left(100 \mathrm{MHz}, \mathrm{CDCl}_{3}\right) \delta_{\mathrm{C}} / \mathrm{ppm}-5.0,-4.6,18.2,22.2,26.0$ (3 C), 28.9, 31.5, 36.1, 36.5, 40.7, $68.5,74.1,127.1$ (2 C), 128.3 (2 C), 132.5, 144.0, 171.4.

HRMS (EI) $\mathrm{m} / z$ calcd for $\mathrm{C}_{21} \mathrm{H}_{33} \mathrm{ClO}_{3} \mathrm{Si}[\mathrm{M}]^{+}:$396.1887, found: 396.2046 .

$[\alpha]^{20}{ }_{589}=+9.31\left(c 0.72, \mathrm{CHCl}_{3}\right)$.

IR $\left(v_{\max } / \mathrm{cm}^{-1}\right): 836,1086,1256,1740,2930$.

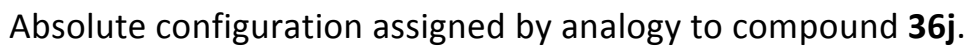




\section{$(-)-(R)-4-H e x y l o x e p a n-2-o n e(41 a)$}

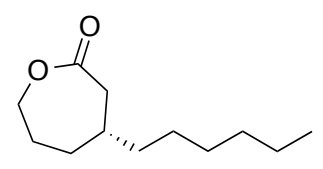

$\mathrm{Cp}_{2} \mathrm{ZrHCl}$ (206 mg, $0.80 \mathrm{mmol}, 2.0$ eq.) was added to a stirred, room temperature, solution of 1-hexene $\left(0.13 \mathrm{~mL}, 4.0 \mathrm{mmol}, 2.5\right.$ eq.) in $\mathrm{CH}_{2} \mathrm{Cl}_{2}(0.40 \mathrm{~mL})$ under an argon atmosphere. After stirring for $30 \mathrm{~min}$ at room temperature, the resulting clear yellow solution was transferred via syringe over about $1 \mathrm{~min}$ to a clear colourless stirred solution of $(\boldsymbol{R})$-B $(30.0 \mathrm{mg}, 0.040 \mathrm{mmol}, 0.10$ eq.) in $\mathrm{Et}_{2} \mathrm{O}(2.0 \mathrm{~mL})$ under an argon atmosphere. The resulting dark mixture was allowed to stir for $10 \mathrm{~min}$ before 6,7-dihydrooxepin-2(5H)-one ( $45 \mathrm{mg}, 0.40 \mathrm{mmol}, 1.0$ eq.) and then TMSCl $(0.25$ $\mathrm{mL}, 2.0 \mathrm{mmol}, 5.0$ eq.) were added via syringe. Stirring at room temperature was continued for 4 $\mathrm{h}$ before the reaction was quenched by the addition of $\mathrm{Et}_{2} \mathrm{O}(c a 3 \mathrm{~mL})$ and then $\mathrm{NH}_{4} \mathrm{Cl}(1 \mathrm{M}$ aq., $c a$ $1.5 \mathrm{~mL}$ ). The mixture was partitioned between water and $\mathrm{Et}_{2} \mathrm{O}$ and the aqueous phase was extracted with $\mathrm{Et}_{2} \mathrm{O}(3 \times 10 \mathrm{~mL})$. The combined organic phase was washed with $\mathrm{NaHCO}_{3}$ (aq. sat., ca $10 \mathrm{~mL})$, dried $\left(\mathrm{MgSO}_{4}\right)$, filtered and concentrated in vacuo. Flash column chromatography of the yellow residue (1:9; EtOAc:petrol; $\mathrm{SiO}_{2}$ ) gave (+)-(R)-4-hexyloxepan-2-one (76 mg, $0.38 \mathrm{mmol}$, $95 \%)$ as a yellow oil.

HPLC analysis of the amide derivative of 41a (see general procedure for details) indicated an enantiomeric excess of 93\% [Chiralpak ${ }^{\circledR}$ IC; flow: $1.0 \mathrm{~mL} / \mathrm{min}$; hexane/i-PrOH: 87:13; $\lambda=210 \mathrm{~nm}$; major enantiomer, $t_{R}=10.14$ min; minor enantiomer, $\left.t_{R}=10.63 \mathrm{~min}\right]$.

${ }^{1} \mathrm{H}$ NMR $\left(400 \mathrm{MHz}, \mathrm{CDCl}_{3}\right) \delta_{\mathrm{H}} / \mathrm{ppm}: 0.87(\mathrm{t}, J=6.6 \mathrm{~Hz}, 3 \mathrm{H}), 1.19-1.38(\mathrm{~m}, 10 \mathrm{H}), 1.39-1.52(\mathrm{~m}, 1 \mathrm{H})$, $1.65-1.82(\mathrm{~m}, 2 \mathrm{H}), 1.85-2.01(\mathrm{~m}, 2 \mathrm{H}), 2.53(\mathrm{dd}, J=13.5,9.8 \mathrm{~Hz}, 1 \mathrm{H}), 2.61(\mathrm{dq}, J=13.5,1.2 \mathrm{~Hz}, 1 \mathrm{H})$, 4.14-4.28 (m, 2H).

${ }^{13} \mathrm{C}$ NMR $\left(100 \mathrm{MHz}, \mathrm{CDCl}_{3}\right) \delta_{\mathrm{c}} / \mathrm{ppm}: 14.0,22.6,26.7,27.7,29.2,31.7,34.0,34.9,36.0,40.0,69.2$, 175.2 .

HRMS (EI) $m / z$ calcd for $\mathrm{C}_{12} \mathrm{H}_{22} \mathrm{NaO}_{2}[\mathrm{M}+\mathrm{Na}]^{+}: 221.1512$, found: 221.1507 .

$[\alpha]_{589}^{20}:-15.5$ (c 1.80, $\left.\mathrm{CHCl}_{3}\right)$.

IR $\left(v_{\max } / \mathbf{c m}^{-1}\right):$ 1731, 2856, 2925, 2955.

Absolute configuration assigned by analogy to compound $\mathbf{4 1 b}$. 


\section{(-)-(R)-4-(4-(Benzyloxy)butyl)oxepan-2-one (41b)}

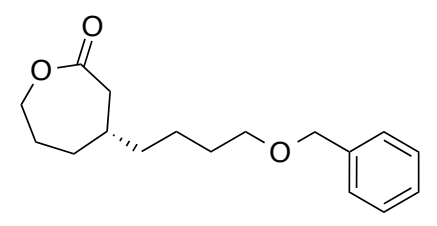

$\mathrm{Cp}_{2} \mathrm{ZrHCl}$ (206 mg, $0.80 \mathrm{mmol}, 2.0$ eq.) was added to a stirred, room temperature, solution of ((but-3-en-1-yloxy)methyl)benzene (162 mg, $4.0 \mathrm{mmol}, 2.5$ eq.) in $\mathrm{CH}_{2} \mathrm{Cl}_{2}(0.40 \mathrm{~mL})$ under an argon atmosphere. After stirring for $30 \mathrm{~min}$ at room temperature, the resulting clear yellow solution was transferred via syringe over about 1 min to a clear colourless stirred solution of $(\boldsymbol{R})$-B (30.0 mg, $0.040 \mathrm{mmol}, 0.10$ eq.) in $\mathrm{Et}_{2} \mathrm{O}(2.0 \mathrm{~mL})$ under an argon atmosphere. The resulting dark mixture was allowed to stir for $10 \mathrm{~min}$ before 6,7-dihydrooxepin-2(5H)-one (45 mg, $0.40 \mathrm{mmol}$, 1.0 eq.) and then $\mathrm{TMSCl}(0.25 \mathrm{~mL}, 2.0 \mathrm{mmol}, 5.0$ eq.) were added via syringe. Stirring at room temperature was continued for $4 \mathrm{~h}$ before the reaction was quenched by the addition of $\mathrm{Et}_{2} \mathrm{O}$ ( $c a$ $3 \mathrm{~mL}$ ) and then $\mathrm{NH}_{4} \mathrm{Cl}\left(1 \mathrm{M}\right.$ aq., ca $1.5 \mathrm{~mL}$ ). The mixture was partitioned between water and $\mathrm{Et}_{2} \mathrm{O}$ and the aqueous phase was extracted with $\mathrm{Et}_{2} \mathrm{O}(3 \times 10 \mathrm{~mL})$. The combined organic phase was washed with $\mathrm{NaHCO}_{3}$ (aq. sat., ca $10 \mathrm{~mL}$ ), dried $\left(\mathrm{MgSO}_{4}\right)$, filtered and concentrated in vacuo. Flash column chromatography of the yellow residue (1:4; EtOAc:petrol; $\mathrm{SiO}_{2}$ ) gave (-)-(R)-4-(4(benzyloxy)butyl)oxepan-2-one (68 mg, $0.25 \mathrm{mmol}, 62 \%$ ) as a white solid.

HPLC analysis indicated an enantiomeric excess of $90 \%$ [Chiralpak ${ }^{\otimes}$ ID; flow: $1.0 \mathrm{~mL} / \mathrm{min}$; hexane/i-PrOH: 90:10; $\lambda=210 \mathrm{~nm}$; minor enantiomer, $t_{R}=32.85 \mathrm{~min}$; major enantiomer, $t_{R}=$ $35.20 \mathrm{~min}]$.

${ }^{1} \mathbf{H}$ NMR $\left(400 \mathrm{MHz}, \mathrm{CDCl}_{3}\right) \delta_{\mathrm{H}} / \mathrm{ppm}: 1.29-1.52(\mathrm{~m}, 5 \mathrm{H}), 1.56-1.66(\mathrm{~m}, 2 \mathrm{H}), 1.70-1.84(\mathrm{~m}, 2 \mathrm{H}), 1.86-$ $2.01(\mathrm{~m}, 2 \mathrm{H}), 2.54(\mathrm{dd}, J=13.7,9.8 \mathrm{~Hz}, 1 \mathrm{H}), 2.62(\mathrm{dq}, J=13.7,1.0 \mathrm{~Hz}, 1 \mathrm{H}), 3.47(\mathrm{t}, J=6.4 \mathrm{~Hz}, 2 \mathrm{H})$, 4.14-4.30 (m, 2H), $4.50(\mathrm{~s}, 2 \mathrm{H}), 7.27-7.39(\mathrm{~m}, 5 \mathrm{H})$.

${ }^{13} \mathrm{C}$ NMR $\left(100 \mathrm{MHz}, \mathrm{CDCl}_{3}\right) \delta_{\mathrm{c}} / \mathrm{ppm}: 23.5,27.6,29.7,33.9,34.8,35.7,40.1,69.2,70.1,72.9,127.5$, 127.6 (2 C), 128.3 (2 C), 138.5, 175.1.

HRMS (EI) $m / z$ calcd for $\mathrm{C}_{17} \mathrm{H}_{24} \mathrm{NaO}_{3}[\mathrm{M}+\mathrm{Na}]^{+}: 299.1618$, found: 299.1610 .

$[\alpha]^{20}{ }_{589}:-17.5\left(c 1.60, \mathrm{CHCl}_{3}\right)$.

IR $\left(v_{\max } / \mathbf{c m}^{-1}\right):$ 1729, 2859, 2932, 3031.

MP $43-58^{\circ} \mathrm{C}$.

Absolute configuration assigned by X-ray crystallography (see Appendix A5). 
$(-)-(R)-4-H e x y l-4,5-d i h y d r o b e n z o[b]$ oxepin-2(3H)-one (42a)

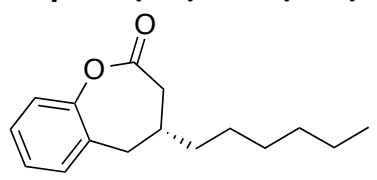

$\mathrm{Cp}_{2} \mathrm{ZrHCl}$ (206 mg, $0.80 \mathrm{mmol}, 2.0$ eq.) was added to a stirred, room temperature, solution of 1-hexene $\left(0.12 \mathrm{~mL}, 1.0 \mathrm{mmol}, 2.5\right.$ eq.) in $\mathrm{CH}_{2} \mathrm{Cl}_{2}(0.40 \mathrm{~mL})$ under an argon atmosphere. After stirring for about $30 \mathrm{~min}$, the resulting clear yellow solution was transferred via syringe over about $1 \mathrm{~min}$ to a clear colourless stirred solution of $(\boldsymbol{R})-\mathbf{B}\left(30.0 \mathrm{mg}, 0.040 \mathrm{mmol}, 0.10\right.$ eq.) in $\mathrm{Et}_{2} \mathrm{O}$ $(2.0 \mathrm{~mL})$ under an argon atmosphere. The resulting dark mixture was allowed to stir for an additional $10 \mathrm{~min}$ before benzo[b]oxepin-2(5H)-one (64.1 $\mathrm{mg}, 0.40 \mathrm{mmol}, 1.0$ eq.) and then TMSCl $(0.25 \mathrm{~mL}, 2.0 \mathrm{mmol}, 5.0$ eq.) were added dropwise via syringe. The reaction was stirred for $6 \mathrm{~h}$ before the reaction was quenched by the addition of $\mathrm{Et}_{2} \mathrm{O}(c a 3 \mathrm{~mL})$ and then $\mathrm{NH}_{4} \mathrm{Cl}(1 \mathrm{M}$. aq., $c a$ $1.5 \mathrm{~mL}$ ). The mixture was partitioned between water and $\mathrm{Et}_{2} \mathrm{O}$ and the aqueous phase extracted with $\mathrm{Et}_{2} \mathrm{O}(3 \times 10 \mathrm{~mL})$. The combined organic phase was washed with $\mathrm{NaHCO}_{3}$ (aq. sat., $c a 10 \mathrm{~mL}$ ), dried $\left(\mathrm{MgSO}_{4}\right)$, filtered and concentrated in vacuo. Flash column chromatography of the residue (5:95; $\mathrm{Et}_{2} \mathrm{O}$ :petrol; $\mathrm{SiO}_{2}$ ) gave (-)-(R)-4-hexyl-4,5-dihydrobenzo[b]oxepin-2(3H)-one (60.9 mg, 0.25 $\mathrm{mmol}, 63 \%)$ as a yellow oil.

HPLC analysis indicated an enantiomeric excess of $88 \%$ [Chiralpak ${ }^{\circledR} \mathrm{IB}$; flow: $1.0 \mathrm{~mL} / \mathrm{min}$; hexane/iPrOH: 99:1; $\lambda=210 \mathrm{~nm}$; major enantiomer, $t_{R}=6.80 \mathrm{~min}$; minor enantiomer, $t_{R}=7.78 \mathrm{~min}$ ].

${ }^{1} \mathrm{H}$ NMR $\left(400 \mathrm{MHz}, \mathrm{CDCl}_{3}\right) \delta_{\mathrm{H}} / \mathrm{ppm} 0.89(\mathrm{t}, J=6.8 \mathrm{~Hz}, 3 \mathrm{H}) 1.20-1.49(\mathrm{~m}, 10 \mathrm{H}), 2.15(\mathrm{dd}, J=12.1$, $6.8 \mathrm{~Hz}, 1 \mathrm{H}), 2.38(\mathrm{dt}, J=13.4,6.7 \mathrm{~Hz}, 1 \mathrm{H}), 2.47-2.58(\mathrm{~m}, 2 \mathrm{H}), 2.95(\mathrm{dd}, J=13.9,6.7 \mathrm{~Hz}, 1 \mathrm{H}), 7.05$ - $7.11(m, 1 H), 7.12-7.20(m, 2 H), 7.25-7.31(m, 1 H)$.

${ }^{13} \mathrm{C}$ NMR $\left(100 \mathrm{MHz}, \mathrm{CDCl}_{3}\right) \delta_{\mathrm{C}} / \mathrm{ppm} 14.1,22.6,26.9,29.1,31.7,34.3,34.6,36.8,38.7,119.2$, 125.6, 128.3, 129.1, 130.3, 151.8, 171.0.

HRMS (ESI) $\mathrm{m} / \mathrm{z}$ calcd for $\mathrm{C}_{16} \mathrm{H}_{22} \mathrm{O}_{2} \mathrm{Na}[\mathrm{M}+\mathrm{Na}]^{+}:$269.1512, found: 269.1506 .

$[\alpha]^{20}{ }_{589}=-23.58\left(c 1.01, \mathrm{CHCl}_{3}\right)$.

IR $\left(v_{\max } / \mathrm{cm}^{-1}\right):$ 760, 1091, 1223, 1761, 2926.

Absolute configuration assigned by analogy to compound $\mathbf{4 1 b}$. 
$(+)-(R)-4-(2-(2-M e t h y l-1,3-d i o x o l a n-2-y l) e t h y l) t e t r a h y d r o-2 H-p y r a n-2-o n e(36 u)$

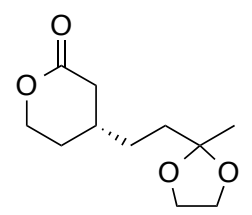

$\mathrm{Cp}_{2} \mathrm{ZrHCl}$ (206 mg, $0.80 \mathrm{mmol}, 2.0$ eq.) was added to a stirred, room temperature, solution of 2-methyl-2-vinyl-1,3-dioxolane (114 mg, $1.0 \mathrm{mmol}, 2.5$ eq.) in $\mathrm{CH}_{2} \mathrm{Cl}_{2}(0.40 \mathrm{~mL})$ under an argon atmosphere. After stirring for $30 \mathrm{~min}$ at room temperature, the resulting clear yellow solution was transferred via syringe over about $1 \mathrm{~min}$ to a clear colourless stirred solution of $(\boldsymbol{R})$-B $(30.0 \mathrm{mg}$, $0.040 \mathrm{mmol}, 0.10$ eq.) in $\mathrm{Et}_{2} \mathrm{O}(2.0 \mathrm{~mL})$ under an argon atmosphere. The resulting dark mixture was allowed to stir for $10 \mathrm{~min}$ before 5,6-dihydro-2H-pyran-2-one ( $34 \mu \mathrm{L}, 0.40 \mathrm{mmol}, 1.0$ eq.) and then $\mathrm{TMSCl}(0.25 \mathrm{~mL}, 2.0 \mathrm{mmol}, 5.0$ eq.) were added via syringe. Stirring at room temperature was continued for $4 \mathrm{~h}$ before the reaction was quenched by the addition of $\mathrm{Et}_{2} \mathrm{O}(c a 3 \mathrm{~mL})$ and then $\mathrm{NH}_{4} \mathrm{Cl}\left(1 \mathrm{M}\right.$ aq., $c a 1.5 \mathrm{~mL}$ ). The mixture was partitioned between water and $\mathrm{Et}_{2} \mathrm{O}$ and the aqueous phase was extracted with $\mathrm{Et}_{2} \mathrm{O}(3 \times 10 \mathrm{~mL})$. The combined organic phase was washed with $\mathrm{NaHCO}_{3}$ (aq. sat., ca $10 \mathrm{~mL}$ ), dried $\left(\mathrm{MgSO}_{4}\right)$, filtered and concentrated in vacuo. Flash column chromatography of the yellow residue (1:4; EtOAc:petrol; $\left.\mathrm{SiO}_{2}\right)$ gave $(+)-(R)-4-(2-(2-m e t h y l-1,3-$ dioxolan-2-yl)ethyl)tetrahydro-2H-pyran-2-one (35 mg, $0.16 \mathrm{mmol}, 41 \%$ ) as a colourless oil.

HPLC analysis of the amide derivative of $\mathbf{3 6 u}$ (see general procedure for details) indicated an enantiomeric excess of $78 \%$ [Chiralpak ${ }^{\circledR} \mathrm{IC}$; flow: $1.0 \mathrm{~mL} / \mathrm{min}$; hexane/i-PrOH: 80:20; $\lambda=210 \mathrm{~nm}$; minor enantiomer, $t_{R}=25.18 \mathrm{~min}$; major enantiomer, $\left.t_{R}=28.86 \mathrm{~min}\right]$.

${ }^{1} \mathrm{H}$ NMR $\left(400 \mathrm{MHz}, \mathrm{CDCl}_{3}\right) \delta_{\mathrm{H}} / \mathrm{ppm}: 1.31(\mathrm{~s}, 3 \mathrm{H}), 1.42-1.56(\mathrm{~m}, 3 \mathrm{H}), 1.64-1.72(\mathrm{~m}, 2 \mathrm{H}), 1.91-2.00$ $(\mathrm{m}, 2 \mathrm{H}), 2.15(\mathrm{dd}, J=17.1,10.0 \mathrm{~Hz}, 1 \mathrm{H}), 2.71(\mathrm{ddd}, J=17.1,5.6,1.7 \mathrm{~Hz}, 1 \mathrm{H}), 3.88-4.00(\mathrm{~m}, 4 \mathrm{H})$, $4.25(\mathrm{td}, J=10.5,3.7 \mathrm{~Hz}, 1 \mathrm{H}), 4.42(\mathrm{ddd}, J=11.3,4.7,3.9 \mathrm{~Hz}, 1 \mathrm{H})$.

${ }^{13} \mathrm{C} \mathrm{NMR}\left(100 \mathrm{MHz}, \mathrm{CDCl}_{3}\right) \delta_{\mathrm{c}} / \mathrm{ppm}: 23.8,28.9,30.3,31.6,36.1,36.6,64.7$ (2 C), 68.4, 109.6, 171.2 .

HRMS (EI) $m / z$ calcd for $\mathrm{C}_{11} \mathrm{H}_{18} \mathrm{NaO}_{4}[\mathrm{M}+\mathrm{Na}]^{+}: 237.1097$, found: 237.1095 .

$[\alpha]^{20}{ }_{589}:+14.6\left(c 0.50, \mathrm{CHCl}_{3}\right)$.

IR $\left(\mathbf{v}_{\max } / \mathrm{cm}^{-1}\right):$ 1731, 2979, 3042.

Absolute configuration assigned by analogy to compound $\mathbf{3 6 j}$. 


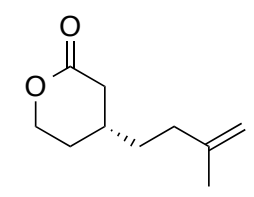

$\mathrm{Cp}_{2} \mathrm{ZrHCl}(3.0 \mathrm{~g}, 12.0 \mathrm{mmol}, 2.0$ eq.) was added to a stirred, room temperature, solution of isoprene (1.5 mL, $15.0 \mathrm{mmol}, 2.5$ eq.) in $\mathrm{CH}_{2} \mathrm{Cl}_{2}(0.40 \mathrm{~mL})$ under an argon atmosphere. After stirring for $30 \mathrm{~min}$ at room temperature, the resulting clear yellow solution was transferred via syringe over about $1 \mathrm{~min}$ to a clear colourless stirred solution of $(\boldsymbol{R})$-B $(451 \mathrm{mg}, 0.6 \mathrm{mmol}, 0.10$ eq.) in $\mathrm{Et}_{2} \mathrm{O}(2.0 \mathrm{~mL})$ under an argon atmosphere. The resulting dark mixture was allowed to stir for $10 \mathrm{~min}$ before 5,6-dihydro-2H-pyran-2-one (520 $\mathrm{LL}, 12.0 \mathrm{mmol}, 1.0$ eq.) and then $\mathrm{TMSCl}(2.4$ $\mathrm{mL}, 30.0 \mathrm{mmol}, 5.0$ eq.) were added via syringe. Stirring at room temperature was continued for 5 $h$ before the reaction was quenched by the addition of $\mathrm{Et}_{2} \mathrm{O}(c a 30 \mathrm{~mL})$ and then $\mathrm{NH}_{4} \mathrm{Cl}(1 \mathrm{M}$ aq., ca $15 \mathrm{~mL}$ ). The mixture was partitioned between water and $\mathrm{Et}_{2} \mathrm{O}$ and the aqueous phase was extracted with $\mathrm{Et}_{2} \mathrm{O}(3 \times 50 \mathrm{~mL})$. The combined organic phase was washed with $\mathrm{NaHCO}_{3}$ (aq. sat., ca $30 \mathrm{~mL})$, dried $\left(\mathrm{MgSO}_{4}\right)$, filtered and concentrated in vacuo. Flash column chromatography of the yellow residue (1:4; $t$-BuOMe:petrol; $\mathrm{SiO}_{2}$ ) gave $(+)-(R)$-4-(3-methylbut-3-en-1-yl)tetrahydro$2 \mathrm{H}$-pyran-2-one (408 mg, $3.1 \mathrm{mmol}, 78 \%$ ) as a yellow oil.

HPLC analysis of the amide derivative of $\mathbf{3 6 v}$ (see general procedure for details) indicated an enantiomeric excess of $88 \%$ [Chiralpak ${ }^{\circledR}$ IC; flow: $1.0 \mathrm{~mL} / \mathrm{min}$; hexane/i-PrOH: 87:13; $\lambda=210 \mathrm{~nm}$; major enantiomer, $t_{R}=17.75 \mathrm{~min}$; minor enantiomer, $\left.t_{R}=19.85 \mathrm{~min}\right]$.

${ }^{1} \mathrm{H}$ NMR $\left(400 \mathrm{MHz}, \mathrm{CDCl}_{3}\right) \delta_{\mathrm{H}} / \mathrm{ppm}: 1.45-1.60(\mathrm{~m}, 3 \mathrm{H}), 1.73(\mathrm{~s}, 3 \mathrm{H}), 1.90-2.10(\mathrm{~m}, 2 \mathrm{H}), 2.05(\mathrm{t}, J=$ $7.3 \mathrm{~Hz}, 2 \mathrm{H}), 2.14(\mathrm{dt}, J=17.4,10.3 \mathrm{~Hz}, 1 \mathrm{H}), 2.71(\mathrm{ddd}, J=17.4,5.9,1.7 \mathrm{~Hz}, 1 \mathrm{H}), 4.25(\mathrm{td}, J=11.3$, $3.4 \mathrm{~Hz}, 1 \mathrm{H}), 4.38-4.45(\mathrm{~m}, 1 \mathrm{H}), 4.71(\mathrm{~d}, J=21.2 \mathrm{~Hz}, 2 \mathrm{H})$.

${ }^{13} \mathrm{C} \mathrm{NMR}\left(100 \mathrm{MHz}, \mathrm{CDCl}_{3}\right) \delta_{\mathrm{C}} / \mathrm{ppm}: 28.8,30.9,33.9,34.4,36.4,38.3,66.4,110.6,144.7,171.3$.

HRMS (EI) $m / z$ calcd for $\mathrm{C}_{10} \mathrm{H}_{16} \mathrm{NaO}_{2}[\mathrm{M}+\mathrm{Na}]^{+}:$191.1043, found: 191.1045 .

$[\alpha]_{589}^{20}:+4.3\left(c\right.$ 1.30, $\left.\mathrm{CHCl}_{3}\right)$.

IR $\left(v_{\max } / \mathrm{cm}^{-1}\right):$ 1649, 1735, 2873, 2920, 2959.

Absolute configuration assigned by analogy to compound $\mathbf{3 6 \mathbf { j }}$. 


\section{(-)-(R)-7-Methyl-4,4a,5,6-tetrahydrocyclopenta[c]pyran-1(3H)-one (44)}<smiles>CC1=C2CCC3CCOC(=O)C3CC2C1</smiles>

A stream of $\mathrm{O}_{3}$ was passed through a solution of $36 \mathrm{v}$ ( $50 \mathrm{mg}, 0.30 \mathrm{mmol}, 1.0$ eq.) in $\mathrm{CH}_{2} \mathrm{Cl}_{2}(5.0$ $\mathrm{mL}$ ) and $\mathrm{MeOH}(2.0 \mathrm{~mL})$ at $-78{ }^{\circ} \mathrm{C}$ until a blue colour persisted. The solution was purged with a stream of $\mathrm{O}_{2}$ until the blue colour faded and then $\mathrm{Me}_{2} \mathrm{~S}(30 \mu \mathrm{L}, 0.31 \mathrm{mmol}, 1.1$ eq.) was added and the solution allowed to warm to RT for $2 \mathrm{~h}$. The resulting solution was concentrated in vacuo to give a yellow oil, which was used without further purification in the following step. The yellow oil was dissolved in benzene $(4.0 \mathrm{~mL})$ and para-toluene sulfonic acid $(51 \mathrm{mg}, 0.30 \mathrm{mmol}, 1.0$ eq.) was added. The resulting solution was fitted with Dean-Stark apparatus and heated under reflux at $100{ }^{\circ} \mathrm{C}$ for $16 \mathrm{~h}$. The reaction was cooled to RT, quenched with $\mathrm{NaHCO}_{3}(10 \mathrm{~mL}$, sat. aq.) and extracted with EtOAc $(3 \times 10 \mathrm{~mL})$. The combined organic layers were washed with brine $(10 \mathrm{~mL}$, sat. aq.), dried over $\mathrm{MgSO}_{4}$, filtered, concentrated in vacuo and purified by column chromatography (9:1; petrol:EtOAc; $\left.\mathrm{SiO}_{2}\right)$ to give $(37 \mathrm{mg}, 0.24 \mathrm{mmol}, 81 \%)$ as a yellow oil.

HPLC analysis indicated an enantiomeric excess of $83 \%$ [Chiralpak ${ }^{\circledR} \mathrm{IA}$; flow: $1.0 \mathrm{~mL} / \mathrm{min}$; hexane/i-PrOH: 99:1; $\lambda=210 \mathrm{~nm}$; minor enantiomer, $t_{R}=20.94$ min; major enantiomer, $t_{R}=22.19$ $\min ]$.

${ }^{1} \mathrm{H}$ NMR $\left(400 \mathrm{MHz}, \mathrm{CDCl}_{3}\right) \delta_{\mathrm{H}} / \mathrm{ppm}: 1.36-1.47(\mathrm{~m}, 1 \mathrm{H}), 1.57(\mathrm{td}, J=12.2,4.9 \mathrm{~Hz}, 1 \mathrm{H}), 1.98(\mathrm{~m}, 1 \mathrm{H})$, $2.14(\mathrm{~s}, 3 \mathrm{H}), 2.21-2.31(\mathrm{~m}, 1 \mathrm{H}), 2.37-2.52(\mathrm{~m}, 1 \mathrm{H}), 2.82-2.95(\mathrm{~m}, 1 \mathrm{H}), 4.20(\mathrm{td}$, $J=12.5,2.9 \mathrm{~Hz}, 1 \mathrm{H}$ ), 4.34 (ddd, $J=11.3,4.7,2.2 \mathrm{~Hz}, 1 \mathrm{H}$ ).

${ }^{13} \mathrm{C}$ NMR $\left(100 \mathrm{MHz}, \mathrm{CDCl}_{3}\right) \delta_{\mathrm{c}} / \mathrm{ppm}: 16.7,30.9,31.0,38.6,43.3,69.5,125.1,160.0,164.3$. HRMS (EI) $m / z$ calcd for $\mathrm{C}_{9} \mathrm{H}_{12} \mathrm{NaO}_{2}[\mathrm{M}+\mathrm{Na}]^{+}: 175.0730$, found: 175.0723 .

$[\alpha]^{20}{ }_{589}:-97.6\left(\mathrm{c} 0.60, \mathrm{CHCl}_{3}\right)$.

IR $\left(v_{\max } / \mathrm{cm}^{-1}\right):$ 1639, 1738, 2873, 2927, 2958.

Absolute configuration assigned by analogy to compound $\mathbf{3 6 j}$. 


\section{Chapter 6: References}

1 Hanessian, S. Total Synthesis of Natural Products: The 'Chiron' Approach, Pergamon Press: Oxford, 1983.

2 Sheldon, R. A. in: Chirotechnoglogy, Marcel Dekker, New York, 1993.

3 Kagan, H. B.; Fiaud, J. C. "New Approaches in Asymmetric Synthesis", Top. Stereochem. 1978, 10, 175;

4 Jacobsen, E. N.; Pfaltz, A.; Yamamoto, H. Comprehensive Asymmetric Catalysis, Springer-Verlag: Berlin/Heidelberg, 1999.

5 (a) Roughley, S. D.; Jordan, A.M. J. Med. Chem. 2011, 54, 3451; (b) Christmann, M.; Bräse, S. Asymmetric synthesis : the essentials. $2^{\text {nd }}$ ed.; Wiley-VCH: Weinheim, 2008, 355.

6 Carey, J. S.; Laffan, D.; Thomson, C.; Williams, M. T. Org. Biomol. Chem. 2006, 4, 2337.

7 Akiyama, T. Chem. Rev. 2007, 107, 5744.

8 (a) Alexakis, A.; Backvall, J. E.; Krause, N.; Pamies, O.; Dieguez, M. Chem. Rev. 2008, 108, 2796; (b) Harutyunyan, S. R.; den Hartog, T.; Geurts, K.; Minnaard, A. J.; Feringa, B. L. Chem. Rev. 2008, 108, 2824; (c) Jerphagnon, T.; Pizzuti, M. G.; Minnaard, A. J.; Feringa, B. L. Chem. Soc. Rev. 2009, 38, 1039.

9 Verkade, J. M. M.; van Hemert, L. J. C.; Quaedflieg, P. J. L. M.; Rutjes, F. P. J. T. Chem. Soc. Rev. 2008, $37,29$. 10 Liu, X.; Fox, J. M. J. Am. Chem. Soc. 2006, 128, 5600 and references therin.

11 Davies, H. L.; Beckwith, R. E. J. Chem. Rev. 2003, 103, 2861.

12 Perlmutter, P. Conjugate Addition Reactions in Organic Synthesis Pergamon, 1992.

13 (a) Rossiter, B. E.; Swingle, N. M. Chem. Rev. 1991, 92, 771; (b) Krause, N.; Gerold, A. Angew. Chem. Int. Ed. 1997, 36, 186.

14 (a) Krause, N.; Hoffmann-Röder, A. Synthesis 2001, 171; (b) Christoffers, J.; Koripelly, G.; Rosiak, A.; Rossle, M. Synthesis 2007, 1279.

15 Gilman, H.; Straley, J. M. Red. Trav. Chim. Pays-Bas 1936, 55, 821.

16 Kharasch, M. S.; Tawney, P. O. J. Am. Chem. Soc. 1941, 63, 2308.

17 (a) Alexakis, A.; Benhaim, C. Eur. J. Org. Chem. 2002, 3221; Thaler, T.; Knochel, P. Angew. Chem. Int. Ed. 2009, 48, 645; (b) Hawner, C.; Alexakis, A. Chem. Commun. 2010, 46, 7295; (c) Wang, S. Y.; Loh, T. P. Chem. Commun. 2010, 46, 8694.

18 Bower, J. F.; Krische, M. J. Handbook of Green Chemistry, Wiley-VCH: Verlag GmbH \& Co. KGaA, 2010.

19 Howell G. P. Org. Process Res. Dev. 2012, 16, 1258.

20 Lum, T. K.; Wang, S. Y.; Loh, T. P. Org. Lett. 2008, 10, 761.

21 Ter Horst, B.; Feringa, B. L.; Minnaard, A. J. Org. Lett. 2007, 9, 3013.

22 Negishi, E. Handbook of Organopalladium Chemistry for Organic Synthesis: Volume 1 \& 2, John Wiley \& Sons, 2003, 2789.

23 Ball, Z. T.; Trost, B. M. Synthesis, 2005, 6, 853.

24 Chemler, S. R.; Trauner, D.; Danishefsky, S. J. Angew. Chem. Int. Ed. 2001, 40, 4544.

25 Negishi, E. Dalton Trans. 2005, 827.

26 Rouhi, A. M. C\&EN news, 2004, 82, 36.

27 (a) Hart, D. W.; Schwartz, J. J. Am. Chem. Soc. 1974, 8115; (b) Schwartz, J.; Labinger J. A. Angew. Chem. Int. 
Ed. 1976, 15, 333; (c) Carr, D. B.; Schwartz, J. J. Am. Chem. Soc. 1979, 3521.

28 (a) Takacs, J. M. In Comp. Organomet. Chem. II, Abel, E. W.; Stone, F. G. A.; Wilkinson, L. S.; Hegedus, L. S. Eds.; Vol 12, chapt 3.1, 1995; (b) Marek, I. New Aspects of Zirconium Containing Organic Compounds, Springer: Berlin, 2005.

29 Hegedus, L. S. Transition metals in the synthesis of complex organic molecules, $2^{\text {nd }}$ ed.; University Science books, 1999.

30 (a) Hayashi, T. Synlett 2001, 879. (b) Yamasaki, K.; Hayashi, T. Chem. Rev. 2003, 103, 2829. (c) Müller, D.; Alexakis, A. Chem. Commun. 2012, 48, 12037.

31 (a) Tian, P.; Dong, H. Q.; Lin G. Q. ACS Catal. 2012, 2, 95; (b) Bolm, C.; Hildebrand, J. P.; Muñiz, K.; Hermanns N. Angew. Chem. Int. Ed. 2001, 40, 3284; (c) Hayashi, T. Bull. Chem. Soc. Jpn. 2004, 77, 13.

32 Takaya, Y.; Ogasawara, M.; Hayashi T. J. Am. Chem. Soc. 1998, 120, 5579.

33 Takaya, Y.; Ogasawara, M.; Hayashi T. Tetrahedron Lett. 1998, 39, 8479.

34 Hickmann, V.; Alcarazo, M.; Fürstner A. J. Am. Chem. Soc. 2010, 132, 11042.

35 Defieber, C.; Paquin, J. F.; Serna, S.; Carreira, E. M. Org. Lett. 2004, 6, 3873.

36 Alexakis, A.; Albrow, V.; Biswas, K.; d'Augustin, M.; Prieto, O.; Woodward S. Chem. Commun. 2005, 2843.

37 Albrow, V. E.; Blake, A. J.; Fryatt, R.; Wilson, C.; Woodward S. Eur. J. Org. Chem. 2006, 2549.

38 Zweifel, G.; Miller, J. A. Org. React. 1984, 32, 375.

39 Vuagnoux-d'Augustin, M.; Alexakis A. Chem. Eur. J. 2007, 13, 9647.

40 Palais, L.; Alexakis A. Chem. Eur. J. 2009, 15, 10473.

41 Muller, D.; Tissot, M.; Alexakis A. Org. Lett. 2011, 13, 3040.

42 May, T. L.; Dabrowski, J. A.; Hoveyda A. H. J. Am. Chem. Soc. 2011, 133, 736.

43 Gao, F.; McGrath, K. P.; Lee, Y.; Hoveyda, A. H. J. Am. Chem. Soc. 2010, 132, 14315.

44 Willcox, D.; Woodward S.; Alexakis A. Chem. Commun. 2014 DOI: 10.1039/c3cc48191c.

45 Oi, S.; Taira, A.; Honma, Y.; Inoue, Y. Org. Lett. 2003, 5, 97.

46 Otomaru, Y.; Hayashi, T. Tetrahedron Asymmetry 2004, 15, 2647.

47 Nakao, Y.; Chen, J.; Imanaka, H.; Hiyama, T.; Ichikawa, Y.; Duan, W. L.; Shintani, R.; Hayashi, T. J. Am. Chem. Soc. 2007, 129, 9137.

48 Shintani, R.; Ichikawa, Y.; Hayashi, T.; Chen, J.; Nakao, Y.; Hiyama, T. Org. Lett. 2007, 9, 4643.

49 Lee, K.; Hoveyda A. H. J. Org. Chem. 2009, 74, 4455.

50 Oi, S.; Sato, T.; Inoue, Y. Tetrahedron Letters 2004, 45, 5051.

51 Nicolaou, K. C.; Tang, W.; Dagneau, P.; Faraoni, R. Angew. Chem. Int. Ed. 2005, 44, 3874.

52 Westmeier, J.; Pfaff, C.; Siewert, J.; von Zezschwitz, P. Adv. Synth. Catal. 2013, 355, 2651.

53 Westmeier, J.; Kress, S.; Pfaff, C.; von Zezschwitz, P. J. Org. Chem. 2013, 78, 10718.

54 Sĕbesta, R. ChemCatChem 2013, 5, 1069.

55 Yoshida, M.; Ohmiya, H.; Sawamura M. J. Am. Chem. Soc. 2012, 134, 11896.

56 Shido, Y.; Yoshida, M.; Tanabe, M.; Ohmiya, H.; Sawamura M. J. Am. Chem. Soc. 2012, 134, 18573.

57 Wipf, P.; Smitrovich, J. H. J. Org. Chem. 1991, 56, 6494. 
58 Wipf, P.; Xu, W.; Smitrovich, J. H.; Lehmann, R.; Venanzi, L. M. Tetrahedron 1994, 50, 1935.

59 Wipf, P.; Takahashi, H. Chem. Commun. 1996, 2675.

60 Maksymowicz, R. M.; Roth, P. M. C.; Fletcher, S. P. Nat. Chem. 2012, 4, 649.

61 Maksymowicz, R. M.; Roth, P. M. C.; Thompson, A. L.; Fletcher, S. P. Chem. Commun. 2013, 49, 4211.

62 Maksymowicz, R. M.; Sidera, M.; Roth, P. M. C.; Fletcher, S. P. Synthesis, 2013, 45, 2662.

63 van Klaveren, M.; Lambert, F.; Eijkelkamp, D.I. F. M.; Grove, D. M.; van Koten, G. Tet. Lett. 1994, 35, 6135.

64 Stangeland, E. L.; Sammakia T. Tetrahedron 1997, 53, 16503.

65 Mizutani, H.; Degrado, S. J.; Hoveyda, A. H. J. Am. Chem. Soc. 2002, 124, 779.

66 (a) Alexakis, A.; Polet, D.; Benhaim, C.; Rosset S. Tetrahedron Asymm. 2004, 15, 2199; (b) Alexakis, A.; Polet, D.; Rosset, S.; March, S. J. Org. Chem. 2004, 69, 5660.

67 López, F.; Harutyunyan, S. R.; Minnaard, A. J.; Feringa, B. L. J. Am. Chem. Soc. 2004, 126, 12784.

68 Hajra, A.; Yoshikai, N.; Nakamura, E. Org. Lett. 2006, 8, 4153.

69 Tauchman, J.; Císařová, I.; Štěpnička, P. Eur. J. Org. Chem. 2010, 4276.

70 Endo, K.; Ogawa, M.; Shibata, T. Angew. Chem. Int. Ed. 2010, 49, 2410.

71 Dohi, K.; Kondo, J.; Yamada, H.; Arakawa, R.; Sakaguchi, S. Eur. J. Org. Chem. 2012, 7143.

72 Magrez, M.; Wencel-Delord, J.; Crévisy, C.; Mauduit, M. Tetrahedron 2012, 68, 3507.

73 Bauer, T.; Majdecki, M.; Jurczak, J. Tetrahedron 2013, 69, 1930.

74 Endo, K.; Hamada, D.; Yakeishi, S.; Shibata, T. Angew. Chem. Int. Ed. 2013, 52, 606.

75 Buchwald, S. L.; LaMaire, S. J.; Nielsen, R. B. Org. Synth. 1993, 71, 77.

76 Salomon, R. G.; Kochi, J. K. J. Am. Chem. Soc. 1973, 95, 1889.

77 Feringa, B. L.; Pineschi, M.; Arnold, L. A.; Imbos, R.; de Vries, A. H. M. Angew. Chem. Int. Ed. 1997, 36, 2620.

78 Alexakis, A.; Krause, N.; Woodward, S. Copper-Catalyzed Asymmetric Synthesis Chapter 2, Wiley-VCH: Weinheim, 2014.

79 Villacorta, G. M.; Rao, C.; Lippard, S. J. J. Am. Chem. Soc. 1988, 110, 3175.

80 Alexakis, A.; Frutos, J.; Mangeney, P. Tetrahedron Asymm. 1993, 4, 2427.

81 Escher, I. H.; Pfaltz, A. Tetrahedron 2000, 56, 2879.

82 Degrado, S. J.; Mizutani, H.; Hoveyda, A. H. J. Am. Chem. Soc. 2001, 123, 755.

83 Feringa, B. L.; Badorrey, R.; Peña, D.; Harutyunyan, S. R.; Minnaard, A. J. Proc. Natl. Acad. Sci. USA 2004, $101,5834$.

84 Alexakis, A.; Frutos, J. C.; Mangeney, P. Tetrahedron Asymm. 1993, 4, 2431.

85 Tissot-Croset, K.; Polet, D.; Gille, S.; Hawner, C.; Alexakis, A. Synthesis 2004, 2586.

86 Smith, C. R.; RajanBabu, T. V. Org. Lett. 2008, 10, 1657.

87 Bunnage, M.; Davies, S.; Parkin, R.; Roberts, R.; Smith, A.; Withey, J. Org. Biomol. Chem. 2004, 3337.

88 Arnold, L. A.; Imbos, R.; Mandoli, A.; de Vries, A. H. M.; Naasz, R.; Feringa, B. L. Tetrahedron 2000, 56, 2865.

89 Trost, B. M.; Silverman, S. M.; Stambuli, J. P. J. Am. Chem. Soc. 2011, 133, 19483. 
90 (a) Jung, M. E.; Maderna, A. Tetrahedron Lett. 2005, 46, 5057; (b) Yanagisawa, A.; Shinohara, A.; Takahashi, H.; Arai, T. Synlett 2007, 1, 141.

91 Reingold, I. D.; Bowerman, C.; John, M.; Walters, R. S.; Daglen, B. C.; Butterfield, A. M.; Gembicky, M. Tetetrahedron Lett. 2006, 47, 1653.

92 Cossy, J.; Lutz, F.; Alauze, V.; Meyer, C. Synlett 2002, 1, 45.

93 (a) Giacobbe, S. A.; Rossi, T. Tetrahedron Asymm. 1996, 7, 3079; (b) Kasatkin, A.; Whitby, R. J. Tetrahedron 2003, 59, 9857.

94 Vrielynck, F. A.; De Clercq, P. J. Molecules 2007, 12, 237.

95 Brown, M. K.; Degrado, S. J.; Hoveyda, A. H. Angew. Chem. Int. Ed. 2005, 44, 5306.

96 Shirakawa, E.; Ikeda, D.; Masui, S.; Yoshida, M.; Hayashi, T. J. Am. Chem. Soc. 2012, 134, 272.

97 Nagashima, F.; Kuba Y.; Asakawa, Y. Chem. Pharm. Bull. 2006, 54, 902.

98 Coia, N.; Mokhtari, N.; Vasse, J. L.; Szymoniak, J. Org. Lett. 2011, 13, 6292.

99 (a) Degrado, S. J.; Mizutani, H.; Hoveyda, A. H. J. Am. Chem. Soc. 2001, 123, 755; (b) Naeemi, Q.; Robert, T.; Kranz, D. P.; Velder, J.;. Schmalz, H. G. Tetrahedron Asymm. 2011, 22, 887.

100 Sidera, M.; Roth, P. M. C.; Maksymowicz, R. M.; Fletcher, S. P. Angew. Chem. Int. Ed. 2013, $52,7995$.

101 Roth, P. M. C.; Sidera, M.; Maksymowicz, R. M.; Fletcher, S. P. Nature Protocols 2014, 9, 104.

102 Reinitzer, F. Monatsh. Chem. 1888, 9, 421.

103 (a) Tachibana, K.; Imaoka, I.; Yoshino, H.; Emura, T.; Kodama, H.; Furuta, Y.; Kato, N.; Nakamura, M.; Ohta, M.; Taniguchi, K.; Ishikura, N.; Nagamuta, M.; Onuma, E.; Sato, H. Bioorg. Med. Chem. 2007, 15, 174; (b) Smith, H. J.; Nicholls, P. J.; Simons, C.; Le Lain, R. Expert Opin. Ther. Pat. 2001, 11, 789; (c) Labrie, C.; Martel, C.; Dufour, J. M.; Levesque, C.; Merand, Y.; Labrie, F. Cancer Res. 1992, 52, 610.

104 Brazier, E. J.; Hogan, P. J.; Leung, C. W.; O’Kearney-McMullan, A.; Norton, A. K.; Powell, L.; Robinson, G. E.; Williams, E. G. Org. Process Res. Dev. 2010, 14, 544.

105 (a) Stephan, E. Org. Prep. Proced. Int. 2006, 38, 217; (b) Krause N.; Thorand, S. Inorg. Chim. Acta 1999, 296, 1.

106 Westermann, J.; Nickisch, K. Angew. Chem. Int. Ed. 1993, 32, 1368.

107 Westermann, J.; Imbery, U.; Nguyen A. T.; Nickisch, K. Eur. J. Inorg. Chem. 1998, 295.

108 Adamczyk, M.; Chen, Y. Y.; Johnson, D. D.; Reddy, R. E. Tetrahedron 1997, 53, 12855.

109 Modi, S. P.; Gardner, J. O.; Milowsky, A.; Wierzba, M.; Forgione, L.; Mazur, P.; Solo, A. J.; Duax, W. L.; Galdecki, Z.; Grochulski, P.; Wawrzak, Z. J. Org. Chem. 1989, 54, 2317.

110 Oreilly, J. M.; Li, N. Y.; Duax, W. L.; Brueggemeier, R. W. J. Med. Chem. 1995, 38, 2842.

111 French, A. N.; Wilson, S. R.; Welch, M. J.; Katzenellenbogen, J. A. Steroids 1993, 58, 157.

112 (a) Das, J. P.; Marek, I. Chem. Commun. 2011, 47, 4593; (b) Trost, B. M.; Jiang, C. H. Synthesis 2006, 369; (c) Douglas, C. J.; Overman, L. E. Proc. Natl. Acad. Sci. USA 2004, 101, 5363; (d) Christoffers, J.; Baro, A. Angew. Chem. Int. Ed. 2003, 42, 1688; (e) Corey, E. J.; Guzman-Perez, A. Angew. Chem. Int. Ed. 1998, 37, 388.

113 d'Augustin M.; Palais L.; Alexakis A. Angew. Chem. Int. Ed. 2005, 44, 1376. 
114 (a) Palais, L.; Mikhel, I. S.; Bournaud, C.; Micouin, L.; Falciola, C. A.; Vuagnoux-d'Augustin, M.; Rosset, S.; Bernardinelli, G.; Alexakis, A. Angew. Chem. Int. Ed. 2007, 46, 7462; (b) Brown, M. K.; Hoveyda, A. H. J. Am. Chem. Soc. 2008, 130, 12904; (c) May, T. L.; Brown, M. K.; Hoveyda, A. H. Angew. Chem. Int. Ed. 2008, $47,7358$.

115 Hird, A. W.; Hoveyda, A. H. J. Am. Chem. Soc. 2005, 127, 14988.

116 Lee, K. S.; Brown, M. K.; Hird, A. W.; Hoveyda, A. H. J. Am. Chem. Soc. 2006, 128, 7182.

117 Brown, M. K.; May, T. L.; Baxter, C. A.; Hoveyda, A. H. Angew. Chem. Int. Ed. 2007, 46, 1097; May, T. L.; Brown, M. K.; Hoveyda, A. H. Angew. Chem. Int. Ed. 2008, 47, 7358.

118 Martin, D.; Kehrli, S.; d’Augustin, M.; Clavier, H.; Mauduit, M.; Alexakis, A. J. Am. Chem. Soc. 2006, 128, 8416; Kehrli, S.; Martin, D.; Rix, D.; Mauduit, M.; Alexakis, A. Chem. Eur. J. 2010, 16, 9890.

119 Matsumoto, Y.; Yamada, K. I.; Tomioka, K. J. Org. Chem. 2008, 73, 4578.

120 Germain, N.; Magrez, M.; Kehrli, S.; Mauduit, M.; Alexakis, A. Eur. J. Org. Chem. 2012, 5301; Tissot, M.; Poggiali, D.; Henon, H.; Muller, D.; Guenee, L.; Mauduit, M.; Alexakis, A. Chem. Eur. J. 2012, $18,8731$.

121 Mak, J. Y. W.; Williams, C. M. Chem. Commun. 2012, 48, 287.

122 Project taken over by Dr Mireia Sidera and Philippe M. C. Roth.

123 More optimisation results and substrate screening can be found at Sidera, M.; Roth, P. M. C.; Maksymowicz, R. M.; Fletcher, S. P. Angew. Chem. Int. Ed. 2013, 52, 7995.

124 (a) Zhang, Q.; Lu, Y.; Ding, Y.; Zhai, J.; Ji, Q.; Ma, W.; Yang, M.; Fan, H.; Long, J.; Tong, Z.; Shi, Y.; Jia, Y.; Han, B.; Zhang, W.; Qiu, C.; Ma, X.; Li, Q.; Shi, Q.; Zhang, H.; Li, D.; Zhang, J.; Lin, J.; Li, L.; Gao, Y.; Chen, Y. J. Med. Chem. 2012, 55, 8757. (b) Held, C.; Fröhlich, R.: Metz, P. Angew. Chem. Int. Ed. 2001, 40, 1058.

125 Yeung, K.; Paterson, I. Chem. Rev. 2005, 105, 4237.

126 (a) Wang, L.; Li, D.; Xu, S.; Cai, H.; Yao, H.; Zhang, Y.; Jiang, J.; Xu, J. Eur. J. Med. Chem. 2012, 52, 242; (b) Harding, W. W.; Schmidt, M.; Tidgewell, K.; Kannan, P.; Holden, K. G.; Dersch, C. M.; Rothman, R. B.; Prisinzano, T. E. Biorg. Med. Chem. Lett. 2006, 16, 3170.

127 Rihs, G.; Spescha, M. Helv. Chim. Acta 1993, 76, 1219.

128 (a) Kanai, M.; Tomioka, K. Tetrahedron Lett. 1995, 36, 4275; (b) Kanai, M.; Nakagawa, Y.; Tomioka, K. Tetrahedron 1999, 55, 3843.

129 (a) Yan, M.; Zhou, Z. Y.; Chan, A. S. C. Chem. Commun. 2000, 115; (b) Liang, L.; Su, L. M.; Li, X. S.; Chan, A. S. C. Tetrahedron Lett. 2003, 44, 7217; (c) Liang, L.; Yan, M.; Li, Y. M.; Chan, A. S. C. Tetrahedron Asymm. 2004, 15, 2575.

130 Reetz, M. T.; Gosberg, A.; Moulin, D. Tetrahedron Lett. 2002, 43, 1189.

131 (a) Clavier, H.; Coutable, L.; Guillemin, J. C.; Mauduit, M. J. Organomet. Chem. 2005, 690, 5237; (b) Clavier, H.; Coutable, L.; Toupet, L.; Guillemin, J. C.; Mauduit, M. J. Eur. J. Inorg, Chem. 2009, 1989.

132 Mao, B.; Fañanás-Mastral, M.; Feringa, B. L. Org. Lett. 2013, 15, 286.

133 Csizmadiová, J.; Mečiarová, M.; Almássy, A.; Horváth, B.; Šebesta, R. J. Organomet. Chem. 2013, 737, 47.

134 Chatterjee, A. K.; Morgan, J. P.; Scholl, M.; Grubbs R. H. J. Am. Chem. Soc. 2000, 122, 3783.

135 Reich, H. J.; Renga, J. M.; Reich, I. L. J. Am. Chem. Soc. 1975, 97, 5434. 
136 Saeki, M.; Toyota, M. Heterocycles 2011, 82, 1705.

137 (a) Liang, K. W.; Chandrasekharam, M.; Li, C. L.; Liu, R. S. J. Org. Chem. 1998, 63, 7289; (b) Bouyssi, D.; Monteiro, N.; Balme, G. Tetrahedron Lett. 1999, 40, 1297.

138 (a) Takacs, J. M.; Myoung, Y. C. Tetrahedron Lett. 1992, 33, 317; (b) Weinges, K.; Ziegler, H. J.; Maurer, W.; Schmidbauer, S. B. Liebigs Ann. Chem. 1993, 1029; (c) Takacs, J. M.; Myoung, Y. C.; Anderson, L. G. J. Org. Chem. 1994, 59, 6928; (d) Nangia, A.; Prasuna, G. Tetrahedron 1996, 52, 3435.

139 Yamane, T.; Takahashi, M.; Ogasawara, K. Synthesis 1995, 444.

140 Pucheault, M.; Darses, S.; Genêt, J. P. Eur. J. Org. Chem. 2002, 3552.

141 a) Robert, T.; Velder, J.; Schmalz, H. G. Angew. Chem. Int. Ed. 2008, 47, 7718; b) Kirk, D. N. Tetrahedron $1986,42,777$. 


\section{Appendix}

\section{A1: Ligand structures}

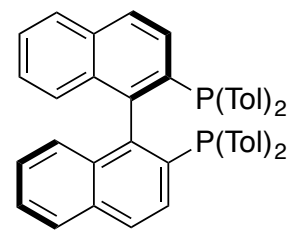

L1<smiles>[Y10][Y20]([Y16])([H])c1cc(OC)nc(OC)c1-c1c(OC)cc(OC)nc1P</smiles>

L7

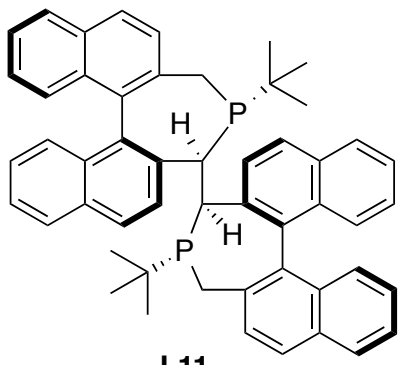

L11

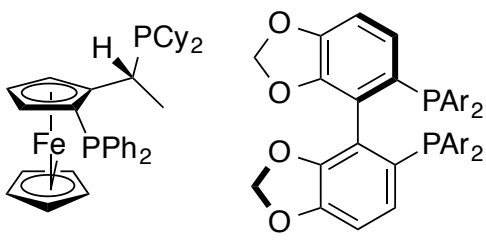

L2

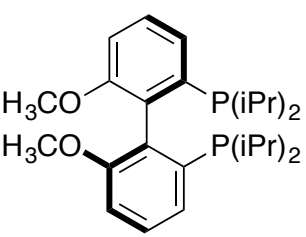

L5<smiles>Pc1ccccc1-c1c(-c2c(-c3ccccc3)ccc3c2OCCO3)ccc2c1OCCO2</smiles>

L6

$\mathrm{Ar}=\mathrm{DTBM}$ L4<smiles>COc1c(Cl)ccc(-c2c(P)ccc(-c3ccccc3)c2OC)c1OC</smiles>

L8<smiles>c1ccc(-c2nccc3cccc(-c4nccc5ccccc45)c23)cc1</smiles>

L12<smiles>FC1(F)Oc2ccc(-c3ccccc3)c(-c3c(-c4ccccc4)ccc4c3OC(F)(F)O4)c2O1</smiles>

L9

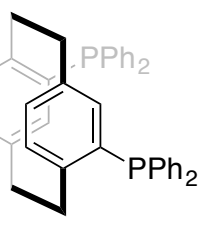

L10

A2: Phosphoramidite Ligands:<smiles>CN(C)P1OC(c2ccccc2)(c2ccccc2)C(c2ccccc2)C2OC(C)(C)OC21</smiles>

L15<smiles>CC(c1ccccc1)N(c1ccccc1)P(Oc1ccc2ccccc2c1-c1c(Oc2ccccc2)ccc2ccccc12)[C@H](C)c1ccccc1</smiles>

L19

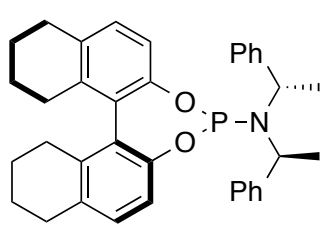

L22

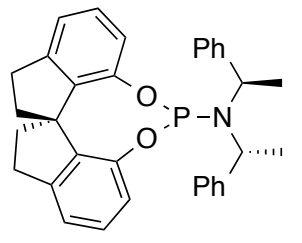

L16<smiles>C[C@H](c1ccccc1)N([C@H](C)c1ccccc1)p1oc2ccccc2c2ccccc2o1</smiles>

L17

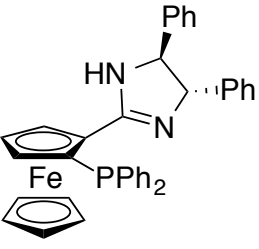

L13<smiles>c1ccc(OPOc2cccc3c2[C@@H]2CCc4cccc(c4-3)O2)cc1</smiles>

L14<smiles>Cc1cc2ccccc2c(-c2c(OP(N(C)C)N(C)C)c(C)cc3ccccc23)c1O</smiles><smiles>C[C@H](c1ccc2ccccc2c1)N(PPc1ccc2ccccc2c1-c1c(O)ccc2ccccc12)C1CCCCC1</smiles>

L21<smiles>CC(C)N(PP)[C@@H](C)c1ccc(Cl)cc1</smiles> 


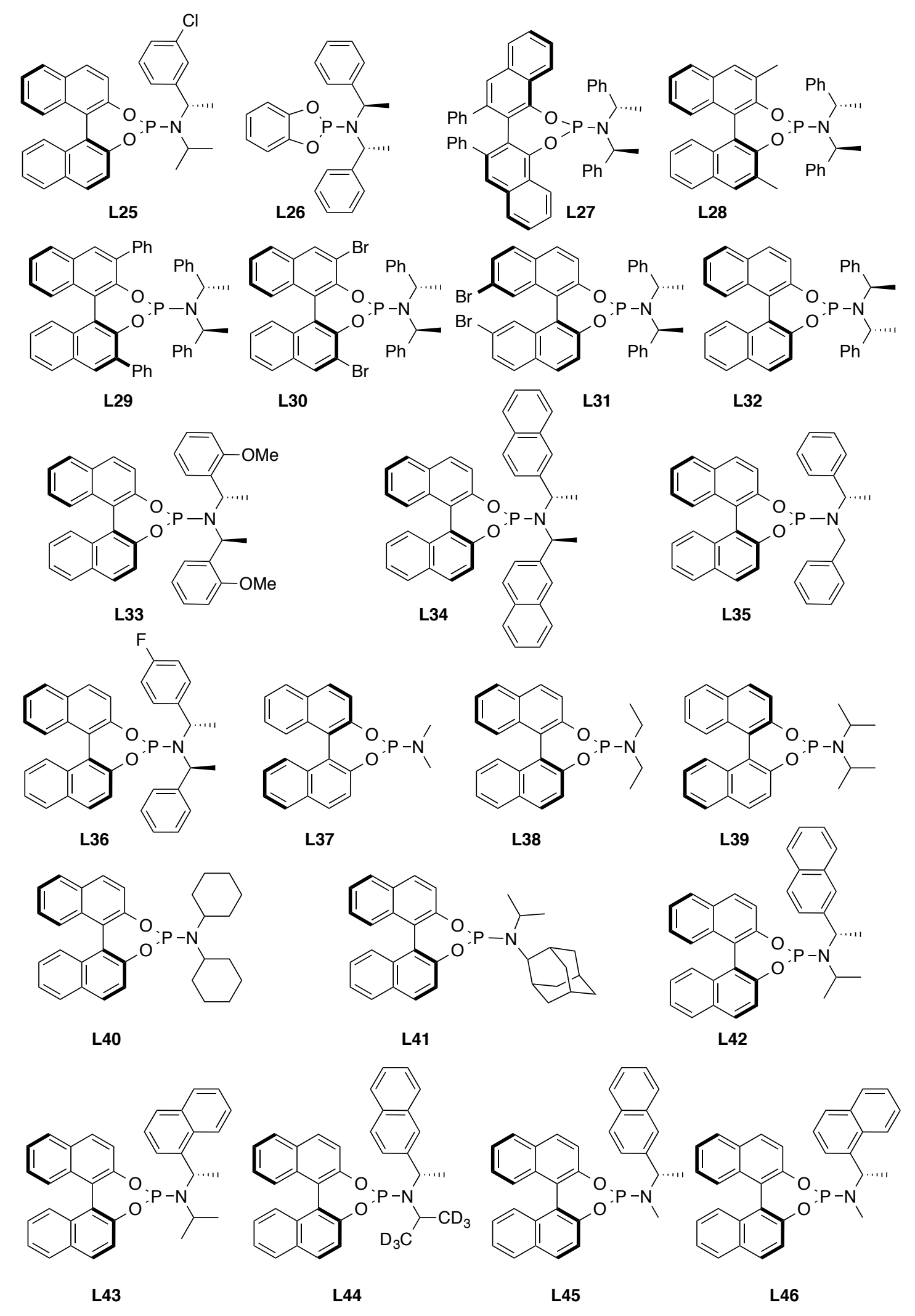



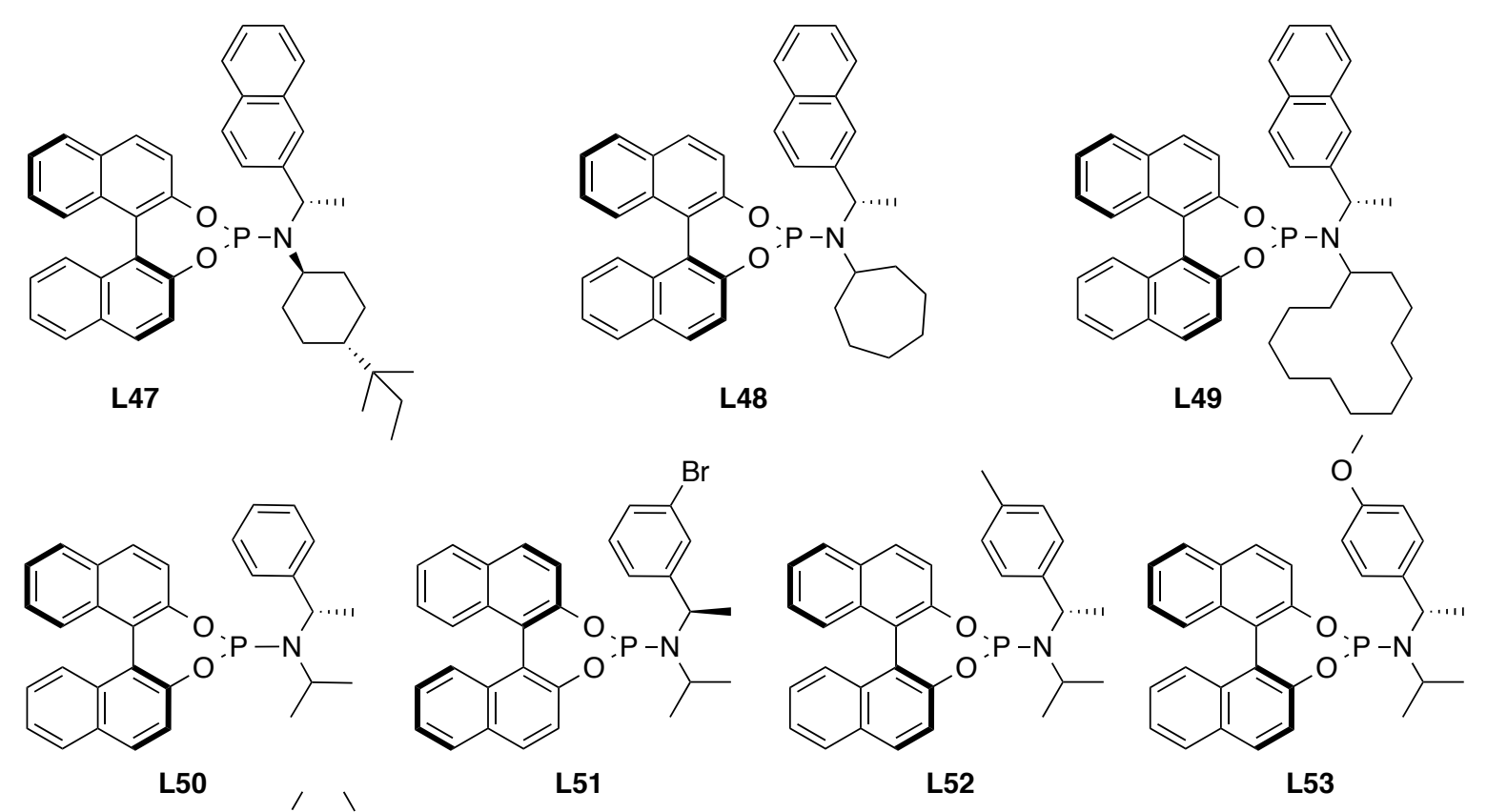<smiles>COc1cccc([C@H](C)N(P)C(C)C)c1Oc1ccc2ccccc2c1-c1c(O)ccc2ccccc12</smiles>

L54

L55

L56<smiles>CC(C)NPc1ccc2ccccc2c1-c1c(O)ccc2ccccc12</smiles><smiles>CC(C)N(P)C1c2ccccc2-c2cccc(O)c21</smiles>

L58

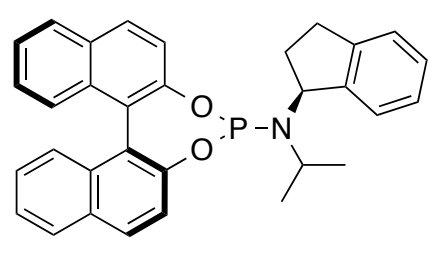

L59

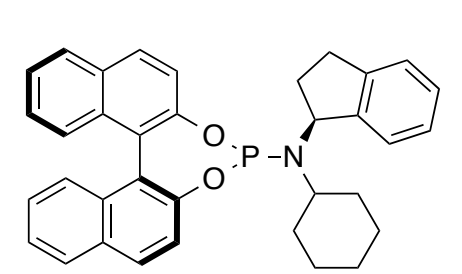

L60

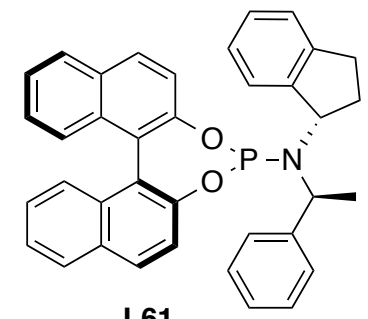

L61<smiles>CC(C)(C)Oc1ccc2ccccc2c1-c1c(O)ccc2ccccc12</smiles>

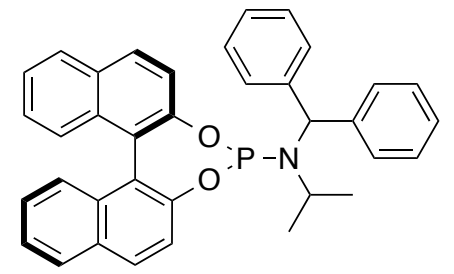

L63

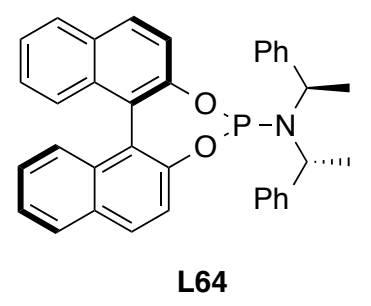

L64 


\section{X-ray Crystallography Data}

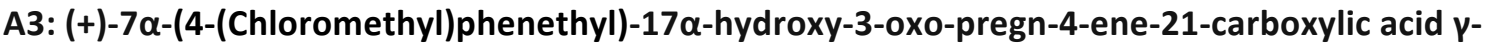
lactone
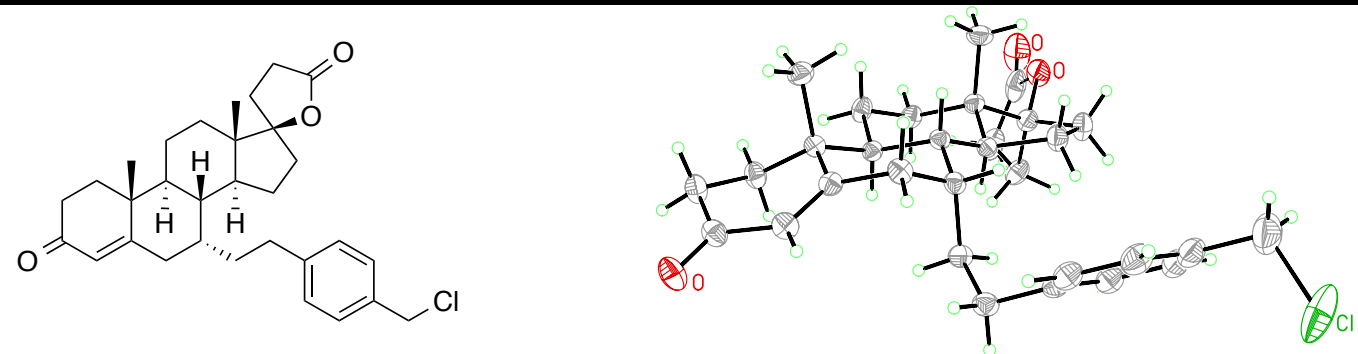

Key data and refinement for compound 32c

Empirical formula

Formula weight

Temperature

Wavelength

Crystal system

Space group

Unit cell dimensions

Volume

Z

Density (calculated)

Absorption coefficient

$\mathrm{F}(000)$

Crystal size

Theta range for data collected Index ranges

Reflections collected

Independent reflections

Completeness to theta $=75.061^{\circ}$

Absorption correction

Max. and min. transmission

Refinement method

Data/restraint/parameters

Goodness-of-fit on $\mathrm{F}^{2}$

Final $\mathrm{R}$ indices [ $\mathrm{I} 2 \mathrm{2sigma}(\mathrm{I})]$

$R$ indices (all data)

Absolute structure parameter

Largest diff. peak and hole
$\mathrm{C}_{31} \mathrm{H}_{39} \mathrm{ClO}_{3}$

495.10

$150 \mathrm{~K}$

$1.54180 \AA$

Orthorhombic

P 212121

$a=10.5479$ (1) $\AA \alpha=90^{\circ}$

$b=12.7584(1) \AA \beta=90^{\circ}$

$c=19.3047(2) \AA \gamma=90^{\circ}$

2597.92(4) $\AA^{3}$

4

$1.266 \mathrm{Mg} / \mathrm{m}^{3}$

$1.534 \mathrm{~mm}^{-1}$

1064

$0.160 \times 0.060 \times 0.060 \mathrm{~mm}^{3}$

4.153 to $76.592^{\circ}$

$-13<=\mathrm{h}<=13,-16<=\mathrm{K}<=16,-20<=\mid<=23$

58125

$5419[R($ int $)=0.028]$

$99.7 \%$

Semi-empirical from equivalents

0.91 and 0.75

Full-matrix least squares on F2

$5398 / 0 / 317$

0.9999

$$
\begin{gathered}
\mathrm{R} 1=0.0274, w R 2=0.0748 \\
R 1=0.0278, w R 2=0.0752 \\
-0.011(11) \\
0.27 \text { and }-0.34 \text { e. } \AA^{-3}
\end{gathered}
$$




\begin{tabular}{|c|c|}
\hline \multicolumn{2}{|c|}{ Key data and refinement for compound $36 \mathbf{j}$} \\
\hline Empirical formula & $\mathrm{C}_{16} \mathrm{H}_{22} \mathrm{O}_{3}$ \\
\hline Formula weight & 262.35 \\
\hline Temperature & $250 \mathrm{~K}$ \\
\hline Wavelength & $1.54180 \AA$ \\
\hline Crystal system & Monoclinic \\
\hline Space group & P 21 \\
\hline \multirow[t]{3}{*}{ Unit cell dimensions } & $a=6.3705(2) \AA \alpha=90^{\circ}$ \\
\hline & $b=30.5650(6) \AA \beta=101.442(3)^{\circ}$ \\
\hline & $c=7.5402(2) \AA \gamma=90^{\circ}$ \\
\hline Volume & $1439.01(7) \AA^{3}$ \\
\hline Z & 4 \\
\hline Density (calculated) & $1.211 \mathrm{Mg} / \mathrm{m}^{3}$ \\
\hline Absorption coefficient & $0.657 \mathrm{~mm}^{-1}$ \\
\hline$F(000)$ & 568 \\
\hline Crystal size & $0.34 \times 0.20 \times 0.10 \mathrm{~mm}^{3}$ \\
\hline Theta range for data collected & 5.790 to $76.453^{\circ}$ \\
\hline Index ranges & $-7<=\mathrm{h}<==7,-38<=\mathrm{K}<=38,0<=\mathrm{l}<=9$ \\
\hline Reflections collected & 5961 \\
\hline Independent reflections & $5961[\mathrm{R}$ (int) $=0.034]$ \\
\hline Completeness to theta $=75.061^{\circ}$ & $99.8 \%$ \\
\hline Absorption correction & Semi-empirical from equivalents \\
\hline Max. and min. transmission & 0.94 and 0.70 \\
\hline Refinement method & Full-matrix least squares on F2 \\
\hline Data/restraint/parameters & $4572 / 1 / 344$ \\
\hline Goodness-of-fit on $\mathrm{F}^{2}$ & 1.0029 \\
\hline Final R indices [I>2sigma(I)] & $R 1=0.0481, w R 2=0.1395$ \\
\hline $\mathrm{R}$ indices (all data) & $R 1=0.0488, w R 2=0.1405$ \\
\hline Largest diff. peak and hole & 0.47 and -0.23 e. $\AA^{-3}$ \\
\hline
\end{tabular}




\begin{tabular}{|c|c|}
\hline \multicolumn{2}{|c|}{ Key data and refinement for compound $41 \mathrm{~b}$} \\
\hline Empirical formula & $\mathrm{C}_{17} \mathrm{H}_{24} \mathrm{O}_{3}$ \\
\hline Formula weight & 276.38 \\
\hline Temperature & $150 \mathrm{~K}$ \\
\hline Wavelength & $1.54180 \AA$ \\
\hline Crystal system & Monoclinic \\
\hline Space group & P 21 \\
\hline \multirow[t]{3}{*}{ Unit cell dimensions } & $a=6.6786(1) \AA \alpha=90^{\circ}$ \\
\hline & $b=7.2868(2) \AA ̊ \Omega=97.140(2)^{\circ}$ \\
\hline & $c=15.4818(3) \AA ̊=90^{\circ}$ \\
\hline Volume & $747.59(3) \AA^{3}$ \\
\hline Z & 2 \\
\hline Density (calculated) & $1.228 \mathrm{Mg} / \mathrm{m}^{3}$ \\
\hline Absorption coefficient & $0.657 \mathrm{~mm}^{-1}$ \\
\hline$F(000)$ & 300 \\
\hline Crystal size & $0.34 \times 0.21 \times 0.03 \mathrm{~mm}^{3}$ \\
\hline Theta range for data collected & 5.760 to $76.129^{\circ}$ \\
\hline Index ranges & $-8<=\mathrm{h}<=8,-9<=\mathrm{K}<=9,-19<=\mathrm{l}<=19$ \\
\hline Reflections collected & 22676 \\
\hline Independent reflections & $3068[\mathrm{R}(\mathrm{int})=0.027]$ \\
\hline Completeness to theta $=75.061^{\circ}$ & $99.6 \%$ \\
\hline Absorption correction & Semi-empirical from equivalents \\
\hline Max. and min. transmission & 0.98 and 0.80 \\
\hline Refinement method & Full-matrix least squares on $\mathrm{F} 2$ \\
\hline Data/restraint/parameters & $3058 / 1 / 182$ \\
\hline Goodness-of-fit on $F^{2}$ & 1.0022 \\
\hline Final $R$ indices [I>2sigma(I)] & $\mathrm{R} 1=0.0329, w R 2=0.0903$ \\
\hline $\mathrm{R}$ indices (all data) & $\mathrm{R} 1=0.0339, \mathrm{wR} 2=0.0916$ \\
\hline Absolute structure parameter & $-0.06(16)$ \\
\hline Largest diff. peak and hole & 0.22 and -0.14 e. $\AA^{-3}$ \\
\hline
\end{tabular}


A6: Example HPLC Traces

(-)-(S)-3-(4-Phenylbutyl)cyclohexanone (2b)
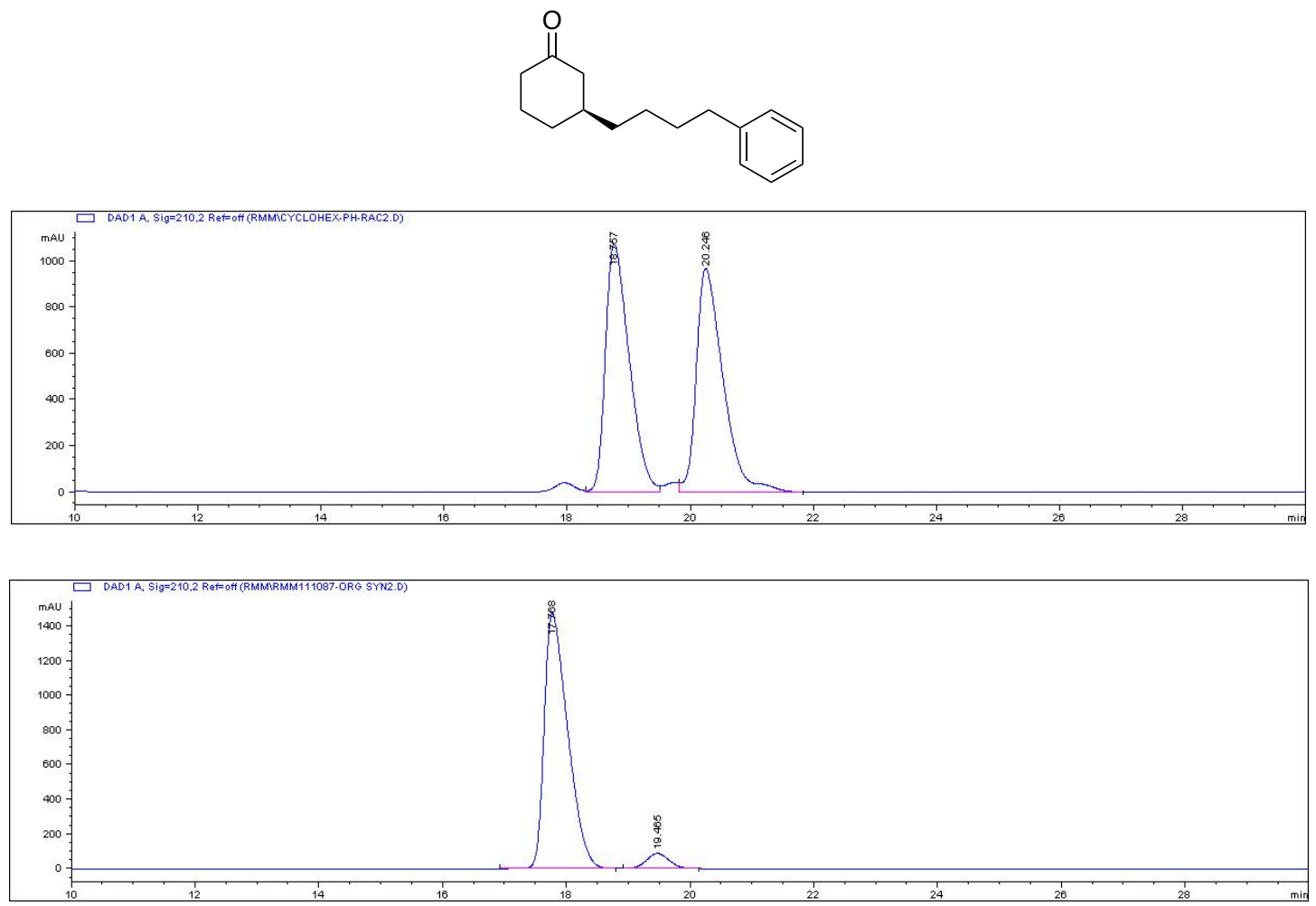

(-)-(R)-4,4-Dimethyl-3-(4-phenylbutyl)cyclohexanone (15a)
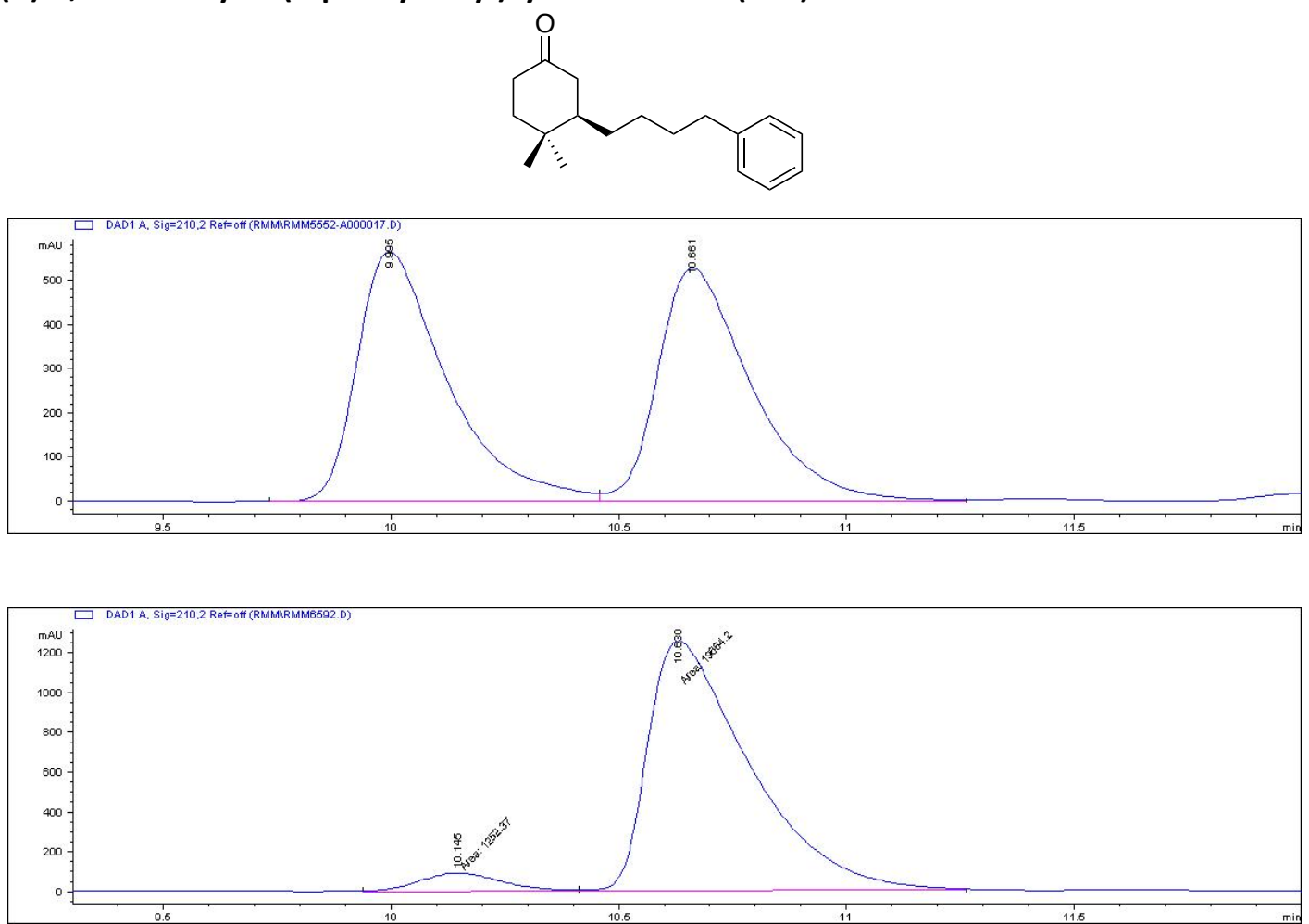